\title{
C-H Activation by Ruthenium(II), Cobalt(III) and Manganese(I) Catalysis
}

\author{
Dissertation \\ for the award of the degree \\ "Doctor rerum naturalium" \\ of the Georg-August-Universität Göttingen

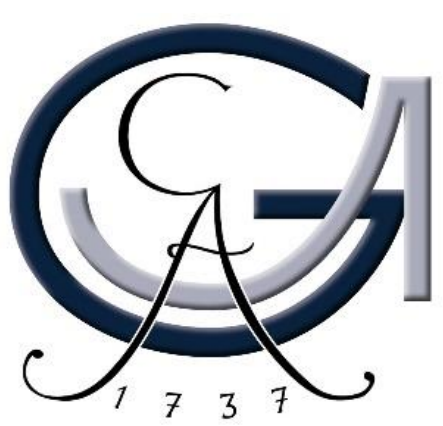

within the doctoral program of chemistry

of the Georg-August-University School of Science (GAUSS)

submitted by

Daniel Zell

from Bad Karlshafen

Göttingen, 2017 



\section{Thesis Committee}

Prof. Dr. Lutz Ackermann, Institute of Organic and Biomolecular Chemistry

PD Dr. Alexander Breder, Institute of Organic and Biomolecular Chemistry

\section{Members of the Examination Board}

Reviewer: Prof. Dr. Lutz Ackermann, Institute of Organic and Biomolecular Chemistry

Second Reviewer: PD Dr. Alexander Breder, Institute of Organic and Biomolecular Chemistry

\section{Further Members of the Examination Board}

Prof. Dr. Konrad Koszinowski, Institute of Organic and Biomolecular Chemistry

Prof. Dr. Dietmar Stalke, Institute of Inorganic Chemistry

Dr. Shoubik Das, Institute of Organic and Biomolecular Chemistry

Dr. Franziska Thomas, Institute of Organic and Biomolecular Chemistry

Date of the Oral Examination: 04.07.2017 



\section{Contents}

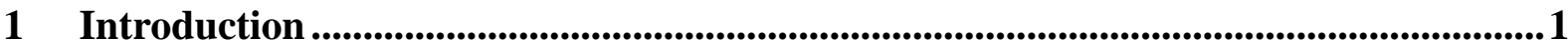

1.1 Transition Metal-Catalyzed C-H Activation .................................................................... 1

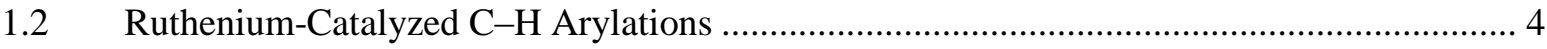

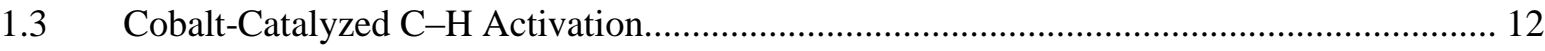

1.3.1 Cobalt(III)-Catalyzed C-H Functionalization................................................................. 13

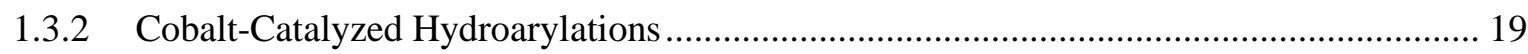

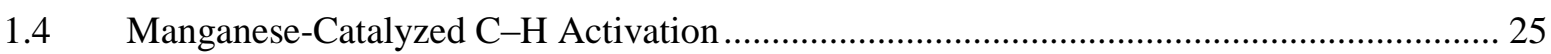

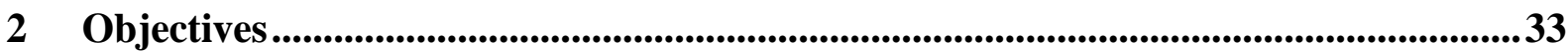

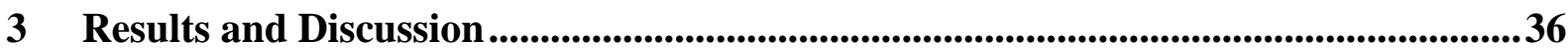

3.1 C-H Arylations by Ruthenium(II)-Phosphinous Acid Catalysts ....................................... 36

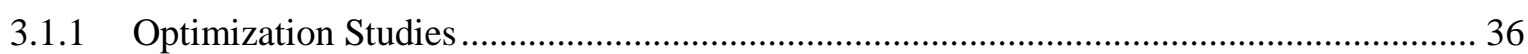

3.1.2 Scope of the C-H Arylation with Aryl Bromides .............................................................. 38

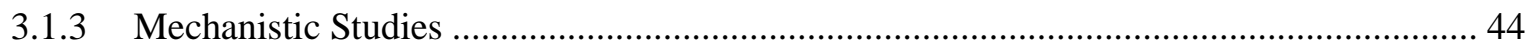

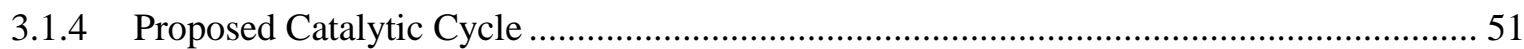

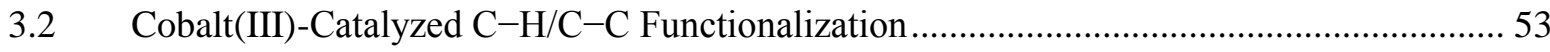

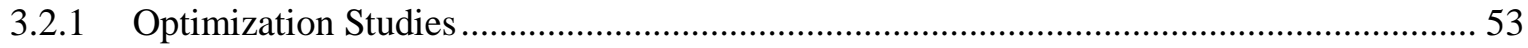

3.2.2 Scope of the Cobalt(III)-Catalyzed C-H/C-C Functionalization ....................................... 55

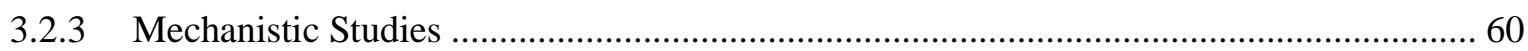

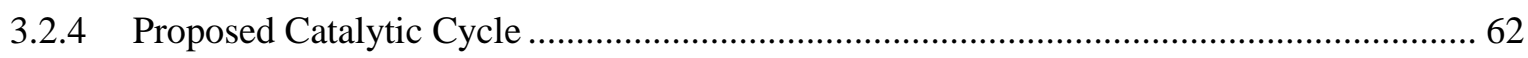

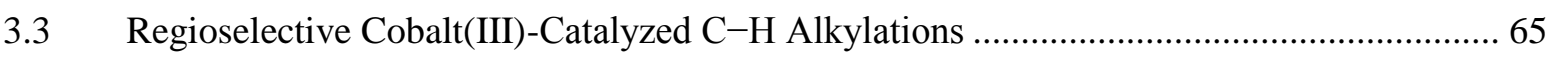

3.3.1 Optimization Studies for the Linear-Selective C-H Alkylation........................................... 65

3.3.2 Optimization Studies for the Branched-Selective C-H Alkylation....................................... 66

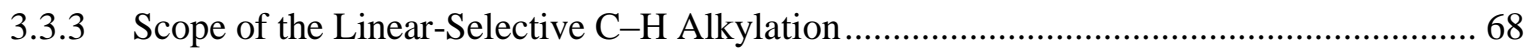

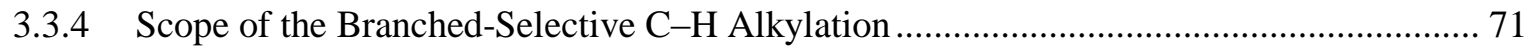

3.3.5 Enantioselective Cobalt(III)-Catalyzed C-H Hydroarylations ........................................... 74

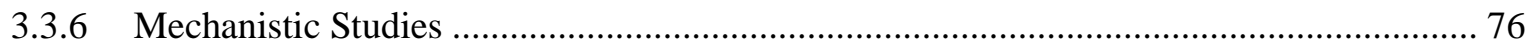

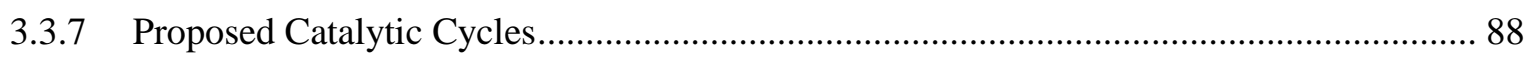

3.4 Cobalt(III)-Catalyzed Allylative and Alkenylative C-H/C-F Functionalizations ................ 93

3.4.1 Optimization Studies for the Allylative C-H/C-F Functionalizations................................. 93

3.4.2 Optimization Studies for the Alkenylative C-H/C-F Functionalization ............................ 96

3.4.3 Scope of the Allylative Cobalt(III)-Catalyzed C-H/C-F Functionalization ....................... 97

3.4.4 Scope of the Alkenylative Cobalt(III)-Catalyzed C-H/C-F Functionalization .................. 99

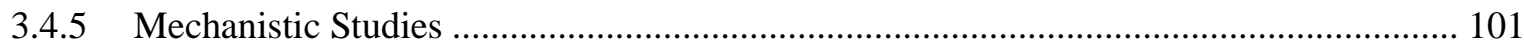




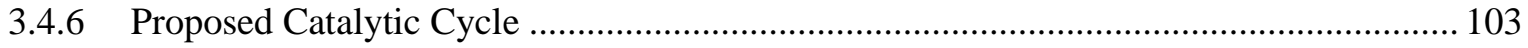

3.5 Manganese(I)-Catalyzed Allylative C-H/C-F Funtionalizations........................................ 105

3.5.1 Optimization Studies for Indoles as Substrates ................................................................. 105

3.5.2 Optimization Studies for Ketimines as Substrates ………............................................... 106

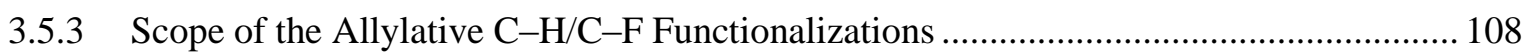

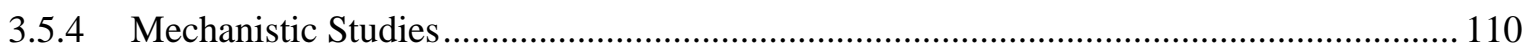

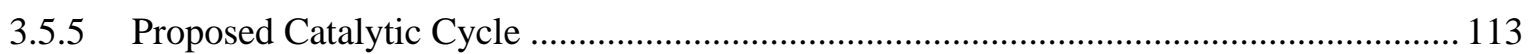

$4 \quad$ Summary and Outlook ........................................................................................................ 115

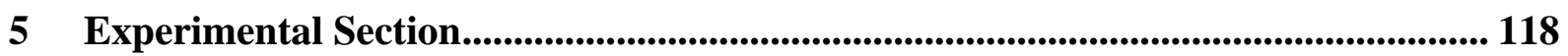

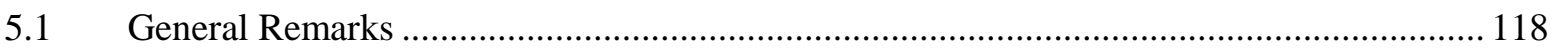

5.1.1 General Procedure A: Ruthenium(II)-PA-Catalyzed C-H Arylation .................................. 121

5.1.2 General Procedure B: Cobalt(III)-Catalyzed C-H/C-C Functionalization........................ 121

5.1.3 General Procedure C: Rhodium(III)-Catalyzed C-H/C-C Functionalization ................... 121

5.1.4 General Procedure D: Cobalt(III)-Catalyzed Linear-selective C-H Alkylation ............... 121

5.1.5 General Procedure E: Cobalt(III)-Catalyzed Branched-Selective C-H Alkylation .......... 121

5.1.6 General Procedure F: Cobalt(III)-Catalyzed Allylative C-H/C-F Functionalization...... 122

5.1.7 General Procedure G: Cobalt(III)-Catalyzed Alkenylative C-H/C-F Functionalization 122

5.1.8 General Procedure H: Manganese(I)-Catalyzed Allylative C-H/C-F Functionalization 122

5.2 Ruthenium(II)-PA-Catalyzed C-H Arylation of (E)-2-Styrylpyridines .................................. 123

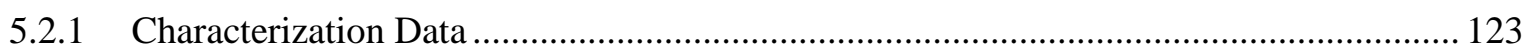

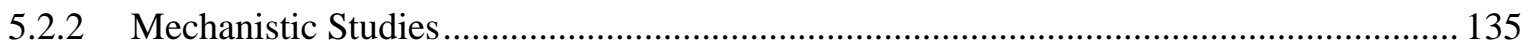

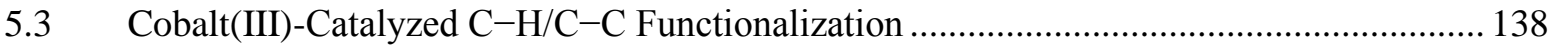

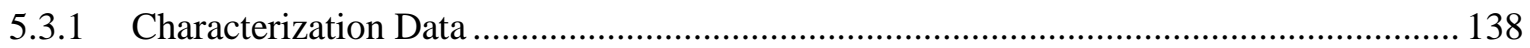

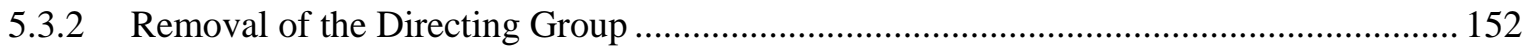

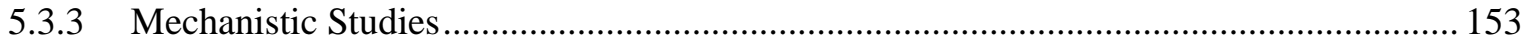

$5.4 \quad$ Regioselective Cobalt(III)-Catalyzed C-H Alkylations..................................................... 157

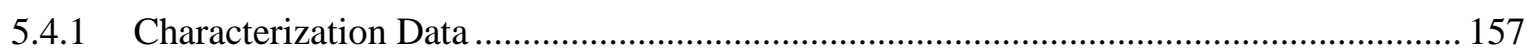

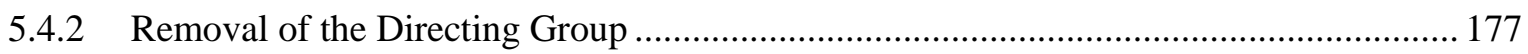

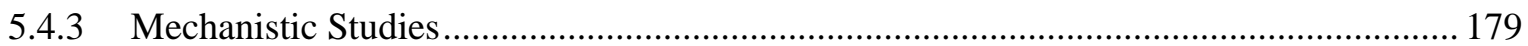

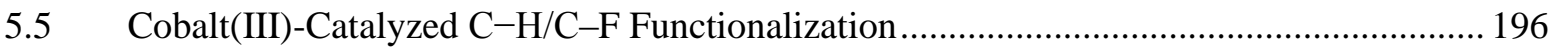

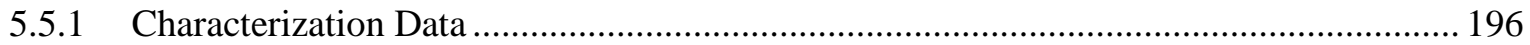

5.5.2 Mechanistic Studies for the Allylative Cobalt(III)-Catalyzed C-H/C-F Activation ....... 208

5.6 Manganese(I)-Catalyzed Allylative C-H/C-F Functionalization ....................................... 212

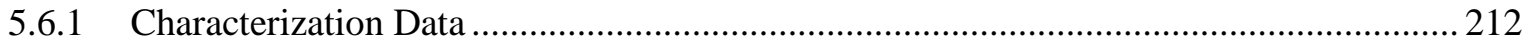

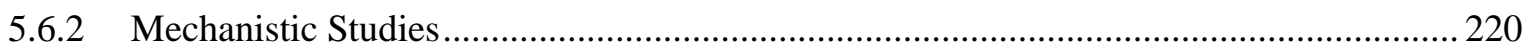

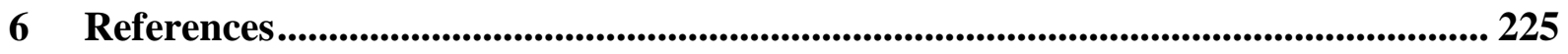




\section{List of Abbreviations}

\begin{tabular}{|c|c|}
\hline Ac & acetyl \\
\hline acac & acetyl acetonate \\
\hline Ad & adamantyl \\
\hline Alk & alkyl \\
\hline AMLA & ambiphilic metal-ligand activation \\
\hline aq. & aqueous \\
\hline $\mathrm{Ar}$ & aryl \\
\hline atm & atmospheric pressure \\
\hline ATR & attenuated total reflectance \\
\hline BHT & 2,6-di-tert-butyl-4-methylphenol \\
\hline BIES & base-assisted internal electrophilic substitution \\
\hline BINAP & 2,2'-bis(diphenylphosphino)-1,1'-binaphthyl \\
\hline $\mathrm{Bn}$ & benzyl \\
\hline Boc & tert-butyloxycarbonyl \\
\hline $\mathrm{Bu}$ & butyl \\
\hline $\mathrm{Bz}$ & benzoyl \\
\hline calc. & calculated \\
\hline cat. & catalytic \\
\hline$c f$. & confer \\
\hline CMD & concerted metalation deprotonation \\
\hline $\operatorname{cod}$ & 1,5-cyclooctadiene \\
\hline conv. & conversion \\
\hline $\mathrm{Cp}$ & cyclopentadienyl \\
\hline $\mathrm{Cp}^{*}$ & pentamethylcyclopentadienyl \\
\hline Cy & cyclohexyl \\
\hline$\delta$ & chemical shift \\
\hline $\mathrm{d}$ & doublet \\
\hline DCE & 1,2-dichloroethane \\
\hline DCM & dichloromethane \\
\hline dd & doublet of doublet \\
\hline DFT & density functional theory \\
\hline DG & directing group \\
\hline DMA & $N, N$-dimethylacetamide \\
\hline DME & 1,2-dimethoxyethane \\
\hline DMF & $\mathrm{N}, \mathrm{N}$-dimethylformamide \\
\hline DMSO & dimethyl sulfoxide \\
\hline DMPU & 1,3-dimethyl-3,4,5,6-tetrahydro-2( $1 H)$-pyrimidinone \\
\hline DPPH & 2,2-diphenyl-1-picrylhydrazyl \\
\hline dt & doublet of triplet \\
\hline $\mathrm{E}$ & electron-withdrawing group \\
\hline Ed. & edition \\
\hline
\end{tabular}


$e e$

e.g.

EI

equiv

ESI

Et

FG

$\mathrm{g}$

GC

GPC

$\mathrm{h}$

Hal

Het

Hept

Hex

HFIP

HPLC

HR-MS

$\mathrm{Hz}$

$i$

IES

IMes

IR

$J$

KIE

L

LDA

LiHMDS

LLHT

$m$

$\mathrm{m}$

M

$[\mathrm{M}]^{+}$

$\mathrm{Me}$

Mes

$\mathrm{mg}$

$\mathrm{MHz}$

min

$\mathrm{mL}$

mmol

M. p.

MS

MTBE

$\mathrm{m} / \mathrm{z}$

NCFS

NCTS

NHC

NMP enantiomeric excess

exempli gratia

electron ionization

equivalent

electronspray ionization

ethyl

functional group

gram

gas chromatography

gel permeation chromatography

hour

halogen

hetero(aryl)

heptyl

hexyl

1,1,1,3,3,3-hexafluoro-2-propanol

high performance liquid chromatography

high resolution mass spectrometry

Hertz

iso

internal electrophilic substitution

1,3-bis(2,4,6-trimethylphenyl)imidazol-2-ylidene

infrared spectroscopy

coupling constant

kinetic isotope effect

ligand

lithium diisopropylamide

lithium bis(trimethylsilyl)amide

ligand-to-ligand hydrogen transfer

meta

multiplet

molar

molecular ion peak

methyl

mesityl

milligram

megahertz

minute

milliliter

millimol

melting point

mass spectrometry

methyl tert-butyl ether

mass-to-charge ratio

$N$-cyano- $N$-(4-fluorophenyl)-4-methylbenzenesulfonamide

$N$-cyano-4-methyl- $N$-phenyl benzenesulfonamide

$\mathrm{N}$-heterocyclic carbene

$N$-methylpyrrolidinone 


\begin{tabular}{ll} 
NMR & nuclear magnetic resonance \\
$o$ & ortho \\
OPV & oil pump vacuum \\
$p$ & para \\
PA & phosphinous acid \\
pent & pentyl \\
Ph & phenyl \\
PMP & para-methoxyphenyl \\
Piv & pivaloyl \\
ppm & parts per million \\
Pr & propyl \\
Py & pyridyl \\
q & quartet \\
ref. & reference \\
r.r. & regioisomeric ratio \\
RT & room temperature \\
s & singlet and second \\
sat. & saturated \\
SET & single electron transfer \\
SPO & secondary phosphine oxide \\
SPS & solvent purification system \\
$t$ & tert \\
t & triplet \\
$T$ & temperature \\
TEMPO & $2,2,6,6$-tetramethylpiperidine- $N$-oxide \\
Tf & trifluoromethanesulfonyl \\
TFE & $2,2,2$-Trifluoroethanol \\
TFA & trifluoroacetic acid \\
THF & tetrahydrofuran \\
TLC & thin layer chromatography \\
TM & transition metal \\
TMP & $3,4,5$-trimethoxyphenyl \\
TMS & trimethylsilyl \\
Ts & para-toluenesulfonyl \\
TS & transition state \\
wt $\%$ & weight percent \\
X & (pseudo)halide \\
& \\
\hline &
\end{tabular}







\section{Introduction}

\subsection{Transition Metal-Catalyzed C-H Activation}

The past five decades have witnessed prosperous advances in the development of highly efficient transition metal-catalyzed $\mathrm{C}-\mathrm{C} / \mathrm{C}-\mathrm{Het}$ bond forming processes that tremendously expanded the toolbox of modern organic synthesis. Thus far, predominantly transition metal-catalyzed cross coupling reactions, such as Hiyama- ${ }^{[1]}$ Kumada-Corriu-,${ }^{[2]}$ Mizoroki-Heck- $-{ }^{[3]}$ Negishi-,${ }^{[4]}$ Sonogashira-,${ }^{[5]}$ Stille-,${ }^{[6]}$ and Suzuki-Miyaura-couplings, ${ }^{[7]}$ have found widespread applications in agrochemical and pharmaceutical industry and streamlined the total synthesis of complex natural products. ${ }^{[8]}$ Consequently, these significant innovations culminated in the Nobel Prize for Chemistry in 2010 for Heck, Negishi and Suzuki.

Despite this enormous progress, cross-coupling reactions are associated with a series of drawbacks like prefunctionalization to often sensitive starting materials and the generation of toxic by-products (Figure 1). ${ }^{[9]}$ However, climate change and severe environmental pollution due to chemicals have created a strong demand for atom- ${ }^{[10]}$ and step-economical ${ }^{[11]}$ processes, giving rise to the concept of green chemistry. ${ }^{[12]}$

In striking contrast, $\mathrm{C}-\mathrm{H}$ bond functionalizations ${ }^{[13]}$ have become an invaluable and powerful strategy to achieve synthetic versatility in excellent atom- and step-economies by obviating typical prefunctionalization steps and directly functionalizing $\mathrm{C}-\mathrm{H}$ bonds.

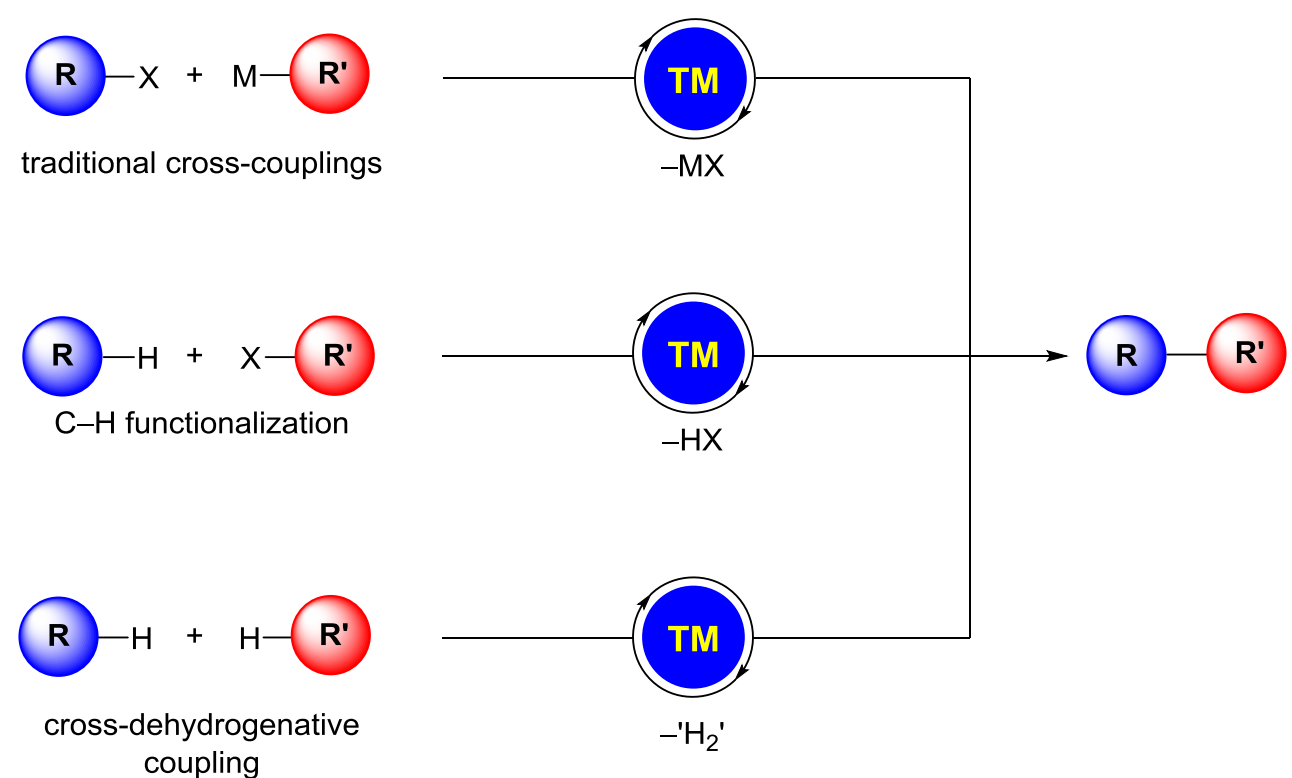

Figure 1. Comparison of general methods for $\mathrm{C}-\mathrm{C} / \mathrm{C}-\mathrm{Het}$ bond formation.

Nonetheless, environmentally benign and sustainable transition metal-catalyzed transformations should not only match substrate availability: the use of earth-abundant $3 \mathrm{~d}$ base and particularly costeffective metals is also of prime importance. Thus, a boost in the development of inter alia 
manganese- ${ }^{[14]}$ and cobalt-catalyzed ${ }^{[15]} \mathrm{C}-\mathrm{H}$ functionalizations has revolutionized sustainable organic synthesis.

The most important basis for the development of new efficient metal-catalyzed $\mathrm{C}-\mathrm{H}$ functionalizations is a detailed mechanistic understanding. Consequently, considerable efforts have been made to elucidate several mechanistically distinct pathways for the crucial C-H activation step (Scheme 1). ${ }^{[16]}$ In this respect, a) oxidative addition was observed for late metals in low oxidation states. Furthermore, for e.g. alkylidene-, amide-, imide-, or alkoxide complexes of early as well as late transition metals, b) 1,2 addition to the $\mathrm{C}-\mathrm{H}$ bond is favored via formal $\left[2_{\sigma}+2_{\pi}\right]$ reaction. ${ }^{[17]}$ In addition, c) an electrophilic substitution mechanism was observed for inter alia $\mathrm{Pd}^{2+}$ and $\mathrm{Pt}^{2+}$ in polar media and involves the electrophilic attack of the metal to carbon. ${ }^{[18]}$ For inter alia actinides, lanthanides, and early transition metals which are not prone to oxidations state changes d) $\sigma$-bond metathesis is a commonly proposed pathway. ${ }^{[16 c]}$ Closely related to $\sigma$-bond metathesis, Periana/Goddard ${ }^{[19]}$ coined the term internal electrophilic substitution (IES) for mechanisms involving electrophilic attack of the metal and deprotonation by e.g. alkoxy-ligands via a four-membered transition state.

a) oxidative addition

b) 1,2-addition
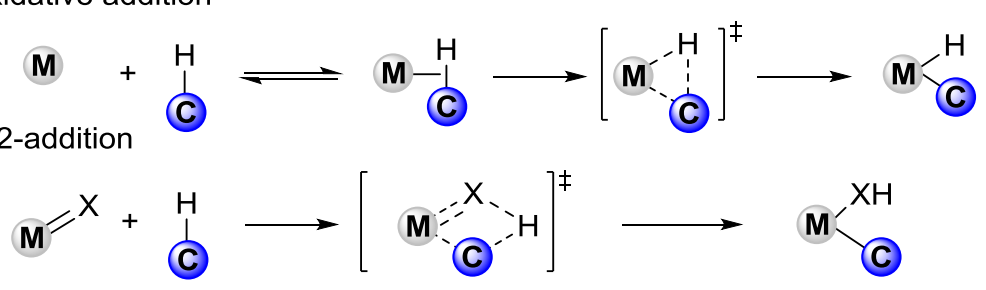

c) electrophilic substitution

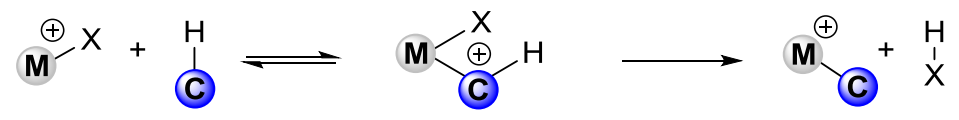

d) $\sigma$-bond metathesis

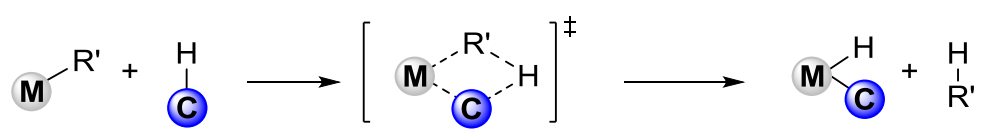

e) internal electrophilic substitution (IES)

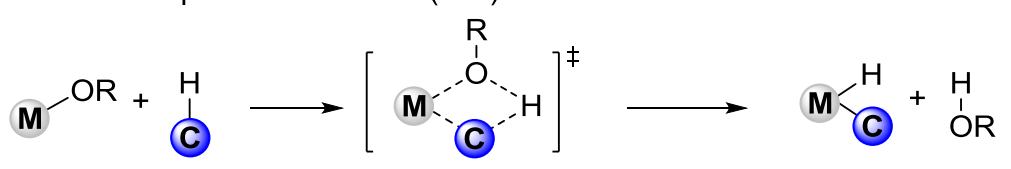

Scheme 1. Common mechanistic pathways for $\mathrm{C}-\mathrm{H}$ activation.

In contrast to the above mentioned mechanistic scenarios, numerous reports have highlighted baseassisted C-H metalation events (Scheme 2). ${ }^{[20]}$ While Fagnou/Gorelsky ${ }^{[20 c]}$ have coined the term CMD (concerted metalation deprotonation), computational studies by MacGregor/Davies ${ }^{[21]}$ revealed an agostic metal-hydrogen interaction, being rationalized by the term ambiphilic metal-ligand activation (AMLA). In both mechanisms, the metalation and deprotonation steps proceed in a concerted fashion via a six-membered transition state (Scheme 2a). Additionally, typical experimental characteristics of 
CMD-type $\mathrm{C}-\mathrm{H}$ functionalizations are often high kinetic isotope effects (KIEs) and a clear preference for acidic $\mathrm{C}-\mathrm{H}$ bonds. ${ }^{[22]}$

Nevertheless, in recent years several reports have emphasized a significant rate acceleration of electrophilic substitution type $\mathrm{C}-\mathrm{H}$ activations by bifunctional acetate or carboxylate additives, which are suggested to proceed via a six-membered transition state. Based on detailed mechanistic studies, this manifold was termed base-assisted internal electrophilic substitution (BIES) (Scheme 2b). Thus, intermolecular competition experiments have revealed a preference for more electron-rich substrates and typically low KIEs have been observed which clearly underline the unique mechanism of this pathway. Hence, in contrast to CMD-type $\mathrm{C}-\mathrm{H}$ activation, the site-selectivity of BIES-type $\mathrm{C}-\mathrm{H}$ transformations was not governed by kinetic $\mathrm{C}-\mathrm{H}$ acidity. ${ }^{[23]}$

a) CMD/AMLA

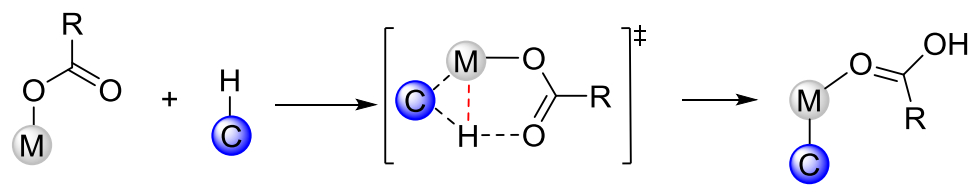

b) BIES

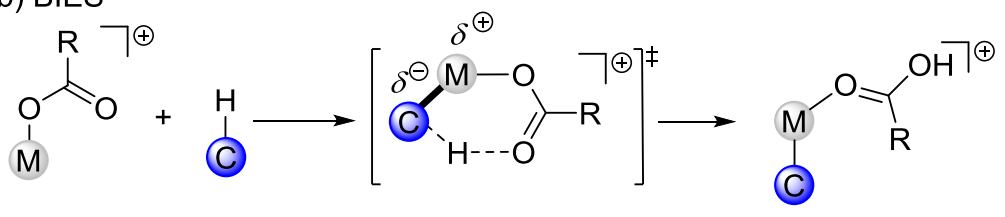

Scheme 2. Base-assisted C-H activation mechanisms.

Moreover, a major challenge in $\mathrm{C}-\mathrm{H}$ functionalizations includes achieving regioselectivity in complex molecules which display $\mathrm{C}-\mathrm{H}$ bonds with similar dissociation energies. Besides the preference for the activation of somewhat more acidic positions e.g. in azoles, a strategy involving Lewis basic functionalities, which direct the transition metal into close proximity, has been well established in the field of $\mathrm{C}-\mathrm{H}$ activation (Scheme 3).
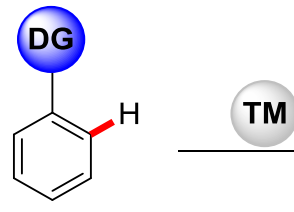

Scheme 3. Regioselective $\mathrm{C}-\mathrm{H}$ activation by directing groups.

In recent years, numerous efforts have been made to explore weak, ${ }^{[24]}$ modifiable or even removable directing groups (Figure 2), ${ }^{[25]}$ which have overall strongly contributed to the versatility of $\mathrm{C}-\mathrm{H}$ functionalizations and their impact on sustainable organic synthesis. 


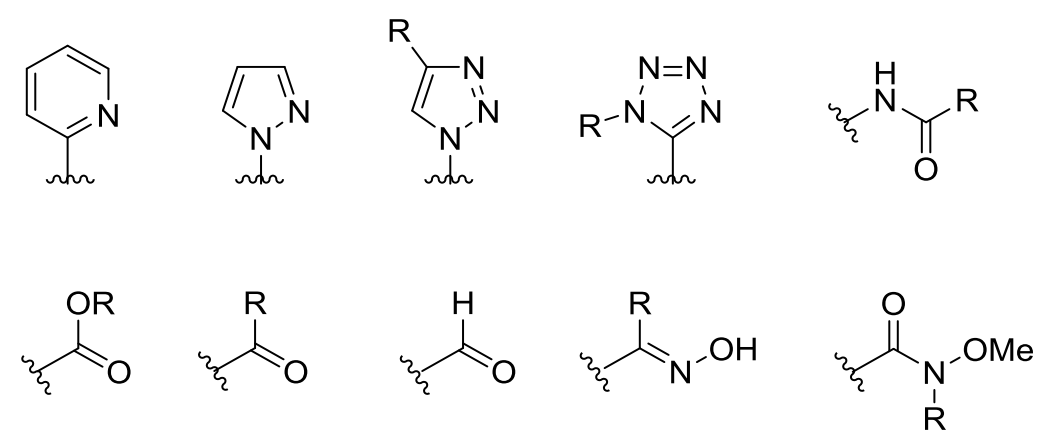

Figure 2. Selected examples of important directing groups in $\mathrm{C}-\mathrm{H}$ activation catalysis.

\subsection{Ruthenium-Catalyzed C-H Arylations}

The development of cost-effective methods for the preparation of biaryls has gained tremendous interest in the past decades since biaryls are common structural motifs in inter alia agrochemically and pharmaceutically relevant molecules. In addition, there is a lack of generally applicable classical methods for their synthesis. ${ }^{[26]}$

Thus far, a number of synthetic methods have been developed for the synthesis of biaryls, with crosscoupling reactions being particularly powerful. ${ }^{[8]}$ As mentioned above, a more sustainable strategy to accomplish $\mathrm{C}-\mathrm{C}$ bond formations involves the direct $\mathrm{C}-\mathrm{H}$ functionalization by cost-effective metals, such as ruthenium. Until now, ruthenium complexes have been employed for numerous $\mathrm{C}-\mathrm{H}$ transformations, including $\mathrm{C}-\mathrm{C}$, but also a wealth of $\mathrm{C}-\mathrm{Het}$ bond formations. ${ }^{[26 \mathrm{~b}, 27]}$

As early as 2001, the first ruthenium-catalyzed $\mathrm{C}-\mathrm{H}$ arylation was reported by Oi/Inoue, who used the dimeric $\left[\mathrm{RuCl}_{2} \text { (benzene) }\right]_{2}$ complex and triphenylphosphine as the crucial additive for the orthoselective $\mathrm{C}-\mathrm{H}$ arylation of 2-phenylpyridine (1a) with aryl bromides 2 (Scheme 4). Remarkably, their studies already revealed that ruthenium(II) catalysts are able to undergo oxidative addition with challenging aryl chlorides 3 as well as aryl triflates $4{ }^{[28]}$ In this regard, the authors disclosed the following reactivity order for the mono-arylation of 2-phenylpyridine (1a): $\mathrm{PhCl}<\mathrm{PhOTf}<\mathrm{PhI}$ $<\mathrm{PhBr}$.

Interestingly, a series of additives, including phosphines, phosphites, and bidendate diphosphines rendered phosphines as the optimal choice. It is noteworthy that the di-arylated product 5aa' was obtained solely by drastically increasing the amount of the aryl bromide 2 . 

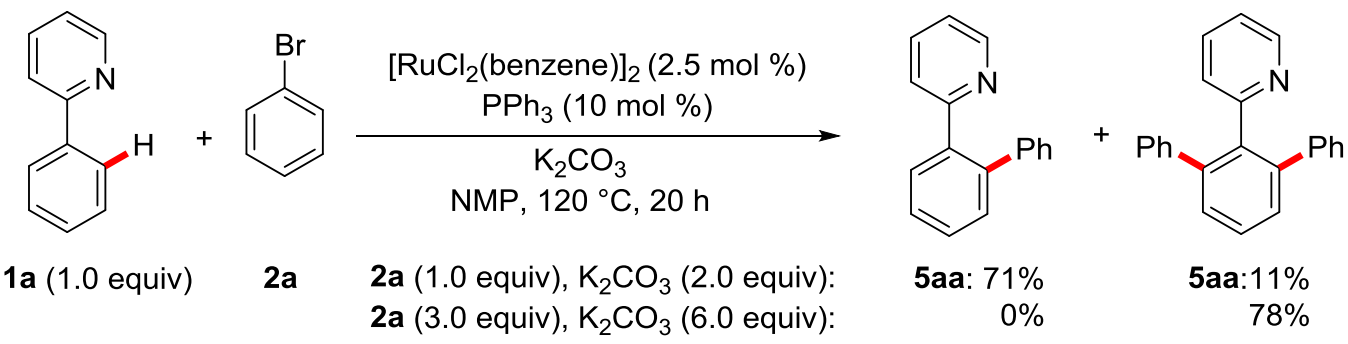

5aa: $71 \%$

$0 \%$

5aa: $11 \%$

$78 \%$

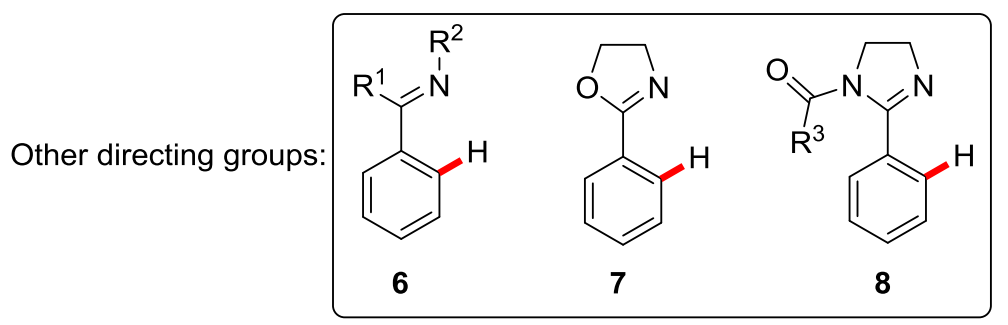

Scheme 4. Ruthenium(II)-catalyzed C-H arylation.

Further studies by Oi/Inoue and coworkers ${ }^{[29]}$ have extended the applicability of these arylation methods to valuable directing groups, such as ketimines $\mathbf{6},{ }^{[30]}$ oxazolines $\mathbf{7}$, and imidazolines $\mathbf{8} .{ }^{[31]}$ Remarkably, the authors accomplished a highly Z-selective ruthenium(II)-catalyzed arylation of $(E)$-2styrylpyridines 9, which displayed a wide substrate scope of arylated (E)-2-styrylpyridines 10 (Scheme 5). ${ }^{[32]}$ Nevertheless, the arylation of 2-vinylpyridines 11 was not achieved and there was no example for acyclic tetrasubstituted alkenes 11. However, the reaction system of Oi/Inoue was later shown to be irreproducible due to impurities in the solvent NMP. ${ }^{[33]}$<smiles>[R]C=Cc1ccccn1</smiles>

9<smiles>C(=C(c1ccccc1)c1ccccn1)c1ccccc1</smiles>

10a: $100 \%$

2a

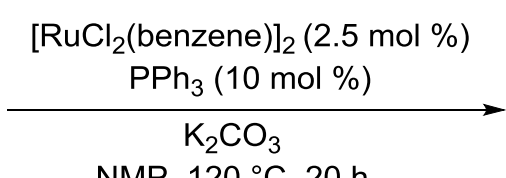

NMP, $120^{\circ} \mathrm{C}, 20 \mathrm{~h}$<smiles>[R]/C(=C/c1ccccn1)c1ccccc1</smiles>

10<smiles>C(=C/c1ccccn1)\c1ccccc1</smiles>

10d: $0 \%$

Scheme 5. Ruthenium(II)-catalyzed arylation of alkenes (E)-styrylpyridines 9.

At around the same time, Kakiuchi and coworkers accomplished a ruthenium(0)-catalyzed $\mathrm{C}-\mathrm{H}$ arylations with arylboronates $\mathbf{1 2}$ as the arylating reagents, utilizing valuable ketones $\mathbf{1 3}$ as the directing group (Scheme 6a). ${ }^{[34]}$ The authors proposed that the ruthenium complex first undergoes oxidative addition with the $\mathrm{C}-\mathrm{H}$ bond, then transmetalation, and finally reductive elimination occur. However, a major drawback of this reaction is the formation of one equivalent of the corresponding 
benzylic alcohols by reduction of ketone 13. Subsequently, the efficiency of this reaction has been improved by the addition of acetone or pinacolone, which suppressed the reduction and thus avoided excess amounts of the ketone 13a (Scheme 6b).

a)

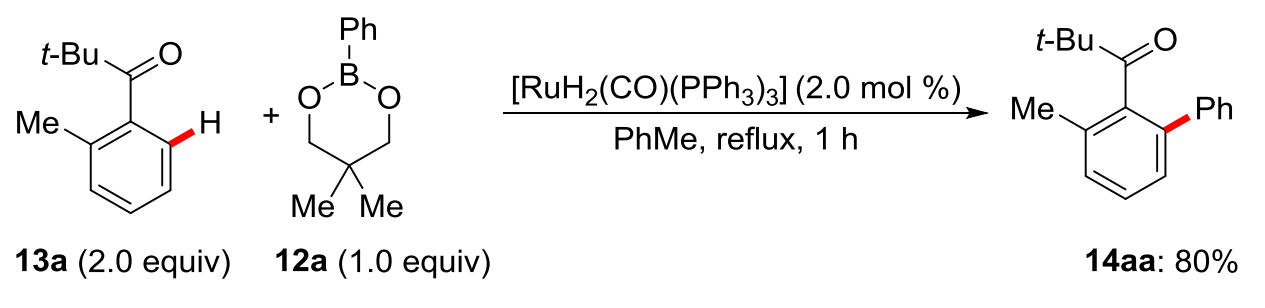

b)

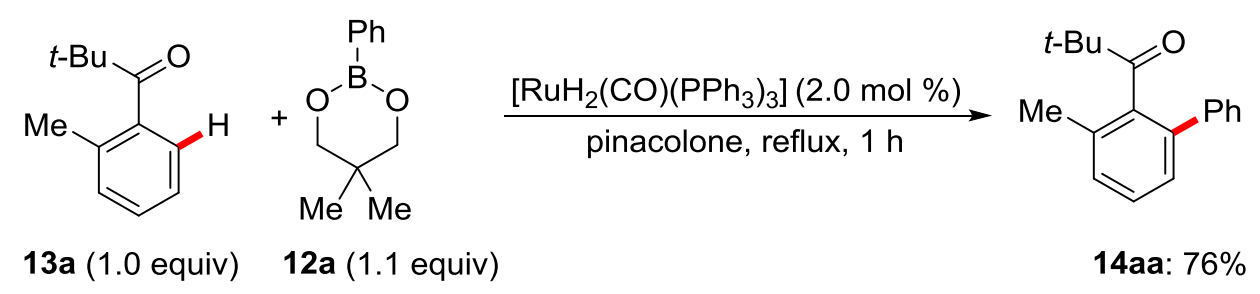

Scheme 6. Ruthenium(0)-catalyzed C-H arylation with arylboronate 12a.

Interestingly, the authors presented compelling evidence for the coordination of the ketone $\mathbf{1 3}$ to the ruthenium-hydride complex, which they inferred from a difference between intra- and intermolecular kinetic isotope effects (KIEs). Under optimal reaction conditions, the authors showed a broad applicability towards electron-donating and electron-withdrawing substituents. Nonetheless, valuable functional groups such as esters, cyano-, chloro-, bromo-, and iodo-substituents not being within the reaction scope represented a major limitation. ${ }^{[35]}$

Despite these undisputed advances, a general and efficient ruthenium-catalyzed arylation using inexpensive aryl chlorides $\mathbf{3}$ had not yet been disclosed at that time, and all protocols were limited to phosphine ligands.

As early as 2005, Ackermann presented a significant breakthrough in ruthenium(II)-catalyzed arylations by employing structurally diverse (heteroatom-substituted) secondary phosphine oxides ${ }^{[36]}$ ((HA)SPOs) as powerful preligands (Scheme 7). 


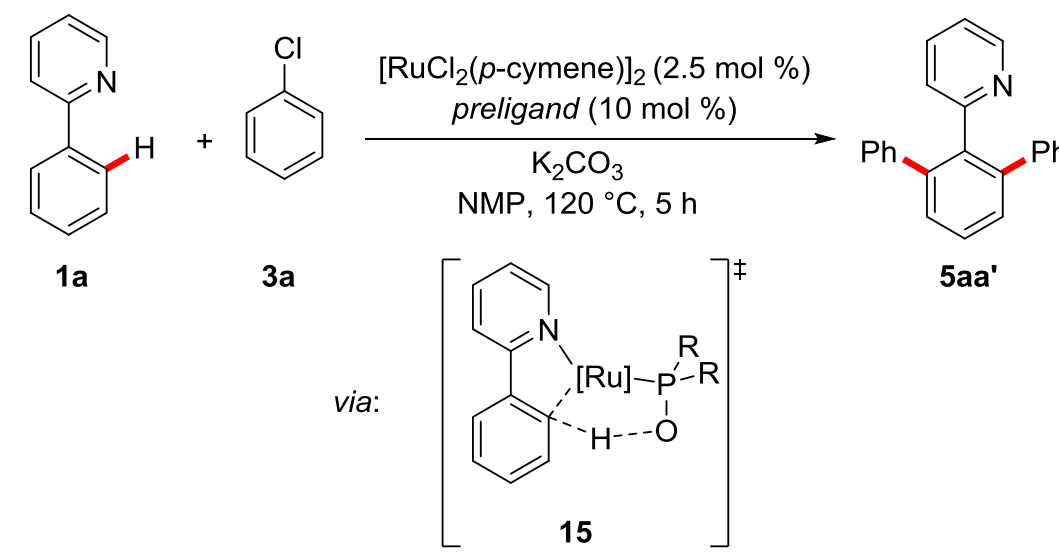

Preligands:

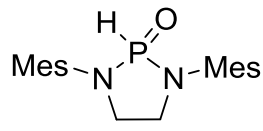

Yield of 5aa':

$61 \%$

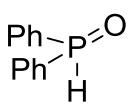

$54 \%$

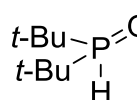

$61 \%$

$$
\begin{gathered}
1-A d-P=0 \\
1-A d^{-}{ }_{1}=0 \\
72 \%(24 h: 98 \%)
\end{gathered}
$$

Scheme 7. Ruthenium(II)-catalyzed C-H arylations using SPOs as additives.

Hence, a large variety of different air-stable SPOs and HASPOs was tested, somewhat counterintuitively rendering the sterically most hindered di-adamantyl-substituted SPO as the optimal additive. Under the optimized reaction conditions a series of valuable functional groups, such as esters, ketones, and nitriles, was well tolerated and, moreover, it was demonstrated that modifiable ketimines $\mathbf{6}$ as the directing group delivered the corresponding mono-arylated products $\mathbf{5}$ in overall higher efficiency than Oi/Inoue had previously achieved by using phosphine additives (vide supra). ${ }^{[30]}$ Thereafter, the mechanistic working mode of the SPO preligands was proposed to be a ruthenium(II)phosphinous acid (PA) cooperative $\mathrm{C}-\mathrm{H}$ activation via a five-membered transition state $\mathbf{1 5}$ (Scheme 7). ${ }^{[36 a]}$

Subsequently, Ackermann and coworkers reported on a (HA)SPO-preligand enabled rutheniumcatalyzed $\mathrm{C}-\mathrm{H}$ arylation with aryl tosylates, highlighting the catalytic system to enable oxidative addition through $\mathrm{C}-\mathrm{O}^{[37]}$ bond cleavage. ${ }^{[36 \mathrm{~b}]}$

Inspired by the successful strategy of applying bifunctional ligands like SPOs, Ackermann and coworkers introduced - for the first time in ruthenium-catalyzed $\mathrm{C}-\mathrm{H}$ functionalizations- carboxylates as additives (Scheme 8$){ }^{[36 a]}$ The mechanistic pathway of the $\mathrm{C}-\mathrm{H}$ activation by carboxylate-assistance was suggested to proceed via a six-membered CMD-type transition state $\mathbf{1 6}$ that had first been proposed by Reutov and coworkers ${ }^{[38]}$ and was thereafter computationally studied by Fagnou/Gorelsky for the palladium-catalyzed C-H activation. ${ }^{\text {[20c, 39] }}$

Hence, a significant rate acceleration by carboxylates as compared to phosphines and phosphates was also observed for the ruthenium(II)-catalyzed $\mathrm{C}-\mathrm{H}$ arylation of triazoles $\mathbf{1 7}$. Notably, triazoles $\mathbf{1 7}$ are valuable directing groups due to their considerable impact on bioorganic chemistry and material sciences. ${ }^{[40]}$ In contrast to previous reports, less polar toluene was successfully employed as the 
solvent, since NMP is hygroscopic and often led to irreproducible results due to carboxylic acid impurities (vide supra). ${ }^{[26 a, 33]}$
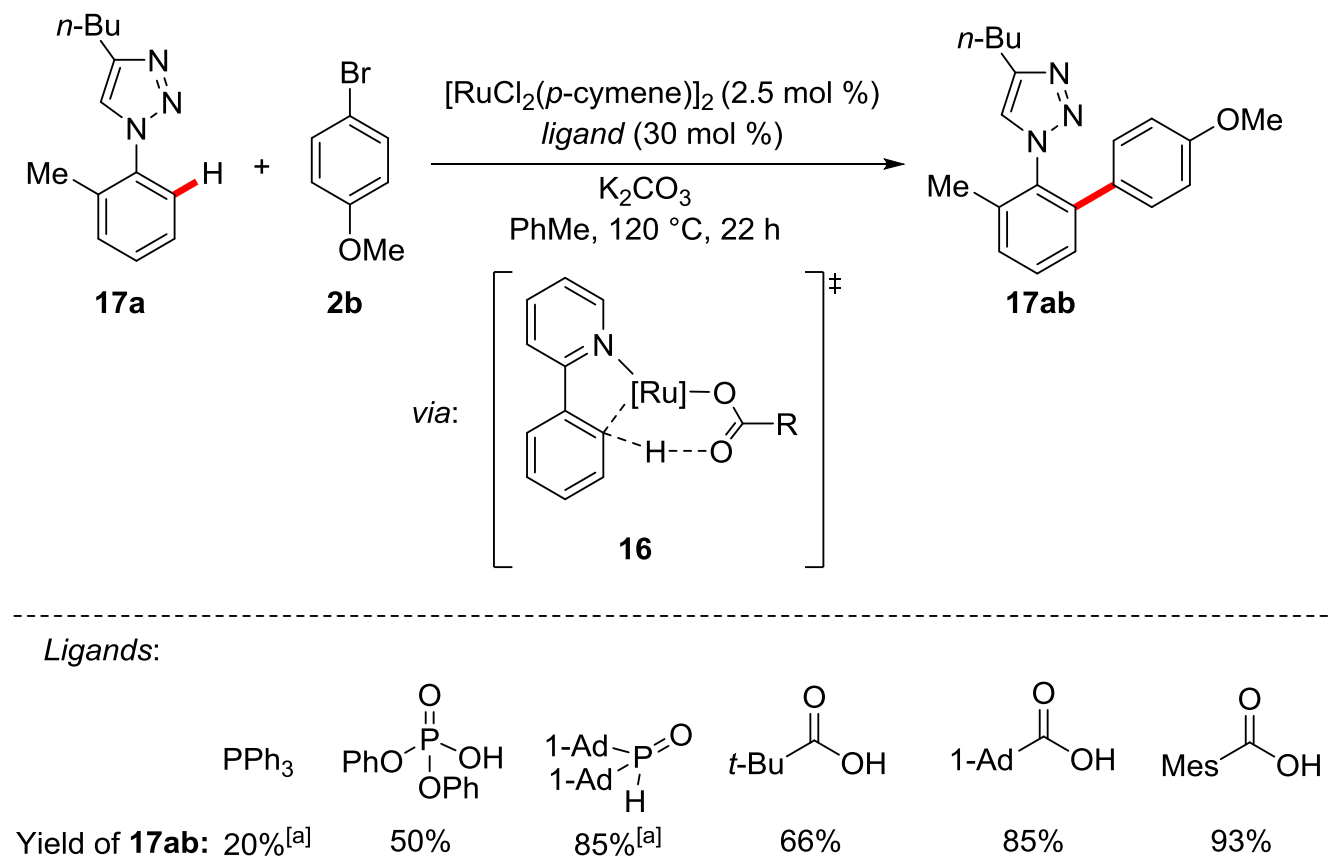

Scheme 8. Ruthenium(II)-catalyzed C-H arylation through carboxylate-assistance. [a] $10 \mathrm{~mol} \%$.

Thereafter, the idea of using carboxylate additives was picked up by Dixneuf and coworkers, as they utilized the well-defined ruthenium(II)-acetate complex $\left[\mathrm{Ru}(\mathrm{OAc})_{2}(p\right.$-cymene $\left.)\right]$ as a competent catalyst for the construction of poly-heterocyclic molecules by $\mathrm{C}-\mathrm{H}$ arylation. ${ }^{[41]}$

The first detailed mechanistic insights for carboxylate-assisted ruthenium-catalyzed $\mathrm{C}-\mathrm{H}$ activations were reported by Ackermann and coworkers in $2010{ }^{[42]}$ The authors achieved the synthesis of a welldefined ruthenium(II)biscarboxylate complex $\left[\mathrm{Ru}\left(\mathrm{O}_{2} \mathrm{CMes}\right)_{2}(p\right.$-cymene $\left.)\right](\mathbf{2 0})$, which displayed an excellent reactivity for the $\mathrm{C}-\mathrm{H}$ arylation of inter alia pyrazoles $\mathbf{1 8}$, triazoles $\mathbf{1 7}$, oxazolines $\mathbf{7}$, imidazoles 19, as well as 2-styrylpyridines $\mathbf{9}$.

Besides these synthetically useful contributions, a set of important mechanistic findings shed light on the reaction mechanism. First, H/D-exchange experiments indicated the $\mathrm{C}-\mathrm{H}$ activation to be reversible (Scheme 9a). Second, the 2-phenylpyridine derivative $\mathbf{1 b}$ was shown to form the ruthenium cyclometalated complex 21 through a stoichiometric reaction with $\left[\mathrm{Ru}\left(\mathrm{O}_{2} \mathrm{CMes}\right)_{2}(p\right.$-cymene) $)$ (20). Third, the complex 21 was demonstrated to be catalytically active and thus delivered the arylated product 5bb (Scheme 9b) in high yield. Finally, various intermolecular competition experiments revealed a clear preference for electron-deficient aryl bromides $\mathbf{2}$, which suggested that the oxidative addition is the turnover-limiting elementary step in this transformation. ${ }^{[42]}$ 
a) H/D-exchange experiment

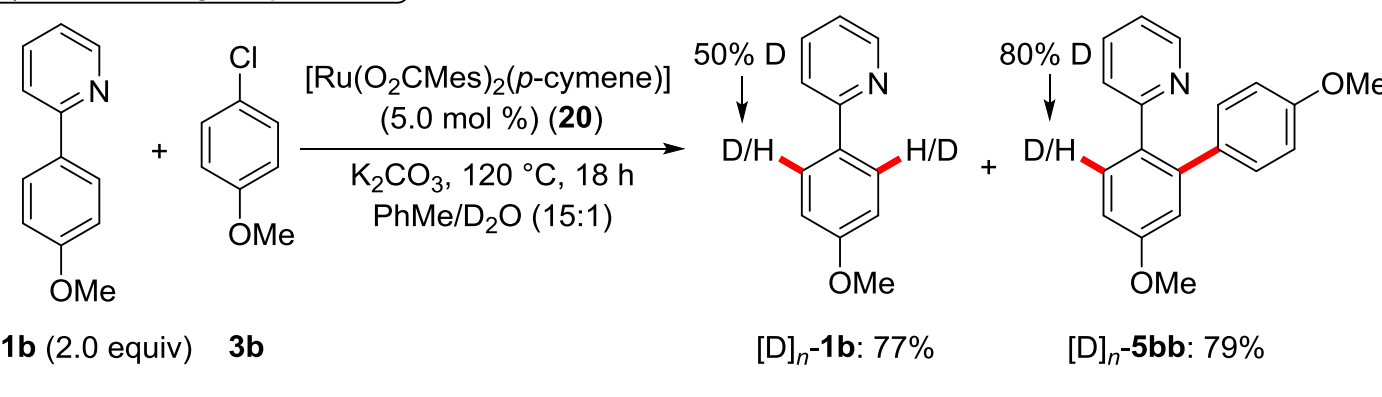

\section{b) isolation of key intermediate 21}<smiles>COc1ccc(-c2ccccn2)cc1</smiles>

1b

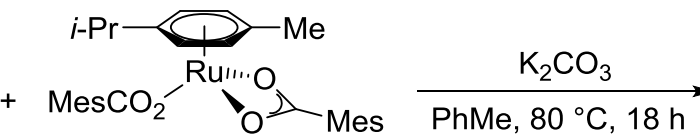

20

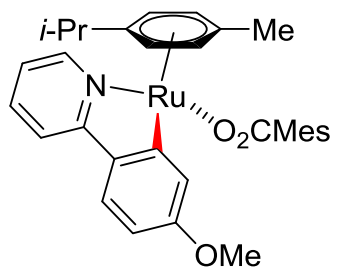

21: $60 \%$<smiles>COc1ccc(-c2ccccn2)cc1</smiles><smiles>COc1ccc(Cl)cc1</smiles>

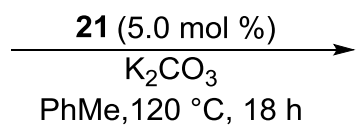

1b (2.0 equiv)<smiles>COc1ccc(-c2cc(OC)ccc2-c2ccccn2)cc1</smiles>

5bb: $78 \%$

Scheme 9. Key mechanistic findings for the carboxylate-assisted $\mathrm{C}-\mathrm{H}$ arylation.

To summarize all these mechanistic results, the authors proposed a general catalytic cycle (Scheme 10), which is initiated by a reversible carboxylate-assisted $\mathrm{C}-\mathrm{H}$ ruthenation via a CMD-type sixmembered transition state 16 to deliver ruthenacycle 23. Subsequently, a turnover-limiting oxidative addition of the aryl halide $\mathbf{2 4}$ to the ruthenium(II) complex $\mathbf{2 3}$ occurs and generates a ruthenium(IV) intermediate 25. Ultimately, a reductive elimination produces the desired arylated product $\mathbf{2 6}$ and regenerates the active ruthenium complex 27. 


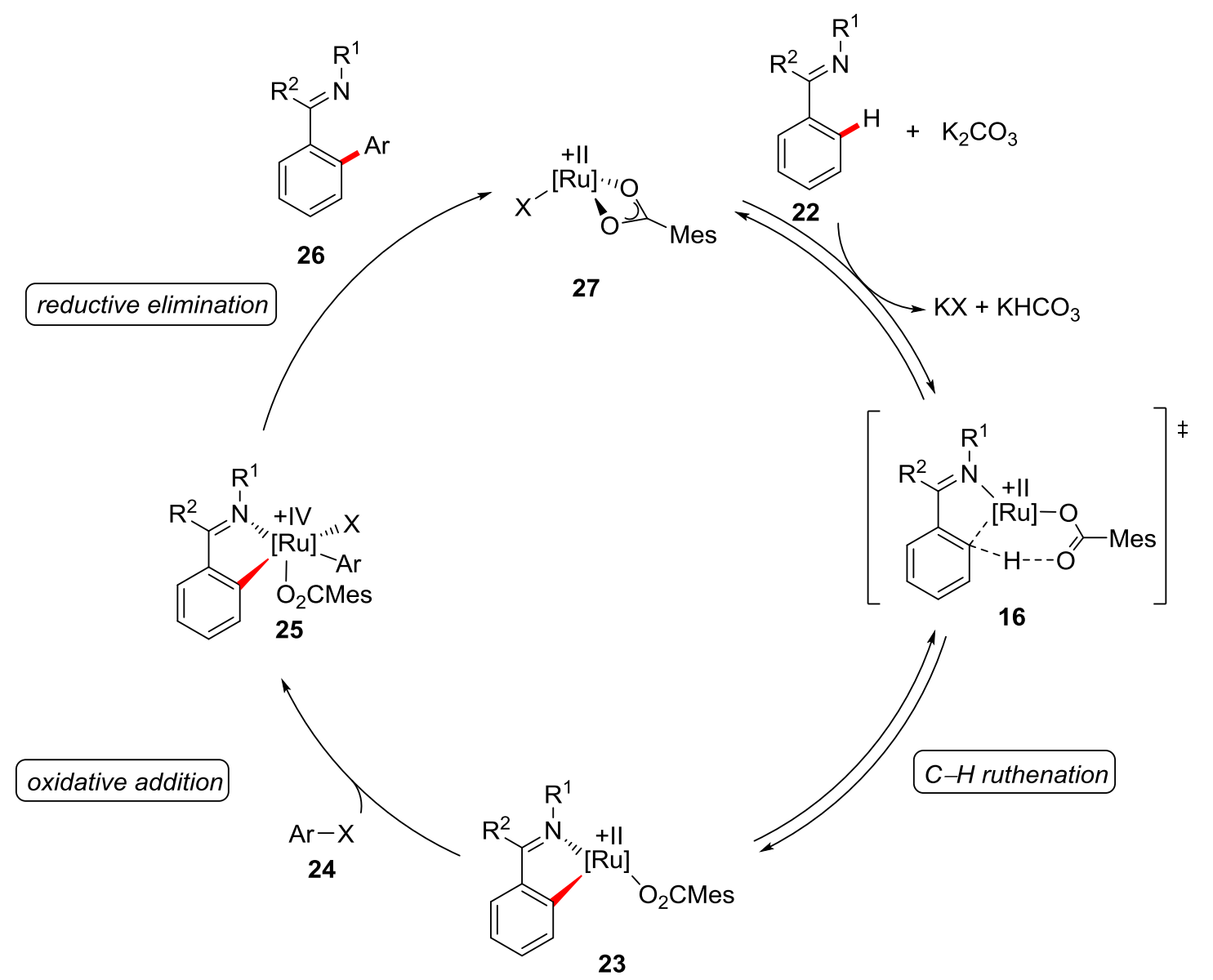

Scheme 10. Proposed catalytic cycle for the carboxylate-assisted ruthenium(II)-catalyzed $\mathrm{C}-\mathrm{H}$ arylation. $\mathrm{X}=\mathrm{Br}^{-}, \mathrm{Cl}^{-}, \mathrm{MesCO}_{2}^{-}$.

Furthermore, the excellent performance and broad applicability of ruthenium(II)-carboxylate complexes was highlighted by regioselective arylations of diversely substituted heterocycles, such as pyrroles 28, thiophenes 29 and indoles $\mathbf{3 0}$, which bear a removable pyrimidyl directing group. ${ }^{[43]}$ Based on seminal observations by Ackermann (vide supra) using water as additive in rutheniumcatalyzed C-H arylations, ${ }^{[36 c]}$ Dixneuf and coworkers demonstrated the robustness of rutheniumcatalyzed $\mathrm{C}-\mathrm{H}$ arylations by establishing water as an economically attractive and environmentally benign reaction medium (Scheme 11). ${ }^{[44]}$ 


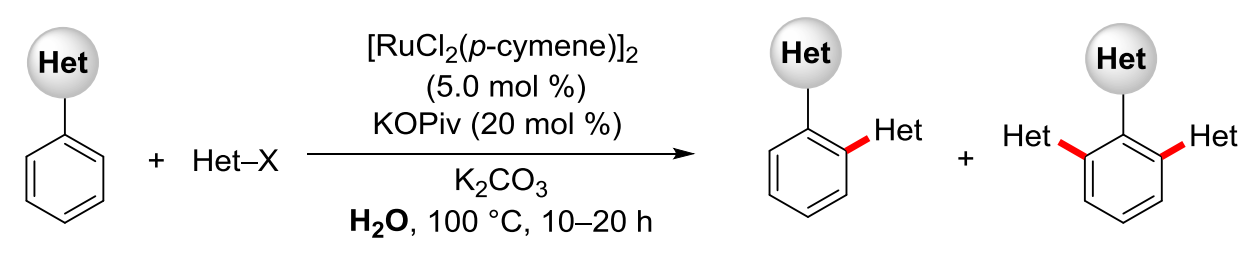

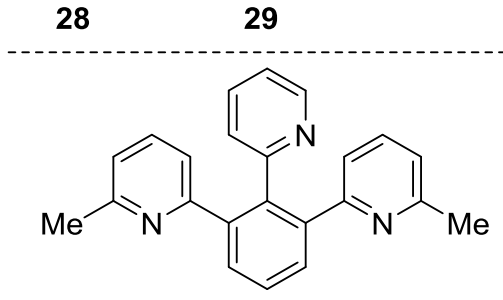

5am: $41 \%$<smiles>c1ccc(-c2cccc(-c3cccs3)c2-c2cccs2)nc1</smiles>

5an: $89 \%$ 
suggested the in situ reduction of the ruthenium(III)-precursor to the catalytically active ruthenium(II)complex..$^{[48]}$

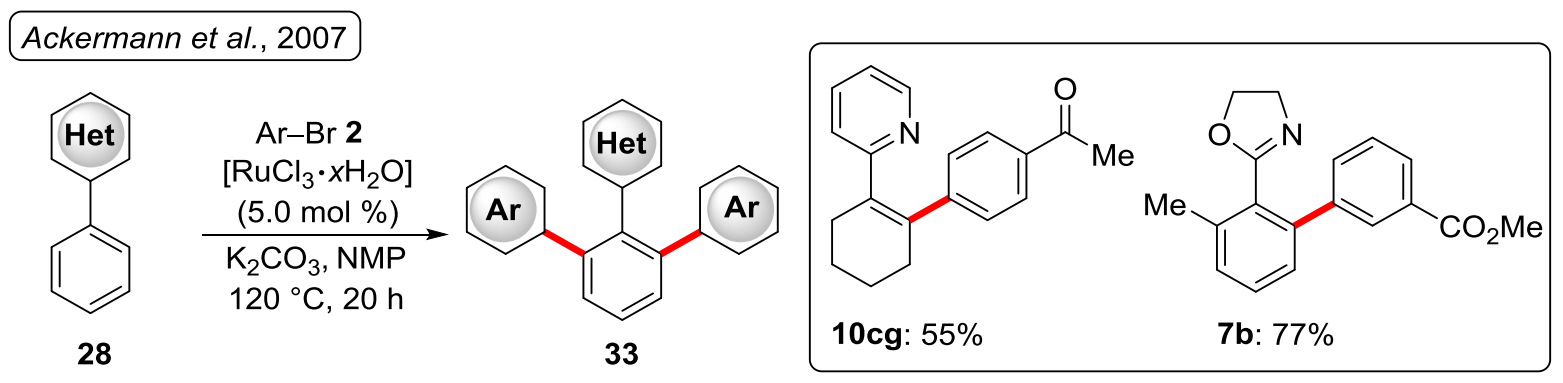

\section{Yu et al., 2010}

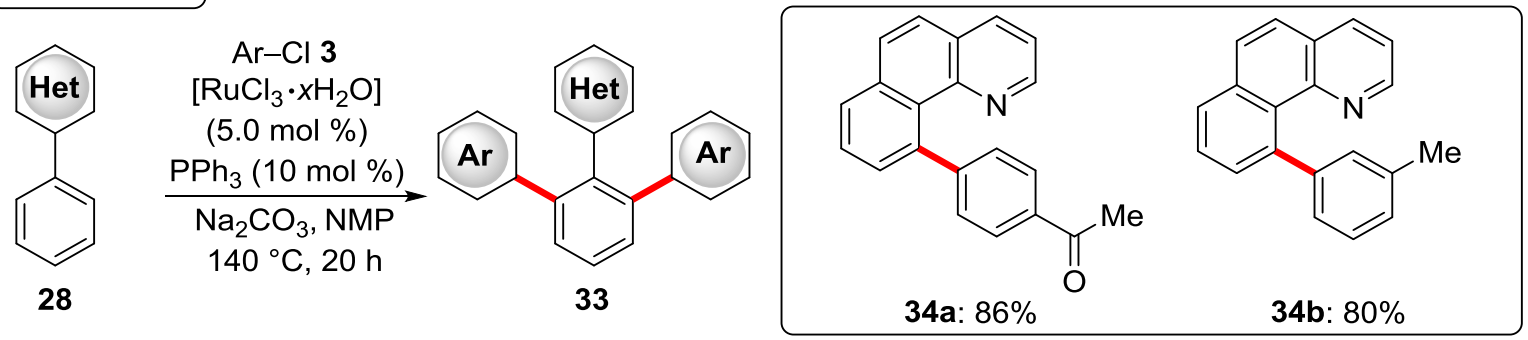

Scheme 13. $\left[\mathrm{RuCl}_{3} \cdot x \mathrm{H}_{2} \mathrm{O}\right]$-catalyzed $\mathrm{C}-\mathrm{H}$ arylations.

\subsection{Cobalt-Catalyzed C-H Activation}

In 1938, Otto Roelen made a groundbreaking advance in homogenous catalysis by reporting on the first metal-catalyzed hydroformylation reaction. ${ }^{[49]}$ Remarkably, after testing a variety of catalytic systems, he suggested that the simple and inexpensive cobalt complex $\left[\mathrm{CoH}(\mathrm{CO})_{4}\right]$ is the optimal catalyst. ${ }^{[50]}$ After these pioneering studies, the following decades have witnessed enormous progress in cobalt catalysis, e.g. the development of the Pauson-Khand-reaction. ${ }^{[51]}$ Nowadays, the great importance of cobalt catalysts is reflected by numerous industrial processes using economically attractive cobalt complexes, such as the Fischer-Tropsch-process ${ }^{[52]}$ and the hydrodesulfurization of petroleum. ${ }^{[53]}$

Due to the earth abundance and thus inexpensiveness ${ }^{[54]}$ of cobalt as well as the beneficial step- and atom-economical features of $\mathrm{C}-\mathrm{H}$ activation catalysis, it appeared particularly attractive to investigate the performance of cobalt catalysts in $\mathrm{C}-\mathrm{H}$ functionalizations. Thus, Murahashi and coworkers accomplished one of the first examples of $\mathrm{C}-\mathrm{H}$ functionalizations in 1955, as they reported on a cobalt-catalyzed carbonylative cyclization of azobenzenes 35. However, extremely harsh conditions were shown to be necessary for achieving overall moderate yields (Scheme 14). ${ }^{[55]}$ 


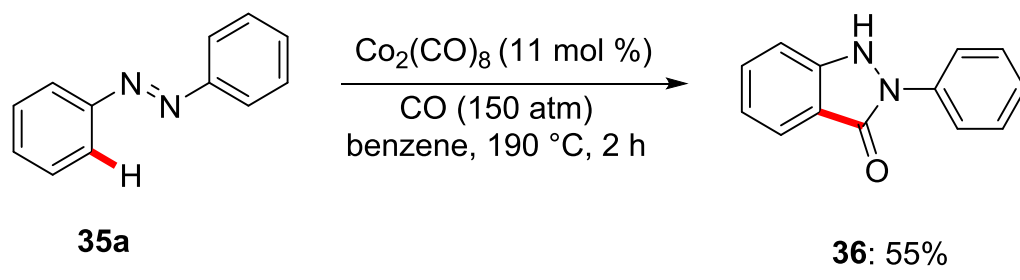

Scheme 14. Cobalt-catalyzed carbonylative cyclization.

Almost 40 years later, one of the first examples for a stoichiometric $\mathrm{C}-\mathrm{H}$ activation by a cobalt complex was reported by Klein and coworkers in 1993 by again using azobenzene (35a) as the substrate and $\left[\mathrm{CoMe}\left(\mathrm{PMe}_{3}\right)_{4}\right]$ as the cobalt source (Scheme 15). ${ }^{[56]}$ The authors suggested that the reaction is initiated by a dissociation of one $\mathrm{PMe}_{3}$ ligand. Subsequently, oxidative addition of the ortho-C-H bond to the cobalt complex occurs, and finally a reductive elimination of methane provides the cyclometalated species $\mathbf{3 7}$.

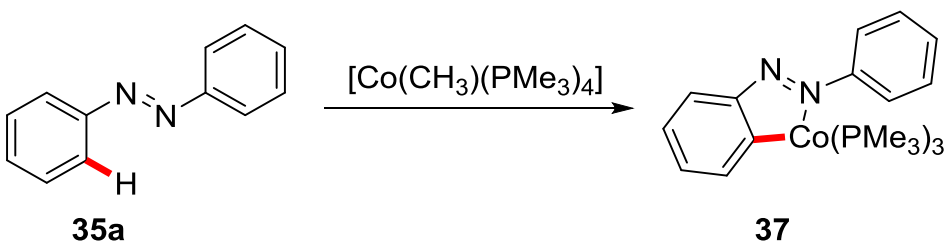

Scheme 15. Stoichiometric formation of a cobaltacycle through $\mathrm{C}-\mathrm{H}$ activation.

Based on these findings, Klein and coworkers reported on the synthesis of several cobaltacycles by $\mathrm{C}-\mathrm{H}$ activation, directed by Lewis basic directing groups with nitrogen-, phosphorous-, sulfur-, or oxygen-functionalities. ${ }^{[57]}$

These fundamental studies paved the way for a blossom of cobalt-catalyzed C-H functionalizations by initially low-valent cobalt catalytic systems, with numerous contributions by inter alia Ackermann and Yoshikai. ${ }^{[15]}$ Despite the broad synthetic applicability of low-valent cobalt-catalyzed C-H functionalizations, one of the major drawbacks of some low-valent cobalt catalytic systems is often the need for stoichiometric amounts of Grignard reagents, which limit the overall atom-efficiency and the substrate scope with respect to base-labile functional groups.

\subsubsection{Cobalt(III)-Catalyzed C-H Functionalization}

Matsunaga/Kanai and coworkers devised an elegant strategy to implement $\left[\mathrm{Co}^{\mathrm{III}} \mathrm{Cp}^{*}\right]$-complexes, ${ }^{[58]}$ which obviate the use of Grignard reagents and usually follow the reactivity features of the wellestablished rhodium(III) C-H activation catalysis. ${ }^{[130,59]}$ As an early example in 2013, they investigated the catalytic performance of several cobalt complexes in addition reactions of 2phenylpyridine (1a) to highly electrophilic sulfonylimines $\mathbf{3 8}$ as well as $\alpha, \beta$-unsaturated ketones, esters and amides 39 (Scheme 16). ${ }^{[60]}$ During their optimization studies, the authors emphasized that only 
$\left[\mathrm{Co}^{\mathrm{III}} \mathrm{Cp}^{*}\right]$ - complexes were powerful catalysts for these transformations, whereas $\mathrm{CoCl}_{2}$ did not furnish the desired products.<smiles></smiles>

$1 \mathbf{a}$<smiles>[R]/C=N\S(=O)(=O)c1cccs1</smiles>

38

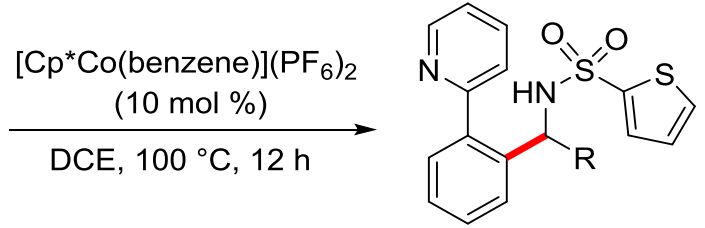

$\mathrm{R}=p-\mathrm{CF}_{3}-\mathrm{C}_{6} \mathrm{H}_{4}(39): 64 \%$

$\mathrm{R}=p-\mathrm{OMe}-\mathrm{C}_{6} \mathrm{H}_{4}(\mathbf{4 0}): 57 \%$<smiles>[C+]c1ccccc1-c1ccccn1</smiles>

$1 \mathrm{a}$<smiles>[R]C=CC(=O)n1cccc1</smiles>

39

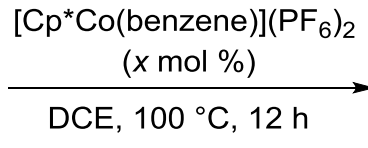

DCE, $100^{\circ} \mathrm{C}, 12 \mathrm{~h}$

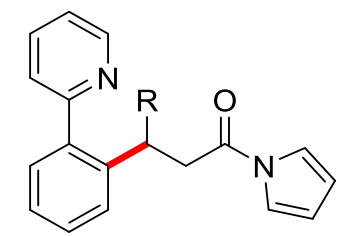

$\mathrm{R}=n-\operatorname{Pr}(\mathbf{4 1}): 91 \%(x=5.0 \mathrm{~mol} \%)$

$\mathrm{R}=\mathrm{Ph}(\mathbf{4 2}): \quad 78 \%(x=10 \mathrm{~mol} \%)$

Scheme 16. Cobalt(III)-catalyzed C-H functionalization and addition to electrophiles.

Under comparably mild reaction conditions, various functional groups, such as enolizable ketones, hydroxyls as well as ester-groups, were tolerated, contrasting the often limited substrate scope of lowvalent cobalt-catalyzed reactions.

Remarkably, a related $\left[\mathrm{Rh}^{\mathrm{III}} \mathrm{Cp}^{*}\right]$-catalyzed $\mathrm{C}-\mathrm{H}$ functionalization ${ }^{[61]}$ was shown to be inefficient in addition reactions to less electrophilic $\alpha, \beta$-unsaturated esters and amides, indicating the unique reactivity profile of $\left[\mathrm{Co}^{\mathrm{III}} \mathrm{Cp}^{*}\right]$-catalysts. Presumably, the higher nucleophilicity of the cobalt-carbon bond enables the addition reaction to even less electrophilic functionalities. In this regard, the Pauling electronegativity of cobalt is $\chi_{\mathrm{F}} \approx 1.9$ and of rhodium it is $\chi_{\mathrm{F}} \approx 2.3$. $^{[62]}$

Capitalizing on this distinct reactivity feature of $\left[\mathrm{Co}^{\mathrm{III}} \mathrm{Cp}^{*}\right]$-complexes, a plethora of new synthetic methods were developed, with partly new reactivities and selectivities as compared to the analogous $\left[\mathrm{Rh}^{\mathrm{III}} \mathrm{Cp}^{*}\right]$-catalysis. Shortly afterwards, the same group reported on a similar reaction with indoles $\mathbf{3 0}$, but using KOAc as the crucial additive to accomplish a significant rate acceleration. ${ }^{[63]}$ The authors proposed that the $\mathrm{C}-\mathrm{H}$ activation elementary step proceeds either via an electrophilic or acetateassisted CMD-type mechanism.

Encouraged by these seminal reports of Matsunaga/Kanai, Ellman and coworkers probed the reactivity of cobalt(III) complexes to aldehyde $\mathbf{4 3}$ functionalities in addition reactions. ${ }^{[64]}$ The reported reaction comprised a sequence of an addition and successive cylization reaction to furnish indazoles 44 with azobenzenes 35 or furanes 45 employing $\alpha, \beta$-unsaturated oximes 46 (Scheme 17). 
a) furanes

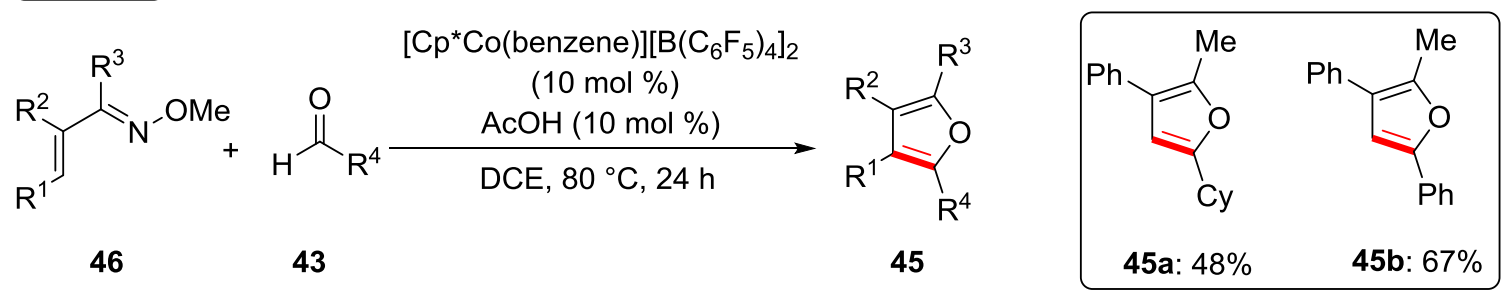

b) indazoles

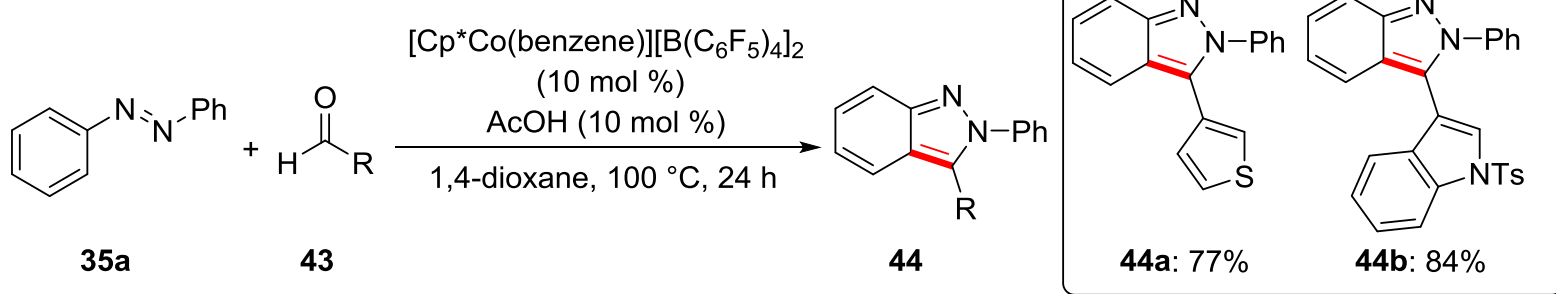

Scheme 17. Cobalt(III)-catalyzed synthesis of a) furanes 45 and b) indazoles 44 .

In addition, the isohypsic synthesis of heterocyles, such as isoquinolines ${ }^{[5]} 49$ (Scheme 18a) and indoles 50 (Scheme 18b) ${ }^{[23 c]}$ by cobalt(III)-catalyzed $\mathrm{C}-\mathrm{H} / \mathrm{N}-\mathrm{O}$ functionalizations was accomplished by Ackermann and coworkers, employing oximes $\mathbf{4 6}$ and nitrones $\mathbf{4 7}$ as the directing groups. These reactions exhibited a broad scope with respect to both substrates. Intriguingly, the overall high efficiency of cobalt(III) catalysis was highlighted by a reaction time of only 15 min (Scheme 18a). Further notable contributions to this reaction type by cobalt(III) catalysis were independently reported by Chang,${ }^{[66]}$ Jiao $^{[67]}$ KanailMatsunaga ${ }^{[68]}$ and Sundaraju.${ }^{[69]}$
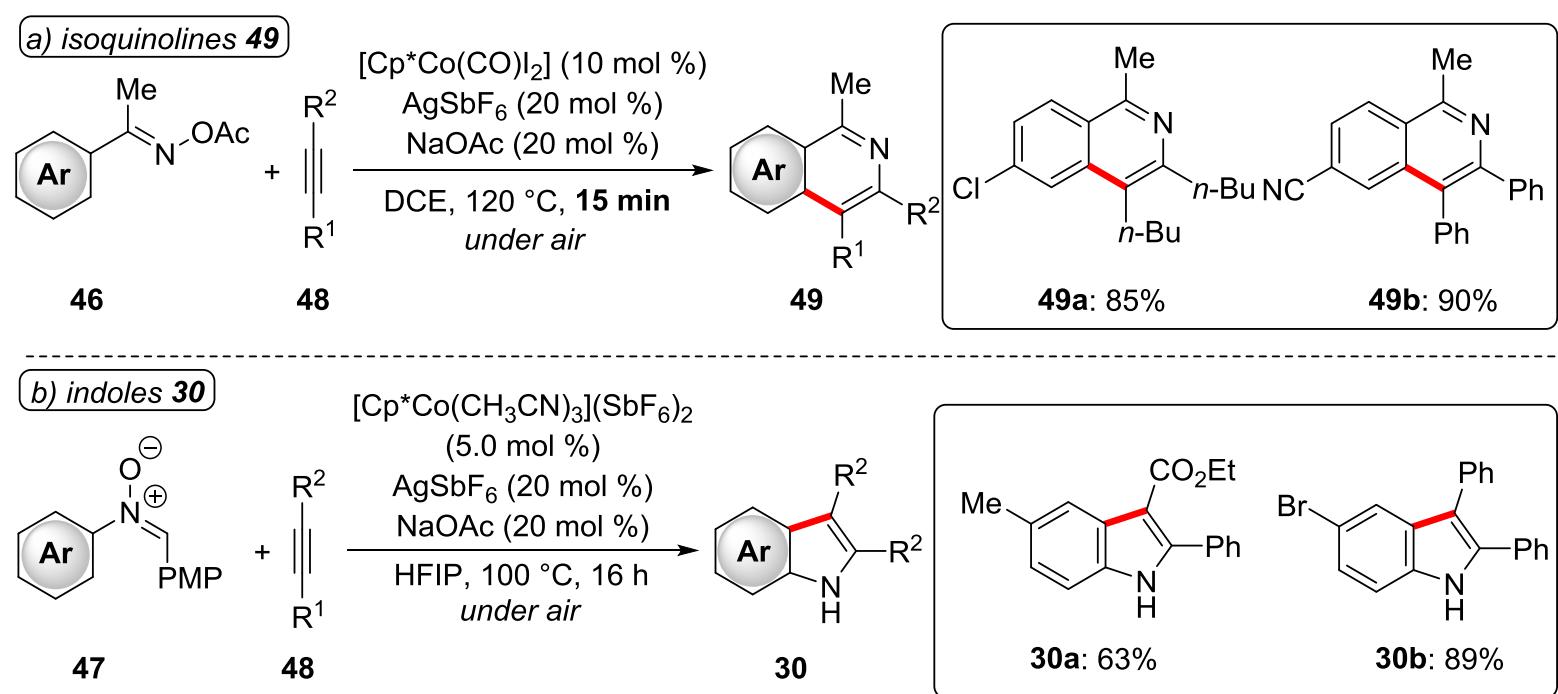

Scheme 18. Cobalt(III)-catalyzed C-H/N-O functionalizations for the synthesis of heterocycles.

The syntheses presented above already indicated the great importance of nitrogen-containing heterocycles. ${ }^{[70]}$ Along this line, Matsunaga/Kanai published an amidation of 2-pyrimidylindoles 50 with sulfonyl azides $\mathbf{5 1}^{[71]}$ (Scheme 19) with ample scope and excellent yields. Thereafter, the same 
group extended the applicability of cobalt(III) catalysis to an analogous phosphoramidation ${ }^{[72]}$ utilizing phosphorylazides 53.

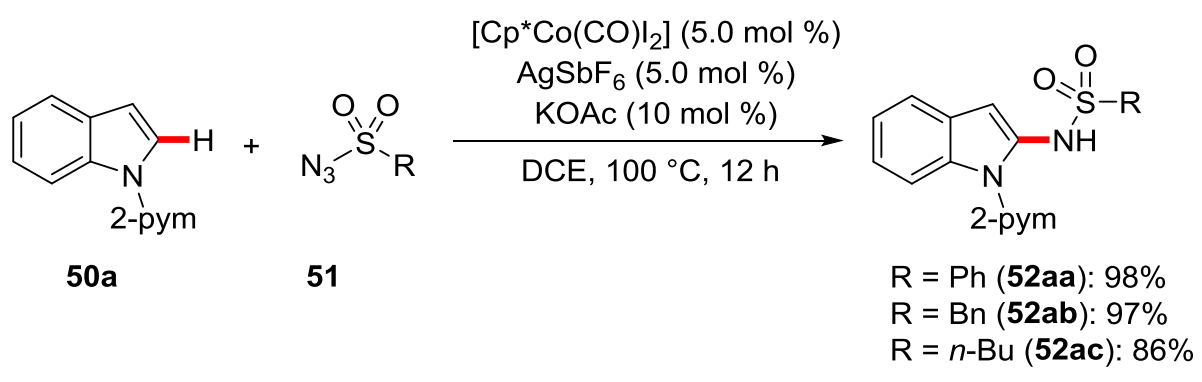

Scheme 19. Cobalt(III)-catalyzed amidation with sulfonyl azides 51.

Another important and easily modifiable nitrogen-containing functional group is the cyano-group. ${ }^{\text {[7] }}$ Accordingly, Ackermann ${ }^{[74]}$ and Glorius $^{[75]}$ independently reported on cobalt(III)-catalyzed cyanations. The reaction system of Ackermann and coworkers is characterized by a low catalyst loading of only $2.5 \mathrm{~mol} \%$ and functionalizations of valuable heteroarenes to inter alia cyanated 2pyrimidylindoles 58/60 and 2-pyrimidylpyrroles 59 (Scheme 20). The corresponding products were obtained in almost quantitative yield, good functional group tolerance, and very good mono/diselectivities.

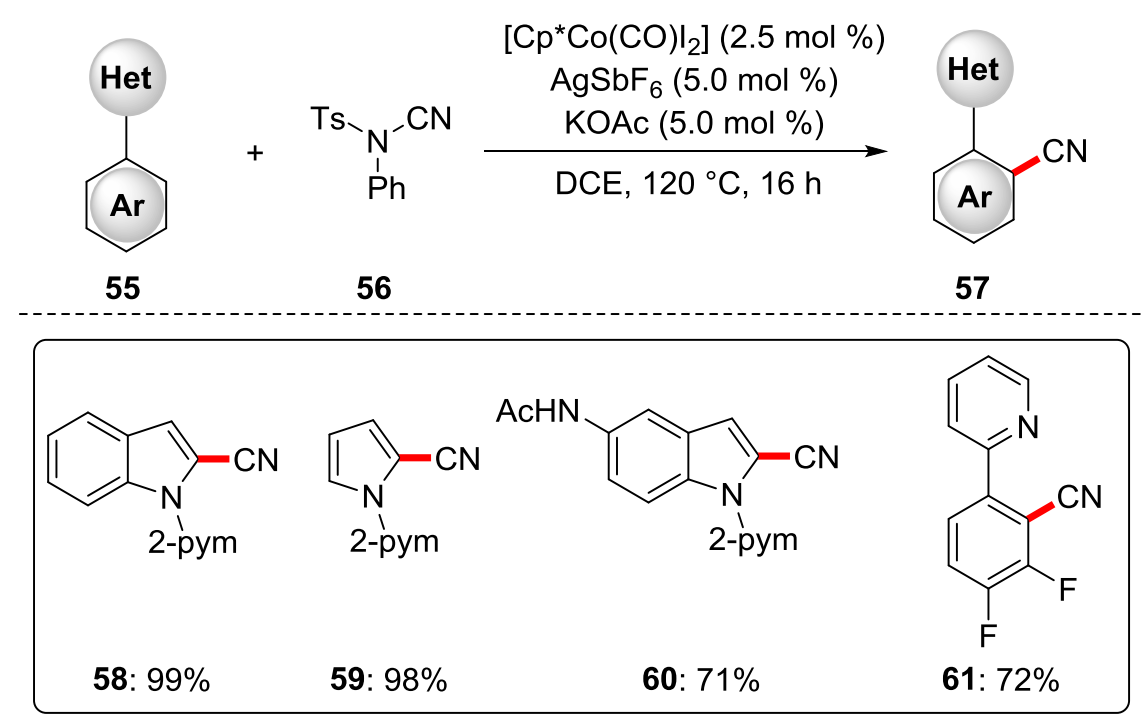

Scheme 20. Cobalt(III)-catalyzed C-H cyanation of heteroarenes.

Moreover, the authors proposed a catalytic cycle of the cobalt(III)-catalyzed $\mathrm{C}-\mathrm{H}$ cyanation (Scheme 21 ), which is initiated by reversible $\mathrm{C}-\mathrm{H}$ cobaltation involving an acetate-assisted deprotonation pathway to deliver cobaltacycle 62a. After coordination of the cyanation reagent 56, a $\beta$-nitrogen elimination takes place. Subsequently, proto-demetalation with the in situ generated acetic acid delivers the cyanated product $\mathbf{6 6}$ and regenerates the catalytically active cobalt(III)-acetate species 62a. 


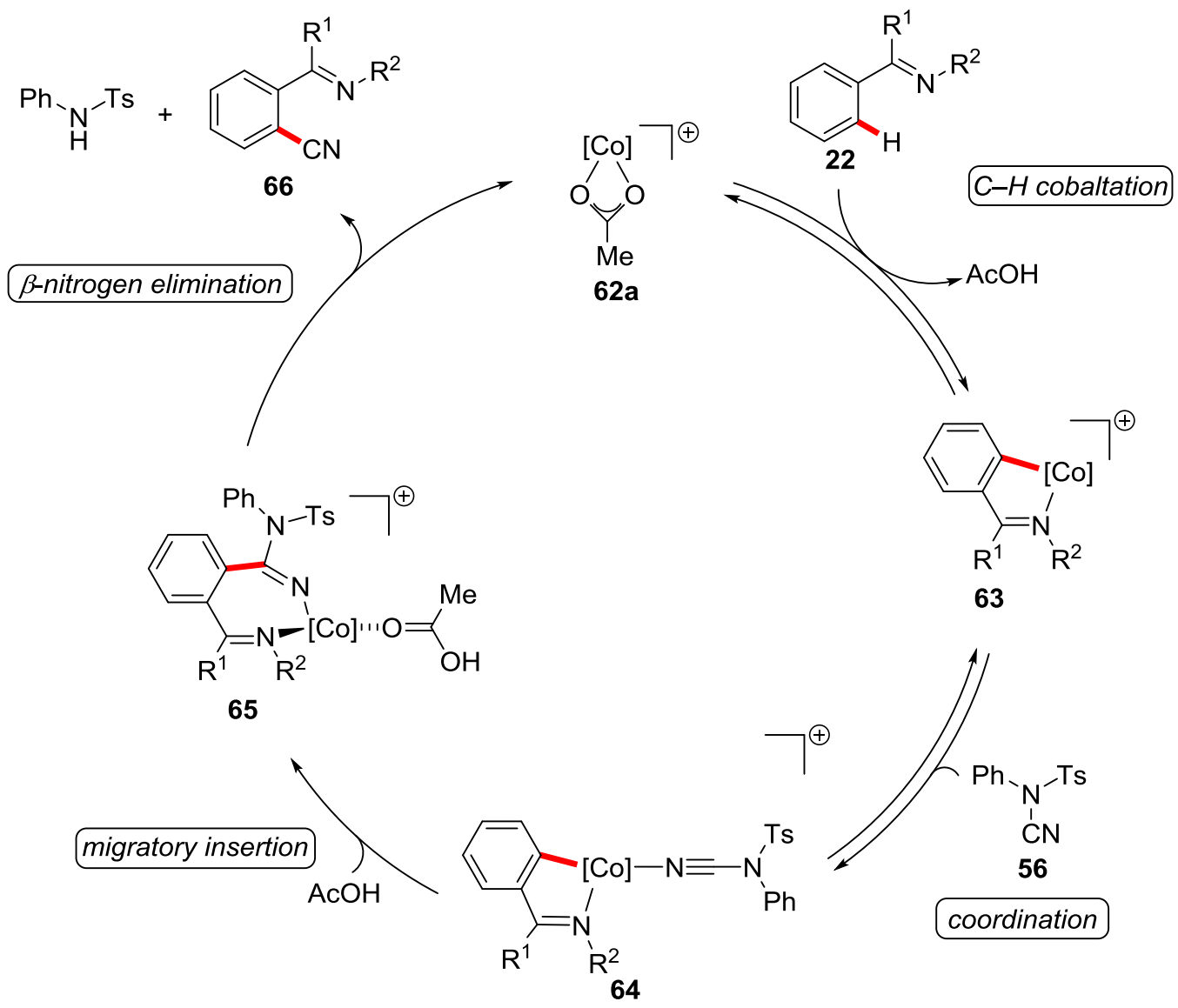

Scheme 21. Proposed catalytic cycle for the cobalt(III)-catalyzed cyanation. [Co] $=\mathrm{Cp}^{*} \mathrm{Co}^{\mathrm{III}}$.

Generally, the $\beta$-nitrogen elimination described above was found to be an energetically preferred step due to the formation of strong metal-nitrogen bonds. Likewise, the same pathway should be favored for oxygen, which is in $\beta$-position to a metal center. Consequently, a process involving $\mathrm{C}-\mathrm{H}$ functionalization and subsequent $\beta$-oxygen elimination is a step-economical approach for installing functionalities, such as synthetically versatile allyl-groups, which have found widespread applications in organic synthesis. ${ }^{[76]}$ In this context, Ackermann, ${ }^{[77]}$ Glorius $^{[75]}$ and Kanai/Matsunaga ${ }^{[78]}$ and coworkers have independently devised several methods merging cobalt(III)-catalyzed $\mathrm{C}-\mathrm{H}$ activation with $\mathrm{C}-\mathrm{O}$ bond cleavage by $\beta$-oxygen elimination to accomplish $\mathrm{C}-\mathrm{H}$ allylations with a variety of directing groups and allylating reagents (Scheme 22). Accompanied by a report on cobalt(III)catalyzed cyanations, brominations, and iodinations, Glorius and coworkers showed that allyl carbonates 67 are competent reagents for the allylation of 2-pyrimidylindoles 50. In a follow-up work, the same group demonstrated that benzamides 68 (Scheme 22a) and acrylamides 69 could also be efficiently allylated. ${ }^{[79]}$ The reaction system of Ackermann and coworkers highlighted the use of simple allyl acetates $\mathbf{7 1}$ to accomplish $\mathrm{C}-\mathrm{H}$ allylations of inter alia 2-pyrimidylindoles $\mathbf{5 0}$ as well as less eletron-rich pyridyl- and pyrimidylarenes. Surprisingly, the crucial $\beta$-oxygen elimination step turned out to be such a strong driving force that even weak leaving groups like hydroxyls could be 
eliminated by using allyl alcohols $\mathbf{7 4}$ directly, as Kanai/Matsunaga and coworkers demonstrated. In addition, the authors emphasized that the reactivity of the cobalt(III) complex was much higher as compared to the well-established complex $\left[\mathrm{Cp}^{*} \mathrm{RhCl}_{2}\right]_{2}$ under otherwise unchanged reaction conditions.
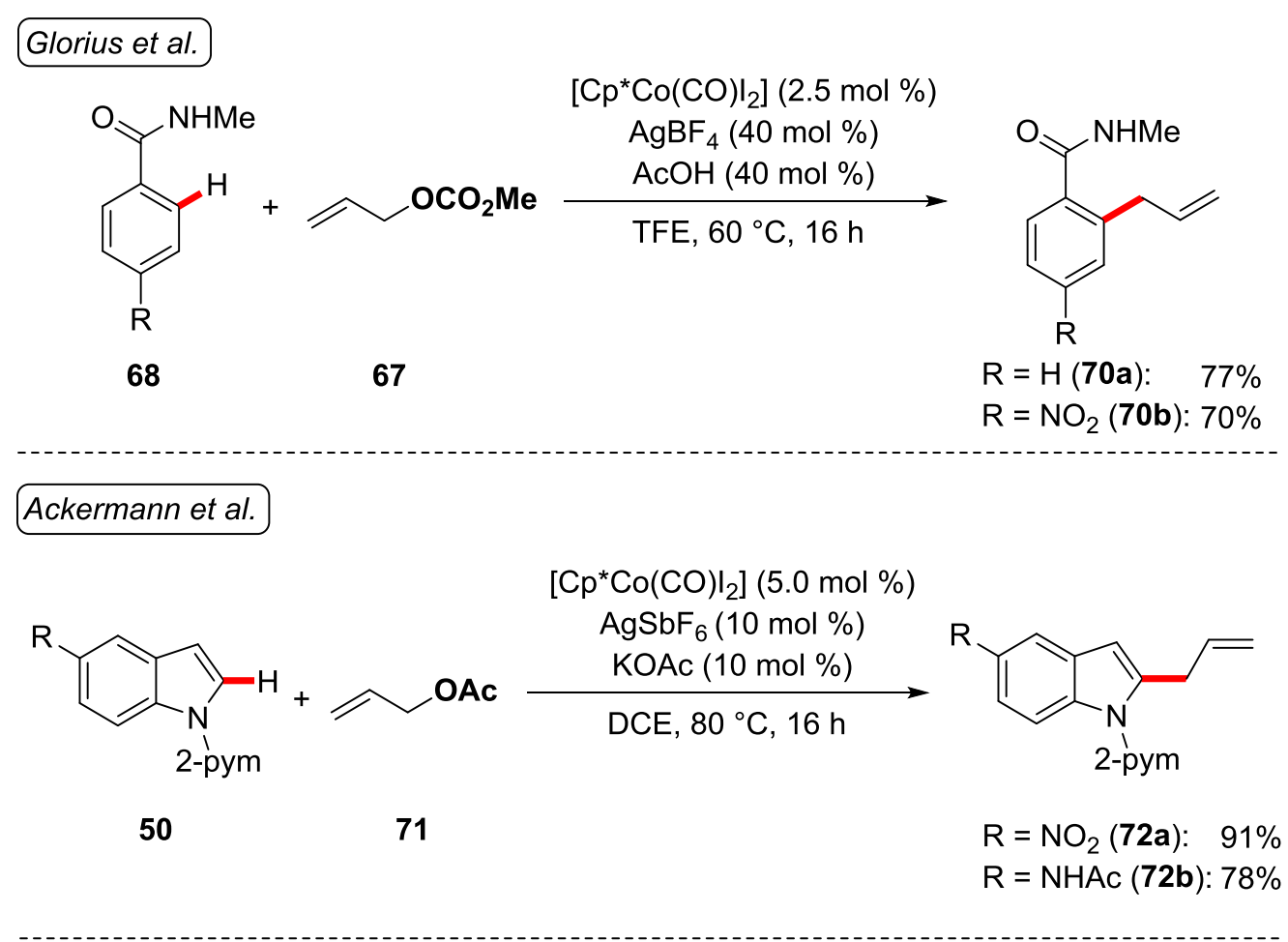

\section{Kanai/Matsunaga et al.}

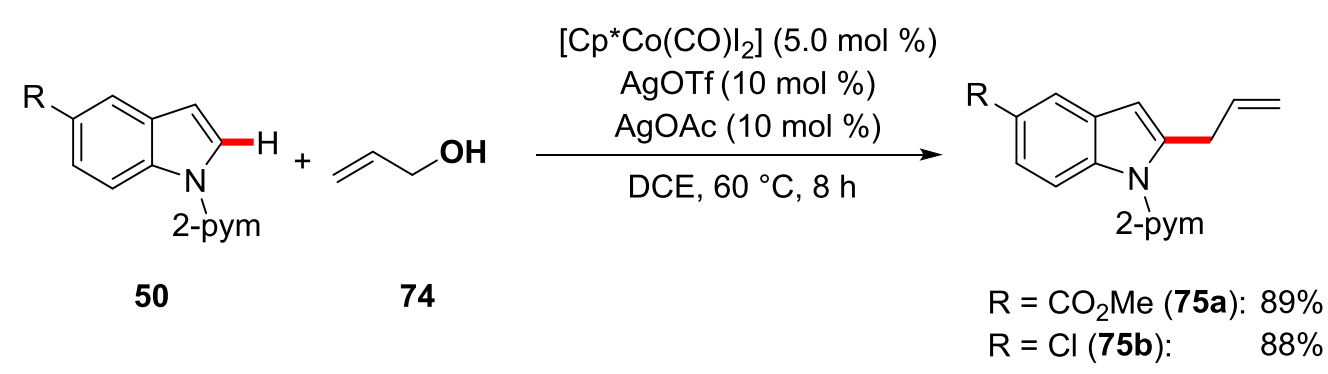

Scheme 22. Cobalt(III)-catalyzed C-H allylations.

Furthermore, a catalytic cycle for the general leaving group OR can be postulated (Scheme 23). Interestingly, all reported catalytic systems featured a significant rate acceleration by carboxylates or carboxylic acid additives, which leads to the assumption that the cationic cobalt-carboxylate $\mathbf{6 2}$ ( $c f$. Scheme 21) might be catalytically active species.

Initially, a base-assisted $\mathrm{C}-\mathrm{H}$ cobaltation takes place, which was shown to be reversible and not turnover-limiting, at least in the case of indole substrates. ${ }^{[77]}$ After reversible coordination of the allyl substrate $\mathbf{7 6}$ to form complex 77, a migratory insertion furnishes intermediate $\mathbf{7 8}$, which undergoes a $\beta$-oxygen elimination step to release the allylated product $\mathbf{7 9}$ and a cobalt(III)-alkoxy species. 
Additionally, Kanai/Matsunaga showed by DFT-studies that a competing $\mathrm{S}_{\mathrm{N}} 2$ pathway is unfavorable. ${ }^{[78]}$ Finally, a reaction of the cobalt(III)-alkoxy species with carboxylic acid regenerates the active cobalt(III)-carboxylate catalyst $\mathbf{6 2}$.

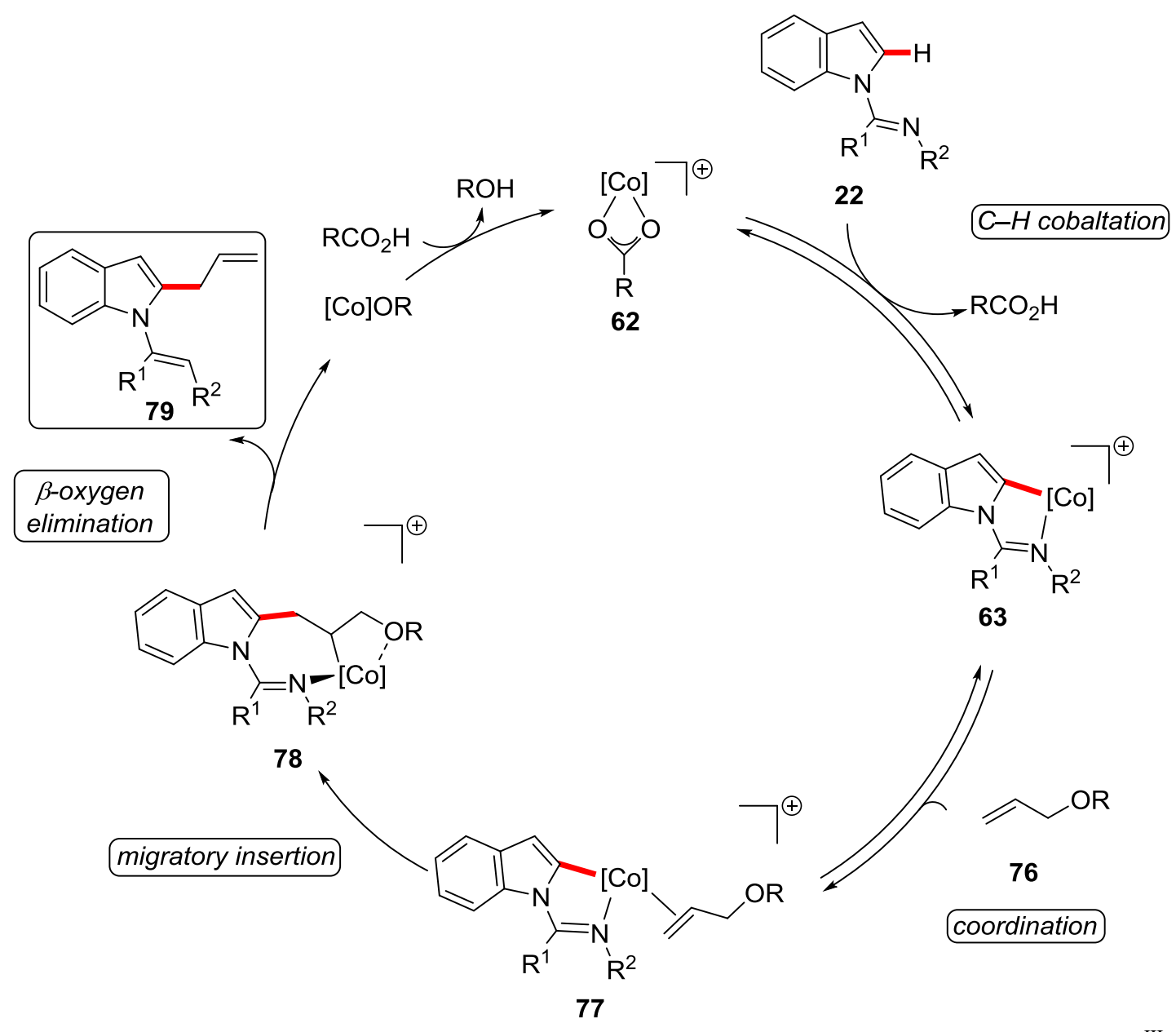

Scheme 23. Proposed catalytic cycle for the cobalt(III)-catalyzed allylation. $[\mathrm{Co}]=\mathrm{Cp}^{*} \mathrm{Co}^{\mathrm{III}}$.

\subsubsection{Cobalt-Catalyzed Hydroarylations}

The first example of a cobalt-catalyzed $\mathrm{C}-\mathrm{H}$ hydroarylation reaction was reported by Kisch and coworkers in 1994, using azobenzenes 35 and tolane 48a as substrates. ${ }^{[80]}$ The corresponding difunctionalized products $\mathbf{8 0}$ were obtained in good isolated yields of around 70-80\% (Scheme 24).<smiles>[R]c1cc([R])c(/N=N/[OH2+])c([2H])c1</smiles>

35

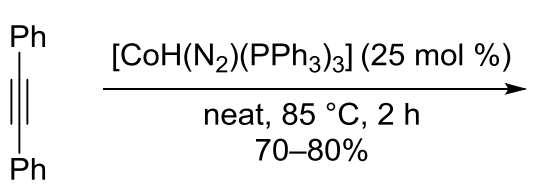

$48 a$

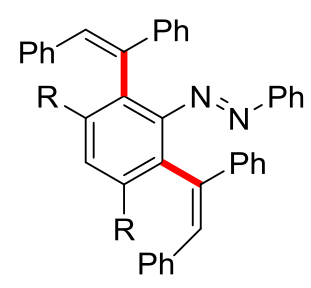

$80(\mathrm{R}=\mathrm{H}, \mathrm{Me}, \mathrm{F}, \mathrm{Cl})$

Scheme 24. Cobalt(I)-catalyzed $\mathrm{C}-\mathrm{H}$ hydroarylation. 
These fundamental findings inspired Yoshikai and coworkers to develop a cobalt-catalyzed $\mathrm{C}-\mathrm{H}$ hydroarylation of internal alkynes 48 (Scheme 25). The catalytic system comprised comparably low amounts of a simple cobalt salt as the catalytic precursor, a phosphine ligand, and stoichiometric amounts of a Grignard reagent as the reductant. In this context, the authors first succeeded in functionalizing 2-phenylpyridines $\mathbf{1}^{[81]}$ Afterwards they extended the substrate scope to ketimines $\mathbf{6}^{[82]}$ and indoles $\mathbf{3 0}$ as well. ${ }^{[83]}$ Under the optimal reaction conditions, the desired alkenylated products $\mathbf{8 1}$ were delivered in very good yields and an excellent regiocontrol was achieved in the case of unsymmetrical alkynes 48. Most of the reactions proceeded at ambient temperature so that even sensitive functional groups such as nitriles were well tolerated.

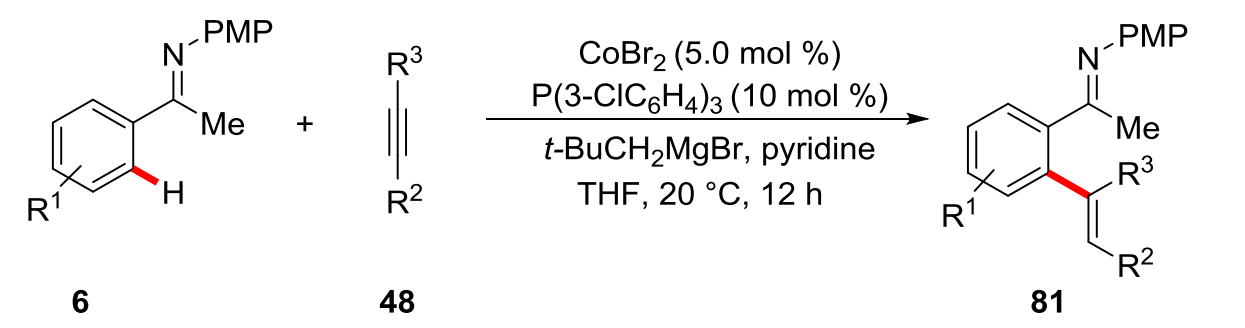

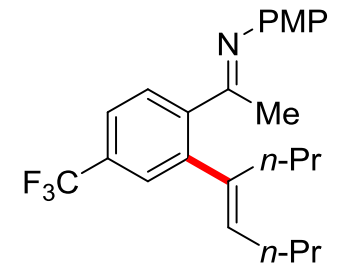

81a: $85 \%$<smiles>CC(=N)/C=C(\C)c1ccccc1/C(C)=N/P=N</smiles>

81b: $81 \%$

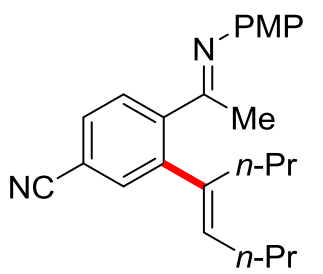

81c: $57 \%$

Scheme 25. Low-valent cobalt-catalyzed $\mathrm{C}-\mathrm{H}$ hydroarylation with ketimines 6.

Thereafter, the excellent performance of low valent cobalt catalysis in hydroarylation reactions was successfully extended to more challenging alkenes by Yoshikai ${ }^{[84]}$ and Nakamura. ${ }^{[85]}$ Yoshikai and coworkers developed a cobalt-catalyzed $\mathrm{C}-\mathrm{H}$ hydroarylation with styrenes $\mathbf{8 2}$ and 2-phenylpyridines $\mathbf{1}$ (Scheme 26). Remarkably, the authors accomplished a complete switch in Markovnikov/antiMarkovnikov selectivity by the judicious choice of the ligand. While bulky NHC ligands afforded linear products $\mathbf{8 3}$, phosphine ligands completely reversed the selectivity and delivered the branched products 84 in overall good to excellent regioselectivities. 


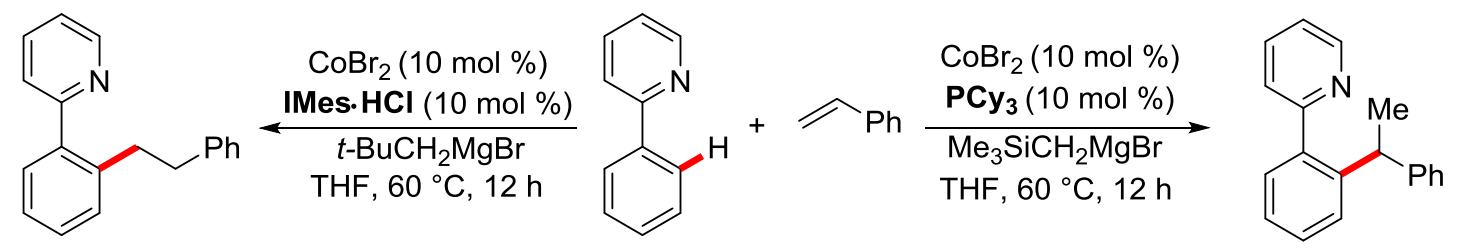

83aa

1a

82a

84aa
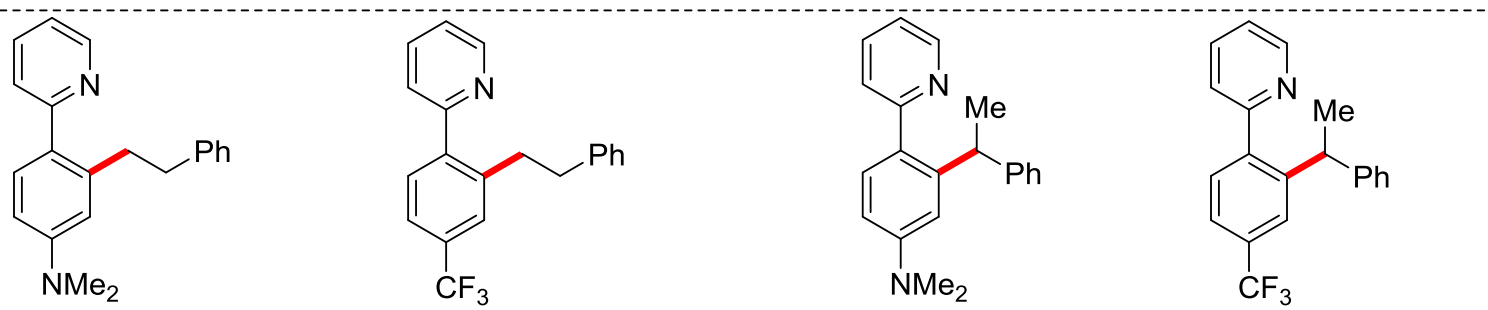

83ca: $46 \%$ (AM:M = 99:1) 83da: $82 \%(A M: M=85: 15)$

84ca: $53 \%$ (AM:M < 1:99) 84da: 31\% (AM:M = 21:79)

Scheme 26. Low-valent cobalt-catalyzed regioselective C-H hydroarylations.

Moreover, the authors conducted deuterium labeling experiments with deuterated 2-phenylpyridine- $d_{5}$ ([D] $\left.]_{5}-1 a\right)$. In the case of the branched product 84aa a higher content of deuterium was observed in the $\alpha$-position of the styrene moiety. In contrast, for the linear product 83aa a larger deuterium incorporation could be detected in $\beta$-position. ${ }^{[84]}$ These findings led to the mechanistic hypothesis that the $\mathrm{C}-\mathrm{H}$ activation via oxidative addition as well as the migratory insertion steps are reversible for both reactions, while the reductive elimination is turnover-limiting as well as regioselectivitydetermining (Scheme 27). The bulky NHC ligand forces the styrene 82 to insert linearly into the carbon-cobalt bond to avoid steric interactions, whereas the linear-selective reaction is controlled by the electronically favored formation of a benzylic cobalt species, ${ }^{[86]}$ as the authors claimed. ${ }^{[84]}$

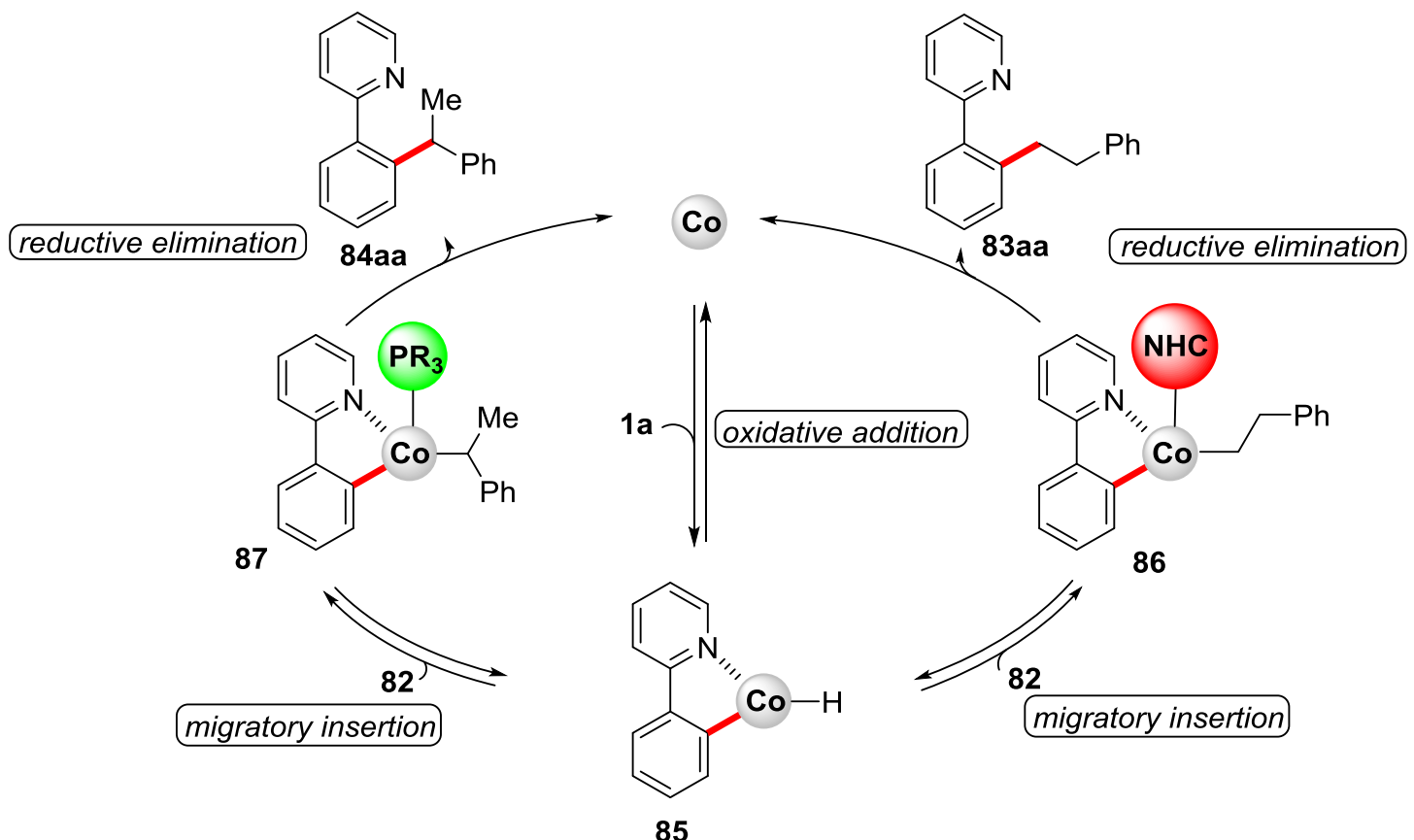

Scheme 27. Proposed mechanism for the regioselective cobalt-catalyzed C-H hydroarylation. 
Thereafter, Nakamura and coworkers disclosed a low-valent cobalt-catalyzed C-H hydroarylation of benzamides 68 with mostly electronically unactivated alkenes $\mathbf{8 8}$. However, the authors also presented a few examples comprising styrenes $\mathbf{8 2}$ (Scheme 28). ${ }^{[85]}$ The catalytic system was characterized by an inexpensive $\mathrm{Co}(\mathrm{acac})_{3}$ catalyst, stoichiometric amounts of a Grignard reagent, and a reaction temperature as low as $25^{\circ} \mathrm{C}$. The authors proposed DMPU to be an essential ligand, which they had previously shown for a related reaction. ${ }^{[87]}$ Importantly, under very mild reaction conditions, even base-labile functional groups like esters were well tolerated. Interestingly, electronically unactivated alkenes $\mathbf{8 8}$ delivered solely the linear-selective product $\mathbf{8 9}$ in excellent yields and regioselectivities. In contrast, the use of styrene (82a) afforded the linear product 89ca in only moderate regioselectivity. Nevertheless, the authors did not propose a mechanistic reason for this interesting change in selectivity, which is obviously related to Yoshikai's observation (vide supra).

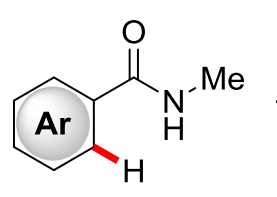

68

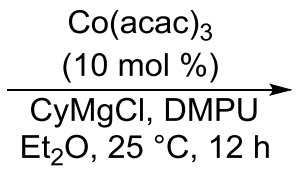

88

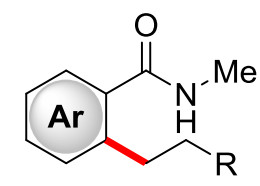

89

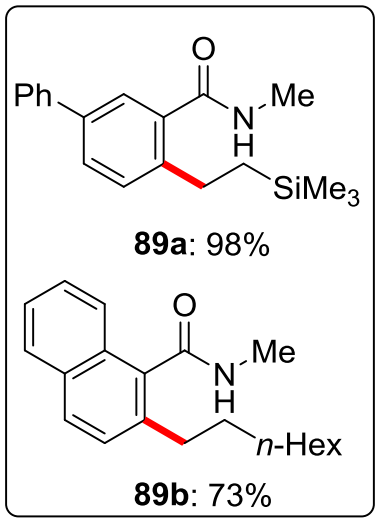

89b: $73 \%$

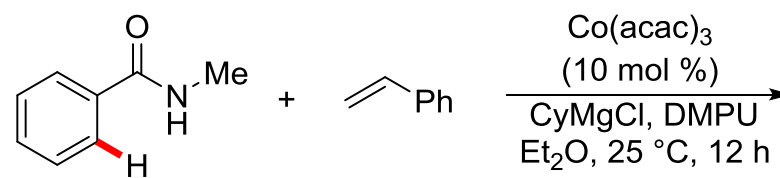

$68 \mathrm{c}$

82a<smiles>CNC(=O)c1ccccc1CCc1ccccc1</smiles>

89ca: $56 \%$ (AM:M = 69:31)

Scheme 28. Low-valent cobalt-catalyzed C-H hydroarylation of benzamides 68 with alkenes 88.

Intriguingly, Yoshikai and coworkers accomplished an enantioselective low-valent cobalt-catalyzed hydroarylation with styrenes $\mathbf{8 2}$ in the $\mathrm{C} 2$-position of indole $\mathbf{9 0}$, which bear a ketimine directing group in C3-position (Scheme 29). ${ }^{[88]}$ The key to success in achieving moderate to good enantioselectivity was the use of phosphoramidite ligands, ${ }^{[89]}$ featuring a BINOL-derived backbone. ${ }^{[88]}$ Overall, good yields and enantioselectivities were obtained, albeit sterically demanding indoles $\mathbf{9 0}$ led to a clear decrease in yield and enantioselectivity, as it was demonstrated for the 7-ethyl substituted indole $\mathbf{9 0 b}$. 

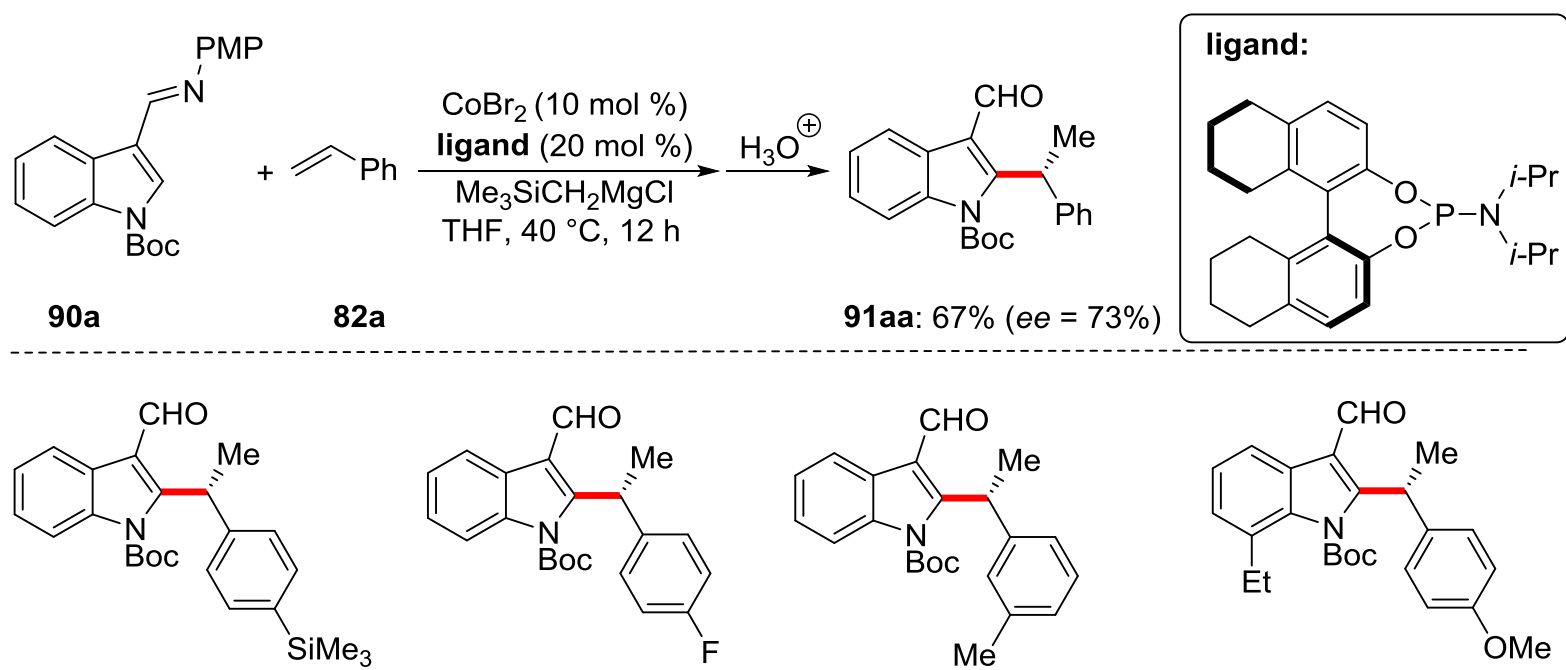

91ab: $48 \%(e e=85 \%)$

91ac: $63 \%(e e=80 \%)$

91ad: $52 \%(e e=85 \%)$

91be: $16 \%(e e=58 \%)$

Scheme 29. Low-valent cobalt catalyzed enantioselective C-H hydroarylation.

Based on a previous report by Fagnou and coworkers on a rhodium(III)-catalyzed C-H hydroarylation $^{[00]}$ with alkynes 48 and low-valent cobalt-catalyzed reaction of Yoshikai, ${ }^{[83]}$ Kanai/Matsunaga and coworkers presented a cobalt(III)-catalyzed hydroarylation of 2carbamoylindoles $92 .{ }^{[91]}$

Remarkably, the authors achieved a full control of chemoselectivity depending on the directing group (Scheme 30). While carbamoyl-substituted indoles 92 furnished the expected hydroarylation products 93 with excellent regioisomeric ratios and a broad scope (Scheme 30a), a morpholine unit on the carbamoyl residue in substrate $\mathbf{9 4}$ almost exclusively afforded the corresponding pyrroloindolones $\mathbf{9 5}$ (Scheme 30b) via a hydroarylation/annulation sequence.

The authors emphasized the unique selectivity features of cobalt(III) catalysis by comparison with the chemoselectivity of the corresponding rhodium(III)-catalyzed hydroarylation. Notably, rhodium(III) catalysis exclusively yielded the hydroarylated product $\mathbf{9 3}$, regardless of the carbamoyl-moiety. 
a) hydroarylation

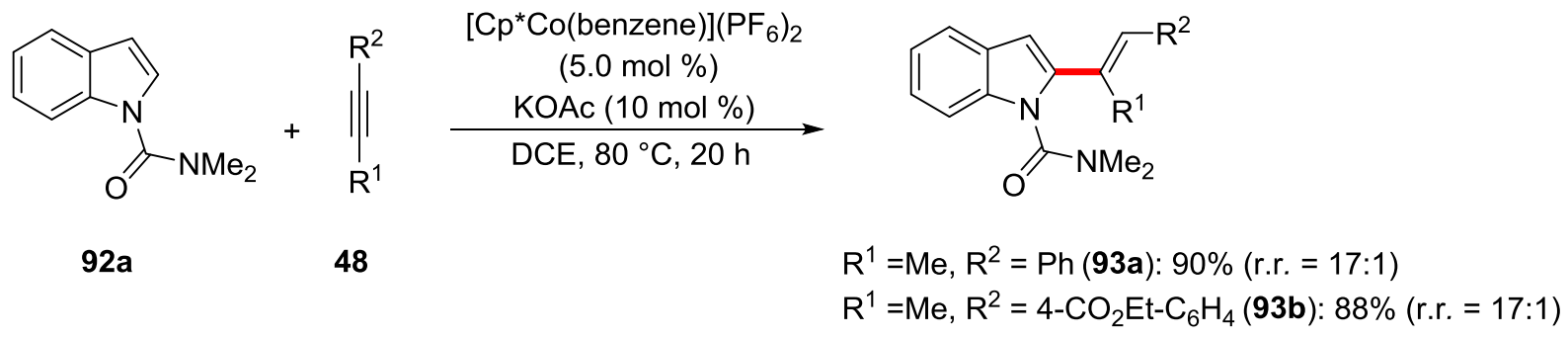

b) hydroarylation/annulation

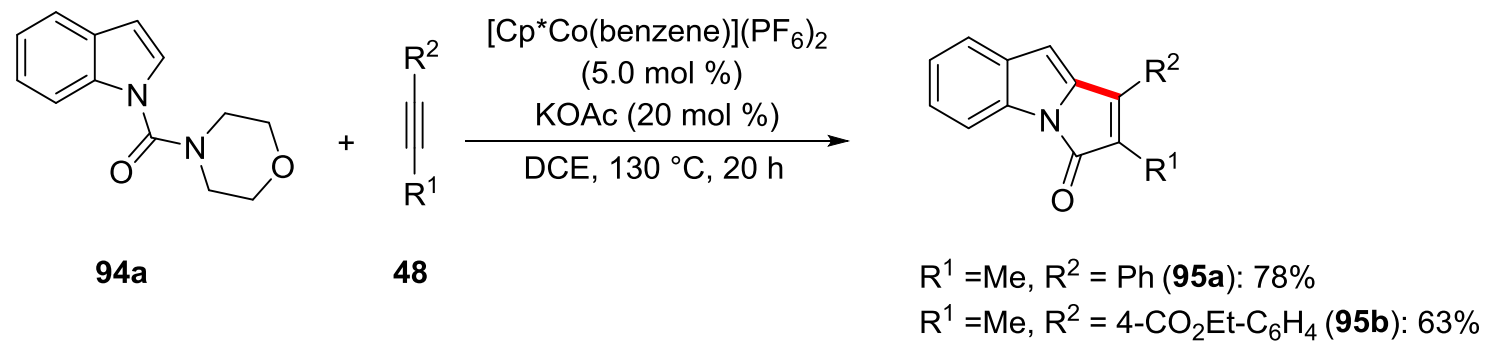

Scheme 30. Cobalt(III)-catalyzed chemoselective C-H hydroarylations with alkynes $\mathbf{4 8 .}$

Finally, the authors proposed a catalytic cycle (Scheme 31), which is initiated by the formation of the catalytically active cobalt(III)-monoacetate species 62a. Subsequently, a reversible cobaltation delivers the cobaltacycle 96 via an acetate-assisted $\mathrm{C}-\mathrm{H}$ activation mechanism. The exact pathway of this $\mathrm{C}-\mathrm{H}$ activation step is presumably not fully concerted, as it was shown by DFT calculations. Rather, a stepwise mechanism was proposed, which is initiated by a weak interaction between cobalt and the C2-position of indole, followed by deprotonation through acetate-assistance. ${ }^{[20]}$ After reversible coordination of the alkyne $\mathbf{4 8}$ and migratory insertion, the crucial cobalt-alkenylintermediate $\mathbf{9 8}$ is produced. Depending on the leaving group ability of the dialkylamine unit, either a proto-demetalation follows, delivering the product $\mathbf{9 3}$, or an annulation reaction by nucleophilic attack of the cobalt-carbon bond with following $\beta$-nitrogen elimination affords the pyrroloindolone 95. In addition, DFT calculations and charge estimation by a natural population analysis led to the assumption that a crucial cobalt(III)-alkenyl intermediate $\mathbf{9 8}$ is more nucleophilic than the analogous rhodium(III)-intermediate due the diminished electrophilicity of cobalt (vide supra). In this respect, a simple change to a better leaving group, such as the morpholino-unit, energetically favoured the successive annulation reaction. 


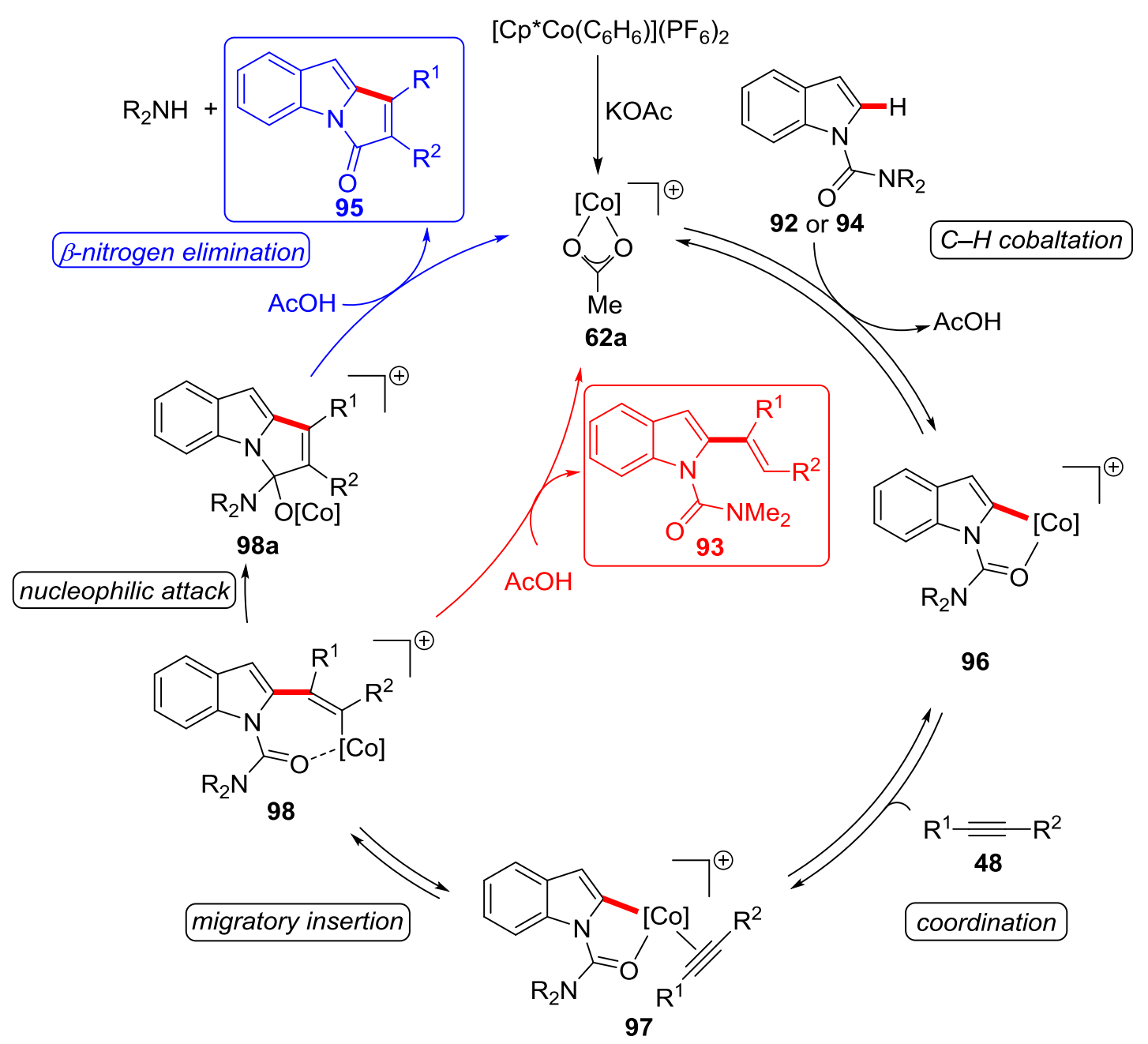

Scheme 31. Postulated catalytic cycle of the cobalt(III)-catalyzed hydroarylation of alkynes. $[\mathrm{Co}]=\mathrm{Cp}^{*} \mathrm{Co}^{\text {III }}$.

\subsection{Manganese-Catalyzed C-H Activation}

The achievement of sustainable chemical processes ideally involves the use of earth-abundant reagents and catalysts. In this regard, manganese represents an ideal candidate for $\mathrm{C}-\mathrm{H}$ activation catalysis, since it is the twelfth most abundant element in the earth crust, and the third most abundant transition metal after iron and titanium. ${ }^{[54]}$ However, manganese could be an appealing metal for new catalysts not only from an economical viewpoint, but also due to its usually low toxicity, ${ }^{[54,92]}$ which matches the principles of green chemistry. ${ }^{[12]}$ In addition, there are several manganese co-factors in enzymes that are involved in the human metabolism of inter alia carbohydrates and amino acids, rendering manganese essential for human life. ${ }^{[93]}$

Thus, by strong analogy with the stoichiometric cobalt-mediated $\mathrm{C}-\mathrm{H}$ activation by Klein and coworkers ( $c f$. chapter 1.3), Stone/Bruce and coworkers presented a manganese-mediated $\mathrm{C}\left(\mathrm{sp}^{2}\right)-\mathrm{H}$ activation of azobenzene (35a) to deliver the manganacycle 99 (Scheme 32). ${ }^{[4]}$ 


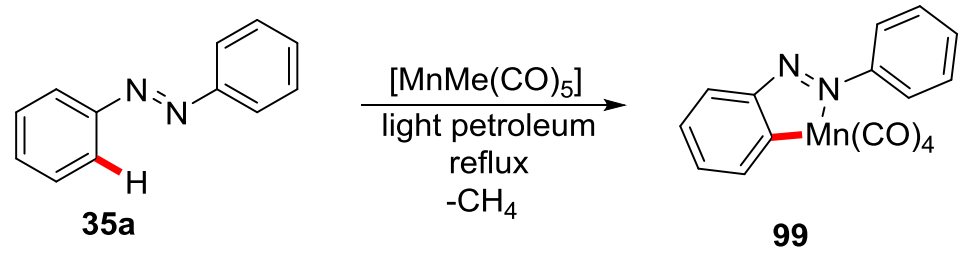

Scheme 32. Stoichiometric manganese(I)-mediated $\mathrm{C}\left(\mathrm{sp}^{2}\right)-\mathrm{H}$ activation.

Thereafter, a number of reactivities with these well-defined manganacycles were disclosed by inter alia Liebeskind for the synthesis of indenoles, ${ }^{[95]}$ and by Nicholson ${ }^{[96]}$ and Woodgate. ${ }^{[97]}$ These accomplishments have already predicted the great potential of manganese in $\mathrm{C}-\mathrm{H}$ activation catalysis. However, almost 40 years had passed since the report of StonelBruce until the first example of manganese-catalyzed C-H activation was reported by Kuninobu/Takai in 2007 (Scheme 33). ${ }^{[98]}$ Here, the authors presented a manganese(I)-catalyzed $\mathrm{C}-\mathrm{H}$ activation of imidazoles $\mathbf{1 9}$ and a subsequent addition reaction of the manganacycle to electrophilic aldehydes $\mathbf{4 3}$. Notably, triethylsilane turned out to be a crucial reagent for catalytic turnover, delivering the silyl-protected benzylic alcohols $\mathbf{1 0 0}$ in overall moderate to good yields. The authors emphasized that only a manganese-catalyst was efficient, whereas other representative metal-catalysts based on inter alia rhenium, ruthenium, and iridiumcomplexes were not active. Within a comprehensive scope it was demonstrated that even sterically demanding substrates like ortho-substituted phenyl-groups led to only a slight decrease in yield.<smiles>Cn1ccnc1-c1ccccc1[18F]</smiles>

$19 a$

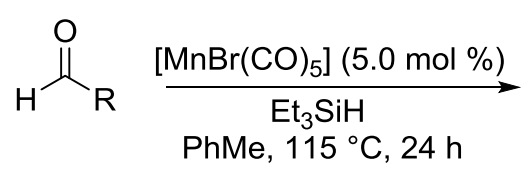

43<smiles>[R]C(OCC)c1ccccc1-c1nccn1C</smiles>

$\mathrm{R}=4-\mathrm{CF}_{3} \mathrm{C}_{6} \mathrm{H}_{4}$ (100aa): $87 \%$

$\mathrm{R}=2-\mathrm{OMeC}_{6} \mathrm{H}_{4}$ (100ab): $59 \%$

$\mathrm{R}=n-\mathrm{C}_{8} \mathrm{H}_{17}(100 \mathrm{ac}): \quad 75 \%$

Scheme 33. Manganese(I)-catalyzed C-H addition to aldehydes 43.

Moreover, a plausible mechanistic scenario was proposed (Scheme 34). The yet undefined structure of the catalytically active manganese(I) species 101 undergoes $\mathrm{C}-\mathrm{H}$ activation by oxidative addition, furnishing the manganese(III)-hydride species 102. Afterwards, migratory insertion of the polar carbonyl bond into the nucleophilic carbon-manganese bond takes place. Finally, triethylsilane promotes a reductive elimination to release the silyl-protected product $\mathbf{1 0 0}$, the active catalyst, and molecular hydrogen. 


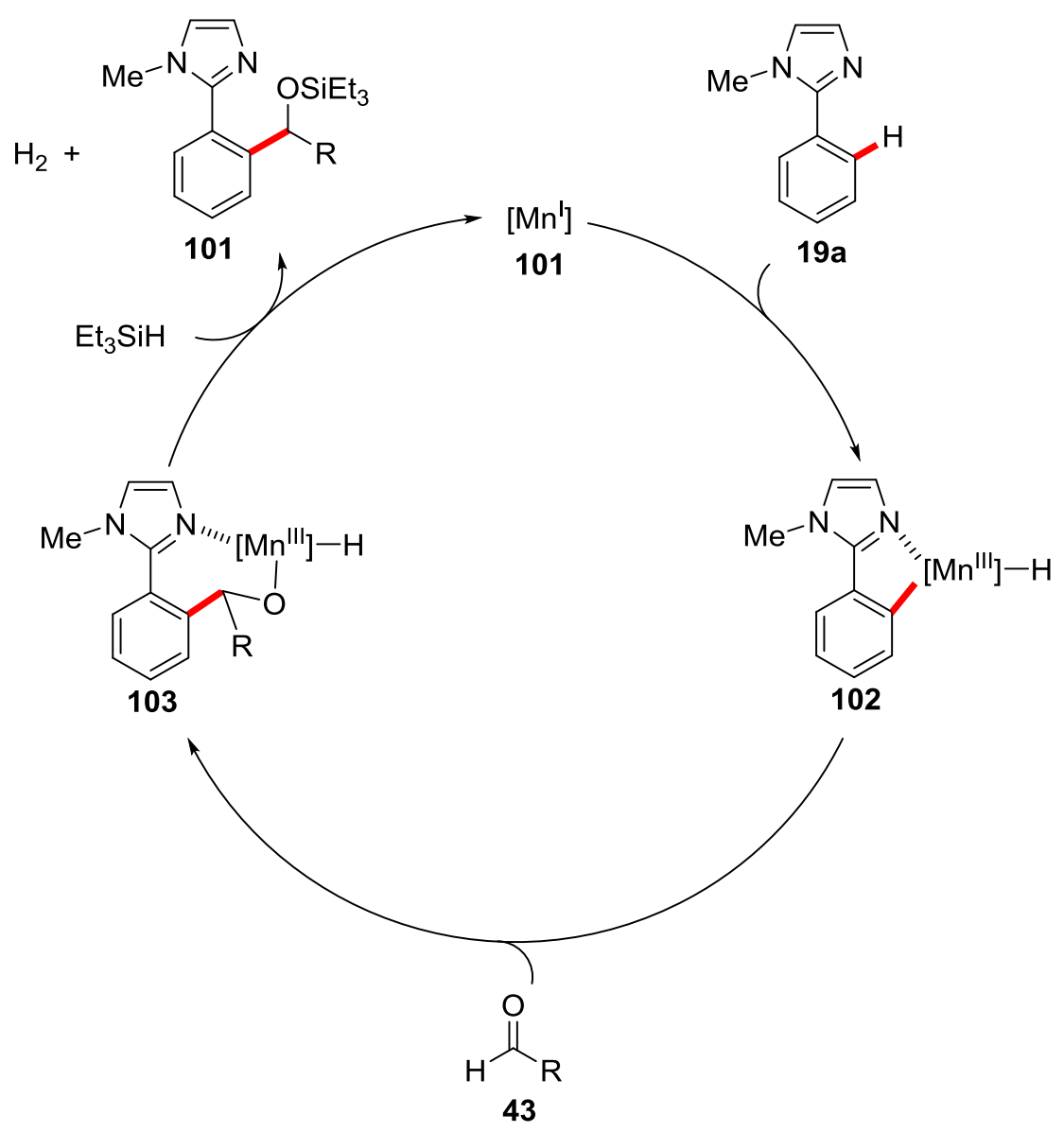

Scheme 34. Proposed catalytic cycle for the manganese(I)-catalyzed C-H addition to aldehydes 43.

Even though this reaction represented a significant breakthrough in manganese $\mathrm{C}-\mathrm{H}$ activation catalysis, a serious limitation was represented by the need for triethylsilane to achieve catalytic turnover. In contrast, Wang and coworkers succeeded in the development of a more general manganese(I)-catalyzed $\mathrm{C}-\mathrm{H}$ addition to aldehydes $\mathbf{4 3}$ by using $\mathrm{ZnBr}_{2}$ and $\mathrm{Me}_{2} \mathrm{Zn}$ (Scheme 35). ${ }^{\text {[9] }}$ The optimal reaction conditions allowed for aromatic as well as olefinic $\mathrm{C}-\mathrm{H}$ activation and various functional groups like halogens, ester-, and methoxy- groups were tolerated. Likewise, detailed mechanistic studies led to the hypothesis that $\mathrm{Me}_{2} \mathrm{Zn}$ was crucial for the in situ formation of the active catalytic species $\left[\mathrm{MnMe}(\mathrm{CO})_{5}\right]$, which facilitates the $\mathrm{C}-\mathrm{H}$ cyclomanganation step via oxidative addition.

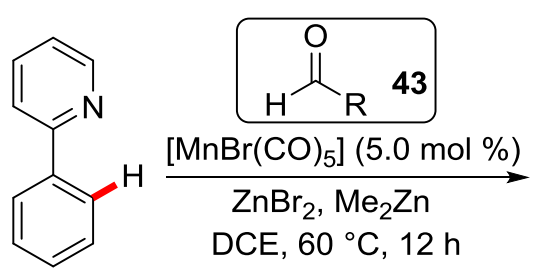

$1 a$<smiles>[R]C(O)c1ccccc1-c1ccccn1</smiles>

104

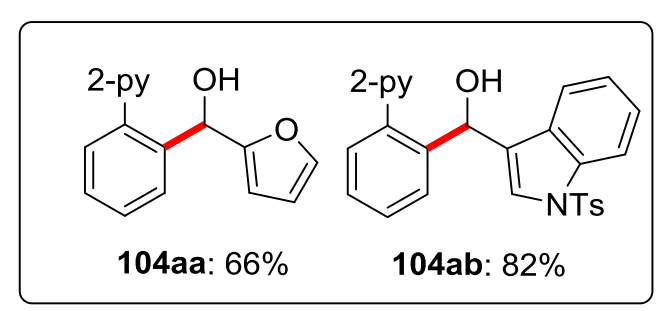

43. 
Additionally, Ackermann and coworkers reported on a manganese(I)-catalyzed aminocarbonylation of heteroarenes through $\mathrm{C}-\mathrm{H}$ addition to furnish isocyanates. ${ }^{[100]}$ The authors presented an ample scope of differently substituted 2-pyridylindoles $\mathbf{1 0 5}$ and a large variety of isocyanates $\mathbf{1 0 6}$ to afford the corresponding aminocarbonylated products $\mathbf{1 0 7}$ in very good yields. Notably, sensitive functional groups, such as ketones and esters, were well tolerated under the optimal reaction conditions.

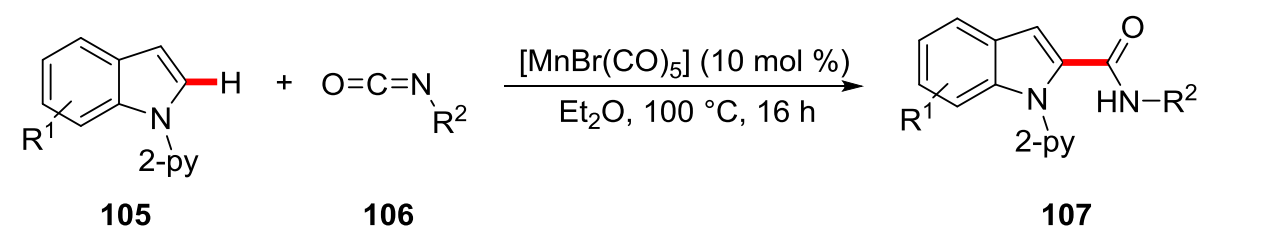

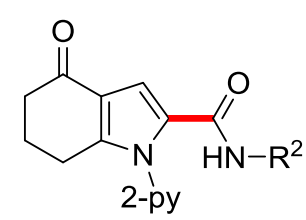

107a: $81 \%$<smiles>COC(=O)c1ccc2cc(C(=O)Nc3ccccc3)n(CC(=O)c3ccccc3)c2c1</smiles>

107b: $82 \%$

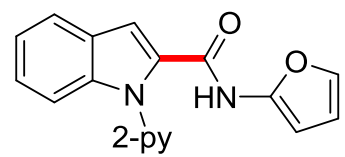

107c: $62 \%$

Scheme 36. Manganese(I)-catalyzed aminocarbonylation.

Recently, Ackermann and coworkers disclosed a manganese(I)-catalyzed cyanation reaction on several heterocycles (Scheme 37). ${ }^{[101]}$ Overall, a variety of functional groups was tolerated, including esters, ketones, and tryptophans. The authors introduced the new and more electrophilic cyanation reagent $\mathrm{N}$ cyano- $N$-(4-fluorophenyl)-4-methylbenzenesulfonamide (NCFS) (108) and compared its reactivity with Beller's well-established $N$-cyano- $N$-phenyl-p-toluenesulfonamide (NCTS) (56). ${ }^{[102]}$ Thus, with respect to the cyanation of very challenging tryptophan 110, NCFS was shown to be a more powerful cyanation reagent than NCTS. ${ }^{[101]}$

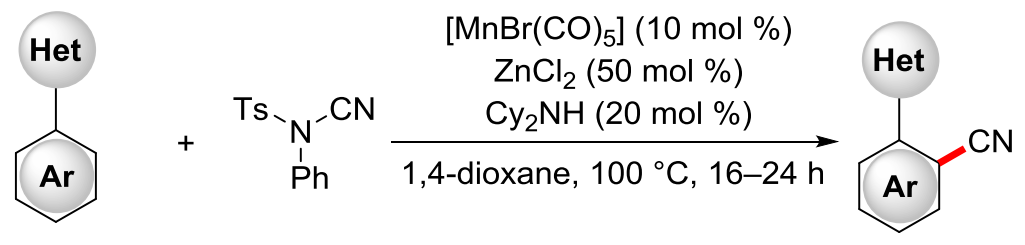

55

57<smiles>N#Cc1cc2ccccc2n1C#N</smiles>

2-pym<smiles>N#Cc1cccn1COn1cccc1</smiles>

59: $86 \%$<smiles>[Y6]n1c(C#N)cc2c1CCCC2=O</smiles>

109: $83 \%$<smiles>CC(=O)[C@H](Cc1c(C#N)n([Tl])c2ccccc12)Nc1ccccc1</smiles>

110: $58 \%$ (NCTS)

110: $68 \%$ (NCFS)

Scheme 37. Manganese(I)-catalyzed C-H cyanation. 
Intermolecular competition experiments with electron-donating and electron-withdrawing substituents indicated a clear preference for electron-rich substrates. Moreover, H/D-exchange experiments and a KIE study revealed a fully reversible $\mathrm{C}-\mathrm{H}$ activation step to be at work. In addition, detailed DFT studies were supportive of $\mathrm{ZnCl}_{2}$ to act as a Lewis acid and to facilitate the turnover-limiting migratory insertion step in a hetero-bimetallic fashion.

However, the reactivity of manganacycles was not only limited to addition reactions with strong electrophiles. Indeed, the insertion of inherently less polar carbon-carbon multiple bonds proved to be viable as well. Thus, Wang and coworkers accomplished the first manganese(I)-catalyzed alkenylation reaction with terminal alkynes 111 using $\left[\mathrm{MnBr}(\mathrm{CO})_{5}\right]$ as the catalyst and $\mathrm{Cy}_{2} \mathrm{NH}$ as the crucial base additive. $^{[103]}$

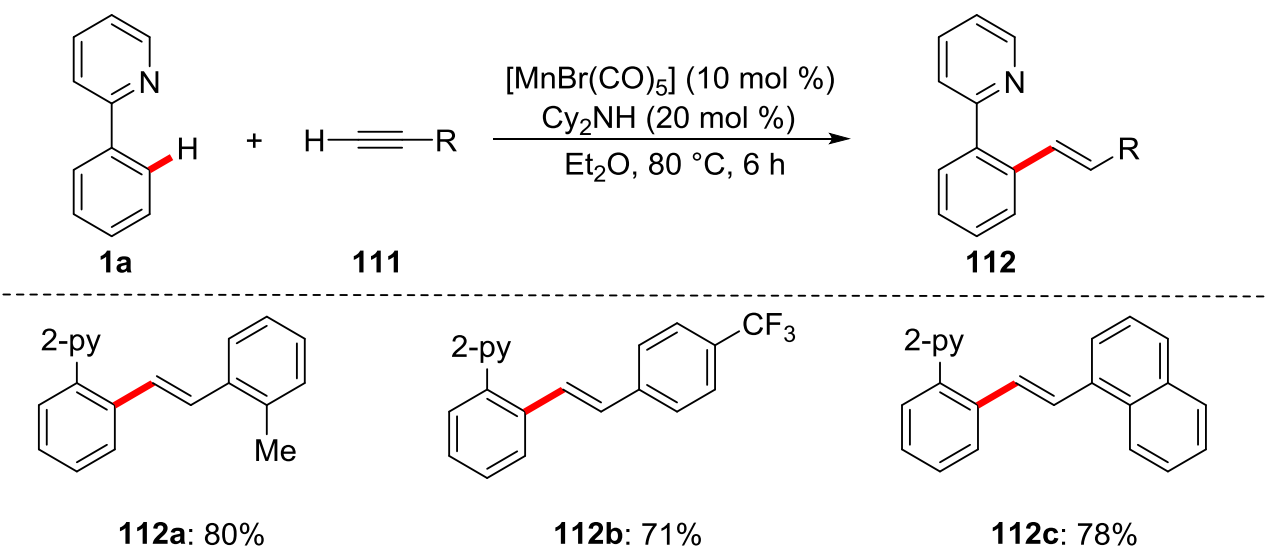

Scheme 38. Manganese(I)-catalyzed C-H alkenylation.

In this reaction, a variety of aryl- as well as alkyl-substituted terminal alkynes $\mathbf{1 1 1}$ reacted smoothly to provide the (E)-configurated alkenes $\mathbf{1 1 2}$ with perfect anti-Markovnikov selectivity. Finally, the authors performed DFT calculations to shed light on the reaction mechanism. Based on these studies, it was proposed that $\mathrm{Cy}_{2} \mathrm{NH}$ facilitates the $\mathrm{C}-\mathrm{H}$ activation step via a base-assisted deprotonative pathway.

Thereafter, the same group extended the manganese(I)-catalyzed hydroarylation protocol to electronically activated $\alpha, \beta$-unsaturated carbonyls 113 (Scheme 39). ${ }^{[104]}$ The reaction proceeded with excellent mono-selectivities, delivering the corresponding products 114 in satisfactory yields. 


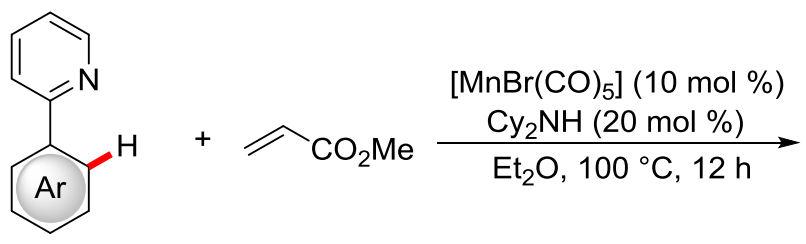

1

113

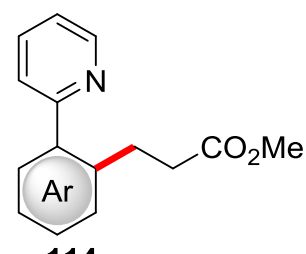

114<smiles>[R7]c1cc(C(=O)OCC)ccc1CCC(C)=O</smiles>

114a: $66 \%$<smiles>COc1ccc([18O])c(CCC(C)=O)c1</smiles>

114b: $63 \%$

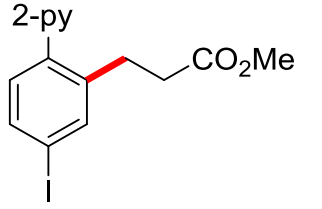

114c: $74 \%$

Scheme 39. Manganese(I)-catalyzed hydroarylation of $\alpha, \beta$-unsaturated carbonyls 113.

Furthermore, Wang and coworkers demonstrated that the $\mathrm{C}-\mathrm{H}$ hydroarylation strategy turned out to be applicable in $\mathrm{C}-\mathrm{H} / \mathrm{N}-\mathrm{H}$ annulation reactions with imines $\mathbf{1 1 5}$ and terminal 111 as well as internal alkynes 48 to deliver isoquinolines 116 (Scheme 40). ${ }^{[105]}$ In addition to a broad substrate scope with good yields and regioselectivities, the authors unraveled large parts of the reaction mechanism.<smiles>N=C(N)c1ccccc1</smiles>

115
$+\mathrm{R}^{1}=\mathrm{R}^{2} \frac{\left[\mathrm{MnBr}(\mathrm{CO})_{5}\right](10 \mathrm{~mol} \%)}{1,4 \text {-dioxane, } 105^{\circ} \mathrm{C}, 12 \mathrm{~h}}$

48 or 111

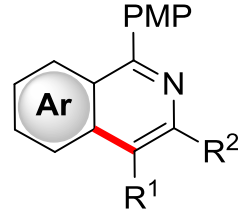

116

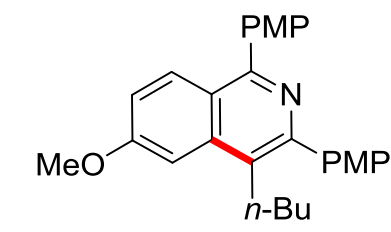

116aa: $94 \%(9: 1)$<smiles>CCCNc1nc(P=P)c2ccc(OC)cc2c1P(CCC)C(C)C</smiles>

116ab: $73 \%$<smiles>COc1ccc2c(P)nc(-c3ccccc3)cc2c1</smiles>

116ac: $87 \%$

Scheme 40. Manganese(I)-catalyzed C-H/N-H annulation.

Thus, the authors proposed a catalytic cycle (Scheme 41), which is commenced by a presumably turnover-limiting $\mathrm{C}-\mathrm{H}$ activation step to form manganacycle 117. After ligand exchange of $\mathrm{CO}$ by alkyne-coordination, a migratory insertion leads to intermediate 119, which is prone to undergo reductive elimination to release the desired product $\mathbf{1 1 6}$ and generates a manganese-hydride species 120. The latter reacts with a new imine $\mathbf{1 1 5}$ to again deliver manganacycle $\mathbf{1 1 7}$ through $\mathrm{C}-\mathrm{H}$ manganation under evolution of hydrogen. 


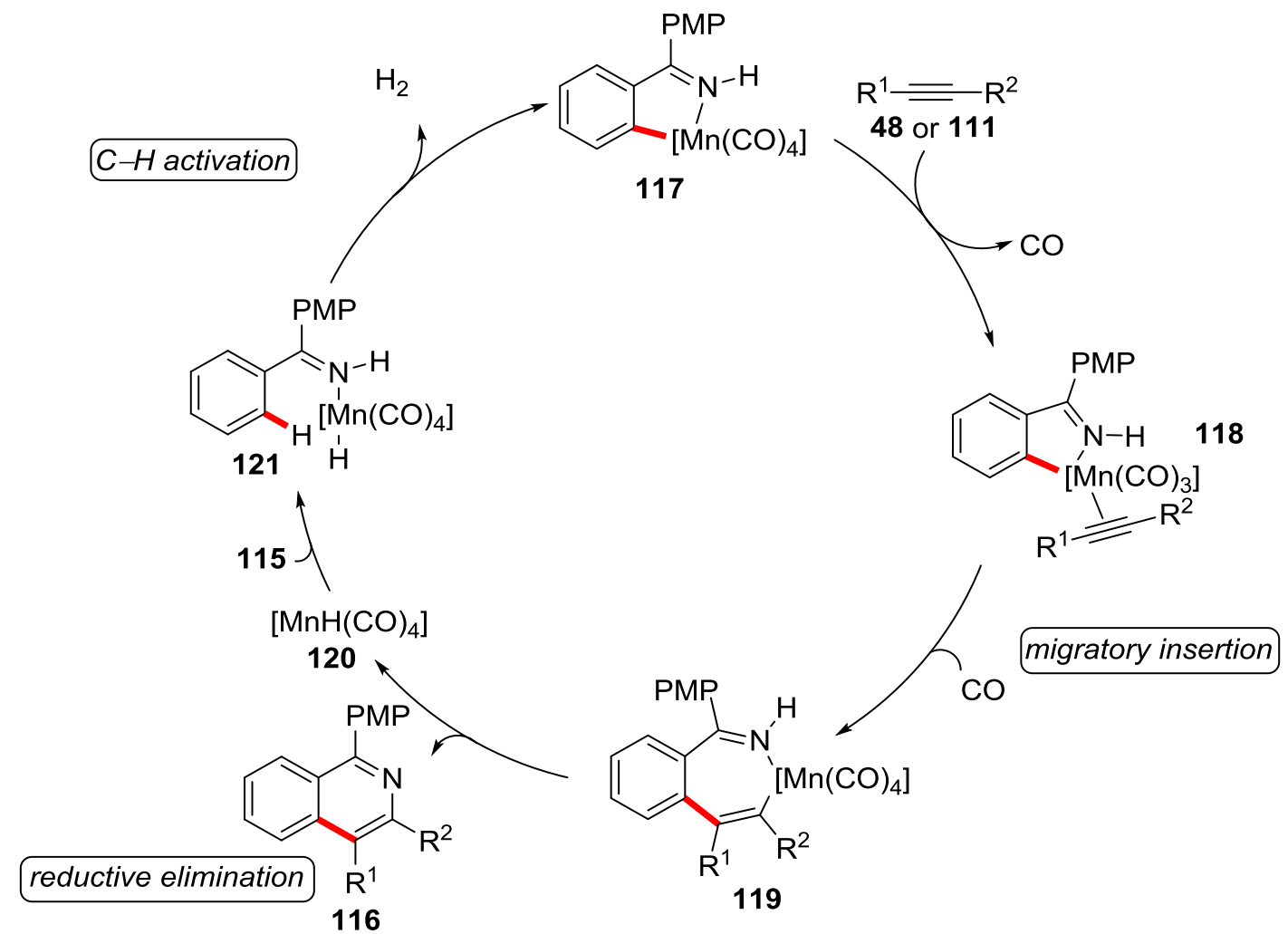

Scheme 41. Postulated catalytic cycle for the manganese(I)-catalyzed C-H/N-H annulation.

Ackermann and coworkers reported on an manganese-catalyzed $\mathrm{C}-\mathrm{H}$ annulation with acrylates ${ }^{[106]}$ to afford highly valuable $\beta$-amino esters ${ }^{[107]} 122$ (Scheme 42 ) in good yields and overall intriguing levels of diastereoselectivities.
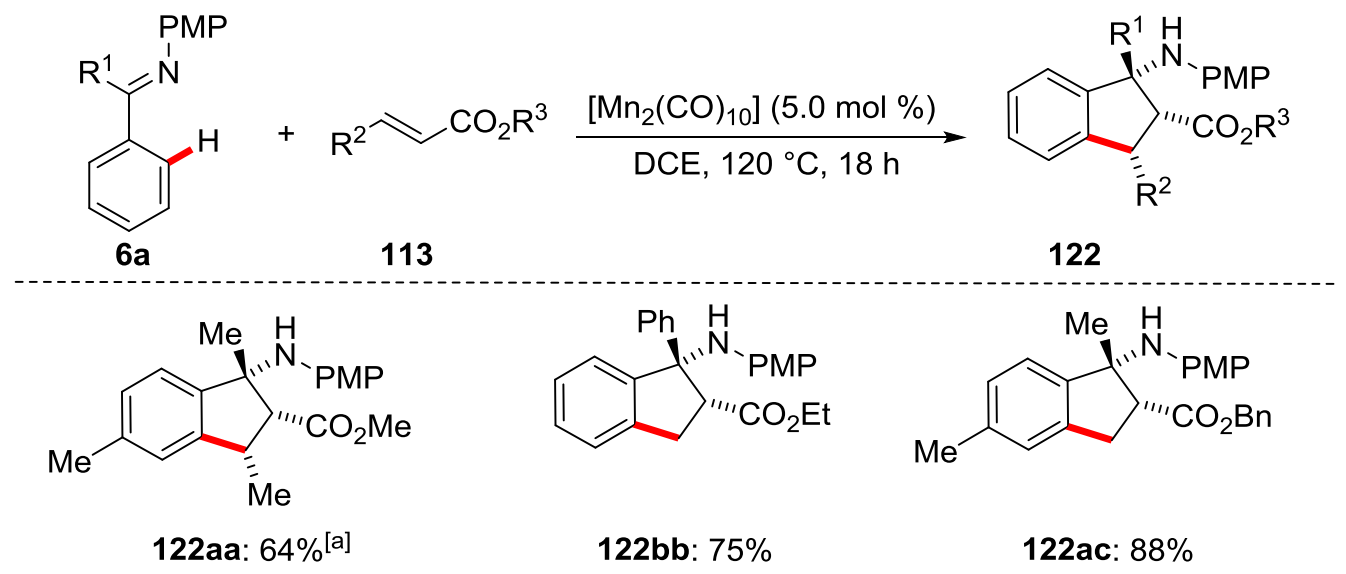

Scheme 42. Manganese-catalyzed synthesis of $\beta$-amino esters via a $\mathrm{C}-\mathrm{H}$ annulation process. [a] $\left[\mathrm{Mn}_{2}(\mathrm{CO})_{10}\right](10 \mathrm{~mol} \%)$ in PhMe.

In addition to the previously described substitutive cobalt(III)-catalyzed $\mathrm{C}-\mathrm{H}$ allylation with allyl carbonates 67 ( $c f$. Scheme 22), manganese catalysis was shown to be a competent alternative to enable $\mathrm{C}-\mathrm{H}$ allylations. 
In this regard, the Ackermann group reported on a $\mathrm{C}-\mathrm{H}$ allylation of ketimines $\mathbf{6}$ with allyl carbonates 67, delivering the corresponding allylated ketones 123 in excellent yields and good diastereoselectivities upon acidic workup (Scheme 43). ${ }^{[108]}$
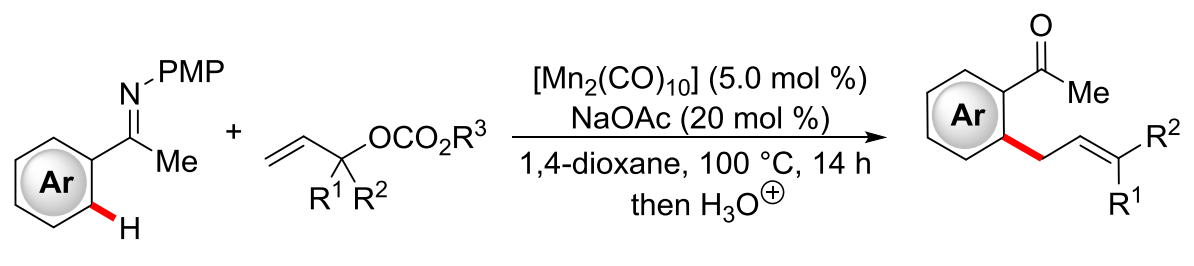

6

67

123<smiles>C=CCc1cc(C#N)ccc1C(C)=O</smiles>

123aa: $87 \%$<smiles>CC(=O)c1ccc(C)cc1C/C=C/c1ccccc1</smiles>

123bb: $68 \%(47: 1)^{[a]}$<smiles>CC(=O)c1ccc(C)cc1CC=C(C)C</smiles>

123bc: $67 \%$ [a,b]

Scheme 43. Manganese-catalyzed $\mathrm{C}-\mathrm{H}$ allylation with allyl carbonates 67. [a] At $120^{\circ} \mathrm{C}$. [b] $\left[\mathrm{Mn}_{2}(\mathrm{CO})_{10}\right](10 \mathrm{~mol} \%)$. 


\section{Objectives}

The development of novel strategies for highly stereoselective transition metal-catalyzed $\mathrm{C}-\mathrm{C}$ bond forming processes is of prime importance for expanding the toolbox of modern organic synthesis. In recent years, $\mathrm{C}-\mathrm{H}$ bond functionalizations ${ }^{[13]}$ have emerged as a powerful reaction class due to their synthetic versatility and excellent atom- ${ }^{[10]}$ and step-economy. ${ }^{[11]}$ Thus, the focus of this thesis is directed towards exploring novel cost-effective and environmentally benign $\mathrm{C}-\mathrm{H}$ functionalizations. ${ }^{[12 b]}$ In this context, particularly the efficiency of catalysts should be studied that are based on rather inexpensive ruthenium as well as earth-abundant base metals such as cobalt and manganese. ${ }^{[54]}$

In the past decade, invaluable advances in ruthenium-catalyzed $\mathrm{C}-\mathrm{H}$ arylations were inter alia achieved by Ackermann and coworkers, highlighting powerful carboxylates as the additives of choice. ${ }^{[20 \mathrm{a}, 109]}$ Nevertheless, also the use of secondary phosphine oxides (SPOs) as preligands has been revealed to be highly beneficial. ${ }^{[36]}$ Within this thesis, the performance of well-defined and easily modifiable ruthenium(II)-phosphinous acid (PA) complexes 124 in the $\mathrm{C}-\mathrm{H}$ arylation of $(E)$ styrylpyridines 9 as challenging acyclic alkene substrates should be explored (Scheme 44). Moreover, detailed mechanistic studies should elucidate the pathways of the $\mathrm{C}-\mathrm{H}$ and the $\mathrm{C}-\mathrm{X}$ bond activation steps.

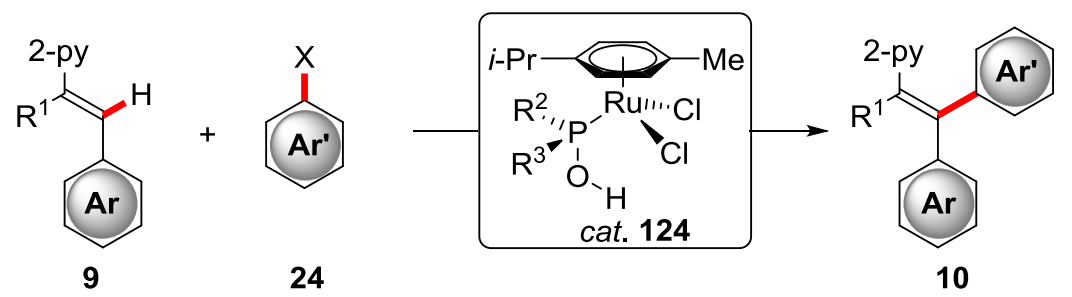

Scheme 44. Ruthenium(II)-PA-catalyzed C-H arylation.

Despite of undisputed progress in the field of cobalt(III)-catalyzed $\mathrm{C}-\mathrm{H}$ bond activations, ${ }^{[15]}$ there is still a lack of methods that merge $\mathrm{C}-\mathrm{H}$ with $\mathrm{C}-\mathrm{C}^{[110]}$ functionalizations in a tandem process. In this regard, vinylcyclopropanes $\mathbf{1 2 5}^{[111]}$ are due to their ring strain ideal synthons for achieving the first cobalt-catalyzed C-H/C-C functionalization, along with detailed mechanistic studies (Scheme 45).

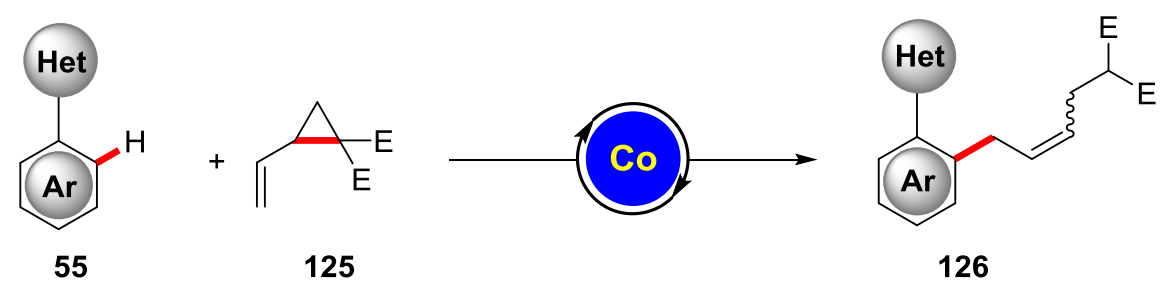

Scheme 45. Cobalt(III)-catalyzed $\mathrm{C}-\mathrm{H} / \mathrm{C}-\mathrm{C}$ functionalization.

So far, there have been numerous reports on transition metal-catalyzed C-H hydroarylations, ${ }^{[12]}$ with a plethora of metals and broad synthetic applicability. Thus far, the control of Markovnikov/anti- 
Markovnikov regioselectivity has relied on the use of expensive ligands ${ }^{[113]}$ and was mostly restricted to electronically activated styrenes $\mathbf{8 2} .^{[114]}$ Within this thesis, a new concept for regiocontrol in $\mathrm{C}-\mathrm{H}$ hydroarylations of unactivated alkenes should be developed (Scheme 46). This approach should encompass the design of reaction conditions to easily switch between branched and linear selectivity.

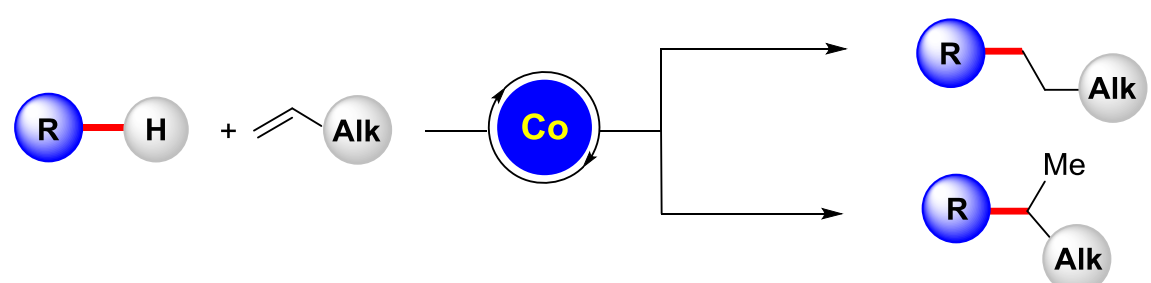

Scheme 46. Control of branched/linear selectivity in cobalt(III)-catalyzed C-H hydroarylations.

There is a strong demand for novel synthetic methods to install fluorine in organic molecules, ${ }^{[115]}$ because fluorine is known to improve several physicochemical properties of e.g. pharmaceuticals, such as metabolic stability, lipophilicity and membrane permeability. ${ }^{[16]}$ So far, several methods for $\mathrm{C}-\mathrm{H} / \mathrm{C}-\mathrm{X}$ functionalizations are known that are based on feasible $\beta$-carbon, ${ }^{[117]}$-oxygen, ${ }^{[75,77-78]}$ or nitrogen $^{[74,91,117 \mathrm{a}]}$ eliminations. Thus, a cobalt(III)-catalyzed $\mathrm{C}-\mathrm{H}$ activation was intended to be combined with $\mathrm{C}-\mathrm{F}$ functionalizations ${ }^{[18]}$ through $\beta$-fluoride elimination ${ }^{[119]}$ using perfluoroalkylalkenes 127 and 1,1-difluoroalkenes 128 (Scheme 47).

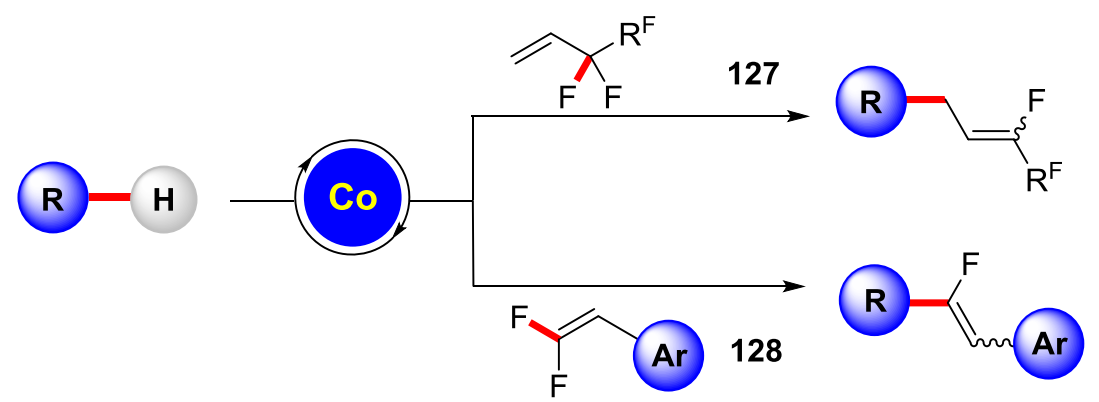

Scheme 47. Cobalt(III)-catalyzed allylative and alkenylative C-H/C-F functionalization.

In recent years, a tremendous progress has been achieved in utilizing manganese $(\mathrm{I})$ catalysts for $\mathrm{C}-\mathrm{H}$ functionalizations ${ }^{[14]}$ with partly unique levels of selectivity control. ${ }^{[117 \mathrm{~d}, 120]}$ Thus, the development of a novel manganese(I)-catalyzed C-H/C-F functionalization was intended (Scheme 48). Particularly, a comparison with the analogous cobalt(III)-catalyzed reaction in terms of reactivities and selectivities should be of prime importance for revealing unique features of metals in $\mathrm{C}-\mathrm{H}$ activation catalysis. 


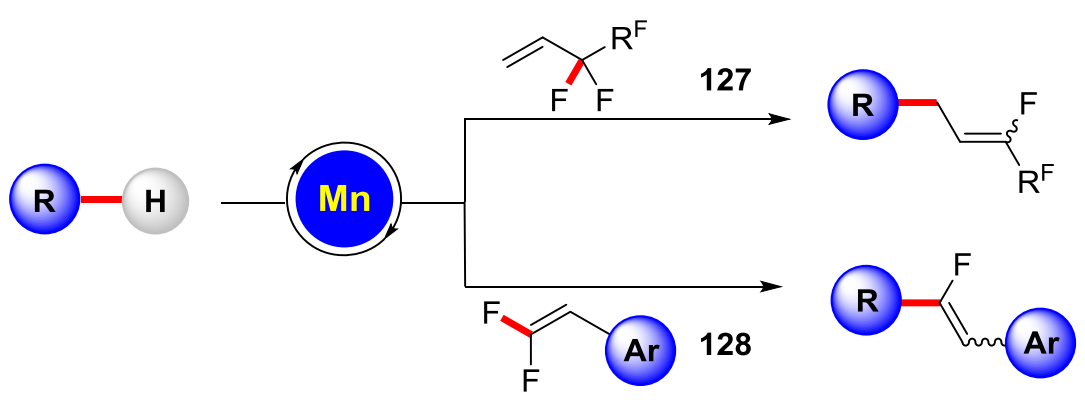

Scheme 48. Manganese(I)-catalyzed allylative and alkenylative $\mathrm{C}-\mathrm{H} / \mathrm{C}-\mathrm{F}$ functionalization. 


\section{Results and Discussion}

\subsection{C-H Arylations by Ruthenium(II)-Phosphinous Acid Catalysts}

The last years have witnessed significant progress in the development of ruthenium catalyzed $\mathrm{C}-\mathrm{H}$ arylations. In this context, especially well-defined ruthenium(II)-carboxylate catalysts have proven to be powerful catalysts with a broad applicability and excellent functional group tolerance. ${ }^{\text {[20a, } 109 a]}$ However, there is still a strong demand for the synthesis of novel and efficient ruthenium catalysts, which could be used for applications in inter alia polymer chemistry and material sciences. ${ }^{[121]}$ For this purpose, highly reactive and bench-stable catalysts are needed which bear an easily modifiable ligand such as phosphinous acid. Thus, the exploration of versatile single-component ruthenium(II)-PA complexes 124, their use in $\mathrm{C}-\mathrm{H}$ arylations, and new mechanistic insights represent a great opportunity to raise the potential of ruthenium-catalyzed $\mathrm{C}-\mathrm{H}$ arylation methods.

\subsubsection{Optimization Studies}

We initiated our optimization studies by probing the effect of different ruthenium(II) catalysts on the desired $\mathrm{C}-\mathrm{H}$ arylation of (E)-2-styrylpyridine (9a) (Table 1). In this context, conventionally used catalytic precursors like $\left[\mathrm{RuCl}_{3} \cdot \mathrm{H}_{2} \mathrm{O}\right]^{[47-48]}$ and $\left[\mathrm{RuCl}_{2}(p \text {-cymene) }]_{2}\right.$ (entries 1 and 2) were clearly outcompeted by well-defined single component phosphinous acid (PA) ruthenium(II) catalysts $\mathbf{1 2 4},{ }^{[122]}$ that have been synthesized following an optimized protocol by $S$. Warratz. ${ }^{[123]}$ It is noteworthy that in the absence of any ruthenium precursor the desired product 10ab was not formed (entry 3). To elucidate the role of the substitution pattern on the phosphorous atom of the phosphinous acid ligand, the reactivity of different ruthenium(II)-PA complexes $\mathbf{1 2 4}$ with aliphatic as well as aromatic substituents has been studied (entries 4-10). Aromatic substituents only led to moderate yields, with a decrease in reactivity for electron-poor substituents (entry 5). In contrast, a clearly higher reactivity was achieved by employing alkyl-substituted PA-ligands (entries 7 and 8). However, a sterically more congested substitution pattern led to only unsatisfactory results (entries 9 and 10), which reveals that a balanced situation between the repulsive steric interactions and electronic parameters of the PA-ligand is necessary for an efficient transformation.

Generally, it can be speculated that electron-donating substituents on the phosphorous atom increase the basicity of the deprotonated phosphinous acid ligand, which acts as internal base during the metalligand cooperated $\mathrm{C}-\mathrm{H}$ activation. ${ }^{[36 a, 123]}$ Consequently, a faster $\mathrm{C}-\mathrm{H}$ activation elementary step would occur. In addition, the contribution of attractive London dispersion interactions ${ }^{[124]}$ to the stabilization of the $\mathrm{C}-\mathrm{H}$ activation transition state should be considered as well, since a significant rate acceleration by using diadamantyl-phosphine oxides as preligands has been observed for previously reported ruthenium(II)-catalyzed C-H arylations. ${ }^{[36]}$ 
Table 1. Optimization for the $\mathrm{C}-\mathrm{H}$ arylation of (E)-2-styrylpyridine (9a) with aryl bromides 2.<smiles>COc1ccc(Br)cc1</smiles>

9a
$2 b$

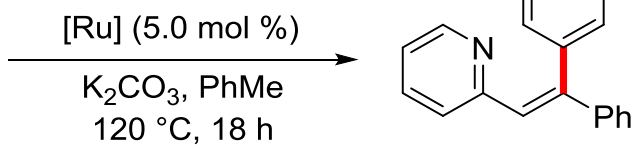

$10 \mathrm{ab}$

\begin{tabular}{|c|c|c|}
\hline Entry & {$[\mathrm{Ru}]$} & yield / \% ${ }^{[\mathrm{a}]}$ \\
\hline 1 & {$\left[\mathrm{RuCl}_{3} \cdot x \mathrm{H}_{2} \mathrm{O}\right]$} & $<5^{[\mathrm{b}]}$ \\
\hline 2 & {$\left[\mathrm{RuCl}_{2}(p \text {-cymene })\right]_{2}$} & 9 \\
\hline 3 & --- & $---[b, c]$ \\
\hline 4 & {$\left[\mathrm{RuCl}_{2}(p\right.$-cymene $\left.)\left(\mathrm{Ph}_{2} \mathrm{POH}\right)\right](\mathbf{1 2 4 a})$} & 77 \\
\hline 5 & {$\left[\mathrm{RuCl}_{2}(p\right.$-cymene $\left.)\left\{\left(p-\mathrm{FC}_{6} \mathrm{H}_{4}\right)_{2} \mathrm{POH}\right\}\right](\mathbf{1 2 4 b})$} & 60 \\
\hline 6 & {$\left[\mathrm{RuCl}_{2}(p\right.$-cymene $\left.)(t-\mathrm{BuPhPOH})\right](\mathbf{1 2 4 c})$} & 51 \\
\hline 7 & {$\left[\mathrm{RuCl}_{2}(p\right.$-cymene $\left.)\left(i-\mathrm{Pr}_{2} \mathrm{POH}\right)\right](\mathbf{1 2 4 d})$} & 92 \\
\hline 8 & {$\left[\mathrm{RuCl}_{2}(p\right.$-cymene $\left.)\left(n-\mathrm{Bu}_{2} \mathrm{POH}\right)\right](124 \mathrm{e})$} & 96 \\
\hline 9 & {$\left[\mathrm{RuCl}_{2}(p\right.$-cymene $\left.)\left(\mathrm{Cy}_{2} \mathrm{POH}\right)\right](\mathbf{1 2 4 f})$} & 53 \\
\hline 10 & {$\left[\mathrm{RuCl}_{2}(p\right.$-cymene $\left.)\left(o-\mathrm{Tol}_{2} \mathrm{POH}\right)\right](\mathbf{1 2 4 g})$} & 71 \\
\hline
\end{tabular}

[a] Reaction conditions: 9a $(0.50 \mathrm{mmol}), \mathbf{2 b}(0.75 \mathrm{mmol}),[\mathrm{Ru}](5.0 \mathrm{~mol} \%), \mathrm{K}_{2} \mathrm{CO}_{3}(1.00 \mathrm{mmol}), \mathrm{PhMe}$ $(0.25 \mathrm{M}), 120^{\circ} \mathrm{C}, 18 \mathrm{~h}$, isolated yield. [b] Determined by GC with $n$-dodecane as the internal standard. [c] $n$ $\mathrm{Bu}_{2} \mathrm{P}(\mathrm{O}) \mathrm{H}(20 \mathrm{~mol} \%)$ as additive.

Encouraged by these preliminary optimization results, the catalytic performance of the optimal ruthenium(II)-PA complex 124e was furthermore tested by reducing the catalyst loading (Table 2), employing slightly higher concentrations and reaction temperatures. Surprisingly, catalyst loadings as low as $0.90 \mathrm{~mol} \%$ still provided an excellent conversion to the desired product 10ab (entry 4), while even lower catalyst loadings led to a slightly decreased reactivity (entry 5). 
Table 2. Reduction of the catalyst loading.<smiles>COc1ccc(Br)cc1</smiles>

$9 a$

2b
$\left[\mathrm{RuCl}_{2}(p\right.$-cymene $\left.)\left(n-\mathrm{Bu}_{2} \mathrm{POH}\right)\right](\mathbf{1 2 4 e})$ ( $x$ mol \%) $\mathrm{K}_{2} \mathrm{CO}_{3}, \mathrm{PhMe}$ $140^{\circ} \mathrm{C}, 18 \mathrm{~h}$<smiles>COc1ccc(/C(=C/c2ccccn2)c2ccccc2)cc1</smiles>

$10 a b$

\begin{tabular}{ccc}
\hline Entry & $x /$ mol $\%$ & yield / $\%^{[\mathrm{a}]}$ \\
\hline 1 & 5.00 & 99 \\
2 & 2.50 & 99 \\
3 & 1.00 & 98 \\
4 & 0.90 & 98 \\
5 & 0.75 & 82 \\
\hline
\end{tabular}

[a] Reaction conditions: 9a $(0.50 \mathrm{mmol}), 2 \mathbf{b}(0.75 \mathrm{mmol}),[\mathrm{Ru}](5.0 \mathrm{~mol} \%), \mathrm{K}_{2} \mathrm{CO}_{3}(1.00 \mathrm{mmol}), \mathrm{PhMe}$ $(1.00 \mathrm{M}), 140{ }^{\circ} \mathrm{C}, 18 \mathrm{~h}$. Conversions determined by means of GC with $n$-dodecane as the internal standard.

\subsubsection{Scope of the C-H Arylation with Aryl Bromides}

With the optimized catalytic system in hand, the substrate scope for the $\mathrm{C}-\mathrm{H}$ arylation of $(E)-2$ styrylpyridine (9a) with aryl bromides $\mathbf{2}$ was explored. To start with, different substitution patterns as well as valuable functional groups on the aryl bromide $\mathbf{2}$ were extensively tested (Table 3). Furthermore, a variation of substituents in para-position of the aryl bromide $\mathbf{2}$ led to overall good to excellent yields. However, no clear reactivity trend with respect to the electronic nature of the aryl bromide 2 (entries 1-8) could be disclosed. Notably, even challenging functional groups, such as esters, amines, as well as enolizable ketones, were well tolerated under the optimal reaction conditions. Furthermore, substituents in meta- and ortho-positions (entries 9 and 10) were well accepted and afforded the desired arylated products $\mathbf{1 0}$ in good yields under slightly altered reaction conditions for the ortho-substituted aryl bromide $\mathbf{2 j}$. Nevertheless, a significant limitation was observed by using the sterically very congested aryl bromide $\mathbf{2 k}$ (entry 11). ${ }^{[125]}$ The electron-rich heteroarene 3-bromothiophene (2l) afforded the corresponding product $\mathbf{1 0 1}$ in very good yield (entry 12), together with detectable amounts of the diastereomer $(Z / E=96: 4)$, which might be rationalized in terms of a thermal isomerization. 
Table 3. Scope of the ruthenium(II)-PA-catalyzed C-H arylation with aryl bromides 2.

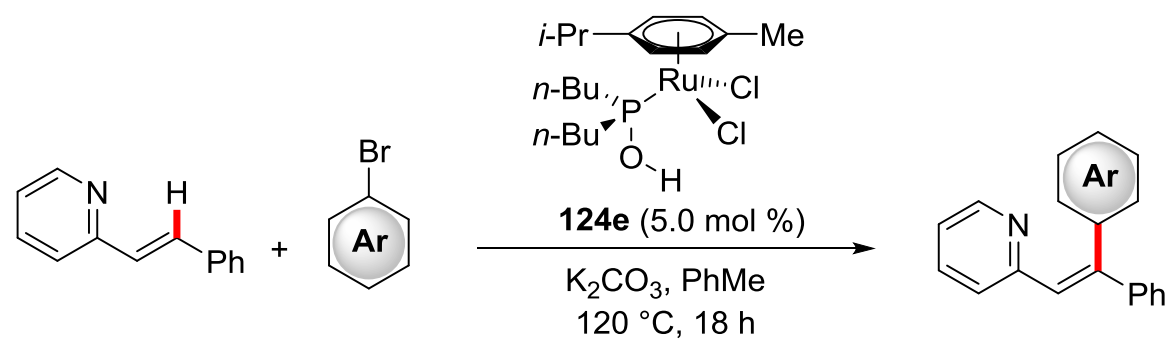

9a

2

\begin{tabular}{|c|c|c|c|c|c|}
\hline Entry & aryl bromide & 2 & product & $10 \mathrm{a}$ & yield / $\%^{[\mathrm{a}]}$ \\
\hline 1 & $-\mathrm{NMe}_{2}$ & $2 c$ & & $10 a c$ & 94 \\
\hline 2 & $\mathrm{OMe}$ & $2 b$ & & 10ab & 96 \\
\hline 3 & & 2d & & 10ad & 93 \\
\hline 4 & & $2 \mathbf{a}$ & & 10aa & 80 \\
\hline 5 & & $2 e$ & & 10ae & 92 \\
\hline 6 & $-\mathrm{CF}_{3}$ & $2 f$ & & 10af & 71 \\
\hline 7 & $\searrow-\mathrm{CO}_{2} \mathrm{Et}$ & $2 \mathrm{~g}$ & & 10ag & 87 \\
\hline
\end{tabular}


8<smiles>CC(=O)c1ccc(Br)cc1</smiles>

9<smiles>COc1cccc(Br)c1</smiles>

10<smiles>COc1ccccc1Br</smiles>

11<smiles>Cc1cc(C)c(Br)c(C)c1</smiles>

12
$2 h$<smiles>CC(=O)c1ccc(C(=Cc2ccccn2)c2ccccc2)cc1</smiles>

$2 \mathbf{i}$<smiles>COc1cccc(/C(=C\c2ccccn2)c2ccccc2)c1</smiles>

$2 \mathbf{j}$<smiles>COc1ccccc1/C(=C\c1ccccn1)c1ccccc1</smiles>

2k

$--$<smiles>C(=C(c1ccccc1)c1ccsc1)c1ccccn1</smiles>

10ai

80

10aj

$81^{[\mathrm{b}]}$

10ah

87

10ak

$-{ }_{--}^{[b]}$

10al

$87^{[\mathrm{c}]}$

[a] Reaction conditions: 9a $(0.50 \mathrm{mmol}), 2(0.75 \mathrm{mmol}),[\mathrm{Ru}](5.0 \mathrm{~mol} \%), \mathrm{K}_{2} \mathrm{CO}_{3}(1.00 \mathrm{mmol}), \mathrm{PhMe}(2.0 \mathrm{~mL}$, $0.25 \mathrm{M}), 120{ }^{\circ} \mathrm{C}, 18 \mathrm{~h}$, isolated yields. [b] At $140{ }^{\circ} \mathrm{C}, \mathrm{PhMe}(1.00 \mathrm{M})$. [c] $\mathrm{Z} / E=96: 4$, determined by means of ${ }^{1} \mathrm{H}-$ NMR spectroscopy.

Having revealed the broad applicability of the reaction with respect to electronic and steric parameters of the aryl bromide $\mathbf{2}$, the substitution pattern of (E)-2-styrylpyridine (9a) was tested next (Table 4). Herein, a slight preference for electron-rich substrates with methyl- (9b) and methoxy-substituents (9c) (entries 1-5) as compared to the electron-poor trifluoromethyl-substituted (E)-2-styrylpyridine (9d) (entries 6-8) was observed, albeit the overall high reactivity of the catalytic system might have levelled this effect. 
Results and Discussion

41

Table 4. Scope of (E)-2-styrylpyridines 9.<smiles>[R]c1ccc(/C=C/c2ccccn2)cc1</smiles>

9

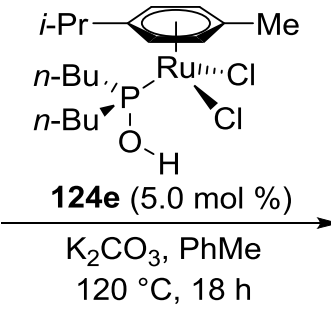

2<smiles>[R]c1ccc(C(=Cc2ccccn2)c2ccc([R])cc2)cc1</smiles>

10

Entry

$\mathrm{R}^{1}$

$\mathrm{R}^{2}$

product

10

yield / \% ${ }^{\text {[a] }}$

1

$\mathrm{Me}$

2

$\mathrm{Me}$

3

$\mathrm{Me}$

4

$\mathrm{OMe}$<smiles>COc1ccc(C(=Cc2ccccn2)c2ccc(C)cc2)cc1</smiles>

$\mathrm{CO}_{2} \mathrm{Et}$

5

$\mathrm{OMe}$
$\mathrm{OMe}$

10bb
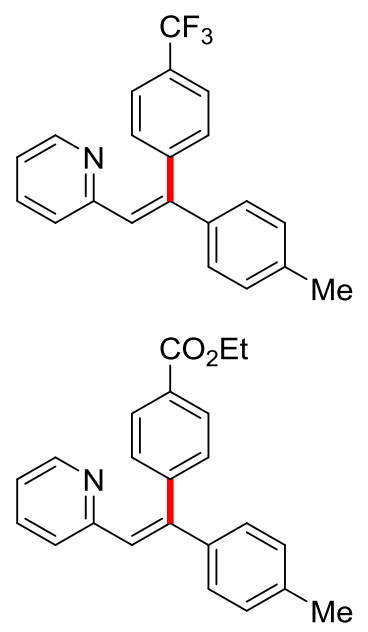<smiles>COc1ccc(C(=Cc2ccccn2)c2ccc(OC)cc2)cc1</smiles>

$\mathrm{OMe}$

$\mathrm{CF}_{3}$<smiles>COc1ccc(C(=Cc2ccccn2)c2ccc(C(F)(F)F)cc2)cc1</smiles>

$10 b g$

10bf

85

10cb

93

90

87

10cf

84 
6

7

8
$\mathrm{CF}_{3}$

$\mathrm{OMe}$<smiles>COc1ccc(C(=Cc2ccccn2)c2ccc(C(F)(F)F)cc2)cc1</smiles>

10db

90

10df

77<smiles>C=C(C)C(=Cc1ccccn1)c1ccc(C(F)(F)F)cc1</smiles>

10dh

78

[a] Reaction conditions: 9 (0.50 mmol), $2(0.75 \mathrm{mmol}),[\mathrm{Ru}](5.0 \mathrm{~mol} \%), \mathrm{K}_{2} \mathrm{CO}_{3}(1.00 \mathrm{mmol}), \mathrm{PhMe}(0.25 \mathrm{M})$, $120{ }^{\circ} \mathrm{C}, 18 \mathrm{~h}$, isolated yield.

It is noteworthy that the sterically encumbered trisubstituted alkene $\mathbf{9 e}$ was successfully converted to the fully substituted acyclic alkene 10eb, displaying the first synthesis of tetrasubstituted acyclic alkenes by ruthenium-catalyzed $\mathrm{C}-\mathrm{H}$ arylation (Scheme 49). Tetrasubstituted alkenes are important key motifs in inter alia pharmaceuticals and thus far considerable efforts have been made to devise highly stereoselective methods for their synthesis. ${ }^{[126]}$

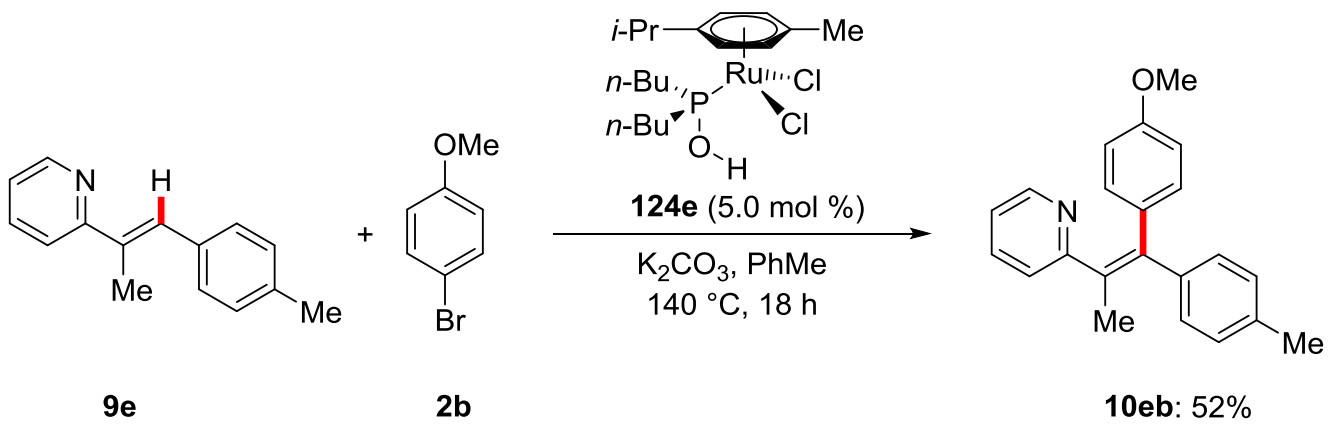

Scheme 49. Ruthenium(II)-PA-catalyzed synthesis of the tetrasubstitued acyclic alkene 10eb.

Given the overall excellent performance of the catalytic system, more challenging aryl chlorides 3 with an inherently higher $\mathrm{C}-\mathrm{Cl}$ dissociation energy ${ }^{[37 \mathrm{~b}]}$ were probed as arylating reagents under otherwise identical reaction conditions (Table 5). In summary, all the tested aryl chlorides 3 were efficiently converted into the desired products $\mathbf{1 0}$ in good to excellent yields, demonstrating that ruthenium(II)-PA complexes are very competent arylating catalysts, disregarding the $\mathrm{C}-\mathrm{X}$ bond 
strength. In this context, it should be emphasized that $S$. Warratz succeeded in using aryl tosylates as arylating reagents by employing a ruthenium(II)-PA complex. ${ }^{[123,127]}$

Table 5. Scope of the ruthenium(II)-PA-catalyzed C-H arylation with aryl chlorides 3.

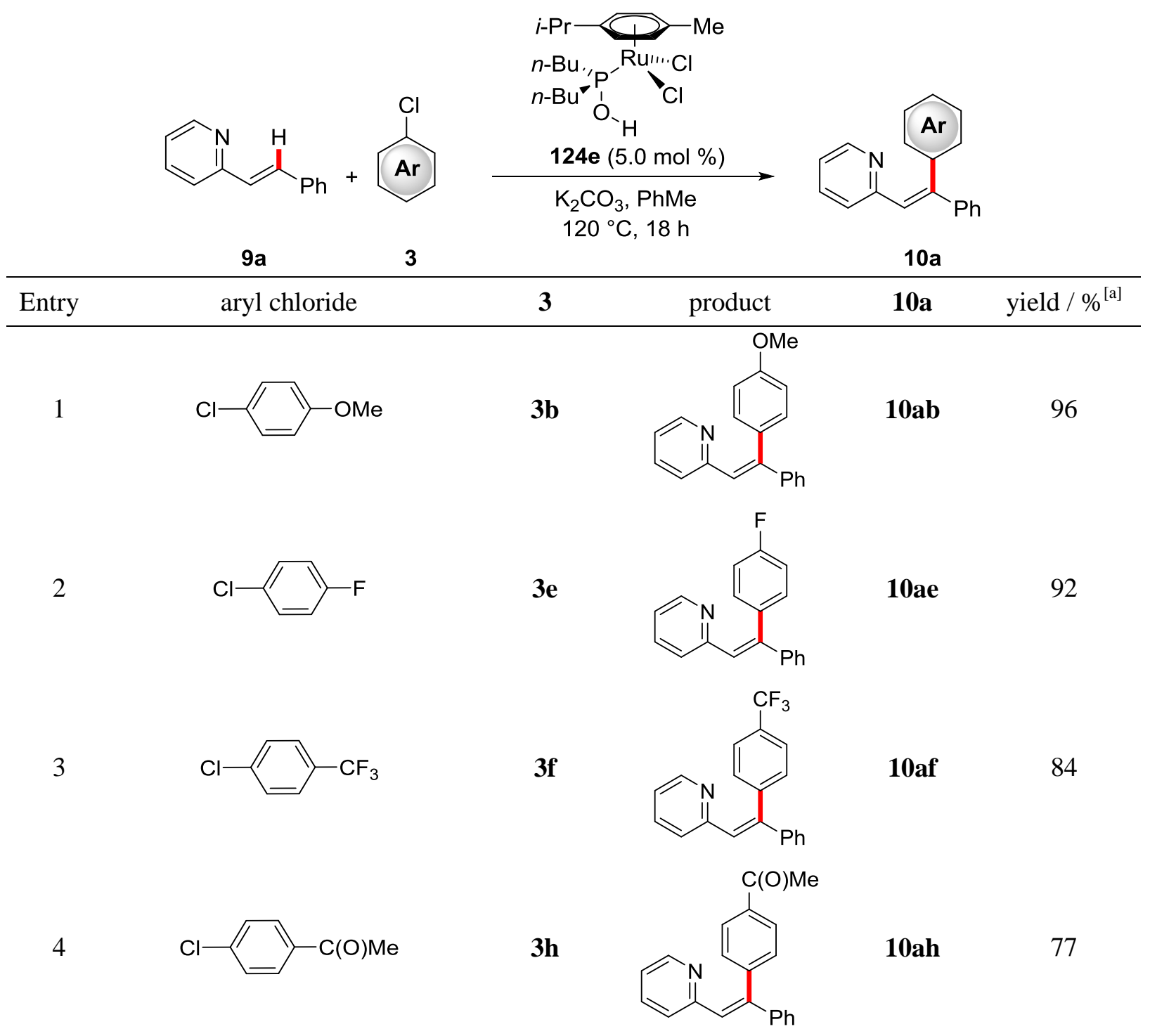

[a] Reaction conditions: 9a $(0.50 \mathrm{mmol}), 3(0.75 \mathrm{mmol})$, [Ru] (5.0 mol \%), $\mathrm{K}_{2} \mathrm{CO}_{3}(1.00 \mathrm{mmol}), \mathrm{PhMe}(0.25 \mathrm{M})$, $120^{\circ} \mathrm{C}, 18 \mathrm{~h}$, isolated yield.

The overall reactivity of the optimized catalytic system seemed to be only moderately affected by the electronic nature of both reagents. In summary, the substitution pattern of the (E)-2-styrylpyridines 9 had a slight impact on the reactivity, whereas the electronics of the organic electrophile exerted only a minor influence. Only the steric effects of the aryl bromide $\mathbf{2}$ had a major influence on the reactivity, with a significant drop in reactivity for di-ortho-substituted aryl bromides $\mathbf{2}$ ( $c f$. Table 3, entry 11). 


\subsubsection{Mechanistic Studies}

\subsubsection{Experiments with Radical Scavengers}

A key step in the mechanism of $\mathrm{C}-\mathrm{H}$ arylation reactions involves the activation and cleavage of $\mathrm{C}-\mathrm{Hal}$ bonds. So far, the mechanism of this process has remained elusive and underexplored, as it has been unclear whether a single electron transfer (SET)-type process is operative. In this context, there are a few reports on oxidative additions of aryl halides to metals, such as nickel, ${ }^{[128]}$ palladium ${ }^{[129]}$ and platinum, ${ }^{[130]}$ proceeding via SET-type activation of the $\mathrm{C}-\mathrm{Hal}$ bond.

To delineate the $\mathrm{C}-\mathrm{Hal}$ bond activation pathway, a set of well-established radical scavengers in stoichiometric amounts was added to the catalytic system (Table 6). In all cases, the catalytic reaction was significantly inhibited, which might suggest that a SET-type $\mathrm{C}-\mathrm{Br}$ cleavage process occurred. Interestingly, a similar observation for ruthenium(II)-catalyzed $\mathrm{C}-\mathrm{H}$ arylations was recently reported by Ackermann and coworkers ${ }^{[131]}$ Nevertheless, no radical adduct of the scavenger and any reaction intermediate could be detected by means of GC- and ${ }^{1}$ H-NMR- analysis. To shed light on this interesting mechanistic pathway, further studies should include for instance spin trapping experiments, since some radical scavengers like TEMPO possibly not only inhibit by radical, but also oxidative processes. $^{[132]}$

Table 6. Experiments with radical scavengers.<smiles>C(=C/c1ccccn1)\c1ccccc1</smiles>

9a<smiles>COc1ccc(Br)cc1</smiles>

2b

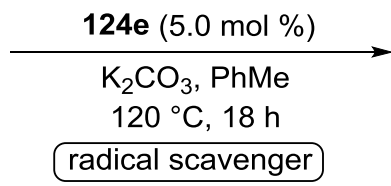

radical scavenger<smiles>COc1ccc(/C(=C\c2ccccn2)c2ccccc2)cc1</smiles>

$10 \mathrm{ab}$

\begin{tabular}{ccc}
\hline Entry & radical scavenger & conversion \\
\hline 1 & TEMPO & $2 \%$ \\
2 & $\mathrm{Ph}_{2} \mathrm{C}=\mathrm{CH}_{2}$ & --- \\
3 & $\mathrm{BHT}$ & --- \\
4 & Galvinoxyl & -- \\
5 & DPPH & -- \\
\hline
\end{tabular}

[a] Reaction conditions: 9a $(0.50 \mathrm{mmol}), \mathbf{2 b}(0.75 \mathrm{mmol}),[\mathrm{Ru}](5.0 \mathrm{~mol} \%), \mathrm{K}_{2} \mathrm{CO}_{3}(1.00 \mathrm{mmol})$, scavenger $(0.50 \mathrm{mmol}), \mathrm{PhMe}(2.0 \mathrm{~mL}, 0.25 \mathrm{M}), 120^{\circ} \mathrm{C}, 18 \mathrm{~h}$. Conversions were determined by $\mathrm{GC}$ with $n$-dodecane as the internal standard. 


\subsubsection{Hammett Plot Analysis}

Besides the exact working mode of the $\mathrm{C}-\mathrm{Hal}$ bond activation, a particularly interesting question in ruthenium(II)-catalyzed $\mathrm{C}-\mathrm{H}$ arylations encompasses the nature of the turnover-limiting step in the catalytic cycle. So far, there has been limited evidence for the oxidative addition to be turnoverlimiting, albeit detailed kinetic studies have not been presented. ${ }^{[42]}$ In this regard, a Hammett plot analysis $^{[133]}$ is a suitable analysis tool, which relies on a logarithmic relationship between the reaction rate $k$ and a specific substitution parameter $\sigma$ of the reacting substrate as well as a reaction parameter $\rho$ (Equation 1).

$$
\log k=\sigma \times \rho
$$

(Equation 1)

Notably, a Hammett plot analysis is particularly interesting for $\mathrm{C}-\mathrm{H}$ arylations with para-substituents on aryl halides because of the overall minor steric effects and the availability of known values for commonly used arylating substrates. ${ }^{[134]}$ Based on the initial reaction rates $k_{\mathrm{X}}$, employing differently para-substituted aryl bromides $\mathbf{2}$ and the known $\sigma_{\mathrm{p}}$-values, the resulting Hammett plots could indicate whether electronic effects of the aryl halide have a considerable impact on the overall reaction rate. Depending on the sign of the slope, it can furthermore elucidate whether oxidative addition or reductive elimination are turnover-limiting. ${ }^{[135]}$

Thus, a Hammett plot analysis of the $\mathrm{C}-\mathrm{H}$ arylation on (E)-2-styrylpyridines 9 with differently parasubstituted aryl bromides 2 was performed by comparing initial rates and correlating them with literature known $\sigma_{\mathrm{p}}$-values (Scheme 50, Figure 3 and Figure 4).<smiles>[X]c1ccc(Br)cc1</smiles>

9a

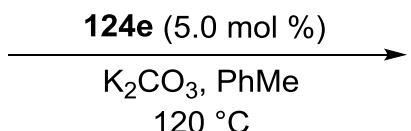

$120^{\circ} \mathrm{C}$<smiles>[X]c1ccc(C(=Cc2ccccn2)c2ccccc2)cc1</smiles>

$10 \mathrm{a}$

Scheme 50. Hammett plot analysis of differently para-substituted aryl bromides 2 . 


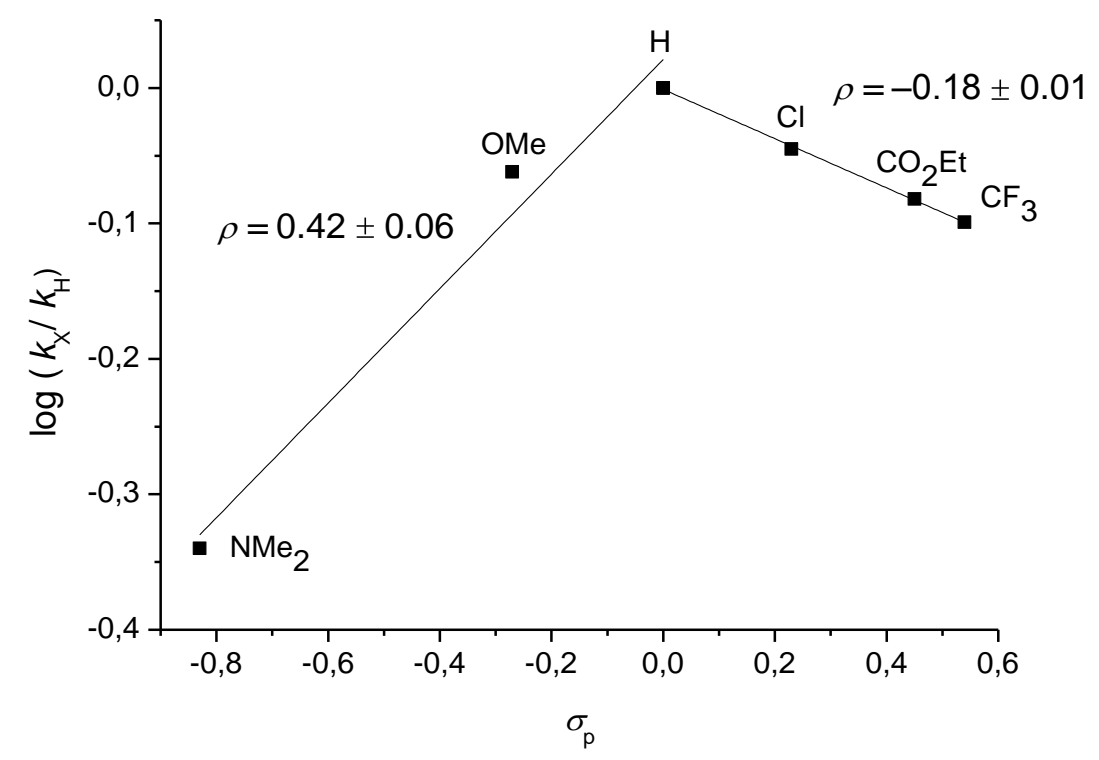

Figure 3. Hammett plot of the ruthenium(II)-PA-catalyzed arylation of (E)-2-styrylpyridine (9a).

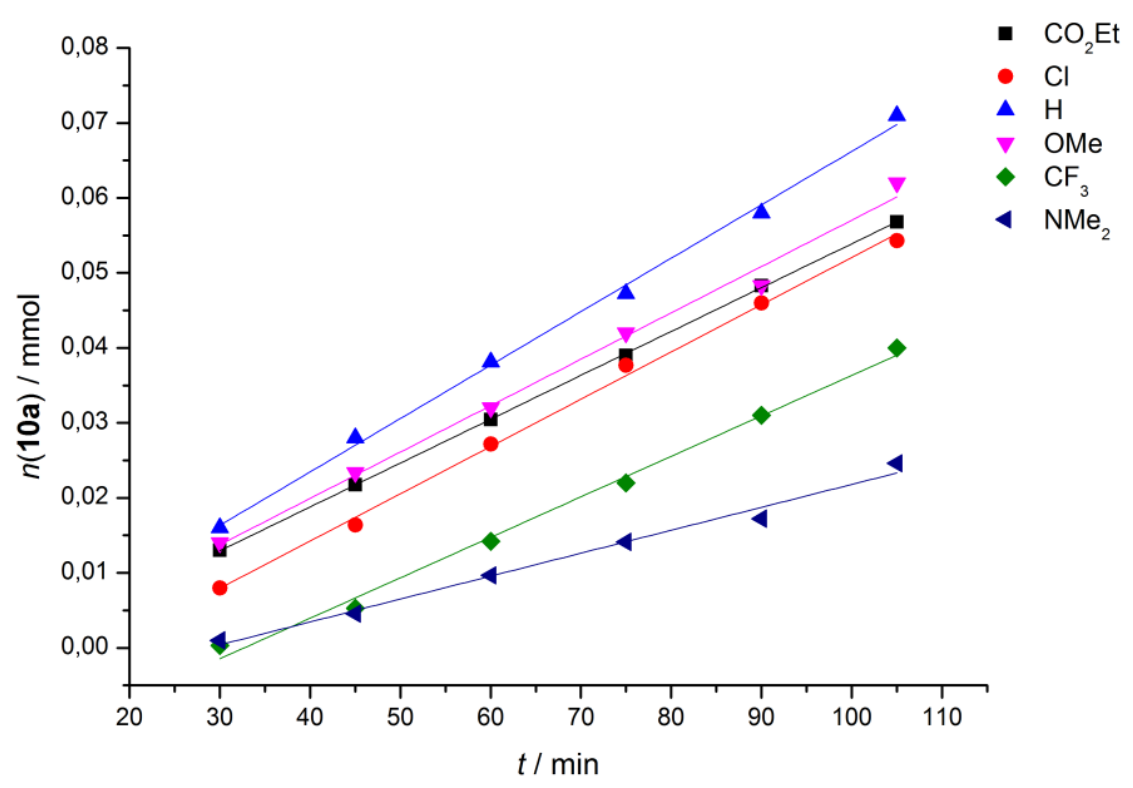

Figure 4. Initial rates using different aryl bromides 2.

Remarkably, the obtained Hammett plot exhibited an unusual V-shape with slopes of $\rho=0.42 \pm 0.06$ and $\rho=-0.18 \pm 0.01$. Likewise, a similar behaviour has been disclosed by Chatani and coworkers for a ruthenium(II)-catalyzed arylation with a bidentate directing group. ${ }^{[135 \mathrm{~b}]}$

This unusual finding can be interpreted by a change in the turnover-limiting step from oxidative addition in the regime of electron-donating substituents to reductive elimination with electrondeficient aryl bromides 2 . Nevertheless, the observed $\rho$-value within the electron-deficient substitution 
regime is not significantly high enough for drawing a reliable conclusion and the absolute reaction rates for electron-deficient aryl bromides 2 were still found to be comparably high. Overall, it can be concluded that rather oxidative addition is the turnover-limiting step, while for highly electrondeficient aryl bromides $\mathbf{2}$ the reductive elimination becomes energetically less feasible.

\subsubsection{KIE Studies}

Based on the results of the Hammett plot analysis, further mechanistic evidence for a turnover-limiting oxidative addition were indeed necessary. For this purpose, a study of the kinetic isotope effect (KIE) could elucidate the impact of the $\mathrm{C}-\mathrm{H}$ activation elementary step on the overall reaction rate.

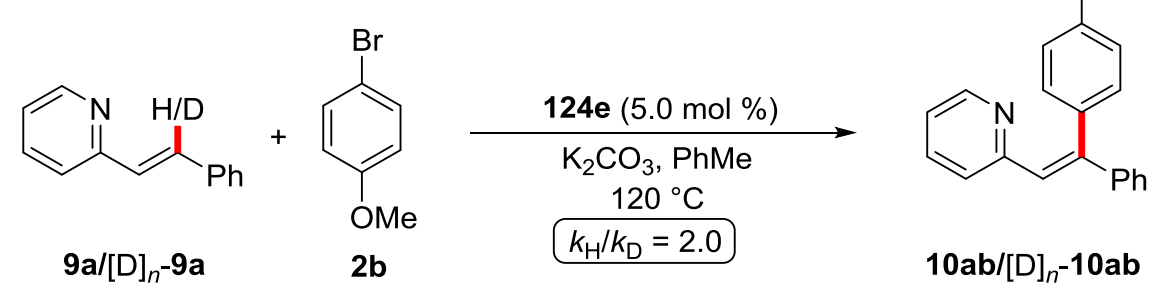

Scheme 51. KIE of the ruthenium(II)-PA-catalyzed arylation.

In this regard, a comparison of the independent reaction rates ${ }^{[136]}$ with $9 \mathbf{a}$ and the isotopically labeled $[D]_{1}-9 a$ was conducted by an in situ ReactIR analysis (Scheme 51, Figure 5 and Figure 6). For this purpose, the initial rates were followed by integration of a characteristic IR-band of the products 10ab and $[\mathrm{D}]_{n}-\mathbf{1 0 a b}$ at $1520 \mathrm{~cm}^{-1}$ during the reaction progress (vide infra). In this way, a KIE of $k_{\mathrm{H}} / k_{\mathrm{D}} \approx 2.0$ was measured.

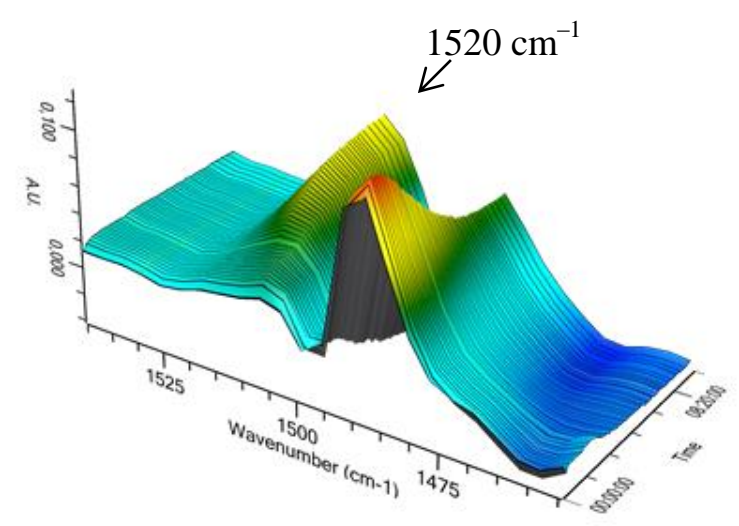

Figure 5. Kinetic profile of the $\mathrm{C}-\mathrm{H}$ arylation followed by in situ-IR analysis.

This observation reveals that the $\mathrm{C}-\mathrm{H}$ cleavage is a kinetically relevant elementary step, either being the turnover-limiting step itself or happening before turnover-limiting step as an equilibrium reaction. The latter assumption is in agreement with studies of $S$. Warratz who detected a significant H/Dexchange in the ortho-positions of 2-phenyloxazoline 7a in a ruthenium(II)-PA catalyzed $\mathrm{C}-\mathrm{H}$ arylation, which indicates a reversible $\mathrm{C}-\mathrm{H}$ activation to be operative. ${ }^{[123]}$ Therefore, the observed KIE 
value can be interpreted in terms of a reversible, but kinetically relevant $\mathrm{C}-\mathrm{H}$ activation before the turnover-limiting formal oxidative addition.

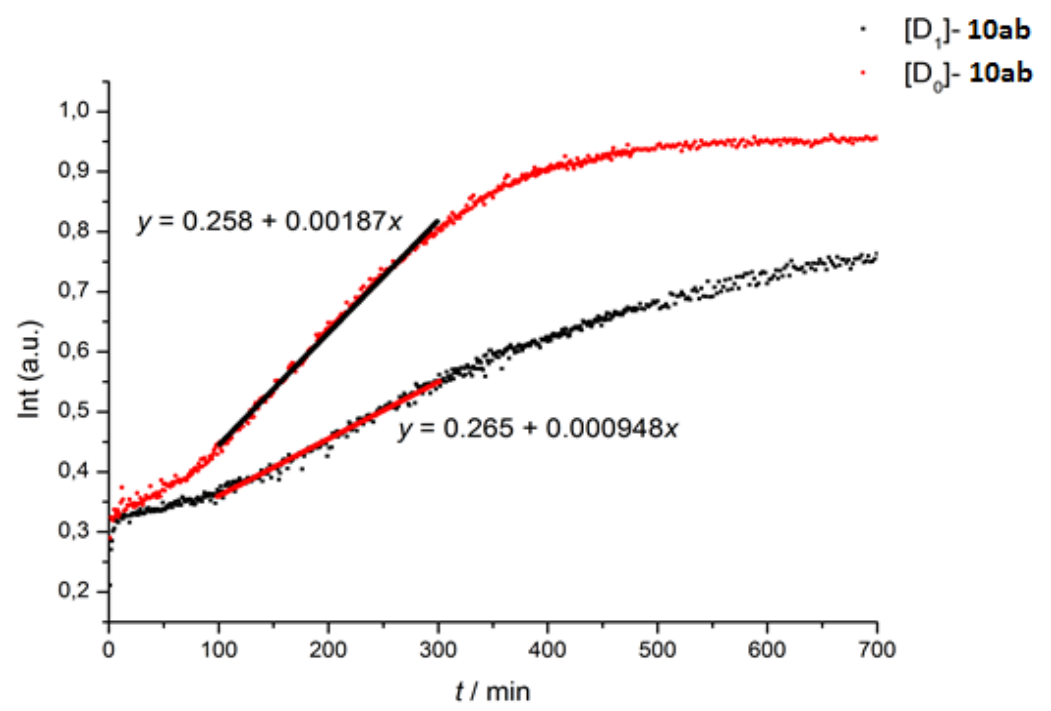

Figure 6. KIE of the ruthenium(II)-PA-catalyzed C-H arylation of 9a by ReactIR analysis.

\subsubsection{Kinetic Reaction Order}

\subsection{Reaction Order with respect to the concentration of $(E)$-2-styrylpyridine $(9 a)$}

Generally, an analysis of the kinetic reaction order in dependence on the concentration of each reactant as well as the catalyst represents a powerful mechanistic tool to elucidate the nature of the turnoverlimiting step. ${ }^{[137]}$

Thus, the overall reaction rate showed a first order dependence $(n=0.87 \pm 0.02)$ on the concentration of $(E)$-2-styrylpyridine (9a), which is a hint for the $\mathrm{C}-\mathrm{H}$ activation elementary step to be kinetically relevant (Figure 7). 


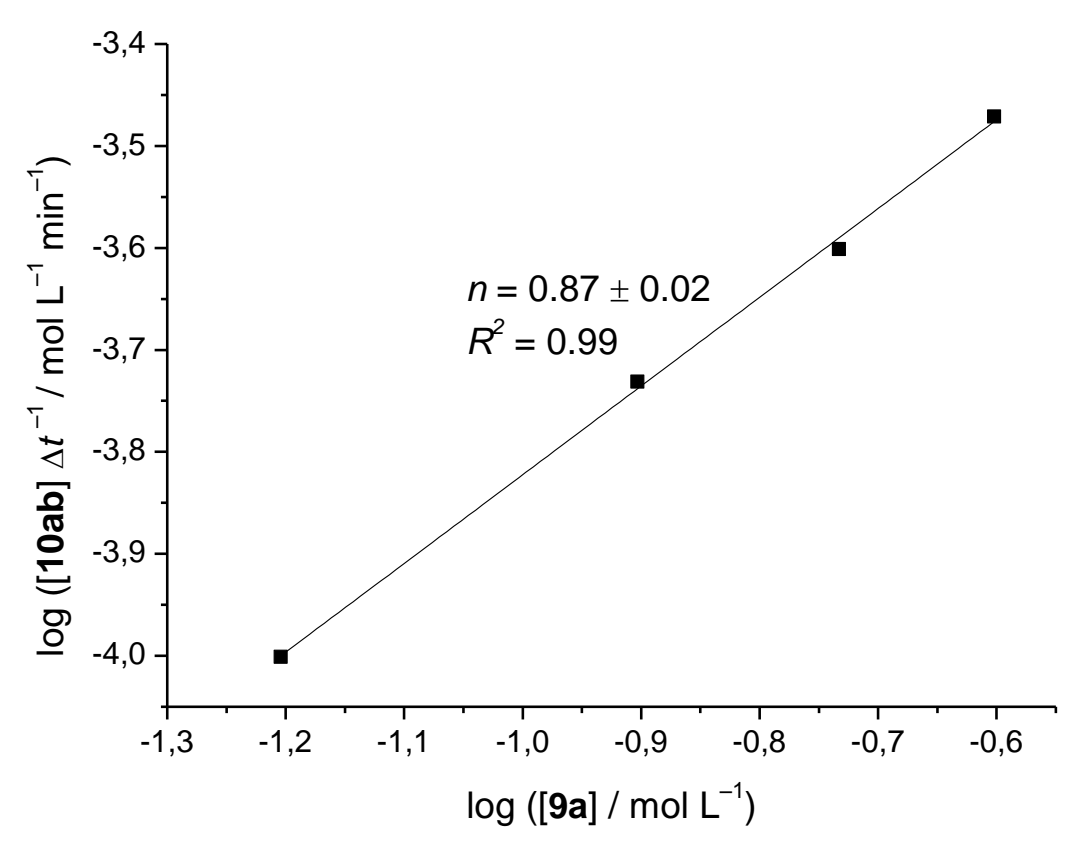

Figure 7. Reaction order $n$ in [(E)-2-styrylpyridine] (9a).

\subsection{Reaction Order with respect to the concentration of 4-bromoanisole ( $2 \mathrm{~b}$ )}

The kinetic order with respect to the concentration of the arylating reagent 4-bromoanisole (2b) exhibited saturation kinetics (Figure 8).

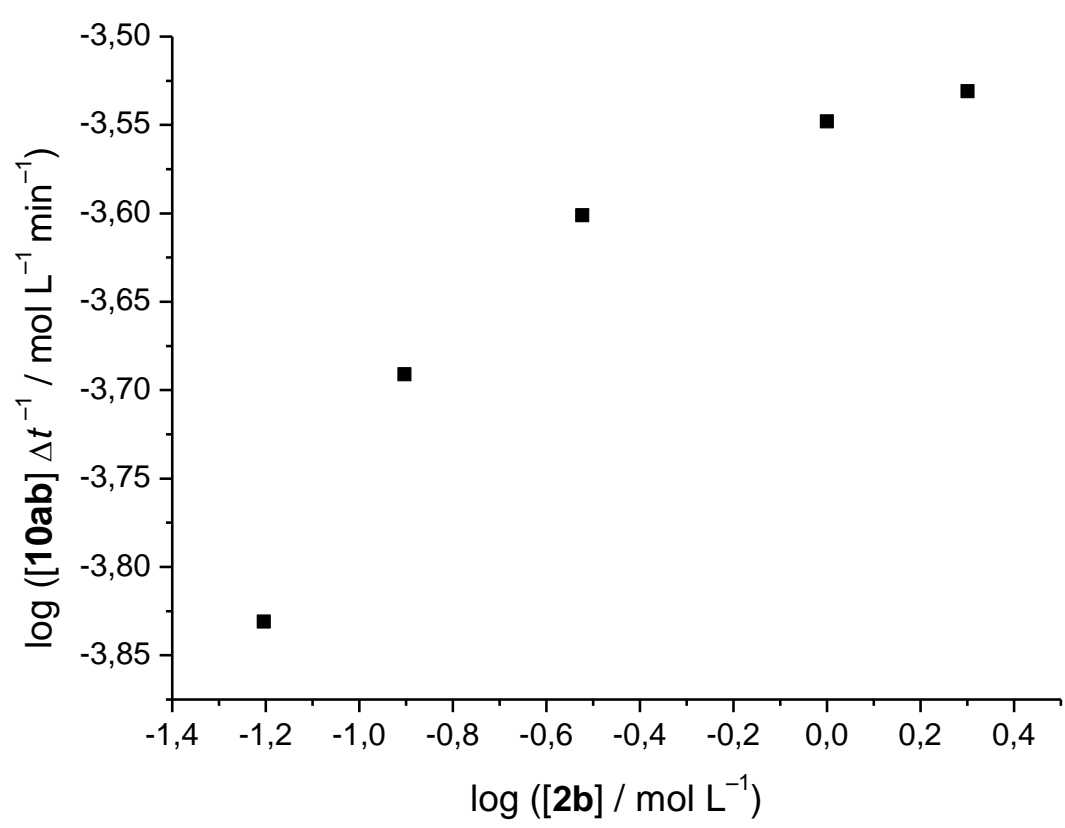

Figure 8. Reaction order $n$ in [4-bromoanisole] (2b).

This observation is indicative of the oxidative addition to be the turnover-limiting step of the catalytic cycle. For the studied reaction, a simplified reaction is depicted in Scheme 52. Assuming that the 
concentration of ruthenacycle $\mathbf{1 2 7}$ follows steady-state kinetics (Equation 2) and the subsequent steps after oxidative addition are fast and neglectable for the reaction rate, the overall rate law for the formation of the product 10ab (Equation 3) simplifies to Equation 4. ${ }^{[137 \mathrm{c}]}$

$$
\begin{gathered}
\frac{d[\mathbf{1 2 7}]}{d t}=k_{1}[\mathbf{9 a}][\mathbf{1 2 6}]-k_{-1}[\mathbf{1 2 7}]-k_{2}[\mathbf{1 2 7}][\mathbf{2 b}]=0 \\
\frac{d[\mathbf{1 0} \mathbf{a b}]}{d t}=k_{1}[\mathbf{2 b}][\mathbf{1 2 7}] \\
\frac{d[\mathbf{1 0 a b}]}{d t}=\frac{k_{1} k_{2}[\mathbf{9 a}][\mathbf{1 2 6}][\mathbf{2 b}]}{k_{-1}+k_{2}[\mathbf{2 b}]}
\end{gathered}
$$

If there is a very high concentration of 4-bromoanisole $(\mathbf{2 b})$, the term $k_{2}[\mathbf{2 b}]$ could become larger than $k_{-1}$ and consequently the overall reaction rate becomes independent on the concentration of the 4bromoanisole (2b). That could be reasonable explanation for the observed saturation kinetics.

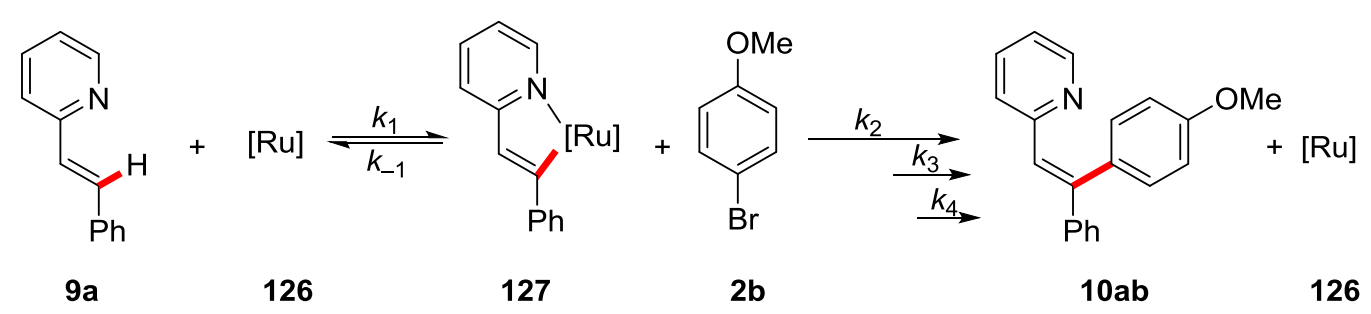

Scheme 52. Simplified kinetic scheme of the ruthenium(II)-PA-catalyzed C-H arylation.

\subsection{Reaction Order with respect to the concentration of complex 124e}

Further kinetic investigations established a first order dependence on the concentration of the singlecomponent ruthenium(II)-PA complex 124e (Figure 9), which reveals the presence of a mononuclear ruthenium-complex within the turnover-limiting step of the catalytic cycle. 


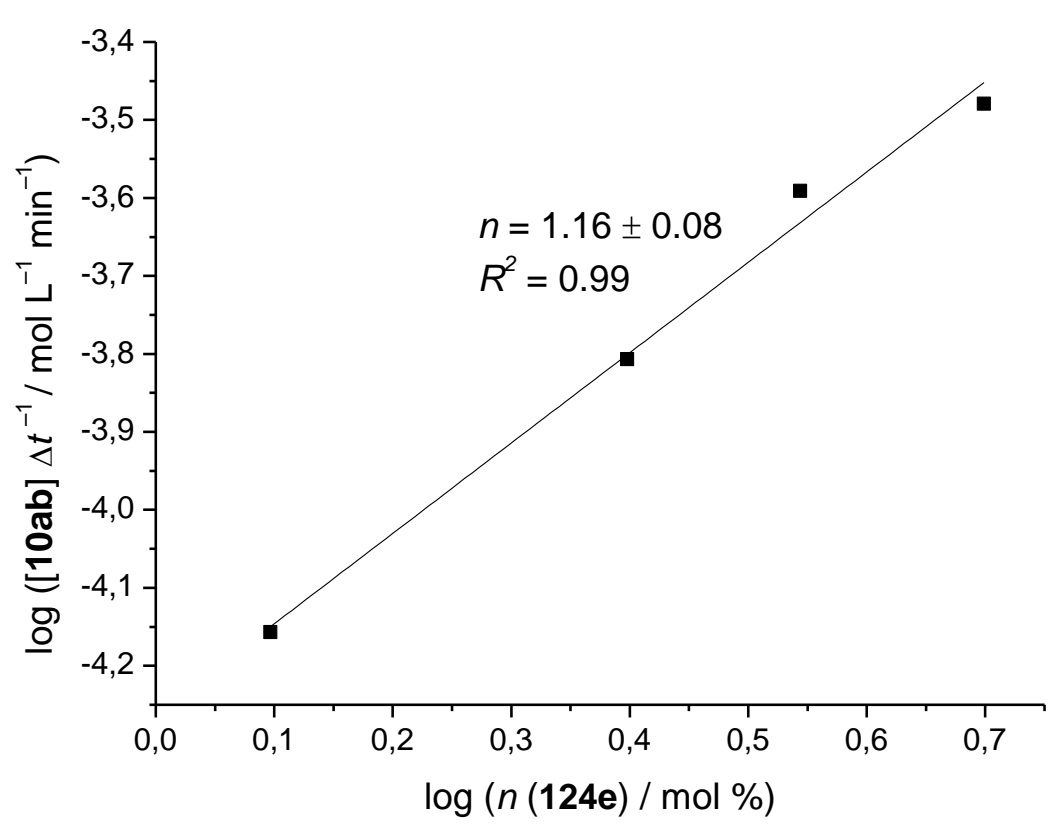

Figure 9. Reaction order in $[\mathbf{1 2 4 e}]$.

\subsubsection{Proposed Catalytic Cycle}

In summary, it can be concluded that the $\mathrm{C}-\mathrm{H}$ activation is a reversible, but kinetically relevant elementary step (Scheme 53). Presumably, the $\mathrm{C}-\mathrm{H}$ activation proceeds by metal-ligand-cooperation, in which the in situ formed phosphinite acts as an internal base for the deprotonation step. Likewise, a Hammett plot analysis, KIE studies as well as kinetic order experiments supported the oxidative addition to be the turnover-limiting step. However, for electron-deficient aryl bromides $\mathbf{2}$ a slight trend to a slower reductive elimination step was observed. Additionally, computational studies involving DFT-calculations, performed by S. J. Garden, confirmed that the PA-ligand assisted the deprotonation during a metal-ligand-cooperative $\mathrm{C}-\mathrm{H}$ activation step (transition state 127). ${ }^{[123]}$

Thereafter, a SET-type oxidative addition of the aryl bromide $\mathbf{2}$ to the cyclometalated complex $\mathbf{1 2 8}$ occurs and furnishes the ruthenium(IV) complex 129. After reductive elimination, the product 10a is released and the active ruthenium(II)-PA catalyst $\mathbf{1 2 6}$ is regenerated.

However, the exact structures of some assumed intermediates have thus far remained unclear and some efforts should be made to investigate possible hapticity changes or even a decoordination of the $p$-cymene ligand in the course of the reaction. In this regard, a possible dissociation of the $p$-cymene ligand has already been proposed in related reactions. ${ }^{[138]}$ Additionally, Ackermann and coworkers reported on $\mathrm{C}-\mathrm{H}$ arylation catalysis which is enabled by a powerful and $p$-cymene-free $\left[\mathrm{RuCl}_{3} \cdot x \mathrm{H}_{2} \mathrm{O}\right]-$ precursor. $^{[47-48]}$ 


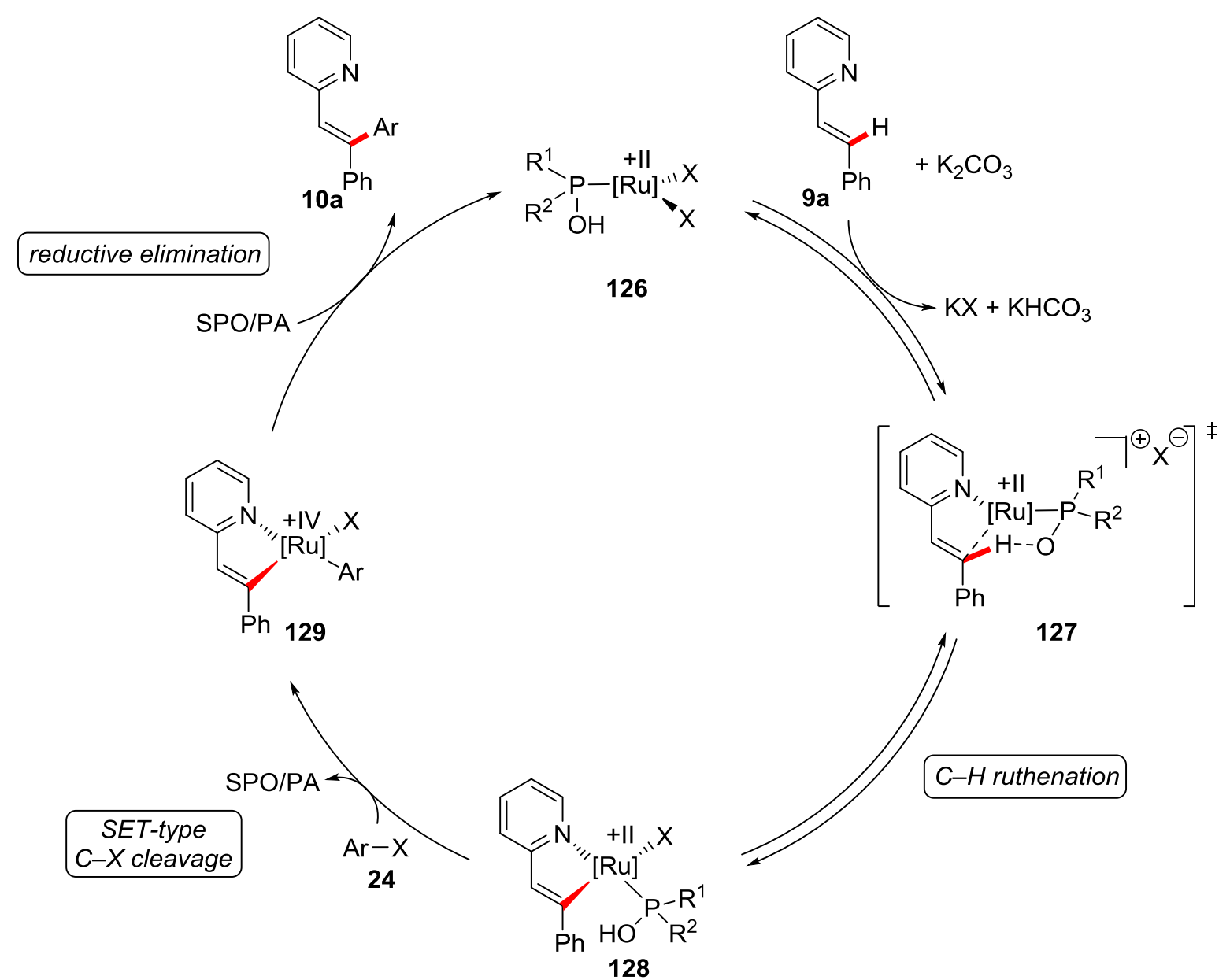

Scheme 53. Proposed catalytic cycle of the ruthenium(II)-PA-catalyzed C-H arylation. $[\mathrm{Ru}]=\mathrm{Ru}(\mathrm{II})(p$-cymene). $\mathrm{X}=\mathrm{Cl}$ or $\mathrm{Br}$. 


\subsection{Cobalt(III)-Catalyzed C-H/C-C Functionalization}

The use of inexpensive $3 \mathrm{~d}$ base metals, such as inter alia manganese ${ }^{[14 a, 139]}$ and cobalt, ${ }^{[15]}$ for $\mathrm{C}-\mathrm{H}$ functionalization reaction has been intensively studied in the last few years. In this regard, not only the cost-efficiency of these metals is of prime importance, but also a systematic study of their unique reactivity profile is essential for making considerable advances in transition metal-catalysis.

There are indeed aspects in $\mathrm{C}-\mathrm{H}$ functionalizations like reactivity and stereoselectivity that can be controlled not only by the judicious choice of ligands, but also by the distinctive features of any metal. In this regard, there are several reports of inter alia Ackermann, ${ }^{[23 c]}$ Glorius, ${ }^{[140]}$ Kanai/Matsunaga,${ }^{[91]}$ and $W_{a n g^{[141]}}$ on higher reactivities and distinct chemoselectivities for cobalt(III) complexes as compared to the corresponding rhodium(III) $\mathrm{C}-\mathrm{H}$ activation catalysis.

Despite these intriguing advances, there is still demand for the exploration of distinct selectivity features in cobalt(III) catalysis. Therefore, the control of diastereoselectivity in $\mathrm{C}-\mathrm{H} / \mathrm{C}-\mathrm{C}$ activations ${ }^{[117 \mathrm{a}, 117 \mathrm{c}, 117 \mathrm{~d}]}$ with vinylcyclopropanes $\mathbf{1 2 5}$ represents a promising objective.

\subsubsection{Optimization Studies}

The optimization studies were commenced by probing the effect of various acetate- and pivalate bases with different alkali metal counter cations (Table 7). Lithium, sodium and potassium acetate as additives delivered the desired product 130aa in satisfactory yields, whereas cesium acetate was by far the least effective additive (entries 1-4). The same trend was observed for pivalate bases, with sodium pivalate being the optimal base for this transformation (entry 6). In contrast, potassium- and cesium pivalate led to a significant drop in reactivity (entries 7 and 8).

Furthermore, no reaction was observed by omitting the cobalt(III) catalyst, which is a clear hint for cobalt catalysis to be operative in this reaction (entry 9). In addition, the silver additive $\mathrm{AgSbF}_{6}$ was found to be essential for catalytic activity (entry 10). Based on previous results in $\mathrm{C}-\mathrm{H}$ activation by cobalt(III) catalysis, no further optimization of the silver additive was performed. ${ }^{[15 a]}$ A reaction without base additive led to only moderate yield and $10 \%$ of a double bond regioisomer was detected, in which the double bond was shifted into conjugation with the indole nucleus (entry 11). In contrast, efficient conversion can only be accomplished in the presence of a base additive, which is a hint for a BIES-type $\mathrm{C}-\mathrm{H}$ activation pathway.

Notably, all tested reaction conditions exclusively furnished the thermodynamically less favored Zdiastereomer in very good selectivities, which is in contrast to $E$-selective manganese(I) ${ }^{[117 \mathrm{a}, 117 \mathrm{~d}]}$ and rhodium(III) catalysis. ${ }^{[117 \mathrm{c}]}$ Furthermore, $Q . B u$ tested different cobalt(III) sources, indicating that silver is not necessary for the reaction (entry 15) and other ligands are inferior to the electron-rich $\mathrm{Cp}^{*}$ (entries 16 and 17). In this context, she detected a slight decrease in E/Z-ratio to 8:1, which could be interesting for future developments of ligand-controlled diastereoselective $\mathrm{C}-\mathrm{H} / \mathrm{C}-\mathrm{C}$ functionalizations. 
Remarkably, the catalytic performance was only moderately affected by decreasing the reaction temperature to $25^{\circ} \mathrm{C}$ (entry 18).

Table 7. Optimization for the cobalt(III)-catalyzed C-H/C-C functionalization.

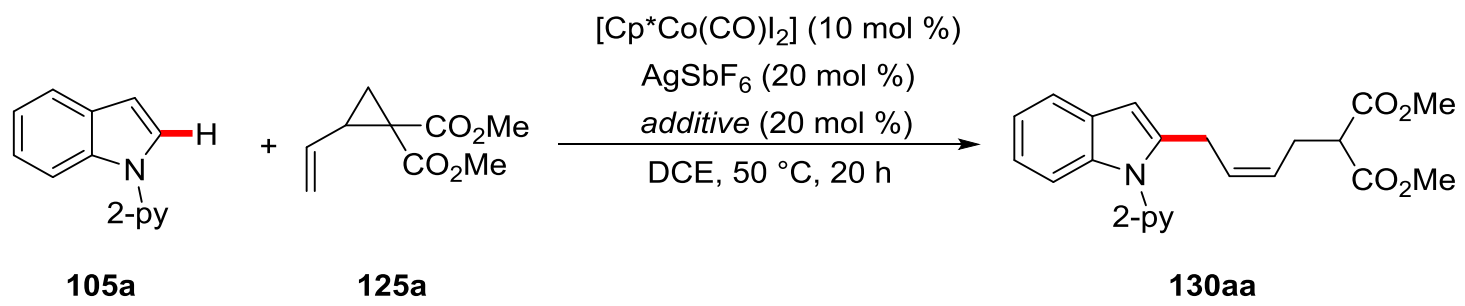

\begin{tabular}{|c|c|c|}
\hline Entry & additive & yield / \% ${ }^{[\mathrm{a}]}$ \\
\hline 1 & LiOAc & 74 \\
\hline 2 & $\mathrm{NaOAc}$ & 88 \\
\hline 3 & KOAc & 76 \\
\hline 4 & $\mathrm{CsOAc}$ & 11 \\
\hline 5 & LiOPiv & 61 \\
\hline 6 & NaOPiv & 93 \\
\hline 7 & KOPiv & 51 \\
\hline 8 & CsOPiv & 39 \\
\hline 9 & NaOPiv & $---[c]$ \\
\hline 10 & --- & $--_{-}^{[\mathrm{d}]}$ \\
\hline 11 & --- & $68^{[\mathrm{e}]}$ \\
\hline 12 & $\mathrm{NaO}_{2} \mathrm{C}\left(3-\mathrm{CF}_{3}\right) \mathrm{C}_{6} \mathrm{H}_{4}$ & 68 \\
\hline 13 & $\mathrm{KO}_{2} \mathrm{CMes}$ & 27 \\
\hline 14 & $\mathrm{Na}(\mathrm{O}-\mathrm{Piv}-\mathrm{Val})$ & 84 \\
\hline 15 & NaOPiv & $91^{[\mathrm{f}]}$ \\
\hline 16 & NaOPiv & $18^{[\mathrm{g}]}$ \\
\hline 17 & NaOPiv & $10^{[\mathrm{h}]}$ \\
\hline 18 & NaOPiv & $64^{[\mathrm{i}]}$ \\
\hline
\end{tabular}

[a] Reaction conditions: 105a $(0.50 \mathrm{mmol}), \mathbf{1 2 5 a}(0.60 \mathrm{mmol}),\left[\mathrm{Cp} * \mathrm{Co}(\mathrm{CO}) \mathrm{I}_{2}\right](10 \mathrm{~mol} \%), \mathrm{AgSbF}_{6}(20 \mathrm{~mol} \%)$, additive $(20 \mathrm{~mol} \%)$, DCE $(1.0 \mathrm{~mL}), 20 \mathrm{~h}$, isolated yield. py = pyridyl, $\mathrm{E}=\mathrm{CO}_{2} \mathrm{Me}$; all $E / Z=1: 11$. [b] Additive $(10 \mathrm{~mol} \%)$. [c] Without [Co]. [d] Without $\mathrm{AgSbF}_{6}$. [e] Double bond regioisomer $(10 \%)$ observed. [f] $\left[\mathrm{Cp} * \mathrm{Co}\left(\mathrm{CH}_{3} \mathrm{CN}\right)_{3}\right]\left(\mathrm{SbF}_{6}\right)_{2}(10 \mathrm{~mol} \%)$. [g] [1,3-( $\left.t-\mathrm{Bu})_{2} \mathrm{C}_{5} \mathrm{H}_{3} \mathrm{Co}(\mathrm{CO}) \mathrm{I}_{2}\right](10 \mathrm{~mol} \%), E / Z=1: 8$. [h] [CpCo(CO) $\left.\mathrm{I}_{2}\right](10 \mathrm{~mol} \%), E / Z=1: 8$. [i] At $25^{\circ} \mathrm{C}$. 


\subsubsection{Scope of the Cobalt(III)-Catalyzed C-H/C-C Functionalization}

Under the optimized reaction conditions for the cobalt(III)-catalyzed $\mathrm{C}-\mathrm{H} / \mathrm{C}-\mathrm{C}$ functionalization, an extensive substrate scope was probed (Table 8). At first, various substituents in C5-position of the indole were tested, such as methoxy, bromo, iodo, and nitro groups (entries 2-5). Notably, all functional groups were fully tolerated, delivering the desired Z-configurated product in very good yields ranging from $88 \%$ to $92 \%$ and good diastereoselectivities.

Furthermore, sterically demanding substituents in C3-position were employed, furnishing the desired products 100fa and 100ga still in good to excellent yield (entries 6 and 7), with a minor drop in diastereoselectivity for product $\mathbf{1 0 0 f a}$, probably due to steric effects. The substituent in C3-position of $\mathbf{1 0 5 g}$ can flexibly rotate and is sterically less demanding than the methyl-substituted analogue. Substituents in C4- and C6-position (entries 8 and 9) were well tolerated, affording the corresponding products 105ha and 105ia in excellent yields. For the C7-substituted product 105ja (entry 10) the diastereoselectivities were somewhat reduced, presumably due to steric interactions, which is in good agreement with the observation for the C3-methyl-substituted product 130fa (entry 6). The pyridyldirecting group of the products $\mathbf{1 3 0}$ was shown to be removable ( $c f$. chapter 5.3.2). However, a chemoselective method, that tolerated the alkene functionality, has not been disclosed so far.

Finally, a sterically more congested substitution pattern on the pyridyl-directing group did not influence the reactivity, with overall very good yields of 130ka and 130la (entries 11 and 12). Only the diastereoselectivity profited somewhat from the changed substitution on the pyridyl-ring, affording the product in excellent selectivities of $Z / E=14: 1$. A reasonable explanation for this unexpected finding could be the involvement of stabilizing London dispersion interactions, ${ }^{[124,142]}$ partly exhibited by the pyridyl-ring, which was highlighted by DFT-calculations of M. Feldt.$^{[143]}$

Table 8. Scope of the cobalt(III)-catalyzed C-H/C-C functionalization of indoles $\mathbf{1 0 5}$.

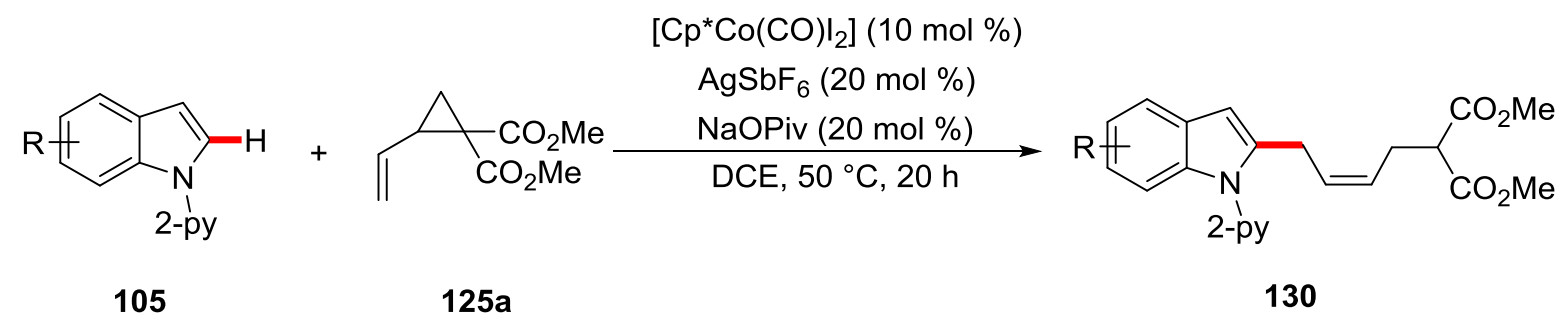

Entry 2-pyridylindole


3<smiles>[Y6]n1ccc2cc(Br)ccc21</smiles><smiles>[R6]n1ccc2cc(I)ccc21</smiles>

5<smiles>[Y6]n1ccc2cc([N+](=O)[O-])ccc21</smiles>

6<smiles>Cc1cn([Te])c2ccccc12</smiles>

7<smiles>CC(=O)Cc1cn([Ga]2CCCCCC2)c2ccccc12</smiles>

8<smiles>COc1cccc2c1ccn2[18O]</smiles>

9<smiles>[R7]n1ccc2ccc(C(OC)OC)cc21</smiles>

10<smiles>[R6]n1ccc2cccc(CC)c21</smiles><smiles>c1ccc2[nH]ccc2c1</smiles>

11<smiles>Cc1ccnc(N)c1</smiles>

12

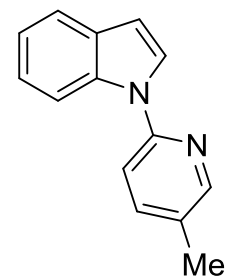

$105 c$

$\mathrm{Br}$<smiles>COC(=O)C(C/C=C\Cc1cc2ccccc2n1C(=O)O)C(C)=O</smiles><smiles>COC(=O)CC(C/C=C\Cc1cc2cc(I)ccc2n1[Pb])C(C)=O</smiles>

130da $14: 1 \quad 89$

105d

$105 \mathrm{e}$<smiles>COCC(C/C=C\Cc1cc2cc([N+](=O)[O-])ccc2n1[18O])C(OC)OC</smiles><smiles>CC(=O)C(C/C=C\Cc1c(C)c2ccccc2n1C(=O)c1ccccc1)C(C)=O</smiles><smiles>CCCCn1c(CC(C)=O)c(C/C=C\CC(C(C)=O)C(C)OCC)c2ccccc21</smiles><smiles>COC(=O)C(C/C=C\Cc1cc2c(OC)cccc2n1P(C)C)C(C)=O</smiles>

$105 i$<smiles></smiles>

130ia $8: 1 \quad 84$

105j<smiles>[Y7]n1c(C/C=C\CC(C(C)=O)C(=O)OC)cc2cccc(CC)c21</smiles>

130ja $\quad 8: 1 \quad 68$

105k<smiles>COCC(C/C=C\Cc1cc2ccccc2n1-c1cc(C)ccn1)C(=O)OC</smiles>

1051

130ea $17: 1 \quad 88$

130fa $5: 1 \quad 93$

130ga 11:1 74

130ha $13: 1 \quad 77$

130ka $14: 1 \quad 81$

130la $14: 1 \quad 92$

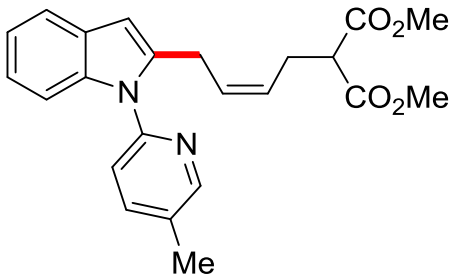

[a] Reaction conditions: $105(0.50 \mathrm{mmol}), \mathbf{1 2 5 a}(0.60 \mathrm{mmol}),\left[\mathrm{Cp} * \mathrm{Co}(\mathrm{CO}) \mathrm{I}_{2}\right](10 \mathrm{~mol} \%), \mathrm{AgSbF}_{6}(20 \mathrm{~mol} \%), \mathrm{NaOPiv}$ (20 mol \%), DCE $(1.0 \mathrm{~mL}), 50{ }^{\circ} \mathrm{C}, 20 \mathrm{~h}$, isolated yield. 
Furthermore, the scope was extended to vinylcyclopropane 125b derived from meldrums acid ${ }^{[144]}$ (Table 9). Herein, a remarkable switch in diastereoselectivity to the $E$-configurated products was found. In all cases, the main isomer was the $E$-configurated product, but the selectivities were overall poor. By comparison with the analogous rhodium(III)-catalyzed reaction, the same reactivity and similar selectivity were obtained, with an even higher E/Z-ratio (entry 1 ).

The reason for this unexpected switch in diastereocontrol is not yet fully elucidated and there is need for detailed computational studies dealing with this kind of substrate-controlled stereoselectivity. In this respect, a detailed understanding of this observation would possibly give rise to the development of a switchable control in diastereoselectivity of $\mathrm{C}-\mathrm{H} / \mathrm{C}-\mathrm{C}$ bond activations by the substitution pattern on the vinylcyclopropane $\mathbf{1 2 5}$.

Table 9. Scope of the cobalt(III)-catalyzed $\mathrm{C}-\mathrm{H} / \mathrm{C}-\mathrm{C}$ functionalization with meldrums acid-derived vinylcyclopropane $\mathbf{1 2 5 b}$.

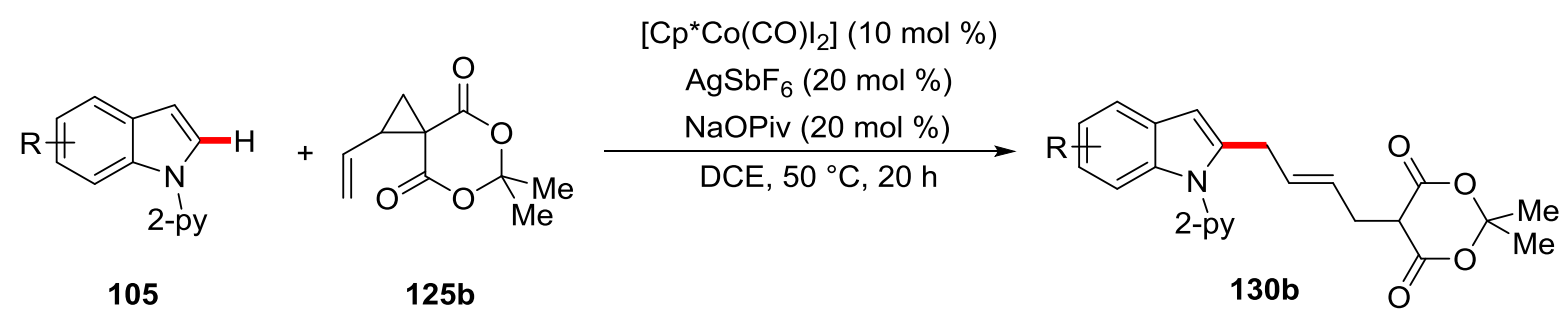

Entry

[a] Reaction conditions: $105(0.50 \mathrm{mmol}), \mathbf{1 2 5 b}(0.60 \mathrm{mmol}),\left[\mathrm{Cp} * \mathrm{Co}(\mathrm{CO}) \mathrm{I}_{2}\right](10 \mathrm{~mol} \%), \mathrm{AgSbF}_{6}(20 \mathrm{~mol} \%), \mathrm{NaOPiv}$ $(20 \mathrm{~mol} \%)$, DCE $(1.0 \mathrm{~mL}), 50{ }^{\circ} \mathrm{C}, 20 \mathrm{~h}$, isolated yield. [b] With $\left[\mathrm{Cp} * \mathrm{Rh}\left(\mathrm{CH}_{3} \mathrm{CN}\right)_{3}\right]\left(\mathrm{SbF}_{6}\right)_{2}(10 \mathrm{~mol} \%)$ product 130ab $(83 \%$, $Z / E=1: 5.9)$ was obtained.

Besides the applicability of this reaction to the functionalization of 2-pyridylindoles $\mathbf{1 0 5}$, also the inherently less electron-rich heterocycles were tested (Table 10). Thus, also 2-phenylpyridines 1 were 
successfully converted with comparable efficiency, but somewhat decreased diastereoselectivities (entries 1 and 2). In contrast, rhodium(III) catalysis furnished the $E$-configurated product 132ba' in lower yield, poor selectivity of $E / Z=2: 1$ and a significant amount of the di-functionalized product 132ba" was observed (entry 3). These findings emphasize the unique diastereo- and site-selectivities of the cobalt(III)-catalyzed $\mathrm{C}-\mathrm{H} / \mathrm{C}-\mathrm{C}$ activation.

In this respect, a reasonable explanation for the mono-selectivity could be the overall shorter cobaltcarbon bond lengths, ${ }^{[17 \mathrm{~b}]}$ which presumably increase steric interactions in catalytic intermediates towards the mono-functionalized product 132ba. Furthermore, 1-(thiophen-2-yl)-1H-pyrazole (131) reacted with moderate yield, but excellent diastereoselectivity (entry 4). In this regard, $Q$. Bu could extend the heteroarene-scope to valuable examples of functionalized phenylpyrazoles $\mathbf{1 8}$ with excellent levels of site- and diastereoselectivity. ${ }^{[117 b]}$

Table 10. Scope of the cobalt(III)-catalyzed C-H/C-C functionalization with 2-phenylpyridines 1.

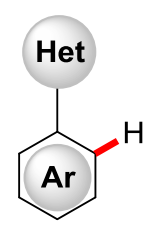

1 or 131

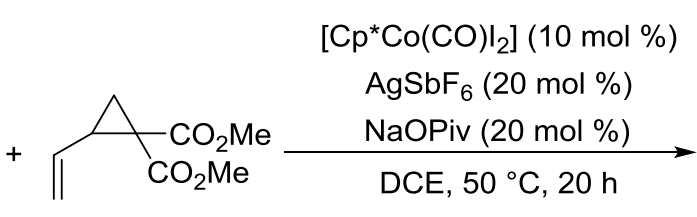

$125 a$

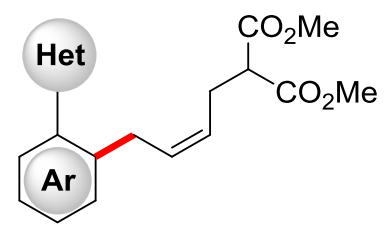

132

Entry heteroarene 1


4

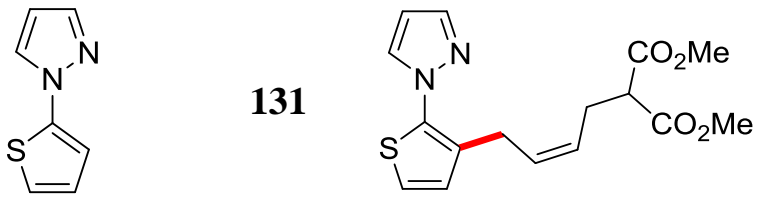

133

$12: 1$

[a] Reaction conditions: 1 or $131(0.50 \mathrm{mmol}), \mathbf{1 2 5 a}(0.60 \mathrm{mmol}),\left[\mathrm{Cp} * \mathrm{Co}(\mathrm{CO}) \mathrm{I}_{2}\right](10 \mathrm{~mol} \%), \mathrm{AgSbF}_{6}(20 \mathrm{~mol} \%), \mathrm{NaOPiv}$ (20 mol \%), DCE $(1.0 \mathrm{~mL}), 20 \mathrm{~h}$, isolated yields. [b] With $\left[\mathrm{Cp} * \mathrm{Rh}\left(\mathrm{CH}_{3} \mathrm{CN}\right)_{3}\right]\left(\mathrm{SbF}_{6}\right)_{2}(10 \mathrm{~mol} \%)$ di-substituted 132ba" $(12 \%, Z / E=1: 3)$ was obtained.

Additionally, some limititations in the scope were disclosed (Scheme 54). Benzamides 68, oximes 48 as well as oxazolines 7 unfortunately delivered no or only traces of the desired products under otherwise identical reaction condition. Moreover, the use of weaker directing groups like tetrazoles $\mathbf{3 1}$ and the functionalization via a six-membered cobaltacycle with substrate 136a turned out to be unfavorable. However, it cannot be excluded that extensive optimization studies on a certain substrate might lead to the desired transformation.

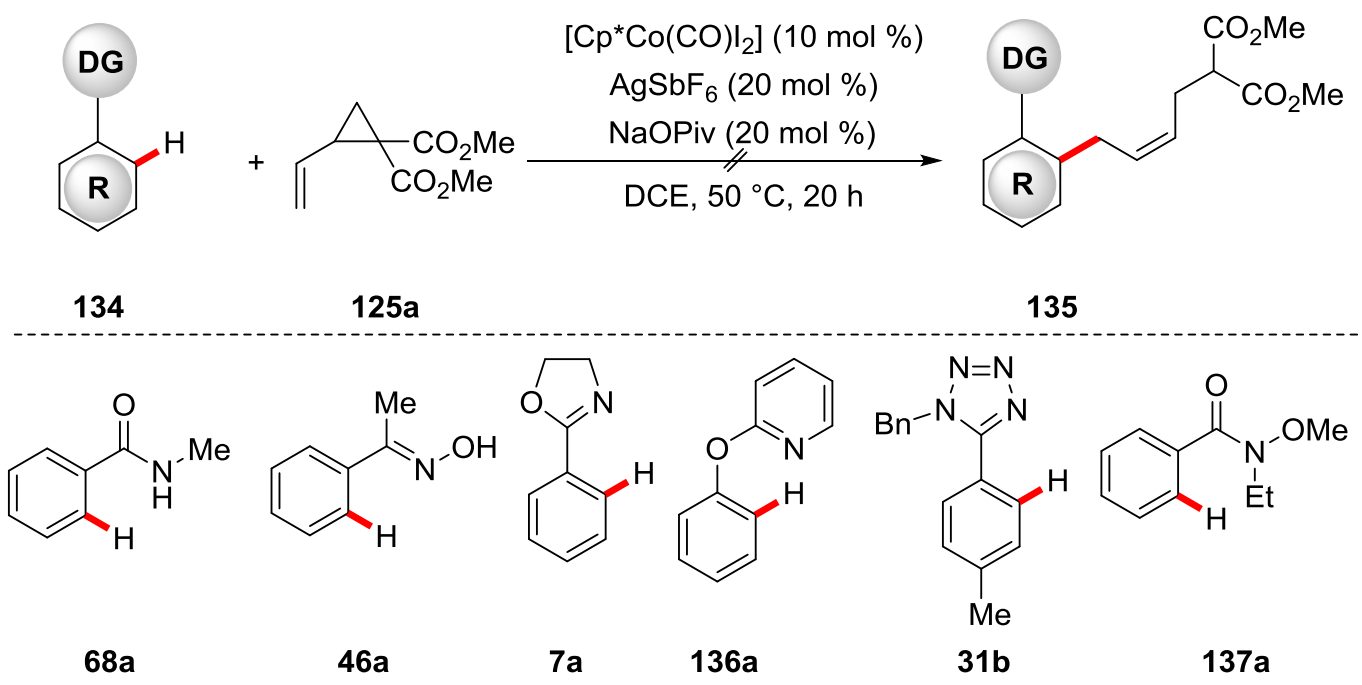

Scheme 54. Limitations of the cobalt(III)-catalyzed C-H/C-C functionalization.

Subsequently, it was explored whether the developed catalytic reaction could proceed under even milder conditions, at an ambient temperature of $25^{\circ} \mathrm{C}$ (Table 11). In this manner, a minor decrease in reactivity was observed, with yields being around $10 \%$ less than under the optimized reaction conditions, except for the unsubstituted substrate 105a (entry 1). Overall, the observed diastereoselectivities are comparable with those at $50{ }^{\circ} \mathrm{C}(c f$. Table 8). 
Table 11. Scope of the cobalt(III)-catalyzed $\mathrm{C}-\mathrm{H} / \mathrm{C}-\mathrm{C}$ functionalization at $25^{\circ} \mathrm{C}$.

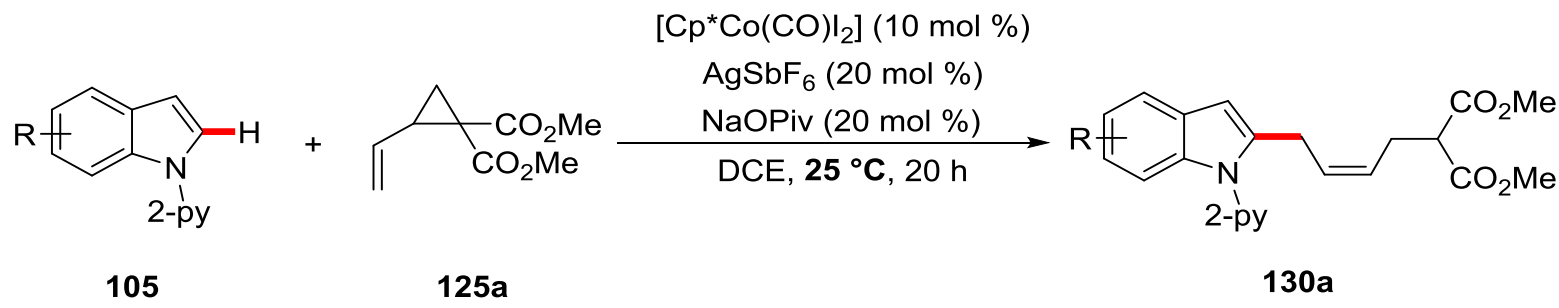

Entry 2-pyridylindole

[a] Reaction conditions: $105(0.50 \mathrm{mmol})$, 125a $(0.60 \mathrm{mmol}),\left[\mathrm{Cp} * \mathrm{Co}(\mathrm{CO}) \mathrm{I}_{2}\right](10 \mathrm{~mol} \%), \mathrm{AgSbF}_{6}(20 \mathrm{~mol} \%), \mathrm{NaOPiv}$ (20 mol \%), DCE $(1.0 \mathrm{~mL}), 25^{\circ} \mathrm{C}, 20 \mathrm{~h}$, isolated yield.

\subsubsection{Mechanistic Studies}

\subsubsection{H/D-Exchange Experiment}

To rationalize the $\mathrm{C}-\mathrm{H}$ activation mechanism, a catalytic reaction in the presence of deuterated cosolvent $\mathrm{CD}_{3} \mathrm{OD}$ was carried out under otherwise unchanged reaction conditions. In the reisolated starting material 105a as well as in the product 130aa a significant H/D-scrambling was observed (Scheme 55). In the case of the reisolated starting material 105a, a deuterium incorporation of $41 \%$ in $\mathrm{C} 2$-position is indicative of a reversible $\mathrm{C}-\mathrm{H}$ activation step.

Furthermore, in the starting material 105a and the product 130aa significant amounts of H/Dexchange in C7- as well as in C3-position of the indole were detected. Deuterium incorporation in C7position can be explained by the formation of a six-membered cobaltacycle, which has been reported 
for indoles and indolines in inter alia copper- ${ }^{[145]}$ rhodium- $^{[146]}$ and iridium-catalyzed ${ }^{[147]} \mathrm{C}-\mathrm{H}$ functionalizations. Nevertheless, the corresponding C7-substituted product could not be detected, which might be due to the unfavored formation of an expected eight-membered intermediate after migratory insertion of the alkene. The H/D-scrambling in C3-position can be explained by a reversible electrophilic activation and following deutero-demetalation.

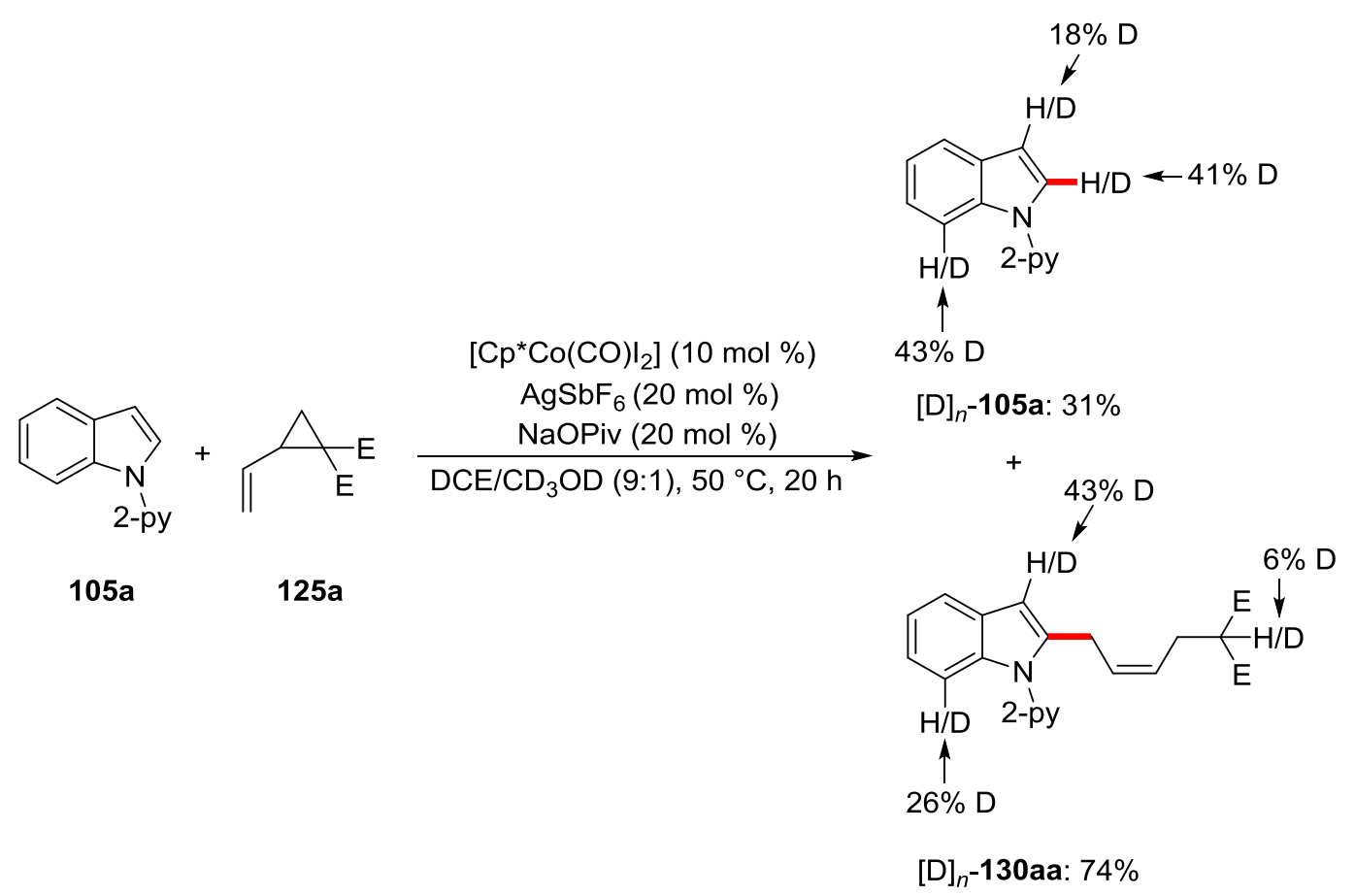

Scheme 55. H/D-exchange study of the cobalt(III)-catalyzed C-H/C-C functionalization. $\mathrm{E}=\mathrm{CO}_{2} \mathrm{Me}$.

\subsection{H/D-exchange in the Absence of Cobalt(III) Catalyst}

Based on the observed deuterium incorporation in C3-position of the indole nucleus, a reaction in the presence of deuterated co-solvent $\mathrm{CD}_{3} \mathrm{OD}$ without the cobalt(III) catalyst and vinylcyclopropane $\mathbf{1 2 5 a}$ was performed. Herein, no detectable H/D-scrambling in any position of the recovered indole 105a (Scheme 56) was observed. These findings emphasize that the $\mathrm{C}-\mathrm{H}$ activation occurs solely via an organometallic cobalt(III) catalysis, and the previously described H/D-scrambling C7-position (vide supra) is a result of cobalt(III)-catalyzed $\mathrm{C}-\mathrm{H}$ activation via a six-membered cobaltacycle.

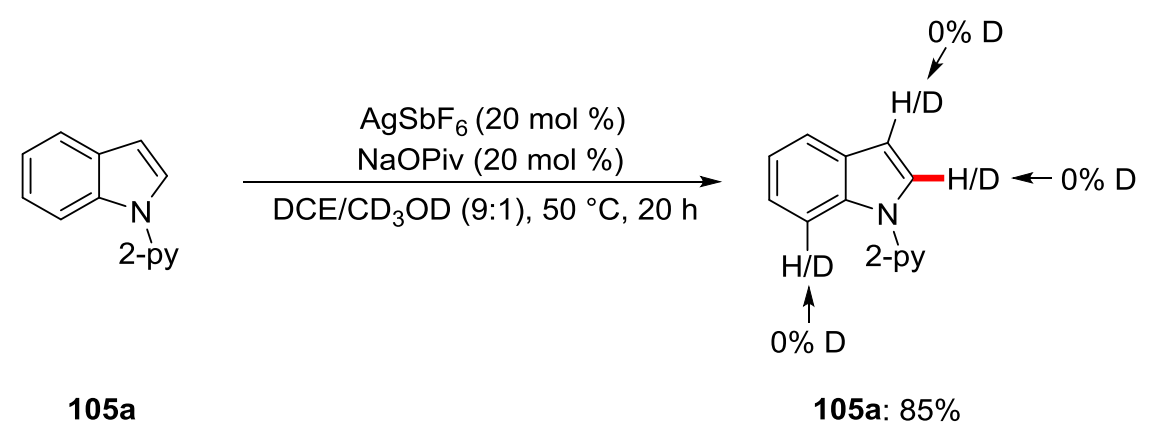

Scheme 56. H/D-exchange study in the absence of the cobalt(III) catalyst. 


\subsubsection{Kinetic Isotope Effect}

To gain a deeper mechanistic understanding of the $\mathrm{C}-\mathrm{H}$ activation elementary step, a KIE-experiment by comparison of independent reaction rates of 2-pyridylindole (105a) and $[\mathrm{D}]_{1}-\mathbf{1 0 5 a}$ was performed (Scheme 57). In this reaction, a KIE of $k_{\mathrm{H}} / k_{\mathrm{D}}=1.3$ was obtained, which suggests a facile and not turnover-limiting $\mathrm{C}-\mathrm{H}$ activation to be operative.

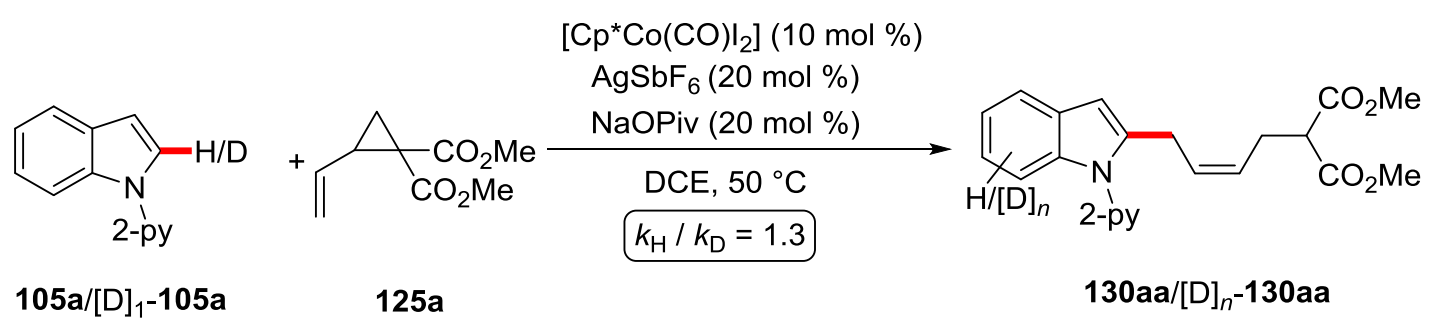

Scheme 57. KIE experiment for the cobalt(III)-catalyzed C-H/C-C functionalization.

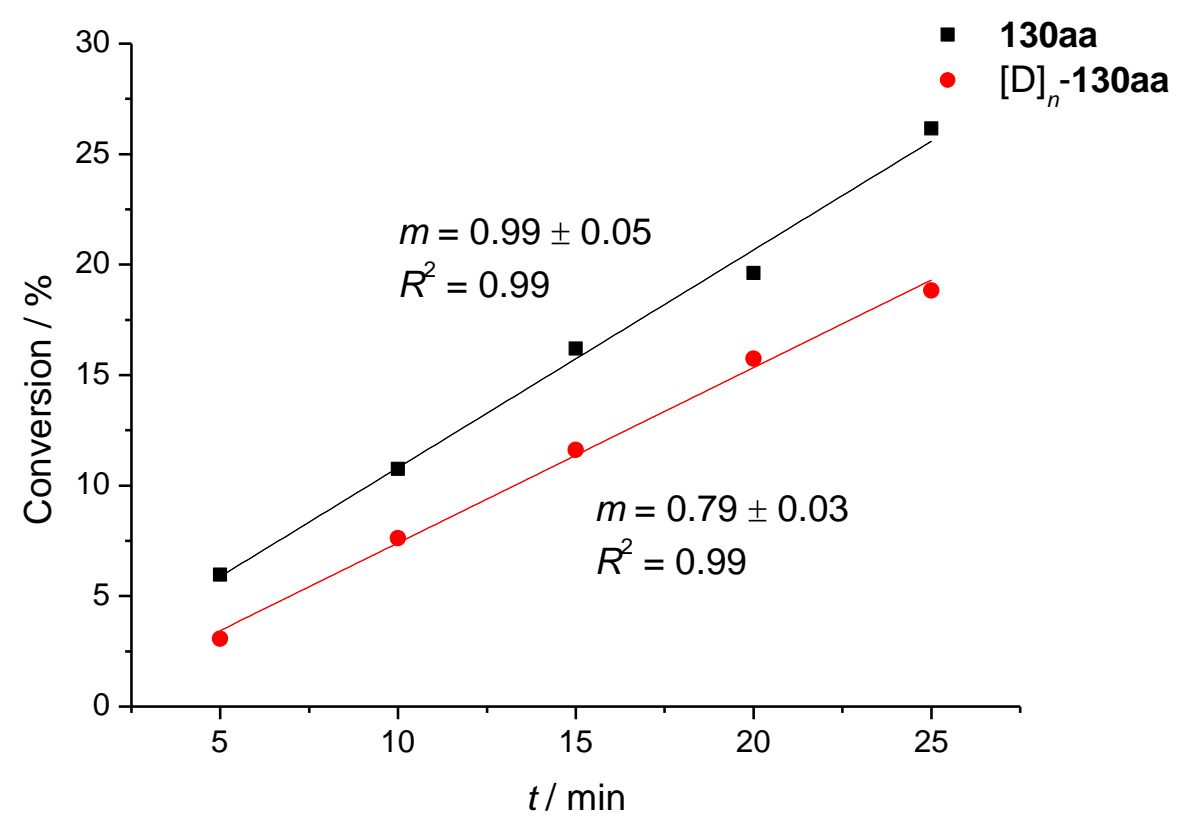

Figure 10. KIE-experiment for the cobalt(III)-catalyzed C-H/C-C functionalization.

\subsubsection{Proposed Catalytic Cycle}

Based on these mechanistic findings, a catalytic cycle was proposed (Scheme 58). The active catalytic species 138 might be a cationic cobalt(III)-pivalate complex formed by reaction between the cobalt(III) precursor and silver hexafluoroantimonate. ${ }^{[91]}$ In this respect, hexafluoroantimonate is a non-nucleophilic and weakly-coordinating anion ${ }^{[148]}$ and it is proposed to energetically stabilize the catalytically active species as well as the following cationic reaction intermediates. The first step comprises a facile and reversible $\mathrm{C}-\mathrm{H}$ cobaltation that is assisted by the pivalate additive and presumably proceeds via a BIES-type mechanistic pathway to furnish the cobaltacycle $\mathbf{1 3 9}$ ( $c f$. Table 
7). For the following steps, extensive computational studies by DFT calculations were performed by M. Feldt. ${ }^{[117 b]}$ Accordingly, a coordination of the vinylcyclopropane 125a leads to intermediate 140, in which a migratory insertion of the coordinated vinylcyclopropane 125a into the carbon-cobalt bond occurs. An oxygen-coordination of the ester group to the cobalt center is suggested to energetically stabilize the following intermediate 141. Thereafter, a diastereoselectivity-determining $\mathrm{C}-\mathrm{C}$ cleavage process leads to the favored Z-configurated intermediate 142.

By applying the energetic span model ${ }^{[149]}$ to the energetic profile, the activation barrier difference for this selectivity-determining $\mathrm{C}-\mathrm{C}$ cleavage process amounts to $6.5 \mathrm{kcal} / \mathrm{mol}$ in favor of the $Z$ configurated transition state. The origin of this diastereocontrol presumably lies in attractive London dispersion interactions that are more pronounced for the compact Z-configurated transition state. ${ }^{[143]}$ Along the same line, the diastereodivergence between cobalt(III) and rhodium(III) catalysis is proposed to be an effect of dispersion interactions due to the smaller carbon-cobalt bonds. The reduced bond lengths in cobalt(III) intermediates of this reaction lead to larger dispersion contributions, which are strongly dependent on the interaction distance $r$ by proportionality to $r^{-6}$. Finally, a protodemetalation step by the in situ formed pivalic acid regenerates the active catalyst and releases the product 130aa. Notably, $Q$. Bu did not observe any post catalytic-isomerization of the double bond, when the $E$-configurated product was subjected to the optimal reaction conditions. ${ }^{[17 b]}$ 


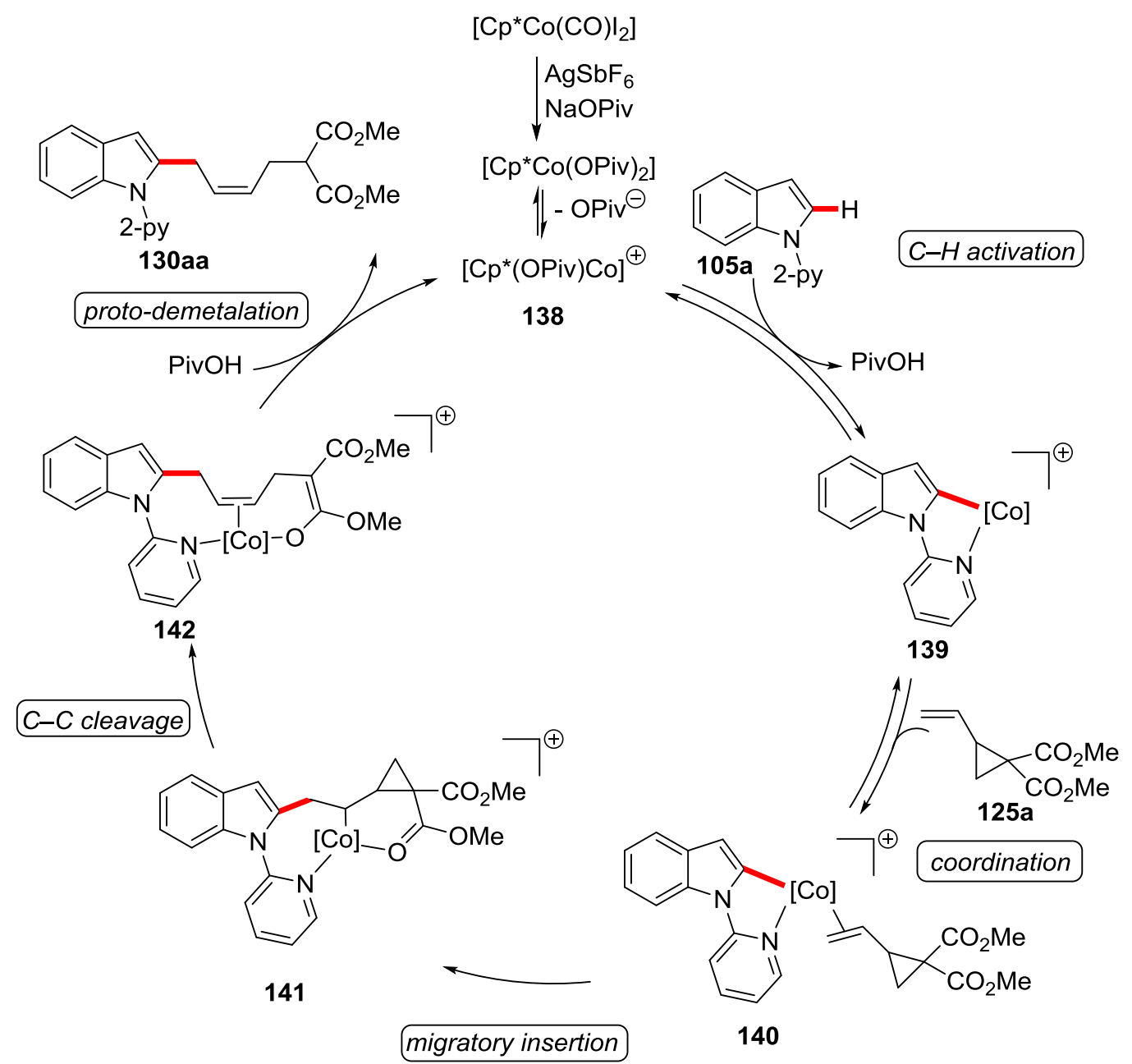

Scheme 58. Proposed catalytic cycle for the cobalt(III)-catalyzed C-H/C-C activation. $[\mathrm{Co}]=\mathrm{Cp}^{*} \mathrm{Co}^{\mathrm{III}}$. 


\subsection{Regioselective Cobalt(III)-Catalyzed C-H Alkylations}

Transition metal-catalyzed $\mathrm{C}-\mathrm{H}$ alkylations by alkene hydroarylation reactions represent a versatile and powerful strategy to achieve highly atom-economical alkylations. ${ }^{[110 c, 150]}$ However, in these reactions there is an inherent requirement for Markovnikov/anti-Markovnikov selectivity control, which has so far been mainly restricted to electronically activated alkenes and the use of complex ligands. ${ }^{[113-114,151]}$ Yoshikai and coworkers have indeed demonstrated a switchable regiocontrol in low valent cobalt-catalyzed $\mathrm{C}-\mathrm{H}$ hydroarylations, but stoichiometric amounts of Grignard reagents were required, which translated into a considerable limitation regarding the substrate scope. ${ }^{[14]}$

Consequently, a new and sustainable concept to achieve regiocontrol in cobalt(III) catalysis is indispensable. The new catalytic system must obviate the use of Grignard reagents, laborious ligand design and should ideally be applicable to hydroarylations with unactivated alkenes. In this regard, a full control of linear/branched selectivity is of prime importance.

\subsubsection{Optimization Studies for the Linear-Selective C-H Alkylation}

The optimization studies for the cobalt(III)-catalyzed anti-Markovnikov-selective $\mathrm{C}-\mathrm{H}$ alkylation were commenced by testing the effect of a few selected acetate and pivalate additives (Table 12, entries 1-5). Among a series of alkali metal cations, especially lithium and sodium performed well, while the cesium analogue delivered the desired linear product 144aa in only unsatisfactory yield or trace amount (entries 3 and 5). Remarkably, acetate bases displayed a slightly higher selectivity up to an excellent ratio of AM:M = 99:1. Thereafter, the effect of different reaction temperatures on the regioselectivity was studied by using pivalic acid as the additive. Interestingly, the selectivity dropped with decreasing temperature in favor of the Markovnikov-product 145aa (entries 6-8). Moreover, the use of sterically much more congested 1-adamantanecarboxylic acid as additive (entries 9-11) led to a further drop in linear selectivity. Notably, by increasing the acid loading up to one equivalent, an intriguing inversion of selectivity (entry 11) towards the branched product 145aa was observed.

In summary, lower temperatures and carboxylic acids as additives tend to favor the formation of the branched product, although the temperature-dependence is much less pronounced.

To achieve an excellent linear selectivity of AM:M > 99:1, the temperature was kept at $120{ }^{\circ} \mathrm{C}$ and no additive was utilized (entry 14). Noteworthily, no reactivity was observed by omitting the essential $\mathrm{AgSbF}_{6}$ or the catalyst (entries 12 and 13). Silver was found to play no role in the catalytic reaction, because silver-free conditions with $\left[\mathrm{Cp} * \mathrm{Co}\left(\mathrm{CH}_{3} \mathrm{CN}\right)_{3}\right]\left(\mathrm{SbF}_{6}\right)_{2}$ as the catalyst still furnished the desired product 144aa in satisfactory yield (entry 15). Additionally, cobalt(III) complexes with differently substituted Cp-ligands performed overall poorly as catalysts (entries 17 and 18). 
Table 12. Optimization studies for the anti-Markovnikov-selective $\mathrm{C}-\mathrm{H}$ alkylation.

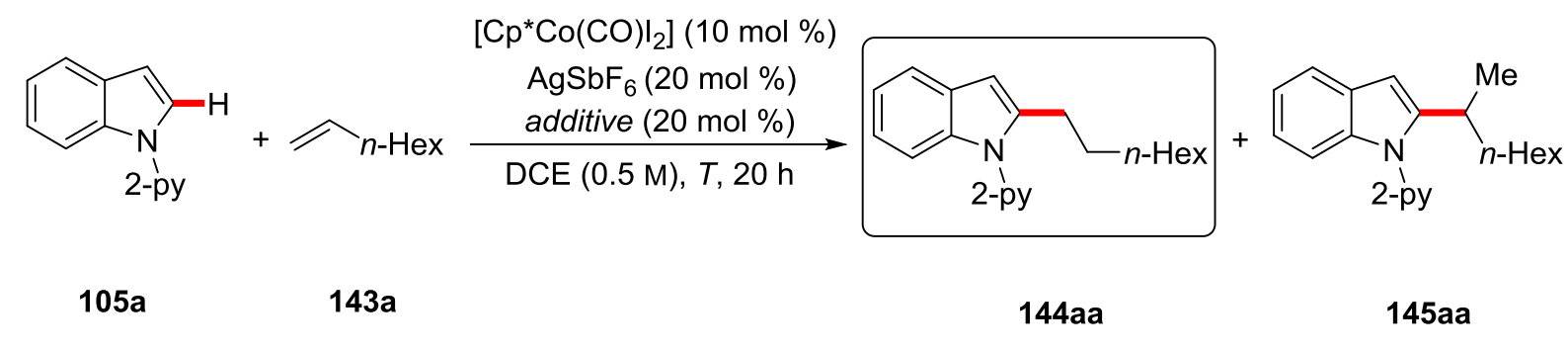

\begin{tabular}{|c|c|c|c|c|}
\hline Entry & additive & $T /{ }^{\circ} \mathrm{C}$ & 144aa: $145 \mathrm{aa}^{[\mathrm{a}]}$ & yield / \% ${ }^{[\mathrm{a}]}$ \\
\hline 1 & $\mathrm{LiOAc}$ & 120 & 99:1 & 82 \\
\hline 2 & $\mathrm{NaOAc}$ & 120 & $98: 2$ & 77 \\
\hline 3 & $\mathrm{CsOAc}$ & 120 & $98: 2$ & 15 \\
\hline 4 & $\mathrm{NaOPiv}$ & 120 & $88: 12$ & 46 \\
\hline 5 & CsOPiv & 120 & --- & traces \\
\hline 6 & $\mathrm{PivOH}$ & 120 & $93: 7$ & 80 \\
\hline 7 & $\mathrm{PivOH}$ & 100 & $85: 15$ & 67 \\
\hline 8 & $\mathrm{PivOH}$ & 60 & $52: 48$ & 56 \\
\hline 9 & $1-\mathrm{AdCO}_{2} \mathrm{H}$ & 120 & $88: 12$ & 82 \\
\hline 10 & $1-\mathrm{AdCO}_{2} \mathrm{H}(40 \mathrm{~mol} \%)$ & 120 & $46: 54$ & 74 \\
\hline 11 & $1-\mathrm{AdCO}_{2} \mathrm{H}(100 \mathrm{~mol} \%)$ & 120 & $37: 63$ & 81 \\
\hline 12 & --- & 120 & --- & $--^{[b]}$ \\
\hline 13 & --- & 120 & --- & $--^{[c]}$ \\
\hline 14 & --- & 120 & $>99: 1$ & 82 \\
\hline 15 & --- & 100 & $>99: 1$ & 75 \\
\hline 16 & --- & 120 & $>99: 1$ & $76^{[\mathrm{d}]}$ \\
\hline 17 & --- & 120 & $95: 5$ & $7^{[\mathrm{e}]}$ \\
\hline 18 & --- & 120 & $>99: 1$ & traces $^{[f]}$ \\
\hline $\begin{array}{l}{ }^{\text {aa] }} \text { Reaction co } \\
\text { additive }(20 \mathrm{~m} \\
\text { spectroscopy. } \\
{ }^{[f]}[\mathrm{CpCo}(\mathrm{CO}) \mathrm{I}\end{array}$ & $\begin{array}{l}\text { 105a }(0.50 \mathrm{mmol}), 143 \mathrm{a}( \\
\mathrm{DCE}(1.0 \mathrm{~mL}), 120{ }^{\circ} \mathrm{C}, 20 \mathrm{~h} \\
\operatorname{lgbF}_{6} \cdot{ }^{[\mathrm{cc}]} \mathrm{No}\left[\mathrm{Cp} * \mathrm{Co}(\mathrm{CO}) \mathrm{I}_{2}\right.\end{array}$ & $\begin{array}{l}\mathrm{mmol} \text {, } \\
\text { ated yiel }\end{array}$ & $\begin{array}{l}\left.\mathrm{o}(\mathrm{CO}) \mathrm{I}_{2}\right](10 \mathrm{~mol} \\
\text { ectivities determin } \\
\left.\mathrm{N})_{3}\right]\left(\mathrm{SbF}_{6}\right)_{2} \cdot{ }^{[\mathrm{ee}]}[1,\end{array}$ & $\begin{array}{l}\mathrm{gSbF}_{6}(20 \mathrm{mc} \\
\text { means of }{ }^{1} \mathrm{H}- \\
)_{2} \mathrm{C}_{5} \mathrm{H}_{3} \mathrm{Co}(\mathrm{C}\end{array}$ \\
\hline
\end{tabular}

\subsubsection{Optimization Studies for the Branched-Selective C-H Alkylation}

With the results from the previous optimization study in hand (vide supra), it was initially tested whether the formation of the Markovnikov-selective product 145aa is mainly controlled by reaction temperature. However, still the linear product 144aa (Table 13, entry 1) was formed in the absence of additives although the reaction temperature was decreased to as low as $50^{\circ} \mathrm{C}$. Furthermore, the steric effect of the additives was investigated by comparing a set of carboxylates (entries 2-6). Therein, it could be pointed out that the sterically encumbered 1-adamantylcarboxylate delivered the branched product 145aa in moderate yield, but very good regioselectivity (entries 3 and 6). Moreover, a series 
of carboxylic acids (entries 7-12) was studied, similarly leading to very good selectivities and moderate yields. The use of bulky pivalic and 1-adamantanecarboxylic acid (entries 8 and 12) furnished the Markovnikov-selective product 145aa in good yields. Notably, an increase of the 1adamantanecarboxylic acids concentration furthermore improved the selectivity up to an excellent ratio of M:AM = 95:5 (entries 14-16). Interestingly, carboxylic acids delivered the branched product 145aa with comparable selectivities, whereas a clear trend in steric effects along a series of carboxylates was observed (vide supra). These observations might indicate that the branched-selective mechanistic pathway is generally preferred by using acid additives. As it had previously been observed (vide supra), no product was formed in the absence of $\mathrm{AgSbF}_{6}$ or the catalyst (entries 17 and 18). Noteworthily, under silver-free conditions and $\left[\mathrm{Cp} * \mathrm{Co}\left(\mathrm{CH}_{3} \mathrm{CN}\right)_{3}\right]\left(\mathrm{SbF}_{6}\right)_{2}$ as the catalyst (entry 19), the product 145aa was still afforded in good yields, but the selectivity decreased slightly to $\mathrm{M}: \mathrm{AM}=75: 25$. In this regard, it can be assumed that the acetonitrile ligands of $\left[\mathrm{Cp} * \mathrm{Co}\left(\mathrm{CH}_{3} \mathrm{CN}\right)_{3}\right]\left(\mathrm{SbF}_{6}\right)_{2}$ reduce the selectivity by hampering the reaction between the catalyst or a catalytic intermediate and the acid additive. As observed before (Table 12), cobalt(III) complexes with differently substituted Cp-ligands performed poorly (entries 20 and 21).

Table 13. Optimization studies for the Markovnikov-selective C-H alkylation.

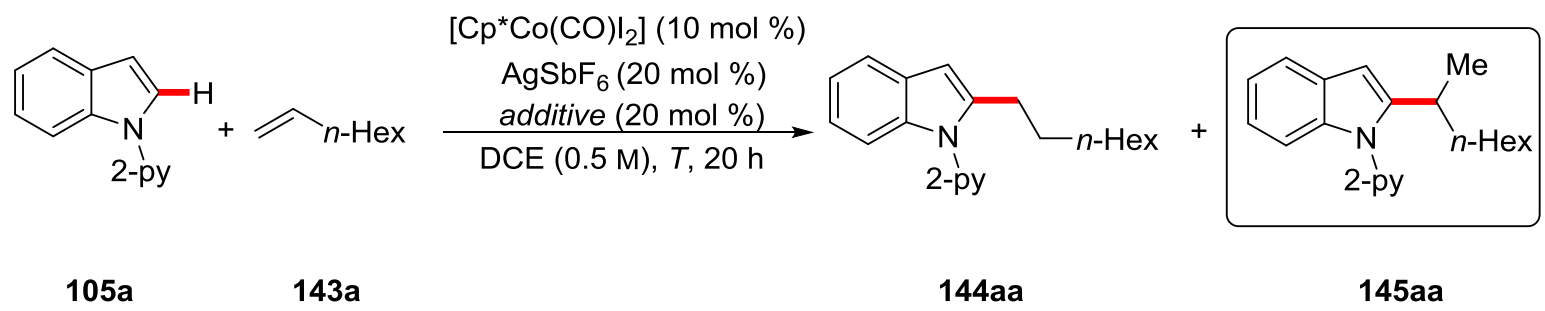

\begin{tabular}{ccccc}
\hline Entry & additive & $T /{ }^{\circ} \mathrm{C}$ & 144aa:145aa $^{[a]}$ & yield / \% \\
\hline 1 & --- & 50 & $>99: 1$ & 9 \\
2 & $\mathrm{LiOAc}$ & 50 & $27: 73$ & 6 \\
3 & $\mathrm{LiO}_{2} \mathrm{C}-(1-\mathrm{Ad})$ & 50 & $8: 92$ & 48 \\
4 & $\mathrm{NaOAc}$ & 50 & $35: 65$ & 11 \\
5 & $\mathrm{NaOPiv}$ & 50 & $22: 78$ & 44 \\
6 & $\mathrm{NaO}_{2} \mathrm{C}-(1-\mathrm{Ad})$ & 50 & $12: 88$ & 50 \\
7 & $\mathrm{AcOH}$ & 50 & $14: 86$ & 43 \\
8 & $\mathrm{PivOH}$ & 50 & $17: 83$ & 68 \\
9 & $\mathrm{CyCO}_{2} \mathrm{H}$ & 50 & $14: 86$ & 58 \\
10 & $\mathrm{C}_{6} \mathrm{H}_{5} \mathrm{CO}_{2} \mathrm{H}$ & 50 & $14: 86$ & 57 \\
11 & $\mathrm{CF}_{3} \mathrm{CO}_{2} \mathrm{H}$ & 50 & --- & --- \\
12 & $1-\mathrm{AdCO}_{2} \mathrm{H}$ & 50 & $13: 87$ & 68 \\
13 & $1-\mathrm{AdCO}_{2} \mathrm{H}$ & 60 & $20: 80$ & 71 \\
14 & $1-\mathrm{AdCO}_{2} \mathrm{H}(40 \mathrm{~mol} \%)^{2}$ & 50 & $10: 90$ & 79
\end{tabular}




\begin{tabular}{|c|c|c|c|c|}
\hline 15 & $1-\mathrm{AdCO}_{2} \mathrm{H}(100 \mathrm{~mol} \%)$ & 50 & $5: 95$ & 80 \\
\hline 16 & $1-\mathrm{AdCO}_{2} \mathrm{H}(100 \mathrm{~mol} \%)$ & 40 & $>1: 99$ & 48 \\
\hline $17^{[\mathrm{b}]}$ & --- & 50 & --- & --- \\
\hline $18^{[\mathrm{c}]}$ & $1-\mathrm{AdCO}_{2} \mathrm{H}$ & 50 & --- & -- \\
\hline $19^{[\mathrm{d}]}$ & $1-\mathrm{AdCO}_{2} \mathrm{H}$ & 50 & $25: 75$ & 65 \\
\hline $20^{[\mathrm{e}]}$ & $1-\mathrm{AdCO}_{2} \mathrm{H}$ & 50 & --- & traces \\
\hline $21^{[\mathrm{f}]}$ & $1-\mathrm{AdCO}_{2} \mathrm{H}$ & 50 & --- & traces \\
\hline
\end{tabular}

\subsubsection{Scope of the Linear-Selective C-H Alkylation}

Under the optimized conditions for the linear-selective $\mathrm{C}-\mathrm{H}$ alkylation, the effect of various 2pyridylindoles 105 was probed (Table 14). Among a series of substituents in C5-position of the indole nucleus, halogens were well tolerated and delivered the corresponding products in good yields (entries 3-5). Even in the presence of a very challenging and redox-active nitro-substituent, a moderate yield of 144ea was achieved (entry 6), although substrate 105e displayed a low solubility in DCE. It is noteworthy that methyl- and ester-substituents in C3-position performed with similar reactivities (entries 7 and 8). Substitution in the C4-position (entries 9 and 10) furnished the desired products $144 \mathrm{ha}$ and $\mathbf{1 4 4 0 a}$ in good yields. Nonetheless, a slight drop in yield was observed for indole 105j bearing an ethyl-substituent in the C7-position, which is possibly due to steric effects (entry 11). Remarkably, the pyridyl-directing group was shown to be removable and the corresponding free indoles were obtained in excellent yields ( $c f$. chapter 5.4.2).

With respect to limitations of the substrate scope, 1-(thiophen-2-yl)-1H-pyrazole (131) was not converted under otherwise unchanged reaction conditions (entry 12). In this regard, V. Müller showed that substrate 131 only reacted by addition of pivalic acid at $140{ }^{\circ} \mathrm{C}$, delivering the corresponding product in good yield and selectivity of AM:M $=55: 45 .{ }^{[152]}$

In addition, 2-pyridyl-1H-pyrrole (146) delivered the desired product 147 with an excellent selectivity of AM:M > 99:1, but only unsatisfactory yields were obtained and significant amounts of difunctionalized product $\mathbf{1 4 7}$ ' were formed (entry 13). In summary, all products were afforded with an excellent linear selectivity of AM:M > 99:1. 
Table 14. Scope of different 2-pyridylindoles 105 under linear-selective reaction conditions.
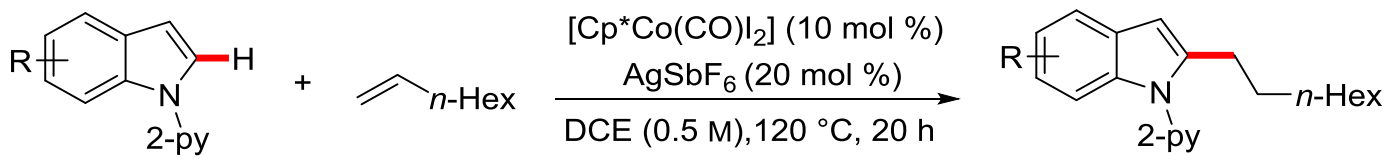

105

$143 a$

144

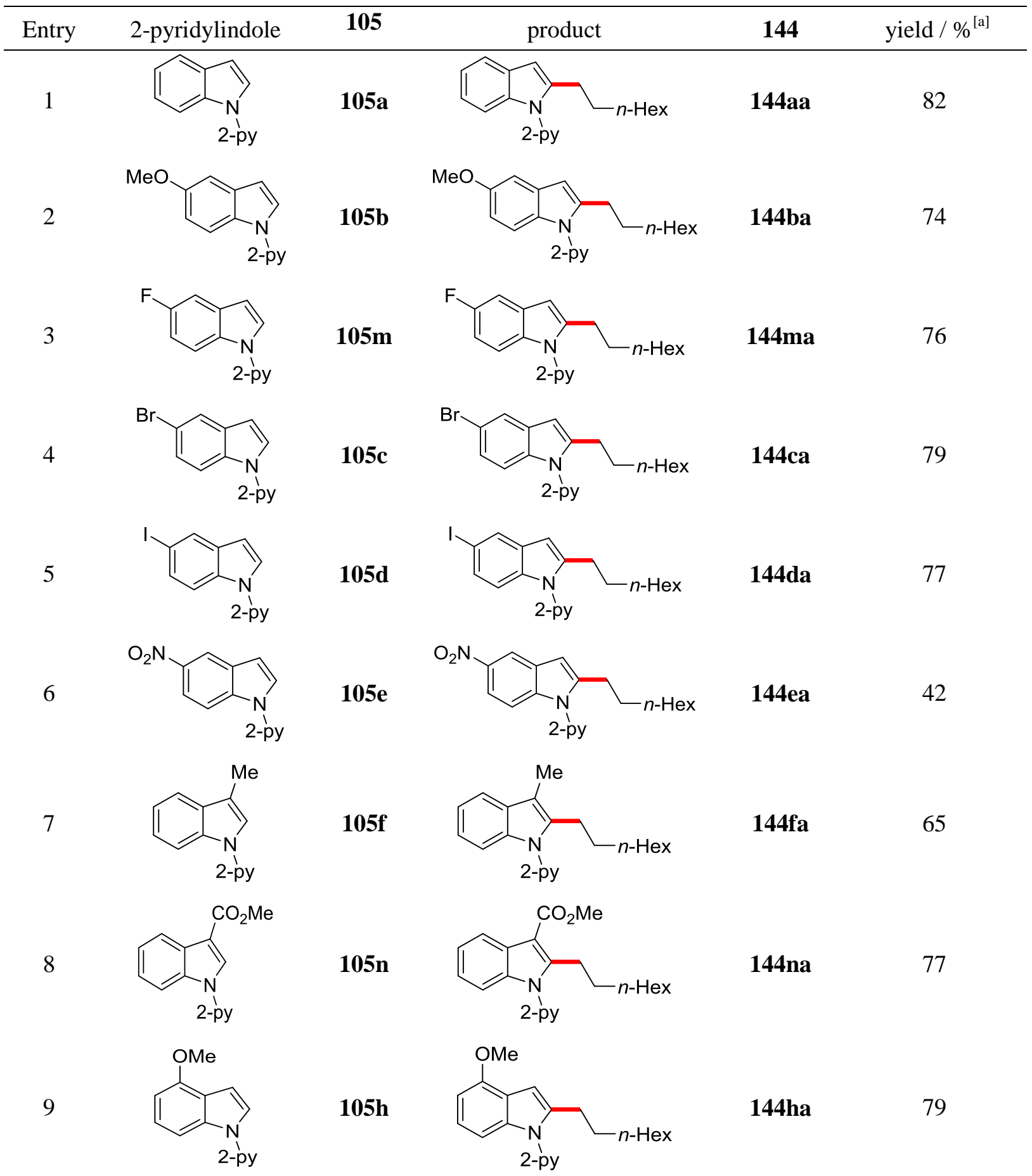


10<smiles>COC(=O)c1cccc2c1ccn2[Te]</smiles>

11<smiles>CCc1cccc2ccn([Te])c12</smiles>

12<smiles></smiles>

13

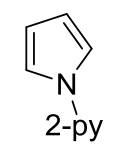

1050

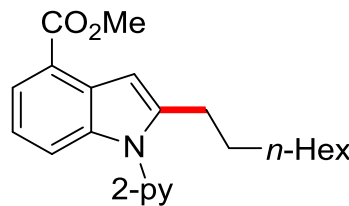

105j

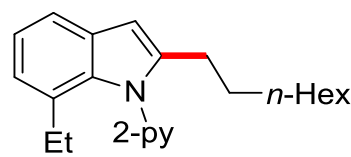

131

146

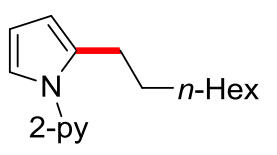

$1440 a$

83

144ja

61

147

$31^{[\mathrm{b}]}$

[a] Reaction conditions: $105(0.50 \mathrm{mmol}), \mathbf{1 4 3 a}(1.50 \mathrm{mmol}),\left[\mathrm{Cp} * \mathrm{Co}(\mathrm{CO}) \mathrm{I}_{2}\right](10 \mathrm{~mol} \%), \mathrm{AgSbF}_{6}(20 \mathrm{~mol} \%)$, DCE $(1.0 \mathrm{~mL}, 0.5 \mathrm{M}), 120{ }^{\circ} \mathrm{C}, 20 \mathrm{~h}$, isolated yield; all AM:M > 99:1, determined by ${ }^{1} \mathrm{H}-\mathrm{NMR}$ spectroscopy. ${ }^{[\mathrm{b}]}$ 45\% of di-substituted product 147' (AM:M > 99:1) was isolated.

Furthermore, alkenes 143 with selected functional groups were examined under the linear-selective reaction regime (Table 15). Initially, allyl benzene (143b) reacted with excellent regioselectivities and no by-products from isomerization of the double bond were observed. Likewise, alkenes with different chain lengths performed well (entries 2-4), with somewhat higher yields for longer alkyl chains, which might be attributed to lower boiling points. The boiling point of 1-hexene (143e) at ambient pressure is $63^{\circ} \mathrm{C},{ }^{[153]}$ presumably leading to evaporation under elevated reaction temperatures. Notably, ester- (143f) and challenging hydroxyl-groups (143g) on the alkene were well tolerated (entries 5 and 6). The good performance of 1,7-octadiene (143h) demonstrated that double bonds did not isomerize during the reaction. In addition, only selective monofunctionalization occurred (entry 7), albeit the overall yield was somewhat diminished. In this regard, the polymerization of 1,7-octadiene (143h) as a potential side reaction should be considered. Furthermore, internal alkenes, such as cis/trans-2-octene (143i), did not react under otherwise unchanged reaction conditions (entry 8).

Table 15. Scope of different alkenes 143 under the linear-selective reaction regime.

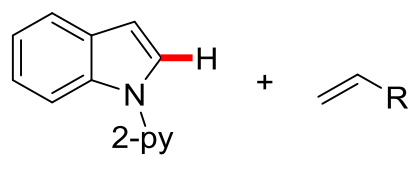

$105 a$

143

$$
\begin{gathered}
\begin{array}{c}
{\left[\mathrm{Cp}^{*} \mathrm{Co}(\mathrm{CO}) \mathrm{I}_{2}\right](10 \mathrm{~mol} \%)} \\
\mathrm{AgSbF}_{6}(20 \mathrm{~mol} \%)
\end{array} \\
\mathrm{DCE}(0.5 \mathrm{M}), 120^{\circ} \mathrm{C}, 20 \mathrm{~h}
\end{gathered}
$$

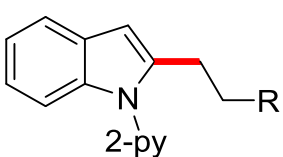

\begin{tabular}{|c|c|c|c|c|c|}
\hline Entry & alkene & 143 & product & 144 & yield $/ \%^{[\mathrm{a}]}$ \\
\hline 1 & & $143 b$ & & 144ab & 85 \\
\hline
\end{tabular}

144 
2

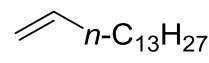

$143 c$

143d

3<smiles>C=COOCl</smiles>

$\gamma_{n-\mathrm{Bu}}$

4

5

$$
\sim \mathrm{r}_{4}^{\mathrm{O}} \mathrm{I}_{\mathrm{O}}^{\mathrm{Me}}
$$$$
-\mathrm{H}_{8} \mathrm{OH}
$$

6<smiles>C=CCCC=C</smiles>

7

8

$\mathrm{Me}_{n} / \mathrm{C}_{n \text {-Pent }}$

$143 i$

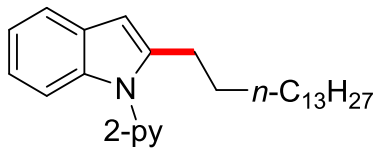

144ac

79

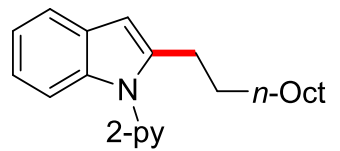

144ad

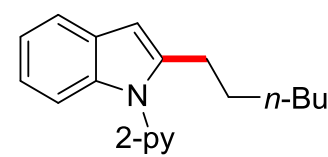

144ae

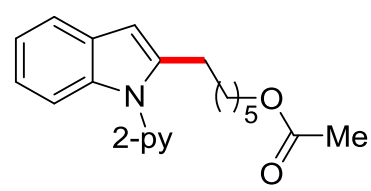

144af

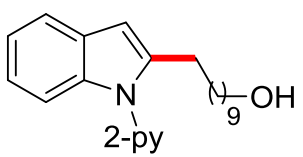

144ag

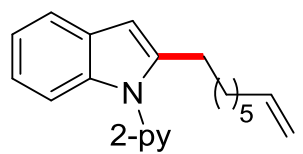

144ah

66

[a] Reaction conditions: 105a $(0.50 \mathrm{mmol}), \mathbf{1 4 3}(1.50 \mathrm{mmol}),\left[\mathrm{Cp} * \mathrm{Co}(\mathrm{CO}) \mathrm{I}_{2}\right](10 \mathrm{~mol} \%), \mathrm{AgSbF}_{6}(20 \mathrm{~mol} \%)$, DCE $(1.0 \mathrm{~mL}, 0.5 \mathrm{M}), 120{ }^{\circ} \mathrm{C}, 20 \mathrm{~h}$, isolated yield; all AM:M > 99:1, determined by ${ }^{1} \mathrm{H}-\mathrm{NMR}$ spectroscopy.

\subsubsection{Scope of the Branched-Selective C-H Alkylation}

The substrate scope under the Markovnikov-selective reaction regime was initially explored by probing various substituents in C5-position, such as methoxy and halogens (Table 16, entries 2-5). Fortunately, all reactions performed with good yields and selectivities in the range of M:AM = 95:5. However, a methyl-substituent in C3-position of the indole nucleus obviously disfavored the formation of the branched product $\mathbf{1 4 5 f a}$ likely due to repulsive steric effects. Thus, the undesired anti-Markovnikov product 144fa was afforded (entry 7). In contrast, substituents in C4- or C7-position again resulted in overall satisfactory yields and very good selectivities (entries 8 and 9). Remarkably, 1-(thiophen-2-yl)-1H-pyrazole (131) was successfully $\mathrm{C}-\mathrm{H}$ alkylated in acceptable selectivity and very good yield at $80{ }^{\circ} \mathrm{C}$ (entry 10). Unfortunately, 2-pyridyl-1H-pyrrole (146) delivered mainly the linear-selective product 149 in lower yield (entry 11).

In summary, only substitution in C3-position affected the branched/linear-selectivity, while different functional groups had no observable impact on the selectivity. The overall reactivity was obviously not controlled by electronic parameters of the indole substrate. Interestingly, the pyridyl-directing group was shown to be removable in an excellent yield ( $c f$. chapter 5.4.2). 
Table 16. Scope of different 2-pyridylindoles 105 and heteroarenes 145 for the branched-selective regime.<smiles>[R][CH]C=C</smiles>

105
$\left[\mathrm{Cp}{ }^{*} \mathrm{Co}(\mathrm{CO}) \mathrm{I}_{2}\right](10 \mathrm{~mol} \%)$ $\underset{1-\mathrm{AdCO}_{2} \mathrm{H} \text { (1.0 equiv) }}{\stackrel{\mathrm{AgSbF}_{6}(20 \mathrm{~mol} \%)}{\longrightarrow}}$ DCE $(1.0 \mathrm{M}), 50^{\circ} \mathrm{C}, 20 \mathrm{~h}$

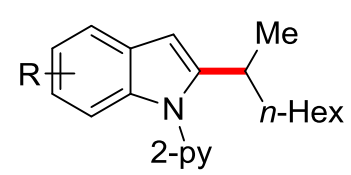

145

\begin{tabular}{|c|c|c|c|c|c|c|}
\hline entry & 2-pyridylindole & 105 & product & 145 & $\mathrm{M}: \mathrm{AM}^{[\mathrm{a}]}$ & yield $/ \%^{[\mathrm{a}]}$ \\
\hline 1 & 2-py & $105 a$ & 2-py & 145aa & $95: 5$ & 80 \\
\hline 2 & $\begin{array}{c}N \\
2-p y\end{array}$ & $105 b$ & 2-py & $145 \mathrm{ba}$ & $94: 6$ & 69 \\
\hline 3 & 2-py & $105 \mathrm{~m}$ & 2-py & 145ma & $96: 4$ & 85 \\
\hline 4 & 2-py & $105 c$ & 2-py & 145ca & $95: 5$ & 80 \\
\hline 5 & 2-py & 105d & 2-py & 145da & $94: 6$ & 82 \\
\hline 7 & 2-py & $105 f$ & 2-py & $144 f a$ & $21: 79$ & 61 \\
\hline 8 & 2-py & $105 h$ & 2-py & 145ha & $95: 5$ & 76 \\
\hline 9 & 2-py & $105 j$ & 2-py & 145ja & $96: 4$ & 71 \\
\hline 10 & & 131 & & 148 & $81: 19$ & $83^{[\mathrm{b}]}$ \\
\hline
\end{tabular}


11

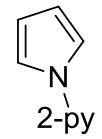

146

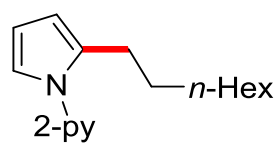

149

40:60

16

[a] Reaction conditions: 105 (0.50 mmol), 143a (1.50 mmol), [Cp*Co(CO) $\left.\mathrm{I}_{2}\right](10 \mathrm{~mol} \%), \mathrm{AgSbF}_{6}(20 \mathrm{~mol} \%)$ and $1-\mathrm{AdCO}_{2} \mathrm{H}(0.50 \mathrm{mmol}), \mathrm{DCE}(0.5 \mathrm{~mL}, 1.0 \mathrm{M}), 50{ }^{\circ} \mathrm{C}, 20 \mathrm{~h}$, isolated yield; all AM:M ratios determined by ${ }^{1} \mathrm{H}-\mathrm{NMR}$ spectroscopy. [b] At $80{ }^{\circ} \mathrm{C}$.

Subsequently, a comprehensive study as to the scope of the alkene 143 was analogously performed under the branched-selective reaction conditions (Table 17). Herein, allylbenzene (143b) delivered the corresponding product 145ab with an outstanding selectivity of M:AM = 99:1 and a good yield of $82 \%$ (entry 1). Thereafter, a variation of the alkyl chain showed no effect on the selectivity, and only a moderate decrease in yield with shorter chain lenght was found (entries 2-4). Remarkably, an estergroup on the alkene 143f was well tolerated and the corresponding product 143af was afforded with an excellent selectivity of M:AM = 99:1 (entry 5). A challenging hydroxyl-group performed moderately (entry 6) with slightly lower yield of the corresponding product 145ag. As observed before, a selective mono-functionalization of 1,7-octadiene 143h was observed with a higher yield of $78 \%$ of the corresponding branched product 145ah than under linear-selective conditions ( $c f$. Table 15, entry 7). This finding could be either explained by a reduced evaporation of 1,7-octadiene (143h) or the diminished polymerization tendency at the lower reaction temperatures. Furthermore, it was shown that internal alkenes, such as cis/trans-2-octene 143i, were not converted under otherwise unchanged reaction conditions (entry 8 ).

Table 17. Scope of different alkenes 143 for the branched-selective regime.

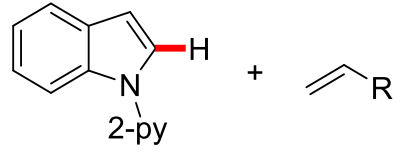

$105 a$

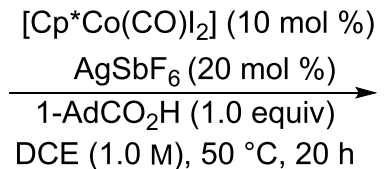

DCE (1.0 M), $50{ }^{\circ} \mathrm{C}, 20 \mathrm{~h}$

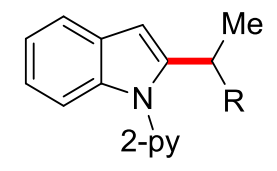

145

\begin{tabular}{|c|c|c|c|c|c|c|}
\hline entry & alkene & 143 & product & 145 & M:AM & yield $/ \%^{[\mathrm{a}]}$ \\
\hline 1 & ${ }^{P h}$ & $143 b$ & 2-py & 145ab & 99:1 & 82 \\
\hline 2 & $n-\mathrm{C}_{13} \mathrm{H}$ & $143 c$ & 2-ру & 145ac & $95: 5$ & 76 \\
\hline
\end{tabular}




3 (43)

[a] Reaction conditions: 105a $(0.50 \mathrm{mmol}), 143$ (1.50 mmol), [Cp*Co(CO) $\left.\mathrm{I}_{2}\right](10 \mathrm{~mol} \%), \mathrm{AgSbF}_{6}(20 \mathrm{~mol} \%)$, 1- $\mathrm{AdCO}_{2} \mathrm{H}(0.50 \mathrm{mmol}), \mathrm{DCE}(0.5 \mathrm{~mL}, 1.0 \mathrm{M}), 50{ }^{\circ} \mathrm{C}, 20 \mathrm{~h}$, isolated yield; all AM:M ratios determined by means of ${ }^{1} \mathrm{H}$-NMR spectroscopy.

\subsubsection{Enantioselective Cobalt(III)-Catalyzed C-H Hydroarylations}

Given the inherent possibility to induce enantioselectivity within the presented branched-selective cobalt(III)-catalyzed $\mathrm{C}-\mathrm{H}$ hydroarylation, extensive optimization studies were performed by employing chiral carboxylic acids (Table 18). In this respect, the use of natural amino acids as chiral additives is particularly attractive because of their synthetic versatility ${ }^{[154]}$ and natural availability. Thus far, a considerable number of $\mathrm{C}-\mathrm{H}$ functionalizations involving metals like inter alia ruthenium $^{[131,155]}$ and palladium ${ }^{[156]}$ have been reported, in which mono-protected amino acids have been utilized as beneficial additives. However, their role in these transformations has been mainly limited to the in situ formation of the corresponding bases for a CMD-type C-H activation pathway. In stark contrast, the utilization of their chirality to enable asymmetric $\mathrm{C}-\mathrm{H}$ functionalizations is restricted to only a few examples. ${ }^{[157]}$ So far, considerable efforts have been made by inter alia Cramer, ${ }^{[158]}$ You $^{[159]}$ and Lam ${ }^{[160]}$ to synthesize costly, but efficient chiral Cp-ligands for asymmetric $\mathrm{C}-\mathrm{H}$ functionalizations.

In the herein described branched-selective $\mathrm{C}-\mathrm{H}$ hydroarylation reaction, the acid additive is presumably not only involved in the BIES-type $\mathrm{C}-\mathrm{H}$ activation, but also serves as a proton source for the product releasing proto-demetalation (vide infra: Scheme 70). If the step before the turnoverlimiting proto-demetalation, namely the migratory insertion of the alkene $\mathbf{1 4 3}$, is a reversible step, then potentially enantioselectivity might be induced by the amino acid ( $c f$. Scheme 70 ). 
To start with, the $N$-pthalimide protected L-isoleucine was tested (Table 18) at different concentrations and reaction temperatures, and indeed an intriguing ee of $23 \%$ (entries 1-3) was observed in product 145aa. Subsequently, different $N$-pthalimide-protected amino acids were probed, with an optimal $e e$ of $25 \%$ for mono-protected L-leucine (entries 4-7). ${ }^{[161]}$ In summary, no clear trend in steric effects of the amino acid backbone was observed. It is noteworthy that different $N$-pivaloyl and -1-adamantoyl protected amino acids delivered the product as racemic mixture (entries 8-11). Additionally, $N$-acetyl, -ester and -t-butyloxycarbonyl (Boc) protecting groups furnished the products in only unsatisfactory enantioselectivities and partly very low branched/linear selectivities (entries 12-16). Moreover, two selected dipeptides were tested, with overall low enantioselectivities and conversions (entries 17 and 18). Interestingly, the stronger Brønsted acid $(R)$-BINOL-phosphoric acid failed to provide the desired product.

In summary, phthalimide was found to be the optimal protecting group. However, the exact reason for that observation has remained unclear. Notably, extensive optimization studies by $V$. Müller and $F$. Pesciaioli concerning different protecting groups, including a structural diversification of the phthalimide-protecting group as well as different amino acids, led thus far to a slight increase in enantioselectivity of up to $36 \% e e .^{[162]}$

Table 18. Optimization studies for the enantioselective cobalt(III)-catalyzed C-H alkylation.

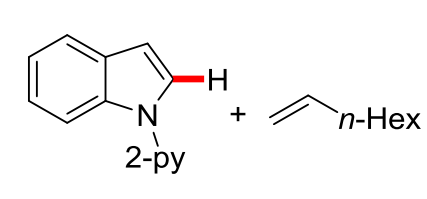

$105 a$

$143 a$
$\left[\mathrm{Cp}^{*} \mathrm{Co}(\mathrm{CO}) \mathrm{I}_{2}\right](10 \mathrm{~mol} \%)$

$\mathrm{AgSbF}_{6}(20 \mathrm{~mol} \%)$ additive $(20 \mathrm{~mol} \%)$ DCE $(0.5 \mathrm{M}), 50^{\circ} \mathrm{C}, 20 \mathrm{~h}$

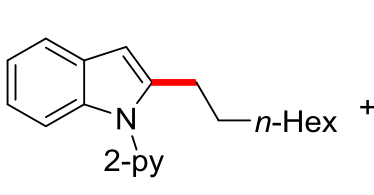

$144 a a$

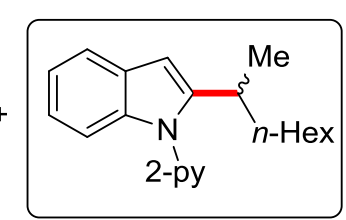

145aa

\begin{tabular}{ccccc}
\hline Entry & additive & 145aa:144aa & $\begin{array}{c}\text { yield / \% } \\
(\text { GC-conversion })\end{array}$ & ee / \% \\
\hline 1 & Phth-Ile-OH & $92: 8$ & $33(67)$ & 23 \\
2 & Phth-Ile-OH & $93: 7$ & $37(69)^{[\mathrm{b}]}$ & 23 \\
3 & Phth-Ile-OH & $98: 2$ & $(45)^{[\mathrm{c}]}$ & 23 \\
$\mathbf{4}$ & Phth-Leu-OH & $\mathbf{9 0 : 1 0}$ & $\mathbf{2 5}(\mathbf{4 1})$ & $\mathbf{2 5}$ \\
5 & Phth-Ala-OH & $89: 11$ & $18(35)$ & 19 \\
6 & Phth-Trp-OH & $93: 7$ & $27(48)$ & 21 \\
7 & Phth-Phe-OH & $93: 7$ & $(67)$ & 21 \\
8 & 1-Ad-Val-OH & --- & $(5)$ & --- \\
9 & 1-Ad- $t$-Leu-OH & $85: 15$ & $25^{[\mathrm{b}]}$ & 1 \\
10 & Piv-Ile-OH & $80: 20$ & $(37)$ & --- \\
11 & Piv-Asp-OH & $45: 55$ & $18^{[\mathrm{b}]}$ & --- \\
12 & Ac-Ile-OH & $80: 20$ & $(33)^{[\mathrm{b}]}$ & 18
\end{tabular}




\begin{tabular}{|c|c|c|c|c|}
\hline 13 & $\mathrm{CO}_{2} \mathrm{Me}-\mathrm{Ile}-\mathrm{OH}$ & $96: 4$ & $(81)$ & 7 \\
\hline 14 & Boc-Ile-OH & $95: 5$ & $(35)$ & 10 \\
\hline 15 & Boc-Tyr-OH & --- & $--^{[b]}$ & --- \\
\hline 16 & $\mathrm{Boc}_{2}-\mathrm{Val}-\mathrm{OH}$ & $90: 10$ & $31^{[\mathrm{b}]}$ & 12 \\
\hline 17 & Boc-Val-Gly-OH & $80: 20$ & $(25)^{[\mathrm{b}]}$ & 3 \\
\hline 18 & Boc-Trp-Gly-OH & 99:1 & $(4)^{[b]}$ & 2 \\
\hline 19 & $(R)$-BINOL-phosphoric acid & --- & --- & --- \\
\hline
\end{tabular}

\subsubsection{Mechanistic Studies}

\subsubsection{H/D-Exchange Experiments}

\subsection{H/D-Exchange for the linear-selective reaction}

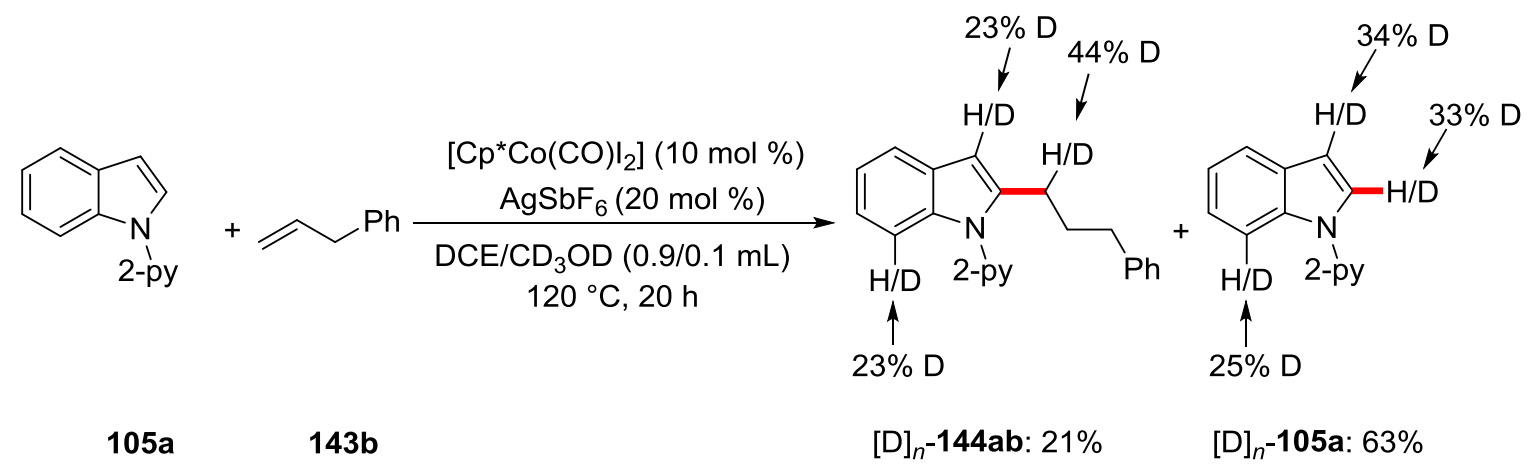

Scheme 59. H/D-exchange under the linear-selective reaction conditions.

To study the mechanism of the $\mathrm{C}-\mathrm{H}$ activation elementary step, an H/D-exchange experiment under the linear-selective reaction regime with $\mathrm{CD}_{3} \mathrm{OD}$ as the co-solvent was conducted. As a result, a significant deuterium incorporation was observed in C3- and C7-positions of the product 144ab and the starting material 105a (Scheme 59), as it had previously been shown for the cobalt(III)-catalyzed $\mathrm{C}-\mathrm{H} / \mathrm{C}-\mathrm{C}$ activation ( $c f$. chapter 3.2.3.1). Importantly, a significant $\mathrm{H} / \mathrm{D}$-exchange in $\mathrm{C} 2$-position of the reisolated starting material 105a suggested a facile and reversible cobalt(III)-catalyzed $\mathrm{C}-\mathrm{H}$ activation event to be operative.

Notably, a considerable amount of deuterium incorporation could be exclusively detected in $\alpha$-position of the alkyl chain in product 144ab. Based on this result, a H/D-exchange reaction with product 144ab under otherwise unchanged reaction conditions resulted again in a significant H/D-scrambling in $\alpha$ - 
position of the alkyl chain (Scheme 60), rendering a post-catalytic H/D-exchange likely. A reasonable mechanistic pathway for this $\mathrm{H} / \mathrm{D}$-exchange could be a cobalt(III)-catalyzed $\mathrm{C}\left(\mathrm{sp}^{3}\right)-\mathrm{H}$ activation. ${ }^{[163]}$

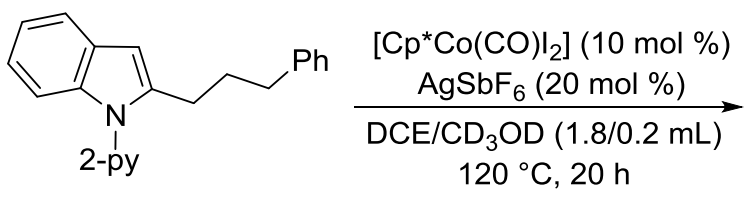

$144 a b$

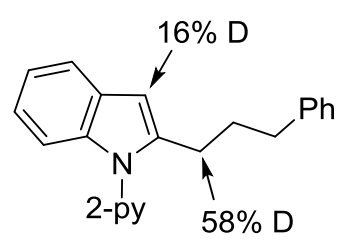

$[\mathrm{D}]_{n}-144 \mathrm{ab}: 67 \%$

Scheme 60. H/D-exchange with product 144ab under the linear-selective reaction conditions.

\subsection{H/D-Exchange for the branched-selective reaction}

Under the Markovnikov-selective reaction conditions a significant H/D scrambling was detected in C3as well in C7-position (Scheme 61). Additionally, the reisolated starting material 105a displayed a moderate $\mathrm{H} / \mathrm{D}$-exchange in $\mathrm{C} 2$-position, however, in slightly less amount than under the linearselective conditions ( $c f$. Scheme 59). Overall, the $\mathrm{C}-\mathrm{H}$ activation under the branched-selective reaction regime seems to be reversible, but proceeds less readily than under linear-selective conditions. Furthermore, a minor deuterium incorporation in the methyl group of the alkyl chain was observed, which might be in agreement with the proposed mechanism to involve a proto-demetalation step (vide infra).

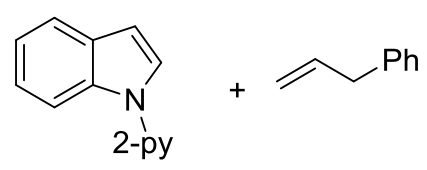

$105 a$

$143 b$
$\left[\mathrm{Cp}{ }^{*} \mathrm{Co}(\mathrm{CO}) \mathrm{I}_{2}\right](10 \mathrm{~mol} \%)$

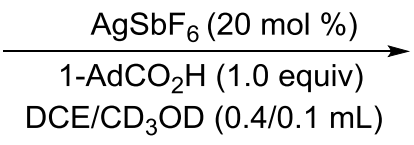

$50{ }^{\circ} \mathrm{C}, 20 \mathrm{~h}$

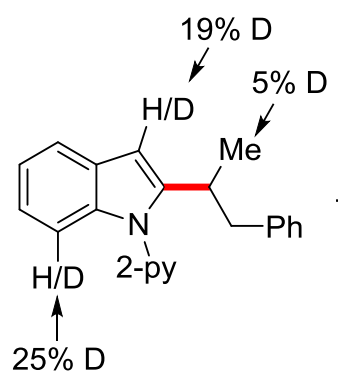

$[\mathrm{D}]_{n}-145 \mathrm{ab}: 41 \%$

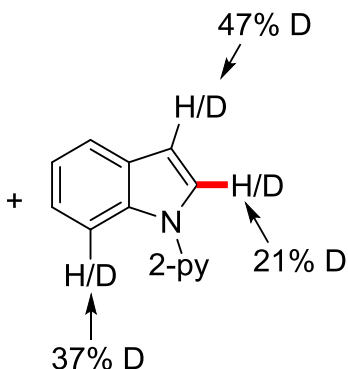

$[\mathrm{D}]_{n}-105 \mathrm{a}: 53 \%$

Scheme 61. H/D-exchange under the branched-selective reaction conditions.

\subsection{H/D-exchange with alkenes 143}

The previously shown experiments revealed that H/D-exchange can occur after the catalytic reaction ( $c f$. Scheme 60) in product 144ab. In addition to this observation, also H/D-exchange with solely the alkene $143 \mathrm{c}$ was studied under the otherwise unchanged reaction conditions.

First, 1-pentadecene (143c) could be reisolated in almost quantitative yield under linear-selective conditions, which showed that no detectable side reactions like dimerization or isomerization of the double bond occurred (Scheme 62a). However, significant H/D-scramblings in both alkene positions were detected. Even under the milder branched-selective reaction conditions, H/D-scrambling was observed (Scheme 62b). Based on these observations, H/D-exchange studies of the cobalt(III)- 
catalyzed $\mathrm{C}-\mathrm{H}$ alkylation should be cautiously interpreted with respect to the prediction of possible mechanistic pathways. However, these preliminary results bear potential for the development of isomerization-free and mild isotope labeling reactions with terminal alkenes. ${ }^{[164]}$

a)
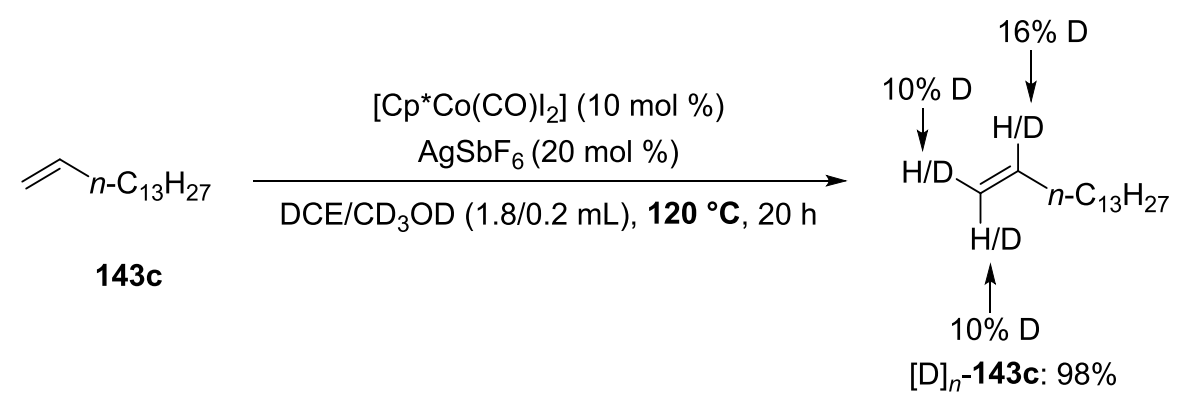

b)
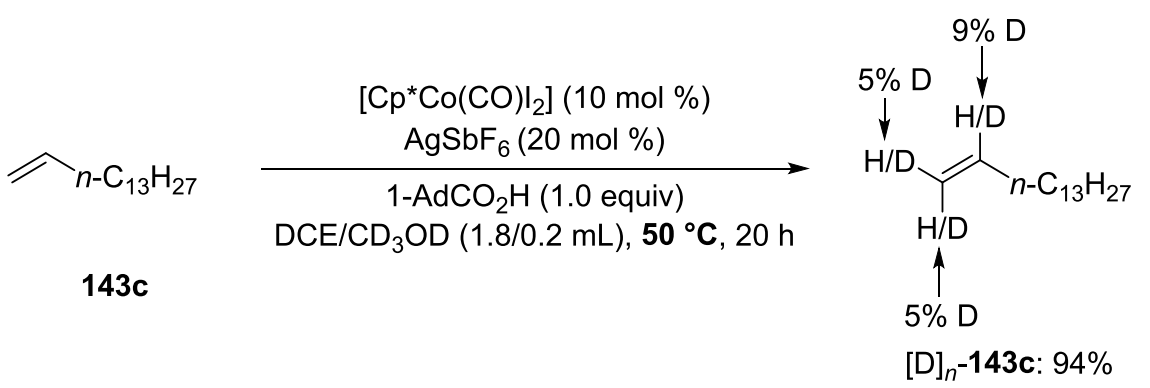

Scheme 62. H/D-exchange with alkene 143c under a) linear- and b) branched-selective conditions.

\subsection{H/D-exchange with $[D]_{1}-105 a$ during the reaction progress}

Under otherwise unchanged linear-selective reaction conditions, but in the absence of any external deuterium-/proton-source, a H/D-exchange experiment was performed with isotopically labeled $[\mathrm{D}]_{1}$ 105a (Scheme 63). Besides the previously observed H/D-scrambling in C2, C3- and C7-positions of the reisolated starting material 105a as well as the product 144aa ( $c f$. Scheme 59) a significant deuteration in $\alpha$-position of the alkyl sidechain was detected.

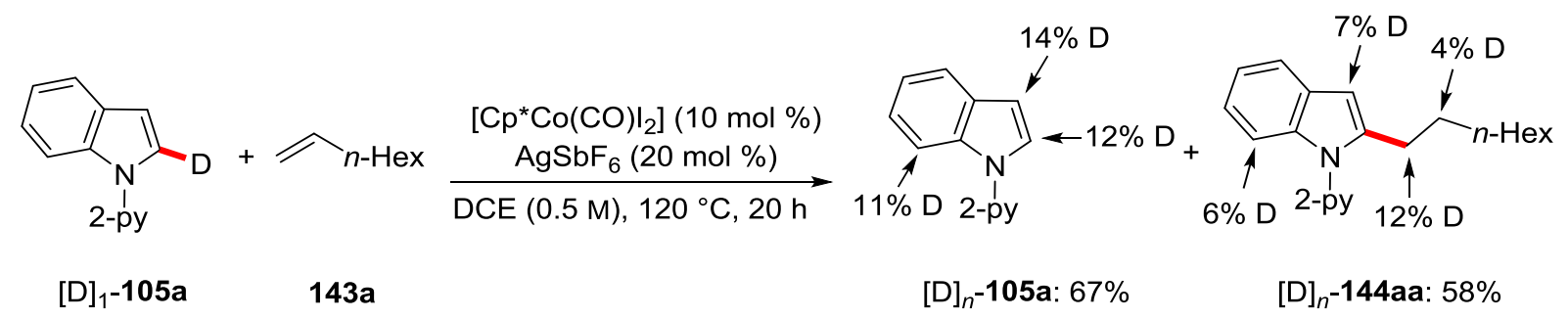

Scheme 63. H/D-exchange with $[\mathrm{D}]_{1}-\mathbf{1 0 5 a}$ under linear-selective reaction conditions.

With respect to the previously observed H/D-exchange study of the product 144ab ( $c f$. Scheme 60), this finding can be most likely explained by post-catalytic deuterium exchange with product 144aa. Remarkably, minor deuteration was detected in $\beta$-position, which might proceed by deuterodemetalation of a catalytically relevant intermediate (vide infra Scheme 69). 


\subsection{H/D-exchange with $[D]_{1}-105 a$}

Moreover, a H/D-exchange reaction with substrate $[\mathrm{D}]_{1}-\mathbf{1 0 5 a}$ was conducted under linear-selective reaction conditions, but in the absence of any alkene (Scheme 64 ). The substrate $[D]_{1}-\mathbf{1 0 5 a}$ could be almost quantitatively reisolated in 93\%, with significant H/D-scramblings in C2-, C3- and C7position. A reasonable explanation for this observation is a reversible $\mathrm{C}-\mathrm{H}$ activation in $\mathrm{C} 2$-position and proto-demetalation, likely by traces of water in the solvent DCE.

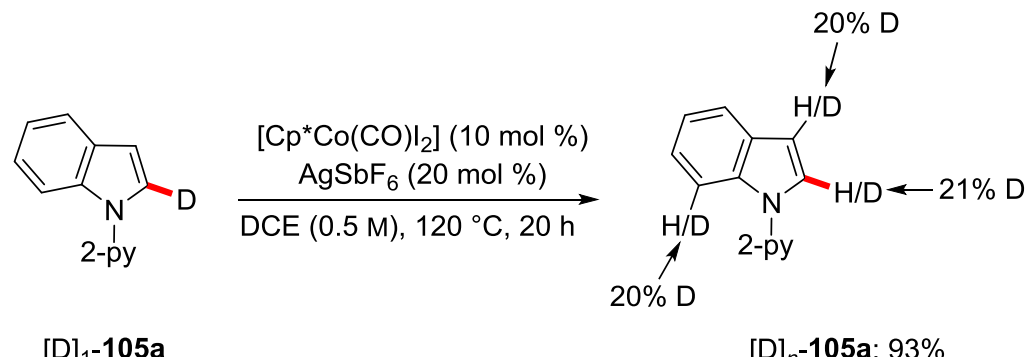

Scheme 64. H/D-exchange experiment with deuterated substrate $[D]_{1}-\mathbf{1 0 5 a}$.

\subsection{Intermolecular competition experiment}

In addition, an intermolecular competition experiment under the branched-selective reaction conditions was carried out by comparing the reaction rates of the electronically distinct 2pyridylindoles $105 \mathrm{~b}$ and $\mathbf{1 0 5 m}$ (Scheme 65). As a result, the electron-rich 5-methoxypyridylindole (105b) reacted preferentially, which possibly supports a BIES-type $\mathrm{C}-\mathrm{H}$ activation.

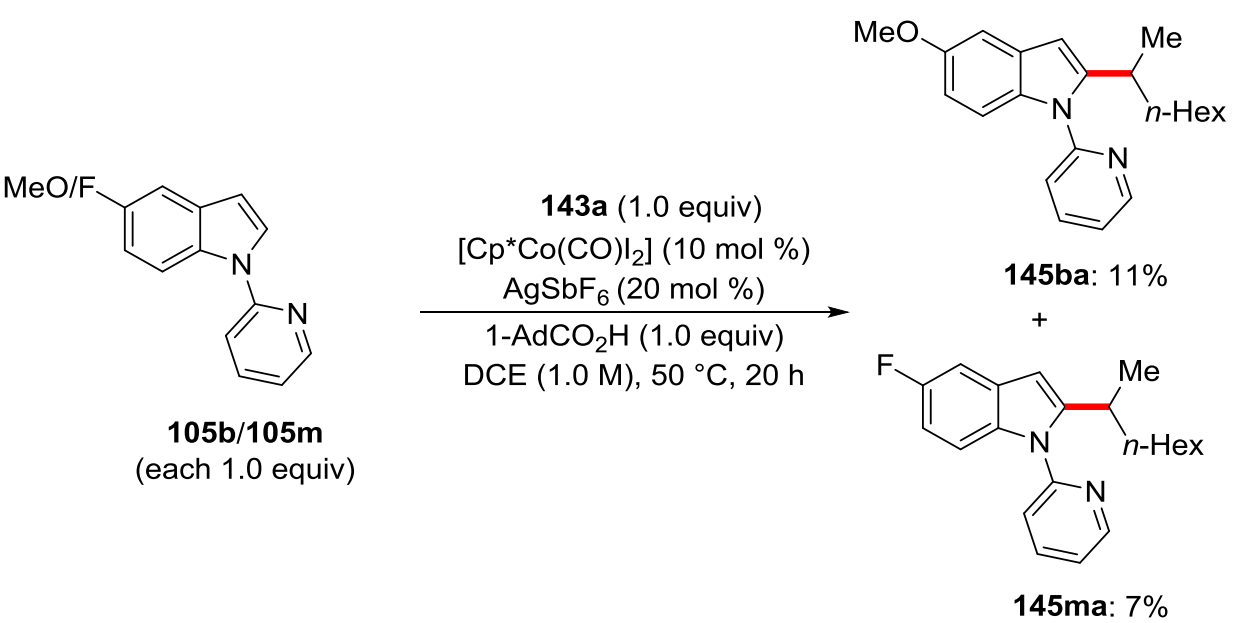

Scheme 65. Intermolecular competition experiment for the branched-selective $\mathrm{C}-\mathrm{H}$ alkylation.

Notably, V. Müller showed a significant preference for the electron-rich 1 -( $p$-tolyl)-1H-pyrazole (18a) as compared to [1-(4-(trifluoromethyl)phenyl]-1H-pyrazole (18b), which is in agreement with the above mentioned findings. Here, the electron-rich pyrazole 18a reacted almost nine times faster than substrate $\mathbf{1 8 b}$. 


\subsubsection{KIE Studies}

\subsection{KIE of the linear-selective reaction}

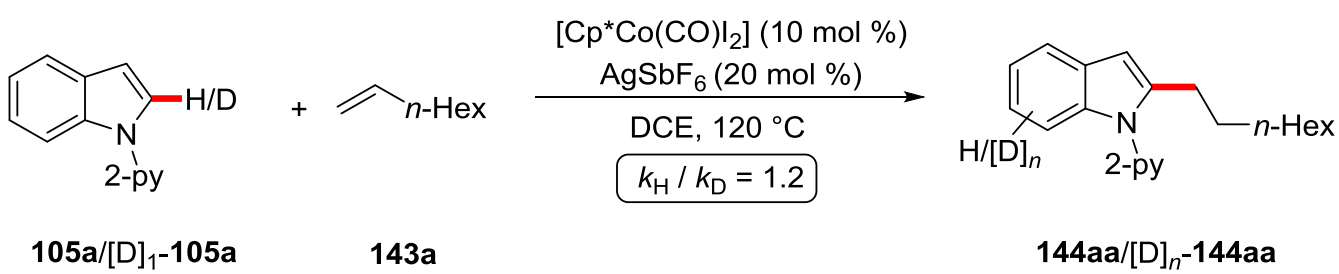

Scheme 66. KIE of the linear-selective reaction.

The kinetic isotope effect (KIE) of the anti-Markovnikov-selective reaction was measured by comparison of independent reaction rates for substrate 105a and its isotopically labeled analogue $[\mathrm{D}]_{1^{-}}$ 105a, resulting in a minor value of $k_{\mathrm{H}} / k_{\mathrm{D}} \approx 1.2$ (Scheme 66). The observed KIE is in good agreement with the results obtained from the H/D-exchange experiments, suggesting the reversible $\mathrm{C}-\mathrm{H}$ activation not to be the turnover-limiting step of the catalytic cycle.

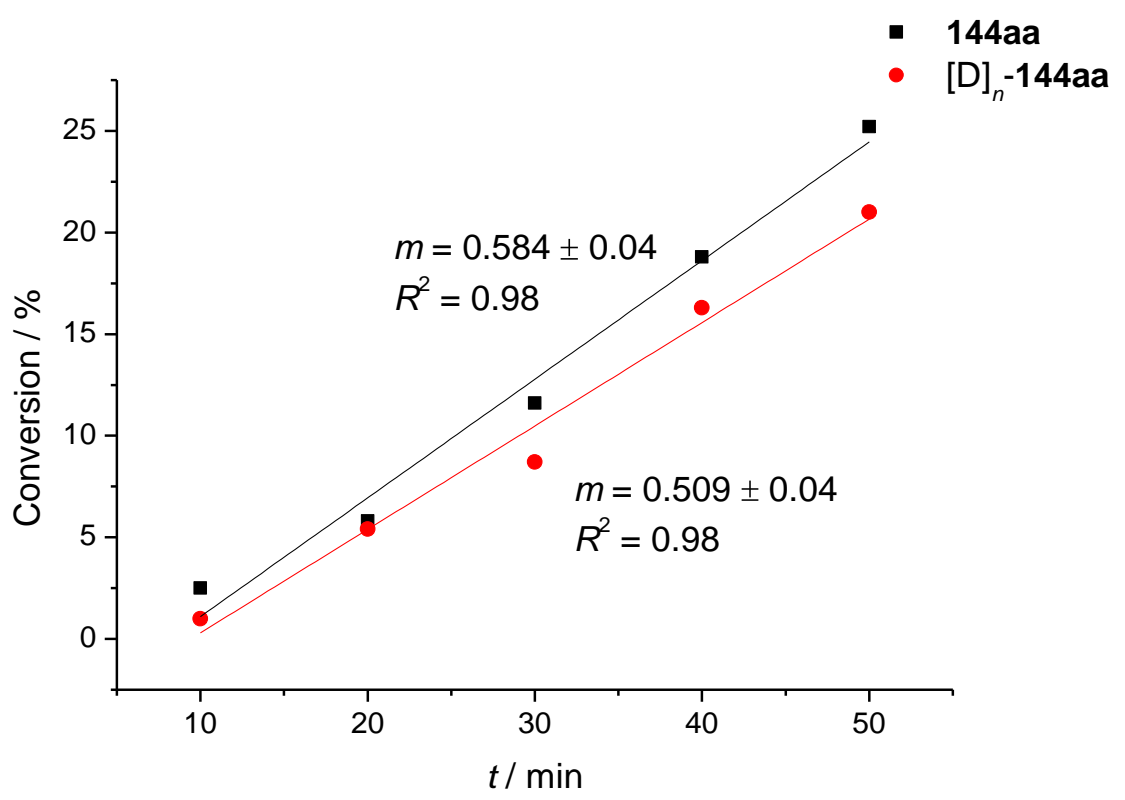

Figure 11. KIE under the linear-selective reaction conditions. 


\subsection{KIE of the branched-selective reaction}

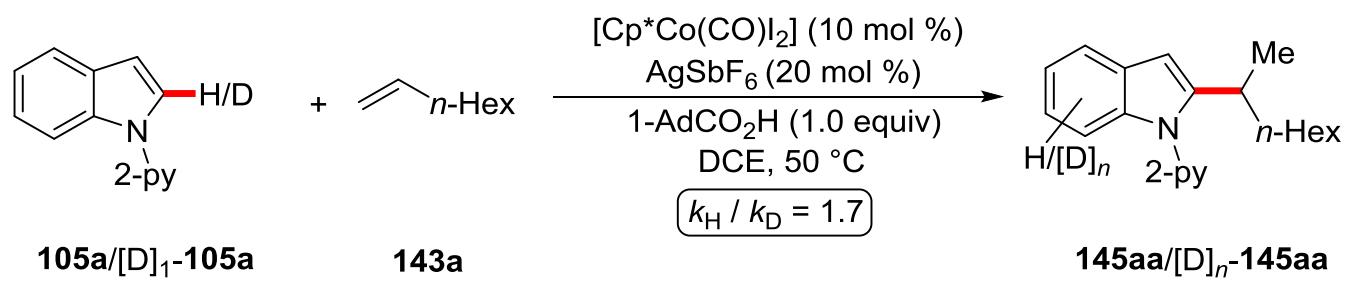

Scheme 67. KIE of the branched-selective reaction.

The kinetic isotope effect (KIE) of the Markovnikov-selective reaction was analogously determined to be $k_{\mathrm{H}} / k_{\mathrm{D}} \approx 1.7$ (Scheme 67 and Figure 12). The comparatively lower H/D-exchange in C2-position of the starting material 105a for the branched-selective reaction (vide supra) and the higher KIE value show that the $\mathrm{C}-\mathrm{H}$ activation step under the Markovnikov-selective reaction regime presumably does not follow an electrophilic activation pathway. Along this line, a rate acceleration by acids as well as carboxylate additives has been observed ( $c f$. Table 13). Overall, these findings might support a BIEStype $\mathrm{C}-\mathrm{H}$ activation mechanism by assistance of the in situ formed 1-adamantylcarboxylate to be operative for the branched-selective reaction regime.

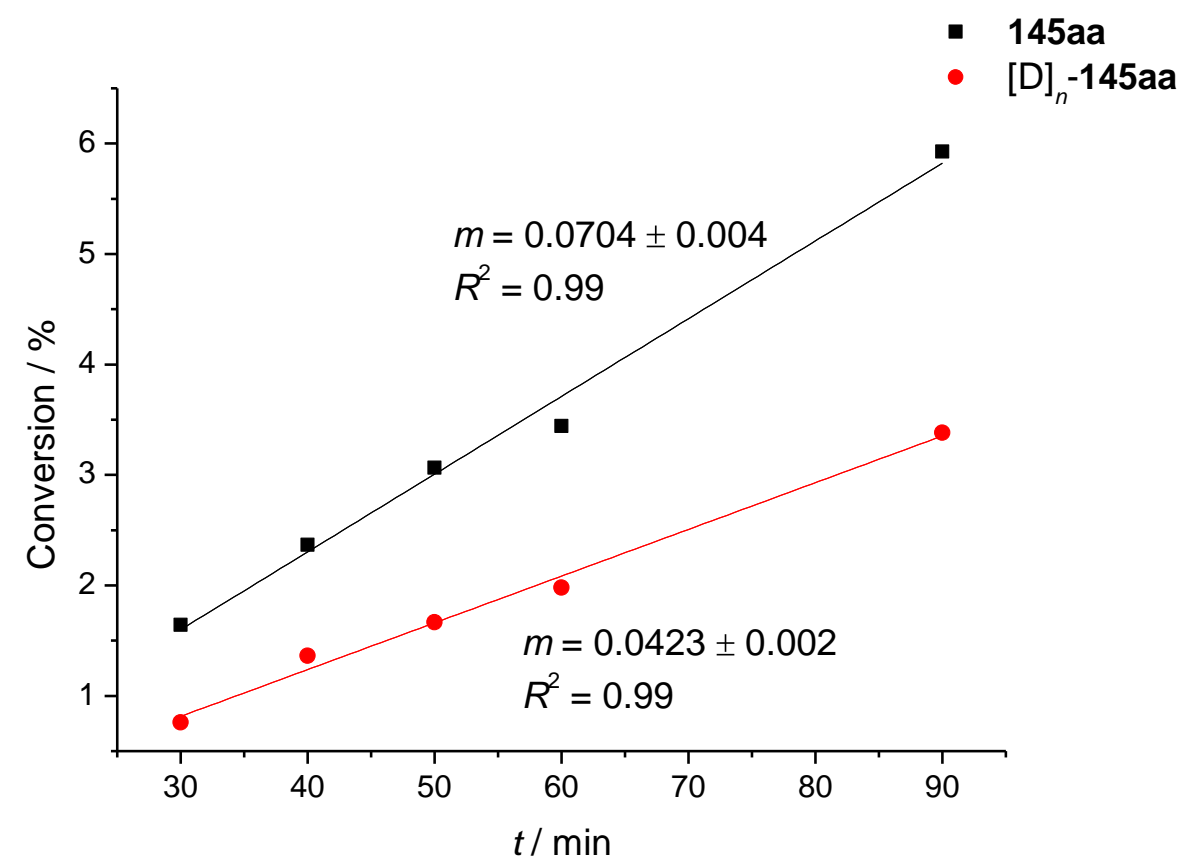

Figure 12. KIE under the branched-selective reaction conditions. 


\subsubsection{Kinetic reaction orders}

\subsection{Kinetic reaction order for the linear-selective reaction}

\subsection{Reaction order with respect to the concentration of 2-pyridylindole (105a)}

The kinetic order of the reaction with respect to the concentration of 2-pyridylindole (105a) equals $n=1.24 \pm 0.11$, which might correspond to a reaction order of one (Figure 13). This result can be interpreted as a clear hint for the participation of 2-pyridylindole (105a) in the turnover-limiting step of the reaction.

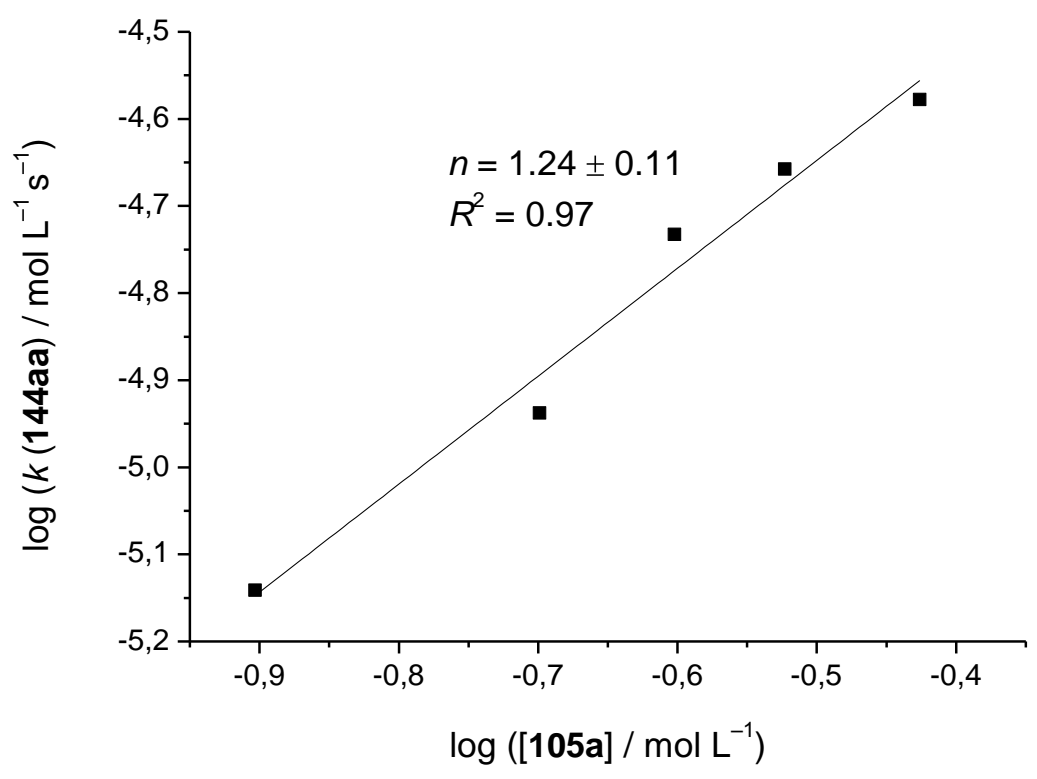

Figure 13. Kinetic order in [2-pyridylindole] (105a) under linear-selective conditions.

\subsection{Reaction order with respect to the concentration of 1-octene (143a)}

Interestingly, a non-integer reaction order ${ }^{[165]}$ of $n=0.30 \pm 0.02$ with respect to the alkeneconcentration 143a was observed (Figure 14), suggesting a complex rate law for this reaction. One possible explanation could be that the step after alkene-insertion is turnover-limiting and both the coordination and the following migratory insertion of the alkene 143a are two interdepending equilibrium reactions, which are kinetically relevant for the following turnover-limiting step. 


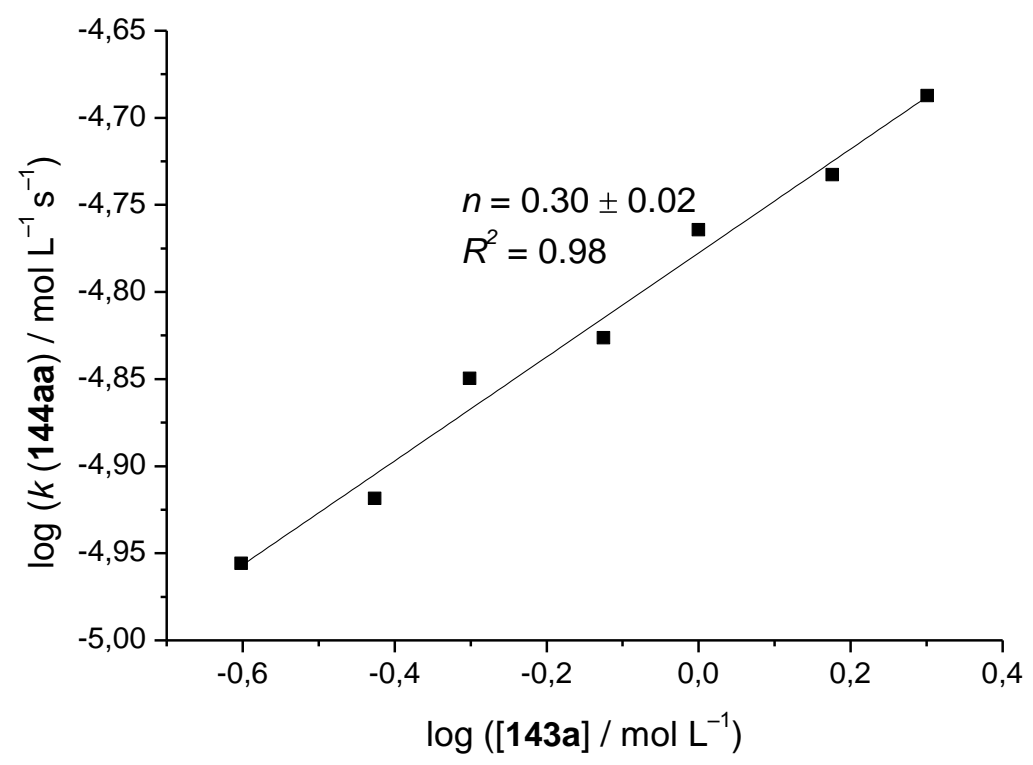

Figure 14. Kinetic order in [1-octene] (143a) under linear-selective conditions.

\subsection{Reaction order with respect to the concentration of $\left[\mathrm{Cp} * \mathrm{Co}(\mathrm{CO}) \mathrm{I}_{2}\right]$}

The reaction order with respect to the catalyst concentration is roughly one, with $n=1.08 \pm 0.12$ (Figure 15), showing that the catalyst is part of the turnover-limiting step of the catalytic cycle.

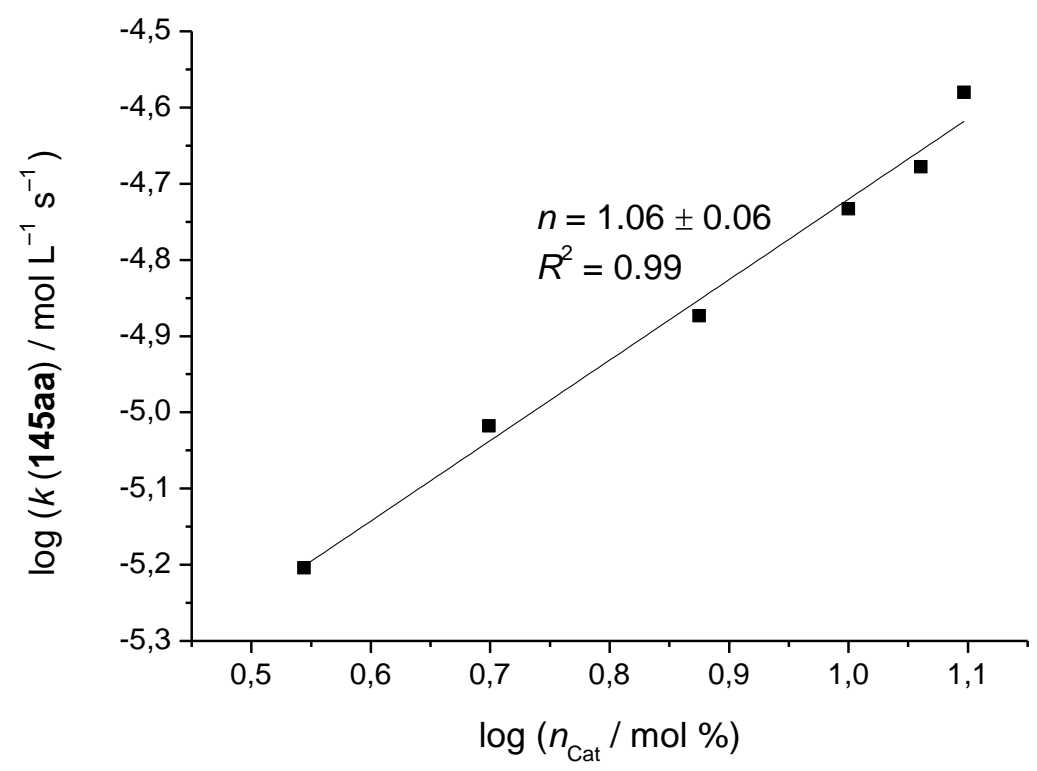

Figure 15. Kinetic order in $\left[\left[\mathrm{Cp} * \mathrm{Co}(\mathrm{CO}) \mathrm{I}_{2}\right]\right]$ under the linear-selective reaction regime.

\subsection{Kinetic reaction order for the branched-selective reaction}

\subsection{Reaction order with respect to the concentration of 2-pyridylindole (105a)}

In stark contrast to the observed first-order-dependence for the linear-selective reaction regime, a zeroorder-dependence with respect to the concentration of 2-pyridylindole (105a) with $n=0.00 \pm 0.09$ was 
observed (Figure 16). Notably, a similar result was obtained by V. Müller, who performed ${ }^{19}$ F-NMR studies of the same reaction system utilizing 5-fluoro-1-(pyridin-2-yl)-1H-indole (105m). ${ }^{[162 b]}$ Based on these results, it can be proposed that the concentration of 2-pyridylindole (105a) is not kinetically relevant and it is neither part of an equilibrium reaction before nor of the turnover-limiting step itself.

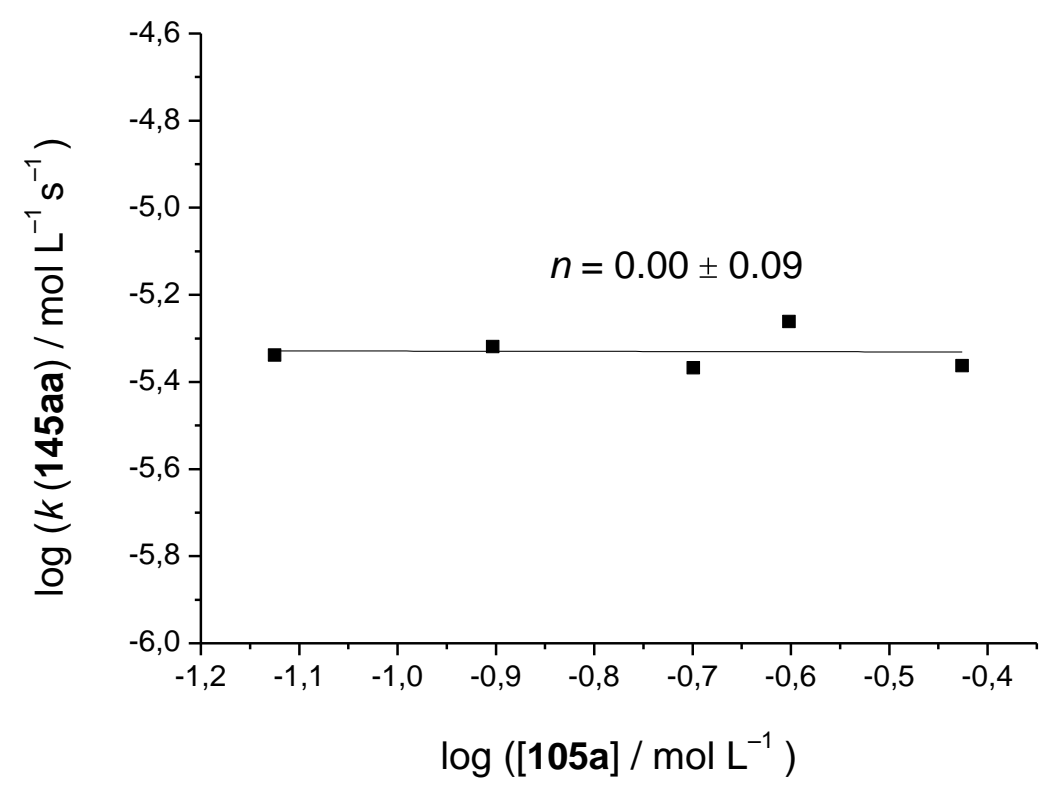

Figure 16. Kinetic order in [2-pyridylindole] (105a) under branched-selective conditions.

\subsection{Reaction order with respect to the concentration of 1-octene (143a)}

In contrast to the non-integer reaction order for the linear-selective regime (cf. Figure 14), the Markovnikov-selective reaction displayed a first order dependence with respect to [1-octene] (143a), which reveals the participation of 1-octene (143a) within or before the turnover-limiting step (Figure 17). 


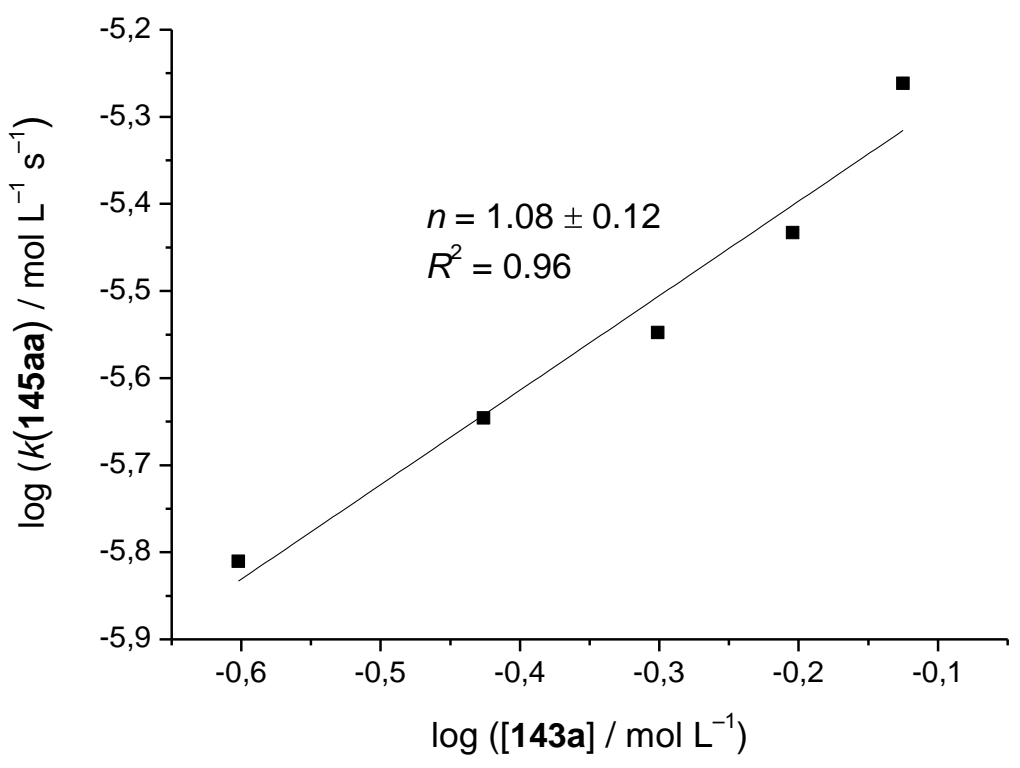

Figure 17. Kinetic order in [1-octene] (143a) under branched-selective conditions.

\subsection{Reaction order with respect to the concentration of $\left[\mathrm{Cp} * \mathrm{Co}(\mathrm{CO}) \mathrm{I}_{2}\right]$}

As for the linear-selective reaction ( $c f$. Figure 15), a first-order dependence was observed with respect to the concentration of the cobalt(III) catalyst (Figure 18).

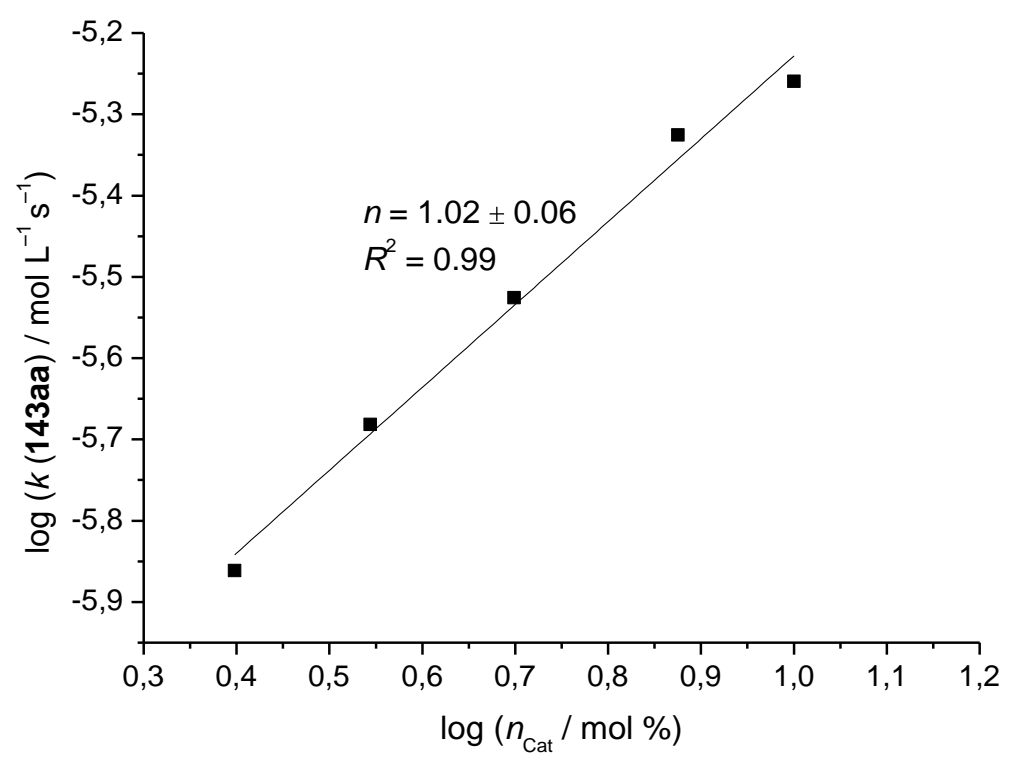

Figure 18. Kinetic order in $\left[\left[\mathrm{Cp} * \mathrm{Co}(\mathrm{CO}) \mathrm{I}_{2}\right]\right]$ under branched-selective conditions.

\subsubsection{Arrhenius Activation Energies}

A common approximation for the measurement of activation barriers $E_{\mathrm{A}}$ of inter alia catalytic reactions is the Arrhenius plot, in which the natural logarithm of the reaction rate $k$ is plotted against 
the inverse reaction temperature $T$ (Equation 5). ${ }^{[166]}$ The slope of the thus obtained graph corresponds to the activation energy $E_{\mathrm{A}}$ divided by the ideal gas constant $R$ and the y-axis intercept represents the natural logarithm of the preexponential factor $A$.

$$
\ln k=\ln (A)-\frac{E_{A}}{R} \times \frac{1}{T}
$$

Thus, the activation energy of the linear-selective reaction was determined to be $E_{\mathrm{A}}=$ $22.3 \pm 1.7 \mathrm{kcal} / \mathrm{mol}$ (Figure 20) and the branched-selective reaction displayed a barrier of $E_{\mathrm{A}}=$ $14.4 \pm 1.6 \mathrm{kcal} / \mathrm{mol}$ (Figure 22), which were obtained by comparison of the initial rates at different reaction temperatures for both reactions (Figure 19 and Figure 21). The difference in activation energy for both reactions amounts to roughly $8.0 \mathrm{kcal} / \mathrm{mol}$, which is in good agreement with the difference in each optimized reaction temperature of $120^{\circ} \mathrm{C}$ and $50{ }^{\circ} \mathrm{C}$, respectively.

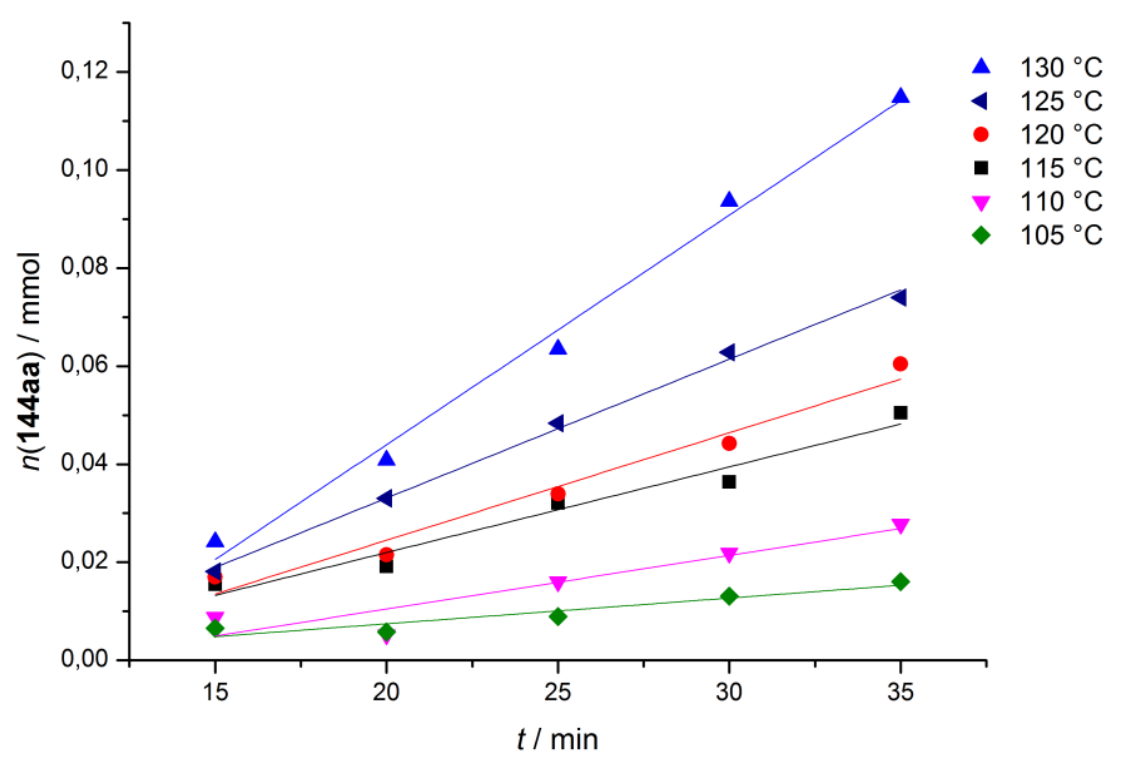

Figure 19. Initial rates at different reaction temperatures for the linear-selective reaction. 


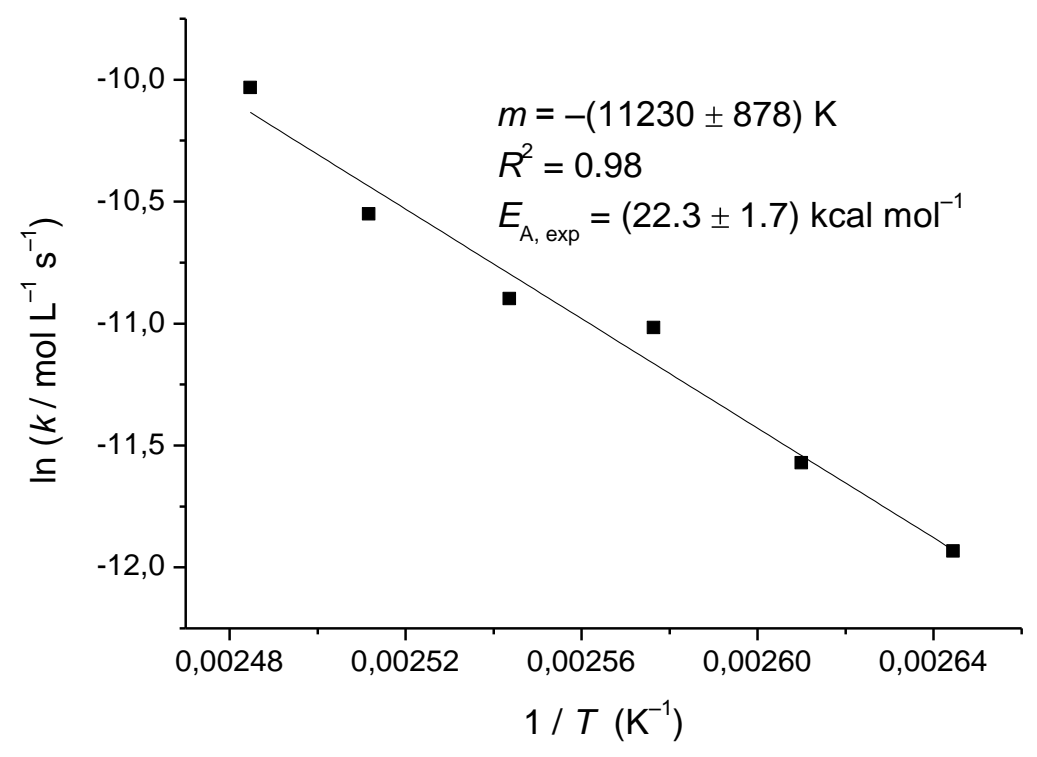

Figure 20. Arrhenius plot for the linear-selective reaction regime.

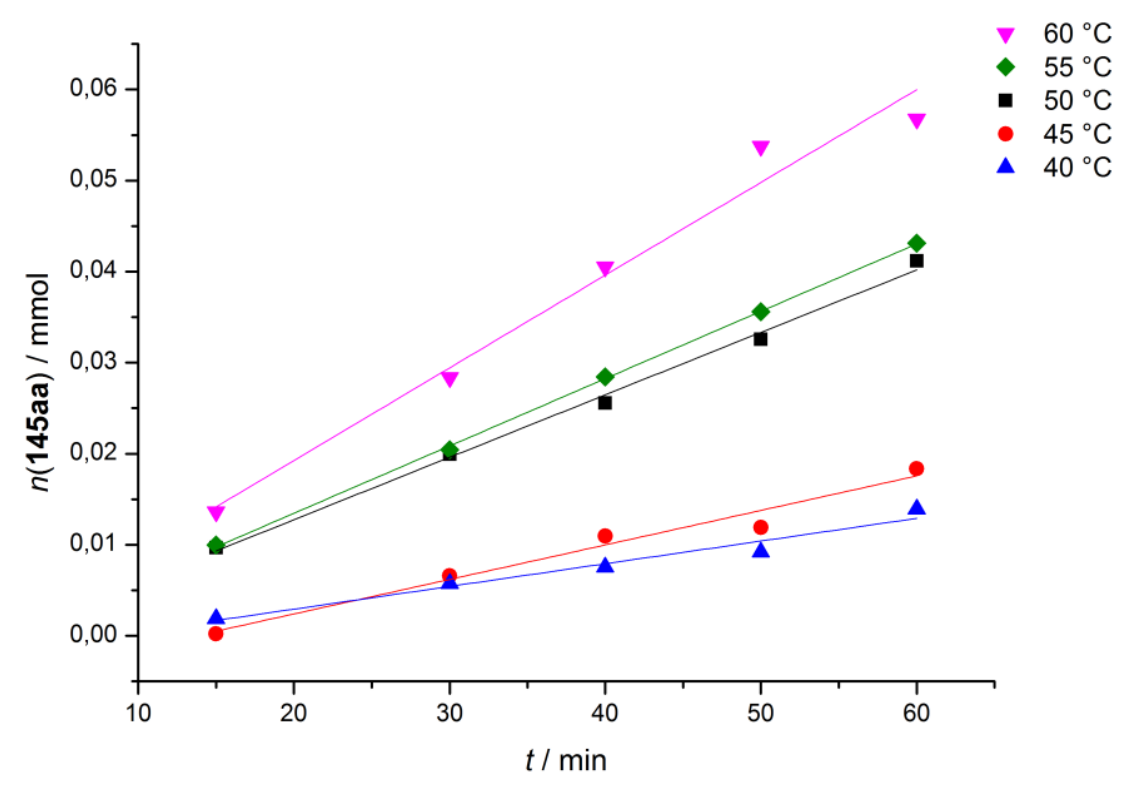

Figure 21. Initial rates at different temperatures for the branched-selective reaction. 


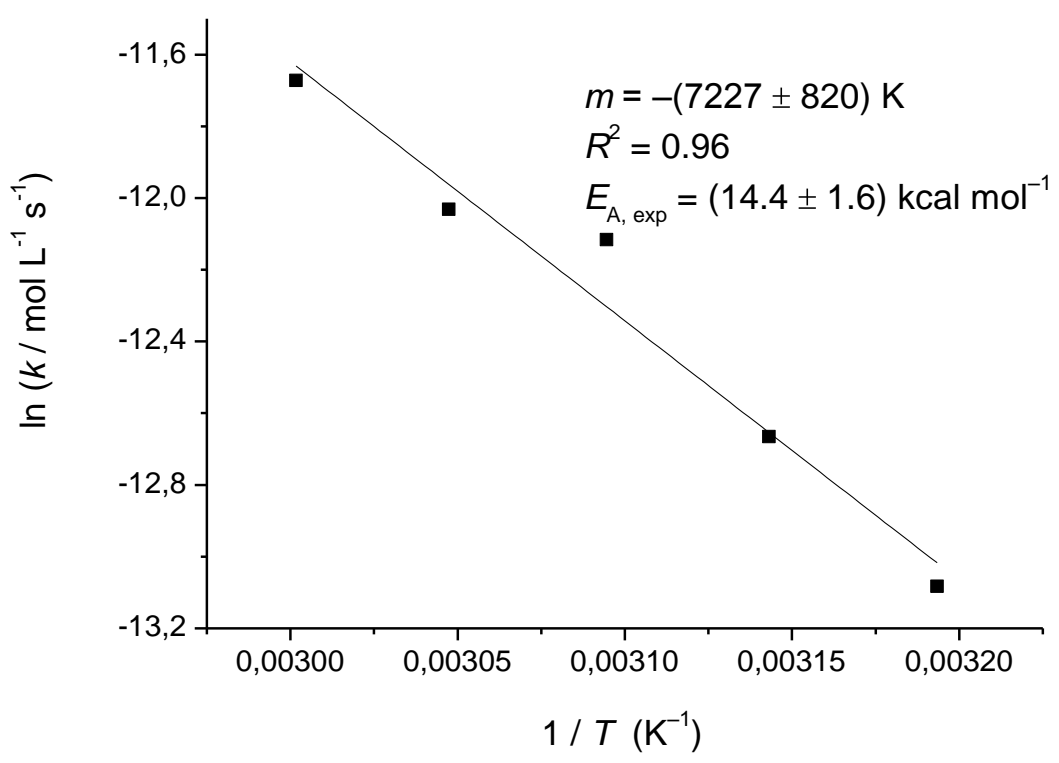

Figure 22. Arrhenius plot for the branched-selective reaction regime.

\subsubsection{Attempted Post-Synthetic Isomerization Studies}

Furthermore, it was studied whether a post-catalytic isomerization through cobalt(III)-catalyzed $\mathrm{C}-\mathrm{C}$ bond cleavage could occur for the linear product 144aa under branched-selective conditions (Scheme 68a) and vice versa (Scheme 68b). Overall, there was no change in connectivity, which indicates that both products are thermodynamically stable under the catalytic reaction conditions.

a)<smiles></smiles>

144aa (AM:M > 99:1)

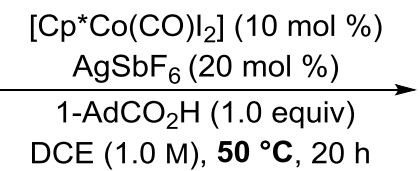

DCE $(1.0 \mathrm{M}), 5^{\circ} \mathrm{C}, 20 \mathrm{~h}$

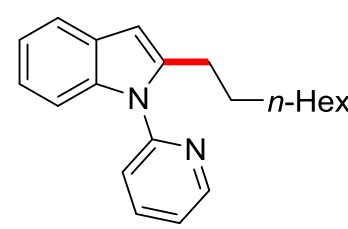

144aa: 95\% (AM:M > 99:1)

b)<smiles>CC(C=O)c1cc2ccccc2n1-c1ccccn1</smiles>

145aa (AM:M = 5:95)

$$
\begin{gathered}
\underset{\left.\mathrm{Cp}^{*} \mathrm{Co}(\mathrm{CO}) \mathrm{I}_{2}\right](10 \mathrm{~mol} \%)}{\mathrm{AgSbF}}(20 \mathrm{~mol} \%) \\
\underset{\mathrm{DCE}(0.5 \mathrm{M}), 120{ }^{\circ} \mathrm{C}, 20 \mathrm{~h}}{\longrightarrow}
\end{gathered}
$$

145aa: $94 \%(A M: M=5: 95)$

Scheme 68. Attempted isomerization of a) the linear 144aa and b) the branched product 145aa.

\subsubsection{Proposed Catalytic Cycles}

In summary, there are several mechanistically distinct characteristics of the presented regiodivergent catalytic reactions. The linear-selective reaction seems to involve a feasible $\mathrm{C}-\mathrm{H}$ activation step which 
is indicated by the significant $\mathrm{H} / \mathrm{D}$-scrambling in $\mathrm{C} 2$-position and the minor $\mathrm{KIE}$ of 1.2, overall rendering the $\mathrm{C}-\mathrm{H}$ activation to be facile and reversible. For the branched-selective reaction $(i)$ a clear rate acceleration was accomplished using carboxylic acids ( $c f$. Table 13), (ii) a reversible H/Dexchange was detected, (iii) a higher KIE of 1.7 was measured and (iv) a clear preference for electronrich substrates was observed. Altogether, these results indicate that the $\mathrm{C}-\mathrm{H}$ activation for the Markovnikov-selective reaction follows a BIES-type mechanistic pathway, which is clearly different from the linear-selective reaction.

The kinetic order studies reveal a first-order-dependence on [2-pyridylindole] (105a) for the linearselective regime, whereas a zero-order-dependence was found for the branched one. With respect to [1-octene] (143a), a non-integer order of roughly 0.3 was observed in the case of the linear-selective reaction, and a first-order-dependence could be shown for the branched-selective one. The kinetic order of the reaction with respect to the catalysts concentration equaled one for both reactions. In summary, these differences clearly reveal that the regiodivergence of the studied reactions is possibly rooted in two mechanisms with distinct rate-determining steps.

To gain a deeper mechanistic insight, DFT-studies were performed in collaboration with S. Grimme and $M$. Bursch. ${ }^{[161]}$ The computational results showed the selectivity control to be a consequence of two mechanistically different pathways for the proto-demetalation elementary step.

On the one hand, linear selectivity is clearly preferred by a ligand-to-ligand hydrogen transfer (LLHT), which enabled the proto-demetalation by delivering the proton from a second substrate 105a to intermediate 153 (Scheme 69, TS1). The energetic difference of both regioisomers amounts to $7.9 \mathrm{kcal} / \mathrm{mol}$. These theoretical results match the experimentally observed regioselectivities as well as the first-order-dependence on substrate 105a because the recoordination with the second 2pyridylindole (105a) seems to be the overall highest calculated activation barrier. 


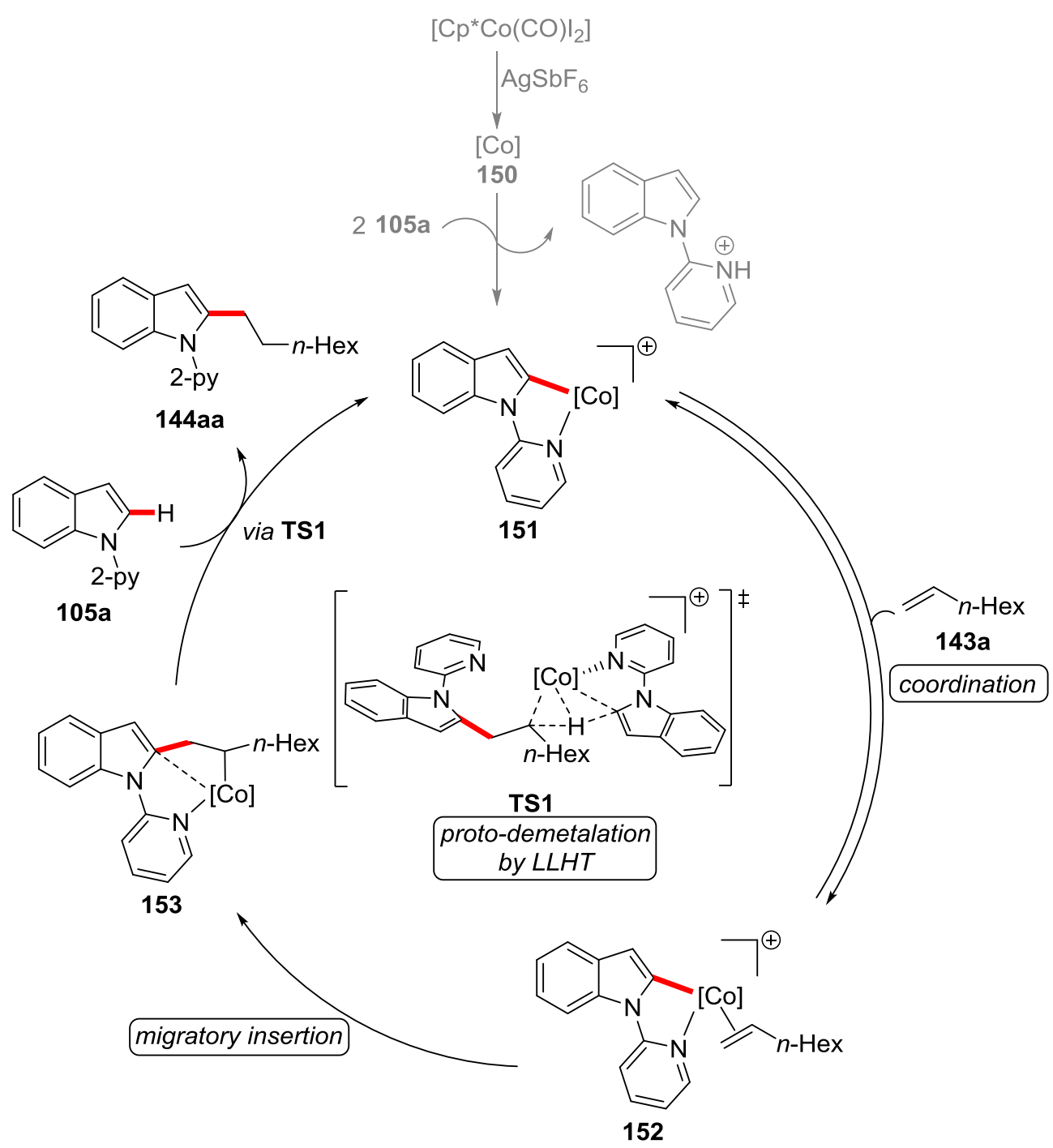

Scheme 69. Proposed catalytic cycle for the linear-selective reaction with only the preferred linear intermediates depicted. $[\mathrm{Co}]=\mathrm{Cp}^{*} \mathrm{Co}^{\mathrm{III}}$.

In the case of the branched-selective reaction, the turnover-limiting proto-demetalation step proceeds via TS2 (Scheme 70) with 1-adamanantanecarboxylic acid as the proton source and results in the formation of the favored branched product 145aa. The energy difference of the overall activation barriers equals $6.5 \mathrm{kcal} / \mathrm{mol}$, which reflects the excellent experimental regioselectivities. A minor amount of linear product 144aa under the branched-selective reaction conditions might possibly be caused by the anti-Markovnikov-selective LLHT mechanism being in parallel operative, but it is clearly the less favored mechanistic pathway under these conditions ( $c f$. Table 13, entry 1).

The regiocontrol in the Markovnikov-selective reaction is presumably caused by steric interactions in the unfavored linear transition state TS2 (Figure 23), which was disclosed by DFT-calculations of $M$. Bursch. The linear product formation is considerably less favored because the alkyl-chain $\mathrm{R}=n$-Hex hinders the approach of the sterically congested 1-adamantanecarboxylic acid, which is needed as proton source for the proto-demetalation step. In this context, it could be shown that attractive London dispersion interactions ${ }^{[124,142]}$ significantly contribute to a stabilization of all catalytically relevant 
intermediates as well as transition states, but they do not compensate for the energetic destabilization by steric interactions and overall do not influence Markovnikov/anti-Markovnikov selectivity. ${ }^{[161]}$

Generally, the proto-demetalation step is kinetically favored by the presence of carboxylic acids as compared to their carboxylate analogues, which can only form the corresponding acid in situ during the BIES-type $\mathrm{C}-\mathrm{H}$ activation. This hypothesis is in good agreement with the previously described observation that acids, even in high concentrations and regardless of their steric demand, favor the branched product formation, whereas only sterically demanding carboxylates lead to comparably good selectivities ( $c f$. Table 13, entries 2-12).

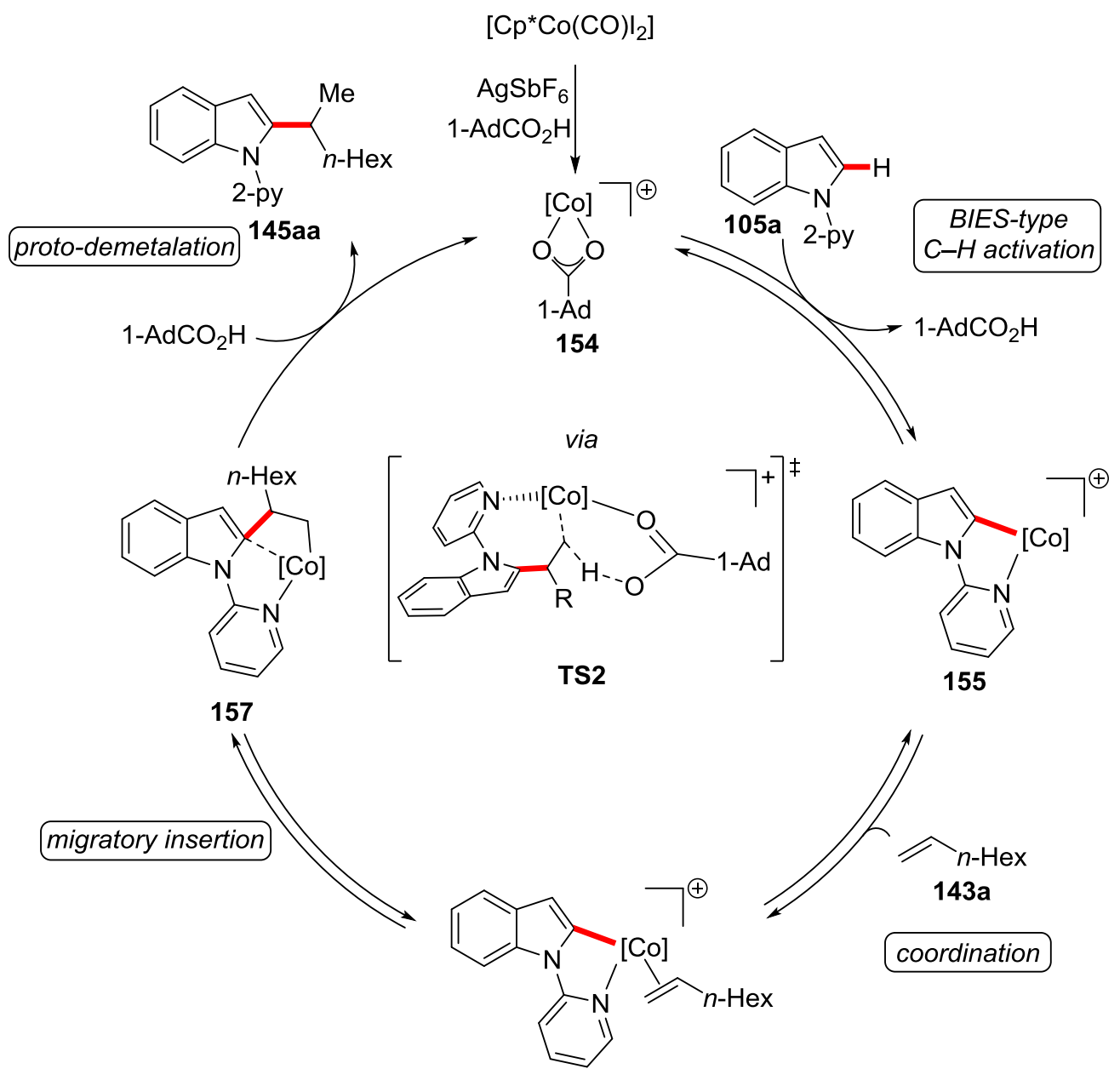

156

Scheme 70. Proposed catalytic cycle for the branched-selective reaction with only the preferred branched intermediates depicted. $[\mathrm{Co}]=\mathrm{Cp}^{*} \mathrm{Co}^{\mathrm{III}}$. 
a)
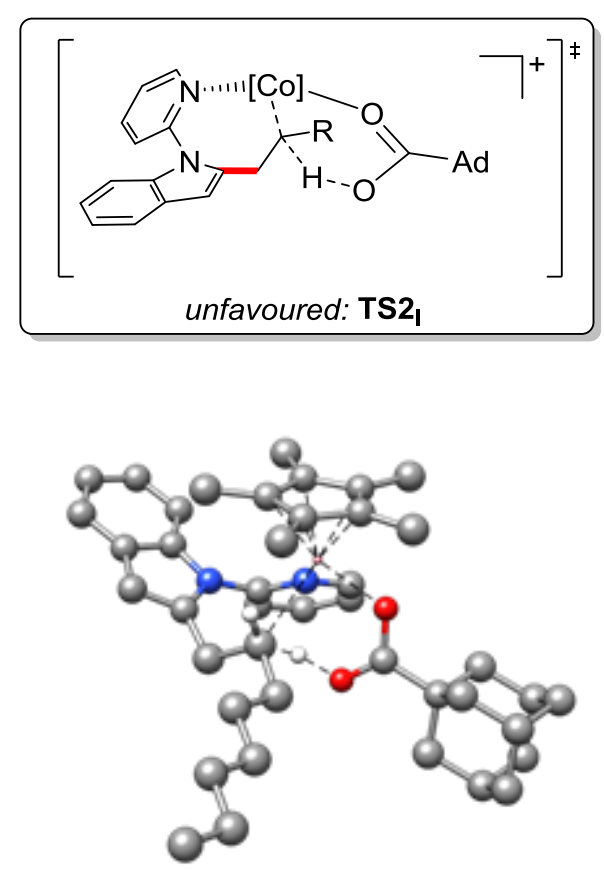

b)
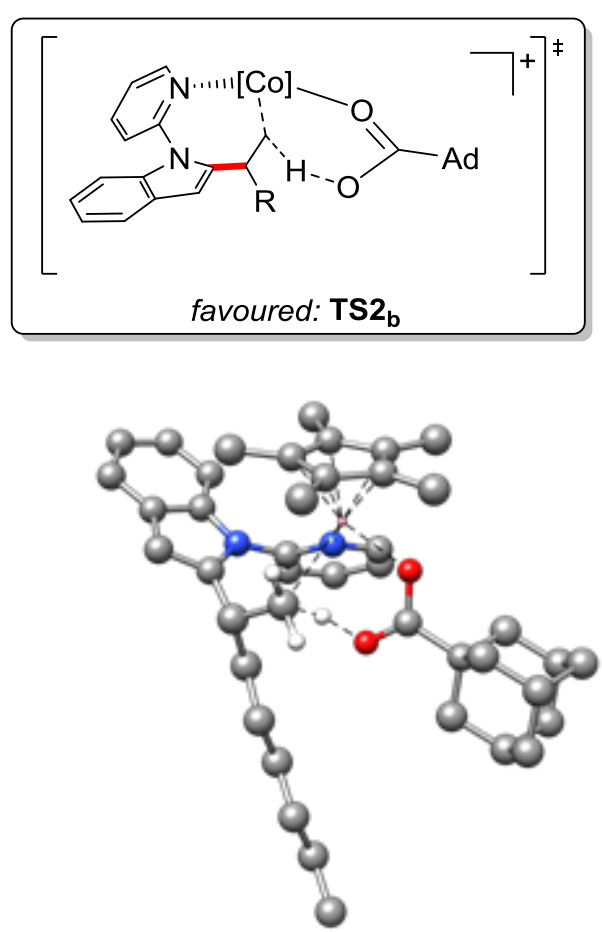

Figure 23. Calculated transition states of the proto-demetalation for the branched-selective reaction with a) the unfavored linear arrangement and b) the energetically favored branched transition state shown; $\mathrm{R}=n$-Hex. ${ }^{[161]}$ 


\subsection{Cobalt(III)-Catalyzed Allylative and Alkenylative C-H/C-F Functionalizations}

The selective synthetis of fluorinated molecules is a major challenge in organic synthesis, since about $20-30 \%$ of all agrochemicals and pharmaceuticals contain fluorine for the improvement of inter alia their metabolic stability and lipophilicity (Figure 24). ${ }^{[18 \mathrm{~h}, 118 \mathrm{k}, 167]}$ Consequently, there is a continued demand for selective and mild conditions to install fluorinated motifs in late-stage modifications of complex molecules. ${ }^{[13 \mathrm{k},}{ }^{118]}$ A particularly sustainable and step-economical access to highly functionalized fluorine-containing molecules can be achieved by merging $\mathrm{C}-\mathrm{H}$ with $\mathrm{C}-\mathrm{F}$ functionalization in a tandem process. ${ }^{[168]}$

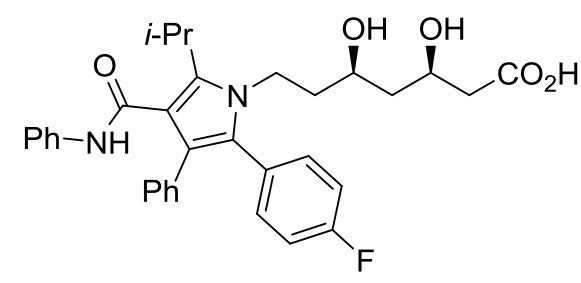

Atorvastatin (lipid-lowering agent)

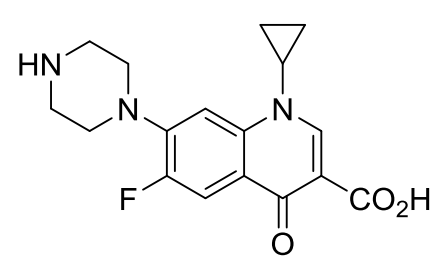

Ciprofloxacin (antibiotic)

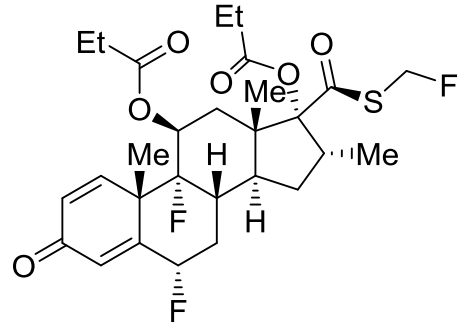

Fluticasone propionate (corticosteroid)

Figure 24. Selected examples of fluorine-containing pharmaceuticals.

\subsubsection{Optimization Studies for the Allylative $\mathrm{C}-\mathrm{H} / \mathrm{C}-\mathrm{F}$ Functionalizations}

The optimization studies for the cobalt(III)-catalyzed C-H/C-F functionalization were started by probing the effect of representative solvents for cobalt(III)-catalyzed $\mathrm{C}-\mathrm{H}$ activation (Table 19). Interestingly, fluorinated solvents ${ }^{[169]}$ such as HFIP and TFE by far outcompeted DCE and MeOH (entries 1-4), with TFE delivering the desired product 158aa in an almost quantitative yield of $97 \%$ with a good diastereoselectivity of $Z / E=86: 14$. According to a report of Berkessel and coworkers on the beneficial effects of fluorinated solvents, ${ }^{[170]}$ it can be assumed that TFE and HFIP form higher order solvent aggregates that significantly stabilize cationic intermediates in catalysis by enhanced $\mathrm{H}$ bond donor abilities. In the absence of any cobalt(III) catalyst, no reactivity was observed (entry 5). Furthermore, a catalyst loading of $0.25 \mathrm{~mol} \%$ still provided the product 158aa in excellent yield, albeit reduced catalyst loadings as low as $0.10 \mathrm{~mol} \%$ seemed to be less efficient (entries 6 and 7). Notably, the reaction still proceeded at $0{ }^{\circ} \mathrm{C}$ and furnished the product in overall moderate yield, but with an improved diastereoselectivity of $Z / E=93: 7$ (entry 8).

Thereafter, various bases were tested, especially mild bases like carbonates. Within a series of alkali metal carbonates, an increased reactivity from lithium to potassium carbonate was found, but with cesium carbonate a significant drop in yield to only $8 \%$ was noticed (entries 4 and 9-12). The preference for potassium as countercation in this reaction was furthermore revealed by obtaining excellent yields with potassium hydrogen carbonate and potassium acetate as the bases (entries 13 
and 14). Among significantly more basic hydroxides, potassium hydroxide was found to be the most efficient base (entries 15 and 16). Based on these findings, it can be assumed that potassiumcontaining bases are generally favored regardless of their $\mathrm{p} K_{\mathrm{B}}$-values.

Importantly, qualitative solubility experiments in TFE as solvent showed an increasing solubility in a row from lithium to cesium fluoride, which is agreement with quantitative experiments of Wynn and coworkers. ${ }^{[171]}$ Thus, by only taking the solubility of fluorides into account, lithium carbonate as base would create the strongest driving force for the reaction. However, the solubility of the corresponding carbonate bases should be considered as well. In this regard, qualitative solubility experiments indicated an increasing solubility from lithium to cesium carbonate, which would lead to the highest reactivity for cesium carbonate. Based on these results, only well-balanced solubilities of the carbonate bases as well as the precipitating alkali metal fluorides can account for this reactivity trend, resulting in the overall highest efficiency for potassium carbonate.

Table 19. Optimization study for the cobalt(III)-catalyzed C-H/C-F functionalization.

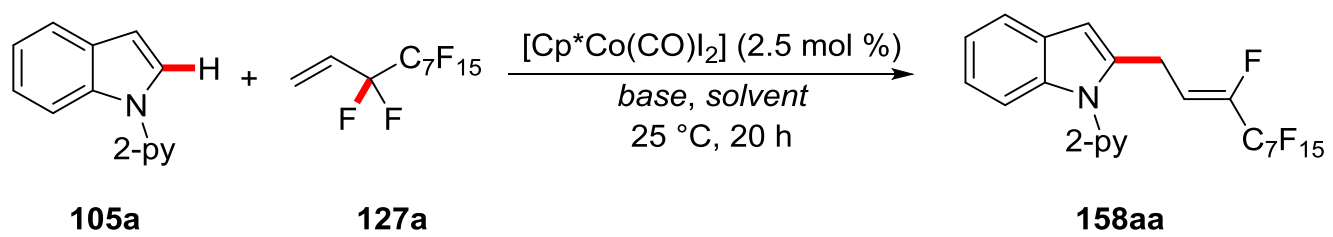

\begin{tabular}{|c|c|c|c|}
\hline Entry & base & solvent & yield / $\%^{[\mathrm{a}]}$ \\
\hline 1 & $\mathrm{~K}_{2} \mathrm{CO}_{3}$ & DCE & 3 \\
\hline 2 & $\mathrm{~K}_{2} \mathrm{CO}_{3}$ & $\mathrm{MeOH}$ & 12 \\
\hline 3 & $\mathrm{~K}_{2} \mathrm{CO}_{3}$ & HFIP & 91 \\
\hline 4 & $\mathrm{~K}_{2} \mathrm{CO}_{3}$ & TFE & 97 \\
\hline 5 & $\mathrm{~K}_{2} \mathrm{CO}_{3}$ & TFE & $--^{[b]}$ \\
\hline 6 & $\mathrm{~K}_{2} \mathrm{CO}_{3}$ & TFE & $95^{[\mathrm{c}]}$ \\
\hline 7 & $\mathrm{~K}_{2} \mathrm{CO}_{3}$ & TFE & $44^{[\mathrm{d}]}$ \\
\hline 8 & $\mathrm{~K}_{2} \mathrm{CO}_{3}$ & TFE & $46^{[\mathrm{e}]}$ \\
\hline 9 & $\mathrm{Li}_{2} \mathrm{CO}_{3}$ & TFE & 18 \\
\hline 10 & $\mathrm{Na}_{2} \mathrm{CO}_{3}$ & TFE & 81 \\
\hline 11 & $\mathrm{Rb}_{2} \mathrm{CO}_{3}$ & TFE & 49 \\
\hline 12 & $\mathrm{Cs}_{2} \mathrm{CO}_{3}$ & TFE & 8 \\
\hline 13 & $\mathrm{KHCO}_{3}$ & TFE & 94 \\
\hline 14 & KOAc & TFE & 87 \\
\hline 15 & $\mathrm{KOH}$ & TFE & 93 \\
\hline
\end{tabular}


16

$\mathrm{Ca}(\mathrm{OH})_{2}$

TFE

45

[a] Reaction conditions: 105a $(0.50 \mathrm{mmol}), 127 \mathrm{a}(0.60 \mathrm{mmol}),[\mathrm{Co}](2.5 \mathrm{~mol} \%)$, base (1.00 equiv), solvent $(0.5 \mathrm{~mL}, 1.0 \mathrm{M}), 25{ }^{\circ} \mathrm{C}, 20 \mathrm{~h}$, isolated yield; all $\mathrm{Z} / E=86: 14$. [b] Without [Co]. [c] $0.25 \mathrm{~mol} \%$ [Co]. [d] $0.10 \mathrm{~mol} \%$ [Co]. [e] At $0{ }^{\circ} \mathrm{C}, Z / E=93: 7$.

To explore this reaction with respect to even more cost-efficient cobalt sources, a comprehensive screening of some well-established cobalt-complexes ${ }^{[15 a]}$ was performed (Table 20). Catalysts with Cp or substituted $(t-\mathrm{Bu})_{2}$-Cp-ligands delivered only traces of the desired product 158aa (entries 2 and 3 ). In striking contrast, the beneficial influence on stereoselectivities by substituents on the $\mathrm{Cp}$-ligand had previously been disclosed by Rovis and coworkers in rhodium(III)-catalyzed C-H activation catalysis. ${ }^{[172]}$ Unfortunately, also simple cobalt salts, such as cobalt(III) fluoride (entry 4) and cobalt(II) bromide (entry 8), failed to furnish any desired product as well as other salts, such as cobalt acetylacetonate and cobalt acetate (entries 5-7). In summary, these findings show the great importance of the $\mathrm{Cp}^{*}$-ligand for efficient catalysis, probably due to its very good donor abilities and rigid structure.

Table 20. Screening of different cobalt sources as catalysts.<smiles>[CH2-]c1cc2ccccc2n1[TeH]</smiles>

$105 a$

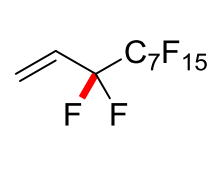

$127 a$

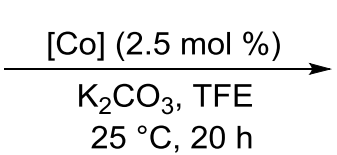

$25^{\circ} \mathrm{C}, 20 \mathrm{~h}$

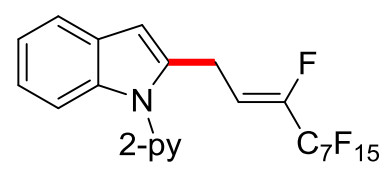

158 aa

\begin{tabular}{ccc}
\hline Entry & {$[\mathrm{Co}]$} & yield / \% \\
\hline 1 & {$\left[\mathrm{Cp} * \mathrm{Co}(\mathrm{CO}) \mathrm{I}_{2}\right]$} & 97 \\
2 & {$\left[\mathrm{CpCo}(\mathrm{CO}) \mathrm{I}_{2}\right]$} & traces \\
3 & {$\left[1,3-(t-\mathrm{Bu})_{2} \mathrm{C}_{5} \mathrm{H}_{3} \mathrm{Co}(\mathrm{CO}) \mathrm{I}_{2}\right]$} & traces \\
4 & $\mathrm{CoF}_{3}$ & -- \\
5 & $\mathrm{Co}(\mathrm{acac})_{3}$ & -- \\
6 & $\mathrm{Co}(\mathrm{acac})_{2}$ & --- \\
7 & $\mathrm{Co}(\mathrm{ac})_{2}$ & --- \\
8 & $\mathrm{CoBr}$ & -- \\
\hline
\end{tabular}

[a] Reaction conditions: 105a $(0.50 \mathrm{mmol}), \mathbf{1 2 7 a}(0.60 \mathrm{mmol})$, [Co] (2.5 mol \%), $\mathrm{K}_{2} \mathrm{CO}_{3}$ (1.00 equiv), solvent

$(0.5 \mathrm{~mL}, 1.0 \mathrm{M}), 25^{\circ} \mathrm{C}, 20 \mathrm{~h}$. Conversions were determined by GC with $n$-dodecane as the internal standard. 


\subsubsection{Optimization Studies for the Alkenylative C-H/C-F Functionalization}

Based on the optimization studies for the allylative $\mathrm{C}-\mathrm{H} / \mathrm{C}-\mathrm{F}$ functionalization, it was studied whether 1,1-difluorostyrenes $\mathbf{1 2 8}$ can be efficiently converted by cobalt(III) catalysis (Table 21). 1,1difluorostyrenes $\mathbf{1 2 8}$ are synthetically easily accessible and biologically relevant substrates. ${ }^{[173]}$ For this purpose, it was initially observed that the reaction performed poorly in the absence of base (entry 1), whereas $\mathrm{AgSbF}_{6}$ had a beneficial influence on the reactivity (entry 2). Thereafter, a significant increase in product yield was achieved by utilizing potassium carbonate as the base (entry 3), while no improvement by additional $\mathrm{AgSbF}_{6}$ (entry 4) was observed. Moreover, the optimal base loading was found to be 1.50 equivalents (entries 5-7). Noteworthily, after screening different metal carbonates the corresponding potassium salt turned out to be optimal (entries 5-11), showing same reactivity trend as it was previously observed for the $\mathrm{C}-\mathrm{H} / \mathrm{C}-\mathrm{F}$ allylative functionalization ( $c f$. Table 19, entries 8-12).

Importantly, a slight increase of the 1,1-difluorostyrenes (128a) concentration furnished the desired alkenylated product 159aa in almost quantitative yield and excellent $Z$-diastereoselectivity (entry 12). Moreover, a control reaction revealed that this reaction is indeed cobalt(III)-catalyzed (entry 13). Subsequently, selected solvents were tested, with an unsatisfactory result for the more expensive perfluorinated HFIP (entry 14). Unfortunately, more economic solvents, such as $\mathrm{MeOH}$ and $\mathrm{H}_{2} \mathrm{O}$, showed no reactivity (entries 16 and 17). It is noteworthy that in all cases excellent diastereoselectivities of $Z / E>$ 99:1 were achieved.

Table 21. Optimization of cobalt(III)-catalyzed alkenylative $\mathrm{C}-\mathrm{H} / \mathrm{C}-\mathrm{F}$ functionalizations.

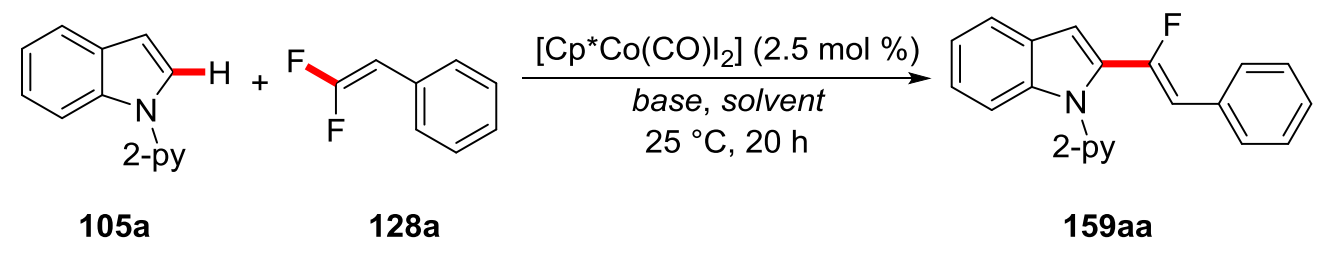

\begin{tabular}{cccc}
\hline Entry & base & solvent & yield $/ \%^{[\mathrm{a}]}$ \\
\hline 1 & --- & TFE & --- \\
2 & --- & TFE & $37^{[\mathrm{b}]}$ \\
3 & $\mathrm{~K}_{2} \mathrm{CO}_{3}$ & TFE & 77 \\
4 & $\mathrm{~K}_{2} \mathrm{CO}_{3}$ & TFE & $73^{[\mathrm{b}]}$ \\
5 & $\mathrm{~K}_{2} \mathrm{CO}_{3}(0.50$ equiv $)$ & TFE & 33 \\
6 & $\mathrm{~K}_{2} \mathrm{CO}_{3}(1.50$ equiv $)$ & TFE & 86 \\
7 & $\mathrm{~K}_{2} \mathrm{CO}_{3}(2.00$ equiv $)$ & TFE & 67 \\
8 & $\mathrm{Li}_{2} \mathrm{CO}_{3}$ & TFE & --
\end{tabular}




$\begin{array}{cccc}9 & \mathrm{Na}_{2} \mathrm{CO}_{3} & \text { TFE } & 18 \\ 10 & \mathrm{Rb}_{2} \mathrm{CO}_{3} & \text { TFE } & 60 \\ 11 & \mathrm{Cs}_{2} \mathrm{CO}_{3} & \text { TFE } & 30 \\ \mathbf{1 2} & \mathrm{K}_{2} \mathrm{CO}_{3} & \text { TFE } & \mathbf{9 8}^{\text {[c] }} \\ 13 & \mathrm{~K}_{2} \mathrm{CO}_{3} & \mathrm{TFE} & --{ }^{[\mathrm{d}]} \\ 14 & \mathrm{~K}_{2} \mathrm{CO}_{3} & \mathrm{HFIP} & 46 \\ 15 & \mathrm{~K}_{2} \mathrm{CO}_{3} & \mathrm{DCE} & -- \\ 16 & \mathrm{~K}_{2} \mathrm{CO}_{3} & \mathrm{MeOH} & --- \\ 17 & \mathrm{~K}_{2} \mathrm{CO}_{3} & \mathrm{H} & ---\end{array}$

[a] Reaction conditions: 105a $(0.50 \mathrm{mmol}), \mathbf{1 2 8 a}(0.60 \mathrm{mmol}),[\mathrm{Co}](2.5 \mathrm{~mol} \%)$, base (1.00 equiv), solvent $(0.5 \mathrm{~mL}, 1.0 \mathrm{M}), 25{ }^{\circ} \mathrm{C}, 20 \mathrm{~h}$, isolated yield; all Z/E-ratios > 99:1, determined by ${ }^{1} \mathrm{H}$-NMR spectroscopy. [b] With $\operatorname{AgSbF}_{6}(5.0 \mathrm{~mol} \%)$. [c] 128a (1.50 equiv). [d] Without [Co].

\subsubsection{Scope of the Allylative Cobalt(III)-Catalyzed C-H/C-F Functionalization}

Under the optimized reaction conditions several heterocycles, including indoles 105, pyrroles 146 and phenylpyrazoles 18 were probed (Table 22). Initially, $1 H, 1 H, 2 H$-perfluorohexene (127b) furnished the corresponding product 158ab in excellent yield and good $Z / E$-ratio (entry 2), displaying a considerable variability with respect to the perfluoroalkyl chain length. Subsequently, various substituents in the C5-position of 2-pyridylindole (105a) were tested, with overall very good yields and good diastereoselectivities (entries 3-7). It is noteworthy that even sterically demanding substrates like 105p were successfully converted although an elevated reaction temperature and higher catalyst loading were necessary (entry 8). Notably, due to the higher reaction temperature the diastereoselectivity dropped to moderate $Z / E=80: 20$. Moreover, it was demonstrated that also 1(pyridin-2-yl)-1H-pyrrole (146) and phenylpyrazoles 18 could deliver the desired products (entries 9-11). In the latter case, an optimal reactivity was achieved by using $\operatorname{AgSbF}_{6}(20 \mathrm{~mol} \%)$ as the additive, which is presumably due to an unfavored BIES-type $\mathrm{C}-\mathrm{H}$ activation step, as the arene is less electron-rich than indoles and pyrroles. 
Table 22. Scope of the cobalt(III)-catalyzed allylative $\mathrm{C}-\mathrm{H} / \mathrm{C}-\mathrm{F}$ funtionalization with different heterocycles.

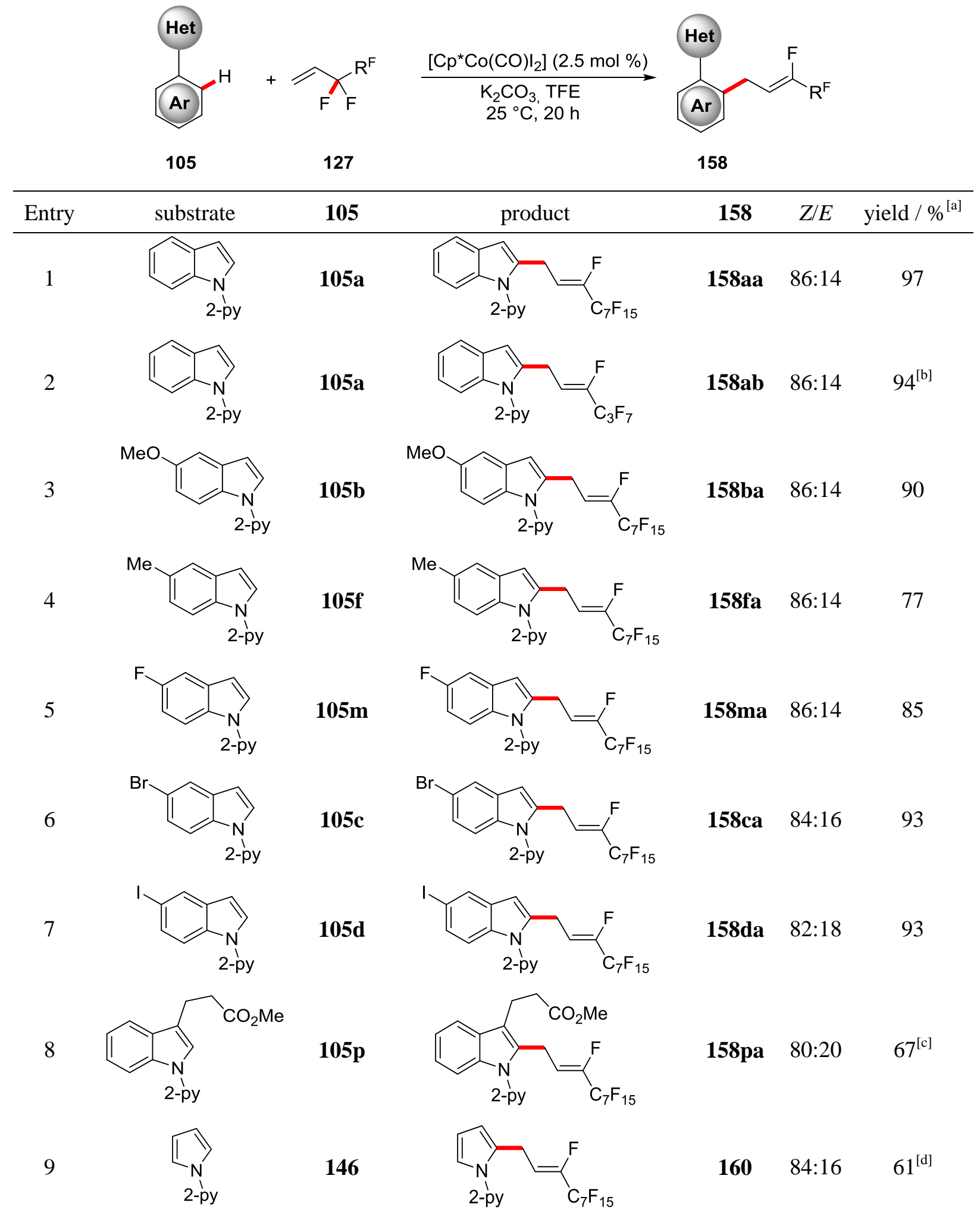


10<smiles>CC(C)(C)c1ccc(-n2cccn2)cc1</smiles>

11<smiles>COc1ccc(-n2ccnc2)cc1</smiles>

18c<smiles>CC(C)(C)c1ccc(-n2cccn2)c(CC=C(F)C(F)(F)F)c1</smiles>

18d<smiles>COc1ccc(-n2cccn2)c(CC=C(F)C(F)(F)F)c1</smiles>

$161 c$

$87: 13$ $79^{[\mathrm{c}, \mathrm{e}]}$

161d $53^{[\mathrm{c}, \mathrm{e}]}$

[a] Reaction conditions: 105a $(0.50 \mathrm{mmol}), \mathbf{1 2 7 a}(0.60 \mathrm{mmol}),[\mathrm{Co}](2.5 \mathrm{~mol} \%), \mathrm{K}_{2} \mathrm{CO}_{3}(1.00$ equiv), TFE $(0.5 \mathrm{~mL}, 1.0 \mathrm{M}), 25{ }^{\circ} \mathrm{C}, 20 \mathrm{~h}$, isolated yield; Z/E-ratio determined by ${ }^{1} \mathrm{H}$-NMR spectroscopy. [b] $1 \mathrm{H}, 1 \mathrm{H}, 2 \mathrm{H}$ perfluorohex-1-ene (127b) $(0.60 \mathrm{mmol})$ was used. [c] At $70{ }^{\circ} \mathrm{C}$ and [Co] $(10 \mathrm{~mol} \%)$. [d] $8 \%$ of di-substituted 160' $(Z / E=84: 16)$ was detected. [e] With $\mathrm{AgSbF}_{6}(20 \mathrm{~mol} \%)$.

\subsubsection{Scope of the Alkenylative Cobalt(III)-Catalyzed C-H/C-F Functionalization}

With the optimized catalytic reaction conditions in hand, the substrate scope with respect to various indoles $\mathbf{1 0 5}$ and 1,1-difluorostyrenes 128 was performed (Table 23). Electron-rich as well as electronpoor indoles reacted in very good yields and excellent diastereoselectivities (entries 1-4). Notably, $V$. Müller and $R$. R. Presa extended the scope to more examples of ortho- and meta-substituted 1,1difluorostyrenes 128. ${ }^{[174]}$ Furthermore, it was demonstrated that the thiophene-derived substrates (entries 5 and 6) performed well, delivering the desired products 159ab and 159ac in almost quantitative yield. However, a slightly decreased diastereoselectivity for the 3-substituted thiophene 128c was observed. Elevated temperatures were necessary to convert the naphthalene-derived substrate 128d (entry 7). In this manner, the sterically even more biased fluorescent anthracenederivative 128e failed to yield the corresponding product, revealing a limitation for this reaction with respect to bulky 1,1-difluoroalkenes $\mathbf{1 2 8}$.

Table 23. Scope of the alkenylative cobalt(III)-catalyzed C-H/C-F functionalization.

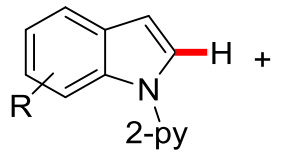

105<smiles>FC(F)=Cc1ccccc1</smiles>

128

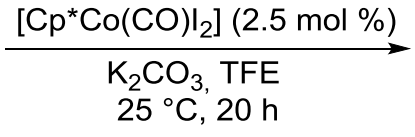

$25^{\circ} \mathrm{C}, 20 \mathrm{~h}$

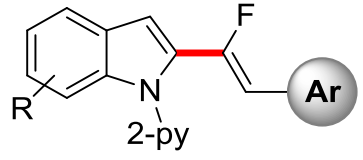

159

Entry indole $\mathbf{1 0 5} 128$

$105 a$

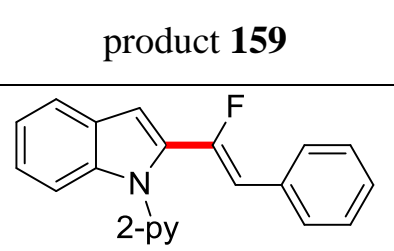

159aa yield / \% $(Z / E)^{[\mathrm{a}]}$ 
2<smiles>COc1ccc2c(ccn2[18O])c1</smiles>

105b

3<smiles>[18OH]n1ccc2cc(F)ccc21</smiles>

$105 \mathrm{~m}$<smiles>O=[Pt]n1ccc2cc(I)ccc21</smiles>

105d

5<smiles>[123I]n1ccc2ccccc21</smiles>

$105 a$<smiles>[18O]n1ccc2ccccc21</smiles>

$105 a$

7<smiles>Cn1ccc2ccccc21</smiles>

$105 a$<smiles>Cn1ccc2ccccc21</smiles>

105a<smiles>FC(F)=Cc1ccccc1</smiles>

128a<smiles>FC(F)=Cc1ccccc1</smiles>

128a<smiles>FC(F)=Cc1ccccc1</smiles>

128a<smiles>FC(F)=Cc1cccs1</smiles>

$128 b$<smiles>FC(F)=Cc1ccsc1</smiles>

$128 c$<smiles>FC(F)=Cc1cccc2ccccc12</smiles>

128d<smiles>FC(F)=Cc1c2ccccc2cc2ccccc12</smiles>

$128 \mathrm{e}$<smiles>COc1ccc2c(c1)cc(/C(F)=C/c1ccccc1)n2[12O]</smiles>

159ba<smiles>FC(=Cc1ccccc1)c1cc2cc(F)ccc2n1[Pb]</smiles>

159ma<smiles>[18O]n1c(/C(F)=C/c2ccccc2)cc2cc(I)ccc21</smiles>
$83^{[b]}$

159da<smiles>[R17]n1c(/C(F)=C/c2cccs2)cc2ccccc21</smiles>
96

159ab<smiles>[R17]n1c(/C(F)=C/c2ccsc2)cc2ccccc21</smiles>

159ac<smiles>[Y8]n1c(/C(F)=C/c2cccc3ccccc23)cc2ccccc21</smiles>

159ad

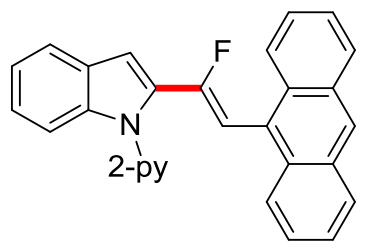

159ae

[a] Reaction conditions: 105a $(0.50 \mathrm{mmol}), \mathbf{1 2 8 a}(0.75 \mathrm{mmol})$, [Co] $(2.5 \mathrm{~mol} \%), \mathrm{K}_{2} \mathrm{CO}_{3}(1.00$ equiv), TFE $(0.5 \mathrm{~mL}, 1.0 \mathrm{M}), 25^{\circ} \mathrm{C}, 20 \mathrm{~h}$, isolated yield; Z/E-ratios in parentheses, determined by ${ }^{1} \mathrm{H}-\mathrm{NMR}$ spetroscopy. [b] At $50{ }^{\circ} \mathrm{C}$. [c] At $70{ }^{\circ} \mathrm{C}$.

Remarkably, under the same reaction conditions as well as the reported one by Loh and coworkers, ${ }^{[168 d]}$ rhodium(III) catalysis was clearly shown to be less efficient. Indeed, the desired product 159aa was delivered with the same excellent diastereoselectivity of $Z / E>99: 1$, but with a considerably reduced efficacy (Scheme 71). The exact reason for this significant difference has yet not been fully elucidated. It can be speculated that the increased nucleophilicity of the cobalt-carbon bond (Pauling-electronegativity of cobalt is $\chi_{\mathrm{F}} \approx 1.9$ and for rhodium it is $\left.\chi_{\mathrm{F}} \approx 2.3\right)^{[62]}$ presumably enables an 
accelerated migratory insertion of the very polarized 1,1-difluorostyrene 128a into the carbon-cobalt bond. ${ }^{[15]}$
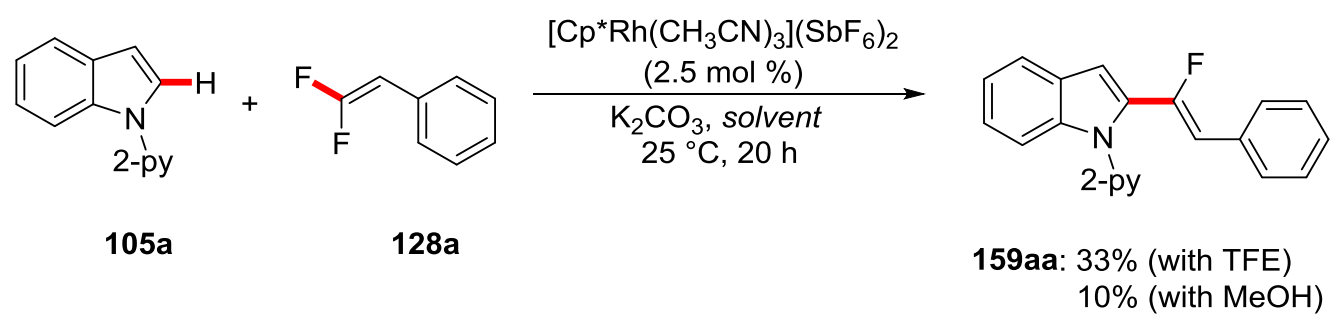

Scheme 71. Performance of a rhodium(III) catalyst in C-H/C-F functionalizations at $25^{\circ} \mathrm{C}$.

\subsubsection{Mechanistic Studies}

\subsubsection{H/D-Exchange Experiments}

Initially, a H/D-exchange study was conducted with deuterated 2-pyridylindole $[\mathrm{D}]_{1}-\mathbf{1 0 5 a}$ in the presence of TFE as solvent and proton source (Scheme 72). In the reisolated starting material 105a and the product 158aa minor amounts of deuterium incorporation in $\mathrm{C} 3$-positions were detected, which was previously observed for the cobalt(III)-catalyzed $\mathrm{C}-\mathrm{H} / \mathrm{C}-\mathrm{C}$ functionalization as well ( $c f$. Scheme 55). This finding might be explained by an electrophilic activation at the C3-position with cobalt(III) and subsequent deutero-demetalation. In contrast to previous observations, no deuteration was detected in the C7-position, which is possibly due to the kinetically unfavored formation of a sixmembered cobaltacycle at the lower reaction temperature. It is noteworthy that almost full protonation in C2-position of the reisolated starting material was observed, which indicated that the $\mathrm{C}-\mathrm{H}$ activation step is fully reversible and not turnover-limiting. Furthermore, it should be mentioned that no H/D-scrambling in any position of the perfluoroalkyl chain could be detected. These results might suggest that the migratory insertion is irreversible.

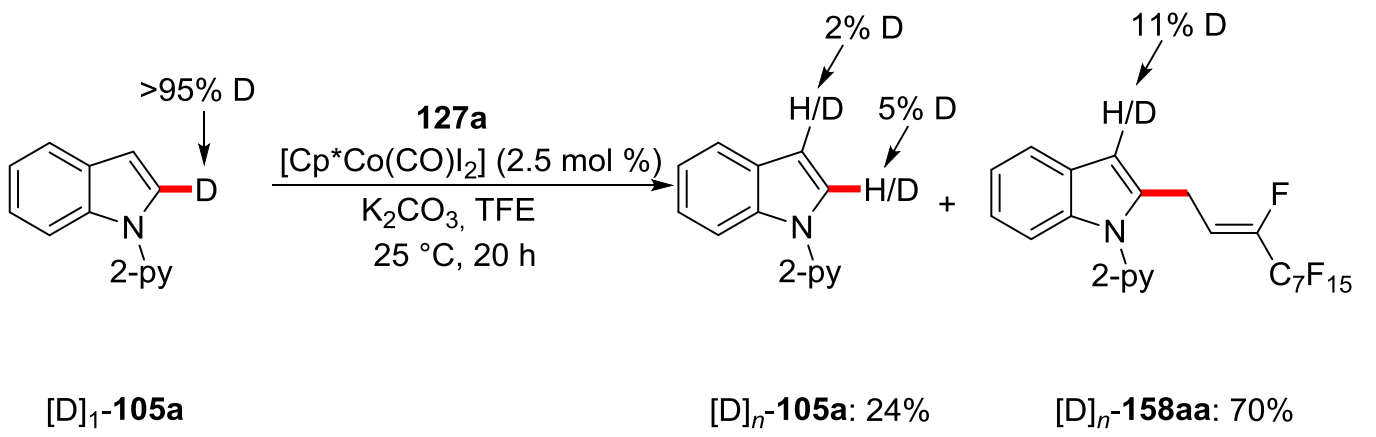

Scheme 72. H/D-exchange study for the cobalt(III)-catalyzed allylative $\mathrm{C}-\mathrm{H} / \mathrm{C}-\mathrm{F}$ functionalization.

\subsubsection{KIE Studies}

The KIE of the allylative cobalt(III)-catalyzed $\mathrm{C}-\mathrm{H} / \mathrm{C}-\mathrm{F}$ functionalization was determined by comparison of independent reaction rates for substrate 105a and its isotopically labeled analogue $[\mathrm{D}]_{1^{-}}$ 105a, resulting in a value of $k_{\mathrm{H}} / k_{\mathrm{D}} \approx 1.5$ (Scheme 73 and Figure 25). The KIE value is in good agreement with the H/D-exchange experiment, which altogether indicate that the $\mathrm{C}-\mathrm{H}$ activation is 
facile and reversible. Nevertheless, the slightly larger KIE as compared to previously studied reactions (cf. Figure 11) leads to the mechanistic hypothesis that the $\mathrm{C}-\mathrm{H}$ activation could be a kinetically relevant equilibrium step before the turnover-limiting migratory insertion.

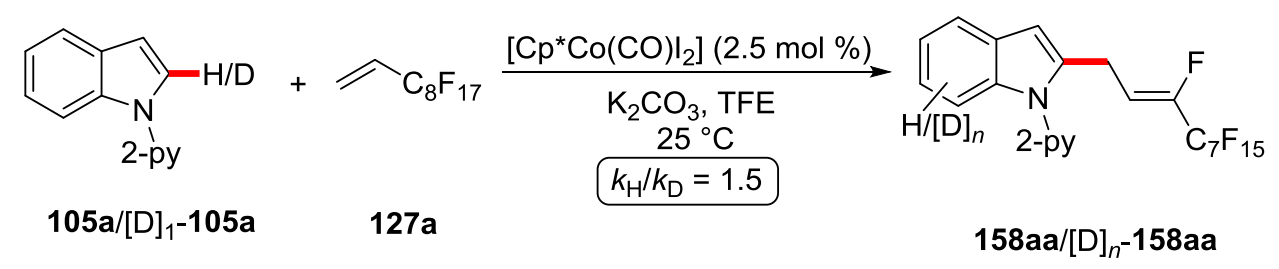

Scheme 73. KIE study of the allylative cobalt(III)-catalyzed C-H/C-F functionalization.

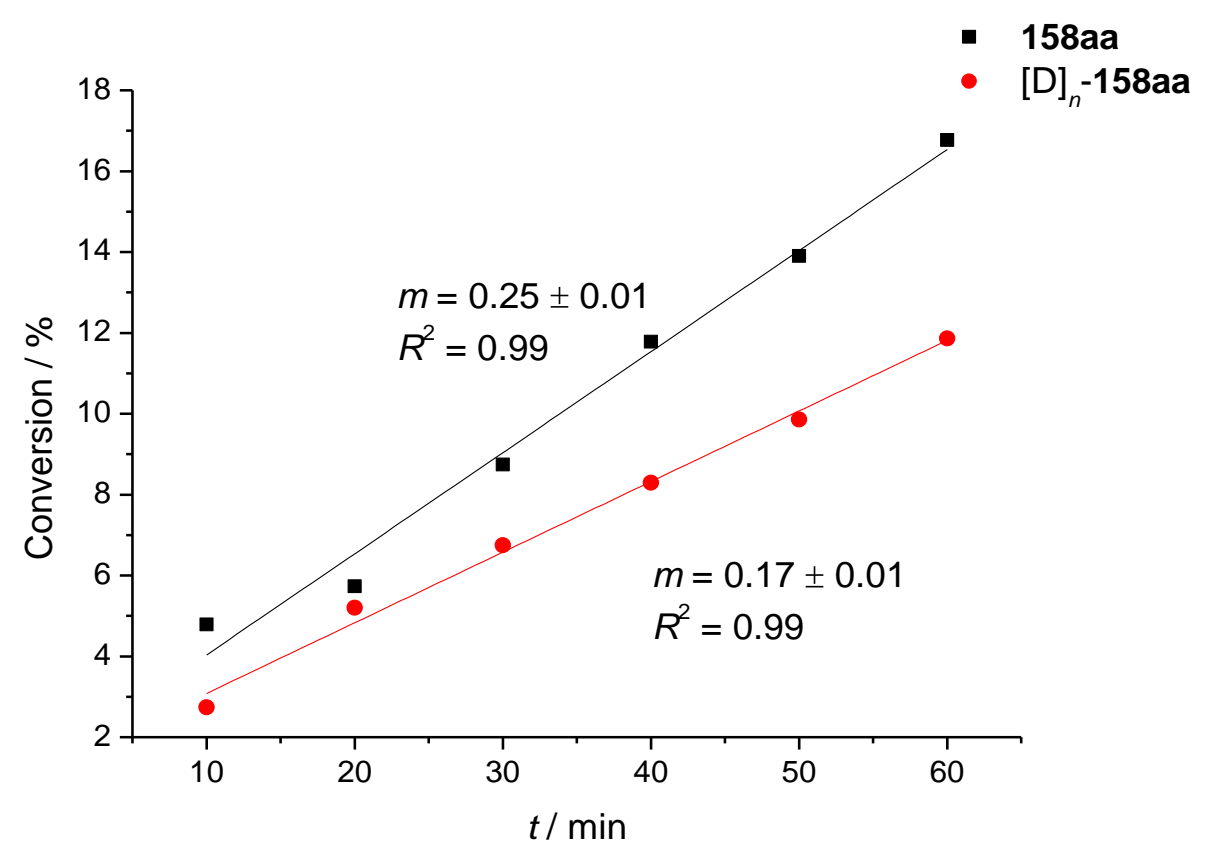

Figure 25. KIE study of the allylative cobalt(III)-catalyzed C-H/C-F functionalization.

\subsubsection{Intermolecular Competition Experiments}

To gain further insight regarding the $\mathrm{C}-\mathrm{H}$ activation mechanism, an intermolecular competition experiment between the electron-rich substrate 18a and electron-poor trifluoromethyl-substituted $\mathbf{1 8 b}$ was conducted (Scheme 74).

Importantly, a strong preference for the electron-rich substrate 18a was found which reacted almost four times faster than the corresponding electron-poor substrate $\mathbf{1 8 b}$. Along with the slightly higher KIE of 1.5, this finding reveals that the $\mathrm{C}-\mathrm{H}$ activation possibly follows a BIES-type pathway, which is clearly favored for electron-rich substrates. Furthermore, a kinetically relevant migratory insertion, which benefits from a more nucleophilic carbon-cobalt bond in electron-rich substrates, could be a reasonable explanation for this reactivity trend. 


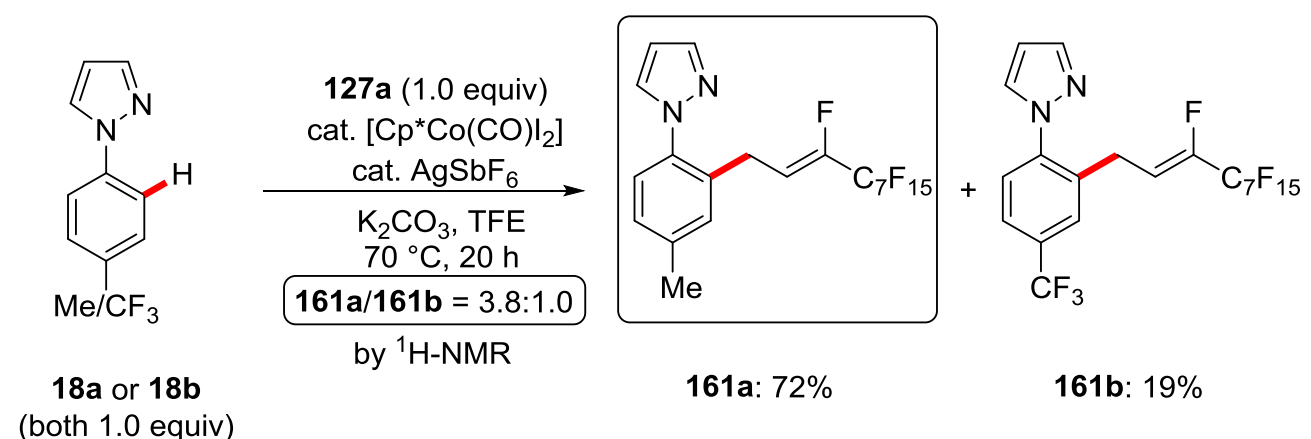

Scheme 74. Intermolecular competition experiment.

\subsubsection{Proposed Catalytic Cycle}

In summary, the $\mathrm{C}-\mathrm{H}$ activation presumably occurs via a reversible and facile BIES-type pathway, in which the $\mathrm{C}-\mathrm{H}$ cleavage likely is assisted by the carbonate, which in situ forms the active cobalt(III)carbonate catalyst 162. Afterwards, the generated cobaltacycle 163 first undergoes a reversible coordination of the perfluoroalkylalkene 127a to furnish intermediate 164. Thereafter, a migratory insertion into the cobalt-carbon bond proceeds to form intermediate $\mathbf{1 6 5}$. The migratory insertion step is proposed to be irreversible because the H/D-exchange study revealed no H/Dscrambling in the perfluoroalkylalkene side-chain (Scheme 72). Afterwards, a process involving the $\beta$ fluoride elimination ${ }^{[119 f]}$ and reaction with the initially formed bicarbonate regenerates the active catalytic species 162 and releases the product 158aa. In principle, the same sequence of elementary steps can be proposed for the alkenylative $\mathrm{C}-\mathrm{H} / \mathrm{C}-\mathrm{F}$ functionalization. 


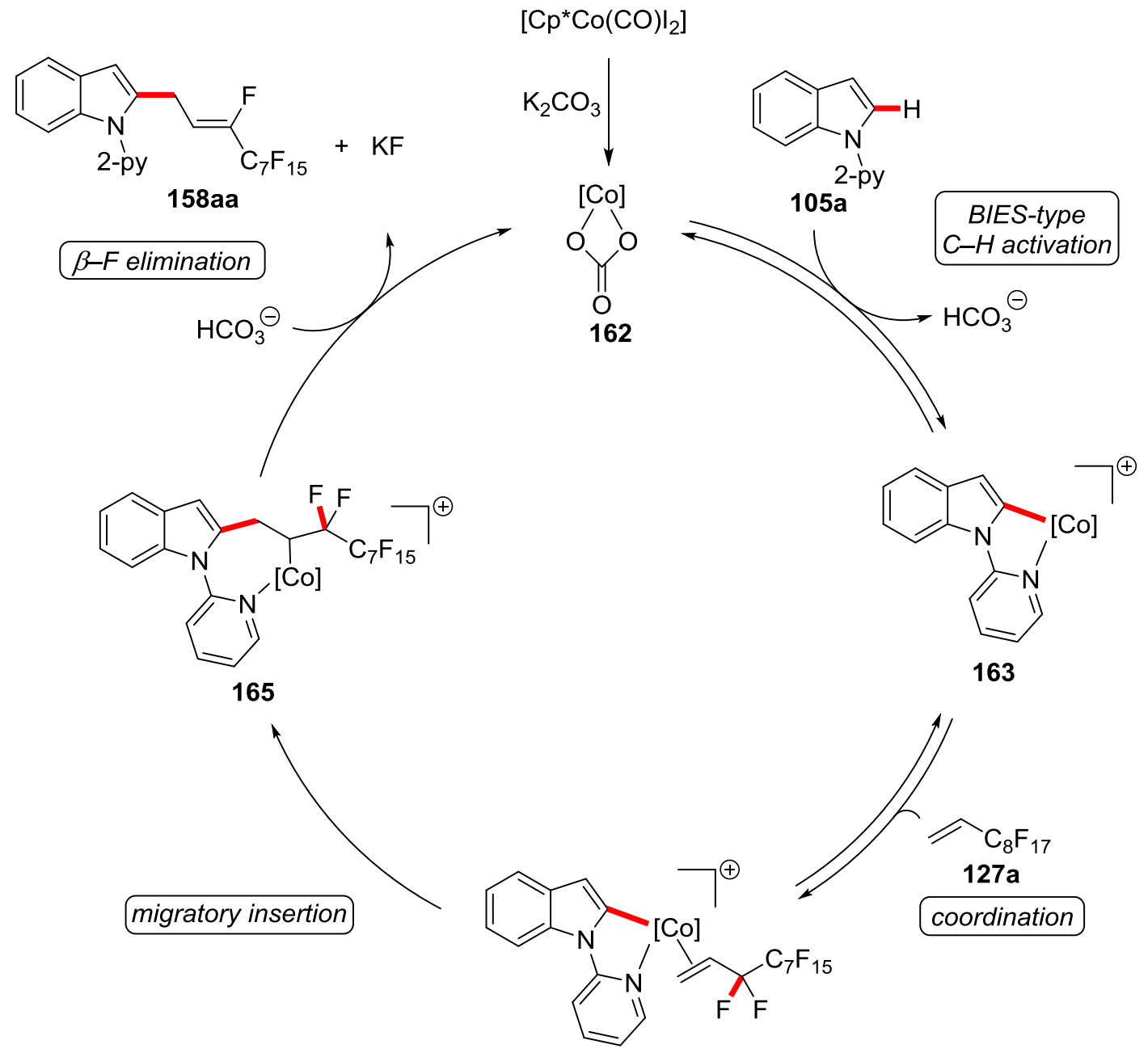

164

Figure 26. Proposed catalytic cycle for the cobalt(III)-catalyzed allylative C-H/C-F functionalization. $[\mathrm{Co}]=\mathrm{Cp}^{*} \mathrm{Co}^{\mathrm{III}}$. 


\subsection{Manganese(I)-Catalyzed Allylative C-H/C-F Funtionalizations}

The use of very inexpensive and less toxic ${ }^{[54,92]}$ manganese complexes in catalysis has been intensively studied in recent years. ${ }^{[14]}$ In this regard, it has been reported that manganese catalysts allowed for $\mathrm{C}-\mathrm{H} / \mathrm{C}-\mathrm{Het}$ functionalizations by $\beta$-oxygen, -nitrogen or -carbon elimination. ${ }^{\text {[108, 117a, 117d] }}$ As it was previously reported, $\beta$-fluoride elimination is a feasible process ${ }^{[119]}$ that allows for the metalcatalyzed installation of fluorine into organic molecules. Thus, it appears to be very attractive to explore the efficiency of manganese catalysts for allylative and alkenylative $\mathrm{C}-\mathrm{H} / \mathrm{C}-\mathrm{F}$ functionalizations ${ }^{[168]}$ on a variety of valuable substrates. Additionally, detailed mechanistic studies should eventually lead to the development of related $\mathrm{C}-\mathrm{H} / \mathrm{C}-\mathrm{Het}$ transformations.

\subsubsection{Optimization Studies for Indoles as Substrates}

The optimization study for the desired transformation was commenced by screening various solvents, initially with unsatisfactory yields of the desired product 158aa (Table 24, entries 1-6). In stark contrast, 1,4-dioxane turned out to be the optimal solvent for this reaction (entry 7) and by far outcompeted other prevalent solvents for manganese(I)-catalyzed $\mathrm{C}-\mathrm{H}$ activations, such as DCE and $n-\mathrm{Bu}_{2} \mathrm{O}$. Subsequently, the effect of bases was probed which were shown to be essential for an efficient conversion (entry 8). Stronger bases like calcium and potassium hydroxide only performed poorly to moderately, with the same observation for potassium phosphate (entries 9-11). These findings are comparable with a related cobalt(III)-catalyzed reaction (cf. chapter 3.4.1) and clearly demonstrate that no direct correlation between reactivity and base strength was observed for this transformation. In this manner, also considerably weaker bases like potassium hydrogen carbonate and sodium acetate, which is a common base in manganese(I)-catalyzed $\mathrm{C}-\mathrm{H}$ activation catalysis, ${ }^{[14]}$ displayed a moderate to good reactivity (entries 12 and 13). As it has been previously observed ( $c f$. Table 7 and Table 24), a comparison between several alkali metal carbonates showed that the reactivity increased from lithium to potassium carbonate, but significantly dropped, when heavier analogues like rubidium and cesium carbonate were employed (entries 15-18). Notably, qualitative solubility experiments in 1,4-dioxane revealed an increasing solubility of alkali metal fluorides as well as carbonates from lithium to cesium salts. This observation is in good agreement with the previously observed trend for the cobalt(III)-catalyzed $\mathrm{C}-\mathrm{H} / \mathrm{C}-\mathrm{F}$ functionalization in TFE ( $c f$. chapter 3.4.1). Finally, a reaction in the absence of the manganese(I) catalyst afforded no detectable amounts of the desired product 158aa (entry 19). It is noteworthy that the diastereoselectivity of $Z / E=90: 10$ was found unaltered by the probed reaction conditions. 
Table 24. Optimization for the manganese(I)-catalyzed allylative $\mathrm{C}-\mathrm{H} / \mathrm{C}-\mathrm{F}$ functionalization.

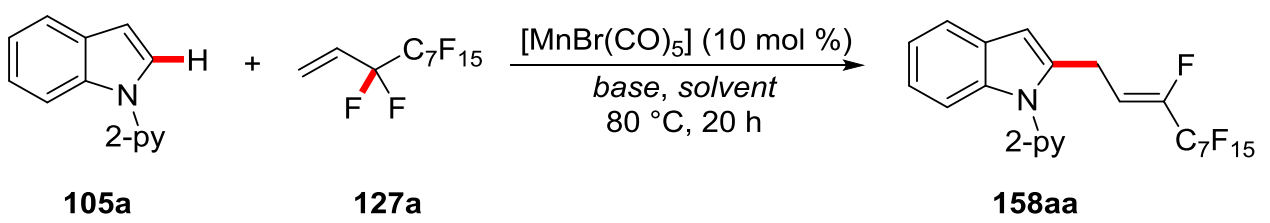

\begin{tabular}{|c|c|c|c|}
\hline Entry & base & solvent & yield / \% ${ }^{[\mathrm{a}]}$ \\
\hline 1 & $\mathrm{~K}_{2} \mathrm{CO}_{3}$ & DCE & 23 \\
\hline 2 & $\mathrm{~K}_{2} \mathrm{CO}_{3}$ & TFE & 8 \\
\hline 3 & $\mathrm{~K}_{2} \mathrm{CO}_{3}$ & $\mathrm{PhMe}$ & 6 \\
\hline 4 & $\mathrm{~K}_{2} \mathrm{CO}_{3}$ & acetone & 23 \\
\hline 5 & $\mathrm{~K}_{2} \mathrm{CO}_{3}$ & $\mathrm{H}_{2} \mathrm{O}$ & 14 \\
\hline 6 & $\mathrm{~K}_{2} \mathrm{CO}_{3}$ & $n-\mathrm{Bu}_{2} \mathrm{O}$ & 14 \\
\hline 7 & $\mathrm{~K}_{2} \mathrm{CO}_{3}$ & 1,4-dioxane & 97 \\
\hline 8 & --- & 1,4-dioxane & 7 \\
\hline 9 & $\mathrm{Ca}(\mathrm{OH})_{2}$ & 1,4-dioxane & 37 \\
\hline 10 & $\mathrm{KOH}$ & 1,4-dioxane & 78 \\
\hline 11 & $\mathrm{~K}_{3} \mathrm{PO}_{4}$ & 1,4-dioxane & 19 \\
\hline 12 & $\mathrm{KHCO}_{3}$ & 1,4-dioxane & 70 \\
\hline 13 & $\mathrm{NaOAc}$ & 1,4-dioxane & 52 \\
\hline 14 & $\mathrm{~K}_{2} \mathrm{CO}_{3}$ & 1,4-dioxane & $45^{[\mathrm{b}]}$ \\
\hline 15 & $\mathrm{Li}_{2} \mathrm{CO}_{3}$ & 1,4-dioxane & 8 \\
\hline 16 & $\mathrm{Na}_{2} \mathrm{CO}_{3}$ & 1,4-dioxane & 92 \\
\hline 17 & $\mathrm{Rb}_{2} \mathrm{CO}_{3}$ & 1,4-dioxane & 25 \\
\hline 18 & $\mathrm{Cs}_{2} \mathrm{CO}_{3}$ & 1,4-dioxane & 5 \\
\hline 19 & $\mathrm{~K}_{2} \mathrm{CO}_{3}$ & 1,4-dioxane & $-{ }^{[c]}$ \\
\hline
\end{tabular}

[a] Reaction conditions: 105a $(0.50 \mathrm{mmol}), 127 \mathbf{a}(0.60 \mathrm{mmol}),\left[\mathrm{MnBr}(\mathrm{CO})_{5}\right](10 \mathrm{~mol} \%)$, base $(0.50 \mathrm{mmol})$, solvent $(0.5 \mathrm{~mL}, 1.0 \mathrm{M}), 20 \mathrm{~h}$, isolated yield; all $\mathrm{Z} / E=90: 10$, determined by ${ }^{1} \mathrm{H}-\mathrm{NMR}$ spectroscopy. [b] At $50{ }^{\circ} \mathrm{C}$. [c] Without $[\mathrm{Mn}]$.

\subsubsection{Optimization Studies for Ketimines as Substrates}

Ketimines 6 are a powerful and transformable class of directing groups ${ }^{[14 a, 175]}$ which could give access to the corresponding ketones $\mathbf{1 6 6}$ after hydrolysis. Thus, a comprehensive optimization study with 
mainly para-methoxyphenyl- (PMP)-substituted ketimines 6 was attempted (Table 25). Indeed, the desired ketone 166aa was furnished even in the absence of any base additive (entry 1). To increase the reactivity, acetate bases were utilized (entries 2 and 3), revealing the sodium salt to be optimal, as it was reported before for manganese-catalyzed $\mathrm{C}-\mathrm{H}$ activations. ${ }^{[14]}$ Furthermore, the influence of the reaction temperature was probed, giving optimal yields at $105^{\circ} \mathrm{C}$ (entry 5). In contrast, higher reaction temperatures, such as $120^{\circ} \mathrm{C}$, led to a slight decrease in yield. However, the observed reactivity difference was overall less pronounced. Interestingly, an increase of the perfluoroalkylalkene 127a concentration turned out to be the optimal condition (entry 8). Importantly, in the absence of manganese(I) catalysis no reactivity was observed (entry 9). A variation of the protecting group to more electron-rich TMP (3,4,5-trimethoxyphenyl) provided the corresponding product 166aa with a slight decrease in yield (entry 10). The dimeric manganese precursor $\left[\mathrm{Mn}_{2}(\mathrm{CO})_{10}\right]$ showed some catalytic activity, albeit the product 166aa was delivered in only unsatisfactory yield (entry 11).

Table 25. Optimization for the allylative $\mathrm{C}-\mathrm{H} / \mathrm{C}-\mathrm{F}$ functionalization of ketimines 6.

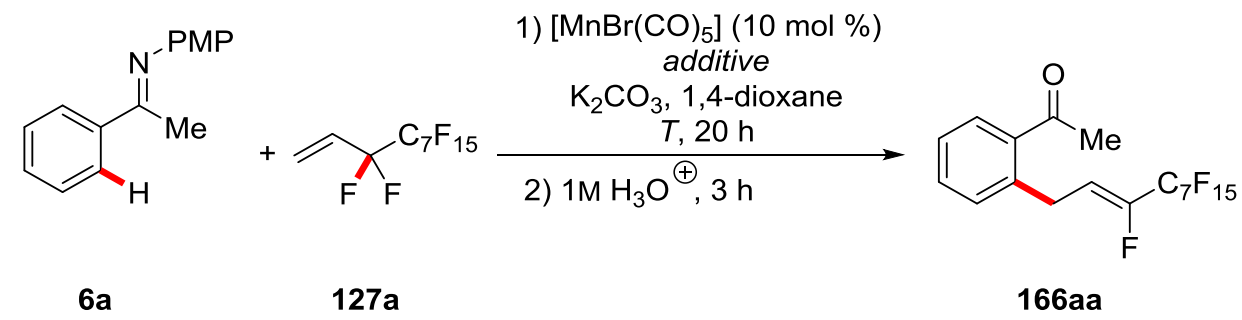

\begin{tabular}{cccc}
\hline Entry & additive & $T /{ }^{\circ} \mathrm{C}$ & yield $/ \%{ }^{[\mathrm{a}]}$ \\
\hline 1 & --- & 120 & $8^{[\mathrm{b}]}$ \\
2 & NaOAc & 120 & 44 \\
3 & KOAc & 120 & 15 \\
4 & NaOAc & 110 & 50 \\
5 & NaOAc & 105 & 53 \\
6 & NaOAc & 90 & 38 \\
7 & NaOAc & 105 & $54^{[\mathrm{c}]}$ \\
8 & NaOAc & 105 & $72^{[\mathrm{d}]}$ \\
9 & NaOAc & 105 & $--\mathrm{-}^{[\mathrm{e}]}$ \\
10 & NaOAc & 105 & $61^{[\mathrm{d} \text { f] }}$ \\
11 & NaOAc & 105 & $32^{[\mathrm{g}]}$
\end{tabular}

[a] Reaction conditions: 6a $(0.50 \mathrm{mmol}), \mathbf{1 2 7 a}(0.75 \mathrm{mmol}),\left[\mathrm{MnBr}(\mathrm{CO})_{5}\right](10 \mathrm{~mol} \%)$, additive $(40 \mathrm{~mol} \%)$, base $(0.50 \mathrm{mmol})$, solvent $(0.50 \mathrm{~mL}, 1.00 \mathrm{M}), 20 \mathrm{~h}$, isolated yield; all $\mathrm{Z} / E=97: 3$, determined by ${ }^{1} \mathrm{H}-\mathrm{NMR}$ 
spectroscopy. [b] Without $\mathrm{K}_{2} \mathrm{CO}_{3}$. [c] 2.00 equiv of 127a. [d] 3.00 equiv of 127a. [e] Without [Mn]. [f] With TMP-substituted ketimine. $[\mathrm{g}]\left[\mathrm{Mn}_{2}(\mathrm{CO})_{10}\right](5.0 \mathrm{~mol} \%)$ as catalyst.

\subsubsection{Scope of the Allylative $\mathrm{C}-\mathrm{H} / \mathrm{C}-\mathrm{F}$ Functionalizations}

Subsequently, an extensive study on the scope for the allylative and alkenylative manganese(I)catalyzed $\mathrm{C}-\mathrm{H} / \mathrm{C}-\mathrm{F}$ functionalization on 2-pyridylindoles 105 was carried out by $U$. Dhawa and $V$. Müller, including various heterocycles like pyrroles, pyrazoles, tryptophan-derivatives, and dipeptides. ${ }^{[176]}$ These results highlighted the broad applicability and excellent functional group tolerance of the optimized catalytic system.

Besides these significant advances, a strong focus has been directed at the generality of the catalytic reaction with respect to valuable ketimines 6 (Table 26). Initially, the change of methyl- to ethylsubstituted ketimine $\mathbf{6 b}$ altered the yield of the desired product $\mathbf{1 6 6 \mathbf { b }}$ only marginally (entries 1 and 2). Subsequently, different substituents in para-position to the directing group were tested $(\mathbf{6} \mathbf{c}-\mathbf{h}$, entries $3-8$ ), revealing no clear trend concerning the electronic properties of the functionalized ketimine $\mathbf{6}$, with overall moderate to good yields. Interestingly, the sterically congested naphthalene-derived ketimine $\mathbf{6} \mathbf{i}$ afforded the corresponding product 166i with moderate yield, but an excellent diastereoselectivity (entry 9). Notably, the meta-fluorine substituted ketimine $\mathbf{6 j}$ delivered the corresponding product via $\mathrm{C}-\mathrm{H}$ activation at the more acidic ortho-position next to fluorine, indicating a CMD-type $\mathrm{C}-\mathrm{H}$ activation pathway to be likely (entry 10). This assumption is in agreement with the finding that there is no clear preference for electron-rich substrates (vide supra) as it would be indicative for a BIES-type $\mathrm{C}-\mathrm{H}$ activation. In contrast, a BIES-type pathway has been previously shown as a potential $\mathrm{C}-\mathrm{H}$ activation mechanism in manganese-catalyzed $\mathrm{C}-\mathrm{H}$ functionalizations. ${ }^{[14 a}$, ${ }^{117 d]}$ Thereafter, a methylene-dioxy substituent of the ketimine $\mathbf{6 k}$ presumably acted as a secondary directing group to direct the manganese(I) catalyst by the Lewis basic oxygen to the sterically more congested position (entry 11). This site-selectivity is well-established for inter alia manganese catalysis. ${ }^{[106,108]}$ In summary, all ketimines 6 reacted with excellent diastereoselectivities and moderate to good yields. 
Table 26. Scope of the manganese(I)-catalyzed allylative $\mathrm{C}-\mathrm{H} / \mathrm{C}-\mathrm{F}$ functionalization of ketimines 6.

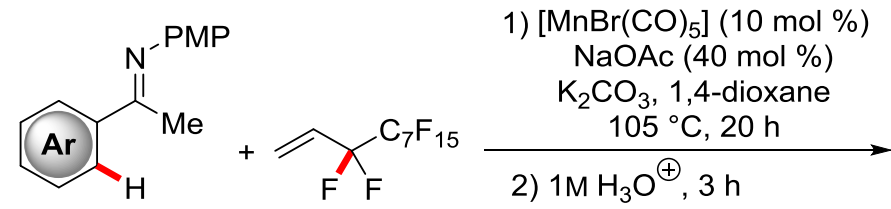

6

$127 a$

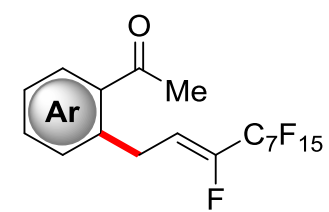

166

\begin{tabular}{|c|c|c|c|c|c|c|}
\hline entry & ketimine & 6 & product & 166 & $Z / E$ & yield $^{[\mathrm{a}]}[\%]$ \\
\hline 1 & & $6 \mathbf{a}$ & & $166 a$ & $97: 3$ & 72 \\
\hline 2 & & $6 b$ & & $166 b$ & $97: 3$ & 65 \\
\hline 3 & & $6 c$ & & $166 \mathrm{c}$ & $97: 3$ & 63 \\
\hline 4 & & 6d & & 166d & $97: 3$ & 51 \\
\hline 5 & & $6 e$ & & $166 e$ & $97: 3$ & 47 \\
\hline 6 & & $6 f$ & & $166 f$ & $97: 3$ & 69 \\
\hline 7 & & $6 g$ & & $166 \mathrm{~g}$ & $97: 3$ & 71 \\
\hline 8 & & $6 h$ & & $166 \mathrm{~h}$ & $97: 3$ & 74 \\
\hline
\end{tabular}


9<smiles>Cc1ccc2ccccc2c1</smiles>

6i<smiles>CC(=O)c1cc2ccccc2cc1CC=C(F)C(F)(F)C(F)(F)C(F)(F)F</smiles>

6j<smiles>CC(=O)c1cccc(F)c1C/C=C(\F)C(F)C(F)(F)C(F)(F)F</smiles><smiles>CC(=O)c1ccc2c(c1C/C=C(\F)C(F)F)OCO2</smiles>

$6 \mathbf{k}$<smiles>CC(=O)c1ccc2c(c1)OCO2</smiles>

166k 98:2

65

[a] Reaction conditions: $6(0.50 \mathrm{mmol}), \mathbf{1 2 7 a}(1.50 \mathrm{mmol}),\left[\mathrm{MnBr}(\mathrm{CO})_{5}\right](10 \mathrm{~mol} \%), \mathrm{NaOAc}(40 \mathrm{~mol} \%)$, $\mathrm{K}_{2} \mathrm{CO}_{3}(0.75 \mathrm{mmol}), 1,4$-dioxane $(0.5 \mathrm{~mL}, 1.0 \mathrm{M}), 105^{\circ} \mathrm{C}, 20 \mathrm{~h}$, isolated yield; $\mathrm{Z} / \mathrm{E}$ ratios determined by ${ }^{1} \mathrm{H}$ NMR spectroscopy.

Moreover, under the optimized reaction conditions, a removable ${ }^{[177]}$ phenylpyrazole $\mathbf{1 8}$ was successfully converted with high yield and good diastereoselectivity (Scheme 75).<smiles>CC(C)(C)c1ccc(-n2ccnc2)cc1</smiles>

$18 \mathrm{c}$<smiles>C=CC(F)(F)C(F)(F)F</smiles>

$127 a$

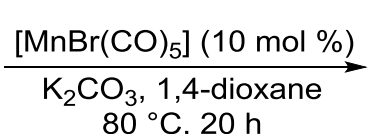
$80^{\circ} \mathrm{C}, 20 \mathrm{~h}$<smiles>CC(C)(C)c1ccc(-n2cccn2)c(CC=C(F)C(F)(F)F)c1</smiles>

161c: $77 \%(Z / E=87: 13)$

Scheme 75. Manganese(I)-catalyzed C-H/C-F functionalization of phenylpyrazoles 18.

\subsubsection{Mechanistic Studies}

\subsubsection{H/D-Exchange Experiments}

To delineate the manganese(I) catalysts mode of action, H/D-exchange experiments in either the presence of fully isotopically labeled $\mathrm{CD}_{3} \mathrm{OD}$ (Scheme 76a) or partly deuterated $\mathrm{CD}_{3} \mathrm{OH}$ (Scheme 76b) were conducted. By using $\mathrm{CD}_{3} \mathrm{OD}$, a minor deuteration in both $\mathrm{C} 3$-positions of the reisolated starting material 105a and the product 158aa was detected. This H/D-exchange presumably proceeded through an electrophilic activation pathway, which is less pronounced for manganese(I) catalysis than in the case of the more electrophilic cobalt(III) catalyst ( $c f$. Scheme 59). Considerably stronger H/D exchange was observed in $\mathrm{C} 2$-position of the reisolated starting material 105a, indicating a reversible $\mathrm{C}-\mathrm{H}$ bond activation step to be operative. In presence of the partly deuterated $\mathrm{CD}_{3} \mathrm{OH}$ (Scheme $76 \mathrm{~b}$ ), no deuterium incorporation in any position was found by means of ${ }^{1} \mathrm{H}-\mathrm{NMR}$ spectroscopy. This 
finding supports that the $\mathrm{H} / \mathrm{D}$-exchange by using $\mathrm{CD}_{3} \mathrm{OD}$ cannot originate from a radical mechanism, but rather from an organometallic $\mathrm{C}-\mathrm{H}$ activation by manganese catalysis.

a)<smiles></smiles>

$105 a$
$127 a$

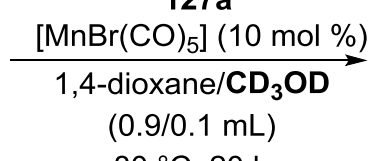
$80^{\circ} \mathrm{C}, 20 \mathrm{~h}$

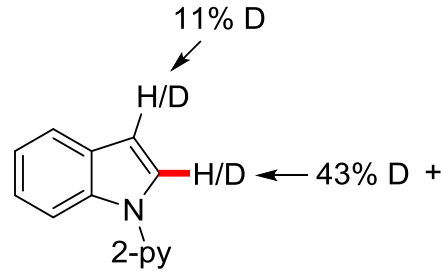

$[D]_{n}-105 a: 35 \%$

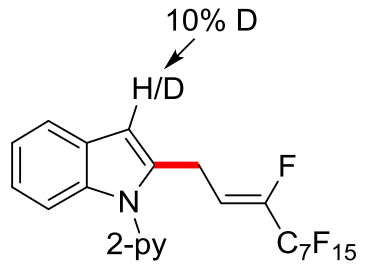

$[D]_{n}-158$ aa: $54 \%(Z / E=90: 10)$

b)<smiles>[Pb]n1ccc2ccccc21</smiles>
$80^{\circ} \mathrm{C}, 20 \mathrm{~h}$

$105 a$

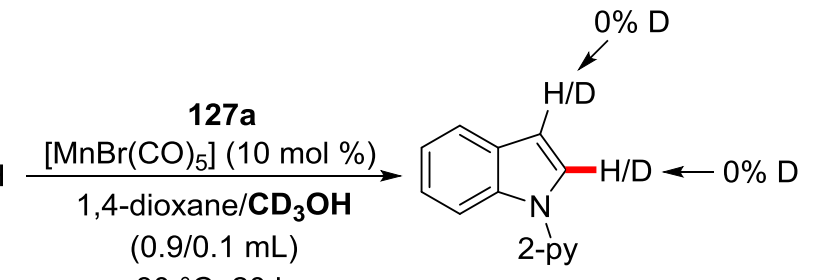

105a: $37 \%$<smiles>O=C(O)c1c(CC=CC(F)C(F)(F)F)n([18O])c2ccccc12</smiles>

158aa: $56 \%(Z / E=90: 10)$

Scheme 76. H/D-exchange experiments with a) $\mathrm{CD}_{3} \mathrm{OD}$ and b) $\mathrm{CD}_{3} \mathrm{OH}$.

\subsubsection{KIE Studies}

Subsequently, a KIE experiment by comparison of the initial rates of independent reactions with substrate 105a and its deuterated analogue $[D]_{1}-\mathbf{1 0 5 a}$ was conducted (Scheme 77 and Figure 27). The experiments revealed a $\mathrm{KIE}$ of $k_{\mathrm{H}} / k_{\mathrm{D}}=1.1$, showing that the $\mathrm{C}-\mathrm{H}$ activation event is facile and not turnover-limiting.

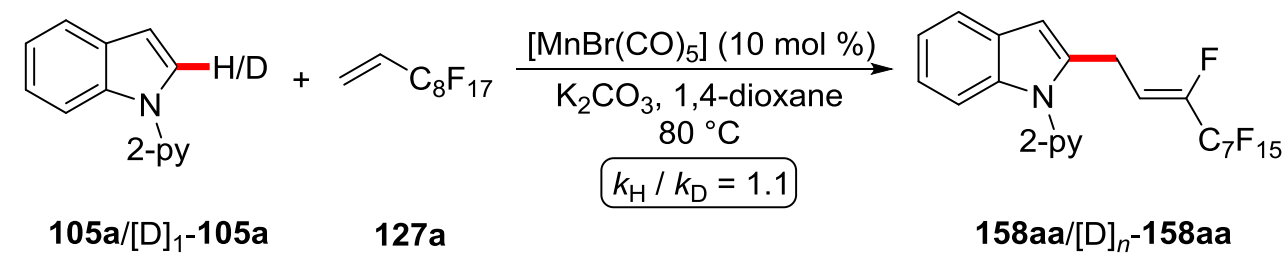

Scheme 77. KIE-experiment for the manganese(I)-catalyzed C-H/C-F functionalization. 


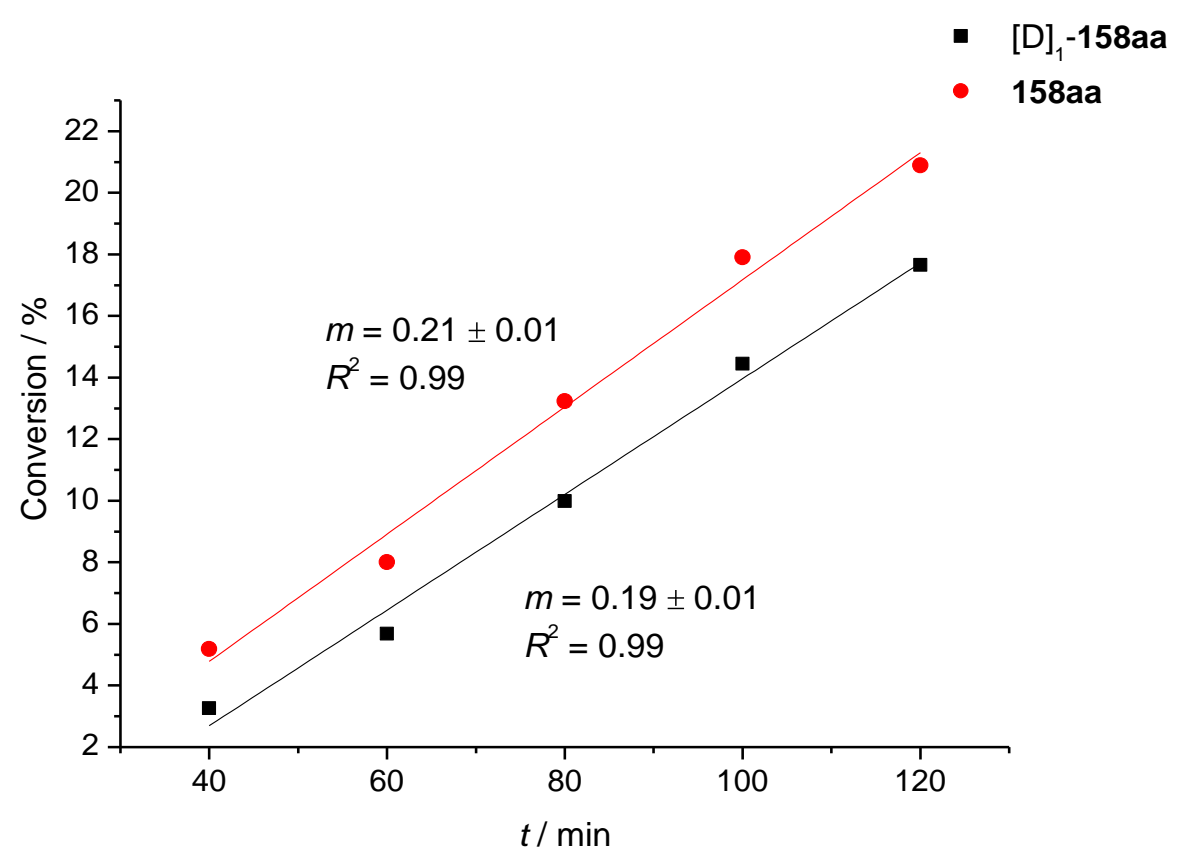

Figure 27. Conversion-time curves for the KIE-experiment.

\subsubsection{Experiments with Cyclometalated Complexes}

Furthermore, it was studied whether the desired product 158aa can be provided by reaction of the perfluoroalkylalkene 127a with the well-defined manganacycle 167 (Scheme 78). The reaction proved viable in an almost quantitative fashion with the same diastereoselectivity as for the catalytic transformation. Based on this result, a manganese(I)-catalyzed $\mathrm{C}-\mathrm{H}$ activation pathway via the formation of manganacycle $\mathbf{1 6 7}$ can be assumed.

Moreover, the importance of the carbonate-base for subsequent elementary steps after the $\mathrm{C}-\mathrm{H}$ activation was studied by performing the same reaction in the absence of base. The corresponding reaction displayed a slight decrease in yield, but the same diastereoselectivity was observed. This observation possibly indicates that the $\mathrm{C}-\mathrm{H}$ activation step is assisted by the carbonate base, which is in full agreement with the poor reactivity of the catalytic reaction by omitting the base ( $c f$. Table 24 entry 8). However, these findings cannot exclude a dual role of the carbonate base: On the one hand it could facilitate the $\mathrm{C}-\mathrm{H}$ activation step, while on the other hand it could participate in a reaction to regenerate the active catalytic species and stabilize the fluoride anion by precipitation of potassium fluoride. 
a)

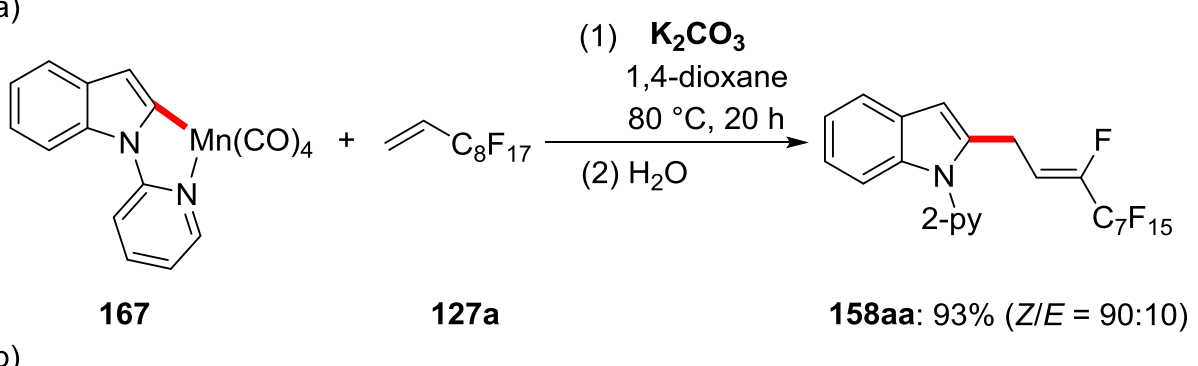

b)

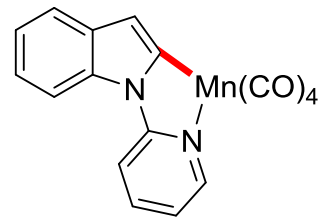

167

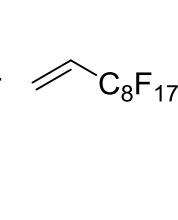

$127 a$ (1)1,4-dioxane $80^{\circ} \mathrm{C}, 20 \mathrm{~h}$

(2) $\mathrm{H}_{2} \mathrm{O}$

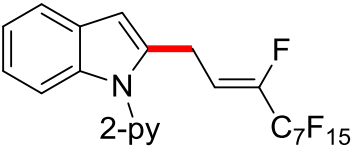

158aa: $82 \%(Z / E=90: 10)$

Scheme 78. Stoichiometric reaction of manganacycle 167 in a) the presence and b) absence of potassium carbonate.

In addition, a catalytic reaction with manganacycle $\mathbf{1 6 7}$ as the catalyst afforded the desired product 158aa in good yield, which indicates that the manganese(I) complex $\mathbf{1 6 7}$ is possibly an intermediate in the catalytic cycle (Scheme 79).

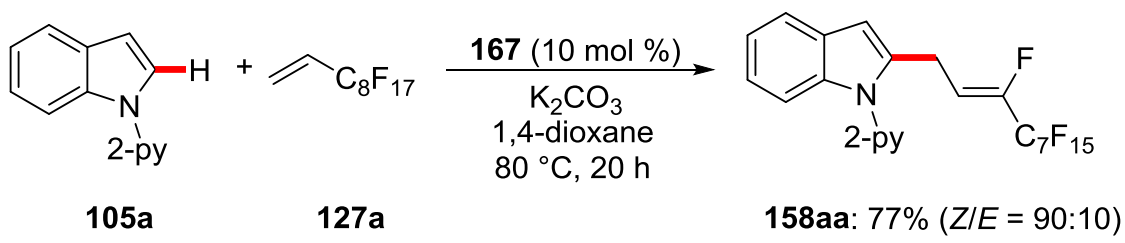

Scheme 79. Catalytic reaction with manganacycle 167 as catalyst.

\subsubsection{Proposed Catalytic Cycle}

In conclusion, the allylative manganese(I)-catalyzed $\mathrm{C}-\mathrm{H} / \mathrm{C}-\mathrm{F}$ functionalization of indoles 105 most likely proceeds via a reversible and facile BIES-type mechanistic pathway, which is supported by the results obtained from the H/D-exchange experiments (Scheme 76) and the KIE study (Scheme 77). The active catalyst 168 is proposed to be a manganese(I) carbonate complex, in which the carbonate assists the deprotonation step in an intramolecular manner (Scheme 80). Likewise, DFT studies by $M$. Bursch and S. Grimme support a BIES-type C-H activation mechanism to proceed and notably to be favored in an intramolecular fashion by carbonate as internal base. ${ }^{[176]}$ These studies contrast previous theoretical results by Maseras and Echavarran, who pointed out that the carbonate-assisted palladiumcatalyzed $\mathrm{C}-\mathrm{H}$ activation proceeds favorably via an intermolecular deprotonation. ${ }^{[178]}$

Thereafter, the manganacycle 169 is coordinated by the perfluoroalkylalkene 127a in a rapid and reversible process. Subsequently, a migratory insertion into the manganese-carbon bond forms intermediate 171. According to the H/D-exchange study, which disclosed no deuterium incorporation 
in any position of the perfluoroalkylalkene side-chain, the migratory insertion is presumably an irreversible step (Scheme 76). Additionally, DFT-calculations by M. Bursch showed that the subsequent $\beta$-fluoride elimination step forms the preferred $Z$-configured product 158aa and regenerate the active catalyst by the initially formed bicarbonate. ${ }^{[176]}$ Future mechanistic investigations, including competition experiments, should reveal the $\mathrm{C}-\mathrm{H}$ activation mechanism in the case of $e$.g. less electronrich substrates like ketimines $\mathbf{6}$. Interestingly, during the scope of ketimines $\mathbf{6}$ a preference for particularly acidic $\mathrm{C}-\mathrm{H}$ bonds was found (Table 26 , entry 10). These findings indicate that there might be rather a CMD- instead of BIES-type C-H activation operative for ketimines 6 .

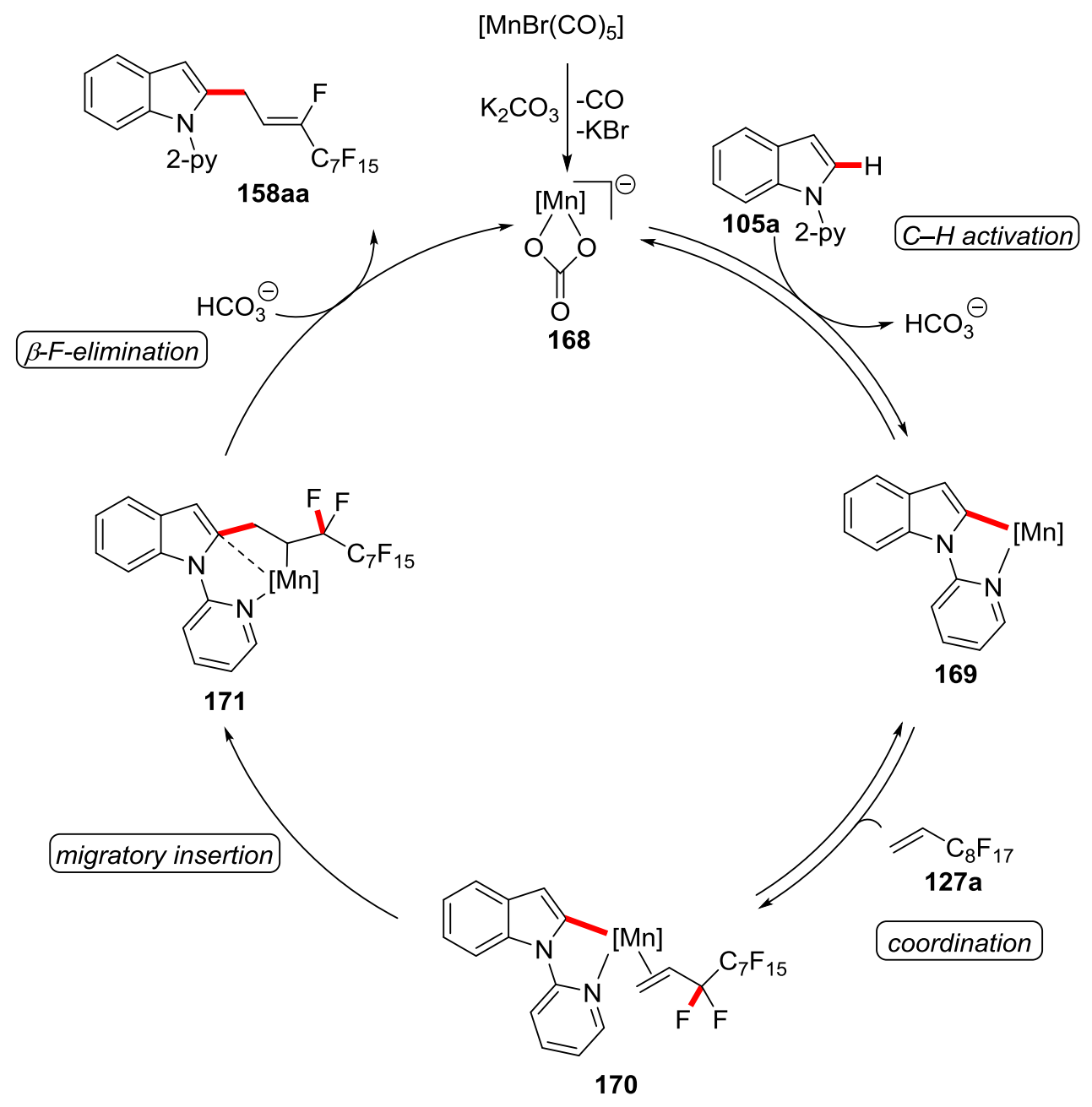

Scheme 80. Proposed catalytic cycle of the allylative manganese(I)-catalyzed C-H/C-F functionalization. $[\mathrm{Mn}]=\mathrm{Mn}^{\mathrm{I}}(\mathrm{CO})_{3}$. 


\section{Summary and Outlook}

The development of highly step- and atom-economical $\mathrm{C}-\mathrm{H}$ functionalizations by inexpensive and earth-abundant metals streamlines organic synthesis and offers new tools for inter alia pharmaceutical, agrochemical chemistry, and material sciences. In this thesis, several new synthetic methods have been devised that reveal the remarkable efficiency and synthetic diversity of ruthenium(II), cobalt(III), and manganese(I) complexes in $\mathrm{C}-\mathrm{H}$ activation catalysis.

In the first project, well-defined single component phosphinous acid (PA) complexes of ruthenium(II) have been employed as powerful catalyst for the $\mathrm{C}-\mathrm{H}$ arylation of valuable and omnipresent alkene motifs, including challenging aryl chlorides $\mathbf{3}$ as arylating reagents (Scheme 81). For the first time, detailed kinetic studies, such as kinetic orders and Hammett plot analyses, revealed the mechanistic nature of the turnover-limiting step and gave hints on a SET-type $\mathrm{C}-\mathrm{X}$ bond activation process. ${ }^{[123]}$

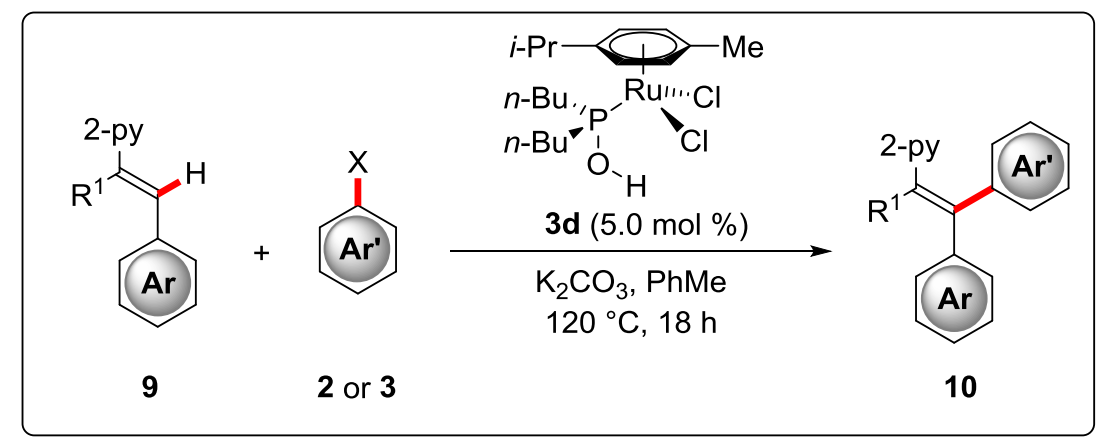

Scheme 81. Ruthenium(II)-PA-catalyzed C-H arylation of alkenes 9.

These findings might give rise to the design of new tailor-made catalysts with enhanced efficiencies by adjusting the electronic features or attractive London dispersion and repulsive steric interactions on the easily modifiable phosphinous acid ligand. In this context, insightful studies concerning steric effects on the reactivity might be conducted, e.g. by comparing the buried volume of several PA ligands, which is a concept that has been developed for inter alia NHC and phosphine ligands by Cavallo. ${ }^{[179]}$

In the next project, a cobalt(III)-catalyzed $\mathrm{C}-\mathrm{H} / \mathrm{C}-\mathrm{C}$ functionalization of valuable heteroarenes and arenes by using vinylcyclopropanes $\mathbf{1 2 5}$ was achieved (Scheme 82). ${ }^{[17 \mathrm{~b}]}$ Under very mild reaction conditions, the thermodynamically less stable $Z$-configurated diastereomer was obtained in excellent yields as well as very good positional- and diastereoselectivities. In striking contrast to the analogous $E$-selective rhodium ${ }^{[117 c]}$ and manganes ${ }^{[117 \mathrm{~d}]}$ catalyses, this reaction appeared to be stereocomplementary, which bears great potential for new uniquely selective synthetic methods based on cobalt(III) catalysis. 


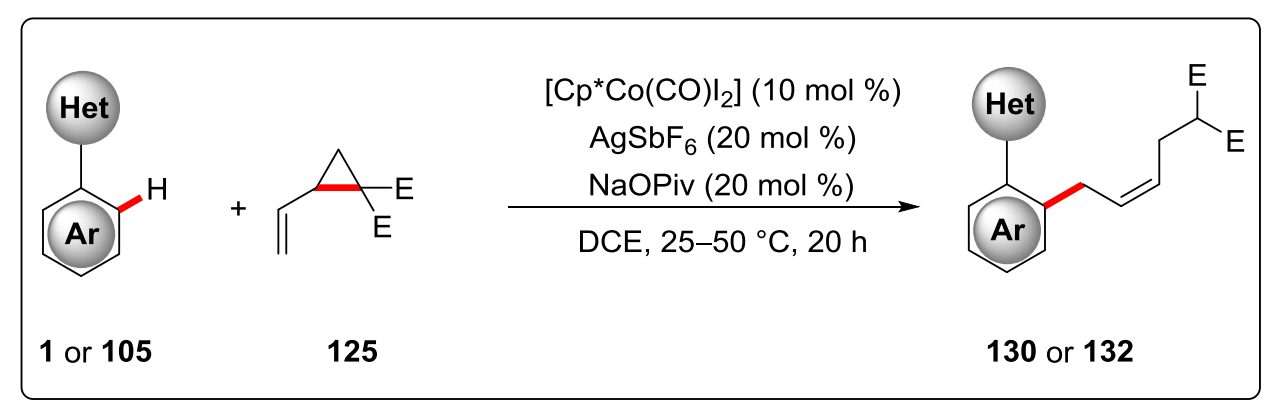

Scheme 82. Cobalt(III)-catalyzed C-H/C-C functionalization.

In the third project, a new concept for Markovnikov/anti-Markovnikov regiocontrol in $\mathrm{C}-\mathrm{H}$ hydroarylations was devised (Scheme 83). ${ }^{[161]}$ Here, the control of regioselectivity was accomplished by a tunable switch of the regiodetermining proto-demetalation step. Within this reaction, an expedient substrate scope was highlighted with excellent and fully complementary branched/linear selectivities, notably using unactivated alkenes. Likewise, extensive mechanistic studies, including detailed kinetic experiments, revealed a switch in the $\mathrm{C}-\mathrm{H}$ activation manifold from a linear-selective LLHT to a branched-selective BIES-type pathway to be stereodiscriminating. Herein, inter alia a change from zeroth-order dependence on the concentration of indoles $\mathbf{1 0 5}$ for the branched- to a first orderdependence for the linear-selective regime supported the mechanistic hypothesis.

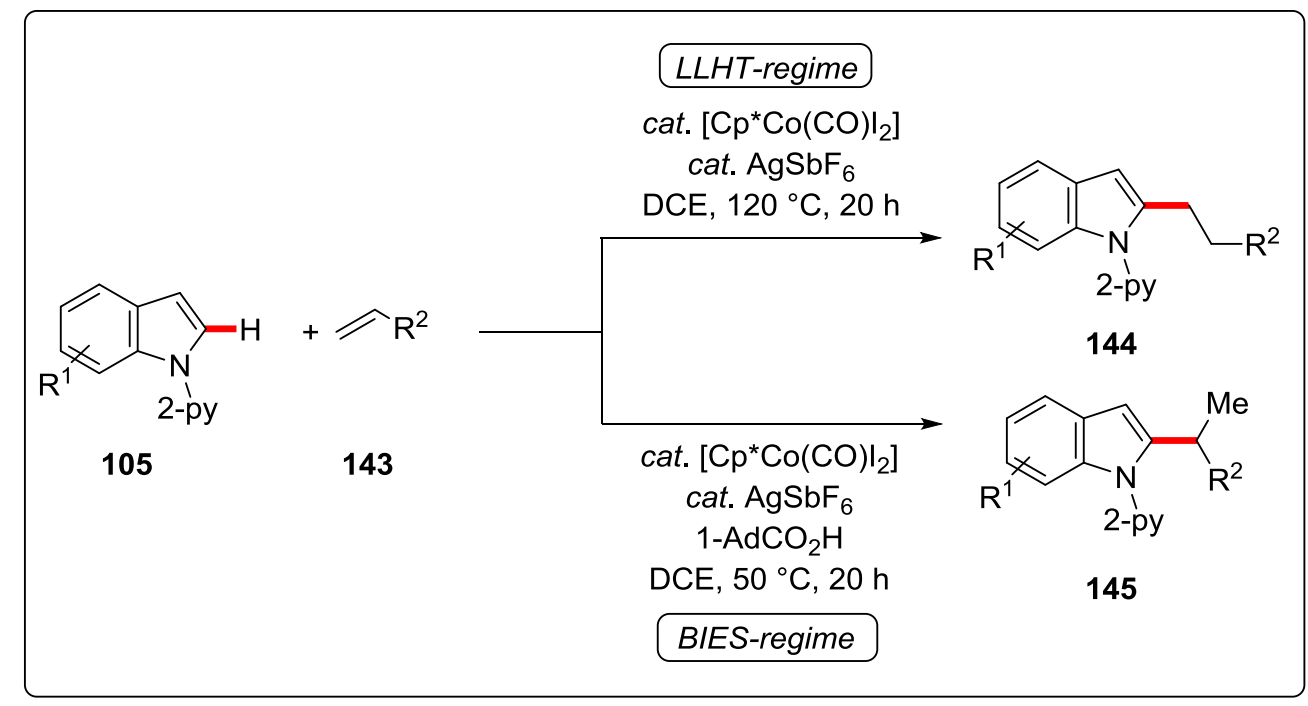

Scheme 83. Cobalt(III)-catalyzed branched/linear-selective C-H hydroarylation.

Based on this mechanistic insight, an enantioselective cobalt(III)-catalyzed hydroarylation protocol was developed by employing monoprotected amino acids (MPAAs). However, yields and enantioselectivities are overall rather moderate, which gives rise to the future development of more reactive and selective catalytic systems $e$.g. by using oligopeptides as additives.

Furthermore, cobalt(III)-catalyzed allylative and alkenylative $\mathrm{C}-\mathrm{H} / \mathrm{C}-\mathrm{F}$ functionalizations were developed (Scheme 84). ${ }^{[174]}$ A diverse set of perfluoroalkylalkenes 127 as well as valuable 1,1difluoroalkenes 128 were successfully converted at $25{ }^{\circ} \mathrm{C}$ with ample scope and excellent functional 
group tolerance. The products displayed good to excellent diastereoselectivities and the catalyst loading could be reduced to $0.25 \mathrm{~mol} \%$ without loss of reactivity.

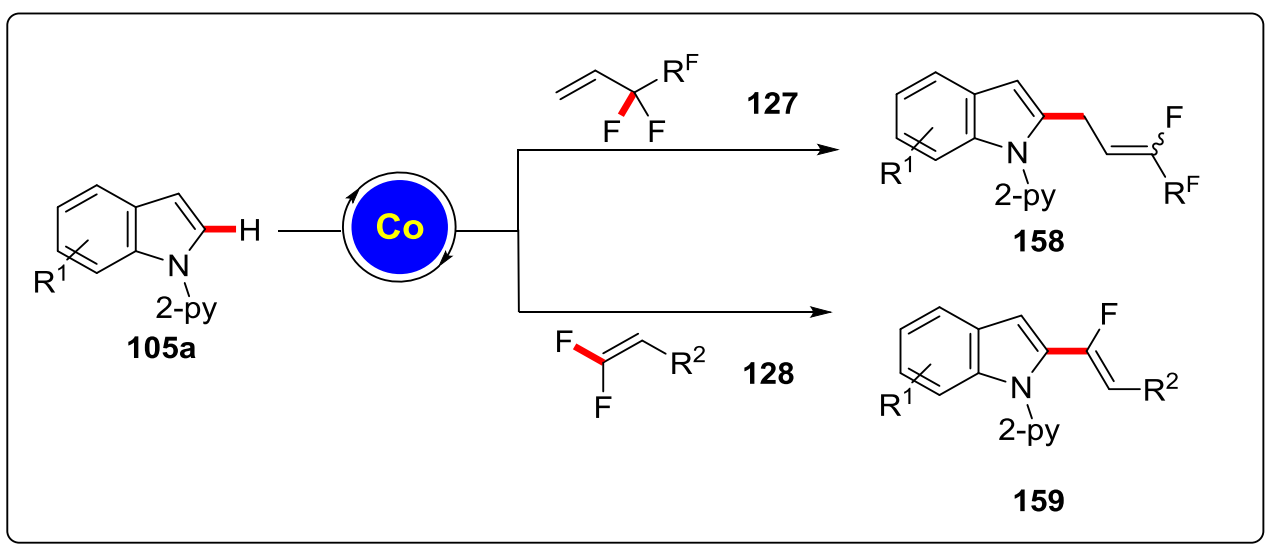

Scheme 84. Cobalt(III)-catalyzed allylative and alkenylative C-H/C-F functionalizations.

Finally, a manganese(I) complex, which obviates the use of costly $\mathrm{Cp}^{*}$ ligands, was shown to be a competent catalyst in allylative and alkenylative $\mathrm{C}-\mathrm{H} / \mathrm{C}-\mathrm{F}$ functionalizations, with overall excellent and improved diastereoselectivities as compared to the analagous cobalt(III) catalysis (Scheme 85). ${ }^{[176]}$ The reaction displayed a broad applicability, including the functionalization of valuable and easily transformable ketimines 6. Regarding a future prospect, the development of e.g. manganese(I)carboxylate complexes might be of great importance for studying the selectivity control in this and related reactions.

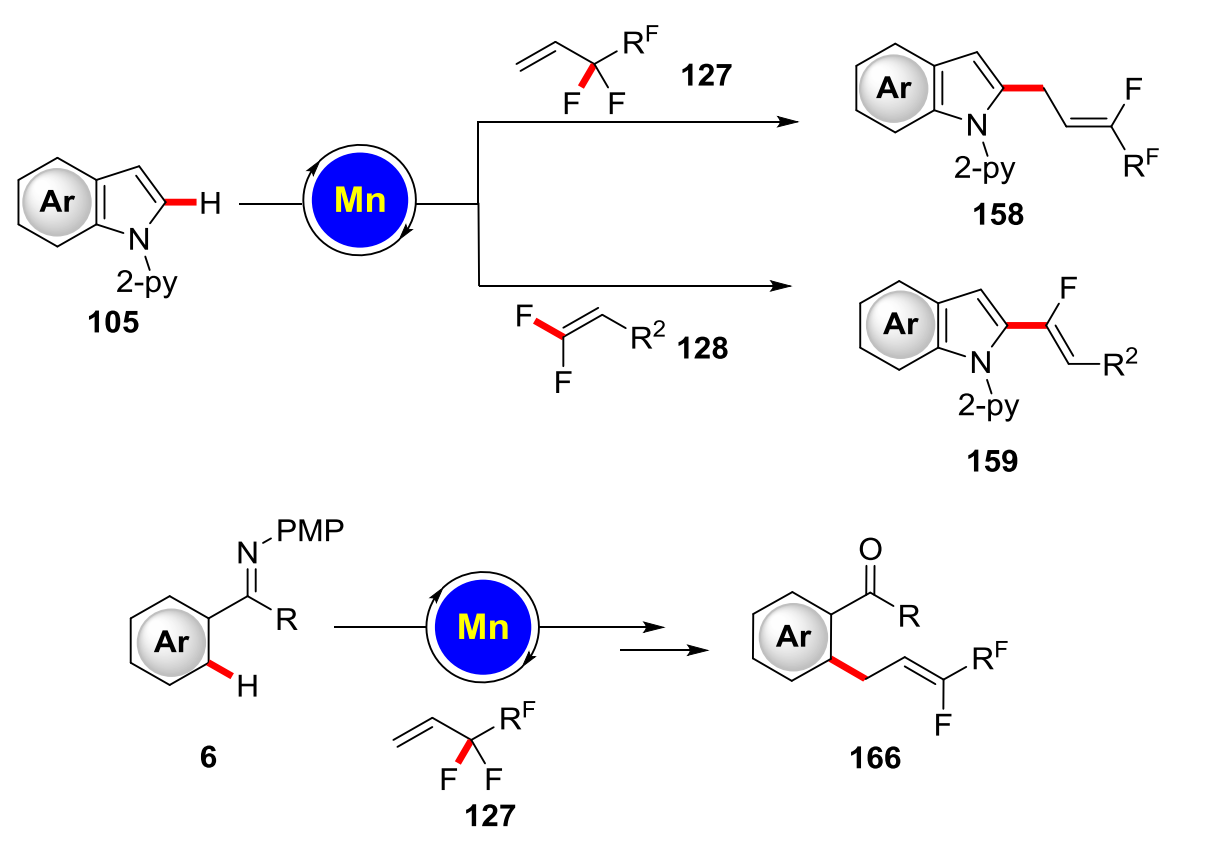

Scheme 85. Manganese(I)-catalyzed allylative and alkenylative C-H/C-F functionalizations. 


\section{Experimental Section}

\subsection{General Remarks}

All reactions involving moisture- or air-sensitive reagents or products were performed under an atmosphere of nitrogen using pre-dried glassware and standard Schlenk techniques. If not otherwise mentioned, yields refer to isolated compounds, estimated to be $>95 \%$ pure as determined by ${ }^{1} \mathrm{H}-\mathrm{NMR}$.

\section{Vacuum}

The following uncorrected pressures were measured on the used vacuum pump: membrane pump vacuum (MPV): 0.5 mbar, oil pump vacuum (OPV): 0.1 mbar.

\section{Melting Points}

Melting points were measured using a Stuart ${ }^{\circledR}$ Melting Point Apparatus SMP3 from BARLOWORLD SCIENTIFIC. Values are uncorrected.

\section{Column and HPLC-Chromatography}

Analytical thin layer chromatography (TLC) was performed on MERCK, silica gel 60 F254 aluminum plates. Detection was performed under UV light at $254 \mathrm{~nm}$ or by treatment with a $\mathrm{KMnO}_{4}$ solution. Chromatographic purification of products was accomplished by flash column chromatography on MERCK silica gel, grade 60 (0.040-0.063 mm and 0.063-0.200 mm, 70-230 mesh ASTM). Analytical High-Performance-Liquid-Chromatography (HPLC) for determination of enantiomeric excess was conducted on an AGILENT 1260 Infinity system with Chiralpak $I C-3^{\circledR}$ and $I F-3^{\circledR}$ from DAICEL (both $3.0 \mu \mathrm{m}$ particle size; $\varnothing=4.6 \mathrm{~mm}$ and length $=250 \mathrm{~mm}$ ) as columns.

\section{Gel permeation chromatography (GPC)}

GPC purifications were performed on a JAI system (JAI-LC-9260 II NEXT) equipped with two sequential columns (JAIGEL-2HR, gradient rate: 5.000; JAIGEL-2.5HR, gradient rate: 20.000; internal diameter $=20 \mathrm{~mm}$; length $=600 \mathrm{~mm}$; Flush rate $=10.0 \mathrm{~mL} / \mathrm{min}$ and chloroform (HPLC-quality with $0.6 \%$ ethanol as stabilizer) was used as the eluent.

\section{Gas Chromatography}

Gas chromatography or coupled gas chromatography-mass spectrometry was performed on a 5890 Series II GC system with mass detector HP 5972 from HEWLETT-PACKARD, a 7890 GC system with/without mass detector $5975 C$ (Triplex-Axis-Detector) or a $7890 B$ BC system coupled with a 5977 mass detector, both systems from AGILENT TECHNOLOGIES. 


\section{Nuclear Magnetic Resonance Spectroscopy}

Nuclear magnetic resonance (NMR) spectra were recorded on VARIAN Inova 500, 600, VARIAN Mercury 300, VX 300, VARIAN Avance 300, VARIAN VNMRS 300 and BRUKER Avance III 300, 400 and $H D 500$ spectrometers. All chemical shifts are given as $\delta$-values in ppm relative to the residual proton peak of the deuterated solvent or its carbon atom, respectively. ${ }^{1} \mathrm{H}$ - and ${ }^{13} \mathrm{C}-\mathrm{NMR}$ spectra were referenced using the residual proton or solvent carbon peak (see table), respectively. ${ }^{13} \mathrm{C}$ - and ${ }^{19} \mathrm{~F}$ NMR were measured as proton-decoupled spectra.

\begin{tabular}{c|cc} 
& ${ }^{1} \mathrm{H}-\mathrm{NMR}$ & ${ }^{13} \mathrm{C}-\mathrm{NMR}$ \\
\hline $\mathrm{CDCl}_{3}$ & 7.26 & 77.16 \\
{$[\mathrm{D}]_{6}-\mathrm{DMSO}$} & 2.50 & 39.52
\end{tabular}

The detected resonance-multiplicities were described by the following abbreviations: s (singlet), $d$ (doublet), $\mathrm{t}$ (triplet), q (quartet), hept (heptet), m (multiplet) or analogous representations. The coupling constants $J$ are reported in Hertz $(\mathrm{Hz})$. Analysis of the recorded spectra was carried out with MestReNova 10 software.

\section{Infrared Spectroscopy}

Infrared spectra were recorded at a BRUKER Alpha-P ATR FT-IR spectrometer. Liquid samples were measured as a film, solid samples neat. The analysis of the spectra was carried out using the software from BRUKER OPUS 6. The absorption is given in wave numbers $\left(\mathrm{cm}^{-1}\right)$ and the spectra were recorded in the range of 4000-400 $\mathrm{cm}^{-1}$. In situ-IR studies were performed on METTLER TOLEDO ReactIRTM 15 with an $i C I R 4.3$ software.

\section{Mass Spectrometry}

Electron ionization (EI) and EI high resolution mass spectra (HR-MS) were measured on a time-offlight mass spectrometer AccuTOF from JOEL. Electrospray ionization (ESI) mass spectra were recorded on an Ion-Trap mass spectrometer $L C Q$ from FINNIGAN, a quadrupole time-of-flight maXis from BRUKER DALTONIC or on a time-of-flight mass spectrometer microTOF from BRUKER DALTONIC. ESI-HRMS spectra were recorded on a BRUKER Apex IV or a BRUKER Daltonic 7T, fourier transform ion cyclotron resonance (FTICR) mass spectrometer. The ratios of mass to charge $(\mathrm{m} / \mathrm{z})$ are indicated and intensities relative to the base peak $(I=100)$ are written in parentheses. 


\section{Solvents}

All solvents for reactions involving moisture-sensitive reagents were dried, distilled and stored under inert atmosphere ( $\mathrm{Ar}$ or $\mathrm{N}_{2}$ ) according to the following standard procedures:

1,2-Dichloroethane was dried over $\mathrm{CaH}_{2}$ for $8 \mathrm{~h}$, degassed and distilled under reduced pressure.

Dichloromethane was purified using a solvent purification system (SPS-800) from M. BRAUN.

Diethyl ether was purified using a solvent purification system (SPS-800) from M. BRAUN.

1,4-Dioxane was distilled from sodium benzophenone ketyl.

1,1,1,3,3,3-Hexafluoropropan-2-ol was distilled from $3 \AA$ molecular sieves.

Methanol was stirred over magnesium turnings at $65^{\circ} \mathrm{C}$ for $3 \mathrm{~h}$ prior to distillation from $\mathrm{Mg}(\mathrm{OMe})_{2}$.

$N$-Methyl-2-pyrrolidone was stirred over $\mathrm{CaH}_{2}$ at $200{ }^{\circ} \mathrm{C}$ for $4 \mathrm{~h}$ and distilled under reduced pressure.

Tetrahydrofuran was purified using a solvent purification system (SPS-800) from M. BRAUN.

Toluene was purified using a solvent purification system (SPS-800) from M. BRAUN.

2,2,2-Trifluoroethanol was stirred over $\mathrm{CaSO}_{4}$ and distilled under reduced pressure.

Water was degassed by repeated Freeze-Pump-Thaw degassing procedure.

\section{Reagents}

Chemicals obtained from commercial sources with purity higher than $95 \%$ were used without further purification. The following literature-known compounds were synthesized based on previously described methods:

(E)-2-Styrylpyridines $\mathbf{9},{ }^{[180]}$ SPOs, ${ }^{[178]}$ ruthenium(II)-PA complexes, ${ }^{[123]}$ ketimines $\mathbf{6},{ }^{[36 c,}{ }^{181]}$ indoles $\mathbf{1 0 5}^{[182]}$ and 2-phenylpyridines $\mathbf{1}^{[183]}$

The following chemicals were kindly provided by the persons named below:

Uttam Dhawa: 4-methoxy-1-(pyridin-2-yl)-1 $H$-indole (105h) and 5-methoxy-(pyridin-2-yl)-1 $H$-indole (105b).

Weiping Liu: (E)-1-(4-methoxyphenyl)- $N$-(4-methoxyphenyl)ethan-1-imine $\quad(\mathbf{6 c}), \quad(E)-1-(4-$ fluorophenyl)- $N$-(4-methoxyphenyl)ethan-1-imine $\quad(\mathbf{6 f}), \quad$ (E)-1-(4-bromophenyl)- $N$-(4methoxyphenyl)ethan-1-imine (6g) and (E)-1-(4-iodophenyl)- $N$-(4-methoxy-phenyl)ethan-1-imine (6h).

Tjark Meyer: $\left[\mathrm{RuCl}_{2}(p\right.$-cymene $\left.)\left(\mathrm{Ph}_{2} \mathrm{POH}\right)\right](\mathbf{1 2 4 a}),\left[\mathrm{RuCl}_{2}(p\right.$-cymene $\left.)\left\{\left(p-\mathrm{FC}_{6} \mathrm{H}_{4}\right)_{2} \mathrm{POH}\right\}\right](\mathbf{1 2 4 b})$.

Valentin Müller: 5-fluoro-1-(pyridin-2-yl)-1H-indole (105m) and 5-bromo-1-(pyridin-2-yl)-1 $H$-indole (105c).

Karsten Rauch: $\left[\mathrm{RuCl}_{2}(p \text {-cymene })\right]_{2}$ and $\left[\mathrm{Ru}\left(\mathrm{O}_{2} \mathrm{CMes}\right)_{2}(p\right.$-cymene $\left.)\right](\mathbf{2 0})$.

Svenja Warratz: $\left[\mathrm{RuCl}_{2}(p\right.$-cymene $\left.)(t-\mathrm{BuPhPOH})\right](\mathbf{1 2 4 c})$ and $\left[\mathrm{RuCl}_{2}(p\right.$-cymene $\left.)\left(o-\mathrm{Tol}_{2} \mathrm{POH}\right)\right](\mathbf{1 2 4 g})$. 


\subsubsection{General Procedure A: Ruthenium(II)-PA-Catalyzed C-H Arylation}

A suspension of (E)-2-styrylpyridine 9 (0.50 mmol, 1.00 equiv), aryl bromide 2 (0.75 mmol, 1.50 equiv), $\left[\mathrm{RuCl}_{2}(p\right.$-cymene $\left.)\left(n-\mathrm{Bu}_{2} \mathrm{POH}\right)\right](\mathbf{1 2 4 \mathrm { e }})(11.7 \mathrm{mg}, 25.0 \mu \mathrm{mol}, 5.00 \mathrm{~mol} \%)$ and $\mathrm{K}_{2} \mathrm{CO}_{3}$ (138 mg, $1.00 \mathrm{mmol}, 2.00$ equiv) in PhMe $(2.00 \mathrm{~mL}, 0.25 \mathrm{M})$ was stirred at $120{ }^{\circ} \mathrm{C}$ for $18 \mathrm{~h}$. At ambient temperature, the mixture was diluted with EtOAc $(5.0 \mathrm{~mL})$, filtered over Celite and the solvents were removed in vacuo. Isolation by column chromatography on silica gel ( $n$-hexane/EtOAc) yielded the corresponding products $\mathbf{1 0}$.

\subsubsection{General Procedure B: Cobalt(III)-Catalyzed C-H/C-C Functionalization}

A suspension of indole 105 ( $0.50 \mathrm{mmol}, 1.00$ equiv), vinylcyclopropanes 125 ( $0.60 \mathrm{mmol}, 1.20$ equiv), [Cp*Co(CO) $\left.\mathrm{I}_{2}\right](23.8 \mathrm{mg}, 50.0 \mu \mathrm{mol}, 10.0 \mathrm{~mol} \%), \mathrm{AgSbF}_{6}(34.4 \mathrm{mg}, 100 \mu \mathrm{mol}, 20.0 \mathrm{~mol} \%)$ and NaOPiv (12.4 mg, $100 \mu \mathrm{mol}, 20.0 \mathrm{~mol} \%)$ in DCE $(1.00 \mathrm{~mL}, 0.50 \mathrm{M})$ was stirred at $50{ }^{\circ} \mathrm{C}$ for $20 \mathrm{~h}$. At ambient temperature, the solvent was removed in vacuo and the remaining residue was purified by column chromatography on silica gel ( $n$-hexane/EtOAc) to yield the desired products $\mathbf{1 3 0}$.

\subsubsection{General Procedure C: Rhodium(III)-Catalyzed C-H/C-C Functionalization}

A suspension of indole 105 ( $0.25 \mathrm{mmol}, 1.00$ equiv), vinylcyclopropane 125 ( $0.30 \mathrm{mmol}, 1.20$ equiv), $\left[\mathrm{Cp} * \mathrm{Rh}\left(\mathrm{CH}_{3} \mathrm{CN}\right)_{3}\right]\left(\mathrm{SbF}_{6}\right)_{2}(19.7 \mathrm{mg}, 25.0 \mu \mathrm{mol}, 10.0 \mathrm{~mol} \%)$ and NaOPiv $(6.20 \mathrm{mg}, 50.0 \mu \mathrm{mol}$, $20.0 \mathrm{~mol} \%)$ in DCE $(1.00 \mathrm{~mL}, 0.25 \mathrm{M})$ was stirred at $50{ }^{\circ} \mathrm{C}$ for $20 \mathrm{~h}$. At ambient temperature, the solvent was removed in vacuo and the remaining residue was purified by column chromatography on silica gel ( $n$-hexane/EtOAc) to afford the desired products $\mathbf{1 3 0}$.

\subsubsection{General Procedure D: Cobalt(III)-Catalyzed Linear-selective C-H Alkylation}

A suspension of indole 105 ( $0.50 \mathrm{mmol}, 1.00$ equiv), alkene 143 (1.50 mmol, 3.00 equiv), $\left[\mathrm{Cp} * \mathrm{Co}(\mathrm{CO}) \mathrm{I}_{2}\right](23.8 \mathrm{mg}, 50.0 \mu \mathrm{mol}, 10.0 \mathrm{~mol} \%)$ and $\mathrm{AgSbF}_{6}(34.4 \mathrm{mg}, 100 \mu \mathrm{mol}, 20.0 \mathrm{~mol} \%)$ in DCE $(1.00 \mathrm{~mL}, 0.50 \mathrm{M})$ was stirred at $120{ }^{\circ} \mathrm{C}$ for $20 \mathrm{~h}$. At ambient temperature, the reaction mixture was diluted with EtOAc $(5.0 \mathrm{~mL})$ and the solvents were removed in vacuo. The remaining residue was purified by column chromatography on silica gel ( $n$-hexane/EtOAc) to afford the desired products 144 .

\subsubsection{General Procedure E: Cobalt(III)-Catalyzed Branched-Selective C-H Alkylation}

A suspension of indole 105 ( $0.50 \mathrm{mmol}, 1.00$ equiv), alkene 143 (1.50 mmol, 3.00 equiv), [Cp*Co(CO) $\left.\mathrm{I}_{2}\right](23.8 \mathrm{mg}, 50.0 \mu \mathrm{mol}, 10.0 \mathrm{~mol} \%), \mathrm{AgSbF}_{6}(34.4 \mathrm{mg}, 100 \mu \mathrm{mol}, 20.0 \mathrm{~mol} \%)$ and 1$\mathrm{AdCO}_{2} \mathrm{H}\left(90.1 \mathrm{mg}, 0.50 \mathrm{mmol}, 1.00\right.$ equiv) in DCE $(0.50 \mathrm{~mL}, 1.00 \mathrm{M})$ was stirred at $50{ }^{\circ} \mathrm{C}$ for $20 \mathrm{~h}$. At ambient temperature, the reaction mixture was diluted with EtOAc $(5.0 \mathrm{~mL})$ and the solvents were removed in vасио. The remaining residue was purified by column chromatography on silica gel ( $n$ hexane/EtOAc) to yield the desired products $\mathbf{1 4 5}$. 


\subsubsection{General Procedure F: Cobalt(III)-Catalyzed Allylative C-H/C-F Functionalization}

A suspension of indole 105 (0.50 mmol, 1.00 equiv), perfluoroalkylalkene 127 (0.60 mmol, 1.20 equiv), [Cp* $\mathrm{Co}(\mathrm{CO}) \mathrm{I}_{2}$ ] (6.00 mg, $\left.12.5 \mu \mathrm{mol}, 2.5 \mathrm{~mol} \%\right)$ and $\mathrm{K}_{2} \mathrm{CO}_{3}(69.1 \mathrm{mg}, 0.50 \mathrm{mmol}$, 1.00 equiv) in TFE $(0.50 \mathrm{~mL}, 1.00 \mathrm{M})$ was stirred at $25{ }^{\circ} \mathrm{C}$ for $20 \mathrm{~h}$. The mixture was diluted with EtOAc $(5.0 \mathrm{~mL})$, filtered over Celite and the solvents were removed in vacuo. Isolation by column chromatography on silica gel ( $n$-hexane/EtOAc) afforded the desired products 158.

\subsubsection{General Procedure G: Cobalt(III)-Catalyzed Alkenylative C-H/C-F Functionalization}

A suspension of indole 105 ( $0.50 \mathrm{mmol}, 1.00$ equiv), 1,1-difluoroalkene 128 ( $0.75 \mathrm{mmol}, 1.50$ equiv), $\left[\mathrm{Cp} * \mathrm{Co}(\mathrm{CO}) \mathrm{I}_{2}\right](6.00 \mathrm{mg}, 12.5 \mu \mathrm{mol}, 2.5 \mathrm{~mol} \%)$ and $\mathrm{K}_{2} \mathrm{CO}_{3}(69.1 \mathrm{mg}, 0.50 \mathrm{mmol}, 1.00$ equiv) in TFE $(0.50 \mathrm{~mL}, 1.00 \mathrm{M})$ was stirred at $25^{\circ} \mathrm{C}$ for $20 \mathrm{~h}$. The mixture was diluted with EtOAc $(5.0 \mathrm{~mL})$, filtered over Celite and the solvents were removed in vacuo. Isolation by column chromatography on silica gel ( $n$-hexane/EtOAc) afforded the desired products 159.

\subsubsection{General Procedure H: Manganese(I)-Catalyzed Allylative C-H/C-F Functionalization}

A suspension of ketimine $6(0.50 \mathrm{mmol}, 1.00$ equiv $)$, perfluoroalkylalkene 127 (1.50 mmol, 3.00 equiv), $\left[\mathrm{MnBr}(\mathrm{CO})_{5}\right](13.7 \mathrm{mg}, 10.0 \mathrm{~mol} \%), \mathrm{NaOAc}(16.4 \mathrm{mg}, 40 \mathrm{~mol} \%)$ and $\mathrm{K}_{2} \mathrm{CO}_{3}(104 \mathrm{mg}$, $0.75 \mathrm{mmol}, 1.50$ equiv) in 1,4 -dioxane $(0.50 \mathrm{~mL}, 1.00 \mathrm{M})$ was stirred at $105^{\circ} \mathrm{C}$ for $20 \mathrm{~h}$. At ambient temperature, the mixture was diluted with EtOAc $(5.0 \mathrm{~mL})$, filtered over Celite and the solvents were removed in vacuo. The remaining residue was purified by column chromatography on silica gel ( $n$ hexane/EtOAc) to furnish the desired products $\mathbf{1 6 6}$. 


\subsection{Ruthenium(II)-PA-Catalyzed C-H Arylation of $(E)$-2-Styrylpyridines}

\subsubsection{Characterization Data}<smiles>C(=C(c1ccccc1)c1ccccc1)c1ccccn1</smiles>

(Z)-2-(2,2-Diphenylvinyl)pyridine (10aa): The general procedure $\mathbf{A}$ was followed using $(E)$-2styrylpyridine (9a) (90.5 mg, $0.50 \mathrm{mmol}, 1.00$ equiv) and 4-bromobenzene (2a) (118 mg, $0.75 \mathrm{mmol}$, 1.50 equiv). Isolation by column chromatography ( $n$-hexane/EtOAc: 20/1) yielded 10aa (103 mg, $80 \%)$ as a colorless solid.

M.p.: $206^{\circ} \mathrm{C}$.

${ }^{1}$ H-NMR $\left(300 \mathrm{MHz}, \mathrm{CDCl}_{3}\right): \delta=8.53(\mathrm{ddd}, J=4.9,1.9,0.9 \mathrm{~Hz}, 1 \mathrm{H}), 7.36-7.27(\mathrm{~m}, 9 \mathrm{H}), 7.23-7.16$ (m, 2H), 7.14 (s, 1H), 6.97 (ddd, $J=7.4,4.9,1.1 \mathrm{~Hz}, 1 \mathrm{H}), 6.65$ (ddd, $J=8.1,1.1,0.9 \mathrm{~Hz}, 1 \mathrm{H}$ ).

${ }^{13}$ C-NMR $\left(75 \mathrm{MHz}, \mathrm{CDCl}_{3}\right): \delta=156.5\left(\mathrm{C}_{\mathrm{q}}\right), 149.2(\mathrm{CH}), 145.8\left(\mathrm{C}_{\mathrm{q}}\right), 142.6\left(\mathrm{C}_{\mathrm{q}}\right), 139.8\left(\mathrm{C}_{\mathrm{q}}\right), 135.3$ $(\mathrm{CH}), 130.2(\mathrm{CH}), 128.8(\mathrm{CH}), 128.7(\mathrm{CH}), 128.3(\mathrm{CH}), 128.1(\mathrm{CH}), 127.8(\mathrm{CH}), 127.7(\mathrm{CH}), 123.7$ $(\mathrm{CH}), 121.1(\mathrm{CH})$.

IR (ATR): 1583, 1560, 1506, 1488, 1219, 774, 762, 698, 552, $504 \mathrm{~cm}^{-1}$.

MS (EI) $m / z$ (relative intensity): 257 ([M] $\left.]^{+}, 40\right), 256$ (100), 182 (10).

HR-MS (ESI): $m / z$ calcd. for $\left[\mathrm{C}_{19} \mathrm{H}_{16} \mathrm{~N}\right]^{+}[\mathrm{M}+\mathrm{H}]^{+} 258.1277$, found 258.1280 .

The spectral data are in accordance with those reported in the literature. ${ }^{[32]}$<smiles>COc1ccc(C(=Cc2ccccn2)c2ccccc2)cc1</smiles>

(Z)-2-[2-(4-Methoxyphenyl)-2-phenylvinyl]pyridine (10ab): The general procedure $\mathbf{A}$ was followed using (E)-2-styrylpyridine (9a) (90.5 mg, $0.50 \mathrm{mmol}, 1.00$ equiv) and 4-bromoanisole (2b) (140 mg, $0.75 \mathrm{mmol}, 1.50$ equiv). Isolation by column chromatography ( $n$-hexane/EtOAc: $20 / 1$ ) yielded 10ab (138 $\mathrm{mg}, 96 \%)$ as a pale yellow solid.

M.p.: $111^{\circ} \mathrm{C}$.

${ }^{1}$ H-NMR $\left(300 \mathrm{MHz}, \mathrm{CDCl}_{3}\right): \delta=8.53(\mathrm{ddd}, J=5.0,1.9,0.9 \mathrm{~Hz}, 1 \mathrm{H}), 7.39-7.29(\mathrm{~m}, 6 \mathrm{H}), 7.16-7.08$ $(\mathrm{m}, 3 \mathrm{H}), 7.04-6.96(\mathrm{~m}, 1 \mathrm{H}), 6.92-6.85(\mathrm{~m}, 2 \mathrm{H}), 6.76(\mathrm{ddd}, J=8.1,1.1,0.9 \mathrm{~Hz}, 1 \mathrm{H}), 3.83(\mathrm{~s}, 3 \mathrm{H})$. 
${ }^{13}$ C-NMR $\left(75 \mathrm{MHz}, \mathrm{CDCl}_{3}\right): \delta=159.1\left(\mathrm{C}_{\mathrm{q}}\right), 156.7\left(\mathrm{C}_{\mathrm{q}}\right), 149.0(\mathrm{CH}), 145.6\left(\mathrm{C}_{\mathrm{q}}\right), 142.7\left(\mathrm{C}_{\mathrm{q}}\right), 135.3$ $(\mathrm{CH}), 131.9\left(\mathrm{C}_{\mathrm{q}}\right), 131.3(\mathrm{CH}), 128.1(\mathrm{CH}), 128.0(\mathrm{CH}), 127.8(\mathrm{CH}), 127.8(\mathrm{CH}), 123.7(\mathrm{CH}), 121.0$ $(\mathrm{CH}), 114.0(\mathrm{CH}), 55.3\left(\mathrm{CH}_{3}\right)$.

IR (ATR): 3054, 1603, 1508, 1465, 1244, 1026, 827, 773, 762, $540 \mathrm{~cm}^{-1}$.

MS (EI) $m / z$ (relative intensity): 287 ([M] $\left.]^{+}, 40\right), 286$ (100), 271 (10), 243 (20).

HR-MS (ESI): $m / z$ calcd. for $\left[\mathrm{C}_{20} \mathrm{H}_{18} \mathrm{NO}\right]^{+}[\mathrm{M}+\mathrm{H}]^{+} 288.1383$, found 288.1391 .

The spectral data are in accordance with those reported in the literature. ${ }^{[184]}$<smiles>CN(C)c1ccc(/C(=C/c2ccccn2)c2ccccc2)cc1</smiles>

(Z)-N,N-dimethyl-4-[1-phenyl-2-(pyridin-2-yl)vinyl]aniline (10ac): The general procedure A was followed using (E)-2-styrylpyridine (9a) $(90.5 \mathrm{mg}, 0.50 \mathrm{mmol}, 1.00$ equiv) and 4-bromo- $N, N$ dimethylaniline (2c) (150 mg, $0.75 \mathrm{mmol}, 1.50$ equiv). Isolation by column chromatography ( $n$ hexane/EtOAc: 20/1) yielded 10ac (141 mg, 94\%) as a yellow solid.

M.p.: $102{ }^{\circ} \mathrm{C}$.

${ }^{1}$ H-NMR $\left(300 \mathrm{MHz}, \mathrm{CDCl}_{3}\right): \delta=8.53(\mathrm{ddd}, J=4.9,1.9,0.9 \mathrm{~Hz}, 1 \mathrm{H}), 7.43-7.21(\mathrm{~m}, 6 \mathrm{H}), 7.09-7.02$ (m, 2H), $7.01(\mathrm{~s}, 1 \mathrm{H}), 6.97(\mathrm{ddd}, J=8.1,1.1,0.9 \mathrm{~Hz}, 1 \mathrm{H}), 6.87(\mathrm{ddd}, J=8.1,8.1,1.1 \mathrm{~Hz}, 1 \mathrm{H}), 6.72-$ $6.62(\mathrm{~m}, 2 \mathrm{H}), 2.98(\mathrm{~s}, 6 \mathrm{H})$.

${ }^{13}$ C-NMR $\left(75 \mathrm{MHz}, \mathrm{CDCl}_{3}\right): \delta=157.3\left(\mathrm{C}_{\mathrm{q}}\right), 149.9\left(\mathrm{C}_{\mathrm{q}}\right), 149.1(\mathrm{CH}), 146.1\left(\mathrm{C}_{\mathrm{q}}\right), 143.5\left(\mathrm{C}_{\mathrm{q}}\right), 135.2$ $(\mathrm{CH}), 131.1(\mathrm{CH}), 128.2(\mathrm{CH}), 128.1(\mathrm{CH}), 127.9(\mathrm{CH}), 127.6(\mathrm{CH}), 127.4\left(\mathrm{C}_{\mathrm{q}}\right), 123.7(\mathrm{CH}), 120.8$ $(\mathrm{CH}), 112.2(\mathrm{CH}), 40.3\left(\mathrm{CH}_{3}\right)$.

IR (ATR): 3046, 1603, 1577, 1558, 1519, 1431, 946, 766, $512 \mathrm{~cm}^{-1}$.

MS (EI) $\mathrm{m} / \mathrm{z}$ (relative intensity): $300\left([\mathrm{M}]^{+}, 60\right), 299$ (100), 283 (10), 149 (10).

HR-MS (ESI): $m / z$ calcd. for $\left[\mathrm{C}_{21} \mathrm{H}_{21} \mathrm{~N}_{2}\right]^{+}[\mathrm{M}+\mathrm{H}]^{+} 301.1699$, found 301.1701 .<smiles>Cc1ccc(/C(=C/c2ccccn2)c2ccccc2)cc1</smiles>

(Z)-2-[2-(4-Methylphenyl)-2-phenylvinyl]pyridine (10ad): The general procedure A was followed using (E)-2-styrylpyridine (9a) (90.5 mg, $0.50 \mathrm{mmol}, 1.00$ equiv) and 4-bromotoluene (2d) (128 mg, 
$0.75 \mathrm{mmol}, 1.50$ equiv). Isolation by column chromatography ( $n$-hexane/EtOAc: $20 / 1)$ yielded 10ad (126 $\mathrm{mg}, 93 \%)$ as a yellow solid.

M.p.: $86^{\circ} \mathrm{C}$.

${ }^{1}$ H-NMR $\left(300 \mathrm{MHz}, \mathrm{CDCl}_{3}\right): \delta=8.54(\mathrm{ddd}, J=4.9,1.9,0.9 \mathrm{~Hz}, 1 \mathrm{H}), 7.37-7.25(\mathrm{~m}, 6 \mathrm{H}), 7.16-7.08$ (m, 5H), 6.97 (ddd, $J=7.5,4.9,1.1 \mathrm{~Hz}, 1 \mathrm{H}), 6.72(\mathrm{ddd}, J=8.1,1.1,0.9 \mathrm{~Hz}, 1 \mathrm{H}), 2.39$ (s, 3H).

${ }^{13}$ C-NMR $\left(75 \mathrm{MHz}, \mathrm{CDCl}_{3}\right): \delta=156.8\left(\mathrm{C}_{\mathrm{q}}\right), 149.2(\mathrm{CH}), 145.8\left(\mathrm{C}_{\mathrm{q}}\right), 142.8\left(\mathrm{C}_{\mathrm{q}}\right), 137.5\left(\mathrm{C}_{\mathrm{q}}\right), 132.8$ $\left(\mathrm{C}_{\mathrm{q}}\right), 135.2(\mathrm{CH}), 130.0(\mathrm{CH}), 129.4(\mathrm{CH}), 128.4(\mathrm{CH}), 128.2(\mathrm{CH}), 127.9(\mathrm{CH}), 127.8(\mathrm{CH}), 123.4$ $(\mathrm{CH}), 121.0(\mathrm{CH}), 21.3\left(\mathrm{CH}_{3}\right)$.

IR (ATR): 3051, 1583, 1459, 775, 762, 697, 589, 521, $404 \mathrm{~cm}^{-1}$.

MS (EI) $\mathrm{m} / z$ (relative intensity): 271 ([M] $\left.{ }^{+}, 45\right), 270$ (100), 254 (15), 127 (10).

HR-MS (ESI): $m / z$ calcd. for $\left[\mathrm{C}_{20} \mathrm{H}_{18} \mathrm{~N}\right]^{+}[\mathrm{M}+\mathrm{H}]^{+} 272.1434$, found 272.1436.

The spectral data are in accordance with those reported in the literature. ${ }^{[184]}$<smiles>Fc1ccc(C(=Cc2ccccc2)c2ccccc2)cc1</smiles>

(Z)-2-[2-(4-Fluorophenyl)-2-phenylvinyl]pyridine (10ae): A modified general procedure A was followed at $140{ }^{\circ} \mathrm{C}$ using (E)-2-styrylpyridine (9a) (90.5 mg, $0.50 \mathrm{mmol}, 1.00$ equiv) and 1-bromo-4fluorobenzene (2e) $(131 \mathrm{mg}, 0.75 \mathrm{mmol}, 1.50$ equiv). Isolation by column chromatography ( $n$ hexane/EtOAc: 20/1) yielded 10ae (127 mg, 92\%) as a pale yellow solid.

M.p.: $95^{\circ} \mathrm{C}$.

${ }^{1} \mathbf{H}-\mathbf{N M R}\left(300 \mathrm{MHz}, \mathrm{CDCl}_{3}\right): \delta=8.53(\mathrm{ddd}, J=4.9,1.9,0.9 \mathrm{~Hz}, 1 \mathrm{H}), 7.42-7.28(\mathrm{~m}, 6 \mathrm{H}), 7.21-7.12$ (m, 3H), 7.09-6.98 (m, 3H), 6.71 (ddd, $J=8.1,1.1,0.9 \mathrm{~Hz}, 1 \mathrm{H})$.

${ }^{13}$ C-NMR $\left(75 \mathrm{MHz}, \mathrm{CDCl}_{3}\right): \delta=162.5\left(\mathrm{~d},{ }^{1} J_{\mathrm{C}-\mathrm{F}}=249 \mathrm{~Hz}, \mathrm{C}_{\mathrm{q}}\right), 156.5\left(\mathrm{C}_{\mathrm{q}}\right), 149.5(\mathrm{CH}), 144.8\left(\mathrm{C}_{\mathrm{q}}\right)$, $142.5\left(\mathrm{C}_{\mathrm{q}}\right), 135.8\left(\mathrm{C}_{\mathrm{q}}\right), 135.5(\mathrm{CH}), 132.0\left(\mathrm{CH}, \mathrm{d},{ }^{3} J_{\mathrm{C}-\mathrm{F}}=8.0 \mathrm{~Hz}\right), 129.0(\mathrm{CH}), 128.4(\mathrm{CH}), 128.2$ $(\mathrm{CH}), 127.8(\mathrm{CH}), 123.8(\mathrm{CH}), 121.3(\mathrm{CH}), 115.8\left(\mathrm{CH}, \mathrm{d},{ }^{2} J_{\mathrm{C}-\mathrm{F}}=21.4 \mathrm{~Hz}\right)$.

${ }^{19}$ F-NMR $\left(282 \mathrm{MHz}, \mathrm{CDCl}_{3}\right): \delta=-114.0(\mathrm{tt}, J=8.7,5.5 \mathrm{~Hz})$.

IR (ATR): 1584, 1560, 1505, 1445, 1219, 1156, 760, 745, 588, $522 \mathrm{~cm}^{-1}$.

MS (EI) $\mathrm{m} / z$ (relative intensity): 275 ([M] $\left.{ }^{+}, 40\right), 274$ (100).

HR-MS (ESI): $m / z$ calcd. for $\left[\mathrm{C}_{19} \mathrm{H}_{15} \mathrm{FN}\right]^{+}[\mathrm{M}+\mathrm{H}]^{+} 276.1183$, found 276.1187.

The spectral data are in accordance with those reported in the literature. ${ }^{[184]}$ 
<smiles>FC(F)(F)c1ccc(C(=Cc2ccccn2)c2ccccc2)cc1</smiles>

(Z)-2-\{2-[4-(Trifluoromethyl)phenyl]-2-phenylvinyl\}pyridine (10af): A modified general procedure A was followed at $140{ }^{\circ} \mathrm{C}$ using (E)-2-styrylpyridine (9a) $(90.5 \mathrm{mg}, 0.50 \mathrm{mmol}, 1.50$ equiv) and 1bromo-4-(trifluoromethyl)benzene (2f) $(169 \mathrm{mg}, 0.75 \mathrm{mmol}, 1.50$ equiv). Isolation by column chromatography ( $n$-hexane/EtOAc: 20/1) yielded 10af (115 mg, 71\%) as a pale yellow solid.

M.p.: $75^{\circ} \mathrm{C}$.

${ }^{1}$ H-NMR $\left(300 \mathrm{MHz}, \mathrm{CDCl}_{3}\right): \delta=8.53(\mathrm{ddd}, J=4.9,1.9,0.9 \mathrm{~Hz}, 1 \mathrm{H}), 7.60(\mathrm{~d}, J=7.9 \mathrm{~Hz}, 2 \mathrm{H}), 7.41-$ $7.27(\mathrm{~m}, 8 \mathrm{H}), 7.20(\mathrm{~s}, 1 \mathrm{H}), 7.03(\mathrm{ddd}, J=7.4,4.9,1.1 \mathrm{~Hz}, 1 \mathrm{H}), 6.72(\mathrm{ddd}, J=8.1,1.1,0.9 \mathrm{~Hz}, 1 \mathrm{H})$.

${ }^{13}$ C-NMR $\left(75 \mathrm{MHz}, \mathrm{CDCl}_{3}\right): \delta=158.8\left(\mathrm{C}_{\mathrm{q}}\right), 149.4(\mathrm{CH}), 144.2\left(\mathrm{C}_{\mathrm{q}}\right) 143.9\left(\mathrm{C}_{\mathrm{q}}\right), 141.9\left(\mathrm{C}_{\mathrm{q}}\right), 135.5$ $(\mathrm{CH}), 130.5(\mathrm{CH}), 129.9\left(\mathrm{q},{ }^{2} J_{\mathrm{C}-\mathrm{F}}=32.8 \mathrm{~Hz}, \mathrm{C}_{\mathrm{q}}\right), 129.4(\mathrm{CH}), 128.3(\mathrm{CH}), 128.3(\mathrm{CH}), 127.7(\mathrm{CH})$, $125.5\left(\mathrm{q},{ }^{3} J_{\mathrm{C}-\mathrm{F}}=3.7 \mathrm{~Hz}, \mathrm{CH}\right), 124.1\left(\mathrm{q},{ }^{1} J_{\mathrm{C}-\mathrm{F}}=274 \mathrm{~Hz}, \mathrm{C}_{\mathrm{q}}\right), 123.8(\mathrm{CH}), 121.5(\mathrm{CH})$.

${ }^{19} \mathbf{F}-\mathbf{N M R}\left(282 \mathrm{MHz}, \mathrm{CDCl}_{3}\right): \delta=-62.5(\mathrm{~m})$.

IR (ATR): 3059, 1583, 1459, 1314, 1152, 1106, 1062, 1017, 742, $718 \mathrm{~cm}^{-1}$.

MS (EI) $m / z$ (relative intensity): $325\left([\mathrm{M}]^{+}, 40\right), 324$ (100).

HR-MS (EI): $m / z$ calcd. for $\left[\mathrm{C}_{20} \mathrm{H}_{14} \mathrm{~F}_{3} \mathrm{~N}\right]^{+}[\mathrm{M}]^{+} 325.1078$, found 325.1074 .<smiles>CCOC(=O)c1ccc(C(=Cc2ccccn2)c2ccccc2)cc1</smiles>

Ethyl (Z)-4-[1-phenyl-2-(pyridin-2-yl)vinyl]benzoate (10ag): The general procedure A was followed using (E)-2-styrylpyridine (9a) $(90.5 \mathrm{mg}, 0.50 \mathrm{mmol}, 1.00$ equiv) and ethyl 4-bromobenzoate (2g) (172 mg, $0.75 \mathrm{mmol}, 1.50$ equiv). Isolation by column chromatography ( $n$-hexane/EtOAc: 20/1) yielded 10ag (143 $\mathrm{mg}, 87 \%)$ as a yellow solid.

M.p.: $144^{\circ} \mathrm{C}$.

${ }^{1} \mathbf{H}-\mathbf{N M R}\left(500 \mathrm{MHz}, \mathrm{CDCl}_{3}\right): \delta=8.50(\mathrm{ddd}, J=4.9,1.9,0.9 \mathrm{~Hz}, 1 \mathrm{H}), 8.04-7.96(\mathrm{~m}, 2 \mathrm{H}), 7.32-7.25$ (m, 6H), 7.29-7.25 (m, 2H), $7.17(\mathrm{~s}, 1 \mathrm{H}), 6.99(\mathrm{ddd}, J=7.5,4.9,1.1 \mathrm{~Hz}, 1 \mathrm{H}), 6.71(\mathrm{ddd}, J=8.1,1.1$, $0.9 \mathrm{~Hz}, 1 \mathrm{H}), 4.38$ (q, $J=7.1 \mathrm{~Hz}, 2 \mathrm{H}), 1.37$ (t, $J=7.1 \mathrm{~Hz}, 3 \mathrm{H})$. 
${ }^{13}$ C-NMR $\left(125 \mathrm{MHz}, \mathrm{CDCl}_{3}\right): \delta=166.4\left(\mathrm{C}_{\mathrm{q}}\right), 156.0\left(\mathrm{C}_{\mathrm{q}}\right), 149.4(\mathrm{CH}), 144.8\left(\mathrm{C}_{\mathrm{q}}\right), 144.7\left(\mathrm{C}_{\mathrm{q}}\right), 141.9$ $\left(\mathrm{C}_{\mathrm{q}}\right), 135.5(\mathrm{CH}), 130.2(\mathrm{CH}), 129.9(\mathrm{CH}), 129.8\left(\mathrm{C}_{\mathrm{q}}\right), 129.3(\mathrm{CH}), 128.3(\mathrm{CH}), 128.2(\mathrm{CH}), 127.7$ $(\mathrm{CH}), 123.8(\mathrm{CH}), 121.4(\mathrm{CH}), 61.0\left(\mathrm{CH}_{2}\right), 14.3\left(\mathrm{CH}_{3}\right)$.

IR (ATR): 3053, 1708, 1604, 1560, 1462, 1273, 1178, 999, 776, $691 \mathrm{~cm}^{-1}$.

MS (EI) $\mathrm{m} / \mathrm{z}$ (relative intensity): $329\left(\left[\mathrm{M}^{+}, 40\right) 328\right.$ (100), 301 (70), 254 (30).

HR-MS (ESI): $m / z$ calcd. for $\left[\mathrm{C}_{22} \mathrm{H}_{20} \mathrm{NO}_{2}\right]^{+}[\mathrm{M}+\mathrm{H}]^{+} 330.1489$, found 330.1490 .<smiles>CC(=O)c1ccc(C(=Cc2ccccn2)c2ccccc2)cc1</smiles>

(Z)-1-\{4-[1-phenyl-2-(pyridin-2-yl)vinyl]phenyl\}ethan-1-one (10ah): A modified general procedure A was followed at $140{ }^{\circ} \mathrm{C}$ using (E)-2-styrylpyridine (9a) $(90.5 \mathrm{mg}, 0.50 \mathrm{mmol}, 1.00$ equiv) and 1-(4bromophenyl)ethan-1-one (2h) (148 mg, $0.75 \mathrm{mmol}, 1.50$ equiv). Isolation by column chromatography (n-hexane/EtOAc: 20/1) yielded 10ah (130 mg, 87\%) as a pale yellow solid.

M.p.: $97^{\circ} \mathrm{C}$.

${ }^{1} \mathbf{H}-\mathbf{N M R}\left(500 \mathrm{MHz}, \mathrm{CDCl}_{3}\right): \delta=8.50$ (ddd, $\left.J=4.9,1.9,0.9 \mathrm{~Hz}, 1 \mathrm{H}\right), 7.93-7.90(\mathrm{~m}, 2 \mathrm{H}), 7.34-7.27$ (m, 8H), 7.17 (s, 1H), 6.99 (ddd, $J=7.6,4.9,1.1 \mathrm{~Hz}, 1 \mathrm{H}), 6.72(\mathrm{ddd}, J=8.1,1.1,0.9 \mathrm{~Hz}, 1 \mathrm{H}), 2.61$ (s, $3 \mathrm{H})$.

${ }^{13}$ C-NMR $\left(125 \mathrm{MHz}, \mathrm{CDCl}_{3}\right): \delta=197.7\left(\mathrm{C}_{\mathrm{q}}\right), 156.0\left(\mathrm{C}_{\mathrm{q}}\right), 149.4(\mathrm{CH}), 145.2\left(\mathrm{C}_{\mathrm{q}}\right), 144.7\left(\mathrm{C}_{\mathrm{q}}\right), 141.9$ $\left(\mathrm{C}_{\mathrm{q}}\right), 136.3\left(\mathrm{C}_{\mathrm{q}}\right), 135.5(\mathrm{CH}), 130.5(\mathrm{CH}), 129.4(\mathrm{CH}), 128.7(\mathrm{CH}), 128.4(\mathrm{CH}), 128.3(\mathrm{CH}), 127.7$ $(\mathrm{CH}), 123.9(\mathrm{CH}), 121.5(\mathrm{CH}), 26.6\left(\mathrm{CH}_{3}\right)$.

IR (ATR): 1681, 1583, 1264, 856, 747, 652, 599, $585 \mathrm{~cm}^{-1}$.

MS (EI) $m / z$ (relative intensity): 299 ([M] $\left.]^{+}, 40\right), 298$ (100), 254 (200).

HR-MS (ESI): $m / z$ calcd. for $\left[\mathrm{C}_{21} \mathrm{H}_{18} \mathrm{NO}\right]^{+}[\mathrm{M}+\mathrm{H}]^{+} 300.1383$, found 300.1386 .

The spectral data are in accordance with those reported in the literature. ${ }^{[184]}$<smiles>COc1cccc(C(=Cc2ccccn2)c2ccccc2)c1</smiles>

(Z)-2-[2-(3-Methoxyphenyl)-2-phenylvinyl]pyridine (10ai): The general procedure A was followed using (E)-2-styrylpyridine (9a) $(90.5 \mathrm{mg}, 0.50 \mathrm{mmol}, 1.00$ equiv) and 3 -bromoanisole (2i) $(140 \mathrm{mg}$, 
$0.75 \mathrm{mmol}, 1.50$ equiv). Isolation by column chromatography ( $n$-hexane/EtOAc: $20 / 1)$ yielded 10ai (115 $\mathrm{mg}, 80 \%)$ as a pale yellow solid.

M.p.: $108^{\circ} \mathrm{C}$.

${ }^{1} \mathbf{H}-\mathbf{N M R}\left(300 \mathrm{MHz}, \mathrm{CDCl}_{3}\right): \delta=8.53(\mathrm{ddd}, J=4.9,1.9,1.0 \mathrm{~Hz}, 1 \mathrm{H}), 7.41-7.34(\mathrm{~m}, 2 \mathrm{H}), 7.32-7.22$ (m, 5H), 7.15 (s, 1H), 6.97 (ddd, $J=7.5,4.9,1.2 \mathrm{~Hz}, 1 \mathrm{H}), 6.89$ (ddd, $J=8.3,2.7,1.0 \mathrm{~Hz}, 1 \mathrm{H}), 6.79$ (ddd, $J=8.1,1.1,0.9 \mathrm{~Hz}, 1 \mathrm{H}), 6.75-6.69(\mathrm{~m}, 1 \mathrm{H}), 3.70$ (s, 3H).

${ }^{13}$ C-NMR $\left(75 \mathrm{MHz}, \mathrm{CDCl}_{3}\right): \delta=159.9\left(\mathrm{C}_{\mathrm{q}}\right), 156.5\left(\mathrm{C}_{\mathrm{q}}\right), 149.2(\mathrm{CH}), 145.5\left(\mathrm{C}_{\mathrm{q}}\right), 142.2\left(\mathrm{C}_{\mathrm{q}}\right), 141.2$ $\left(\mathrm{C}_{\mathrm{q}}\right), 135.3(\mathrm{CH}), 129.8(\mathrm{CH}), 128.6(\mathrm{CH}), 128.2(\mathrm{CH}), 128.0(\mathrm{CH}), 127.6(\mathrm{CH}), 123.6(\mathrm{CH}), 122.5$ $(\mathrm{CH}), 121.2(\mathrm{CH}), 115.1(\mathrm{CH}), 113.7(\mathrm{CH}), 55.2\left(\mathrm{CH}_{3}\right)$.

IR (ATR): 3051, 1580, 1485, 1461, 1430, 1229, 1041, 872, 742, $551 \mathrm{~cm}^{-1}$.

MS (EI) $m / z$ (relative intensity): 287 ([M] $\left.]^{+}, 20\right), 286$ (100), 271 (10), 243 (20).

HR-MS (ESI): $m / z$ calcd. for $\left[\mathrm{C}_{20} \mathrm{H}_{18} \mathrm{NO}\right]^{+}[\mathrm{M}+\mathrm{H}]^{+} 288.1383$, found 288.1393 .<smiles>COc1ccccc1/C(=C\c1ccccn1)c1ccccc1</smiles>

(Z)-2-[2-(2-Methoxyphenyl)-2-phenylvinyl]pyridine (10aj): A modified general procedure A was followed at $140{ }^{\circ} \mathrm{C}$ in $\mathrm{PhMe}(0.50 \mathrm{~mL}, 1.00 \mathrm{M})$ using (E)-2-styrylpyridine (9a) (90.5 mg, $0.50 \mathrm{mmol}$, 1.00 equiv) and 2-bromoanisole (2j) $(140 \mathrm{mg}, 0.75 \mathrm{mmol}, 1.50$ equiv). Isolation by column chromatography ( $n$-hexane/EtOAc: 20/1) yielded 10aj (116 mg, 81\%) as a pale yellow solid.

M.p.: $114^{\circ} \mathrm{C}$.

${ }^{1} \mathbf{H}-\mathbf{N M R}\left(500 \mathrm{MHz}, \mathrm{CDCl}_{3}\right): \delta=8.51(\mathrm{ddd}, J=4.9,2.0,0.9 \mathrm{~Hz}, 1 \mathrm{H}), 7.38-7.33(\mathrm{~m}, 3 \mathrm{H}), 7.30-7.23$ $(\mathrm{m}, 5 \mathrm{H}), 7.08(\mathrm{dd}, J=7.6,2.0 \mathrm{~Hz}, 1 \mathrm{H}), 6.97-6.92(\mathrm{~m}, 3 \mathrm{H}), 6.70(\mathrm{ddd}, J=8.1,1.1,0.9 \mathrm{~Hz}, 1 \mathrm{H}), 3.58$ (s, 3H).

${ }^{13}$ C-NMR $\left(125 \mathrm{MHz}, \mathrm{CDCl}_{3}\right): \delta=157.3\left(\mathrm{C}_{\mathrm{q}}\right), 156.6\left(\mathrm{C}_{\mathrm{q}}\right), 149.1(\mathrm{CH}), 142.1\left(\mathrm{C}_{\mathrm{q}}\right), 141.8\left(\mathrm{C}_{\mathrm{q}}\right), 135.3$ $(\mathrm{CH}), 131.2(\mathrm{CH}), 129.3(\mathrm{CH}), 129.3(\mathrm{CH}), 128.7\left(\mathrm{C}_{\mathrm{q}}\right), 128.1(\mathrm{CH}), 127.6(\mathrm{CH}), 126.7(\mathrm{CH}), 122.7$ $(\mathrm{CH}), 121.1(\mathrm{CH}), 121.0(\mathrm{CH}), 111.5(\mathrm{CH}), 55.6\left(\mathrm{CH}_{3}\right)$.

IR (ATR): 3056, 1582, 1490, 1461, 1433, 1247, 1160, 1047, 756, $695 \mathrm{~cm}^{-1}$.

MS (EI) $m / z$ (relative intensity): 287 ([M] $\left.]^{+}, 20\right), 270$ (10), 257 (30), 256 (100).

HR-MS (ESI): $m / z$ calcd. for $\left[\mathrm{C}_{20} \mathrm{H}_{18} \mathrm{NO}\right]^{+}[\mathrm{M}+\mathrm{H}]^{+} 288.1383$, found 288.1385 . 
<smiles>C(=C(c1ccccc1)c1ccsc1)c1ccccn1</smiles>

(Z)-2-[2-Phenyl-2-(thiophen-3-yl)vinyl]pyridine (10al): The general procedure A was followed using (E)-2-styrylpyridine (9a) (90.5 mg, $0.50 \mathrm{mmol}, 1.00$ equiv) and 3-bromothiophene (91) (122 mg, $0.75 \mathrm{mmol}, 1.50$ equiv). Isolation by column chromatography ( $n$-hexane/EtOAc: 20/1) yielded 10al (114 $\mathrm{mg}, 87 \%, Z / E=96: 4)$ as a pale brown solid.

M.p.: $87^{\circ} \mathrm{C}$.

${ }^{1}$ H-NMR $\left(500 \mathrm{MHz}, \mathrm{CDCl}_{3}\right.$ ): $\delta=8.53$ (ddd, $\left.J=4.9,1.9,0.9 \mathrm{~Hz}, 1 \mathrm{H}\right), 7.40-7.34$ (m, 4H), 7.33-7.27 (m, 3H), 7.10 (s, 1H), 7.07 (dd, $J=3.0,1.3 \mathrm{~Hz}, 1 \mathrm{H}), 7.01(\mathrm{ddd}, J=7.5,4.9,1.1 \mathrm{~Hz}, 1 \mathrm{H}), 6.87$ (dd, $J=4.9,1.3 \mathrm{~Hz}, 1 \mathrm{H}), 6.80(\mathrm{dd}, J=8.1,1.1,0.9 \mathrm{~Hz}, 1 \mathrm{H})$.

${ }^{13}$ C-NMR $\left(125 \mathrm{MHz}, \mathrm{CDCl}_{3}\right): \delta=156.6\left(\mathrm{C}_{\mathrm{q}}\right), 149.2(\mathrm{CH}), 142.2\left(\mathrm{C}_{\mathrm{q}}\right), 140.4\left(\mathrm{C}_{\mathrm{q}}\right), 139.8\left(\mathrm{C}_{\mathrm{q}}\right), 135.4$ $(\mathrm{CH}), 129.2(\mathrm{CH}), 129.1(\mathrm{CH}), 128.2(\mathrm{CH}), 128.1(\mathrm{CH}), 127.6(\mathrm{CH}), 125.7(\mathrm{CH}), 125.1(\mathrm{CH}), 123.5$ $(\mathrm{CH}), 121.3(\mathrm{CH})$.

IR (ATR): 3049, 1582, 1560, 1461, 1431, 863, 760, 743, $701 \mathrm{~cm}^{-1}$.

MS (EI) $\mathrm{m} / \mathrm{z}$ (relative intensity): 263 ([M] $\left.]^{+}, 40\right), 262$ (100), 230 (25), 186 (25).

HR-MS (EI): $m / z$ calcd. for $\left[\mathrm{C}_{17} \mathrm{H}_{13} \mathrm{NS}\right]^{+}[\mathrm{M}]^{+} 263.0769$, found 263.0765 .<smiles>COc1ccc(/C(=C\c2ccccn2)c2ccc(C)cc2)cc1</smiles>

(Z)-2-[2-(4-Methoxyphenyl)-2-(p-tolyl)vinyl]pyridine (10bb): The general procedure A was followed using (E)-2-(4-methylstyryl)pyridine (9b) $(97.7 \mathrm{mg}, 0.50 \mathrm{mmol}, 1.00$ equiv) and 4bromoanisole (2b) (140 mg, $0.75 \mathrm{mmol}, 1.50$ equiv). Isolation by column chromatography ( $n$ hexane/EtOAc: 20/1) yielded $\mathbf{1 0 b b}(136 \mathrm{mg}, 90 \%)$ as a colorless solid.

M.p.: $69^{\circ} \mathrm{C}$.

${ }^{1}$ H-NMR $\left(300 \mathrm{MHz}, \mathrm{CDCl}_{3}\right): \delta=8.51(\mathrm{ddd}, J=4.9,1.9,0.9 \mathrm{~Hz}, 1 \mathrm{H}), 7.31-7.26(\mathrm{~m}, 1 \mathrm{H}), 7.25-7.22$ (m, 2H), 7.14-7.07 (m, 4H), $7.06(\mathrm{~s}, 1 \mathrm{H}), 6.95(\mathrm{ddd}, J=7.5,4.9,1.1 \mathrm{~Hz}, 1 \mathrm{H}), 6.88-6.82(\mathrm{~m}, 2 \mathrm{H}), 6.72$ (ddd, $J=8.1,1.1,0.9 \mathrm{~Hz}, 1 \mathrm{H}), 3.82(\mathrm{~s}, 3 \mathrm{H}), 2.84(\mathrm{~s}, 3 \mathrm{H})$. 
${ }^{13}$ C-NMR $\left(75 \mathrm{MHz}, \mathrm{CDCl}_{3}\right): \delta=159.2\left(\mathrm{C}_{\mathrm{q}}\right), 157.0\left(\mathrm{C}_{\mathrm{q}}\right), 149.2(\mathrm{CH}), 145.4\left(\mathrm{C}_{\mathrm{q}}\right), 140.0\left(\mathrm{C}_{\mathrm{q}}\right), 137.9$ $\left(\mathrm{C}_{\mathrm{q}}\right), 135.2(\mathrm{CH}), 132.2\left(\mathrm{C}_{\mathrm{q}}\right), 131.3(\mathrm{CH}), 128.9(\mathrm{CH}), 127.7(\mathrm{CH}), 127.5(\mathrm{CH}), 123.6(\mathrm{CH}), 120.8$ $(\mathrm{CH}), 114.6(\mathrm{CH}), 55.2\left(\mathrm{CH}_{3}\right), 21.1\left(\mathrm{CH}_{3}\right)$.

IR (ATR): 3051, 1581, 1507, 1460, 1181, 1024, 848, 824, 745, $531 \mathrm{~cm}^{-1}$.

MS (EI) $\mathrm{m} / \mathrm{z}$ (relative intensity): $301\left([\mathrm{M}]^{+}, 40\right), 300$ (100), 257 (20).

HR-MS (ESI): $m / z$ calcd. for $\left[\mathrm{C}_{21} \mathrm{H}_{20} \mathrm{NO}\right]^{+}[\mathrm{M}+\mathrm{H}]^{+} 302.1539$, found 302.1543 .<smiles>Cc1ccc(C(=Cc2ccccn2)c2ccc(C(F)(F)F)cc2)cc1</smiles>

(Z)-2-\{2-(p-Tolyl)-2-[4-(trifluoromethyl)phenyl]vinyl\}pyridine (10bf): The general procedure A was followed using (E)-2-(4-methylstyryl)pyridine (9b) $(97.7 \mathrm{mg}, 0.50 \mathrm{mmol}, 1.00$ equiv) and 1bromo-4-(trifluoromethyl)benzene (2f) $(169 \mathrm{mg}, 0.75 \mathrm{mmol}, 1.50$ equiv). Isolation by column chromatography ( $n$-hexane/EtOAc: $20 / 1)$ yielded 10bf (144 mg, 85\%) as an off-white solid.

M.p.: $89^{\circ} \mathrm{C}$.

${ }^{1}$ H-NMR $\left(300 \mathrm{MHz}, \mathrm{CDCl}_{3}\right): \delta=8.50(\mathrm{ddd}, J=4.9,1.9,0.9 \mathrm{~Hz}, 1 \mathrm{H}), 7.61-7.54(\mathrm{~m}, 2 \mathrm{H}), 7.34-7.28$ (m, 3H), 7.21-7.16 (m, 2H), 7.15 (s, 1H), 7.14-7.10 (m, 2H), 7.00 (ddd, $J=7.5,4.9,1.1 \mathrm{~Hz}, 1 \mathrm{H}), 6.67$ (ddd, $J=8.1,1.1,0.9 \mathrm{~Hz}, 1 \mathrm{H}), 2.35(\mathrm{~s}, 3 \mathrm{H})$.

${ }^{13} \mathrm{C}-\mathrm{NMR}\left(125 \mathrm{MHz}, \mathrm{CDCl}_{3}\right): \delta=156.0\left(\mathrm{C}_{\mathrm{q}}\right), 149.3(\mathrm{CH}), 144.3\left(\mathrm{C}_{\mathrm{q}}\right), 144.0\left(\mathrm{C}_{\mathrm{q}}\right), 139.0\left(\mathrm{C}_{\mathrm{q}}\right), 138.4$ $\left(\mathrm{C}_{\mathrm{q}}\right), 135.5(\mathrm{CH}), 130.6(\mathrm{CH}), 129.8\left(\mathrm{q},{ }^{2} J_{\mathrm{C}-\mathrm{F}}=32.4 \mathrm{~Hz}, \mathrm{C}_{\mathrm{q}}\right), 129.1(\mathrm{CH}), 128.6(\mathrm{CH}), 127.6(\mathrm{CH})$, $125.5\left(\mathrm{q},{ }^{3} J_{\mathrm{C}-\mathrm{F}}=3.8 \mathrm{~Hz}, \mathrm{CH}\right), 124.0\left(\mathrm{q},{ }^{1} J_{\mathrm{C}-\mathrm{F}}=271 \mathrm{~Hz}, \mathrm{C}_{\mathrm{q}}\right), 123.7(\mathrm{CH}), 121.4(\mathrm{CH}), 21.1\left(\mathrm{CH}_{3}\right)$.

${ }^{19} \mathbf{F}-\mathrm{NMR}\left(282 \mathrm{MHz}, \mathrm{CDCl}_{3}\right): \delta=-62.5(\mathrm{~m})$.

IR (ATR): 3071, 1582, 1460, 1407, 1321, 1161, 1065, 856, $837 \mathrm{~cm}^{-1}$.

MS (EI) $m / z$ (relative intensity): 339 ([M] $\left.{ }^{+}, 35\right), 338$ (100), 268 (10).

HR-MS (ESI): $m / z$ calcd. for $\left[\mathrm{C}_{21} \mathrm{H}_{17} \mathrm{~F}_{3} \mathrm{~N}\right]^{+}[\mathrm{M}+\mathrm{H}]^{+} 340.1308$, found: 340.1311 . 
<smiles>CCOC(=O)c1ccc(C(=Cc2ccccn2)c2ccc(C)cc2)cc1</smiles>

(Z)-Ethyl-4-[2-(pyridin-2-yl)-1-(p-tolyl)vinyl]benzoate (10bg): The general procedure A was followed using (E)-2-(4-methylstyryl)pyridine (9b) $(97.7 \mathrm{mg}, 0.50 \mathrm{mmol}, 1.00$ equiv) and ethyl 4bromobenzoate $(\mathbf{2 g})(172 \mathrm{mg}, 0.75 \mathrm{mmol}, 1.50$ equiv). Isolation by column chromatography ( $n$ hexane/EtOAc: 20/1) yielded $\mathbf{1 0 b g}(149 \mathrm{mg}, 87 \%)$ as yellow solid.

M.p.: $72{ }^{\circ} \mathrm{C}$.

${ }^{1} \mathbf{H}-\mathbf{N M R}\left(300 \mathrm{MHz}, \mathrm{CDCl}_{3}\right): \delta=8.50(\mathrm{ddd}, J=4.9,1.9,0.9 \mathrm{~Hz}, 1 \mathrm{H}), 8.03-7.96(\mathrm{~m}, 2 \mathrm{H}), 7.30-7.24$ (m, 3H), 7.22-7.17 (m, 2H), 7.15 (s, 1H), 7.13-7.08 (m, 2H), 6.97 (ddd, $J=7.4,4.9,1.1 \mathrm{~Hz}, 1 \mathrm{H}), 6.66$ (ddd, $J=8.1,1.1,0.9 \mathrm{~Hz}, 1 \mathrm{H}), 4.37$ (q, $J=7.3 \mathrm{~Hz}, 2 \mathrm{H}), 2.35$ (s, 3H), 1.39 (t, $J=7.3 \mathrm{~Hz}, 3 \mathrm{H}$ ).

${ }^{13}$ C-NMR $\left(125 \mathrm{MHz}, \mathrm{CDCl}_{3}\right): \delta=166.4\left(\mathrm{C}_{\mathrm{q}}\right), 156.2\left(\mathrm{C}_{\mathrm{q}}\right), 149.3(\mathrm{CH}), 145.0\left(\mathrm{C}_{\mathrm{q}}\right), 144.7\left(\mathrm{C}_{\mathrm{q}}\right), 139.0$ $\left(\mathrm{C}_{\mathrm{q}}\right), 138.2\left(\mathrm{C}_{\mathrm{q}}\right), 135.4(\mathrm{CH}), 130.2(\mathrm{CH}), 129.8(\mathrm{CH}), 129.7\left(\mathrm{C}_{\mathrm{q}}\right), 129.1(\mathrm{CH}), 128.4(\mathrm{CH}), 127.6$ $(\mathrm{CH}), 123.7(\mathrm{CH}), 121.3(\mathrm{CH}), 61.0\left(\mathrm{CH}_{2}\right), 21.1\left(\mathrm{CH}_{3}\right), 14.3\left(\mathrm{CH}_{3}\right)$.

IR (ATR): 2980, 1709, 1581, 1460, 1268, 1099, 813, 772, $746 \mathrm{~cm}^{-1}$.

MS (EI) $\mathrm{m} / \mathrm{z}$ (relative intensity): 343 ([M] $\left.]^{+}, 40\right), 342$ (100), 314 (65), 268 (15).

HR-MS (ESI): $m / z$ calcd. for $\left[\mathrm{C}_{23} \mathrm{H}_{22} \mathrm{NO}_{2}\right]^{+}[\mathrm{M}+\mathrm{H}]^{+} 344.1645$, found: 344.1645 .<smiles>COc1ccc(C(=Cc2ccccn2)c2ccc(OC)cc2)cc1</smiles>

2-[2,2-Bis(4-methoxyphenyl)vinyl]pyridine $(10 \mathrm{cb})$ : The general procedure A was followed using (E)-2-(4-methoxystyryl)pyridine (9c) (106 mg, $0.50 \mathrm{mmol}, 1.00$ equiv) and 4-bromoanisole (2b) (140 mg, $0.75 \mathrm{mmol}, 1.50$ equiv). Isolation by column chromatography ( -hexane/EtOAc: 20/1) yielded 10cb (147 $\mathrm{mg}, 93 \%)$ as a colorless solid.

M.p.: $89^{\circ} \mathrm{C}$.

${ }^{1}$ H-NMR $\left(300 \mathrm{MHz}, \mathrm{CDCl}_{3}\right): \delta=8.49$ (ddd, $\left.J=4.9,1.9,0.9 \mathrm{~Hz}, 1 \mathrm{H}\right), 7.32-7.22(\mathrm{~m}, 3 \mathrm{H}), 7.12-7.07$ (m, 2H), 7.01 (s, 1H), 6.93 (ddd, $J=7.5,4.9,1.1 \mathrm{~Hz}, 1 \mathrm{H}), 6.87-6.80$ (m, 4H), 6.69 (ddd, $J=8.1,1.1$, $0.9 \mathrm{~Hz}, 1 \mathrm{H}), 3.81(\mathrm{~s}, 3 \mathrm{H}), 3.78(\mathrm{~s}, 3 \mathrm{H})$. 
${ }^{13}$ C-NMR $\left(125 \mathrm{MHz}, \mathrm{CDCl}_{3}\right): \delta=159.7\left(\mathrm{C}_{\mathrm{q}}\right), 159.3\left(\mathrm{C}_{\mathrm{q}}\right), 157.2\left(\mathrm{C}_{\mathrm{q}}\right), 149.2(\mathrm{CH}), 145.1\left(\mathrm{C}_{\mathrm{q}}\right), 135.5$ $\left(\mathrm{C}_{\mathrm{q}}\right), 135.2(\mathrm{CH}), 132.2\left(\mathrm{C}_{\mathrm{q}}\right), 131.4(\mathrm{CH}), 129.1(\mathrm{CH}), 126.7(\mathrm{CH}), 123.5(\mathrm{CH}), 120.8(\mathrm{CH}), 114.1$ $(\mathrm{CH}), 113.6(\mathrm{CH}), 55.2\left(\mathrm{CH}_{3}\right), 55.1\left(\mathrm{CH}_{3}\right)$.

IR (ATR): 2999, 1601, 1507, 1459, 1240, 1172, 1148, $826 \mathrm{~cm}^{-1}$.

MS (EI) $m / z$ (relative intensity): $317\left(\left[\mathrm{M}^{+}, 40\right), 316\right.$ (100), 273 (15).

HR-MS (ESI): $m / z$ calcd. for $\left[\mathrm{C}_{21} \mathrm{H}_{20} \mathrm{NO}_{2}\right]^{+}[\mathrm{M}+\mathrm{H}]^{+} 318.1489$, found 318.1489.

The spectral data are in accordance with those reported in the literature. ${ }^{[42]}$<smiles>COc1ccc(/C(=C/c2ccccn2)c2ccc(C(F)(F)F)cc2)cc1</smiles>

(E)-2-\{2-(4-Methoxyphenyl)-2-[4-(trifluoromethyl)phenyl]vinyl\}pyridine (10cf): The general procedure A was followed using (E)-2-(4-methoxystyryl)pyridine (9c) (106 mg, $0.50 \mathrm{mmol}$, 1.00 equiv) and 1-bromo-4-(trifluoromethyl)benzene (2f) (169 mg, $0.75 \mathrm{mmol}, 1.50$ equiv). Isolation by column chromatography ( $n$-hexane/EtOAc: $20 / 1)$ yielded 10cf (149 mg, 84\%) as a white solid.

M.p.: $83^{\circ} \mathrm{C}$.

${ }^{1} \mathbf{H}-\mathbf{N M R}\left(500 \mathrm{MHz}, \mathrm{CDCl}_{3}\right): \delta=8.48(\mathrm{ddd}, J=4.9,1.9,0.9 \mathrm{~Hz}, 1 \mathrm{H}), 7.59-7.56(\mathrm{~m}, 2 \mathrm{H}), 7.32-7.28$ (m, 3H), 7.25-7.21 (m, 2H), $7.11(\mathrm{~s}, 1 \mathrm{H}), 6.96(\mathrm{ddd}, J=7.5,4.9,1.0 \mathrm{~Hz}, 1 \mathrm{H}), 6.86-6.82(\mathrm{~m}, 2 \mathrm{H}), 6.65$ (ddd, $J=8.1,1.0,0.9 \mathrm{~Hz}, 1 \mathrm{H}), 3.78(\mathrm{~s}, 3 \mathrm{H})$.

${ }^{13}$ C-NMR $\left(125 \mathrm{MHz}, \mathrm{CDCl}_{3}\right): \delta=159.5\left(\mathrm{C}_{\mathrm{q}}\right), 156.3\left(\mathrm{C}_{\mathrm{q}}\right), 149.4(\mathrm{CH}), 144.1\left(\mathrm{C}_{\mathrm{q}}\right), 143.8\left(\mathrm{C}_{\mathrm{q}}\right), 135.4$ $(\mathrm{CH}), 134.3\left(\mathrm{C}_{\mathrm{q}}\right), 130.6(\mathrm{CH}), 129.7\left(\mathrm{q},{ }^{2} J_{\mathrm{C}-\mathrm{F}}=32.1 \mathrm{~Hz}, \mathrm{C}_{\mathrm{q}}\right), 128.9(\mathrm{CH}), 127.7(\mathrm{CH}), 125.4\left(\mathrm{q},{ }^{3} J_{\mathrm{C}-\mathrm{F}}=\right.$ $3.5 \mathrm{~Hz}, \mathrm{CH}), 124.2\left(\mathrm{q},{ }^{1} J_{\mathrm{C}-\mathrm{F}}=273 \mathrm{~Hz}, \mathrm{C}_{\mathrm{q}}\right), 123.6(\mathrm{CH}), 121.2(\mathrm{CH}), 113.8(\mathrm{CH}), 55.2\left(\mathrm{CH}_{3}\right)$.

${ }^{19} \mathbf{F}-\mathrm{NMR}\left(282 \mathrm{MHz}, \mathrm{CDCl}_{3}\right): \delta=-62.3(\mathrm{~m})$.

IR (ATR): 2963, 1608, 1579, 1510, 1409, 1239, 1108, 993, 801, $557 \mathrm{~cm}^{-1}$.

MS (EI) $m / z$ (relative intensity): 355 ([M] $\left.]^{+}, 30\right), 354$ (100), 311 (25).

HR-MS (ESI): $\mathrm{m} / z$ calcd. for $\left[\mathrm{C}_{21} \mathrm{H}_{17} \mathrm{~F}_{3} \mathrm{NO}\right]^{+}[\mathrm{M}+\mathrm{H}]^{+} 356.1257$, found 356.1255.

The spectral data are in accordance with those reported in the literature. ${ }^{[36 a]}$ 
<smiles>COc1ccc(/C(=C/c2ccccn2)c2ccc(C(F)(F)F)cc2)cc1</smiles>

(Z)-2-[2-(4-Methoxyphenyl)-2-(4-trifluoromethyl)phenylvinyl]pyridine (10db): The general procedure A was followed using (E)-2-[4-(trifluoromethyl)styryl]pyridine (9d) (125 mg, $0.50 \mathrm{mmol}$, 1.00 equiv) and 1-bromoanisole (2b) $(140 \mathrm{mg}, 0.75 \mathrm{mmol}, 1.50$ equiv). Isolation by column chromatography ( $n$-hexane/EtOAc: $20 / 1)$ yielded 10db (158 mg, 90\%) as a brown solid. M.p.: $82^{\circ} \mathrm{C}$.

${ }^{1} \mathbf{H}-\mathbf{N M R}\left(300 \mathrm{MHz}, \mathrm{CDCl}_{3}\right): \delta=8.53(\mathrm{ddd}, J=4.9,1.9,0.9 \mathrm{~Hz}, 1 \mathrm{H}), 7.56-7.54(\mathrm{~m}, 2 \mathrm{H}), 7.47-7.44$ (m, 2H), 7.32 (ddd, $J=8.1,7.5,1.9 \mathrm{~Hz}, 1 \mathrm{H}), 7.11(\mathrm{~s}, 1 \mathrm{H}), 7.10-7.04(\mathrm{~m}, 2 \mathrm{H}), 7.00$ (ddd, $J=7.5,4.9$, $1.1 \mathrm{~Hz}, 1 \mathrm{H}), 6.90-6.83(\mathrm{~m}, 2 \mathrm{H}), 6.78(\mathrm{ddd}, J=8.1,1.1,0.9 \mathrm{~Hz}, 1 \mathrm{H}), 3.80(\mathrm{~s}, 3 \mathrm{H})$.

${ }^{13}$ C-NMR $\left(75 \mathrm{MHz}, \mathrm{CDCl}_{3}\right): \delta=159.5\left(\mathrm{C}_{\mathrm{q}}\right), 156.3\left(\mathrm{C}_{\mathrm{q}}\right), 149.4(\mathrm{CH}), 146.6\left(\mathrm{C}_{\mathrm{q}}\right), 144.1\left(\mathrm{C}_{\mathrm{q}}\right), 135.4$ $(\mathrm{CH}), 131.3(\mathrm{CH}), 131.1\left(\mathrm{C}_{\mathrm{q}}\right), 130.1(\mathrm{CH}), 129.8\left(\mathrm{q},{ }^{2} J_{\mathrm{C}-\mathrm{F}}=32.1 \mathrm{~Hz}, \mathrm{C}_{\mathrm{q}}\right), 128.1(\mathrm{CH}), 125.1\left(\mathrm{q},{ }^{3} J_{\mathrm{C}-\mathrm{F}}=\right.$ $3.5 \mathrm{~Hz}, \mathrm{CH}), 124.1\left(\mathrm{q},{ }^{1} J_{\mathrm{C}-\mathrm{F}}=273 \mathrm{~Hz}, \mathrm{C}_{\mathrm{q}}\right), 123.8(\mathrm{CH}), 121.5(\mathrm{CH}), 114.3(\mathrm{CH}), 55.2\left(\mathrm{CH}_{3}\right)$.

${ }^{19} \mathbf{F}-\mathrm{NMR}\left(282 \mathrm{MHz}, \mathrm{CDCl}_{3}\right): \delta=-62.5(\mathrm{~m})$.

IR (ATR): 2963, 1608, 1579, 1510, 1409, 1239, 1108, 993, 801, $557 \mathrm{~cm}^{-1}$.

MS (EI) $\mathrm{m} / z$ (relative intensity): 355 ([M] $\left.{ }^{+}, 30\right), 354$ (100), 311 (25).

HR-MS (EI): $m / z$ calcd. for $\left[\mathrm{C}_{21} \mathrm{H}_{16} \mathrm{~F}_{3} \mathrm{NO}\right]^{+}[\mathrm{M}]^{+} 355.1184$, found 355.1187 .

The spectral data are in accordance with those reported in the literature. ${ }^{[36 a]}$<smiles>FC(F)(F)c1ccc(C(=Cc2ccccn2)c2ccc(C(F)(F)F)cc2)cc1</smiles>

2-\{2,2-Bis[4-(trifluoromethyl)phenyl]vinyl\}pyridine (10df): The general procedure A was followed using (E)-2-[4-(trifluoromethyl)styryl]pyridine (9d) (125 mg, $0.50 \mathrm{mmol}, 1.00$ equiv) and 1-bromo-4(trifluoromethyl)benzene (2f) (169 $\mathrm{mg}, 0.75 \mathrm{mmol}, 1.50$ equiv). Isolation by column chromatography (n-hexane/EtOAc: $20 / 1)$ yielded 10df (151 mg, 77\%) as a colorless solid.

M.p.: $109^{\circ} \mathrm{C}$. 
${ }^{1} \mathbf{H}-\mathbf{N M R}\left(300 \mathrm{MHz}, \mathrm{CDCl}_{3}\right): \delta=8.52(\mathrm{ddd}, J=4.9,1.8,0.9 \mathrm{~Hz}, 1 \mathrm{H}), 7.67-7.59(\mathrm{~m}, 4 \mathrm{H}), 7.47-7.38$ (m, 3H), 7.37-7.32 (m, 2H), 7.26 (s, 1H), 7.09 (ddd, $J=7.5,4.9,1.1 \mathrm{~Hz}, 1 \mathrm{H}), 6.70$ (ddd, $J=8.1,1.1$, $0.9 \mathrm{~Hz}, 1 \mathrm{H})$.

${ }^{13}$ C-NMR $\left(75 \mathrm{MHz}, \mathrm{CDCl}_{3}\right): \delta=155.3\left(\mathrm{C}_{\mathrm{q}}\right), 149.6(\mathrm{CH}), 145.3\left(\mathrm{C}_{\mathrm{q}}\right), 143.0\left(\mathrm{C}_{\mathrm{q}}\right), 142.9\left(\mathrm{C}_{\mathrm{q}}\right), 135.7$ $(\mathrm{CH}), 131.3(\mathrm{CH}), 130.5(\mathrm{CH}), 130.0\left(\mathrm{q},{ }^{2} J_{\mathrm{C}-\mathrm{F}}=32.5 \mathrm{~Hz}, \mathrm{C}_{\mathrm{q}}\right), 130.0\left(\mathrm{q},{ }^{2} J_{\mathrm{C}-\mathrm{F}}=32.5 \mathrm{~Hz}, \mathrm{C}_{\mathrm{q}}\right), 128.0$ $(\mathrm{CH}), 125.8\left(\mathrm{q},{ }^{3} J_{\mathrm{C}-\mathrm{F}}=3.8 \mathrm{~Hz}, \mathrm{CH}\right), 125.4\left(\mathrm{q},{ }^{3} J_{\mathrm{C}-\mathrm{F}}=3.8 \mathrm{~Hz}, \mathrm{CH}\right), 124.0(\mathrm{CH}), 123.9\left(\mathrm{q},{ }^{1} J_{\mathrm{C}-\mathrm{F}}=\right.$ $\left.275 \mathrm{~Hz}, \mathrm{C}_{\mathrm{q}}\right), 123.8\left(\mathrm{q},{ }^{1} J_{\mathrm{C}-\mathrm{F}}=275 \mathrm{~Hz}, \mathrm{C}_{\mathrm{q}}\right), 122.0(\mathrm{CH})$.

${ }^{19} \mathbf{F}-\mathrm{NMR}\left(282 \mathrm{MHz}, \mathrm{CDCl}_{3}\right): \delta=-62.6(\mathrm{~m})$.

IR (ATR): 1578, 1319, 1163, 1103, 1064, 1017, 837, 769, $739 \mathrm{~cm}^{-1}$.

MS (EI) $m / z$ (relative intensity): 393 ([M] $\left.]^{+}, 40\right), 392$ (100), 322 (15), 248 (10).

HR-MS (ESI): $m / z$ calcd. for $\left[\mathrm{C}_{21} \mathrm{H}_{14} \mathrm{~F}_{6} \mathrm{~N}\right]^{+}[\mathrm{M}+\mathrm{H}]^{+} 394.1025$, found 394.1026.<smiles>CC(=O)c1ccc(C(=Cc2ccccn2)c2ccc(C(F)(F)F)cc2)cc1</smiles>

(E)-1-\{4-[2-(Pyridin-2-yl)-1-(4-(trifluoromethyl)phenyl)vinyl]phenyl\}ethan-1-one (10dh): The general procedure $\mathbf{A}$ was followed using (E)-2-[4-(trifluoromethyl)styryl]pyridine (9d) (125 mg, $0.50 \mathrm{mmol}, 1.00$ equiv) and 1-(4-bromophenyl)ethan-1-one (2h) (148 mg, $0.75 \mathrm{mmol}, 1.50$ equiv). Isolation by column chromatography ( $n$-hexane/EtOAc: 20/1) yielded 10dh (143 $\mathrm{mg}, 78 \%$ ) as a colorless solid.

M.p.: $101^{\circ} \mathrm{C}$.

${ }^{1} \mathbf{H}-\mathbf{N M R}\left(300 \mathrm{MHz}, \mathrm{CDCl}_{3}\right): \delta=8.52(\mathrm{ddd}, J=4.9,1.9,0.9 \mathrm{~Hz}, 1 \mathrm{H}), 7.96-7.90(\mathrm{~m}, 2 \mathrm{H}), 7.57-7.54$ (m, 2H), $7.40(\mathrm{~d}, J=8.1 \mathrm{~Hz}, 2 \mathrm{H}), 7.34(\mathrm{ddd}, J=8.1,7.5,1.9 \mathrm{~Hz}, 1 \mathrm{H}), 7.30-7.25(\mathrm{~m}, 2 \mathrm{H}), 7.21$ (s, $1 \mathrm{H}), 7.03$ (ddd, $J=7.5,4.9,1.1 \mathrm{~Hz}, 1 \mathrm{H}), 6.73(\mathrm{ddd}, J=8.1,1.1,0.9 \mathrm{~Hz}, 1 \mathrm{H}), 2.63$ (s, 3H).

${ }^{13}$ C-NMR $\left(75 \mathrm{MHz}, \mathrm{CDCl}_{3}\right): \delta=197.6\left(\mathrm{C}_{\mathrm{q}}\right), 155.4\left(\mathrm{C}_{\mathrm{q}}\right), 149.5(\mathrm{CH}), 146.3\left(\mathrm{C}_{\mathrm{q}}\right), 144.3\left(\mathrm{C}_{\mathrm{q}}\right), 143.3$ $\left(\mathrm{C}_{\mathrm{q}}\right), 136.6(\mathrm{CH}), 135.7\left(\mathrm{C}_{\mathrm{q}}\right), 131.2(\mathrm{CH}), 130.4(\mathrm{CH}), 130.3\left(\mathrm{q},{ }^{2} J_{\mathrm{C}-\mathrm{F}}=32.5 \mathrm{~Hz}, \mathrm{C}_{\mathrm{q}}\right), 128.8(\mathrm{CH})$, $128.0(\mathrm{CH}), 125.3\left(\mathrm{q},{ }^{3} J_{\mathrm{C}-\mathrm{F}}=3.8 \mathrm{~Hz}, \mathrm{CH}\right), 124.1\left(\mathrm{q},{ }^{1} J_{\mathrm{C}-\mathrm{F}}=270 \mathrm{~Hz}, \mathrm{C}_{\mathrm{q}}\right), 124.0(\mathrm{CH}), 121.9(\mathrm{CH}), 26.6$ $\left(\mathrm{CH}_{3}\right)$.

${ }^{19}$ F-NMR $\left(282 \mathrm{MHz}, \mathrm{CDCl}_{3}\right): \delta=-62.6(\mathrm{~m})$.

IR (ATR): 1672, 1322, 1268, 1162, 1136, 1109, 1017, 834, 740, $607 \mathrm{~cm}^{-1}$.

MS (EI) $m / z$ (relative intensity): 367 ([M] $\left.]^{+}, 30\right), 366$ (100), 322 (15).

HR-MS (ESI): $\mathrm{m} / z$ calcd. for $\left[\mathrm{C}_{22} \mathrm{H}_{17} \mathrm{~F}_{3} \mathrm{NO}\right]^{+}[\mathrm{M}+\mathrm{H}]^{+} 368.1257$, found 368.1259. 
<smiles>COc1ccc(C(=C(C)c2ccccn2)c2ccc(C)cc2)cc1</smiles>

(Z)-2-[1-(4-Methoxyphenyl)-1-(p-tolyl)prop-1-en-2-yl]pyridine (10eb): A modified general procedure $\mathbf{A}$ was followed at $140{ }^{\circ} \mathrm{C}$ in $\mathrm{PhMe}(0.50 \mathrm{~mL}, 1.00 \mathrm{M})$ using (E)-2-[1-(4methylphenyl)prop-1-en-2-yl]pyridine (9e) $(113 \mathrm{mg}, 0.50 \mathrm{mmol}, 1.00$ equiv) and 4-bromoanisole (2b) (140 mg, $0.75 \mathrm{mmol}, 1.50$ equiv). Isolation by column chromatography ( $n$-hexane/EtOAc: 20/1) yielded 10eb $(82.0 \mathrm{mg}, 52 \%, Z / E=12: 1)$ as a pale red solid.

M.p.: $105^{\circ} \mathrm{C}$.

${ }^{1}$ H-NMR $\left(500 \mathrm{MHz}, \mathrm{CDCl}_{3}\right): \delta=8.55(\mathrm{ddd}, J=4.9,1.9,0.9 \mathrm{~Hz}, 1 \mathrm{H}), 7.30(\mathrm{ddd}, J=8.1,7.5,1.9 \mathrm{~Hz}$, 1H), 7.15-7.12 (m, 4H), 6.97 (ddd, $J=7.5,4.9,1.1 \mathrm{~Hz}, 1 \mathrm{H}), 6.86(\mathrm{ddd}, J=8.1,1.1,0.9 \mathrm{~Hz}, 1 \mathrm{H})$, 6.78-6.72 (m, 2H), 6.59-6.54 (m, 2H), 3.68 (s, 3H), 2.35 (s, 3H), 2.20 (s, 3H).

${ }^{13}$ C-NMR $\left(125 \mathrm{MHz}, \mathrm{CDCl}_{3}\right): \delta=162.6\left(\mathrm{C}_{\mathrm{q}}\right), 158.0\left(\mathrm{C}_{\mathrm{q}}\right), 148.9(\mathrm{CH}), 140.7\left(\mathrm{C}_{\mathrm{q}}\right), 140.3\left(\mathrm{C}_{\mathrm{q}}\right), 136.4$ $\left(\mathrm{C}_{\mathrm{q}}\right), 135.4\left(\mathrm{C}_{\mathrm{q}}\right), 134.8\left(\mathrm{C}_{\mathrm{q}}\right), 131.8(\mathrm{CH}), 129.8(\mathrm{CH}), 129.7(\mathrm{CH}), 128.7(\mathrm{CH}), 125.4(\mathrm{CH}), 120.8$ $(\mathrm{CH}), 113.0(\mathrm{CH}), 55.0\left(\mathrm{CH}_{3}\right), 21.5\left(\mathrm{CH}_{3}\right), 21.2\left(\mathrm{CH}_{3}\right)$.

IR (ATR): 3050, 1580, 1505, 1460, 1289, 1239, 1175, 746, $572 \mathrm{~cm}^{-1}$.

MS (EI) $\mathrm{m} / \mathrm{z}$ (relative intensity): 315 ([M] $\left.{ }^{+}, 40\right), 314$ (100), 300 (10), 206 (10).

HR-MS (ESI): $\mathrm{m} / z$ calcd. for $\left[\mathrm{C}_{22} \mathrm{H}_{22} \mathrm{NO}\right]^{+}[\mathrm{M}+\mathrm{H}]^{+} 316.1696$, found 316.1697 .

\subsubsection{Mechanistic Studies}

\subsubsection{Experiments with Radical Scavengers}

A suspension of (E)-2-styrylpyridine (9a) $(90.5 \mathrm{mg}, 0.50 \mathrm{mmol}, 1.00$ equiv), 4-bromoanisole (2b) (140 mg, $0.75 \mathrm{mmol}, 1.50$ equiv), $\left[\mathrm{RuCl}_{2}(p\right.$-cymene $\left.)\left(n-\mathrm{Bu}_{2} \mathrm{POH}\right)\right]$ (124e) (11.7 mg, $25.0 \mu \mathrm{mol}$, $5.00 \mathrm{~mol} \%), \mathrm{K}_{2} \mathrm{CO}_{3}$ (138 mg, $1.00 \mathrm{mmol}, 2.00$ equiv), a radical scavenger ( $0.50 \mathrm{mmol}, 1.00$ equiv) and $n$-dodecane $(25 \mu \mathrm{L})$ as the internal standard in PhMe $(2.00 \mathrm{~mL}, 0.25 \mathrm{M})$ was heated at $120{ }^{\circ} \mathrm{C}$ for $18 \mathrm{~h}$. At ambient temperature, the conversion was measured by GC.

\subsubsection{KIE Studies}

Two parallel reactions of (E)-2-styrylpyridine $(\mathbf{9 a})\left(90.5 \mathrm{mg}, 0.50 \mathrm{mmol}, 1.00\right.$ equiv) or $[\mathrm{D}]_{1}-9 \mathbf{a}$ (91.5 mg, $0.50 \mathrm{mmol}, 1.00$ equiv), 4-bromoanisole (2b) (140 mg, $0.75 \mathrm{mmol}),\left[\mathrm{RuCl}_{2}(p\right.$-cymene $)(n$ $\left.\left.\mathrm{Bu}_{2} \mathrm{POH}\right)\right](\mathbf{1 2 4 e})(11.7 \mathrm{mg}, 25.0 \mu \mathrm{mol}, 5.0 \mathrm{~mol} \%), \mathrm{K}_{2} \mathrm{CO}_{3}(138 \mathrm{mg}, 1.00 \mathrm{mmol})$ and $n$-dodecane $(25 \mu \mathrm{L})$ in PhMe $(2.0 \mathrm{~mL})$ were placed in a $25 \mathrm{~mL}$ Schlenk tube, equipped with the sensor of a ReactIR ${ }^{\mathrm{TM}} 15$ machine, and heated at $120^{\circ} \mathrm{C}$ under nitrogen atmosphere. The KIE was determined by 
following the corresponding initial reaction rates, which were obtained by integration of the vibrational band of the products $\mathbf{1 0 a b}$ and $[\mathrm{D}]_{1} \mathbf{- 1 0 a b}$ at $1520 \mathrm{~cm}^{-1}$, respectively.

\subsubsection{Hammett Plot Analyses}

\subsection{Hammett Plot with (E)-2-styrylpyridine (9a)}

A suspension of (E)-2-styrylpyridine (9a) $\left(90.5 \mathrm{mg}, 0.50 \mathrm{mmol}, 1.00\right.$ equiv), $p-X-\mathrm{C}_{6} \mathrm{H}_{4} \mathrm{Br} \quad 2$ (0.75 mmol, 1.50 equiv), $\left[\mathrm{RuCl}_{2}(p\right.$-cymene $\left.)\left(n-\mathrm{Bu}_{2} \mathrm{POH}\right)\right](\mathbf{1 2 4 e})(11.7 \mathrm{mg}, 25.0 \mu \mathrm{mol}, 5.00 \mathrm{~mol} \%)$, $\mathrm{K}_{2} \mathrm{CO}_{3}(138 \mathrm{mg}, 1.00 \mathrm{mmol}, 2.00$ equiv) and $n$-dodecane $(25 \mu \mathrm{L})$ in $\mathrm{PhMe}(2.0 \mathrm{~mL}, 0.25 \mathrm{M})$ was stirred at $120{ }^{\circ} \mathrm{C}$. Periodic aliquots $(25 \mu \mathrm{L})$ were removed by a syringe and the conversions were analyzed by GC up to ca. 10-20\% conversion.

Table 27. Reaction rates $k_{\mathrm{X}}$ and $\sigma_{\mathrm{p}}$-values for the Hammett plot analysis with aryl bromides 2 .

\begin{tabular}{ccccc}
\hline Entry & substituent $\boldsymbol{X}$ & $\boldsymbol{\sigma}_{\mathbf{p}}$ & $\boldsymbol{k}_{\mathbf{X}} / \boldsymbol{\mu} \mathbf{m o l ~ \mathbf { ~ m i n } ^ { - 1 }}$ & $\boldsymbol{\operatorname { l o g }}\left(\boldsymbol{k}_{\mathbf{X}} / \boldsymbol{k}_{\mathbf{H}}\right)$ \\
\hline 1 & $\mathrm{NMe}_{2}$ & -0.83 & 0.319 & -0.340 \\
2 & $\mathrm{OMe}$ & -0.27 & 0.606 & -0.062 \\
3 & $\mathrm{H}$ & 0 & 0.699 & 0 \\
4 & $\mathrm{Cl}$ & 0.23 & 0.630 & -0.045 \\
5 & $\mathrm{CO}_{2} \mathrm{Et}$ & 0.45 & 0.579 & -0.082 \\
6 & $\mathrm{CF}_{3}$ & 0.54 & 0.557 & -0.099 \\
\hline
\end{tabular}

\subsubsection{Kinetic Order Analysis}

\subsection{Kinetic reaction order with respect to the concentration of $(E)$-2-styrylpyridine (9a)}

The kinetic order with respect to the concentration of $(E)$-2-styrylpyridine (9a) was examined by applying the initial rate method. A suspension of 9a $(0.500,0.370,0.250,0.125 \mathrm{mmol})$, 4bromoanisole (2b) $\quad(140 \mathrm{mg}, \quad 0.75 \mathrm{mmol}), \quad\left[\mathrm{RuCl}_{2}(p\right.$-cymene $\left.)\left(n-\mathrm{Bu}_{2} \mathrm{POH}\right)\right] \quad(\mathbf{1 2 4 e}) \quad(11.7 \mathrm{mg}$, $25.0 \mu \mathrm{mol}), \mathrm{K}_{2} \mathrm{CO}_{3}(138 \mathrm{mg}, 1.00 \mathrm{mmol})$ and $n$-dodecane $(25 \mu \mathrm{L})$ in PhMe $(2.0 \mathrm{~mL})$ was stirred at $120{ }^{\circ} \mathrm{C}$. Periodic aliquots $(25 \mu \mathrm{L})$ were removed by a syringe and analyzed by GC up to ca. $10-20 \%$ conversion.

Table 28. Kinetic reaction order with respect to the concentration of $9 \mathbf{a}$.

\begin{tabular}{|c|c|c|c|c|}
\hline Entry & $c / \mathbf{m o l ~ L}^{-1}$ & $k / \mathrm{mol} \mathrm{L}^{-1} \min ^{-1}$ & $\log \left(c / \mathrm{mol} \mathrm{L}^{-1}\right)$ & $\log \left(k / \mathrm{mol} \mathrm{L}^{-1} \min ^{-1}\right)$ \\
\hline 1 & 0.250 & 3.319 & 0.602 & -3.479 \\
\hline 2 & 0.185 & 2.500 & 0.733 & -3.602 \\
\hline 3 & 0.125 & 1.841 & -0.903 & -3.735 \\
\hline 4 & 0.063 & 0.989 & -1.200 & -4.004 \\
\hline
\end{tabular}




\subsection{Kinetic reaction order with respect to the concentration of 4-bromoanisole ( $2 \mathrm{~b})$}

The kinetic order with respect to the concentration of 4-bromoanisole (2b) was examined by the initial rate method. A suspension of (E)-2-Styrylpyridine (9a) $(90.5 \mathrm{mg}, 0.50 \mathrm{mmol})$, 4-bromoanisole (2b) (4.00, 2.00, 0.600, 0.250, $0.125 \mathrm{mmol}),\left[\mathrm{RuCl}_{2}\left(p\right.\right.$-cymene) $\left.\left(n-\mathrm{Bu}_{2} \mathrm{POH}\right)\right](\mathbf{1 2 4 e})(11.7 \mathrm{mg}, 25.0 \mu \mathrm{mol})$, $\mathrm{K}_{2} \mathrm{CO}_{3}(138 \mathrm{mg}, 1.00 \mathrm{mmol})$ and $n$-dodecane $(25 \mu \mathrm{L})$ in $\mathrm{PhMe}(2.0 \mathrm{~mL})$ was stirred at $120{ }^{\circ} \mathrm{C}$. Periodic aliquots $(25 \mu \mathrm{L})$ were removed by a syringe and analyzed by GC up to ca. 10-20\% conversion.

Table 29. Kinetic reaction order with respect to the concentration of $\mathbf{2 b}$.

\begin{tabular}{|c|c|c|c|c|}
\hline Entry & $c / \mathbf{m o l ~ L}^{-1}$ & $k / \operatorname{mol~L}^{-1} \min ^{-1}$ & $\log \left(c / \mathrm{mol} \mathrm{L}^{-1}\right)$ & $\log \left(k / \operatorname{mol~L}^{-1} \min ^{-1}\right)$ \\
\hline 1 & 2.000 & 2.937 & 0.301 & -3.532 \\
\hline 2 & 1.000 & 2.831 & 0 & -3.548 \\
\hline 3 & 0.300 & 2.483 & -0.523 & -3.605 \\
\hline 4 & 0.125 & 2.023 & -0.903 & -3.694 \\
\hline 5 & 0.063 & 1.469 & -1.201 & -3.833 \\
\hline
\end{tabular}

\subsection{Kinetic reaction order with respect to concentration of $\left[\mathrm{RuCl}_{2}(p\right.$-cymene)(n- $\left.\left.\mathrm{Bu}_{2} \mathrm{POH}\right)\right](124 \mathrm{e})$}

The kinetic order with respect to the concentration of $\left[\mathrm{RuCl}_{2}(p\right.$-cymene $\left.)\left(n-\mathrm{Bu}_{2} \mathrm{POH}\right)\right](\mathbf{1 2 4 e})$ was examined by the initial rate method. A suspension of (E)-2-styrylpyridine (9a) $(90.5 \mathrm{mg}, 0.50 \mathrm{mmol}$, 1.00 equiv), 4-bromoanisole (2b) (140 mg, $0.75 \mathrm{mmol}, 1.50$ equiv), $\left[\mathrm{RuCl}_{2}\left(p\right.\right.$-cymene)( $n$ - $\left.\left.\mathrm{Bu}_{2} \mathrm{POH}\right)\right]$ (124e) $(1.25,2.50,3.50,5.00 \mathrm{~mol} \%), \mathrm{K}_{2} \mathrm{CO}_{3}(138 \mathrm{mg}, 1.00 \mathrm{mmol}, 1.00$ equiv) and $n$-dodecane $(25 \mu \mathrm{L})$ in PhMe $(2.0 \mathrm{~mL})$ was stirred at $120^{\circ} \mathrm{C}$. Periodic aliquots $(25 \mu \mathrm{L})$ were removed by a syringe and analyzed by GC up to ca. $10-20 \%$ conversion.

Table 30. Kinetic reaction order with respect to the concentration of $\mathbf{1 2 4 e}$.

\begin{tabular}{|c|c|c|c|c|}
\hline Entry & $c / \mathbf{m o l ~ L}^{-1}$ & $k / \operatorname{mol~L}^{-1} \min ^{-1}$ & $\log \left(c / \mathrm{mol} \mathrm{L}^{-1}\right)$ & $\log \left(k / \mathrm{mol} \mathrm{L}^{-1} \min ^{-1}\right)$ \\
\hline 1 & 1.25 & 0.697 & 0.097 & -4.157 \\
\hline 2 & 2.50 & 1.560 & 0.398 & -3.807 \\
\hline 3 & 3.50 & 2.565 & 0.544 & -3.591 \\
\hline 4 & 5.00 & 3.319 & 0.699 & -3.479 \\
\hline
\end{tabular}




\subsection{Cobalt(III)-Catalyzed C-H/C - C Functionalization}

\subsubsection{Characterization Data}

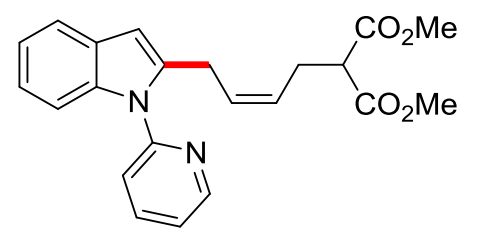

(Z)-Dimethyl 2-\{4-[1-(pyridin-2-yl)-1H-indol-2-yl]but-2-en-1-yl\}malonate (130aa): The general procedure B was followed using 1-(pyridin-2-yl)-1H-indole (105a) $(97.1 \mathrm{mg}, 0.50 \mathrm{mmol}$ ) and dimethyl 2-vinylcyclopropane-1,1-dicarboxylate (125a) $(110 \mathrm{mg}, 0.60 \mathrm{mmol})$. Isolation by column chromatography ( $n$-hexane/EtOAc: $3 / 1)$ yielded 130aa $(176 \mathrm{mg}, 93 \%, E / Z=1: 11)$ as a yellow oil.

${ }^{1} \mathbf{H}-\mathbf{N M R}\left(300 \mathrm{MHz}, \mathrm{CDCl}_{3}\right): \delta=8.64(\mathrm{ddd}, J=4.9,1.9,0.8 \mathrm{~Hz}, 1 \mathrm{H}), 7.86(\mathrm{ddd}, J=8.0,7.6,1.9 \mathrm{~Hz}$, $1 \mathrm{H}), 7.59-7.53(\mathrm{~m}, 1 \mathrm{H}), 7.46-7.41(\mathrm{~m}, 1 \mathrm{H}), 7.36-7.27(\mathrm{~m}, 2 \mathrm{H}), 7.15-7.09(\mathrm{~m}, 2 \mathrm{H}), 6.43$ (d, $J=$ $0.7 \mathrm{~Hz}, 1 \mathrm{H}), 5.64$ (dtt, $J=10.8,7.2,1.0 \mathrm{~Hz}, 1 \mathrm{H}), 5.40$ (dtt, $J=10.8,7.6,1.0 \mathrm{~Hz}, 1 \mathrm{H}), 3.69$ (s, 6H), $3.65(\mathrm{~d}, J=7.2 \mathrm{~Hz}, 2 \mathrm{H}), 3.35(\mathrm{t}, J=7.7 \mathrm{~Hz}, 1 \mathrm{H}), 2.62(\mathrm{ddd}, J=7.7,7.6,1.0 \mathrm{~Hz}, 1.83 \mathrm{H}, Z), 2.54$ (ddd, $J=7.7,7.6,1.0 \mathrm{~Hz}, 0.17 \mathrm{H}, E)$.

${ }^{13}$ C-NMR $\left(125 \mathrm{MHz}, \mathrm{CDCl}_{3}\right): \delta=169.0\left(\mathrm{C}_{\mathrm{q}}\right), 151.1\left(\mathrm{C}_{\mathrm{q}}\right), 149.5(\mathrm{CH}), 139.2\left(\mathrm{C}_{\mathrm{q}}\right), 138.1(\mathrm{CH}), 137.2$ $\left(\mathrm{C}_{\mathrm{q}}\right), 128.8(\mathrm{CH}), 128.4\left(\mathrm{C}_{\mathrm{q}}\right), 126.2(\mathrm{CH}), 121.9(\mathrm{CH}), 121.7(\mathrm{CH}), 120.8(\mathrm{CH}), 120.5(\mathrm{CH}), 119.9$ $(\mathrm{CH}), 109.9(\mathrm{CH}), 102.7(\mathrm{CH}), 52.5\left(\mathrm{CH}_{3}\right), 51.5(\mathrm{CH}), 31.7\left(\mathrm{CH}_{2}, E\right), 30.9\left(\mathrm{CH}_{2}, E\right), 26.7\left(\mathrm{CH}_{2}, \mathrm{Z}\right)$, $25.9\left(\mathrm{CH}_{2}, \mathrm{Z}\right)$.

IR (ATR): 1732, 1586, 1469, 1436, 1150, $745 \mathrm{~cm}^{-1}$.

MS (EI) $m / z$ (relative intensity): 378 ([M] $\left.]^{+}, 30\right), 247$ (100), 219 (90), 206 (70).

HR-MS (EI): $m / z$ calcd. for $\left[\mathrm{C}_{22} \mathrm{H}_{22} \mathrm{~N}_{2} \mathrm{O}_{4}\right]^{+}[\mathrm{M}]^{+} 378.1574$, found 378.1578 .<smiles>COCC(C/C=C\Cc1cc2cc(OC)ccc2n1-c1ccccn1)C(C)=O</smiles>

\section{(Z)-Dimethyl 2-\{4-[5-methoxy-1-(pyridin-2-yl)-1H-indol-2-yl]but-2-en-1-yl\}malonate (130ba):}

The general procedure $\mathbf{B}$ was followed using 5-methoxy-1-(pyridin-2-yl)- $1 H$-indole (105b) (112 mg, $0.50 \mathrm{mmol}$ ) and dimethyl 2-vinylcyclopropane-1,1-dicarboxylate (125a) (110 mg, $0.60 \mathrm{mmol}$ ). Isolation by column chromatography ( $n$-hexane/EtOAc: $3 / 1)$ yielded 130ba $(188 \mathrm{mg}, 92 \%, E / Z=$ 1:13) as a colorless oil.

${ }^{1}$ H-NMR $\left(500 \mathrm{MHz}, \mathrm{CDCl}_{3}\right): \delta=8.61(\mathrm{ddd}, J=4.9,1.9,0.8 \mathrm{~Hz}, 1 \mathrm{H}), 7.85(\mathrm{ddd}, J=8.1,7.5,1.9 \mathrm{~Hz}$, $1 \mathrm{H}), 7.40(\mathrm{ddd}, J=8.1,1.0,0.8 \mathrm{~Hz}, 1 \mathrm{H}), 7.26(\mathrm{ddd}, J=7.5,4.9,1.0 \mathrm{~Hz}, 1 \mathrm{H}), 7.24-7.20(\mathrm{~m}, 1 \mathrm{H}), 7.01$ $(\mathrm{d}, J=2.6 \mathrm{~Hz}, 1 \mathrm{H}), 6.76(\mathrm{dd}, J=8.9,2.6 \mathrm{~Hz}, 1 \mathrm{H}), 6.34(\mathrm{~d}, J=0.8 \mathrm{~Hz}, 1 \mathrm{H}), 5.61(\mathrm{dtt}, J=10.7,7.6$, 
$1.5 \mathrm{~Hz}, 1 \mathrm{H}), 5.38(\mathrm{dtt}, J=10.7,7.7,1.0 \mathrm{~Hz}, 1 \mathrm{H}), 3.82(\mathrm{~s}, 3 \mathrm{H}), 3.68(\mathrm{~s}, 6 \mathrm{H}), 3.58(\mathrm{~d}, J=7.6 \mathrm{~Hz}, 2 \mathrm{H})$, $3.34(\mathrm{t}, J=7.7 \mathrm{~Hz}, 1 \mathrm{H}), 2.61$ (ddd, $J=7.7,7.6,1.5 \mathrm{~Hz}, 1.86 \mathrm{H}, Z), 2.52$ (ddd, $J=7.7,7.6,1.5 \mathrm{~Hz}$, $0.14 \mathrm{H}, E)$.

${ }^{13}$ C-NMR $\left(125 \mathrm{MHz}, \mathrm{CDCl}_{3}\right): \delta=169.2\left(\mathrm{C}_{\mathrm{q}}\right), 154.8\left(\mathrm{C}_{\mathrm{q}}\right), 151.4\left(\mathrm{C}_{\mathrm{q}}\right), 149.5(\mathrm{CH}), 139.8\left(\mathrm{C}_{\mathrm{q}}\right), 138.2$ $(\mathrm{CH}), 132.4\left(\mathrm{C}_{\mathrm{q}}\right), 129.1\left(\mathrm{C}_{\mathrm{q}}\right), 129.0(\mathrm{CH}), 126.3(\mathrm{CH}), 121.7(\mathrm{CH}), 120.6(\mathrm{CH}), 111.3(\mathrm{CH}), 110.9$ $(\mathrm{CH}), 102.7(\mathrm{CH}), 102.1(\mathrm{CH}), 55.8\left(\mathrm{CH}_{3}\right), 52.5\left(\mathrm{CH}_{3}\right), 51.5(\mathrm{CH}), 31.7\left(\mathrm{CH}_{2}, E\right), 30.6\left(\mathrm{CH}_{2}, E\right), 26.7$ $\left(\mathrm{CH}_{2}, \mathrm{Z}\right), 25.9\left(\mathrm{CH}_{2}, \mathrm{Z}\right)$.

IR (ATR): 1732, 1582, 1470, 1434, 1202, 1030, $839 \mathrm{~cm}^{-1}$.

MS (EI) $\mathrm{m} / z$ (relative intensity): 408 ([M] $\left.]^{+}, 30\right), 277$ (80), 249 (100), 236 (40).

HR-MS (EI): $m / z$ calcd. for $\left[\mathrm{C}_{23} \mathrm{H}_{24} \mathrm{~N}_{2} \mathrm{O}_{5}\right]^{+}[\mathrm{M}]^{+} 408.1685$, found 408.1700 .

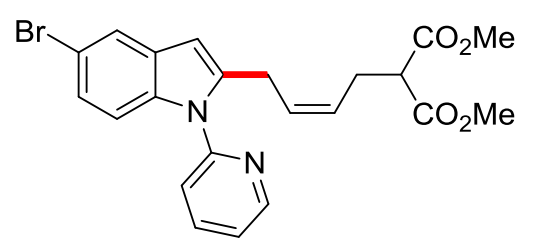

(Z)-Dimethyl 2-\{4-[5-bromo-1-(pyridin-2-yl)-1H-indol-2-yl]but-2-en-1-yl\}malonate (130ca): The general procedure $\mathbf{B}$ was followed using 5-bromo-1-(pyridin-2-yl)-1H-indole (105c) (137 mg, $0.50 \mathrm{mmol}$ ) and dimethyl 2-vinylcyclopropane-1,1-dicarboxylate (125a) (110 mg, $0.60 \mathrm{mmol}$ ). Isolation by column chromatography ( $n$-hexane/EtOAc: $3 / 1)$ yielded 130ca $(203 \mathrm{mg}, 89 \%, E / Z=1: 9)$ as a yellow oil.

${ }^{1}$ H-NMR $\left(400 \mathrm{MHz}, \mathrm{CDCl}_{3}\right): \delta=8.65(\mathrm{ddd}, J=4.9,2.0,0.8 \mathrm{~Hz}, 1 \mathrm{H}), 7.89(\mathrm{ddd}, J=7.9,7.5,2.0 \mathrm{~Hz}$, 1H), 7.66-7.63 (m, 1H), 7.39 (ddd, $J=7.9,1.0,0.8 \mathrm{~Hz}, 1 \mathrm{H}), 7.35-7.30(\mathrm{~m}, 1 \mathrm{H}), 7.19-7.16$ (m, 2H), $6.34(\mathrm{~d}, J=0.8 \mathrm{~Hz}, 1 \mathrm{H}), 5.59(\mathrm{dtt}, J=10.8,7.6,1.4 \mathrm{~Hz}, 1 \mathrm{H}), 5.39(\mathrm{dtt}, J=10.8,7.6,1.2 \mathrm{~Hz}, 1 \mathrm{H}), 3.68$ (s, 6H), $3.60(\mathrm{~d}, J=7.6 \mathrm{~Hz}, 2 \mathrm{H}), 3.34(\mathrm{t}, J=7.6 \mathrm{~Hz}, 1 \mathrm{H}), 2.58$ (ddd, $J=7.6,7.6,1.4 \mathrm{~Hz}, 1.80 \mathrm{H}, Z$ ), 2.52 (ddd, $J=7.6,7.6,1.4 \mathrm{~Hz}, 0.20 \mathrm{H}, E$ ).

${ }^{13}$ C-NMR $\left(100 \mathrm{MHz}, \mathrm{CDCl}_{3}\right): \delta=169.2\left(\mathrm{C}_{\mathrm{q}}\right), 150.9\left(\mathrm{C}_{\mathrm{q}}\right), 149.8(\mathrm{CH}), 140.7\left(\mathrm{C}_{\mathrm{q}}\right), 138.5(\mathrm{CH}), 136.0$ $\left(\mathrm{C}_{\mathrm{q}}\right), 130.2\left(\mathrm{C}_{\mathrm{q}}\right), 128.5(\mathrm{CH}), 127.8(\mathrm{CH}), 124.6(\mathrm{CH}), 122.4(\mathrm{CH}), 121.0(\mathrm{CH}), 113.8\left(\mathrm{C}_{\mathrm{q}}\right), 111.6$ $(\mathrm{CH}), 102.2(\mathrm{CH}), 95.7(\mathrm{CH}), 52.6\left(\mathrm{CH}_{3}\right), 51.4(\mathrm{CH}), 31.6\left(\mathrm{CH}_{2}, E\right), 30.8\left(\mathrm{CH}_{2}, E\right), 26.7\left(\mathrm{CH}_{2}, \mathrm{Z}\right)$, $25.9\left(\mathrm{CH}_{2}, \mathrm{Z}\right)$.

IR (ATR): 1731, 1586, 1470, 1435, 1148, 783, $734 \mathrm{~cm}^{-1}$.

MS (EI) $\mathrm{m} / z$ (relative intensity): 456 ([M, $\left.\left.{ }^{79} \mathrm{Br}\right]^{+}, 25\right), 325$ (100), 297 (90), 286 (40), 245 (55).

HR-MS (EI): $\mathrm{m} / z$ calcd. for $\left[\mathrm{C}_{22} \mathrm{H}_{21}{ }^{79} \mathrm{BrN}_{2} \mathrm{O}_{4}\right]^{+}[\mathrm{M}]^{+} 456.0679$, found 456.0689 . 


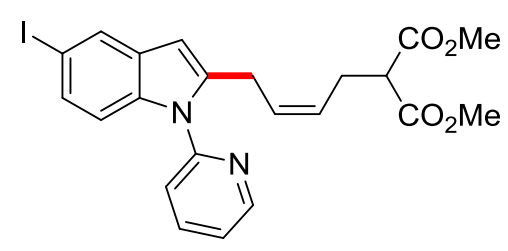

(Z)-Dimethyl 2-\{4-[5-iodo-1-(pyridin-2-yl)-1H-indol-2-yl]but-2-en-1-yl\}malonate (130da): The general procedure $\mathbf{B}$ was followed using 5-iodo-1-(pyridin-2-yl)-1H-indole (105d) (160 mg, $0.50 \mathrm{mmol}$ ) and dimethyl 2-vinylcyclopropane-1,1-dicarboxylate (125a) $(110 \mathrm{mg}, 0.60 \mathrm{mmol})$. Isolation by column chromatography ( $n$-hexane/EtOAc: $3 / 1)$ yielded 130da $(224 \mathrm{mg}, 89 \%, E / Z=$ $1: 14)$ as a yellow oil.

${ }^{1}$ H-NMR $\left(500 \mathrm{MHz}, \mathrm{CDCl}_{3}\right): \delta=8.64(\mathrm{ddd}, J=4.9,1.9,0.8 \mathrm{~Hz}, 1 \mathrm{H}), 7.89(\mathrm{ddd}, J=7.9,7.5,2.1 \mathrm{~Hz}$, $1 \mathrm{H}), 7.86(\mathrm{~d}, J=1.6 \mathrm{~Hz}, 1 \mathrm{H}), 7.39-7.30(\mathrm{~m}, 3 \mathrm{H}), 7.08-7.04(\mathrm{~m}, 1 \mathrm{H}), 6.32(\mathrm{~d}, J=0.8 \mathrm{~Hz}, 1 \mathrm{H}), 5.59$ $(\mathrm{dtt}, J=10.8,7.6,1.4 \mathrm{~Hz}, 1 \mathrm{H}), 5.39(\mathrm{dtt}, J=10.8,7.4,1.0 \mathrm{~Hz}, 1 \mathrm{H}), 3.68(\mathrm{~s}, 6 \mathrm{H}), 3.58(\mathrm{~d}, J=7.6 \mathrm{~Hz}$, 2H), 3.33 (t, $J=7.5 \mathrm{~Hz}, 1 \mathrm{H}), 2.58$ (ddd, $J=7.5,7.4,1.4 \mathrm{~Hz}, 1.87 \mathrm{H}, Z), 2.52(\mathrm{ddd}, J=7.5,7.4,1.4 \mathrm{~Hz}$, $0.13 \mathrm{H}, E)$.

${ }^{13} \mathrm{C}-\mathrm{NMR}\left(125 \mathrm{MHz}, \mathrm{CDCl}_{3}\right): \delta=169.2\left(\mathrm{C}_{\mathrm{q}}\right), 150.8\left(\mathrm{C}_{\mathrm{q}}\right), 149.8(\mathrm{CH}), 140.3\left(\mathrm{C}_{\mathrm{q}}\right), 138.5(\mathrm{CH}), 136.5$ $\left(\mathrm{C}_{\mathrm{q}}\right), 130.9\left(\mathrm{C}_{\mathrm{q}}\right), 130.0(\mathrm{CH}), 128.7(\mathrm{CH}), 128.4(\mathrm{CH}), 126.7(\mathrm{CH}), 122.4(\mathrm{CH}), 120.1(\mathrm{CH}), 112.1$ $(\mathrm{CH}), 101.9(\mathrm{CH}), 84.1\left(\mathrm{C}_{\mathrm{q}}\right), 52.5\left(\mathrm{CH}_{3}\right), 51.4(\mathrm{CH}), 31.7\left(\mathrm{CH}_{2}, E\right), 30.8\left(\mathrm{CH}_{2}, E\right), 26.7\left(\mathrm{CH}_{2}, \mathrm{Z}\right), 25.9$ $\left(\mathrm{CH}_{2}, \mathrm{Z}\right)$.

IR (ATR): 1731, 1586, 1470, 1436, 1149, 784, $730 \mathrm{~cm}^{-1}$.

MS (EI) $\mathrm{m} / \mathrm{z}$ (relative intensity): 504 ([M] $\left.{ }^{+}, 40\right), 373$ (100), 345 (95), 332 (30), 245 (45).

HR-MS (EI): $m / z$ calcd. for $\left[\mathrm{C}_{22} \mathrm{H}_{21} \mathrm{IN}_{2} \mathrm{O}_{4}\right]^{+}[\mathrm{M}]^{+}$504.0541, found 504.0549.

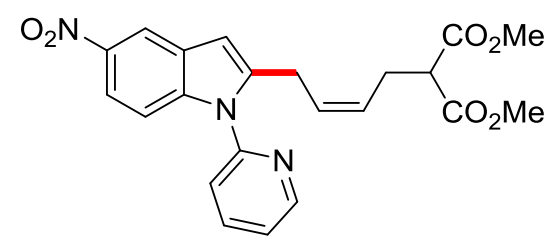

(Z)-Dimethyl 2-\{4-[5-nitro-1-(pyridin-2-yl)-1H-indol-2-yl]but-2-en-1-yl\}malonate (130ea): The general procedure $\mathbf{B}$ was followed using 5-nitro-1-(pyridin-2-yl)-1H-indole (105e) (120 mg, $0.50 \mathrm{mmol}$ ) and dimethyl 2-vinylcyclopropane-1,1-dicarboxylate (125a) (110 mg, $0.60 \mathrm{mmol})$. Isolation by column chromatography ( $n$-hexane/EtOAc: $3 / 1)$ yielded 130ea $(187 \mathrm{mg}, 88 \%, E / Z=1: 17)$ as a yellow oil.

${ }^{1} \mathbf{H}-\mathbf{N M R}\left(300 \mathrm{MHz}, \mathrm{CDCl}_{3}\right): \delta=8.67$ (ddd, $\left.J=4.8,2.0,0.9 \mathrm{~Hz}, 1 \mathrm{H}\right), 8.48-8.43(\mathrm{~m}, 1 \mathrm{H}), 8.00-7.91$ (m, 2H), 7.44-7.38 (m, 2H), 7.28-7.24 (m, 1H), $6.55(\mathrm{~d}, J=0.8 \mathrm{~Hz}, 1 \mathrm{H}), 5.59$ (dtt, $J=10.8,7.5$, $1.4 \mathrm{~Hz}, 1 \mathrm{H}), 5.43$ (dtt, $J=10.8,7.5,1.0 \mathrm{~Hz}, 1 \mathrm{H}), 3.67$ (s, 6H), 3.59 (d, $J=7.5 \mathrm{~Hz}, 1.89 \mathrm{H}, Z), 3.49$ (d, $J=7.5 \mathrm{~Hz}, 0.11 \mathrm{H}, E), 3.35(\mathrm{t}, J=7.7 \mathrm{~Hz}, 1 \mathrm{H}), 2.58(\mathrm{ddd}, J=7.7,7.5,1.4 \mathrm{~Hz}, 2 \mathrm{H})$. 
${ }^{13}$ C-NMR $\left(125 \mathrm{MHz}, \mathrm{CDCl}_{3}\right): \delta=169.0\left(\mathrm{C}_{\mathrm{q}}\right), 150.0\left(\mathrm{C}_{\mathrm{q}}\right), 149.9(\mathrm{CH}), 142.8\left(\mathrm{C}_{\mathrm{q}}\right), 142.3\left(\mathrm{C}_{\mathrm{q}}\right), 140.1$ $\left(\mathrm{C}_{\mathrm{q}}\right), 138.7(\mathrm{CH}), 127.7\left(\mathrm{C}_{\mathrm{q}}\right), 127.6(\mathrm{CH}), 127.2(\mathrm{CH}), 123.1(\mathrm{CH}), 121.1(\mathrm{CH}), 117.3(\mathrm{CH}), 116.8$ $(\mathrm{CH}), 110.0(\mathrm{CH}), 104.0(\mathrm{CH}), 52.5\left(\mathrm{CH}_{3}\right), 51.3(\mathrm{CH}), 31.5\left(\mathrm{CH}_{2}, E\right), 30.9\left(\mathrm{CH}_{2}, E\right), 26.7\left(\mathrm{CH}_{2}, \mathrm{Z}\right)$, $25.9\left(\mathrm{CH}_{2}, \mathrm{Z}\right)$.

IR (ATR): 1732, 1587, 1511, 1436, 1326, 908, $727 \mathrm{~cm}^{-1}$.

MS (EI) $m / z$ (relative intensity): 423 ([M] $\left.]^{+}, 20\right), 292$ (100), 264 (80), 251 (40), 205 (25).

HR-MS (EI): $m / z$ calcd. for $\left[\mathrm{C}_{22} \mathrm{H}_{21} \mathrm{~N}_{3} \mathrm{O}_{6}\right]^{+}[\mathrm{M}]^{+} 423.1425$, found 423.1420 .

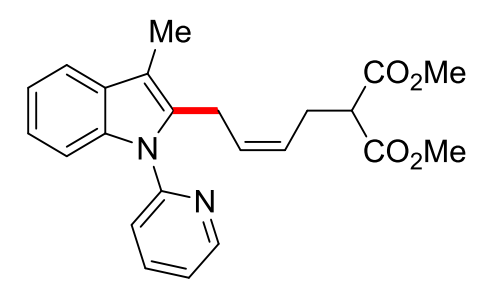

(Z)-Dimethyl 2-\{4-[3-methyl-1-(pyridin-2-yl)-1H-indol-2-yl]but-2-en-1-yl\}malonate (130fa): The general procedure B was followed using 3-methyl-1-(pyridin-2-yl)-1H-indole (105f) (104 mg, $0.50 \mathrm{mmol}$ ) and dimethyl 2-vinylcyclopropane-1,1-dicarboxylate (2a) $(110 \mathrm{mg}, 0.60 \mathrm{mmol})$. Isolation by column chromatography ( $n$-hexane/EtOAc: $3 / 1)$ yielded $130 \mathrm{fa}(182 \mathrm{mg}, 93 \%, E / Z=1: 5)$ as a yellow oil.

${ }^{1} \mathrm{H}-\mathrm{NMR}\left(500 \mathrm{MHz}, \mathrm{CDCl}_{3}\right): \delta=8.61(\mathrm{ddd}, J=4.9,1.9,0.8 \mathrm{~Hz}, 1 \mathrm{H}), 7.85(\mathrm{ddd}, J=8.0,7.5,2.0 \mathrm{~Hz}$, 1H), 7.54-7.51 (m, 1H), 7.39 (ddd, $J=8.0,1.0,0.8 \mathrm{~Hz}, 1 \mathrm{H}), 7.34-7.25(\mathrm{~m}, 2 \mathrm{H}), 7.15-7.10(\mathrm{~m}, 2 \mathrm{H})$, $5.48(\mathrm{dtt}, J=15.2,7.6,1.4 \mathrm{~Hz}, 0.17 \mathrm{H}, E), 5.34(\mathrm{dtt}, J=10.6,7.6,1.4 \mathrm{~Hz}, 0.83 \mathrm{H}, Z), 5.18-5.09$ (m, $1 \mathrm{H}), 3.70(\mathrm{~s}, 5.02 \mathrm{H}, Z), 3.69(\mathrm{~d}, J=7.6 \mathrm{~Hz}, 2 \mathrm{H}), 3.63(\mathrm{~s}, 0.98 \mathrm{H}, E), 3.25(\mathrm{t}, J=7.6 \mathrm{~Hz}, 1 \mathrm{H}), 2.50$ (ddd, $J=7.6,7.6,1.4 \mathrm{~Hz}, 1.68 \mathrm{H}, Z), 2.42(\mathrm{ddd}, J=7.6,7.6,1.4 \mathrm{~Hz}, 0.32 \mathrm{H}, E), 2.31$ (s, 2.52H, Z), 2.27 (s, $0.48 \mathrm{H}, E)$.

${ }^{13}$ C-NMR $\left(125 \mathrm{MHz}, \mathrm{CDCl}_{3}\right): \delta=169.2\left(\mathrm{C}_{\mathrm{q}}\right), 151.6\left(\mathrm{C}_{\mathrm{q}}\right), 149.6\left(\mathrm{C}_{\mathrm{q}}\right), 138.2(\mathrm{CH}), 136.5\left(\mathrm{C}_{\mathrm{q}}\right), 134.7$ $(\mathrm{CH}), 129.8\left(\mathrm{C}_{\mathrm{q}}\right), 129.3(\mathrm{CH}), 125.0(\mathrm{CH}), 121.9(\mathrm{CH}), 121.6(\mathrm{CH}), 121.0(\mathrm{CH}), 120.2(\mathrm{CH}), 118.2$ $\left(\mathrm{C}_{\mathrm{q}}\right), 110.3(\mathrm{CH}), 109.8(\mathrm{CH}), 52.5\left(\mathrm{CH}_{3}\right), 51.4(\mathrm{CH}), 31.6\left(\mathrm{CH}_{2}, E\right), 28.0\left(\mathrm{CH}_{2}, E\right), 26.7\left(\mathrm{CH}_{2}, \mathrm{Z}\right)$, $23.5\left(\mathrm{CH}_{2}, \mathrm{Z}\right), 8.7\left(\mathrm{CH}_{3}\right)$.

IR (ATR): 1732, 1586, 1470, 1457, 1255, 1125, 1148, $738 \mathrm{~cm}^{-1}$. MS (EI) $\mathrm{m} / \mathrm{z}$ (relative intensity): 392 ([M] $\left.{ }^{+}, 45\right), 261$ (70), 233 (100), 220 (65).

HR-MS (EI): $m / z$ calcd. for $\left[\mathrm{C}_{23} \mathrm{H}_{24} \mathrm{~N}_{2} \mathrm{O}_{4}\right]^{+}[\mathrm{M}]^{+} 392.1736$, found 392.1725 . 


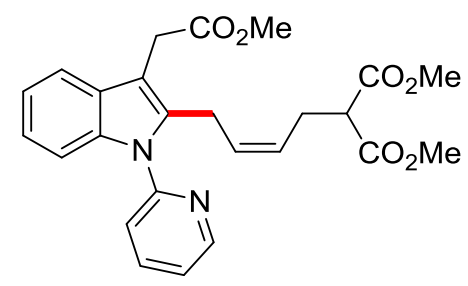

(Z)-Dimethyl 2-\{4-[3-(2-methoxy-2-oxoethyl)-1-(pyridin-2-yl)-1H-indol-2-yl]but-2-en-1-yl\}malonate (130ga): The general procedure $\mathbf{B}$ was followed using methyl 2-(1-(pyridin-2-yl)-1H-indol-3yl)acetate $(\mathbf{1 0 5 g})(133 \mathrm{mg}, 0.50 \mathrm{mmol})$ and dimethyl 2-vinylcyclopropane-1,1-dicarboxylate (125a) (110 mg, $0.60 \mathrm{mmol}$ ). Isolation by column chromatography ( $n$-hexane/EtOAc: $3 / 1)$ yielded 130ga (167 $\mathrm{mg}, 74 \%, E / Z=1: 11)$ as a colorless oil.

${ }^{1}$ H-NMR $\left(500 \mathrm{MHz}, \mathrm{CDCl}_{3}\right): \delta=8.63(\mathrm{ddd}, J=4.9,2.0,0.9 \mathrm{~Hz}, 1 \mathrm{H}), 7.86(\mathrm{ddd}, J=8.0,7.4,2.0 \mathrm{~Hz}$, $1 \mathrm{H}), 7.60-7.57(\mathrm{~m}, 1 \mathrm{H}), 7.40$ (ddd, $J=8.0,0.9,0.9 \mathrm{~Hz}, 1 \mathrm{H}), 7.31-7.26(\mathrm{~m}, 2 \mathrm{H}), 7.17-7.11(\mathrm{~m}, 2 \mathrm{H})$, $5.36(\mathrm{dtt}, J=10.7,7.6,1.4 \mathrm{~Hz}, 1 \mathrm{H}), 5.16(\mathrm{dtt}, J=10.7,7.6,1.4 \mathrm{~Hz}, 1 \mathrm{H}), 3.78(\mathrm{~s}, 2 \mathrm{H}), 3.73(\mathrm{~d}, J=$ $7.6 \mathrm{~Hz}, 2 \mathrm{H}), 3.70(\mathrm{~s}, 6 \mathrm{H}), 3.66(\mathrm{~s}, 3 \mathrm{H}), 3.25(\mathrm{t}, J=7.6 \mathrm{~Hz}, 1 \mathrm{H}), 2.46(\mathrm{ddd}, J=7.6,7.6,1.4 \mathrm{~Hz}, 1.83 \mathrm{H}$, $Z$ ), 2.40 (ddd, $J=7.6,7.6,1.4 \mathrm{~Hz}, 0.17 \mathrm{H}, E)$.

${ }^{13} \mathrm{C}-\mathrm{NMR}\left(125 \mathrm{MHz}, \mathrm{CDCl}_{3}\right): \delta=172.0\left(\mathrm{C}_{\mathrm{q}}\right), 169.2\left(\mathrm{C}_{\mathrm{q}}\right), 151.2\left(\mathrm{C}_{\mathrm{q}}\right), 149.7(\mathrm{CH}), 138.3(\mathrm{CH}), 136.4$ $\left(\mathrm{C}_{\mathrm{q}}\right), 129.2(\mathrm{CH}), 128.3(\mathrm{CH}), 125.4\left(\mathrm{C}_{\mathrm{q}}\right), 122.2(\mathrm{CH}), 122.1\left(\mathrm{C}_{\mathrm{q}}\right), 121.3(\mathrm{CH}), 120.7(\mathrm{CH}), 118.4$ $(\mathrm{CH}), 110.0(\mathrm{CH}), 107.5(\mathrm{CH}), 95.5\left(\mathrm{C}_{\mathrm{q}}\right), 52.5\left(\mathrm{CH}_{3}\right), 51.9\left(\mathrm{CH}_{3}\right), 51.3(\mathrm{CH}), 31.5\left(\mathrm{CH}_{2}, E\right), 30.3$ $\left(\mathrm{CH}_{2}\right), 28.1\left(\mathrm{CH}_{2}, E\right), 26.6\left(\mathrm{CH}_{2}, \mathrm{Z}\right), 23.6\left(\mathrm{CH}_{2}, \mathrm{Z}\right)$.

IR (ATR): 1730, 1586, 1470, 1458, 1147, $731 \mathrm{~cm}^{-1}$.

MS (EI) $m / z$ (relative intensity): 450 ([M] $\left.]^{+}, 30\right) 319$ (80), 291 (100), 259 (40), 245 (45), 219 (50).

HR-MS (EI): $m / z$ calcd. for $\left[\mathrm{C}_{25} \mathrm{H}_{26} \mathrm{~N}_{2} \mathrm{O}_{6}\right]^{+}[\mathrm{M}]^{+} 450.1791$, found 450.1796 .<smiles>COc1cccc2c1cc(C/C=C\CC(C(C)=O)C(C)=O)n2-c1ccccn1</smiles>

(Z)-Dimethyl 2-\{4-[4-methoxy-1-(pyridin-2-yl)-1H-indol-2-yl\}but-2-en-1-yl]malonate (130ha): The general procedure $\mathbf{B}$ was followed using 4-methoxy-1-(pyridin-2-yl)- $1 H$-indole (105h) (112 mg, $0.50 \mathrm{mmol}$ ) and dimethyl 2-vinylcyclopropane-1,1-dicarboxylate (125a) (110 mg, $0.60 \mathrm{mmol})$. Isolation by column chromatography ( $n$-hexane/EtOAc: $3 / 1)$ yielded 130ha $(157 \mathrm{mg}, 77 \%, E / Z=$ 1:13) as a colorless oil.

${ }^{1}$ H-NMR $\left(500 \mathrm{MHz}, \mathrm{CDCl}_{3}\right): \delta=8.61(\mathrm{ddd}, J=4.9,1.9,0.8 \mathrm{~Hz}, 1 \mathrm{H}), 7.85(\mathrm{ddd}, J=8.1,7.5,2.0 \mathrm{~Hz}$, $1 \mathrm{H}), 7.41(\mathrm{ddd}, J=8.1,1.0,0.8 \mathrm{~Hz}, 1 \mathrm{H}), 7.29(\mathrm{ddd}, J=7.5,4.9,1.0 \mathrm{~Hz}, 1 \mathrm{H}), 7.05-7.00(\mathrm{~m}, 1 \mathrm{H})$, 6.94-6.92 (m, 1H), $6.54(\mathrm{~d}, J=7.9 \mathrm{~Hz}, 1 \mathrm{H}), 6.51(\mathrm{~d}, J=0.8 \mathrm{~Hz}, 1 \mathrm{H}), 5.61(\mathrm{dtt}, J=10.8,7.6,1.4 \mathrm{~Hz}$, 
1H), $5.37(\mathrm{dtt}, J=10.8,7.7,1.0 \mathrm{~Hz}, 1 \mathrm{H}), 3.93(\mathrm{~s}, 3 \mathrm{H}), 3.68(\mathrm{~s}, 6 \mathrm{H}), 3.61(\mathrm{~d}, J=7.6 \mathrm{~Hz}, 1.86 \mathrm{H}, Z)$, $3.51(\mathrm{~d}, J=7.6 \mathrm{~Hz}, 0.14 \mathrm{H}, E), 3.34(\mathrm{t}, J=7.7 \mathrm{~Hz}, 1 \mathrm{H}), 2.60$ (ddd, $J=7.7,7.7,1.4 \mathrm{~Hz}, 1.86 \mathrm{H}, Z), 2.52$ (ddd, $J=7.7,7.7,1.4 \mathrm{~Hz}, 0.14 \mathrm{H}, E$ ).

${ }^{13}$ C-NMR $\left(125 \mathrm{MHz}, \mathrm{CDCl}_{3}\right): \delta=169.2\left(\mathrm{C}_{\mathrm{q}}\right), 152.6\left(\mathrm{C}_{\mathrm{q}}\right), 151.4\left(\mathrm{C}_{\mathrm{q}}\right), 149.5(\mathrm{CH}), 139.6\left(\mathrm{C}_{\mathrm{q}}\right), 138.2$ $(\mathrm{CH}), 137.7\left(\mathrm{C}_{\mathrm{q}}\right), 128.8(\mathrm{CH}), 126.2(\mathrm{CH}), 122.5(\mathrm{CH}), 122.1(\mathrm{CH}), 121.0(\mathrm{CH}), 118.7\left(\mathrm{C}_{\mathrm{q}}\right), 103.5$ $(\mathrm{CH}), 100.7(\mathrm{CH}), 99.8(\mathrm{CH}), 55.3\left(\mathrm{CH}_{3}\right), 52.5\left(\mathrm{CH}_{3}\right), 51.5(\mathrm{CH}), 31.6\left(\mathrm{CH}_{2}, E\right), 30.7\left(\mathrm{CH}_{2}, E\right), 26.7$ $\left(\mathrm{CH}_{2}, \mathrm{Z}\right), 25.9\left(\mathrm{CH}_{2}, \mathrm{Z}\right)$.

IR (ATR): 1732, 1587, 1495, 1469, 1255, 1148, 765, $728 \mathrm{~cm}^{-1}$.

MS (EI) $\mathrm{m} / z$ (relative intensity): 408 ([M] $\left.{ }^{+}, 45\right), 277$ (60), 249 (100), 236 (30).

HR-MS (EI): $m / z$ calcd. for $\left[\mathrm{C}_{23} \mathrm{H}_{24} \mathrm{~N}_{2} \mathrm{O}_{5}\right]^{+}[\mathrm{M}]^{+} 408.1680$, found 408.1689 .

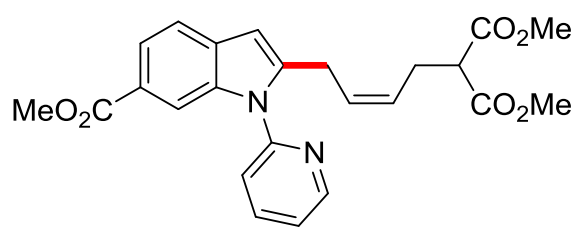

(Z)-Dimethyl 2-\{4-[6-(methoxycarbonyl)-1-(pyridin-2-yl)-1H-indol-2-yl]but-2-en-1-yl\}-malonate (130ia): The general procedure $\mathbf{B}$ was followed using methyl 1-(pyridin-2-yl)-1H-indole-6carboxylate (105i) (126 mg, $0.50 \mathrm{mmol})$ and dimethyl 2-vinylcyclopropane-1,1-dicarboxylate (125a) $(110 \mathrm{mg}, 0.60 \mathrm{mmol})$. Isolation by column chromatography ( $n$-hexane/EtOAc: $3 / 1)$ yielded 130ia (183 $\mathrm{mg}, 84 \%, E / Z=1: 8)$ as a colorless oil.

${ }^{1} \mathbf{H}-\mathbf{N M R}\left(500 \mathrm{MHz}, \mathrm{CDCl}_{3}\right): \delta=8.65(\mathrm{ddd}, J=4.9,1.9,0.8 \mathrm{~Hz}, 1 \mathrm{H}), 8.00-7.98$ (m, 1H), 7.91 (ddd, $J$ $=7.9,7.5,2.0 \mathrm{~Hz}, 1 \mathrm{H}), 7.80(\mathrm{ddd}, J=8.2,1.4,0.8 \mathrm{~Hz}, 1 \mathrm{H}), 7.56-7.53(\mathrm{~m}, 1 \mathrm{H}), 7.45(\mathrm{dd}, J=7.9$, $1.0 \mathrm{~Hz}, 1 \mathrm{H}), 7.35-7.32(\mathrm{~m}, 1 \mathrm{H}), 6.45(\mathrm{~d}, J=0.7 \mathrm{~Hz}, 1 \mathrm{H}), 5.61$ (dtt, $J=10.8,7.6,1.5 \mathrm{~Hz}, 1 \mathrm{H}), 5.41$ (dtt, $J=10.8,7.6,1.0 \mathrm{~Hz}, 1 \mathrm{H}), 3.85$ (s, 3H), 3.69 (s, $0.70 \mathrm{H}, E), 3.67$ (s, 5.30H, Z), 3.64 (d, $J=7.6 \mathrm{~Hz}$, 2H), 3.34 (t, $J=7.7 \mathrm{~Hz}, 1 \mathrm{H}), 2.59$ (ddd, $J=7.7,7.6,1.5 \mathrm{~Hz}, 1.77 \mathrm{H}, Z), 2.53$ (ddd, $J=7.7,7.6,1.5 \mathrm{~Hz}$, $0.23 \mathrm{H}, E)$.

${ }^{13}$ C-NMR $\left(125 \mathrm{MHz}, \mathrm{CDCl}_{3}\right): \delta=169.1\left(\mathrm{C}_{\mathrm{q}}\right), 168.0\left(\mathrm{C}_{\mathrm{q}}\right), 150.6\left(\mathrm{C}_{\mathrm{q}}\right), 149.8(\mathrm{CH}), 143.0\left(\mathrm{C}_{\mathrm{q}}\right), 138.7$ $(\mathrm{CH}), 136.7\left(\mathrm{C}_{\mathrm{q}}\right), 132.2\left(\mathrm{C}_{\mathrm{q}}\right), 128.2(\mathrm{CH}), 126.9(\mathrm{CH}), 123.4\left(\mathrm{C}_{\mathrm{q}}\right), 122.6(\mathrm{CH}), 121.8(\mathrm{CH}), 121.2$ $(\mathrm{CH}), 119.5(\mathrm{CH}), 112.2(\mathrm{CH}), 102.9(\mathrm{CH}), 52.5\left(\mathrm{CH}_{3}, \mathrm{Z}\right), 52.4\left(\mathrm{CH}_{3}, E\right), 51.8\left(\mathrm{CH}_{3}, \mathrm{Z}\right), 51.7\left(\mathrm{CH}_{3}\right.$, E), $51.3(\mathrm{CH}, \mathrm{Z}), 50.7(\mathrm{CH}, E), 31.6\left(\mathrm{CH}_{2}, E\right), 30.8\left(\mathrm{CH}_{2}, E\right), 26.7\left(\mathrm{CH}_{2}, \mathrm{Z}\right), 25.9\left(\mathrm{CH}_{2}, Z\right)$.

IR (ATR): 1731, 1710, 1587, 1470, 1434, 1233, 909, $725 \mathrm{~cm}^{-1}$.

MS (EI) $\mathrm{m} / \mathrm{z}$ (relative intensity): 436 ([M] $\left.]^{+}, 15\right), 305$ (40), 277 (100), 265 (20).

HR-MS (EI): $m / z$ calcd. for $\left[\mathrm{C}_{24} \mathrm{H}_{24} \mathrm{~N}_{2} \mathrm{O}_{6}\right]^{+}[\mathrm{M}]^{+} 436.1629$, found 436.1630 . 


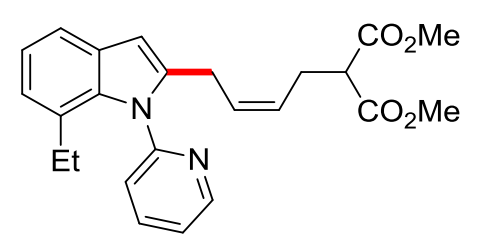

(Z)-Dimethyl 2-\{4-[7-ethyl-1-(pyridin-2-yl)-1H-indol-2-yl]but-2-en-1-yl\}malonate (130ja): The general procedure $\mathbf{B}$ was followed using 7-ethyl-1-(pyridin-2-yl)-1 $H$-indole $(\mathbf{1 0 5 j}) \quad(110 \mathrm{mg}$, $0.50 \mathrm{mmol}$ ) and dimethyl 2-vinylcyclopropane-1,1-dicarboxylate (125a) (110 mg, $0.60 \mathrm{mmol})$. Isolation by column chromatography ( $n$-hexane/EtOAc: $3 / 1)$ yielded 130ja $(138 \mathrm{mg}, 68 \%, E / Z=1: 8)$ as a yellow oil.

${ }^{1}$ H-NMR $\left(500 \mathrm{MHz}, \mathrm{CDCl}_{3}\right): \delta=8.64(\mathrm{ddd}, J=4.8,2.0,0.9 \mathrm{~Hz}, 1 \mathrm{H}), 7.84(\mathrm{ddd}, J=8.0,7.4,2.0 \mathrm{~Hz}$, $1 \mathrm{H}), 7.42-7.38(\mathrm{~m}, 2 \mathrm{H}), 7.33$ (ddd, $J=7.7,1.0,0.9 \mathrm{~Hz}, 1 \mathrm{H}), 7.05(\mathrm{dd}, J=7.6,7.6 \mathrm{~Hz}, 1 \mathrm{H}), 6.94-6.91$ (m, 1H), 6.37 (d, $J=0.8 \mathrm{~Hz}, 1 \mathrm{H}), 5.61(\mathrm{dtt}, J=10.8,7.5,1.4 \mathrm{~Hz}, 1 \mathrm{H}), 5.37(\mathrm{dtt}, J=10.8,7.5,1.0 \mathrm{~Hz}$, 1H), 3.69 (s, 0.70H, E), 3.67 (s, 5.30H, Z), 3.33 (t, $J=7.5 \mathrm{~Hz}, 1 \mathrm{H}), 3.25$ (d, $J=7.5 \mathrm{~Hz}, 2 \mathrm{H}), 2.25$ (ddd, $J=7.5,7.5,1.4 \mathrm{~Hz}, 2 \mathrm{H}), 2.14(\mathrm{q}, J=7.7 \mathrm{~Hz}, 2 \mathrm{H}), 0.91(\mathrm{t}, J=7.7 \mathrm{~Hz}, 3 \mathrm{H})$.

${ }^{13} \mathbf{C}-\mathbf{N M R}\left(125 \mathrm{MHz}, \mathrm{CDCl}_{3}\right): \delta=169.2\left(\mathrm{C}_{\mathrm{q}}\right), 153.3\left(\mathrm{C}_{\mathrm{q}}\right), 149.3(\mathrm{CH}), 140.0\left(\mathrm{C}_{\mathrm{q}}\right), 137.8(\mathrm{CH}), 136.1$ $\left(\mathrm{C}_{\mathrm{q}}\right), 129.3\left(\mathrm{C}_{\mathrm{q}}\right), 128.7(\mathrm{CH}), 127.4\left(\mathrm{C}_{\mathrm{q}}\right), 126.3(\mathrm{CH}), 124.1(\mathrm{CH}), 123.3(\mathrm{CH}), 122.2(\mathrm{CH}), 120.3$ $(\mathrm{CH}), 117.9(\mathrm{CH}), 102.0(\mathrm{CH}), 52.5\left(\mathrm{CH}_{3}\right), 51.4(\mathrm{CH}), 31.6\left(\mathrm{CH}_{2}, E\right), 30.7\left(\mathrm{CH}_{2}, E\right), 26.7\left(\mathrm{CH}_{2}, \mathrm{Z}\right)$, $25.9\left(\mathrm{CH}_{2}, \mathrm{Z}\right), 24.8\left(\mathrm{CH}_{2}\right), 14.5\left(\mathrm{CH}_{3}\right)$.

IR (ATR): $1733,1584,1436,1150,909,729 \mathrm{~cm}^{-1}$.

MS (EI) $\mathrm{m} / z$ (relative intensity): 406 ([M] $\left.]^{+}, 60\right), 275$ (90), 247 (100), 234 (45), 219 (30).

HR-MS (EI): $m / z$ calcd. for $\left[\mathrm{C}_{24} \mathrm{H}_{26} \mathrm{~N}_{2} \mathrm{O}_{4}\right]^{+}[\mathrm{M}]^{+} 406.1887$, found 406.1888 .

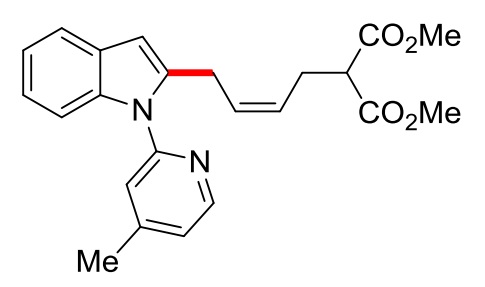

(Z)-Dimethyl 2-\{4-[1-(5-methylpyridin-2-yl)-1H-indol-2-yl]but-2-en-1-yl\}malonate (130ka): The general procedure $\mathbf{B}$ was followed using 1-(5-methylpyridin-2-yl)-1H-indole (105k) (104 mg, $0.50 \mathrm{mmol}$ ) and dimethyl 2-vinylcyclopropane-1,1-dicarboxylate (125a) (110 mg, $0.60 \mathrm{mmol})$. Isolation by column chromatography ( $n$-hexane/EtOAc: $3 / 1)$ yielded 130ka $(159 \mathrm{mg}, 81 \%, E / Z=$ $1: 14)$ as an orange oil.

${ }^{1} \mathbf{H}-\mathbf{N M R}\left(500 \mathrm{MHz}, \mathrm{CDCl}_{3}\right): \delta=8.49(\mathrm{ddd}, J=4.9,1.9,0.8 \mathrm{~Hz}, 1 \mathrm{H}), 7.57-7.52(\mathrm{~m}, 1 \mathrm{H}), 7.32-7.28$ $(\mathrm{m}, 1 \mathrm{H}), 7.26-7.22(\mathrm{~m}, 1 \mathrm{H}), 7.13-7.08(\mathrm{~m}, 3 \mathrm{H}), 6.40(\mathrm{~d}, J=0.7 \mathrm{~Hz}, 1 \mathrm{H}), 5.64(\mathrm{dtt}, J=10.7,7.5$, $1.4 \mathrm{~Hz}, 1 \mathrm{H}), 5.43-5.35(\mathrm{~m}, 1 \mathrm{H}), 3.68(\mathrm{~s}, 6 \mathrm{H}), 3.63(\mathrm{~d}, J=7.5 \mathrm{~Hz}, 2 \mathrm{H}), 3.35(\mathrm{t}, J=7.7 \mathrm{~Hz}, 1 \mathrm{H}), 2.61$ (ddd, $J=7.7,7.6,1.4 \mathrm{~Hz}, 1.87 \mathrm{H}, Z), 2.54(\mathrm{ddd}, J=7.7,7.6,1.4 \mathrm{~Hz}, 0.13 \mathrm{H}, E), 2.42$ (s, 3H). 
${ }^{13}$ C-NMR $\left(125 \mathrm{MHz}, \mathrm{CDCl}_{3}\right): \delta=169.2\left(\mathrm{C}_{\mathrm{q}}\right), 151.3\left(\mathrm{C}_{\mathrm{q}}\right), 149.8\left(\mathrm{C}_{\mathrm{q}}\right), 149.2(\mathrm{CH}), 139.3\left(\mathrm{C}_{\mathrm{q}}\right), 137.3$ $\left(\mathrm{C}_{\mathrm{q}}\right), 129.0(\mathrm{CH}), 128.4\left(\mathrm{C}_{\mathrm{q}}\right), 126.2(\mathrm{CH}), 123.1(\mathrm{CH}), 121.7(\mathrm{CH}), 121.6(\mathrm{CH}), 120.5(\mathrm{CH}), 119.9$ $(\mathrm{CH}), 110.0(\mathrm{CH}), 102.5(\mathrm{CH}), 52.4\left(\mathrm{CH}_{3}\right), 51.4(\mathrm{CH}), 31.6\left(\mathrm{CH}_{2}, E\right), 30.9\left(\mathrm{CH}_{2}, E\right), 26.7\left(\mathrm{CH}_{2}, \mathrm{Z}\right)$, $25.8\left(\mathrm{CH}_{2}, \mathrm{Z}\right), 21.0\left(\mathrm{CH}_{3}\right)$.

IR (ATR): 1732, 1603, 1457, 1433, 1151, 910, $729 \mathrm{~cm}^{-1}$.

MS (EI) $m / z$ (relative intensity): 392 ([M] $\left.{ }^{+}, 25\right), 261$ (100), 233 (55), 220 (50).

HR-MS (EI): $\mathrm{m} / z$ calcd. for $\left[\mathrm{C}_{23} \mathrm{H}_{24} \mathrm{~N}_{2} \mathrm{O}_{4}\right]^{+}[\mathrm{M}]^{+} 392.1736$, found 392.1729.

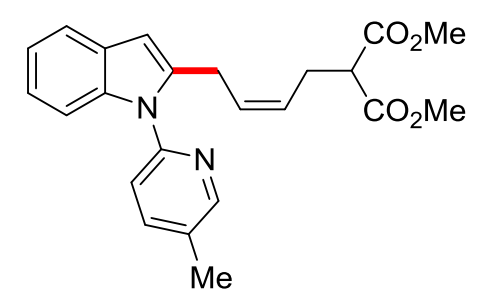

(Z)-Dimethyl 2-\{4-[1-(4-methylpyridin-2-yl)-1H-indol-2-yl]but-2-en-1-yl\}malonate (130la): The general procedure $\mathbf{B}$ was followed using 1-(4-methylpyridin-2-yl)- $1 H$-indole (105l) (104 mg, $0.50 \mathrm{mmol}$ ) and dimethyl 2-vinylcyclopropane-1,1-dicarboxylate (125a) $(110 \mathrm{mg}, 0.60 \mathrm{mmol})$. Isolation by column chromatography ( $n$-hexane/EtOAc: $3 / 1)$ yielded $1301 a(180 \mathrm{mg}, 92 \%, E / Z=1: 14)$ as an orange oil.

${ }^{1} \mathbf{H}-\mathbf{N M R}\left(500 \mathrm{MHz}, \mathrm{CDCl}_{3}\right): \delta=8.46(\mathrm{ddd}, J=4.9,1.9,0.8 \mathrm{~Hz}, 1 \mathrm{H}), 7.55-7.52(\mathrm{~m}, 1 \mathrm{H}), 7.33-7.30$ (m, 1H), 7.28-7.24 (m, 1H), 7.12-7.07 (m, 3H), $6.40(\mathrm{~d}, J=0.8 \mathrm{~Hz}, 1 \mathrm{H}), 5.63(\mathrm{dtt}, J=10.7,7.5$, $1.4 \mathrm{~Hz}, 1 \mathrm{H}), 5.43-5.35(\mathrm{~m}, 1 \mathrm{H}), 3.68(\mathrm{~s}, 6 \mathrm{H}), 3.63(\mathrm{~d}, J=7.5 \mathrm{~Hz}, 2 \mathrm{H}), 3.35(\mathrm{t}, J=7.7 \mathrm{~Hz}, 1 \mathrm{H}), 2.60$ (ddd, $J=7.7,7.7,1.4 \mathrm{~Hz}, 1.87 \mathrm{H}, Z), 2.57$ (ddd, $J=7.7,7.7,1.4 \mathrm{~Hz}, 0.13 \mathrm{H}, E), 2.44$ (s, 3H).

${ }^{13}$ C-NMR $\left(125 \mathrm{MHz}, \mathrm{CDCl}_{3}\right): \delta=169.2\left(\mathrm{C}_{\mathrm{q}}\right), 149.8(\mathrm{CH}), 148.8\left(\mathrm{C}_{\mathrm{q}}\right), 139.2\left(\mathrm{C}_{\mathrm{q}}\right), 138.8(\mathrm{CH}), 137.4$ $\left(\mathrm{C}_{\mathrm{q}}\right), 131.8\left(\mathrm{C}_{\mathrm{q}}\right), 128.9(\mathrm{CH}), 128.3\left(\mathrm{C}_{\mathrm{q}}\right), 126.2(\mathrm{CH}), 121.6(\mathrm{CH}), 120.5(\mathrm{CH}), 120.4(\mathrm{CH}), 119.9$ $(\mathrm{CH}), 110.0(\mathrm{CH}), 102.2(\mathrm{CH}), 52.4\left(\mathrm{CH}_{3}\right), 51.4(\mathrm{CH}), 31.7\left(\mathrm{CH}_{2}, E\right), 30.9\left(\mathrm{CH}_{2}, E\right), 26.7\left(\mathrm{CH}_{2}, \mathrm{Z}\right)$, $25.9\left(\mathrm{CH}_{2}, \mathrm{Z}\right), 21.1\left(\mathrm{CH}_{3}\right)$.

IR (ATR): 1732, 1483, 1456, 1275, 1150, 1205, $785 \mathrm{~cm}^{-1}$.

MS (EI) $m / z$ (relative intensity): 392 ([M] $\left.]^{+}, 20\right), 261$ (100), 233 (75), 220 (60).

HR-MS (EI): $\mathrm{m} / z$ calcd. for $\left[\mathrm{C}_{23} \mathrm{H}_{24} \mathrm{~N}_{2} \mathrm{O}_{4}\right]^{+}[\mathrm{M}]^{+} 392.1736$, found 392.1736 . 


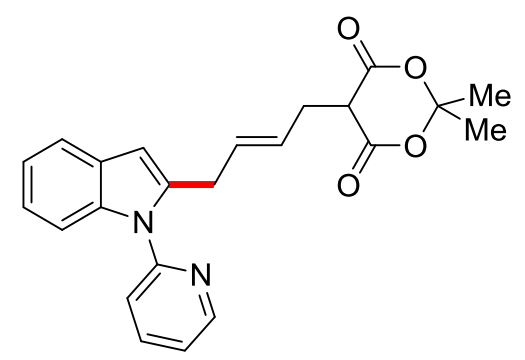

(E)-2,2-Dimethyl-5-\{4-[1-(pyridin-2-yl)-1H-indol-2-yl]but-2-en-1-yl\}-1,3-dioxane-4,6-dione

(130ab): The general procedure $\mathbf{B}$ was followed using 1-(pyridin-2-yl)- $1 H$-indole (105a) $(97.0 \mathrm{mg}$, $0.50 \mathrm{mmol}$ and 6,6-dimethyl-1-vinyl-5,7-dioxaspiro[2.5]octane-4,8-dione $\quad(\mathbf{1 2 5 b}) \quad(118 \mathrm{mg}$, $0.60 \mathrm{mmol}$ ). Isolation by column chromatography ( $n$-hexane/EtOAc: $3 / 1)$ yielded 130ab (162 mg, $83 \%, E / Z=3.7: 1)$ as an orange oil.

${ }^{1} \mathbf{H}-\mathbf{N M R}\left(500 \mathrm{MHz}, \mathrm{CDCl}_{3}\right): \delta=8.63(\mathrm{ddd}, J=4.9,1.9,0.8 \mathrm{~Hz}, 1 \mathrm{H}), 7.85(\mathrm{ddd}, J=8.0,7.4,2.0 \mathrm{~Hz}$, $1 \mathrm{H}), 7.57-7.52(\mathrm{~m}, 1 \mathrm{H}), 7.40(\mathrm{ddd}, J=8.0,1.0,0.8 \mathrm{~Hz}, 1 \mathrm{H}), 7.33-7.27(\mathrm{~m}, 2 \mathrm{H}), 7.12-7.07(\mathrm{~m}, 2 \mathrm{H})$, $6.42(\mathrm{~d}, J=0.8 \mathrm{~Hz}, 1 \mathrm{H}), 5.72(\mathrm{dtt}, J=15.2,6.7,1.2 \mathrm{~Hz}, 0.79 \mathrm{H}, E), 5.64(\mathrm{dtt}, J=10.7,7.2,1.4 \mathrm{~Hz}$, $0.21 \mathrm{H}, Z), 5.50$ (dtt, $J=10.7,7.2,1.6 \mathrm{~Hz}, 0.21 \mathrm{H}, Z$ ), $5.42(\mathrm{dtt}, J=15.2,7.3,1.2 \mathrm{~Hz}, 0.79 \mathrm{H}, E), 3.70$ $(\mathrm{d}, J=7.2 \mathrm{~Hz}, 0.42 \mathrm{H}, Z), 3.55(\mathrm{~d}, J=6.7 \mathrm{~Hz}, 1.58 \mathrm{H}, E), 3.46(\mathrm{t}, J=7.7 \mathrm{~Hz}, 1 \mathrm{H}), 2.74(\mathrm{ddd}, J=7.7$, 7.2, $1.4 \mathrm{~Hz}, 2 \mathrm{H}), 1.71(\mathrm{~s}, 6 \mathrm{H})$.

${ }^{13}$ C-NMR $\left(125 \mathrm{MHz}, \mathrm{CDCl}_{3}\right): \delta=165.0\left(\mathrm{C}_{\mathrm{q}}\right), 151.4\left(\mathrm{C}_{\mathrm{q}}\right), 149.6(\mathrm{CH}), 139.2\left(\mathrm{C}_{\mathrm{q}}\right), 138.3(\mathrm{CH}), 137.3$ $\left(\mathrm{C}_{\mathrm{q}}\right), 131.5(\mathrm{CH}), 128.6\left(\mathrm{C}_{\mathrm{q}}\right), 126.4(\mathrm{CH}), 122.2(\mathrm{CH}), 121.9(\mathrm{CH}), 121.0(\mathrm{CH}), 120.7(\mathrm{CH}), 120.1$ $(\mathrm{CH}), 110.1(\mathrm{CH}), 105.0\left(\mathrm{C}_{\mathrm{q}}\right), 103.0(\mathrm{CH}), 46.3(\mathrm{CH}), 30.9\left(\mathrm{CH}_{2}, E\right), 29.0\left(\mathrm{CH}_{2}, E\right), 28.3\left(\mathrm{CH}_{3}\right), 26.8$ $\left(\mathrm{CH}_{3}\right), 26.5\left(\mathrm{CH}_{2}, \mathrm{Z}\right), 25.9\left(\mathrm{CH}_{2}, \mathrm{Z}\right)$.

IR (ATR): 1782, 1743, 1586, 1469, 1284, 908, $727 \mathrm{~cm}^{-1}$.

MS (EI) $m / z$ (relative intensity): 390 ([M] $\left.{ }^{+}, 30\right), 287$ (35), 259 (40), 247 (100), 219 (80), 206 (85).

HR-MS (EI): $m / z$ calcd. for $\left[\mathrm{C}_{23} \mathrm{H}_{22} \mathrm{~N}_{2} \mathrm{O}_{4}\right]^{+}[\mathrm{M}]^{+} 390.1580$, found: 390.1567 .

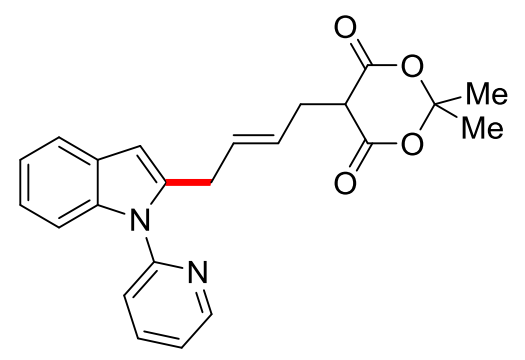

(E)-2,2-Dimethyl-5-\{4-[1-(pyridin-2-yl)-1H-indol-2-yl]but-2-en-1-yl\}-1,3-dioxane-4,6-dione

(130ab): The general procedure $\mathbf{C}$ was followed using 1-(pyridin-2-yl)-1H-indole (105a) $(97.0 \mathrm{mg}$, $0.50 \mathrm{mmol})$ and 6,6-dimethyl-1-vinyl-5,7-dioxaspiro[2.5]octane-4,8-dione $\quad(\mathbf{1 2 5 b}) \quad(118 \mathrm{mg}$, 
$0.60 \mathrm{mmol}$ ). Isolation by column chromatography ( $n$-hexane/EtOAc: $3 / 1)$ yielded 130ab (162 mg, $83 \%, E / Z=5.9: 1)$ as an orange oil.

${ }^{1} \mathbf{H}-\mathbf{N M R}\left(500 \mathrm{MHz}, \mathrm{CDCl}_{3}\right): \delta=8.63(\mathrm{ddd}, J=4.9,1.9,0.8 \mathrm{~Hz}, 1 \mathrm{H}), 7.85(\mathrm{ddd}, J=8.0,7.4,2.0 \mathrm{~Hz}$, $1 \mathrm{H}), 7.57-7.52(\mathrm{~m}, 1 \mathrm{H}), 7.40(\mathrm{ddd}, J=8.0,1.0,0.9 \mathrm{~Hz}, 1 \mathrm{H}), 7.33-7.27(\mathrm{~m}, 2 \mathrm{H}), 7.12-7.07(\mathrm{~m}, 2 \mathrm{H})$, $6.42(\mathrm{~d}, J=0.8 \mathrm{~Hz}, 1 \mathrm{H}), 5.72(\mathrm{dtt}, J=15.2,6.7,1.2 \mathrm{~Hz}, 0.86 \mathrm{H}, E), 5.64(\mathrm{dtt}, J=10.7,7.2,1.4 \mathrm{~Hz}$, $0.14 \mathrm{H}, Z), 5.50$ (dtt, $J=10.7,7.2,1.6 \mathrm{~Hz}, 0.14 \mathrm{H}, Z$ ), $5.42(\mathrm{dtt}, J=15.2,7.3,1.2 \mathrm{~Hz}, 0.86 \mathrm{H}, E), 3.70$ $(\mathrm{d}, J=7.2 \mathrm{~Hz}, 0.29 \mathrm{H}, Z), 3.55(\mathrm{~d}, J=6.7 \mathrm{~Hz}, 1.71 \mathrm{H}, E), 3.46(\mathrm{t}, J=7.7 \mathrm{~Hz}, 1 \mathrm{H}), 2.74$ (ddd, $J=7.7$, 7.2, $1.4 \mathrm{~Hz}, 2 \mathrm{H}), 1.71(\mathrm{~s}, 6 \mathrm{H})$.

${ }^{13}$ C-NMR $\left(125 \mathrm{MHz}, \mathrm{CDCl}_{3}\right): \delta=165.0\left(\mathrm{C}_{\mathrm{q}}\right), 151.4\left(\mathrm{C}_{\mathrm{q}}\right), 149.6(\mathrm{CH}), 139.2\left(\mathrm{C}_{\mathrm{q}}\right), 138.3(\mathrm{CH}), 137.3$ $\left(\mathrm{C}_{\mathrm{q}}\right), 131.5(\mathrm{CH}), 128.6\left(\mathrm{C}_{\mathrm{q}}\right), 126.4(\mathrm{CH}), 122.2(\mathrm{CH}), 121.9(\mathrm{CH}), 121.0(\mathrm{CH}), 120.7(\mathrm{CH}), 120.1$ $(\mathrm{CH}), 110.1(\mathrm{CH}), 105.0\left(\mathrm{C}_{\mathrm{q}}\right), 103.0(\mathrm{CH}), 46.3(\mathrm{CH}), 30.9\left(\mathrm{CH}_{2}, E\right), 29.0\left(\mathrm{CH}_{2}, E\right), 28.3\left(\mathrm{CH}_{3}\right), 26.8$ $\left(\mathrm{CH}_{3}\right), 26.5\left(\mathrm{CH}_{2}, \mathrm{Z}\right), 25.9\left(\mathrm{CH}_{2}, Z\right)$.

IR (ATR): 1782, 1743, 1586, 1469, 1284, 908, $727 \mathrm{~cm}^{-1}$.

MS (EI) m/z (relative intensity): 390 ([M] $\left.]^{+}, 30\right), 287$ (35), 259 (40), 247 (100), 219 (80), 206 (85).

HR-MS (EI): $m / z$ calcd. for $\left[\mathrm{C}_{23} \mathrm{H}_{22} \mathrm{~N}_{2} \mathrm{O}_{4}\right]^{+}[\mathrm{M}]^{+} 390.1580$, found: 390.1567 .

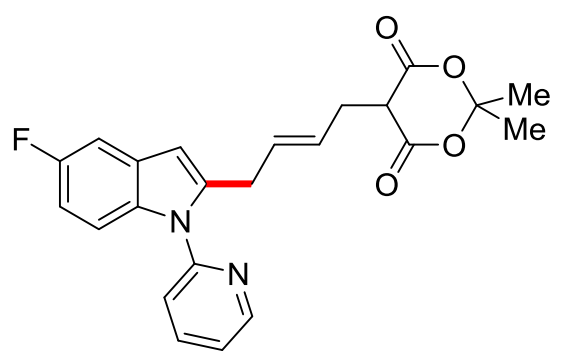

\section{(E)-5-\{4-[5-fluoro-1-(pyridin-2-yl)-1H-indol-2-yl]but-2-en-1-yl\}-2,2-dimethyl-1,3-dioxane-4,6-}

dione $(130 \mathrm{mb})$ : The general procedure $\mathbf{B}$ was followed using 5-fluoro-1-(pyridin-2-yl)-1 $H$-indole (130m) (106 mg, $0.50 \mathrm{mmol})$ and 6,6-dimethyl-1-vinyl-5,7-dioxaspiro[2.5]octane-4,8-dione (125b) $(118 \mathrm{mg}, 0.60 \mathrm{mmol})$. Isolation by column chromatography ( $n$-hexane/EtOAc: $3 / 1)$ yielded $130 \mathrm{mb}$ (165 $\mathrm{mg}, 81 \%, E / Z=2.6: 1)$ as an orange oil.

${ }^{1}$ H-NMR $\left(300 \mathrm{MHz}, \mathrm{CDCl}_{3}\right): \delta=8.62(\mathrm{ddd}, J=4.9,1.9,0.8 \mathrm{~Hz}, 1 \mathrm{H}), 7.85(\mathrm{ddd}, J=8.0,7.4,2.0 \mathrm{~Hz}$, $1 \mathrm{H}), 7.42-7.27(\mathrm{~m}, 2 \mathrm{H}), 7.24-7.14(\mathrm{~m}, 2 \mathrm{H}), 6.82(\mathrm{dt}, J=9.2,2.6 \mathrm{~Hz}, 1 \mathrm{H}), 6.36(\mathrm{~d}, J=0.8 \mathrm{~Hz}, 1 \mathrm{H})$, $5.71(\mathrm{dtt}, J=15.3,6.9,1.4 \mathrm{~Hz}, 0.72 \mathrm{H}, E), 5.65$ (dtt, $J=10.6,7.5,1.4 \mathrm{~Hz}, 0.28 \mathrm{H}, Z), 5.54-5.37$ (m, $1 \mathrm{H}), 3.67(\mathrm{~d}, J=7.5 \mathrm{~Hz}, 0.56 \mathrm{H}, Z), 3.52(\mathrm{~d}, J=6.9 \mathrm{~Hz}, 1.44 \mathrm{H}, E), 3.47$ (t, $J=7.7 \mathrm{~Hz}, 1 \mathrm{H}), 2.78$ (ddd, $J=7.7,7.6,1.4 \mathrm{~Hz}, 0.55 \mathrm{H}, Z), 2.74$ (ddd, $J=7.7,7.6,1.4 \mathrm{~Hz}, 1.45 \mathrm{H}, E), 1.75-1.64$ (m, 6H).

${ }^{13}$ C-NMR $\left(125 \mathrm{MHz}, \mathrm{CDCl}_{3}\right): \delta=165.0\left(\mathrm{C}_{\mathrm{q}}\right), 158.5\left(\mathrm{~d},{ }^{1} J_{\mathrm{C}-\mathrm{F}}=236 \mathrm{~Hz}, \mathrm{C}_{\mathrm{q}}\right), 151.2\left(\mathrm{C}_{\mathrm{q}}\right), 149.6(\mathrm{CH})$, $140.8\left(\mathrm{C}_{\mathrm{q}}\right), 138.4(\mathrm{CH}), 133.9\left(\mathrm{C}_{\mathrm{q}}\right), 131.2(\mathrm{CH}), 129.0\left(\mathrm{~d},{ }^{3} J_{\mathrm{C}-\mathrm{F}}=10.4 \mathrm{~Hz}, \mathrm{C}_{\mathrm{q}}\right), 126.7(\mathrm{CH}), 122.3$ $(\mathrm{CH}), 121.0(\mathrm{CH}), 120.7(\mathrm{CH}), 110.9\left(\mathrm{~d},{ }^{3} J_{\mathrm{C}-\mathrm{F}}=10.1 \mathrm{~Hz}, \mathrm{CH}\right), 109.8\left(\mathrm{~d},{ }^{2} J_{\mathrm{C}-\mathrm{F}}=26.3 \mathrm{~Hz}, \mathrm{CH}\right), 105.0$ 
$\left(\mathrm{C}_{\mathrm{q}}\right), 103.0\left(\mathrm{~d},{ }^{4} J_{\mathrm{C}-\mathrm{F}}=4.2 \mathrm{~Hz}, \mathrm{CH}\right), 46.2(\mathrm{CH}), 30.9\left(\mathrm{CH}_{2}, E\right), 29.0\left(\mathrm{CH}_{2}, E\right), 28.3\left(\mathrm{CH}_{3}\right), 26.8\left(\mathrm{CH}_{3}\right)$, $26.5\left(\mathrm{CH}_{2}, \mathrm{Z}\right), 25.9\left(\mathrm{CH}_{2}, \mathrm{Z}\right)$.

${ }^{19}$ F-NMR $\left(282 \mathrm{MHz}, \mathrm{CDCl}_{3}\right): \delta=-124.0(\mathrm{~m})$.

IR (ATR): 1782, 1745, 1585, 1469, 1288, 1174, 775, $729 \mathrm{~cm}^{-1}$.

MS (EI) m/z (relative intensity): 408 ([M] $\left.{ }^{+}, 10\right) 324$ (15), 305 (15), 277 (25), 265 (85), 237 (100), 224 (80).

HR-MS (EI): $m / z$ calcd. for $\left[\mathrm{C}_{23} \mathrm{H}_{21} \mathrm{FN}_{2} \mathrm{O}_{4}\right]^{+}[\mathrm{M}]^{+} 408.1485$, found 408.1491 .

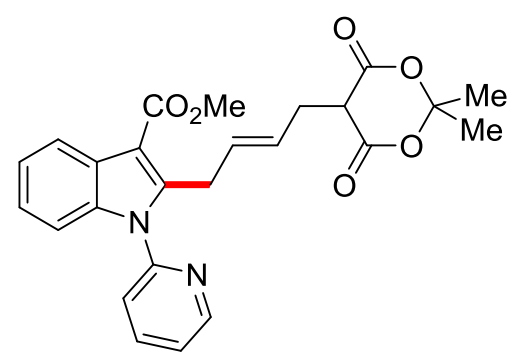

(Z)-Methyl-2-\{4-[2,2-dimethyl-4,6-dioxo-1,3-dioxan-5-yl]but-2-en-1-yl\}-1-(pyridin-2-yl)-1H-

indole-3-carboxylate (130nb): The general procedure $\mathbf{B}$ was followed using methyl 1-(pyridin-2-yl)$1 H$-indole-3-carboxylate $\quad(\mathbf{1 3 0 n}) \quad(126 \mathrm{mg}, \quad 0.50 \mathrm{mmol}) \quad$ and $\quad 6,6$-dimethyl-1-vinyl-5,7dioxaspiro[2.5]octane-4,8-dione (125b) (118 $\mathrm{mg}, 0.60 \mathrm{mmol})$. Isolation by column chromatography ( $n$-hexane/EtOAc: $3 / 1)$ yielded 130nb $(168 \mathrm{mg}, 75 \%, E / Z=2.4: 1)$ as an orange oil.

${ }^{1}$ H-NMR $\left(500 \mathrm{MHz}, \mathrm{CDCl}_{3}\right): \delta=8.68(\mathrm{ddd}, J=4.9,1.9,0.8 \mathrm{~Hz}, 1 \mathrm{H}), 8.17-8.12$ (m, 1H), 7.91 (ddd, $J$ = 8.0, 7.4, $2.0 \mathrm{~Hz}, 1 \mathrm{H}), 7.41-7.39(\mathrm{~m}, 2 \mathrm{H}), 7.24(\mathrm{ddd}, J=9.3,6.8,1.5 \mathrm{~Hz}, 1 \mathrm{H}), 7.18-7.10(\mathrm{~m}, 2 \mathrm{H})$, $5.66(\mathrm{dtt}, J=15.2,7.4,1.2 \mathrm{~Hz}, 0.71 \mathrm{H}, E), 5.50-5.42(\mathrm{~m}, 0.58 \mathrm{H}, Z), 5.23(\mathrm{dtt}, J=15.2,7.2,1.2 \mathrm{~Hz}$, $0.71 \mathrm{H}, E), 3.98(\mathrm{~s}, 3 \mathrm{H}), 3.92(\mathrm{~d}, J=7.4 \mathrm{~Hz}, 2 \mathrm{H}), 3.72(\mathrm{t}, J=7.5 \mathrm{~Hz}, 0.29 \mathrm{H}, Z), 3.44(\mathrm{t}, J=7.5 \mathrm{~Hz}$, $0.71 \mathrm{H}, E), 2.64(\mathrm{ddd}, J=7.5,7.2,1.2 \mathrm{~Hz}, 2 \mathrm{H}), 1.76-1.60(\mathrm{~m}, 6 \mathrm{H})$.

${ }^{13}$ C-NMR $\left(125 \mathrm{MHz}, \mathrm{CDCl}_{3}\right): \delta=166.1\left(\mathrm{C}_{\mathrm{q}}\right), 165.2\left(\mathrm{C}_{\mathrm{q}}, Z\right), 164.9\left(\mathrm{C}_{\mathrm{q}}, E\right), 150.1(\mathrm{CH}), 150.0\left(\mathrm{C}_{\mathrm{q}}\right)$, $146.9\left(\mathrm{C}_{\mathrm{q}}, \mathrm{Z}\right), 146.0\left(\mathrm{C}_{\mathrm{q}}, E\right), 138.7(\mathrm{CH}), 136.9\left(\mathrm{C}_{\mathrm{q}}\right), 130.8(\mathrm{CH}), 126.6\left(\mathrm{C}_{\mathrm{q}}\right), 126.3(\mathrm{CH}), 123.6(\mathrm{CH})$, $123.1(\mathrm{CH}), 122.6(\mathrm{CH}), 122.5(\mathrm{CH}), 121.8(\mathrm{CH}), 110.4(\mathrm{CH}), 106.0\left(\mathrm{C}_{\mathrm{q}}\right), 104.9\left(\mathrm{C}_{\mathrm{q}}\right), 50.6(\mathrm{CH}), 46.5$ $\left(\mathrm{CH}_{3}, \mathrm{Z}\right), 46.3\left(\mathrm{CH}_{3}, E\right), 29.0\left(\mathrm{CH}_{2}, E\right), 28.9\left(\mathrm{CH}_{2}, E\right), 28.8\left(\mathrm{CH}_{3}\right), 26.7\left(\mathrm{CH}_{3}\right), 24.7\left(\mathrm{CH}_{2}, \mathrm{Z}\right), 23.8$ $\left(\mathrm{CH}_{2}, \mathrm{Z}\right)$.

IR (ATR): 1746, 1694, 1537, 1457, 1283, 1189, 1099, 909, $727 \mathrm{~cm}^{-1}$.

MS (EI) $m / z$ (relative intensity): 448 ([M] $\left.]^{+}, 10\right), 364$ (10), 305 (45), 277 (70), 264 (30), 245 (40).

HR-MS (EI): $m / z$ calcd. for $\left[\mathrm{C}_{25} \mathrm{H}_{24} \mathrm{~N}_{2} \mathrm{O}_{6}\right]^{+}[\mathrm{M}]^{+} 448.1634$, found 448.1631 . 
<smiles>CC(=O)C(C/C=C\Cc1ccccc1-c1ccccn1)C(C)=O</smiles>

(Z)-Dimethyl 2-\{4-[2-(pyridin-2-yl)phenyl]but-2-en-1-yl\}malonate (131aa): The general procedure B was followed using 2-phenylpyridine (1a) $(77.6 \mathrm{mg}, 0.50 \mathrm{mmol})$ and dimethyl 2-vinylcyclopropane1,1-dicarboxylate $\mathbf{( 1 2 5 a )}(110 \mathrm{mg}, \quad 0.60 \mathrm{mmol})$. Isolation by column chromatography ( $n$ hexane/EtOAc: $3 / 1)$ yielded 131aa (146 mg, 86\%, E/Z = 1:9) as a colorless oil.

${ }^{1}$ H-NMR $\left(400 \mathrm{MHz}, \mathrm{CDCl}_{3}\right): \delta=8.68-8.63(\mathrm{~m}, 1 \mathrm{H}), 7.73$ (ddd, $\left.J=8.0,7.8,1.9 \mathrm{~Hz}, 1 \mathrm{H}\right), 7.38-7.29$ (m, 3H), 7.29-7.20 (m, 3H), 5.59 (dtt, $J=15.1,7.5,1.4 \mathrm{~Hz}, 0.10 \mathrm{H}, E), 5.51$ (dtt, $J=10.7,7.5,1.4 \mathrm{~Hz}$, $0.90 \mathrm{H}, Z), 5.32-5.20(\mathrm{~m}, 1 \mathrm{H}), 3.69$ (s, $5.40 \mathrm{H}, Z), 3.63$ (s, $0.60 \mathrm{H}, E), 3.50$ (d, J=7.5 Hz, $1.80 \mathrm{H}, Z$ ), $3.50(\mathrm{~d}, J=7.5 \mathrm{~Hz}, 0.20 \mathrm{H}, E), 3.31(\mathrm{t}, J=7.7 \mathrm{~Hz}, 1 \mathrm{H}), 2.56(\mathrm{ddd}, J=7.7,7.7,1.4 \mathrm{~Hz}, 2 \mathrm{H})$.

${ }^{13}$ C-NMR $\left(100 \mathrm{MHz}, \mathrm{CDCl}_{3}\right): \delta=169.3\left(\mathrm{C}_{\mathrm{q}}\right), 159.9\left(\mathrm{C}_{\mathrm{q}}\right), 149.2(\mathrm{CH}), 140.3\left(\mathrm{C}_{\mathrm{q}}\right), 138.3\left(\mathrm{C}_{\mathrm{q}}\right), 136.2$ $(\mathrm{CH}), 131.8(\mathrm{CH}), 129.8(\mathrm{CH}), 129.6(\mathrm{CH}), 128.5(\mathrm{CH}), 126.2(\mathrm{CH}), 125.0(\mathrm{CH}), 124.2(\mathrm{CH}), 121.7$ $(\mathrm{CH}), 52.5\left(\mathrm{CH}_{3}\right), 51.6(\mathrm{CH}), 31.0\left(\mathrm{CH}_{2}\right), 26.7\left(\mathrm{CH}_{2}\right)$.

IR (ATR): 1732, 1586, 1435, 1231, 1196, 1150, 1024, $751 \mathrm{~cm}^{-1}$.

MS (EI) $\mathrm{m} / z$ (relative intensity): $339\left([\mathrm{M}]^{+}, 15\right), 308$ (100).

HR-MS (ESI): $m / z$ calcd. for $\left[\mathrm{C}_{20} \mathrm{H}_{22} \mathrm{NO}_{4}\right]^{+}[\mathrm{M}+\mathrm{H}]^{+} 340.1543$, found 340.1547 .<smiles>COC(=O)C(C/C=C\Cc1cc(OC)ccc1-c1ccccn1)C(C)=O</smiles>

(Z)-Dimethyl 2-\{4-[5-methoxy-2-(pyridin-2-yl)phenyl]but-2-en-1-yl\}malonate $\quad$ (131ba): The general procedure $\mathbf{B}$ was followed using 2-(4-methoxyphenyl)pyridine (1b) $(92.5 \mathrm{mg}, 0.50 \mathrm{mmol})$ and dimethyl 2-vinylcyclopropane-1,1-dicarboxylate (125a) $(110 \mathrm{mg}, 0.60 \mathrm{mmol})$. Isolation by column chromatography ( $n$-hexane/EtOAc: $3 / 1)$ yielded $\mathbf{1 3 1}$ ba $(138 \mathrm{mg}, 75 \%, E / Z=1: 4)$ as a colorless oil.

${ }^{1} \mathbf{H}-\mathbf{N M R}\left(600 \mathrm{MHz}, \mathrm{CDCl}_{3}\right): \delta=8.68-8.63(\mathrm{~m}, 1 \mathrm{H}), 7.69(\mathrm{ddd}, J=8.0,7.6,1.9 \mathrm{~Hz}, 1 \mathrm{H}), 7.34-7.27$ $(\mathrm{m}, 2 \mathrm{H}), 7.18(\mathrm{ddd}, J=7.6,4.9,1.2 \mathrm{~Hz}, 1 \mathrm{H}), 6.82-6.75(\mathrm{~m}, 2 \mathrm{H}), 5.58(\mathrm{dtt}, J=15.8,7.4,1.2 \mathrm{~Hz}$, $0.20 \mathrm{H}, E), 5.51$ (dtt, $J=10.7,7.6,1.2 \mathrm{~Hz}, 0.80 \mathrm{H}, Z), 5.32-5.24(\mathrm{~m}, 1 \mathrm{H}), 3.80(\mathrm{~s}, 3 \mathrm{H}), 3.68(\mathrm{~s}, 4.80 \mathrm{H}$, Z), $3.65(\mathrm{~s}, 1.20 \mathrm{H}, E), 3.51(\mathrm{~d}, J=7.4 \mathrm{~Hz}, 1.59 \mathrm{H}, Z), 3.39(\mathrm{~d}, J=7.4 \mathrm{~Hz}, 0.41 \mathrm{H}, E), 3.31(\mathrm{t}, J=$ $7.7 \mathrm{~Hz}, 1 \mathrm{H}), 2.57$ (ddd, $J=7.7,7.6,1.2 \mathrm{~Hz}, 1.61 \mathrm{H}, Z), 2.53$ (ddd, $J=7.7,7.6,1.2 \mathrm{~Hz}, 0.39 \mathrm{H}, E$ ). 
${ }^{13}$ C-NMR $\left(125 \mathrm{MHz}, \mathrm{CDCl}_{3}\right): \delta=169.2\left(\mathrm{C}_{\mathrm{q}}\right), 159.5\left(\mathrm{C}_{\mathrm{q}}\right), 149.0(\mathrm{CH}), 139.8\left(\mathrm{C}_{\mathrm{q}}\right), 139.5\left(\mathrm{C}_{\mathrm{q}}\right), 136.2$ $(\mathrm{CH}), 132.9\left(\mathrm{C}_{\mathrm{q}}\right), 132.4(\mathrm{CH}), 131.6(\mathrm{CH}), 125.1(\mathrm{CH}), 124.1(\mathrm{CH}), 121.3(\mathrm{CH}), 115.1(\mathrm{CH}), 111.4$ $(\mathrm{CH}), 55.2\left(\mathrm{CH}_{3}\right), 52.4\left(\mathrm{CH}_{3}\right), 51.5(\mathrm{CH}), 36.2\left(\mathrm{CH}_{2}, E\right), 31.7\left(\mathrm{CH}_{2}, E\right), 31.1\left(\mathrm{CH}_{2}, \mathrm{Z}\right), 26.6\left(\mathrm{CH}_{2}, \mathrm{Z}\right)$.

IR (ATR): 1732, 1606, 1587, 1428, 1231, 1149, 988, $787 \mathrm{~cm}^{-1}$.

MS (EI) $\mathrm{m} / z$ (relative intensity): 369 ([M] $\left.{ }^{+}, 10\right), 238$ (100), 197 (40), 193 (10), 167 (75).

HR-MS (EI): $m / z$ calcd. for $\left[\mathrm{C}_{21} \mathrm{H}_{23} \mathrm{NO}_{5}\right]^{+}[\mathrm{M}]^{+} 369.1576$, found 369.1573 .<smiles>COc1ccc(-c2ccccn2)c(C/C=C/CC(C(C)=O)C(C)=O)c1</smiles>

(E)-Dimethyl 2-\{4-[5-methoxy-2-(pyridin-2-yl)phenyl]but-2-en-1-yl\}malonate (131ba'): The general procedure $\mathbf{C}$ was followed using 2-(4-methoxyphenyl)pyridine (1b) (46.3 $\mathrm{mg}, 0.25 \mathrm{mmol}$ ) and dimethyl 2-vinylcyclopropane-1,1-dicarboxylate (125a) $(55.2 \mathrm{mg}, 0.30 \mathrm{mmol})$. Isolation by column chromatography ( $n$-hexane/EtOAc: $5 / 1 \rightarrow 3 / 1 \rightarrow 2 / 1$ ) yielded 131ba' $(41.6 \mathrm{mg}, 45 \%, E / Z=2: 1)$ and 131ba" (16.6 mg, 12\%, E/Z =3:1) as yellow oils.

${ }^{1} \mathbf{H}-\mathbf{N M R}\left(500 \mathrm{MHz}, \mathrm{CDCl}_{3}\right): \delta=8.68-8.63(\mathrm{~m}, 1 \mathrm{H}), 7.68(\mathrm{ddd}, J=8.0,7.8,1.9 \mathrm{~Hz}, 1 \mathrm{H}), 7.34-7.27$ (m, 2H), $7.18(\mathrm{ddd}, J=7.8,4.9,1.2 \mathrm{~Hz}, 1 \mathrm{H}), 6.83-6.75(\mathrm{~m}, 2 \mathrm{H}), 5.61-5.48(\mathrm{~m}, 1 \mathrm{H}), 5.33-5.23(\mathrm{~m}$, 1H), 3.82 (s, 3H), 3.69 (s, 1.80H, Z), 3.67 (s, 4.20H, E), 3.51 (d, J = 7.4 Hz, 0.60H, Z), 3.39 (d, $J=7.4 \mathrm{~Hz}, 1.40 \mathrm{H}, E), 3.34(\mathrm{t}, J=7.7 \mathrm{~Hz}, 1 \mathrm{H}), 2.58(\mathrm{ddd}, J=7.7,7.6,1.4 \mathrm{~Hz}, 0.60 \mathrm{H}, Z), 2.53$ (ddd, $J=7.7,7.6,1.4 \mathrm{~Hz}, 1.40 \mathrm{H}, E)$.

${ }^{13}$ C-NMR $\left(125 \mathrm{MHz}, \mathrm{CDCl}_{3}\right): \delta=169.2\left(\mathrm{C}_{\mathrm{q}}\right), 159.9\left(\mathrm{C}_{\mathrm{q}}\right), 149.0(\mathrm{CH}), 139.8\left(\mathrm{C}_{\mathrm{q}}\right), 139.9\left(\mathrm{C}_{\mathrm{q}}\right), 136.0$ $(\mathrm{CH}), 132.4\left(\mathrm{C}_{\mathrm{q}}\right), 132.2(\mathrm{CH}), 131.1(\mathrm{CH}), 125.1(\mathrm{CH}), 124.2(\mathrm{CH}), 121.3(\mathrm{CH}), 115.3(\mathrm{CH}), 111.5$ $(\mathrm{CH}), 55.3\left(\mathrm{CH}_{3}\right), 52.7\left(\mathrm{CH}_{3}\right), 51.3(\mathrm{CH}), 36.3\left(\mathrm{CH}_{2}, E\right), 31.8\left(\mathrm{CH}_{2}, E\right), 31.2\left(\mathrm{CH}_{2}, \mathrm{Z}\right), 26.7\left(\mathrm{CH}_{2}, \mathrm{Z}\right)$.

IR (ATR): 1732, 1586, 1468, 1436, 1256, 1150, 1170, $747 \mathrm{~cm}^{-1}$.

MS (ESI) $m / z$ (relative intensity): 370 ([M + H $\left.]^{+}, 10\right), 238$ (100), 197 (40).

HR-MS (ESI): $\mathrm{m} / z$ calcd. for $\left[\mathrm{C}_{21} \mathrm{H}_{24} \mathrm{NO}_{5}\right]^{+}[\mathrm{M}+\mathrm{H}]^{+} 370.1649$, found 370.1651 . 
<smiles>COC(C)=O</smiles>

(2E,Z,2' $E, Z)$-Tetramethyl [5-methoxy-2-(pyridin-2-yl)-1,3-phenylene]bis(but-2-ene-4,1-diyl)]dimalonate (131ba"):

${ }^{1}$ H-NMR $\left(600 \mathrm{MHz}, \mathrm{CDCl}_{3}\right): \delta=8.70-8.62(\mathrm{~m}, 1 \mathrm{H}), 7.72-7.65(\mathrm{~m}, 1 \mathrm{H}), 7.23-7.12(\mathrm{~m}, 2 \mathrm{H}), 6.66-$ $6.59(\mathrm{~m}, 2 \mathrm{H}), 5.50-5.42(\mathrm{~m}, 2 \mathrm{H}), 5.24(\mathrm{dtt}, J=10.7,7.6,1.2 \mathrm{~Hz}, 1 \mathrm{H}, Z), 5.14(\mathrm{dtt}, J=15.2,7.7$, $1.4 \mathrm{~Hz}, 1 \mathrm{H}, E), 3.79$ (s, 3H), $3.66(\mathrm{~s}, 12 \mathrm{H}), 3.33$ (t, $J=7.7 \mathrm{~Hz}, 1.51 \mathrm{H}, E), 3.26$ (t, $J=7.7 \mathrm{~Hz}, 0.49 \mathrm{H}$, Z), 3.08 (d, $J=7.6 \mathrm{~Hz}, 0.95 \mathrm{H}, Z$ ), 2.94 (d, $J=7.6 \mathrm{~Hz}, 3.05 \mathrm{H}, E), 2.49$ (ddd, $J=7.7,7.7,1.2 \mathrm{~Hz}$, $3.05 \mathrm{H}, E), 2.42(\mathrm{ddd}, J=7.7,7.6,1.2 \mathrm{~Hz}, 0.95 \mathrm{H}, Z)$.

${ }^{13}$ C-NMR $\left(125 \mathrm{MHz}, \mathrm{CDCl}_{3}\right): \delta=169.1\left(\mathrm{C}_{\mathrm{q}}\right), 159.1\left(\mathrm{C}_{\mathrm{q}}\right), 158.7\left(\mathrm{C}_{\mathrm{q}}\right), 149.3(\mathrm{CH}), 139.7\left(\mathrm{C}_{\mathrm{q}}\right), 135.7$ $(\mathrm{CH}), 132.0\left(\mathrm{C}_{\mathrm{q}}\right), 126.5(\mathrm{CH}), 125.0(\mathrm{CH}), 121.6(\mathrm{CH}), 121.6(\mathrm{CH}), 112.4(\mathrm{CH}), 55.2\left(\mathrm{CH}_{3}\right), 52.4$ $\left(\mathrm{CH}_{3}\right), 51.5(\mathrm{CH}, E), 51.5(\mathrm{CH}, \mathrm{Z}), 36.8\left(\mathrm{CH}_{2}, E\right), 31.8\left(\mathrm{CH}_{2}, E\right), 31.6\left(\mathrm{CH}_{2}, \mathrm{Z}\right), 26.6\left(\mathrm{CH}_{2}, \mathrm{Z}\right)$.

IR (ATR): 1730, 1602, 1459, 1434, 1230, 1148, 1021, $753 \mathrm{~cm}^{-1}$.

MS (EI) $\mathrm{m} / \mathrm{z}$ (relative intensity): $553\left([\mathrm{M}]^{+}, 10\right), 522$ (10), 422 (100).

HR-MS (EI): $m / z$ calcd. for $\left[\mathrm{C}_{30} \mathrm{H}_{35} \mathrm{NO}_{9}\right]^{+}[\mathrm{M}]^{+}$553.2312, found 553.2310 .<smiles>COC(C)CC/C=C\Cc1ccsc1-n1cccn1</smiles>

(Z)-Dimethyl 2-\{4-[2-(1H-pyrazol-1-yl)thiophen-3-yl]but-2-en-1-yl\}malonate (133): The general procedure $\mathbf{B}$ was followed using 1-(thiophen-2-yl)-1H-pyrazole (131) $(75.0 \mathrm{mg}, 0.50 \mathrm{mmol})$ and dimethyl 2-vinylcyclopropane-1,1-dicarboxylate $(\mathbf{1 2 5 a})(110 \mathrm{mg}, 0.60 \mathrm{mmol})$. Isolation by column chromatography ( $n$-hexane/EtOAc: $3 / 1)$ yielded $133(67.0 \mathrm{mg}, 40 \%, E / Z=12: 1)$ as a colorless oil.

${ }^{1}$ H-NMR $\left(500 \mathrm{MHz}, \mathrm{CDCl}_{3}\right): \delta=7.70(\mathrm{dd}, J=1.9,0.5 \mathrm{~Hz}, 1 \mathrm{H}), 7.63(\mathrm{dd}, J=2.5,0.5 \mathrm{~Hz}, 1 \mathrm{H}), 7.11$ $(\mathrm{d}, J=5.5 \mathrm{~Hz}, 1 \mathrm{H}), 6.84(\mathrm{~d}, J=5.5 \mathrm{~Hz}, 1 \mathrm{H}), 6.42(\mathrm{dd}, J=2.5,1.9 \mathrm{~Hz}, 1 \mathrm{H}), 5.56(\mathrm{dtt}, J=10.6,7.6$, $1.0 \mathrm{~Hz}, 1 \mathrm{H}), 5.38(\mathrm{dtt}, J=10.6,7.6,1.0 \mathrm{~Hz}, 1 \mathrm{H}), 3.71(\mathrm{~s}, 6 \mathrm{H}), 3.40-3.24(\mathrm{~m}, 3 \mathrm{H}), 2.66$ (ddd, $J=7.6$, 7.6, $1.0 \mathrm{~Hz}, 1.84 \mathrm{H}, Z), 2.60$ (ddd, $J=7.6,7.6,1.0 \mathrm{~Hz}, 0.16 \mathrm{H}, E)$.

${ }^{13}$ C-NMR $\left(75 \mathrm{MHz}, \mathrm{CDCl}_{3}\right): \delta=169.2\left(\mathrm{C}_{\mathrm{q}}\right), 141.2(\mathrm{CH}), 137.5\left(\mathrm{C}_{\mathrm{q}}\right), 133.3\left(\mathrm{C}_{\mathrm{q}}\right), 131.7(\mathrm{CH}), 130.0$ $(\mathrm{CH}), 127.7(\mathrm{CH}), 126.0(\mathrm{CH}), 121.9(\mathrm{CH}), 107.0(\mathrm{CH}), 52.6\left(\mathrm{CH}_{3}\right), 51.4(\mathrm{CH}), 31.7\left(\mathrm{CH}_{2}, E\right), 30.7$ $\left(\mathrm{CH}_{2}, E\right), 26.9\left(\mathrm{CH}_{2}, \mathrm{Z}\right), 25.8\left(\mathrm{CH}_{2}, \mathrm{Z}\right)$.

IR (ATR): 2953, 1733, 1515, 1457, 1233, 1154, $755 \mathrm{~cm}^{-1}$.

MS (EI) $\mathrm{m} / z$ (relative intensity): 334 ([M] $\left.{ }^{+}, 10\right), 303$ (20), 203 (100).

HR-MS (ESI) $m / z$ calcd. for $\left[\mathrm{C}_{16} \mathrm{H}_{18} \mathrm{~N}_{2} \mathrm{O}_{4} \mathrm{~S}\right]^{+}[\mathrm{M}]^{+} 334.0987$, found 334.0988 . 


\subsubsection{Removal of the Directing Group}

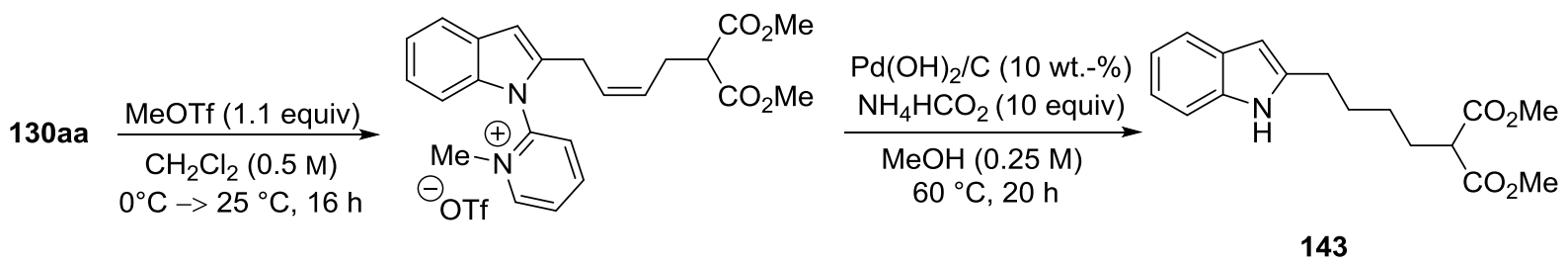

To a solution of $\mathbf{1 3 0 a a}\left(189 \mathrm{mg}, 0.50 \mathrm{mmol}, 1.00\right.$ equiv) in $\mathrm{CH}_{2} \mathrm{Cl}_{2}(1.0 \mathrm{~mL})$ was added $\mathrm{MeOTf}$ $\left(90.3 \mathrm{mg}, 60.0 \mu \mathrm{L}, 0.55 \mathrm{mmol}, 1.10\right.$ equiv) dropwise at $0{ }^{\circ} \mathrm{C}$. After $30 \mathrm{~min}$ the mixture was allowed to warm up to $25^{\circ} \mathrm{C}$ and stirred for $16 \mathrm{~h}$. After removal of the solvent in vacuo $\mathrm{Pd}(\mathrm{OH})_{2} / \mathrm{C}(27.1 \mathrm{mg}$, $10 \mathrm{wt} .-\%)$ and ammonium formate $(315 \mathrm{mg}, 5.00 \mathrm{mmol}, 10.0$ equiv) were added. The mixture was diluted in $\mathrm{MeOH}(2.0 \mathrm{~mL}, 0.25 \mathrm{M})$ and heated at $60{ }^{\circ} \mathrm{C}$ for $20 \mathrm{~h}$. After addition of EtOAc $(10 \mathrm{~mL})$ at ambient temperature, the mixture was filtered through a short pad of Celite and the solvents were removed in vacuo. The crude mixture was purified by flash column chromatography on silica gel ( $n$ hexane/EtOAc: 3/1) to yield $\mathbf{1 4 3}$ (127 $\mathrm{mg}, 84 \%)$ as a pale yellow solid.

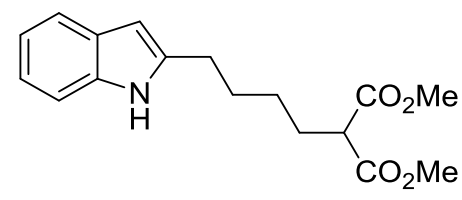

Dimethyl 2-[4-(1H-indol-2-yl)butyl]malonate (143):

M.p.: $75^{\circ} \mathrm{C}$

${ }^{1}$ H-NMR $\left(400 \mathrm{MHz}, \mathrm{CDCl}_{3}\right): \delta=8.08(\mathrm{~s}, 1 \mathrm{H}), 7.56-7.52(\mathrm{~m}, 1 \mathrm{H}), 7.32-7.26(\mathrm{~m}, 1 \mathrm{H}), 7.15-7.05(\mathrm{~m}$, 2H), $6.22(\mathrm{~d}, J=1.2 \mathrm{~Hz}, 1 \mathrm{H}), 3.74(\mathrm{~s}, 6 \mathrm{H}), 3.44(\mathrm{t}, J=7.7 \mathrm{~Hz}, 2 \mathrm{H}), 2.73(\mathrm{t}, J=7.3 \mathrm{~Hz}, 1 \mathrm{H}), 2.03-1.94$ (m, 2H), 1.78-1.68 (m, 2H), 1.47-1.36 (m, 2H).

${ }^{13} \mathbf{C}-\mathbf{N M R}\left(100 \mathrm{MHz}, \mathrm{CDCl}_{3}\right): \delta=169.8\left(\mathrm{C}_{\mathrm{q}}\right), 139.3\left(\mathrm{C}_{\mathrm{q}}\right), 135.8\left(\mathrm{C}_{\mathrm{q}}\right), 128.7\left(\mathrm{C}_{\mathrm{q}}\right), 120.8(\mathrm{CH}), 119.6$ $(\mathrm{CH}), 119.4(\mathrm{CH}), 110.3(\mathrm{CH}), 99.3(\mathrm{CH}), 52.4\left(\mathrm{CH}_{3}\right), 51.4(\mathrm{CH}), 28.6\left(\mathrm{CH}_{2}\right), 28.3\left(\mathrm{CH}_{2}\right), 27.5\left(\mathrm{CH}_{2}\right)$, $26.6\left(\mathrm{CH}_{2}\right)$.

IR (ATR): 3387, 1726, 1459, 1289, 1139, 742, $640 \mathrm{~cm}^{-1}$.

MS (EI) $m / z$ (relative intensity): 303 ([M] $\left.]^{+}, 30\right), 144$ (30), 130 (100).

HR-MS (EI) $\mathrm{m} / z$ calcd. for $\left[\mathrm{C}_{17} \mathrm{H}_{21} \mathrm{NO}_{4}\right]^{+}[\mathrm{M}]^{+}$303.1471, found 303.1465 . 


\subsubsection{Mechanistic Studies}

\subsubsection{H/D-Exchange Experiment}

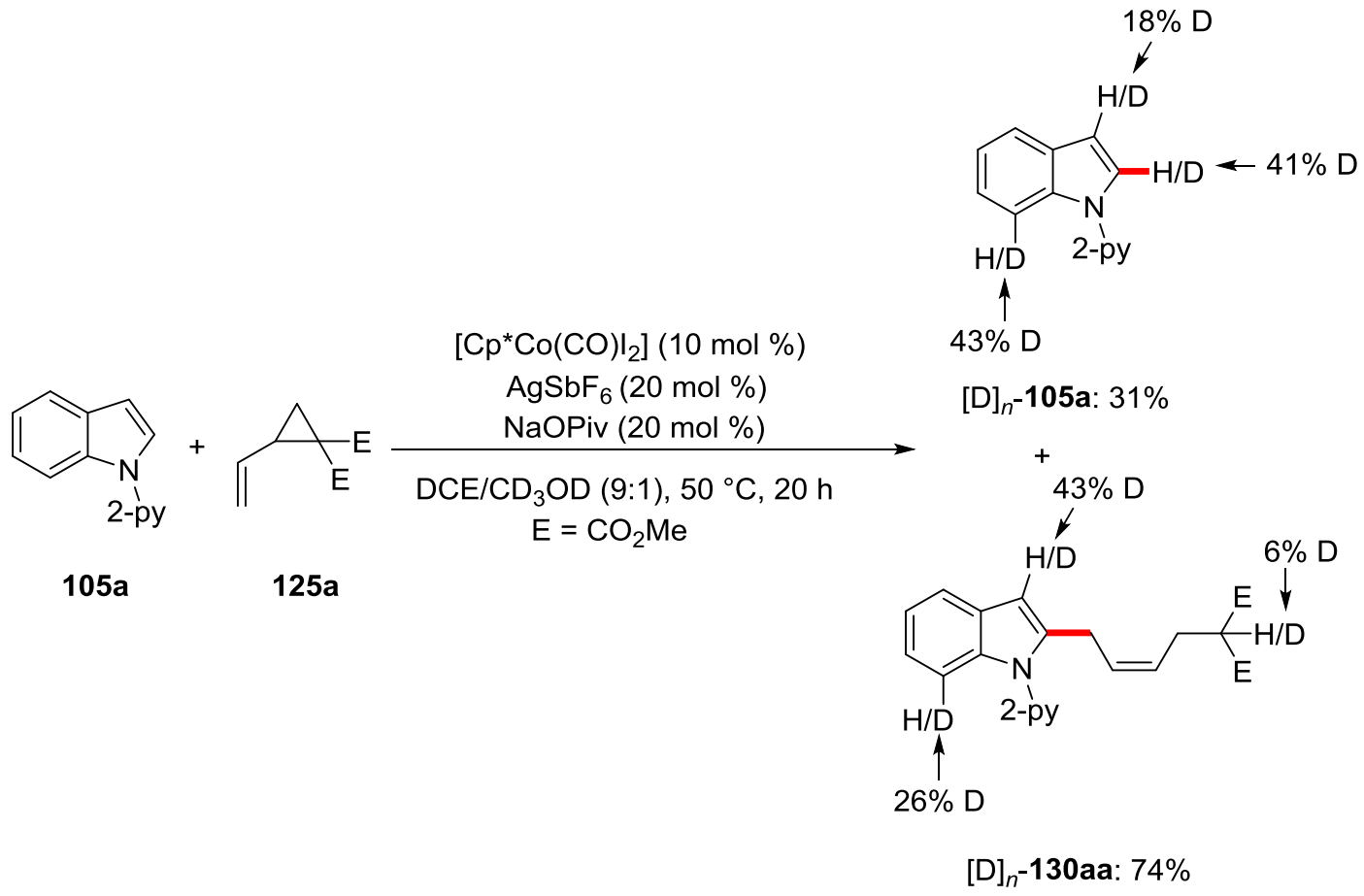

A suspension of 105a (146 mg, $0.75 \mathrm{mmol}, 1.50$ equiv), 125a (91.7 mg, $0.50 \mathrm{mmol}, 1.00$ equiv), [Cp* $\left.\mathrm{Co}(\mathrm{CO}) \mathrm{I}_{2}\right]$ (23.8 mg, $\left.10.0 \mathrm{~mol} \%\right), \mathrm{AgSbF}_{6}(34.4 \mathrm{mg}, 20.0 \mathrm{~mol} \%)$ and $\mathrm{NaOPiv}(12.4 \mathrm{mg}$, $100 \mu \mathrm{mol}, 20 \mathrm{~mol} \%)$ in DCE $(1.8 \mathrm{~mL})$ and $\mathrm{CD}_{3} \mathrm{OD}(0.2 \mathrm{~mL})$ was stirred at $50{ }^{\circ} \mathrm{C}$ for $20 \mathrm{~h}$. After removal of the solvents, the crude mixture was purified by column chromatography on silica gel ( $n$ hexane/EtOAc: $10: 1 \rightarrow 5: 1 \rightarrow 3: 1)$ to yield $[\mathrm{D}]_{n}-\mathbf{1 3 0 a a}(140 \mathrm{mg}, 74 \%, E / Z=1: 5)$ and $[\mathrm{D}]_{n}-\mathbf{1 0 5 a}$ (45.2 $\mathrm{mg}, 31 \%$ reis.) as yellow oils. 

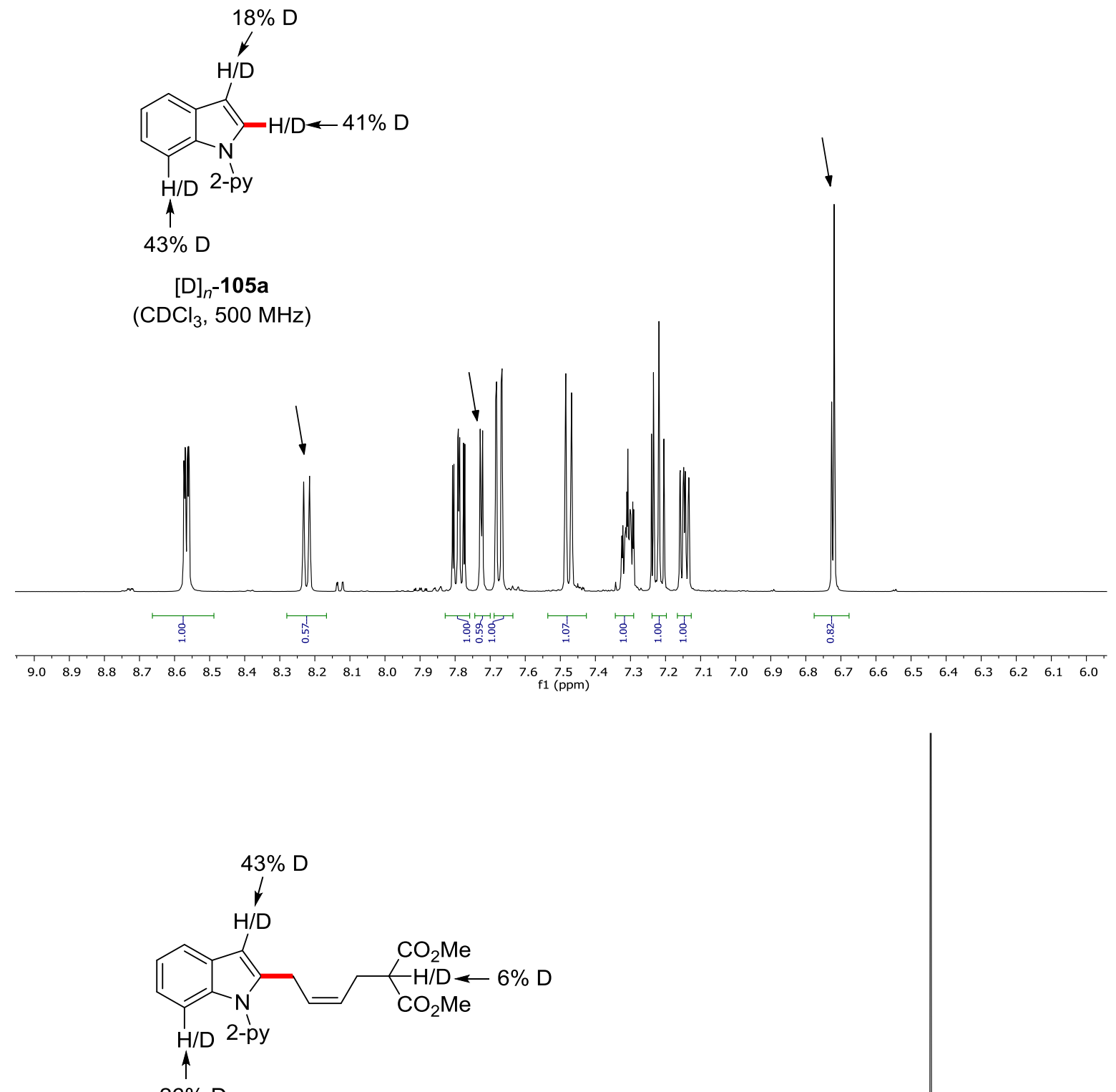

$26 \%$ D

$[D]_{n}-130$ aa

$\left(\mathrm{CDCl}_{3}, 500 \mathrm{MHz}\right)$

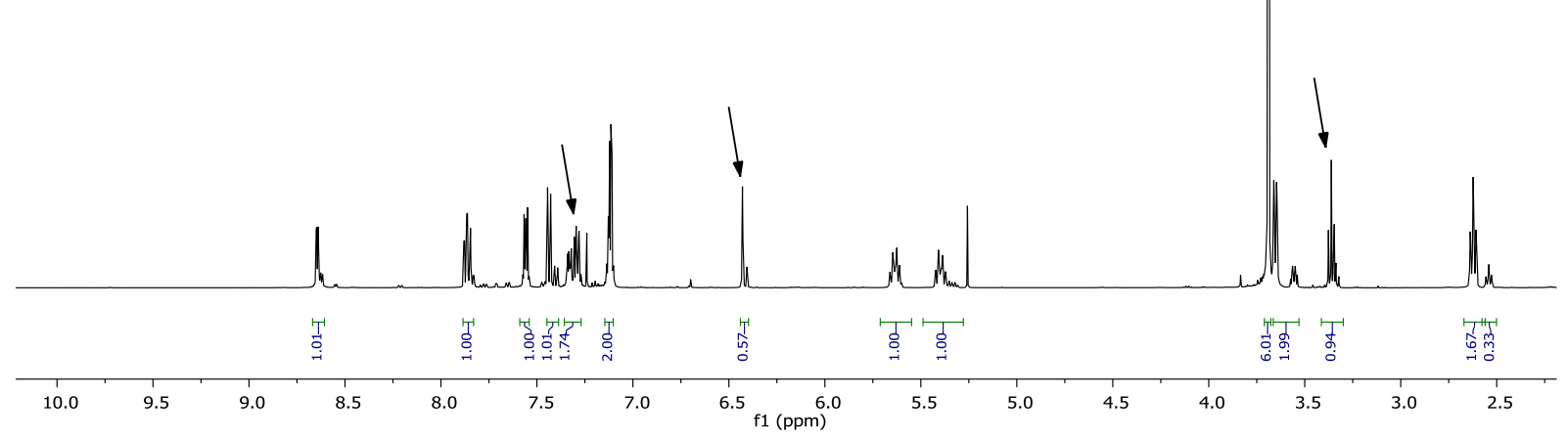




\subsubsection{H/D-exchange in the absence of the catalyst}

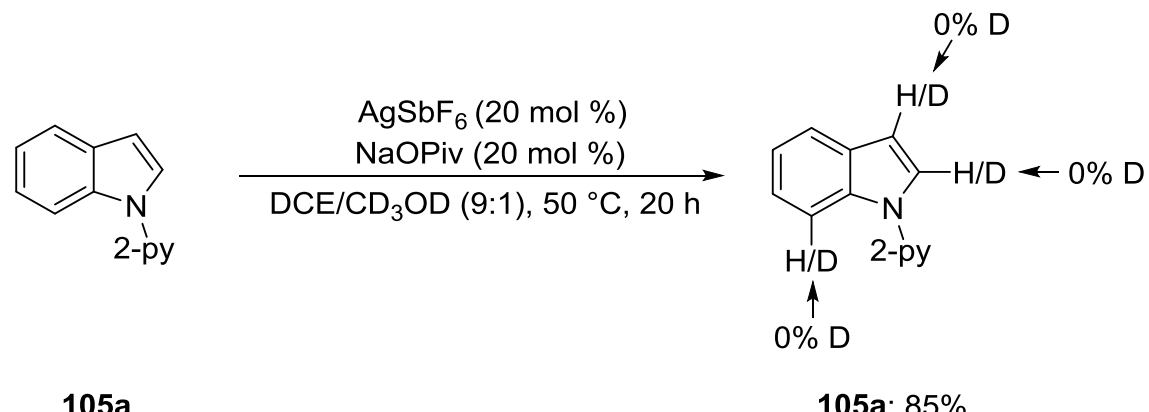

A suspension of 105a (97.1 mg, $0.50 \mathrm{mmol}, 1.00$ equiv), $\mathrm{AgSbF}_{6}(34.4 \mathrm{mg}, 20.0 \mathrm{~mol} \%$ ) and NaOPiv $(12.4 \mathrm{mg}, 100 \mu \mathrm{mol}, 20 \mathrm{~mol} \%)$ in DCE $(1.8 \mathrm{~mL})$ and $\mathrm{CD}_{3} \mathrm{OD}(0.2 \mathrm{~mL})$ was stirred at $50{ }^{\circ} \mathrm{C}$ for $20 \mathrm{~h}$. After removal of the solvents, the crude mixture was purified by column chromatography on silica gel ( $n$-hexane/EtOAc: $10: 1)$ to afford $\mathbf{1 0 5 a}(82.9 \mathrm{mg}, 85 \%$ reisolated).

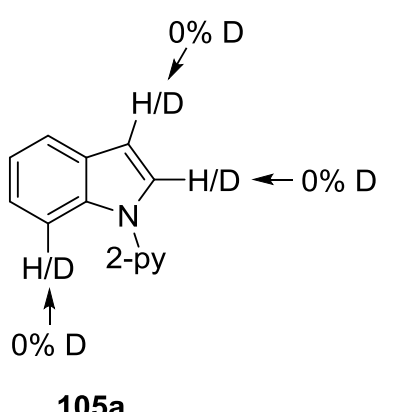

$\left(\mathrm{CDCl}_{3}, 500 \mathrm{MHz}\right)$

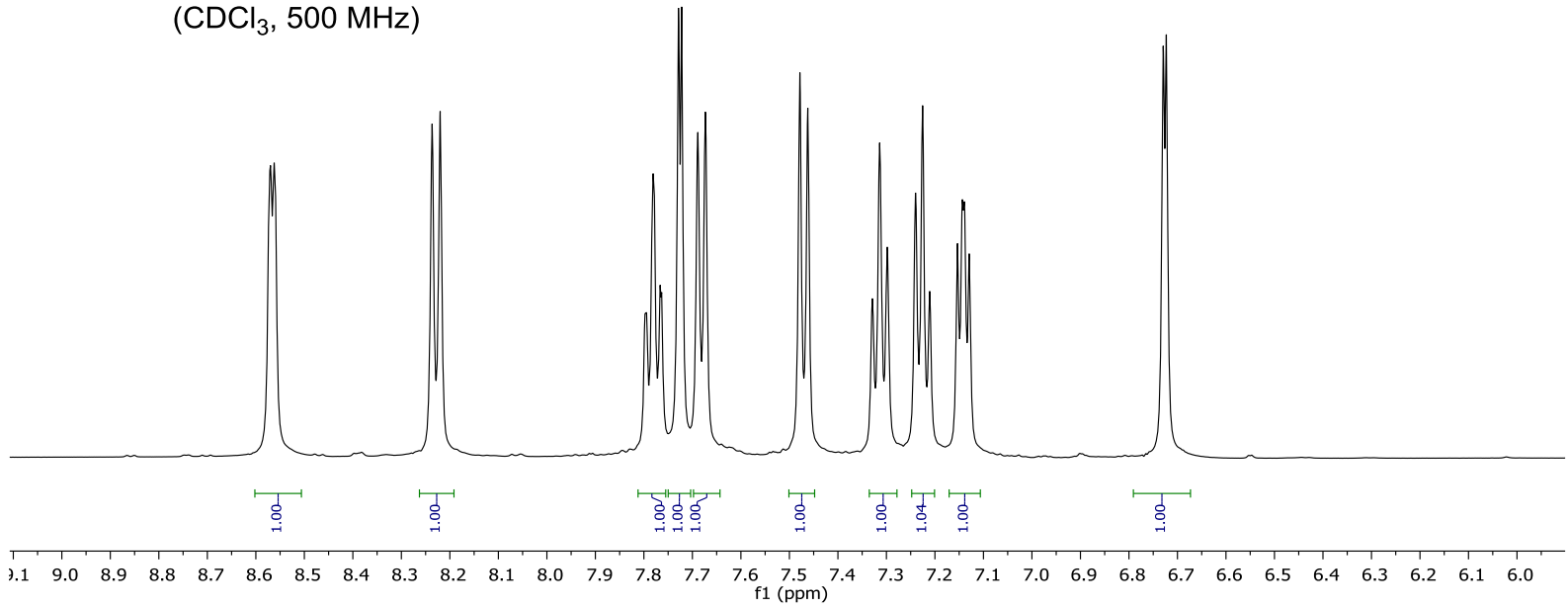




\subsubsection{Kinetic Isotope Effect}

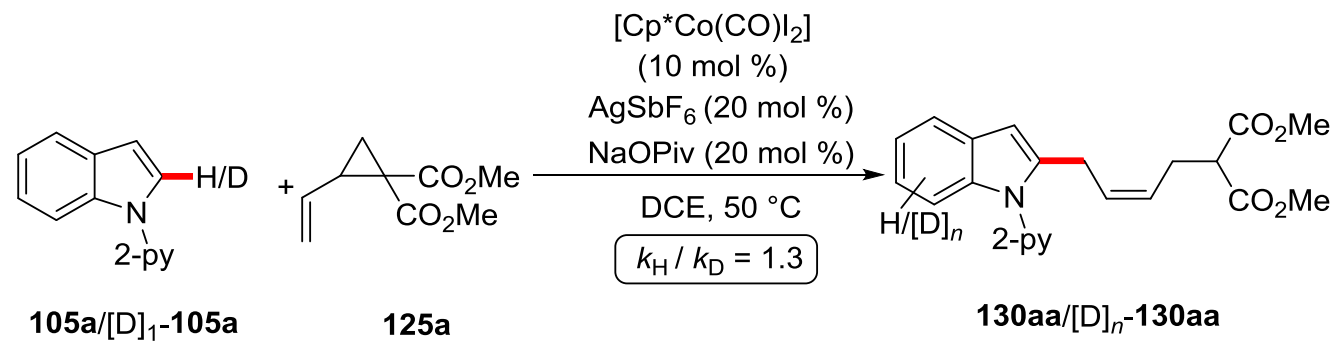

Two parallel reactions of $\mathbf{1 0 5 a}$ or $[\mathrm{D}]_{1}-\mathbf{1 0 5} \mathbf{a}^{[9]}$ were performed to determine the KIE value by comparison of the initial reaction rates. A suspension of $\mathbf{1 0 5 a}$ ( $97.1 \mathrm{mg}, 0.50 \mathrm{mmol}, 1.00$ equiv) or $[D]_{1}-\mathbf{1 0 5 a}$ (97.6 mg, $0.50 \mathrm{mmol}, 1.00$ equiv), 125a (110 mg, $0.60 \mathrm{mmol}, 1.20$ equiv), [Cp*Co(CO) $\left.\mathrm{I}_{2}\right]$ (23.8 mg, $10 \mathrm{~mol} \%), \operatorname{AgSbF}_{6}(34.4 \mathrm{mg}, 20 \mathrm{~mol} \%)$, NaOPiv (12.4 mg, $\left.20 \mathrm{~mol} \%\right)$ and $n$-dodecane $(30 \mu \mathrm{L})$ in DCE $(2.0 \mathrm{~mL})$ was stirred at $50{ }^{\circ} \mathrm{C}$. Periodic aliquots $(25 \mu \mathrm{L})$ were removed to provide the following conversions as determined by $\mathrm{GC}$-analysis:

Table 31. Conversion-time table for the KIE measurement.

\begin{tabular}{c|ccccc}
\hline$t / \min$ & 5 & 10 & 15 & 20 & 25 \\
\hline 130aa / \% & 6.0 & 10.8 & 16.2 & 19.6 & 26.2 \\
{$[\mathrm{D}]_{n}$-130aa / \% } & 3.1 & 7.6 & 11.6 & 15.7 & 18.8 \\
\hline
\end{tabular}




\subsection{Regioselective Cobalt(III)-Catalyzed C-H Alkylations}

\subsubsection{Characterization Data}

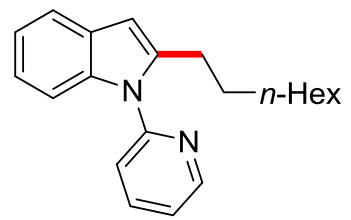

2-n-Octyl-1-(pyridin-2-yl)-1H-indole (144aa): The general procedure D was followed using 1(pyridin-2-yl)-1 $H$-indole (105a) $(97.1 \mathrm{mg}, 0.50 \mathrm{mmol})$ and oct-1-ene (143a) $(168 \mathrm{mg}, 1.50 \mathrm{mmol})$. Isolation by column chromatography ( $n$-hexane/EtOAc: $25 / 1)$ yielded $144 a(125 \mathrm{mg}, 82 \%$ ) as a colorless oil.

${ }^{1}$ H-NMR $\left(600 \mathrm{MHz}, \mathrm{CDCl}_{3}\right): \delta=8.65(\mathrm{ddd}, J=5.0,2.1,0.9 \mathrm{~Hz}, 1 \mathrm{H}), 7.87(\mathrm{ddd}, J=7.8,7.5,2.1 \mathrm{~Hz}$, $1 \mathrm{H}), 7.62-7.56(\mathrm{~m}, 1 \mathrm{H}), 7.42$ (ddd, $J=7.8,1.0,0.9 \mathrm{~Hz}, 1 \mathrm{H}), 7.36-7.27(\mathrm{~m}, 2 \mathrm{H}), 7.16-7.10(\mathrm{~m}, 2 \mathrm{H})$, $6.46(\mathrm{dt}, J=0.9,0.9 \mathrm{~Hz}, 1 \mathrm{H}), 2.86(\mathrm{t}, J=7.7 \mathrm{~Hz}, 2 \mathrm{H}), 1.58-1.50(\mathrm{~m}, 2 \mathrm{H}), 1.31-1.17(\mathrm{~m}, 10 \mathrm{H}), 0.85(\mathrm{t}$, $J=7.1 \mathrm{~Hz}, 3 \mathrm{H})$.

${ }^{13}$ C-NMR $\left(125 \mathrm{MHz}, \mathrm{CDCl}_{3}\right): \delta=151.5\left(\mathrm{C}_{\mathrm{q}}\right), 149.5(\mathrm{CH}), 141.7\left(\mathrm{C}_{\mathrm{q}}\right), 138.1(\mathrm{CH}), 137.2\left(\mathrm{C}_{\mathrm{q}}\right), 128.6$ $\left(\mathrm{C}_{\mathrm{q}}\right), 121.9(\mathrm{CH}), 121.4(\mathrm{CH}), 121.0(\mathrm{CH}), 120.4(\mathrm{CH}), 119.7(\mathrm{CH}), 109.9(\mathrm{CH}), 102.0(\mathrm{CH}), 31.9$ $\left(\mathrm{CH}_{2}\right), 29.4\left(\mathrm{CH}_{2}\right), 29.3\left(\mathrm{CH}_{2}\right), 29.2\left(\mathrm{CH}_{2}\right), 28.7\left(\mathrm{CH}_{2}\right), 27.5\left(\mathrm{CH}_{2}\right), 22.7\left(\mathrm{CH}_{2}\right), 14.2\left(\mathrm{CH}_{3}\right)$.

IR (ATR): 2925, 2854, 1584, 1468, 1455, 780, 755, $733 \mathrm{~cm}^{-1}$.

MS (EI) $m / z$ (relative intensity): 306 ([M] $\left.{ }^{+}, 25\right), 221$ (25), 207 (100), 196 (45), 168 (30).

HR-MS (EI): $m / z$ calcd. for $\left[\mathrm{C}_{21} \mathrm{H}_{26} \mathrm{~N}_{2}\right]^{+}[\mathrm{M}]^{+}$306.2096, found 306.2103.

The analytical data are in accordance with those reported in literature. ${ }^{[185]}$

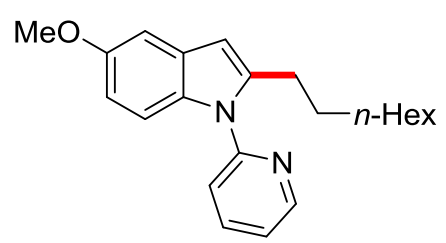

5-Methoxy-2-n-octyl-1-(pyridin-2-yl)-1H-indole (144ba): The general procedure D was followed using 5-methoxy-1-(pyridin-2-yl)-1 $H$-indole (105b) $(112 \mathrm{mg}, 0.50 \mathrm{mmol})$ and oct-1-ene (143a) (168 mg, $1.50 \mathrm{mmol}$ ). Isolation by column chromatography ( $n$-hexane/EtOAc: $25 / 1$ ) yielded 144ba (125 mg, 74\%) as a yellow oil.

${ }^{1}$ H-NMR $\left(400 \mathrm{MHz}, \mathrm{CDCl}_{3}\right): \delta=8.63(\mathrm{ddd}, J=5.0,2.1,0.9 \mathrm{~Hz}, 1 \mathrm{H}), 7.83(\mathrm{ddd}, J=7.9,7.5,2.1 \mathrm{~Hz}$, $1 \mathrm{H}), 7.38(\mathrm{ddd}, J=7.9,1.0,0.9 \mathrm{~Hz}, 1 \mathrm{H}), 7.29-7.23(\mathrm{~m}, 2 \mathrm{H}), 7.06-7.04(\mathrm{~m}, 1 \mathrm{H}), 6.77(\mathrm{dd}, J=8.9$, $2.5 \mathrm{~Hz}, 1 \mathrm{H}), 6.38(\mathrm{dt}, J=0.8,0.8 \mathrm{~Hz}, 1 \mathrm{H}), 3.85(\mathrm{~s}, 3 \mathrm{H}), 2.83(\mathrm{t}, J=7.6 \mathrm{~Hz}, 2 \mathrm{H}), 1.64-1.48(\mathrm{~m}, 2 \mathrm{H})$, $1.34-1.21(\mathrm{~m}, 10 \mathrm{H}), 0.88(\mathrm{t}, J=6.8 \mathrm{~Hz}, 3 \mathrm{H})$. 
${ }^{13}$ C-NMR $\left(100 \mathrm{MHz}, \mathrm{CDCl}_{3}\right): \delta=154.7\left(\mathrm{C}_{\mathrm{q}}\right), 151.6\left(\mathrm{C}_{\mathrm{q}}\right), 149.5(\mathrm{CH}), 142.3\left(\mathrm{C}_{\mathrm{q}}\right), 138.1(\mathrm{CH}), 132.3$ $\left(\mathrm{C}_{\mathrm{q}}\right), 129.1\left(\mathrm{C}_{\mathrm{q}}\right), 121.7(\mathrm{CH}), 120.7(\mathrm{CH}), 110.9(\mathrm{CH}), 110.8(\mathrm{CH}), 102.1(\mathrm{CH}), 101.9(\mathrm{CH}), 55.8$ $\left(\mathrm{CH}_{3}\right), 31.8\left(\mathrm{CH}_{2}\right), 29.2\left(\mathrm{CH}_{2}\right), 29.2\left(\mathrm{CH}_{2}\right), 29.1\left(\mathrm{CH}_{2}\right), 28.6\left(\mathrm{CH}_{2}\right), 27.5\left(\mathrm{CH}_{2}\right), 22.6\left(\mathrm{CH}_{2}\right), 14.0$ $\left(\mathrm{CH}_{3}\right)$.

IR (ATR): 2924, 2853, 1582, 1470, 1450, 1173, $772 \mathrm{~cm}^{-1}$.

MS (EI) $\mathrm{m} / \mathrm{z}$ (relative intensity): 336 ([M] $\left.]^{+}, 40\right), 251$ (20), 237 (100), 222 (15).

HR-MS (EI): $m / z$ calcd. for $\left[\mathrm{C}_{22} \mathrm{H}_{28} \mathrm{~N}_{2} \mathrm{O}\right]^{+}[\mathrm{M}]^{+} 336.2202$, found 336.2193 .

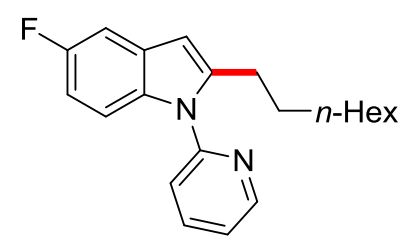

5-Fluoro-2-n-octyl-1-(pyridin-2-yl)-1 $\boldsymbol{H}$-indole (144ma): The general procedure D was followed using 5-fluoro-1-(pyridin-2-yl)-1H-indole (105m) (106 mg, $0.50 \mathrm{mmol})$ and oct-1-ene (143a) (168 mg, $1.50 \mathrm{mmol}$ ). Isolation by column chromatography ( $n$-hexane/EtOAc: $20 / 1)$ yielded $\mathbf{1 4 4 m a}$ (123 mg, 76\%) as a colorless oil.

${ }^{1}$ H-NMR $\left(300 \mathrm{MHz}, \mathrm{CDCl}_{3}\right): \delta=8.65(\mathrm{ddd}, J=4.8,2.1,1.0 \mathrm{~Hz}, 1 \mathrm{H}), 7.83(\mathrm{ddd}, J=8.0,7.5,2.1 \mathrm{~Hz}$, $1 \mathrm{H}), 7.37$ (ddd, $J=8.0,1.3,1.0 \mathrm{~Hz}, 1 \mathrm{H}), 7.31(\mathrm{ddd}, J=7.5,4.8,1.3 \mathrm{~Hz}, 1 \mathrm{H}), 7.26-7.20(\mathrm{~m}, 2 \mathrm{H})$, $7.88-7.80(\mathrm{~m}, 1 \mathrm{H}), 6.39$ (dt, $J=0.8,0.8 \mathrm{~Hz}, 1 \mathrm{H}), 2.80(\mathrm{t}, J=7.6 \mathrm{~Hz}, 2 \mathrm{H}), 1.63-1.50(\mathrm{~m}, 2 \mathrm{H}), 1.34-$ $1.17(\mathrm{~m}, 10 \mathrm{H}), 0.88(\mathrm{t}, J=6.8 \mathrm{~Hz}, 3 \mathrm{H})$.

${ }^{13} \mathrm{C}-\mathrm{NMR}\left(125 \mathrm{MHz}, \mathrm{CDCl}_{3}\right): \delta=158.3\left(\mathrm{~d},{ }^{1} J_{\mathrm{C}-\mathrm{F}}=235 \mathrm{~Hz}, \mathrm{C}_{\mathrm{q}}\right), 151.2\left(\mathrm{C}_{\mathrm{q}}\right), 149.5(\mathrm{CH}), 143.3\left(\mathrm{C}_{\mathrm{q}}\right)$, $138.2(\mathrm{CH}), 133.7\left(\mathrm{C}_{\mathrm{q}}\right), 128.9\left(\mathrm{~d},{ }^{3} J_{\mathrm{C}-\mathrm{F}}=10.2 \mathrm{~Hz}, \mathrm{C}_{\mathrm{q}}\right), 122.1(\mathrm{CH}), 120.9(\mathrm{CH}), 110.7\left(\mathrm{~d},{ }^{3} J_{\mathrm{C}-\mathrm{F}}=\right.$ $10.0 \mathrm{~Hz}, \mathrm{CH}), 109.2\left(\mathrm{~d},{ }^{2} J_{\mathrm{C}-\mathrm{F}}=24.1 \mathrm{~Hz}, \mathrm{CH}\right), 104.6\left(\mathrm{~d},{ }^{2} J_{\mathrm{C}-\mathrm{F}}=24.2 \mathrm{~Hz}, \mathrm{CH}\right), 101.9\left(\mathrm{~d},{ }^{4} J_{\mathrm{C}-\mathrm{F}}=4.1 \mathrm{~Hz}\right.$, $\mathrm{CH}), 31.8\left(\mathrm{CH}_{2}\right), 29.3\left(\mathrm{CH}_{2}\right), 29.3\left(\mathrm{CH}_{2}\right), 29.1\left(\mathrm{CH}_{2}\right), 28.6\left(\mathrm{CH}_{2}\right), 27.6\left(\mathrm{CH}_{2}\right), 22.7\left(\mathrm{CH}_{2}\right), 14.1\left(\mathrm{CH}_{3}\right)$. ${ }^{19}$ F-NMR $\left(282 \mathrm{MHz}, \mathrm{CDCl}_{3}\right): \delta=-124.2(\mathrm{~m})$.

IR (ATR): 2924, 2854, 1584, 1469, 1436, 1176, $775 \mathrm{~cm}^{-1}$.

MS (EI) $m / z$ (relative intensity): 324 ([M] $\left.]^{+}, 30\right), 239$ (25), 225 (100).

HR-MS (EI): $m / z$ calcd. for $\left[\mathrm{C}_{21} \mathrm{H}_{25} \mathrm{FN}_{2}\right]^{+}[\mathrm{M}]^{+} 324.2002$, found 324.1996 .

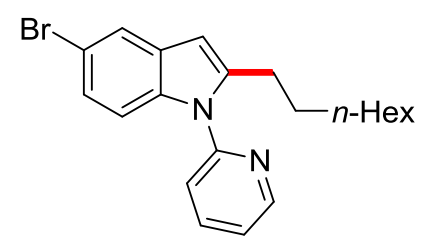

5-Bromo-2-n-octyl-1-(pyridin-2-yl)-1H-indole (144ca): The general procedure D was followed using 5-bromo-1-(pyridin-2-yl)-1H-indole (105c) (136 mg, $0.50 \mathrm{mmol}$ ) and oct-1-ene (143a) (168 mg, 
$1.50 \mathrm{mmol}$ ). Isolation by column chromatography ( $\mathrm{n}$-hexane/EtOAc: $25 / 1)$ yielded 144ca (152 mg, $79 \%)$ as a colorless oil.

${ }^{1}$ H-NMR $\left(300 \mathrm{MHz}, \mathrm{CDCl}_{3}\right): \delta=8.64(\mathrm{ddd}, J=4.9,2.1,0.9 \mathrm{~Hz}, 1 \mathrm{H}), 7.85(\mathrm{ddd}, J=8.0,7.4,2.1 \mathrm{~Hz}$, $1 \mathrm{H}), 7.67(\mathrm{dd}, J=1.5,0.9 \mathrm{~Hz}, 1 \mathrm{H}), 7.34(\mathrm{ddd}, J=8.0,0.9,0.9 \mathrm{~Hz}, 1 \mathrm{H}), 7.32$ (ddd, $J=7.4,4.9$, $0.9 \mathrm{~Hz}, 1 \mathrm{H}), 7.18-7.16(\mathrm{~m}, 2 \mathrm{H}), 6.37(\mathrm{dt}, J=0.8,0.8 \mathrm{~Hz}, 1 \mathrm{H}), 2.79(\mathrm{t}, J=7.8 \mathrm{~Hz}, 2 \mathrm{H}), 1.58-1.48(\mathrm{~m}$, 2H), 1.31-1.19 (m, 10H), 0.87 (t, $J=7.1 \mathrm{~Hz}, 3 \mathrm{H})$.

${ }^{13}$ C-NMR $\left(125 \mathrm{MHz}, \mathrm{CDCl}_{3}\right): \delta=150.9\left(\mathrm{C}_{\mathrm{q}}\right), 149.5(\mathrm{CH}), 143.0\left(\mathrm{C}_{\mathrm{q}}\right), 138.6(\mathrm{CH}), 135.8\left(\mathrm{C}_{\mathrm{q}}\right), 130.2$ $\left(\mathrm{C}_{\mathrm{q}}\right), 124.0(\mathrm{CH}), 122.2(\mathrm{CH}), 122.2(\mathrm{CH}), 120.9(\mathrm{CH}), 113.5\left(\mathrm{C}_{\mathrm{q}}\right), 111.5(\mathrm{CH}), 101.3(\mathrm{CH}), 31.8$ $\left(\mathrm{CH}_{2}\right), 29.3\left(\mathrm{CH}_{2}\right), 29.3\left(\mathrm{CH}_{2}\right), 28.5\left(\mathrm{CH}_{2}\right), 28.5\left(\mathrm{CH}_{2}\right), 27.4\left(\mathrm{CH}_{2}\right), 22.7\left(\mathrm{CH}_{2}\right), 12.1\left(\mathrm{CH}_{3}\right)$.

IR (ATR): 2924, 2853, 1486, 1469, 1456, 864, $780 \mathrm{~cm}^{-1}$.

MS (EI) $\mathrm{m} / z$ (relative intensity): 384 ([M( $\left.\left.\left.{ }^{79} \mathrm{Br}\right)\right]^{+}, 20\right), 301$ (15), 287 (100), 219 (20), 205 (30).

HR-MS (EI): $m / z$ calcd. for $\left[\mathrm{C}_{21} \mathrm{H}_{25}{ }^{79} \mathrm{BrN}_{2}\right]^{+}[\mathrm{M}]^{+}$384.1201, found 384.1197.

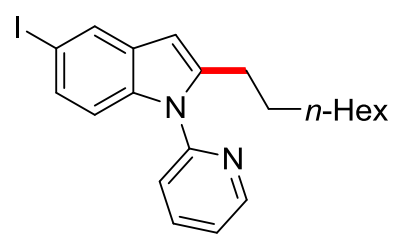

5-Iodo-2-n-octyl-1-(pyridin-2-yl)-1H-indole (144da): The general procedure $\mathrm{D}$ was followed using 5-iodo-1-(pyridin-2-yl)-1 $H$-indole (144d) $(160 \mathrm{mg}, 0.50 \mathrm{mmol})$ and oct-1-ene (143a) (168 mg, $1.50 \mathrm{mmol}$ ). Isolation by column chromatography ( $n$-hexane/EtOAc: $25 / 1)$ yielded 144da (167 mg, $77 \%)$ as a yellowish oil.

${ }^{1}$ H-NMR $\left(300 \mathrm{MHz}, \mathrm{CDCl}_{3}\right): \delta=8.66(\mathrm{ddd}, J=5.0,2.1,0.9 \mathrm{~Hz}, 1 \mathrm{H}), 7.90-7.81(\mathrm{~m}, 2 \mathrm{H}), 7.37-7.29$ $(\mathrm{m}, 3 \mathrm{H}), 7.06(\mathrm{ddd}, J=8.2,0.9,0.9 \mathrm{~Hz}, 1 \mathrm{H}), 6.35(\mathrm{dt}, J=0.8,0.8 \mathrm{~Hz}, 1 \mathrm{H}), 2.79(\mathrm{t}, J=7.8 \mathrm{~Hz}, 2 \mathrm{H})$, $1.58-1.48$ (m, 2H), 1.34-1.15 (m, 10H), 0.87 (t, $J=7.1 \mathrm{~Hz}, 3 \mathrm{H})$.

${ }^{13}$ C-NMR $\left(125 \mathrm{MHz}, \mathrm{CDCl}_{3}\right): \delta=150.9\left(\mathrm{C}_{\mathrm{q}}\right), 149.5(\mathrm{CH}), 142.6\left(\mathrm{C}_{\mathrm{q}}\right), 138.2(\mathrm{CH}), 136.3\left(\mathrm{C}_{\mathrm{q}}\right), 131.0$ $\left(\mathrm{C}_{\mathrm{q}}\right), 129.6(\mathrm{CH}), 128.4(\mathrm{CH}), 122.2(\mathrm{CH}), 120.9(\mathrm{CH}), 112.0(\mathrm{CH}), 101.0(\mathrm{CH}), 84.0\left(\mathrm{C}_{\mathrm{q}}\right), 31.8$ $\left(\mathrm{CH}_{2}\right), 29.3\left(\mathrm{CH}_{2}\right), 29.2\left(\mathrm{CH}_{2}\right), 28.5\left(\mathrm{CH}_{2}\right), 27.4\left(\mathrm{CH}_{2}\right), 27.4\left(\mathrm{CH}_{2}\right), 22.7\left(\mathrm{CH}_{2}\right), 14.9\left(\mathrm{CH}_{3}\right)$.

IR (ATR): 2923, 2852, 1585, 1469, 1455, 867, $780 \mathrm{~cm}^{-1}$.

MS (EI) $\mathrm{m} / \mathrm{z}$ (relative intensity): $432\left([\mathrm{M}]^{+}, 60\right), 347$ (20), 333 (100), 206 (40).

HR-MS (EI): $m / z$ calcd. for $\left[\mathrm{C}_{21} \mathrm{H}_{25} \mathrm{IN}_{2}\right]^{+}[\mathrm{M}]^{+} 432.1062$, found 432.1052 . 


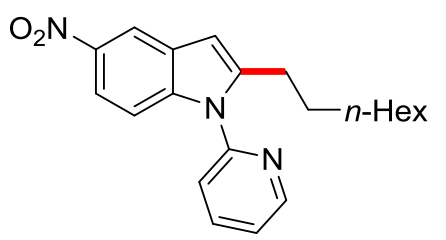

5-Nitro-2-n-octyl-1-(pyridin-2-yl)-1H-indole (144ea): The general procedure $\mathrm{D}$ was followed using 5-nitro-1-(pyridin-2-yl)-1H-indole (105e) (120 mg, $0.50 \mathrm{mmol})$ and oct-1-ene (143a) (168 mg, $1.50 \mathrm{mmol})$. Isolation by column chromatography ( $n$-hexane/EtOAc: 10/1) yielded 144ea $(73.7 \mathrm{mg}$, $42 \%)$ as a yellowish oil.

${ }^{1}$ H-NMR $\left(300 \mathrm{MHz}, \mathrm{CDCl}_{3}\right): \delta=8.66(\mathrm{ddd}, J=5.0,2.1,0.9 \mathrm{~Hz}, 1 \mathrm{H}), 8.48(\mathrm{~d}, J=2.1 \mathrm{~Hz}, 1 \mathrm{H}), 8.03-$ $7.91(\mathrm{~m}, 2 \mathrm{H}), 7.45-7.37(\mathrm{~m}, 2 \mathrm{H}), 7.25(\mathrm{ddd}, J=8.5,0.9,0.6 \mathrm{~Hz}, 1 \mathrm{H}), 6.35(\mathrm{dt}, J=0.8,0.8 \mathrm{~Hz}, 1 \mathrm{H})$, $2.76(\mathrm{t}, J=7.8 \mathrm{~Hz}, 2 \mathrm{H}), 1.61-1.51(\mathrm{~m}, 2 \mathrm{H}), 1.30-1.15(\mathrm{~m}, 10 \mathrm{H}), 0.84(\mathrm{t}, J=7.1 \mathrm{~Hz}, 3 \mathrm{H})$.

${ }^{13}$ C-NMR $\left(125 \mathrm{MHz}, \mathrm{CDCl}_{3}\right): \delta=150.3\left(\mathrm{C}_{\mathrm{q}}\right), 149.9(\mathrm{CH}), 145.2\left(\mathrm{C}_{\mathrm{q}}\right), 142.3\left(\mathrm{C}_{\mathrm{q}}\right), 140.1\left(\mathrm{C}_{\mathrm{q}}\right), 138.6$ $(\mathrm{CH}), 127.7\left(\mathrm{C}_{\mathrm{q}}\right), 123.1(\mathrm{CH}), 121.3(\mathrm{CH}), 117.2(\mathrm{CH}), 116.6(\mathrm{CH}), 110.0(\mathrm{CH}), 103.3(\mathrm{CH}), 31.8$ $\left(\mathrm{CH}_{2}\right), 29.3\left(\mathrm{CH}_{2}\right), 29.3\left(\mathrm{CH}_{2}\right), 29.1\left(\mathrm{CH}_{2}\right), 28.3\left(\mathrm{CH}_{2}\right), 27.5\left(\mathrm{CH}_{2}\right), 22.7\left(\mathrm{CH}_{2}\right), 14.1\left(\mathrm{CH}_{3}\right)$.

IR (ATR): 2926, 2855, 1586, 1512, 1470, 1331, $784 \mathrm{~cm}^{-1}$.

MS (EI) $m / z$ (relative intensity): 351 ([M] $\left.]^{+}, 20\right), 264$ (30), 252 (100), 206 (40).

HR-MS (EI): $m / z$ calcd. for $\left[\mathrm{C}_{21} \mathrm{H}_{25} \mathrm{~N}_{3} \mathrm{O}_{2}\right]^{+}[\mathrm{M}]^{+}$351.1947, found 351.1945 .

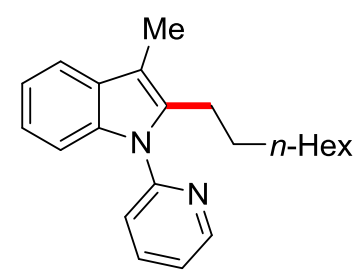

3-Methyl-2-n-octyl-1-(pyridin-2-yl)-1H-indole (144fa): The general procedure D was followed using 3-methyl-1-(pyridin-2-yl)-1 $H$-indole (105f) (104 mg, $0.50 \mathrm{mmol}$ ) and oct-1-ene (143a) (168 mg, $1.50 \mathrm{mmol}$ ). Isolation by column chromatography ( $n$-hexane/EtOAc: 20/1) yielded 144fa (104 mg, $65 \%)$ as a colorless oil.

${ }^{1} \mathbf{H}-\mathbf{N M R}\left(300 \mathrm{MHz}, \mathrm{CDCl}_{3}\right): \delta=8.64(\mathrm{ddd}, J=4.9,2.0,0.9 \mathrm{~Hz}, 1 \mathrm{H}), 7.85(\mathrm{ddd}, J=7.9,7.4,2.0 \mathrm{~Hz}$, $1 \mathrm{H}), 7.56-7.51(\mathrm{~m}, 1 \mathrm{H}), 7.41(\mathrm{ddd}, J=7.9,1.0,0.9 \mathrm{~Hz}, 1 \mathrm{H}), 7.33-7.26(\mathrm{~m}, 2 \mathrm{H}), 7.16-7.08(\mathrm{~m}, 2 \mathrm{H})$, $2.89(\mathrm{t}, J=7.2 \mathrm{~Hz}, 2 \mathrm{H}), 2.31(\mathrm{~s}, 3 \mathrm{H}), 1.32-1.13(\mathrm{~m}, 12 \mathrm{H}), 0.85(\mathrm{t}, J=6.7 \mathrm{~Hz}, 3 \mathrm{H})$.

${ }^{13} \mathrm{C}-\mathrm{NMR}\left(125 \mathrm{MHz}, \mathrm{CDCl}_{3}\right): \delta=151.9\left(\mathrm{C}_{\mathrm{q}}\right), 149.4(\mathrm{CH}), 138.0(\mathrm{CH}), 137.2\left(\mathrm{C}_{\mathrm{q}}\right), 136.5\left(\mathrm{C}_{\mathrm{q}}\right), 129.4$ $\left(\mathrm{C}_{\mathrm{q}}\right), 121.6(\mathrm{CH}), 121.5(\mathrm{CH}), 120.9(\mathrm{CH}), 120.0(\mathrm{CH}), 118.0(\mathrm{CH}), 109.7(\mathrm{CH}), 109.7\left(\mathrm{C}_{\mathrm{q}}\right), 31.9$ $\left(\mathrm{CH}_{2}\right), 29.4\left(\mathrm{CH}_{2}\right), 29.2\left(\mathrm{CH}_{2}\right), 29.2\left(\mathrm{CH}_{2}\right), 29.2\left(\mathrm{CH}_{2}\right), 24.8\left(\mathrm{CH}_{2}\right), 22.7\left(\mathrm{CH}_{2}\right), 14.2\left(\mathrm{CH}_{3}\right), 8.9\left(\mathrm{CH}_{3}\right)$. IR (ATR): 2923, 2854, 1585, 1470, 1436, 1362, $738 \mathrm{~cm}^{-1}$.

MS (EI) $\mathrm{m} / z$ (relative intensity): 320 ([M] $\left.]^{+}, 50\right), 235$ (30), 221 (100), 207 (25).

HR-MS (EI): $m / z$ calcd. for $\left[\mathrm{C}_{22} \mathrm{H}_{28} \mathrm{~N}_{2}\right]^{+}[\mathrm{M}]^{+} 320.2252$, found 320.2251 . 


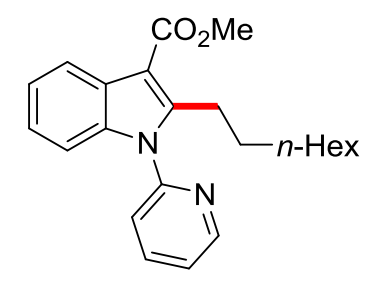

Methyl 2-n-octyl-1-(pyridin-2-yl)-1H-indole-3-carboxylate (144na): The general procedure D was followed using methyl 1-(pyridin-2-yl)-1 $H$-indole-3-carboxylate (105n) $(126 \mathrm{mg}, 0.50 \mathrm{mmol})$ and oct1-ene (143a) (168 mg, $1.50 \mathrm{mmol}$ ). Isolation by column chromatography ( $n$-hexane/EtOAc: 5/1) yielded 144na (140 $\mathrm{mg}, 77 \%)$ as a colorless oil.

${ }^{1}$ H-NMR $\left(300 \mathrm{MHz}, \mathrm{CDCl}_{3}\right): \delta=8.69(\mathrm{ddd}, J=4.9,2.1,0.9 \mathrm{~Hz}, 1 \mathrm{H}), 8.18(\mathrm{ddd}, J=7.9,7.7,0.9 \mathrm{~Hz}$, $1 \mathrm{H}), 7.91$ (ddd, $J=7.9,0.9,0.9 \mathrm{~Hz}, 1 \mathrm{H}), 7.45-7.38(\mathrm{~m}, 2 \mathrm{H}), 7.28-7.21(\mathrm{~m}, 1 \mathrm{H}), 7.18-7.08(\mathrm{~m}, 2 \mathrm{H})$, $3.97(\mathrm{~s}, 3 \mathrm{H}), 2.81(\mathrm{t}, J=7.8 \mathrm{~Hz}, 2 \mathrm{H}), 1.60-1.47(\mathrm{~m}, 2 \mathrm{H}), 1.27-1.10(\mathrm{~m}, 10 \mathrm{H}), 0.86(\mathrm{t}, J=6.8 \mathrm{~Hz}, 3 \mathrm{H})$. ${ }^{13}$ C-NMR $\left(125 \mathrm{MHz}, \mathrm{CDCl}_{3}\right): \delta=165.9\left(\mathrm{C}_{\mathrm{q}}\right), 150.2\left(\mathrm{C}_{\mathrm{q}}\right), 149.9\left(\mathrm{C}_{\mathrm{q}}\right), 149.8(\mathrm{CH}), 138.4(\mathrm{CH}), 136.7$ $\left(\mathrm{C}_{\mathrm{q}}\right), 126.6\left(\mathrm{C}_{\mathrm{q}}\right), 123.4(\mathrm{CH}), 122.6(\mathrm{CH}), 122.3(\mathrm{CH}), 122.2(\mathrm{CH}), 121.4(\mathrm{CH}), 110.1(\mathrm{CH}), 105.3$ $\left(\mathrm{C}_{\mathrm{q}}\right), 50.8\left(\mathrm{CH}_{3}\right), 31.8\left(\mathrm{CH}_{2}\right), 29.5\left(\mathrm{CH}_{2}\right), 29.4\left(\mathrm{CH}_{2}\right), 29.1\left(\mathrm{CH}_{2}\right), 29.0\left(\mathrm{CH}_{2}\right), 25.9\left(\mathrm{CH}_{2}\right), 22.6\left(\mathrm{CH}_{2}\right)$, $14.1\left(\mathrm{CH}_{3}\right)$.

IR (ATR): 2924, 2853, 1697, 1587, 1468, 1434, 1182, $1076 \mathrm{~cm}^{-1}$.

MS (EI) $m / z$ (relative intensity): 364 ([M] $]^{+}, 35$ ), 305 (20), 265 (35), 250 (30), 207 (100).

HR-MS (EI): $m / z$ calcd. for $\left[\mathrm{C}_{23} \mathrm{H}_{28} \mathrm{~N}_{2} \mathrm{O}_{2}\right]^{+}[\mathrm{M}]^{+} 364.2148$, found 364.2151.

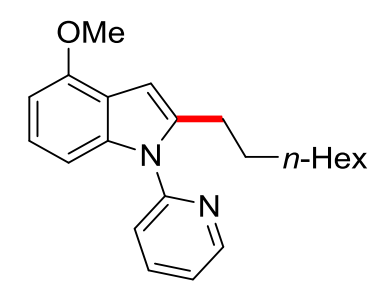

4-Methoxy-2-n-octyl-1-(pyridin-2-yl)-1H-indole (144ha): The general procedure D was followed using 4-methoxy-1-(pyridin-2-yl)-1 $H$-indole (105h) (112 mg, $0.50 \mathrm{mmol})$ and oct-1-ene (143a) $(168 \mathrm{mg}, 1.50 \mathrm{mmol})$. Isolation by column chromatography ( $n$-hexane/EtOAc: $25 / 1)$ yielded 144ha (132 $\mathrm{mg}, 79 \%)$ as a colorless oil.

${ }^{1} \mathbf{H}-\mathbf{N M R}\left(300 \mathrm{MHz}, \mathrm{CDCl}_{3}\right): \delta=8.64(\mathrm{ddd}, J=5.0,2.1,0.9 \mathrm{~Hz}, 1 \mathrm{H}), 7.85(\mathrm{ddd}, J=8.0,7.5,2.1 \mathrm{~Hz}$, $1 \mathrm{H}), 7.40(\mathrm{ddd}, J=8.0,1.0,0.9 \mathrm{~Hz}, 1 \mathrm{H}), 7.32-7.27(\mathrm{~m}, 2 \mathrm{H}), 7.08-6.92(\mathrm{~m}, 1 \mathrm{H}), 6.62-6.52(\mathrm{~m}, 2 \mathrm{H})$, $3.96(\mathrm{~s}, 3 \mathrm{H}), 2.81(\mathrm{t}, J=7.6 \mathrm{~Hz}, 2 \mathrm{H}), 1.61-1.51(\mathrm{~m}, 2 \mathrm{H}), 1.32-1.21(\mathrm{~m}, 10 \mathrm{H}), 0.88(\mathrm{t}, J=6.8 \mathrm{~Hz}, 3 \mathrm{H})$. ${ }^{13}$ C-NMR $\left(125 \mathrm{MHz}, \mathrm{CDCl}_{3}\right): \delta=152.4\left(\mathrm{C}_{\mathrm{q}}\right), 151.6\left(\mathrm{C}_{\mathrm{q}}\right), 149.4(\mathrm{CH}), 140.1\left(\mathrm{C}_{\mathrm{q}}\right), 138.4\left(\mathrm{C}_{\mathrm{q}}\right), 138.0$ $(\mathrm{CH}), 122.1(\mathrm{CH}), 121.9(\mathrm{CH}), 121.1(\mathrm{CH}), 118.9\left(\mathrm{C}_{\mathrm{q}}\right), 103.6(\mathrm{CH}), 100.7(\mathrm{CH}), 99.0(\mathrm{CH}), 55.4$ $\left(\mathrm{CH}_{3}\right), 31.8\left(\mathrm{CH}_{2}\right), 29.3\left(\mathrm{CH}_{2}\right), 29.3\left(\mathrm{CH}_{2}\right), 29.1\left(\mathrm{CH}_{2}\right), 28.6\left(\mathrm{CH}_{2}\right), 27.4\left(\mathrm{CH}_{2}\right), 22.7\left(\mathrm{CH}_{2}\right), 14.1$ $\left(\mathrm{CH}_{3}\right)$. 
IR (ATR): 2923, 2854, 1585, 1470, 1435, 779, $738 \mathrm{~cm}^{-1}$.

MS (EI) $m / z$ (relative intensity): 336 ([M] $\left.{ }^{+}, 45\right), 251$ (20), 237 (100), 221 (25).

HR-MS (EI): $m / z$ calcd. for $\left[\mathrm{C}_{22} \mathrm{H}_{28} \mathrm{~N}_{2} \mathrm{O}\right]^{+}[\mathrm{M}]^{+} 336.2202$, found 336.2205 .

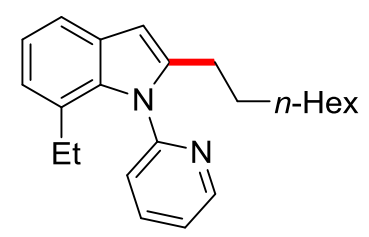

7-Ethyl-2-n-octyl-1-(pyridin-2-yl)-1H-indole (144ja): The general procedure $\mathrm{D}$ was followed using 7-ethyl-1-(pyridin-2-yl)-1 $H$-indole (105j) $(111 \mathrm{mg}, 0.50 \mathrm{mmol})$ and oct-1-ene (143a) (168 mg, $1.50 \mathrm{mmol}$ ). Isolation by column chromatography ( -hexane/EtOAc: 20/1) yielded 144ja (102 mg, $61 \%$ ) as a colorless oil.

${ }^{1}$ H-NMR $\left(300 \mathrm{MHz}, \mathrm{CDCl}_{3}\right): \delta=8.65(\mathrm{ddd}, J=4.9,2.1,0.9 \mathrm{~Hz}, 1 \mathrm{H}), 7.82(\mathrm{ddd}, J=7.9,7.6,2.1 \mathrm{~Hz}$, 1H), 7.45-7.36 (m, 2H), $7.32(\mathrm{ddd}, J=7.6,1.0,0.9 \mathrm{~Hz}, 1 \mathrm{H}), 7.06(\mathrm{~d}, J=7.7 \mathrm{~Hz}, 1 \mathrm{H}), 6.96-6.90$ (m, $1 \mathrm{H}), 6.40$ (dt, $J=0.8,0.8 \mathrm{~Hz}, 1 \mathrm{H}), 2.45$ (t, $J=7.6 \mathrm{~Hz}, 2 \mathrm{H}), 2.15$ (q, $J=7.5 \mathrm{~Hz}, 2 \mathrm{H}), 1.60-1.49$ (m, 2H), 1.32-1.19 (m, 10H), 0.94 (t, $J=7.5 \mathrm{~Hz}, 3 \mathrm{H}), 0.87$ (t, $J=7.1 \mathrm{~Hz}, 3 \mathrm{H})$.

${ }^{13}$ C-NMR $\left(125 \mathrm{MHz}, \mathrm{CDCl}_{3}\right): \delta=153.5\left(\mathrm{C}_{\mathrm{q}}\right), 149.0(\mathrm{CH}), 142.5\left(\mathrm{C}_{\mathrm{q}}\right), 137.5(\mathrm{CH}), 135.9\left(\mathrm{C}_{\mathrm{q}}\right), 129.4$ $\left(\mathrm{C}_{\mathrm{q}}\right), 127.3\left(\mathrm{C}_{\mathrm{q}}\right), 124.0(\mathrm{CH}), 123.2(\mathrm{CH}), 121.8(\mathrm{CH}), 120.3(\mathrm{CH}), 117.7(\mathrm{CH}), 101.3(\mathrm{CH}), 31.9$ $\left(\mathrm{CH}_{2}\right), 29.4\left(\mathrm{CH}_{2}\right), 29.3\left(\mathrm{CH}_{2}\right), 29.2\left(\mathrm{CH}_{2}\right), 28.4\left(\mathrm{CH}_{2}\right), 27.2\left(\mathrm{CH}_{2}\right), 24.9\left(\mathrm{CH}_{2}\right), 22.7\left(\mathrm{CH}_{2}\right), 14.6$ $\left(\mathrm{CH}_{3}\right), 14.1\left(\mathrm{CH}_{3}\right)$.

IR (ATR): 2926, 2855, 1582, 1467, 1435, 1340, 798, $741 \mathrm{~cm}^{-1}$.

MS (EI) $\mathrm{m} / \mathrm{z}$ (relative intensity): 334 ([M] $\left.{ }^{+}, 40\right), 249$ (80), 235 (100), 219 (30).

HR-MS (EI): $m / z$ calcd. for $\left[\mathrm{C}_{23} \mathrm{H}_{30} \mathrm{~N}_{2}\right]^{+}[\mathrm{M}]^{+} 334.2409$, found 334.2413 .

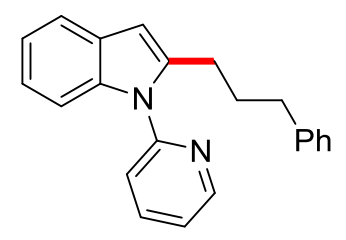

2-(3-Phenylpropyl)-1-(pyridin-2-yl)-1H-indole (144ab): The general procedure D was followed using 1-(pyridin-2-yl)-1H-indole (105a) $(97.1 \mathrm{mg}, 0.50 \mathrm{mmol})$ and allylbenzene (143b) (177 mg, $1.50 \mathrm{mmol}$ ). Isolation by column chromatography ( $n$-hexane/EtOAc: $30 / 1)$ yielded 144ab (133 mg, $85 \%)$ as a colorless oil.

${ }^{1} \mathbf{H}-\mathbf{N M R}\left(400 \mathrm{MHz}, \mathrm{CDCl}_{3}\right): \delta=8.65(\mathrm{ddd}, J=5.0,2.1,0.9 \mathrm{~Hz}, 1 \mathrm{H}), 7.85(\mathrm{ddd}, J=8.0,7.5,2.1 \mathrm{~Hz}$, $1 \mathrm{H}), 7.62-7.56(\mathrm{~m}, 1 \mathrm{H}), 7.43-7.35(\mathrm{~m}, 2 \mathrm{H}), 7.32-7.25(\mathrm{~m}, 3 \mathrm{H}), 7.22-7.12(\mathrm{~m}, 5 \mathrm{H}), 6.51(\mathrm{dt}, J=0.8$, $0.8 \mathrm{~Hz}, 1 \mathrm{H}), 2.93(\mathrm{t}, J=7.7 \mathrm{~Hz}, 2 \mathrm{H}), 2.66(\mathrm{t}, J=7.5 \mathrm{~Hz}, 2 \mathrm{H}), 1.93(\mathrm{tt}, J=7.7,7.5 \mathrm{~Hz}, 2 \mathrm{H})$. 
${ }^{13}$ C-NMR $\left(100 \mathrm{MHz}, \mathrm{CDCl}_{3}\right): \delta=151.4\left(\mathrm{C}_{\mathrm{q}}\right), 149.6(\mathrm{CH}), 141.9\left(\mathrm{C}_{\mathrm{q}}\right), 141.0\left(\mathrm{C}_{\mathrm{q}}\right), 138.1(\mathrm{CH}), 137.2$ $\left(\mathrm{C}_{\mathrm{q}}\right), 128.5\left(\mathrm{C}_{\mathrm{q}}\right), 128.3(\mathrm{CH}), 128.2(\mathrm{CH}), 125.7(\mathrm{CH}), 121.9(\mathrm{CH}), 121.6(\mathrm{CH}), 120.9(\mathrm{CH}), 120.5$ $(\mathrm{CH}), 119.8(\mathrm{CH}), 110.0(\mathrm{CH}), 102.3(\mathrm{CH}), 35.3\left(\mathrm{CH}_{2}\right), 30.2\left(\mathrm{CH}_{2}\right), 26.9\left(\mathrm{CH}_{2}\right)$.

IR (ATR): 3025, 2932, 1584, 1468, 1455, 735, $698 \mathrm{~cm}^{-1}$.

MS (EI) $m / z$ (relative intensity): 312 ([M] $\left.]^{+}, 20\right), 221$ (100), 206 (40), 91 (20).

HR-MS (EI): $m / z$ calcd. for $\left[\mathrm{C}_{22} \mathrm{H}_{20} \mathrm{~N}_{2}\right]^{+}[\mathrm{M}]^{+} 312.1626$, found 312.1624 .

The analytical data are in accordance with those reported in literature. ${ }^{[186]}$

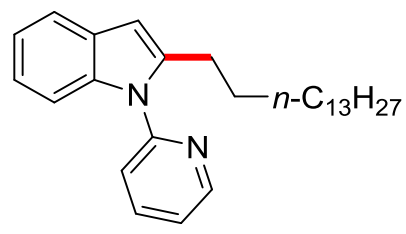

2-n-Pentadecyl-1-(pyridin-2-yl)-1H-indole (144ac): The general procedure $\mathrm{D}$ was followed using 1(pyridin-2-yl)-1H-indole (105a) $(97.1 \mathrm{mg}, 0.50 \mathrm{mmol})$ and pentadec-1-ene (143c) (316 mg, $1.50 \mathrm{mmol}$ ). Isolation by column chromatography ( $n$-hexane/EtOAc: $25 / 1)$ yielded 144ac (159 mg, $79 \%)$ as a colorless oil.

${ }^{1} \mathbf{H}-\mathbf{N M R}\left(300 \mathrm{MHz}, \mathrm{CDCl}_{3}\right): \delta=8.67(\mathrm{ddd}, J=5.0,2.1,0.9 \mathrm{~Hz}, 1 \mathrm{H}), 7.86(\mathrm{ddd}, J=8.0,7.5,2.1 \mathrm{~Hz}$, $1 \mathrm{H}), 7.62-7.56(\mathrm{~m}, 1 \mathrm{H}), 7.42(\mathrm{ddd}, J=8.0,1.0,0.9 \mathrm{~Hz}, 1 \mathrm{H}), 7.36-7.27(\mathrm{~m}, 2 \mathrm{H}), 7.16-7.10(\mathrm{~m}, 2 \mathrm{H})$, $6.46(\mathrm{dt}, J=0.8,0.8 \mathrm{~Hz}, 1 \mathrm{H}), 2.86(\mathrm{t}, J=7.7 \mathrm{~Hz}, 2 \mathrm{H}), 1.65-1.53(\mathrm{~m}, 2 \mathrm{H}), 1.35-1.19(\mathrm{~m}, 24 \mathrm{H}), 0.92(\mathrm{t}$, $J=7.1 \mathrm{~Hz}, 3 \mathrm{H})$.

${ }^{13}$ C-NMR $\left(125 \mathrm{MHz}, \mathrm{CDCl}_{3}\right): \delta=151.5\left(\mathrm{C}_{\mathrm{q}}\right), 149.4(\mathrm{CH}), 141.6\left(\mathrm{C}_{\mathrm{q}}\right), 138.0(\mathrm{CH}), 137.1\left(\mathrm{C}_{\mathrm{q}}\right), 128.6$ $\left(\mathrm{C}_{\mathrm{q}}\right), 121.8(\mathrm{CH}), 121.4(\mathrm{CH}), 121.0(\mathrm{CH}), 120.4(\mathrm{CH}), 119.7(\mathrm{CH}), 109.9(\mathrm{CH}), 102.0(\mathrm{CH}), 31.9$ $\left(\mathrm{CH}_{2}\right), 29.7\left(\mathrm{CH}_{2}\right), 29.7\left(\mathrm{CH}_{2}\right), 29.7\left(\mathrm{CH}_{2}\right), 29.7\left(\mathrm{CH}_{2}\right), 29.6\left(\mathrm{CH}_{2}\right), 29.6\left(\mathrm{CH}_{2}\right), 29.5\left(\mathrm{CH}_{2}\right), 29.4$ $\left(\mathrm{CH}_{2}\right), 29.4\left(\mathrm{CH}_{2}\right), 29.3\left(\mathrm{CH}_{2}\right), 28.6\left(\mathrm{CH}_{2}\right), 27.5\left(\mathrm{CH}_{2}\right), 22.7\left(\mathrm{CH}_{2}\right), 14.0\left(\mathrm{CH}_{3}\right)$.

IR (ATR): 2921, 2851, 1485, 1469, 1456, 780, $735 \mathrm{~cm}^{-1}$.

MS (EI) $m / z$ (relative intensity): 404 ([M] $\left.]^{+}, 35\right), 221$ (55), 207 (100), 195 (15).

HR-MS (EI): $m / z$ calcd. for $\left[\mathrm{C}_{28} \mathrm{H}_{40} \mathrm{~N}_{2}\right]^{+}[\mathrm{M}]^{+}$404.3191, found 404.3182 .

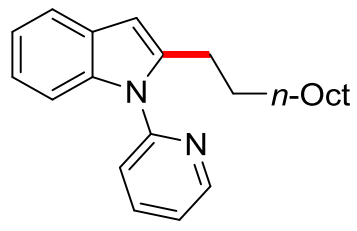

2-n-Decyl-1-(pyridin-2-yl)-1H-indole (144ad): The general procedure D was followed using 1(pyridin-2-yl)-1H-indole (105a) $(97.1 \mathrm{mg}, 0.50 \mathrm{mmol})$ and dec-1-ene (143d) $(210 \mathrm{mg}, 1.50 \mathrm{mmol})$. 
Isolation by column chromatography ( $n$-hexane/EtOAc: $25 / 1)$ yielded 144ad (125 $\mathrm{mg}, 75 \%$ ) as a colorless oil.

${ }^{1} \mathbf{H}-\mathbf{N M R}\left(300 \mathrm{MHz}, \mathrm{CDCl}_{3}\right): \delta=8.66(\mathrm{ddd}, J=5.0,2.1,0.9 \mathrm{~Hz}, 1 \mathrm{H}), 7.86(\mathrm{ddd}, J=8.0,7.5,2.1 \mathrm{~Hz}$, $1 \mathrm{H}), 7.62-7.56(\mathrm{~m}, 1 \mathrm{H}), 7.42(\mathrm{ddd}, J=8.0,1.0,0.9 \mathrm{~Hz}, 1 \mathrm{H}), 7.36-7.27(\mathrm{~m}, 2 \mathrm{H}), 7.16-7.10(\mathrm{~m}, 2 \mathrm{H})$, $6.45(\mathrm{dt}, J=0.8,0.8 \mathrm{~Hz}, 1 \mathrm{H}), 2.86(\mathrm{t}, J=7.7 \mathrm{~Hz}, 2 \mathrm{H}), 1.65-1.51(\mathrm{~m}, 2 \mathrm{H}), 1.35-1.18(\mathrm{~m}, 14 \mathrm{H}), 0.89$ (t, $J=7.1 \mathrm{~Hz}, 3 \mathrm{H})$.

${ }^{13} \mathrm{C}-\mathrm{NMR}\left(125 \mathrm{MHz}, \mathrm{CDCl}_{3}\right): \delta=151.5\left(\mathrm{C}_{\mathrm{q}}\right), 149.4(\mathrm{CH}), 141.6\left(\mathrm{C}_{\mathrm{q}}\right), 138.0(\mathrm{CH}), 137.1\left(\mathrm{C}_{\mathrm{q}}\right), 128.6$ $\left(\mathrm{C}_{\mathrm{q}}\right), 121.8(\mathrm{CH}), 121.4(\mathrm{CH}), 121.0(\mathrm{CH}), 120.4(\mathrm{CH}), 119.7(\mathrm{CH}), 109.9(\mathrm{CH}), 102.0(\mathrm{CH}), 31.9$ $\left(\mathrm{CH}_{2}\right), 29.6\left(\mathrm{CH}_{2}\right), 29.6\left(\mathrm{CH}_{2}\right), 29.5\left(\mathrm{CH}_{2}\right), 29.4\left(\mathrm{CH}_{2}\right), 29.3\left(\mathrm{CH}_{2}\right), 28.6\left(\mathrm{CH}_{2}\right), 27.5\left(\mathrm{CH}_{2}\right), 22.7$ $\left(\mathrm{CH}_{2}\right), 14.1\left(\mathrm{CH}_{3}\right)$.

IR (ATR): 2923, 2852, 1585, 1468, 1455, 780, $735 \mathrm{~cm}^{-1}$.

MS (EI) $m / z$ (relative intensity): 334 ([M] $\left.]^{+}, 45\right), 249$ (30), 235 (100), 221 (45).

HR-MS (EI): $m / z$ calcd. for $\left[\mathrm{C}_{23} \mathrm{H}_{30} \mathrm{~N}_{2}\right]^{+}[\mathrm{M}]^{+} 334.2409$, found 334.2407 .

The analytical data are in accordance with those reported in literature. ${ }^{[186]}$

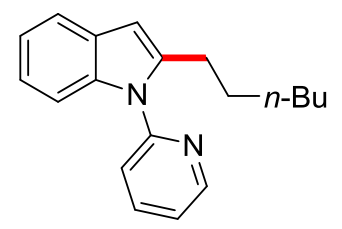

2-n-Hexyl-1-(pyridin-2-yl)-1H-indole (145ae): The general procedure D was followed using 1(pyridin-2-yl)-1H-indole (105a) $(97.1 \mathrm{mg}, 0.50 \mathrm{mmol})$ and hex-1-ene (143e) (126 mg, $1.50 \mathrm{mmol}$ ). Isolation by column chromatography ( $n$-hexane/EtOAc: $25 / 1)$ yielded 144ae $(90.5 \mathrm{mg}, 65 \%$ ) as a colorless oil.

${ }^{1} \mathbf{H}-\mathbf{N M R}\left(400 \mathrm{MHz}, \mathrm{CDCl}_{3}\right): \delta=8.67$ (ddd, $\left.J=5.0,2.1,0.9 \mathrm{~Hz}, 1 \mathrm{H}\right), 7.87$ (ddd, $J=8.0,7.5,2.1 \mathrm{~Hz}$, $1 \mathrm{H}), 7.62-7.56(\mathrm{~m}, 1 \mathrm{H}), 7.42(\mathrm{ddd}, J=8.0,1.0,0.9 \mathrm{~Hz}, 1 \mathrm{H}), 7.36-7.27(\mathrm{~m}, 2 \mathrm{H}), 7.16-7.10(\mathrm{~m}, 2 \mathrm{H})$, $6.46(\mathrm{dt}, J=0.9,0.9 \mathrm{~Hz}, 1 \mathrm{H}), 2.85(\mathrm{t}, J=7.7 \mathrm{~Hz}, 2 \mathrm{H}), 1.63-1.53(\mathrm{~m}, 2 \mathrm{H}), 1.36-1.20(\mathrm{~m}, 6 \mathrm{H}), 0.87(\mathrm{t}$, $J=7.1 \mathrm{~Hz}, 3 \mathrm{H})$.

${ }^{13}$ C-NMR $\left(100 \mathrm{MHz}, \mathrm{CDCl}_{3}\right): \delta=151.5\left(\mathrm{C}_{\mathrm{q}}\right), 149.5(\mathrm{CH}), 141.7\left(\mathrm{C}_{\mathrm{q}}\right), 138.1(\mathrm{CH}), 137.2\left(\mathrm{C}_{\mathrm{q}}\right), 128.6$ $\left(\mathrm{C}_{\mathrm{q}}\right), 121.9(\mathrm{CH}), 121.4(\mathrm{CH}), 121.1(\mathrm{CH}), 120.5(\mathrm{CH}), 119.8(\mathrm{CH}), 110.0(\mathrm{CH}), 102.0(\mathrm{CH}), 31.5$ $\left(\mathrm{CH}_{2}\right), 28.9\left(\mathrm{CH}_{2}\right), 28.5\left(\mathrm{CH}_{2}\right), 27.4\left(\mathrm{CH}_{2}\right), 22.5\left(\mathrm{CH}_{2}\right), 14.0\left(\mathrm{CH}_{3}\right)$.

IR (ATR): 2926, 2856, 1584, 1468, 1455, 779, $735 \mathrm{~cm}^{-1}$.

MS (EI) $\mathrm{m} / \mathrm{z}$ (relative intensity): $278\left([\mathrm{M}]^{+}, 25\right), 221$ (20), 207 (100).

HR-MS (EI): $m / z$ calcd. for $\left[\mathrm{C}_{19} \mathrm{H}_{22} \mathrm{~N}_{2}\right]^{+}[\mathrm{M}]^{+} 278.1783$, found 278.1788 .

The analytical data are in accordance with those reported in literature. ${ }^{[186]}$ 


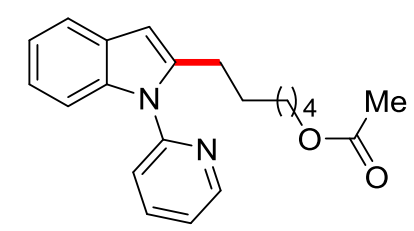

6-[1-(Pyridin-2-yl)-1H-indol-2-yl]hexyl acetate (144af): The general procedure $\mathbf{D}$ was followed using 1-(pyridin-2-yl)-1H-indole (105a) $(97.1 \mathrm{mg}, 0.50 \mathrm{mmol})$ and hex-5-en-1-yl acetate (143f) $(213 \mathrm{mg}, 1.50 \mathrm{mmol})$. Isolation by column chromatography ( $n$-hexane/EtOAc: 10/1) yielded 144af (128 $\mathrm{mg}, 76 \%)$ as a yellowish oil.

${ }^{1}$ H-NMR $\left(300 \mathrm{MHz}, \mathrm{CDCl}_{3}\right): \delta=8.65(\mathrm{ddd}, J=5.0,2.1,0.9 \mathrm{~Hz}, 1 \mathrm{H}), 7.85(\mathrm{ddd}, J=8.0,7.5,2.1 \mathrm{~Hz}$, 1H), 7.60-7.55 (m, 1H), $7.42(\mathrm{ddd}, J=8.0,1.0,0.9 \mathrm{~Hz}, 1 \mathrm{H}), 7.33-7.24(\mathrm{~m}, 2 \mathrm{H}), 7.15-7.08(\mathrm{~m}, 2 \mathrm{H})$, $6.44(\mathrm{dt}, J=0.8,0.8 \mathrm{~Hz}, 1 \mathrm{H}), 4.01(\mathrm{t}, J=6.9 \mathrm{~Hz}, 2 \mathrm{H}), 2.84(\mathrm{t}, J=7.4 \mathrm{~Hz}, 2 \mathrm{H}), 2.02(\mathrm{~s}, 3 \mathrm{H}), 1.63-1.53$ (m, 4H), 1.36-1.28 (m, 4H).

${ }^{13}$ C-NMR $\left(125 \mathrm{MHz}, \mathrm{CDCl}_{3}\right): \delta=170.9\left(\mathrm{C}_{\mathrm{q}}\right), 151.4\left(\mathrm{C}_{\mathrm{q}}\right), 149.4(\mathrm{CH}), 141.3\left(\mathrm{C}_{\mathrm{q}}\right), 138.1(\mathrm{CH}), 137.1$ $\left(\mathrm{C}_{\mathrm{q}}\right), 128.5\left(\mathrm{C}_{\mathrm{q}}\right), 121.9(\mathrm{CH}), 121.4(\mathrm{CH}), 121.0(\mathrm{CH}), 120.4(\mathrm{CH}), 119.7(\mathrm{CH}), 109.9(\mathrm{CH}), 102.0$ $(\mathrm{CH}), 64.4\left(\mathrm{CH}_{2}\right), 28.9\left(\mathrm{CH}_{2}\right), 28.4\left(\mathrm{CH}_{2}\right), 28.4\left(\mathrm{CH}_{2}\right), 27.3\left(\mathrm{CH}_{2}\right), 25.6\left(\mathrm{CH}_{2}\right), 21.0\left(\mathrm{CH}_{3}\right)$.

IR (ATR): 2933, 1736, 1585, 1470, 1456, 1242, 783, $748 \mathrm{~cm}^{-1}$.

MS (EI) $\mathrm{m} / \mathrm{z}$ (relative intensity): $336\left([\mathrm{M}]^{+}, 25\right), 221$ (25), 207 (100).

HR-MS (EI): $\mathrm{m} / z$ calcd. for $\left[\mathrm{C}_{21} \mathrm{H}_{24} \mathrm{~N}_{2} \mathrm{O}_{2}\right]^{+}[\mathrm{M}]^{+} 336.1838$, found 336.1838 .

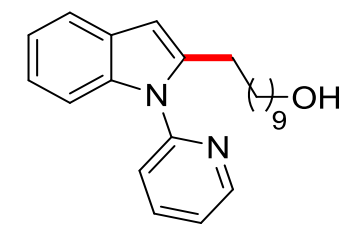

10-[1-(Pyridin-2-yl)-1H-indol-2-yl]decan-1-ol (144ag): The general procedure D was followed using 1-(pyridin-2-yl)-1H-indole (105a) (97.1 mg, $0.50 \mathrm{mmol})$ and dec-9-en-1-ol (143g) (234 mg, $1.50 \mathrm{mmol}$ ). Isolation by column chromatography ( $n$-hexane/EtOAc: $5 / 1)$ yielded 144ag (117 mg, $67 \%)$ as a yellowish oil.

${ }^{1}$ H-NMR $\left(300 \mathrm{MHz}, \mathrm{CDCl}_{3}\right): \delta=8.64(\mathrm{ddd}, J=5.0,2.1,0.9 \mathrm{~Hz}, 1 \mathrm{H}), 7.87(\mathrm{ddd}, J=8.0,7.4,2.1 \mathrm{~Hz}$, $1 \mathrm{H}), 7.59-7.54(\mathrm{~m}, 1 \mathrm{H}), 7.41$ (ddd, $J=8.0,1.0,0.9 \mathrm{~Hz}, 1 \mathrm{H}), 7.33-7.27(\mathrm{~m}, 2 \mathrm{H}), 7.14-7.07(\mathrm{~m}, 2 \mathrm{H})$, $6.43(\mathrm{dt}, J=0.8,0.8 \mathrm{~Hz}, 1 \mathrm{H}), 3.57(\mathrm{t}, J=6.7 \mathrm{~Hz}, 2 \mathrm{H}), 2.82(\mathrm{t}, J=7.6 \mathrm{~Hz}, 2 \mathrm{H}), 1.74(\mathrm{bs}, 1 \mathrm{H}), 1.60$ $1.45(\mathrm{~m}, 4 \mathrm{H}), 1.31-1.19(\mathrm{~m}, 12 \mathrm{H})$.

${ }^{13}$ C-NMR $\left(125 \mathrm{MHz}, \mathrm{CDCl}_{3}\right): \delta=151.4\left(\mathrm{C}_{\mathrm{q}}\right), 149.4(\mathrm{CH}), 141.6\left(\mathrm{C}_{\mathrm{q}}\right), 138.1(\mathrm{CH}), 137.1\left(\mathrm{C}_{\mathrm{q}}\right), 128.5$ $\left(\mathrm{C}_{\mathrm{q}}\right), 121.9(\mathrm{CH}), 121.4(\mathrm{CH}), 121.1(\mathrm{CH}), 120.4(\mathrm{CH}), 119.7(\mathrm{CH}), 109.9(\mathrm{CH}), 101.9(\mathrm{CH}), 62.7$ $\left(\mathrm{CH}_{2}\right), 32.7\left(\mathrm{CH}_{2}\right), 29.5\left(\mathrm{CH}_{2}\right), 29.5\left(\mathrm{CH}_{2}\right), 29.4\left(\mathrm{CH}_{2}\right), 29.3\left(\mathrm{CH}_{2}\right), 29.3\left(\mathrm{CH}_{2}\right), 28.6\left(\mathrm{CH}_{2}\right), 27.4$ $\left(\mathrm{CH}_{2}\right), 25.7\left(\mathrm{CH}_{2}\right)$. 
IR (ATR): 3375, 2925, 2852, 1699, 1585, 1469, 1455, 782, $736 \mathrm{~cm}^{-1}$.

MS (EI) $\mathrm{m} / z$ (relative intensity): 350 ([M] $\left.{ }^{+}, 15\right), 221$ (25), 207 (100).

HR-MS (EI): $m / z$ calcd. for $\left[\mathrm{C}_{23} \mathrm{H}_{30} \mathrm{~N}_{2} \mathrm{O}\right]^{+}[\mathrm{M}]^{+} 350.2358$, found 350.2354 .

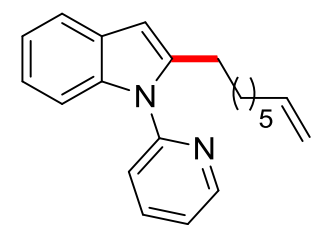

2-(Oct-7-en-1-yl)-1-(pyridin-2-yl)-1H-indole (144ah): The general procedure D was followed using 1-(pyridin-2-yl)-1H-indole (105a) $(97.1 \mathrm{mg}, 0.50 \mathrm{mmol})$ and octa-1,7-diene (143h) (165 mg, $1.50 \mathrm{mmol}$ ). Isolation by column chromatography ( $n$-hexane/EtOAc: 40/1) yielded 144ah (99.8 mg, $66 \%)$ as a colorless oil.

${ }^{1} \mathbf{H}-\mathbf{N M R}\left(500 \mathrm{MHz}, \mathrm{CDCl}_{3}\right): \delta=8.65(\mathrm{ddd}, J=4.9,2.1,0.9 \mathrm{~Hz}, 1 \mathrm{H}), 7.86(\mathrm{ddd}, J=7.8,7.8,2.1 \mathrm{~Hz}$, 1H), 7.63-7.57 (m, 1H), 7.43 (ddd, $J=7.8,1.0,0.9 \mathrm{~Hz}, 1 \mathrm{H}), 7.37-7.32(\mathrm{~m}, 1 \mathrm{H}), 7.23$ (ddd, $J=7.3$, $5.2,0.9 \mathrm{~Hz}, 1 \mathrm{H}), 7.17-7.10(\mathrm{~m}, 2 \mathrm{H}), 6.47$ (dt, $J=0.8,0.8 \mathrm{~Hz}, 1 \mathrm{H}), 5.81$ (ddt, $J=17.0,10.2,6.6 \mathrm{~Hz}$, $1 \mathrm{H}), 5.00(\mathrm{dd}, J=17.0,2.0 \mathrm{~Hz}, 1 \mathrm{H}), 4.95(\mathrm{dd}, J=10.2,2.0 \mathrm{~Hz}, 1 \mathrm{H}), 2.86(\mathrm{t}, J=7.8 \mathrm{~Hz}, 2 \mathrm{H}), 2.03$ (tt, $J=7.8,7.7 \mathrm{~Hz}, 2 \mathrm{H}), 1.62-1.52(\mathrm{~m}, 2 \mathrm{H}), 1.40-1.22(\mathrm{~m}, 6 \mathrm{H})$.

${ }^{13} \mathrm{C}-\mathrm{NMR}\left(125 \mathrm{MHz}, \mathrm{CDCl}_{3}\right): \delta=151.5\left(\mathrm{C}_{\mathrm{q}}\right), 149.5(\mathrm{CH}), 141.6\left(\mathrm{C}_{\mathrm{q}}\right), 139.0(\mathrm{CH}), 138.1(\mathrm{CH}), 137.2$ $\left(\mathrm{C}_{\mathrm{q}}\right), 128.6\left(\mathrm{C}_{\mathrm{q}}\right), 121.9(\mathrm{CH}), 121.4(\mathrm{CH}), 121.1(\mathrm{CH}), 120.5(\mathrm{CH}), 119.8(\mathrm{CH}), 114.1\left(\mathrm{CH}_{2}\right), 110.0$ $(\mathrm{CH}), 102.0(\mathrm{CH}), 33.7\left(\mathrm{CH}_{2}\right), 29.0\left(\mathrm{CH}_{2}\right), 28.7\left(\mathrm{CH}_{2}\right), 28.6\left(\mathrm{CH}_{2}\right), 28.4\left(\mathrm{CH}_{2}\right), 27.4\left(\mathrm{CH}_{2}\right)$.

IR (ATR): 2926, 2855, 1585, 1469, 1436, 1346, 780, $737 \mathrm{~cm}^{-1}$.

MS (EI) $\mathrm{m} / \mathrm{z}$ (relative intensity): 304 ([M] $\left.]^{+}, 100\right), 221$ (20), 207 (100).

HR-MS (EI): $m / z$ calcd. for $\left[\mathrm{C}_{21} \mathrm{H}_{24} \mathrm{~N}_{2}\right]^{+}[\mathrm{M}]^{+} 304.1939$, found 304.1935 .

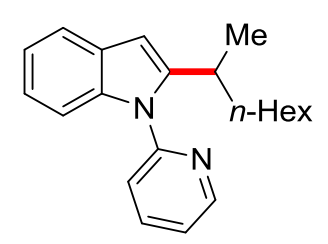

2-(Octan-2-yl)-1-(pyridin-2-yl)-1H-indole (145aa): The general procedure $\mathbf{E}$ was followed using 1(pyridin-2-yl)-1H-indole (105a) (97.1 mg, $0.50 \mathrm{mmol}$ ) and oct-1-ene (143a) (168 mg, $1.50 \mathrm{mmol}$ ). Isolation by column chromatography ( $n$-hexane/EtOAc: $25 / 1)$ yielded 145aa $(122 \mathrm{mg}, 80 \%, 95: 5)$ as a colorless oil. After purification by HPLC pure 145aa could be isolated (110 mg, 72\%, >99:1).

${ }^{1}$ H-NMR $\left(300 \mathrm{MHz}, \mathrm{CDCl}_{3}\right.$ ): $\delta=8.67$ (ddd, $\left.J=4.9,2.0,0.9 \mathrm{~Hz}, 1 \mathrm{H}\right), 7.86(\mathrm{ddd}, J=7.9,7.5,2.0 \mathrm{~Hz}$, $1 \mathrm{H}), 7.59-7.53(\mathrm{~m}, 1 \mathrm{H}), 7.40(\mathrm{ddd}, J=7.9,1.0,0.9 \mathrm{~Hz}, 1 \mathrm{H}), 7.32(\mathrm{ddd}, J=7.5,4.9,1.0 \mathrm{~Hz}, 1 \mathrm{H})$, $7.24-7.21(\mathrm{~m}, 1 \mathrm{H}), 7.12-7.06(\mathrm{~m}, 2 \mathrm{H}), 6.46(\mathrm{dd}, J=0.8,0.8 \mathrm{~Hz}, 1 \mathrm{H}), 3.16(\mathrm{tq}, J=7.6,7.6 \mathrm{~Hz}, 0.95 \mathrm{H}$, 
M), $2.86(\mathrm{t}, J=7.7 \mathrm{~Hz}, 0.10 \mathrm{H}, \mathrm{AM}), 1.64-1.55(\mathrm{~m}, 1 \mathrm{H}), 1.46-1.33(\mathrm{~m}, 1 \mathrm{H}), 1.25-1.10(\mathrm{~m}, 11 \mathrm{H}), 0.82$ (t, $J=6.8 \mathrm{~Hz}, 3 \mathrm{H})$.

${ }^{13}$ C-NMR $\left(125 \mathrm{MHz}, \mathrm{CDCl}_{3}\right): \delta=151.6\left(\mathrm{C}_{\mathrm{q}}\right), 149.6(\mathrm{CH}), 147.4\left(\mathrm{C}_{\mathrm{q}}\right), 138.1(\mathrm{CH}), 137.2\left(\mathrm{C}_{\mathrm{q}}\right), 128.5$ $\left(\mathrm{C}_{\mathrm{q}}\right), 122.1(\mathrm{CH}), 121.7(\mathrm{CH}), 121.3(\mathrm{CH}), 120.4(\mathrm{CH}), 119.8(\mathrm{CH}), 109.9(\mathrm{CH}), 99.8(\mathrm{CH}), 37.2$ $\left(\mathrm{CH}_{2}\right), 31.7\left(\mathrm{CH}_{2}\right), 30.8(\mathrm{CH}), 29.3\left(\mathrm{CH}_{2}\right), 27.1\left(\mathrm{CH}_{2}\right), 22.7\left(\mathrm{CH}_{2}\right), 20.6\left(\mathrm{CH}_{3}\right), 14.1\left(\mathrm{CH}_{3}\right)$.

IR (ATR): 2925, 2854, 1584, 1468, 1435, 780, $733 \mathrm{~cm}^{-1}$.

MS (EI) $m / z$ (relative intensity): 306 ([M] $\left.{ }^{+}, 20\right), 221$ (55), 207 (100).

HR-MS (EI): $m / z$ calcd. for $\left[\mathrm{C}_{21} \mathrm{H}_{26} \mathrm{~N}_{2}\right]^{+}[\mathrm{M}]^{+}$306.2096, found 306.2083.

\section{Asymmetric cobalt(III)-catalyzed C-H hydroarylation:}

A suspension of 2-pyridylindole (105a) $(0.50 \mathrm{mmol}, 1.00$ equiv), oct-1-ene (143a) (168 mg, $1.50 \mathrm{mmol}),\left[\mathrm{Cp} * \mathrm{Co}(\mathrm{CO}) \mathrm{I}_{2}\right] \quad(23.8 \mathrm{mg}, 50.0 \mu \mathrm{mol}, 10.0 \mathrm{~mol} \%), \mathrm{AgSbF}_{6} \quad(34.4 \mathrm{mg}, 100 \mu \mathrm{mol}$, $20.0 \mathrm{~mol} \%)$ and Phth-L-Leu-OH (130 mg, $0.50 \mathrm{mmol}, 1.00$ equiv) in DCE $(0.50 \mathrm{~mL}, 1.00 \mathrm{M})$ was stirred at $50{ }^{\circ} \mathrm{C}$ for $20 \mathrm{~h}$. At ambient temperature, the reaction mixture was diluted with EtOAc $(5.0 \mathrm{~mL})$ and the solvents were removed in vacuo. The remaining residue was purified by column chromatography on silica gel ( $n$-hexane/EtOAc) to yield the desired product 145aa $(57.1 \mathrm{mg}, 37 \%$, $\mathrm{M}: \mathrm{AM}=93: 7)$. The enantiomeric excess was determined by analytical HPLC (Chiralpak ${ }^{\circledR}$ IF-3, $n$ hexane/EtOAc 90:10, flow rate $=0.5 \mathrm{~mL} / \mathrm{min}, \lambda=280 \mathrm{~nm}$ ) to be $25 \%$ ee .

\section{Racemic Mixture:}

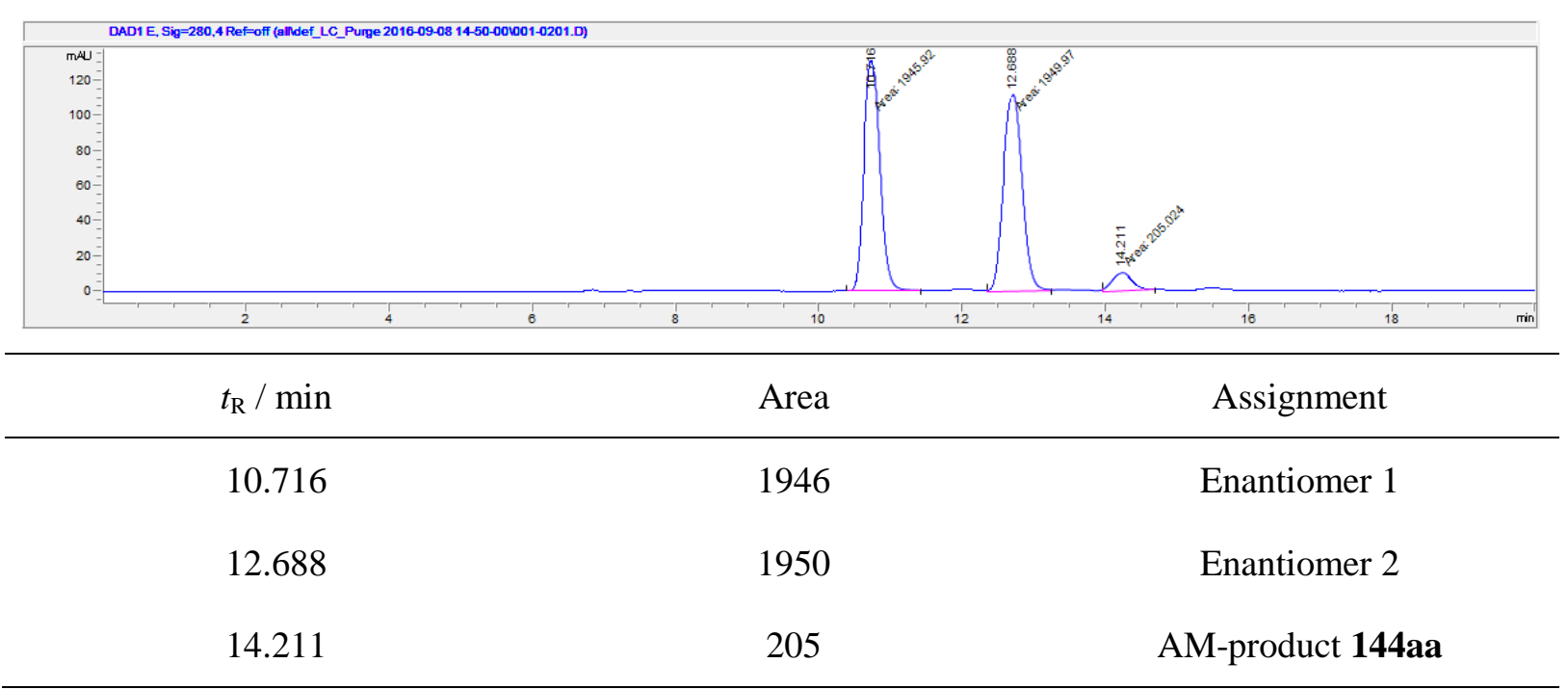


Enantiomeric excess of 25\%:

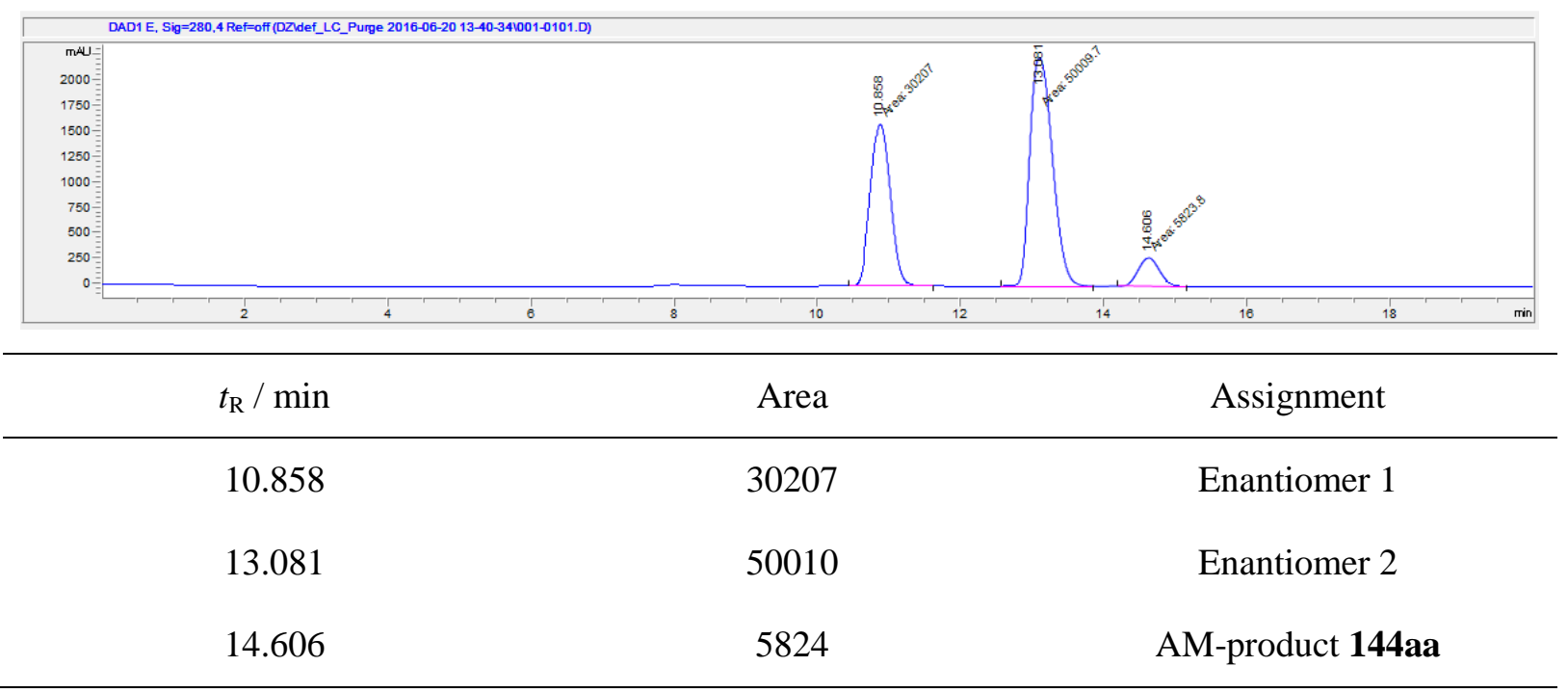<smiles>COc1ccc2c(c1)cc(C(C)CO)n2-c1ccccn1</smiles>

5-Methoxy-2-(octan-2-yl)-1-(pyridin-2-yl)-1H-indole (145ba): The general procedure $\mathbf{E}$ was followed using 5-methoxy-1-(pyridin-2-yl)- $1 H$-indole (105b) $(112 \mathrm{mg}, 0.50 \mathrm{mmol})$ and oct-1-ene (143a) $(168 \mathrm{mg}, 1.50 \mathrm{mmol})$. Isolation by column chromatography ( $n$-hexane/EtOAc: $25 / 1$ ) yielded 145ba (116 mg, 69\%, 94:6) as a colorless oil.

${ }^{1}$ H-NMR $\left(400 \mathrm{MHz}, \mathrm{CDCl}_{3}\right): \delta=8.65(\mathrm{ddd}, J=4.9,2.1,1.0 \mathrm{~Hz}, 1 \mathrm{H}), 7.85(\mathrm{ddd}, J=7.9,7.4,2.1 \mathrm{~Hz}$, $1 \mathrm{H}), 7.38(\mathrm{ddd}, J=7.9,1.0,0.9 \mathrm{~Hz}, 1 \mathrm{H}), 7.29(\mathrm{ddd}, J=7.4,4.9,0.9 \mathrm{~Hz}, 1 \mathrm{H}), 7.17(\mathrm{dd}, J=8.9,0.9 \mathrm{~Hz}$, 1H), 7.08-7.04 (m, 1H), 6.76 (dd, $J=8.9,2.5 \mathrm{~Hz}, 1 \mathrm{H}), 6.39$ (dd, $J=0.8,0.8 \mathrm{~Hz}, 1 \mathrm{H}), 3.86(\mathrm{~s}, 3 \mathrm{H})$, $3.18(\mathrm{tq}, J=6.8,6.8 \mathrm{~Hz}, 0.94 \mathrm{H}, \mathrm{M}), 2.83(\mathrm{t}, J=7.6 \mathrm{~Hz}, 0.12 \mathrm{H}, \mathrm{AM}), 1.67-1.34(\mathrm{~m}, 2 \mathrm{H}), 1.30-1.08$ $(\mathrm{m}, 11 \mathrm{H}), 0.84(\mathrm{t}, J=7.1 \mathrm{~Hz}, 3 \mathrm{H})$.

${ }^{13}$ C-NMR $\left(100 \mathrm{MHz}, \mathrm{CDCl}_{3}\right): \delta=154.7\left(\mathrm{C}_{\mathrm{q}}\right), 151.3\left(\mathrm{C}_{\mathrm{q}}\right), 149.6(\mathrm{CH}), 148.1\left(\mathrm{C}_{\mathrm{q}}\right), 138.1(\mathrm{CH}), 132.4$ $\left(\mathrm{C}_{\mathrm{q}}\right), 129.0\left(\mathrm{C}_{\mathrm{q}}\right), 121.9(\mathrm{CH}), 121.4(\mathrm{CH}), 111.0(\mathrm{CH}), 110.7(\mathrm{CH}), 102.1(\mathrm{CH}), 99.6(\mathrm{CH}), 55.8$ $\left(\mathrm{CH}_{3}\right), 37.1\left(\mathrm{CH}_{2}\right), 31.6\left(\mathrm{CH}_{2}\right), 30.8(\mathrm{CH}), 29.2\left(\mathrm{CH}_{2}\right), 27.0\left(\mathrm{CH}_{2}\right), 22.6\left(\mathrm{CH}_{2}\right), 20.5\left(\mathrm{CH}_{3}\right), 14.0$ $\left(\mathrm{CH}_{3}\right)$.

IR (ATR): 2926, 2855, 1582, 1470, 1435, 1172, $770 \mathrm{~cm}^{-1}$.

MS (EI) $m / z$ (relative intensity): 336 ([M] $\left.]^{+}, 55\right), 251$ (85), 237 (100).

HR-MS (EI+): $m / z$ calcd. for $\left[\mathrm{C}_{22} \mathrm{H}_{28} \mathrm{~N}_{2} \mathrm{O}\right]^{+}[\mathrm{M}]^{+}$336.2202, found 336.2213 


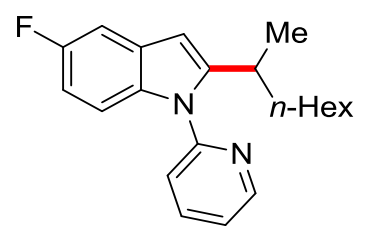

5-Fluoro-2-(octan-2-yl)-1-(pyridin-2-yl)-1H-indole (145ma): The general procedure $\mathbf{E}$ was followed using 5-fluoro-1-(pyridin-2-yl)-1H-indole (105m) (106 mg, $0.50 \mathrm{mmol})$ and oct-1-ene (143a) $(168 \mathrm{mg}, 1.50 \mathrm{mmol})$. Isolation by column chromatography ( $n$-hexane/EtOAc: $20 / 1)$ yielded 145ma (138 $\mathrm{mg}, 85 \%, 96: 4)$ as a colorless oil.

${ }^{1}$ H-NMR $\left(500 \mathrm{MHz}, \mathrm{CDCl}_{3}\right): \delta=8.68(\mathrm{ddd}, J=4.9,2.1,1.0 \mathrm{~Hz}, 1 \mathrm{H}), 7.88(\mathrm{ddd}, J=7.9,7.4,2.1 \mathrm{~Hz}$, 1H), 7.39 (ddd, $J=7.9,1.0,1.0 \mathrm{~Hz}, 1 \mathrm{H}), 7.34$ (ddd, $J=7.4,4.9,1.0 \mathrm{~Hz}, 1 \mathrm{H}), 7.24$ (dd, $J=9.1,2.4 \mathrm{~Hz}$, $1 \mathrm{H}), 7.17(\mathrm{dd}, J=8.9,4.5 \mathrm{~Hz}, 1 \mathrm{H}), 6.85(\mathrm{dd}, J=8.9,2.4 \mathrm{~Hz}, 1 \mathrm{H}), 6.44(\mathrm{dd}, J=0.8,0.8 \mathrm{~Hz}, 1 \mathrm{H}), 3.16$ (tq, $J=6.8,6.8 \mathrm{~Hz}, 0.96 \mathrm{H}, \mathrm{M}), 2.80(\mathrm{t}, J=7.6 \mathrm{~Hz}, 0.08 \mathrm{H}, \mathrm{AM}), 1.70-1.59(\mathrm{~m}, 1 \mathrm{H}), 1.50-1.36(\mathrm{~m}$, $1 \mathrm{H}), 1.32-1.06(\mathrm{~m}, 11 \mathrm{H}), 0.84(\mathrm{t}, J=7.1 \mathrm{~Hz}, 3 \mathrm{H})$.

${ }^{13}$ C-NMR $\left(125 \mathrm{MHz}, \mathrm{CDCl}_{3}\right): \delta=158.3\left(\mathrm{~d},{ }^{1} J_{\mathrm{C}-\mathrm{F}}=235 \mathrm{~Hz}, \mathrm{C}_{\mathrm{q}}\right), 151.4\left(\mathrm{C}_{\mathrm{q}}\right), 149.7(\mathrm{CH}), 149.1\left(\mathrm{C}_{\mathrm{q}}\right)$, $138.3(\mathrm{CH}), 133.8\left(\mathrm{C}_{\mathrm{q}}\right), 128.9\left(\mathrm{~d},{ }^{3} J_{\mathrm{C}-\mathrm{F}}=10.1 \mathrm{~Hz}, \mathrm{C}_{\mathrm{q}}\right), 122.3(\mathrm{CH}), 121.6(\mathrm{CH}), 110.6\left(\mathrm{~d},{ }^{3} J_{\mathrm{C}-\mathrm{F}}=\right.$ $10.0 \mathrm{~Hz}, \mathrm{CH}), 109.1\left(\mathrm{~d},{ }^{2} J_{\mathrm{C}-\mathrm{F}}=24.2 \mathrm{~Hz}, \mathrm{CH}\right), 104.7\left(\mathrm{~d},{ }^{2} J_{\mathrm{C}-\mathrm{F}}=24.1 \mathrm{~Hz}, \mathrm{CH}\right), 99.6\left(\mathrm{~d},{ }^{4} J_{\mathrm{C}-\mathrm{F}}=4.3 \mathrm{~Hz}\right.$, $\mathrm{CH}), 37.0\left(\mathrm{CH}_{2}\right), 31.6\left(\mathrm{CH}_{2}\right), 30.8(\mathrm{CH}), 29.1\left(\mathrm{CH}_{2}\right), 27.0\left(\mathrm{CH}_{2}\right), 22.5\left(\mathrm{CH}_{2}\right), 20.5\left(\mathrm{CH}_{3}\right), 14.0\left(\mathrm{CH}_{3}\right)$.

${ }^{19} \mathbf{F}-\mathbf{N M R}\left(282 \mathrm{MHz}, \mathrm{CDCl}_{3}\right): \delta=-124.2(\mathrm{~m})$.

IR (ATR): 2926, 2855, 1584, 1469, 1435, 1177, $774 \mathrm{~cm}^{-1}$.

MS (EI) $m / z$ (relative intensity): 324 ([M] $\left.]^{+}, 20\right), 239$ (55), 225 (100).

HR-MS (EI): $m / z$ calcd. for $\left[\mathrm{C}_{21} \mathrm{H}_{25} \mathrm{FN}_{2}\right]^{+}[\mathrm{M}]^{+} 324.2002$, found 324.2010 .<smiles>CC(C)c1cc2cc(Br)ccc2n1-c1ccccn1</smiles>

5-Bromo-2-(octan-2-yl)-1-(pyridin-2-yl)-1H-indole (145ca): The general procedure $\mathbf{E}$ was followed using 5-bromo-1-(pyridin-2-yl)- $1 H$-indole (105c) $(136 \mathrm{mg}, 0.50 \mathrm{mmol})$ and oct-1-ene (143a) (168 mg, $1.50 \mathrm{mmol}$ ). Isolation by column chromatography ( $n$-hexane/EtOAc: $25 / 1)$ yielded 145ca (154 mg, $80 \%, 95: 5)$ as a colorless oil.

${ }^{1} \mathbf{H}-\mathbf{N M R}\left(400 \mathrm{MHz}, \mathrm{CDCl}_{3}\right): \delta=8.66$ (ddd, $\left.J=4.9,2.1,0.9 \mathrm{~Hz}, 1 \mathrm{H}\right), 7.87$ (ddd, $J=7.9,7.4,2.1 \mathrm{~Hz}$, $1 \mathrm{H}), 7.70(\mathrm{dd}, J=1.9,0.9 \mathrm{~Hz}, 1 \mathrm{H}), 7.34(\mathrm{ddd}, J=7.9,0.9,0.9 \mathrm{~Hz}, 1 \mathrm{H}), 7.32$ (ddd, $J=7.4,4.9$, $0.9 \mathrm{~Hz}, 1 \mathrm{H}), 7.20-7.08(\mathrm{~m}, 2 \mathrm{H}), 6.38(\mathrm{dd}, J=0.8,0.8 \mathrm{~Hz}, 1 \mathrm{H}), 3.14(\mathrm{tq}, J=6.9,6.9 \mathrm{~Hz}, 0.95 \mathrm{H}, \mathrm{M})$, $2.79(\mathrm{t}, J=7.8 \mathrm{~Hz}, 0.10 \mathrm{H}, \mathrm{AM}), 1.64-1.37(\mathrm{~m}, 2 \mathrm{H}), 1.29-1.10(\mathrm{~m}, 11 \mathrm{H}), 0.84(\mathrm{t}, J=7.1 \mathrm{~Hz}, 3 \mathrm{H})$. 
${ }^{13}$ C-NMR $\left(100 \mathrm{MHz}, \mathrm{CDCl}_{3}\right): \delta=151.1\left(\mathrm{C}_{\mathrm{q}}\right), 149.7(\mathrm{CH}), 148.8\left(\mathrm{C}_{\mathrm{q}}\right), 138.3(\mathrm{CH}), 136.0\left(\mathrm{C}_{\mathrm{q}}\right), 130.2$ $\left(\mathrm{C}_{\mathrm{q}}\right), 124.0(\mathrm{CH}), 122.5(\mathrm{CH}), 122.3(\mathrm{CH}), 121.6(\mathrm{CH}), 113.5\left(\mathrm{C}_{\mathrm{q}}\right), 111.4(\mathrm{CH}), 99.2(\mathrm{CH}), 36.9$ $\left(\mathrm{CH}_{2}\right), 31.6\left(\mathrm{CH}_{2}\right), 30.8(\mathrm{CH}), 29.1\left(\mathrm{CH}_{2}\right), 26.9\left(\mathrm{CH}_{2}\right), 22.5\left(\mathrm{CH}_{2}\right), 20.4\left(\mathrm{CH}_{3}\right), 14.0\left(\mathrm{CH}_{3}\right)$.

IR (ATR): 2925, 2855, 1585, 1470, 1437, 863, 781, $735 \mathrm{~cm}^{-1}$.

MS (EI) $m / z$ (relative intensity): 384 ([M( $\left.\left.\left({ }^{79} \mathrm{Br}\right)\right]^{+}, 25\right), 299$ (45), 287 (100), 219 (75), 205 (15).

HR-MS (EI+): $m / z$ calcd. for $\left[\mathrm{C}_{21} \mathrm{H}_{25}{ }^{79} \mathrm{BrN}_{2}\right]^{+}[\mathrm{M}]^{+} 384.1201$, found 384.1206.

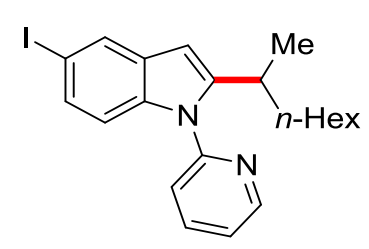

5-Iodo-2-(octan-2-yl)-1-(pyridin-2-yl)-1H-indole (145da): The general procedure $\mathbf{E}$ was followed using 5-iodo-1-(pyridin-2-yl)-1 $H$-indole (105d) (160 mg, $0.50 \mathrm{mmol})$ and oct-1-ene (143a) $(168 \mathrm{mg}$, $1.50 \mathrm{mmol}$ ). Isolation by column chromatography ( $n$-hexane/EtOAc: $25 / 1)$ yielded 145da (178 mg, $82 \%, 94: 6)$ as a colorless oil.

${ }^{1} \mathbf{H}-\mathbf{N M R}\left(300 \mathrm{MHz}, \mathrm{CDCl}_{3}\right): \delta=8.66(\mathrm{ddd}, J=5.0,2.1,0.9 \mathrm{~Hz}, 1 \mathrm{H}), 7.91-7.84(\mathrm{~m}, 2 \mathrm{H}), 7.38-7.32$ $(\mathrm{m}, 3 \mathrm{H}), 6.99(\mathrm{dd}, J=8.6,0.9 \mathrm{~Hz}, 1 \mathrm{H}), 6.36(\mathrm{dd}, J=0.8,0.8 \mathrm{~Hz}, 1 \mathrm{H}), 3.12(\mathrm{tq}, J=6.8,6.8 \mathrm{~Hz}, 0.94 \mathrm{H}$, M), $2.79(\mathrm{t}, J=7.8 \mathrm{~Hz}, 0.12 \mathrm{H}, \mathrm{AM}), 1.64-1.36(\mathrm{~m}, 2 \mathrm{H}), 1.26-1.09(\mathrm{~m}, 11 \mathrm{H}), 0.83(\mathrm{t}, J=7.1 \mathrm{~Hz}, 3 \mathrm{H})$. ${ }^{13}$ C-NMR $\left(125 \mathrm{MHz}, \mathrm{CDCl}_{3}\right): \delta=151.0\left(\mathrm{C}_{\mathrm{q}}\right), 149.7(\mathrm{CH}), 148.3\left(\mathrm{C}_{\mathrm{q}}\right), 138.3(\mathrm{CH}), 136.4\left(\mathrm{C}_{\mathrm{q}}\right), 131.0$ $\left(\mathrm{C}_{\mathrm{q}}\right), 129.6(\mathrm{CH}), 128.5(\mathrm{CH}), 122.4(\mathrm{CH}), 121.6(\mathrm{CH}), 112.0(\mathrm{CH}), 98.9(\mathrm{CH}), 83.9\left(\mathrm{C}_{\mathrm{q}}\right), 37.0\left(\mathrm{CH}_{2}\right)$, $31.7\left(\mathrm{CH}_{2}\right), 30.8(\mathrm{CH}), 29.2\left(\mathrm{CH}_{2}\right), 27.0\left(\mathrm{CH}_{2}\right), 22.6\left(\mathrm{CH}_{2}\right), 20.5\left(\mathrm{CH}_{3}\right), 14.2\left(\mathrm{CH}_{3}\right)$.

IR (ATR): 2924, 2854, 1584, 1469, 1436, 866, $781 \mathrm{~cm}^{-1}$.

MS (EI) $m / z$ (relative intensity): 432 ([M] $\left.]^{+}, 60\right), 348$ (45), 333 (100), 219 (65), 206 (20).

HR-MS (EI): $m / z$ calcd. for $\left[\mathrm{C}_{21} \mathrm{H}_{25} \mathrm{IN}_{2}\right]^{+}[\mathrm{M}]^{+} 432.1062$, found 432.1067 .

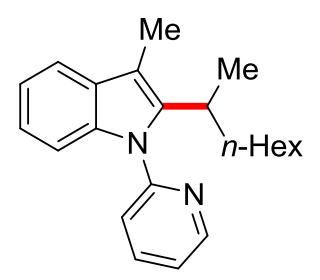

3-Methyl-2-(octan-2-yl)-1-(pyridin-2-yl)-1H-indole (145fa): The general procedure $\mathbf{E}$ was followed using methyl 3-methyl-1-(pyridin-2-yl)- $1 H$-indole (105f) $(104 \mathrm{mg}, 0.50 \mathrm{mmol})$ and oct-1-ene (143a) $(168 \mathrm{mg}, 1.50 \mathrm{mmol}$ ). Isolation by column chromatography ( $n$-hexane/EtOAc: $20 / 1)$ yielded $\mathbf{1 4 5 f a}$ (97.6 $\mathrm{mg}, 61 \%, 21: 79)$ as a colorless oil.

${ }^{1}$ H-NMR $\left(300 \mathrm{MHz}, \mathrm{CDCl}_{3}\right.$ ): $\delta=8.63$ (ddd, $\left.J=4.9,2.0,0.9 \mathrm{~Hz}, 1 \mathrm{H}\right), 7.85$ (ddd, $J=7.9,7.4,2.0 \mathrm{~Hz}$, $1 \mathrm{H}), 7.56-7.51(\mathrm{~m}, 1 \mathrm{H}), 7.41(\mathrm{dt}, J=7.9,0.9 \mathrm{~Hz}, 1 \mathrm{H}), 7.33-7.26(\mathrm{~m}, 2 \mathrm{H}), 7.16-7.08(\mathrm{~m}, 2 \mathrm{H}), 3.03-$ $2.93(\mathrm{~m}, 0.2 \mathrm{H}, \mathrm{M}), 2.89$ (t, $J=7.2 \mathrm{~Hz}, 1.6 \mathrm{H}, \mathrm{AM}), 2.38$ (s, 0.6H, M), 2.31 (s, 2.4H, AM), 1.35-1.12 $(\mathrm{m}, 12 \mathrm{H}), 0.84(\mathrm{t}, J=6.7 \mathrm{~Hz}, 3 \mathrm{H})$. 
${ }^{13}$ C-NMR $\left(125 \mathrm{MHz}, \mathrm{CDCl}_{3}\right): \delta=152.1\left(\mathrm{C}_{\mathrm{q}}, \mathrm{M}\right), 151.9\left(\mathrm{C}_{\mathrm{q}}, \mathrm{AM}\right), 149.5(\mathrm{CH}, \mathrm{M}), 149.4(\mathrm{CH}, \mathrm{AM})$, $138.0(\mathrm{CH}, \mathrm{AM}), 137.9(\mathrm{CH}, \mathrm{M}), 137.2\left(\mathrm{C}_{\mathrm{q}}, \mathrm{AM}\right), 137.2\left(\mathrm{C}_{\mathrm{q}}, \mathrm{M}\right), 136.5\left(\mathrm{C}_{\mathrm{q}}, \mathrm{AM}\right), 136.5\left(\mathrm{C}_{\mathrm{q}}, \mathrm{M}\right)$, $129.9\left(\mathrm{C}_{\mathrm{q}}, \mathrm{M}\right), 129.4\left(\mathrm{C}_{\mathrm{q}}, \mathrm{AM}\right), 121.5(\mathrm{CH}, \mathrm{M}), 122.1(\mathrm{CH}, \mathrm{M}), 121.6(\mathrm{CH}, \mathrm{AM}), 121.5(\mathrm{CH}, \mathrm{AM})$, $121.4(\mathrm{CH}, \mathrm{M}), 121.0(\mathrm{CH}, \mathrm{AM}), 120.0(\mathrm{CH}, \mathrm{AM}), 119.8$ (CH, M), $118.0(\mathrm{CH}, \mathrm{AM}), 117.6(\mathrm{CH}, \mathrm{M})$, $109.9(\mathrm{CH}, \mathrm{M}), 109.7(\mathrm{CH}, \mathrm{AM}), 109.7\left(\mathrm{C}_{\mathrm{q}}, \mathrm{AM}\right), 108.2\left(\mathrm{C}_{\mathrm{q}}, \mathrm{M}\right), 36.0\left(\mathrm{CH}_{2}, \mathrm{M}\right), 31.9\left(\mathrm{CH}_{2}, \mathrm{AM}\right)$, $31.8\left(\mathrm{CH}_{2}, \mathrm{M}\right), 31.7(\mathrm{CH}, \mathrm{M}), 29.4\left(\mathrm{CH}_{2}, \mathrm{AM}\right), 29.2\left(\mathrm{CH}_{2}, \mathrm{AM}\right), 29.2\left(\mathrm{CH}_{2}, \mathrm{AM}\right), 29.2\left(\mathrm{CH}_{2}, \mathrm{AM}\right)$, $27.9\left(\mathrm{CH}_{2}, \mathrm{M}\right), 24.8\left(\mathrm{CH}_{2}, \mathrm{AM}\right), 22.7\left(\mathrm{CH}_{2}, \mathrm{AM}\right), 20.1\left(\mathrm{CH}_{3}, \mathrm{M}\right), 14.2\left(\mathrm{CH}_{3}, \mathrm{AM}\right), 14.2\left(\mathrm{CH}_{3}, \mathrm{M}\right), 9.6$ $\left(\mathrm{CH}_{3}, \mathrm{M}\right), 8.9\left(\mathrm{CH}_{3}, \mathrm{AM}\right)$.

IR (ATR): 2924, 2854, 1586, 1470, 1436, 1362, 779, $739 \mathrm{~cm}^{-1}$.

MS (EI) $m / z$ (relative intensity): 320 ([M] $\left.]^{+}, 55\right), 235$ (30), 221 (100), 207 (30).

HR-MS (EI): $m / z$ calcd. for $\left[\mathrm{C}_{22} \mathrm{H}_{28} \mathrm{~N}_{2}\right]^{+}[\mathrm{M}]^{+} 320.2252$, found 320.2253 .

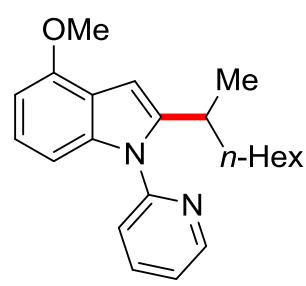

4-Methoxy-2-(octan-2-yl)-1-(pyridin-2-yl)-1H-indole (145ha): The general procedure $\mathbf{E}$ was followed using 4-methoxy-1-(pyridin-2-yl)- $1 H$-indole (105h) $(112 \mathrm{mg}, 0.50 \mathrm{mmol})$ and oct-1-ene (143a) (168 mg, $1.50 \mathrm{mmol}$ ). Isolation by column chromatography ( $n$-hexane/EtOAc: $25 / 1$ ) yielded 145ha $(128 \mathrm{mg}, 76 \%, 95: 5)$ as a yellowish oil.

${ }^{1}$ H-NMR $\left(400 \mathrm{MHz}, \mathrm{CDCl}_{3}\right): \delta=8.65(\mathrm{ddd}, J=4.9,2.1,1.0 \mathrm{~Hz}, 1 \mathrm{H}), 7.87(\mathrm{ddd}, J=7.9,7.4,2.1 \mathrm{~Hz}$, 1H), 7.39 (ddd, $J=7.9,1.1,1.0 \mathrm{~Hz}, 1 \mathrm{H}), 7.32$ (ddd, $J=7.4,4.9,1.1 \mathrm{~Hz}, 1 \mathrm{H}), 7.01$ (dd, $J=8.3,7.8 \mathrm{~Hz}$, $1 \mathrm{H}), 6.86(\mathrm{dd}, J=8.3,0.8 \mathrm{~Hz}, 1 \mathrm{H}), 6.63-6.47(\mathrm{~m}, 2 \mathrm{H}), 3.97(\mathrm{~s}, 3 \mathrm{H}), 3.13(\mathrm{tq}, J=6.8,6.8 \mathrm{~Hz}, 0.95 \mathrm{H}$, M), $2.78(\mathrm{t}, J=7.6 \mathrm{~Hz}, 0.10 \mathrm{H}, \mathrm{AM}), 1.64-1.57(\mathrm{~m}, 1 \mathrm{H}), 1.42-1.34(\mathrm{~m}, 1 \mathrm{H}), 1.25-1.09(\mathrm{~m}, 11 \mathrm{H}), 0.81$ (t, $J=7.3 \mathrm{~Hz}, 3 \mathrm{H})$.

${ }^{13}$ C-NMR $\left(100 \mathrm{MHz}, \mathrm{CDCl}_{3}\right): \delta=152.6\left(\mathrm{C}_{\mathrm{q}}\right), 151.8\left(\mathrm{C}_{\mathrm{q}}\right), 149.6(\mathrm{CH}), 146.1\left(\mathrm{C}_{\mathrm{q}}\right), 138.6\left(\mathrm{C}_{\mathrm{q}}\right), 138.2$ $(\mathrm{CH}), 122.2(\mathrm{CH}), 122.1(\mathrm{CH}), 121.8(\mathrm{CH}), 118.8\left(\mathrm{C}_{\mathrm{q}}\right), 103.6(\mathrm{CH}), 100.6(\mathrm{CH}), 96.8(\mathrm{CH}), 55.4$ $\left(\mathrm{CH}_{3}\right), 37.1\left(\mathrm{CH}_{2}\right), 31.7\left(\mathrm{CH}_{2}\right), 30.7(\mathrm{CH}), 29.2\left(\mathrm{CH}_{2}\right), 27.0\left(\mathrm{CH}_{2}\right), 22.6\left(\mathrm{CH}_{2}\right), 20.6\left(\mathrm{CH}_{3}\right), 14.0$ $\left(\mathrm{CH}_{3}\right)$.

IR (ATR): 2926, 2855, 1586, 1470, 1440, 1258, $764 \mathrm{~cm}^{-1}$.

MS (EI) $\mathrm{m} / z$ (relative intensity): $336\left([\mathrm{M}]^{+}, 55\right), 251$ (85), 237 (100).

HR-MS (EI): $m / z$ calcd. for $\left[\mathrm{C}_{22} \mathrm{H}_{28} \mathrm{~N}_{2} \mathrm{O}\right]^{+}[\mathrm{M}]^{+} 336.2202$, found 336.2213 . 


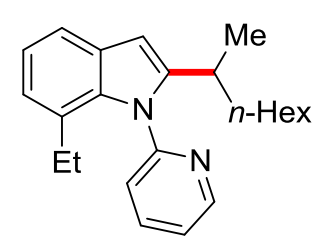

7-Ethyl-2-(octan-2-yl)-1-(pyridin-2-yl)-1H-indole (145ja): The general procedure $\mathbf{E}$ was followed using 7-ethyl-1-(pyridin-2-yl)-1H-indole (105j) $(111 \mathrm{mg}, 0.50 \mathrm{mmol})$ and oct-1-ene (143a) $(168 \mathrm{mg}$, $1.50 \mathrm{mmol}$ ). Isolation by column chromatography ( -hexane/EtOAc: 20/1) yielded 145ja (118 mg, $71 \%, 96: 4)$ as a colorless oil.

${ }^{1} \mathbf{H}-\mathbf{N M R}\left(300 \mathrm{MHz}, \mathrm{CDCl}_{3}\right): \delta=8.65(\mathrm{ddd}, J=4.9,2.0,0.9 \mathrm{~Hz}, 1 \mathrm{H}), 7.82(\mathrm{ddd}, J=7.8,7.6,2.0 \mathrm{~Hz}$, $1 \mathrm{H}), 7.44(\mathrm{dd}, J=7.8,1.4 \mathrm{~Hz}, 1 \mathrm{H}), 7.40(\mathrm{ddd}, J=7.6,4.9,0.9 \mathrm{~Hz}, 1 \mathrm{H}), 7.33(\mathrm{ddd}, J=7.8,0.9,0.9 \mathrm{~Hz}$, $1 \mathrm{H}), 7.06(\mathrm{t}, J=7.7 \mathrm{~Hz}, 1 \mathrm{H}), 6.96-6.90(\mathrm{~m}, 1 \mathrm{H}), 6.40(\mathrm{dd}, J=0.8,0.8 \mathrm{~Hz}, 1 \mathrm{H}), 2.52(\mathrm{tq}, J=6.8$, $6.8 \mathrm{~Hz}, 0.96 \mathrm{H}, \mathrm{M}), 2.45$ (t, $J=7.6 \mathrm{~Hz}, 0.08 \mathrm{H}, \mathrm{AM}), 2.12$ (q, $J=7.5 \mathrm{~Hz}, 2 \mathrm{H}), 1.72-1.59(\mathrm{~m}, 1 \mathrm{H})$, $1.46-1.35(\mathrm{~m}, 1 \mathrm{H}), 1.27-1.10(\mathrm{~m}, 11 \mathrm{H}), 0.94(\mathrm{t}, J=7.5 \mathrm{~Hz}, 3 \mathrm{H}), 0.84(\mathrm{t}, J=7.1 \mathrm{~Hz}, 3 \mathrm{H})$.

${ }^{13} \mathbf{C}-\mathrm{NMR}\left(125 \mathrm{MHz}, \mathrm{CDCl}_{3}\right): \delta=153.6\left(\mathrm{C}_{\mathrm{q}}\right), 149.0(\mathrm{CH}), 148.3\left(\mathrm{C}_{\mathrm{q}}\right), 137.4(\mathrm{CH}), 135.7\left(\mathrm{C}_{\mathrm{q}}\right), 129.4$ $\left(\mathrm{C}_{\mathrm{q}}\right), 127.4\left(\mathrm{C}_{\mathrm{q}}\right), 124.4(\mathrm{CH}), 123.3(\mathrm{CH}), 121.7(\mathrm{CH}), 120.3(\mathrm{CH}), 117.7(\mathrm{CH}), 99.2(\mathrm{CH}), 37.7$ $\left(\mathrm{CH}_{2}\right), 31.7(\mathrm{CH}), 31.1\left(\mathrm{CH}_{2}\right), 29.3\left(\mathrm{CH}_{2}\right), 27.3\left(\mathrm{CH}_{2}\right), 24.8\left(\mathrm{CH}_{2}\right), 22.7\left(\mathrm{CH}_{2}\right), 21.3\left(\mathrm{CH}_{3}\right), 14.7$ $\left(\mathrm{CH}_{3}\right), 14.1\left(\mathrm{CH}_{3}\right)$.

IR (ATR): 2925, 2854, 1583, 1467, 1435, 797, $740 \mathrm{~cm}^{-1}$.

MS (EI) $m / z$ (relative intensity): 334 ([M] $\left.]^{+}, 50\right) 249$ (40), 235 (100), 221 (40).

HR-MS (EI): $m / z$ calcd. for $\left[\mathrm{C}_{23} \mathrm{H}_{30} \mathrm{~N}_{2}\right]^{+}[\mathrm{M}]^{+} 334.2409$, found 334.2396 .<smiles>CC(Cc1ccccc1)c1cc2ccccc2n1-c1ccccc1</smiles>

2-(1-Phenylpropan-2-yl)-1-(pyridin-2-yl)-1H-indole (145ab): The general procedure $\mathbf{E}$ was followed using 1-(pyridin-2-yl)-1H-indole (105a) $(97.1 \mathrm{mg}, 0.50 \mathrm{mmol})$ and allylbenzene (143b) (177 mg, $1.50 \mathrm{mmol}$ ). Isolation by column chromatography ( $n$-hexane/EtOAc: $30 / 1$ ) yielded 145ab $(127 \mathrm{mg}, 82 \%, 99: 1)$ as a colorless oil.

${ }^{1} \mathbf{H}-\mathbf{N M R}\left(400 \mathrm{MHz}, \mathrm{CDCl}_{3}\right): \delta=8.69(\mathrm{ddd}, J=4.9,2.1,0.9 \mathrm{~Hz}, 1 \mathrm{H}), 7.84(\mathrm{ddd}, J=7.9,7.5,2.1 \mathrm{~Hz}$, $1 \mathrm{H}), 7.66-7.55(\mathrm{~m}, 1 \mathrm{H}), 7.33(\mathrm{ddd}, J=7.5,4.9,1.0 \mathrm{~Hz}, 1 \mathrm{H}), 7.28(\mathrm{ddd}, J=7.9,1.0,0.9 \mathrm{~Hz}, 1 \mathrm{H})$, 7.24-7.21 (m, 1H), 7.18-7.08 (m, 5H), 6.93-6.88 (m, 2H), $6.52(\mathrm{dd}, J=0.8,0.8 \mathrm{~Hz}, 1 \mathrm{H}), 3.59-3.46$ 
(m, 0.99H, M), $3.00(\mathrm{dd}, J=13.2,5.3 \mathrm{~Hz}, 1 \mathrm{H}), 2.86(\mathrm{t}, J=7.7 \mathrm{~Hz}, 0.02 \mathrm{H}, \mathrm{AM}), 2.56(\mathrm{dd}, J=13.2$, $8.7 \mathrm{~Hz}, 1 \mathrm{H}), 1.23(\mathrm{~d}, J=7.0 \mathrm{~Hz}, 3 \mathrm{H})$.

${ }^{13}$ C-NMR $\left(100 \mathrm{MHz}, \mathrm{CDCl}_{3}\right): \delta=151.6\left(\mathrm{C}_{\mathrm{q}}\right), 149.7(\mathrm{CH}), 146.5\left(\mathrm{C}_{\mathrm{q}}\right), 140.3\left(\mathrm{C}_{\mathrm{q}}\right), 138.3(\mathrm{CH}), 137.3$ $\left(\mathrm{C}_{\mathrm{q}}\right), 129.0(\mathrm{CH}), 128.5\left(\mathrm{C}_{\mathrm{q}}\right), 128.1(\mathrm{CH}), 125.9(\mathrm{CH}), 122.2(\mathrm{CH}), 121.7(\mathrm{CH}), 121.6(\mathrm{CH}), 120.5$ $(\mathrm{CH}), 120.1(\mathrm{CH}), 109.9(\mathrm{CH}), 100.4(\mathrm{CH}), 43.8\left(\mathrm{CH}_{2}\right), 32.8(\mathrm{CH}), 19.3\left(\mathrm{CH}_{3}\right)$.

IR (ATR): 3025, 2932, 1584, 1468, 1437, 735, $698 \mathrm{~cm}^{-1}$.

MS (EI) $\mathrm{m} / z$ (relative intensity): $312\left([\mathrm{M}]^{+}, 20\right), 221$ (35), 207 (100).

HR-MS (EI): $m / z$ calcd. for $\left[\mathrm{C}_{22} \mathrm{H}_{20} \mathrm{~N}_{2}\right]^{+}[\mathrm{M}]^{+} 312.1626$, found 312.1621 .

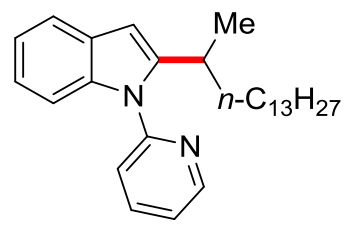

2-(Pentadecan-2-yl)-1-(pyridin-2-yl)-1H-indole (145ac): The general procedure $\mathbf{E}$ was followed using 1-(pyridin-2-yl)-1H-indole (105a) $(97.1 \mathrm{mg}, 0.50 \mathrm{mmol})$ and pentadec-1-ene (143c) $(316 \mathrm{mg}$, $1.50 \mathrm{mmol}$ ). Isolation by column chromatography ( $n$-hexane/EtOAc: $25 / 1$ ) yielded 145ac (154 mg, $76 \%, 95: 5)$ as a yellowish oil.

${ }^{1}$ H-NMR $\left(300 \mathrm{MHz}, \mathrm{CDCl}_{3}\right): \delta=8.69(\mathrm{ddd}, J=5.0,2.1,0.9 \mathrm{~Hz}, 1 \mathrm{H}), 7.87(\mathrm{ddd}, J=8.0,7.5,2.1 \mathrm{~Hz}$, 1H), 7.62-7.56 (m, 1H), 7.42 (ddd, $J=8.0,1.0,0.9 \mathrm{~Hz}, 1 \mathrm{H}), 7.36-7.27$ (m, 2H), 7.16-7.10 (m, 2H), $6.50(\mathrm{dd}, J=0.8,0.8 \mathrm{~Hz}, 1 \mathrm{H}), 3.22(\mathrm{tq}, J=6.8,6.8 \mathrm{~Hz}, 0.95 \mathrm{H}, \mathrm{M}), 2.86(\mathrm{t}, J=7.7 \mathrm{~Hz}, 0.10 \mathrm{H}, \mathrm{AM})$, $1.72-1.62(\mathrm{~m}, 1 \mathrm{H}), 1.50-1.40(\mathrm{~m}, 1 \mathrm{H}), 1.35-1.12(\mathrm{~m}, 25 \mathrm{H}), 0.93(\mathrm{t}, J=7.1 \mathrm{~Hz}, 3 \mathrm{H})$.

${ }^{13}$ C-NMR $\left(125 \mathrm{MHz}, \mathrm{CDCl}_{3}\right): \delta=151.7\left(\mathrm{C}_{\mathrm{q}}\right), 149.6(\mathrm{CH}), 147.4\left(\mathrm{C}_{\mathrm{q}}\right), 138.2(\mathrm{CH}), 137.4\left(\mathrm{C}_{\mathrm{q}}\right), 128.6$ $\left(\mathrm{C}_{\mathrm{q}}\right), 122.1(\mathrm{CH}), 121.4(\mathrm{CH}), 121.0(\mathrm{CH}), 120.4(\mathrm{CH}), 119.7(\mathrm{CH}), 109.7(\mathrm{CH}), 99.7(\mathrm{CH}), 37.1$ $\left(\mathrm{CH}_{2}\right), 32.0\left(\mathrm{CH}_{2}\right), 30.8(\mathrm{CH}), 29.7\left(\mathrm{CH}_{2}\right), 29.7\left(\mathrm{CH}_{2}\right), 29.7\left(\mathrm{CH}_{2}\right), 29.7\left(\mathrm{CH}_{2}\right), 29.7\left(\mathrm{CH}_{2}\right), 29.6$ $\left(\mathrm{CH}_{2}\right), 29.5\left(\mathrm{CH}_{2}\right), 29.4\left(\mathrm{CH}_{2}\right), 27.1\left(\mathrm{CH}_{2}\right), 22.7\left(\mathrm{CH}_{2}\right), 20.6\left(\mathrm{CH}_{3}\right), 14.2\left(\mathrm{CH}_{3}\right)$.

IR (ATR): 2923, 2852, 1585, 1468, 1456, 780, $735 \mathrm{~cm}^{-1}$.

MS (EI) $\mathrm{m} / z$ (relative intensity): 404 ([M] $\left.{ }^{+}, 35\right), 221$ (55), 207 (100), 195 (15).

HR-MS (EI): $m / z$ calcd. for $\left[\mathrm{C}_{28} \mathrm{H}_{40} \mathrm{~N}_{2}\right]^{+}[\mathrm{M}]^{+}$404.3191, found 404.3182 .

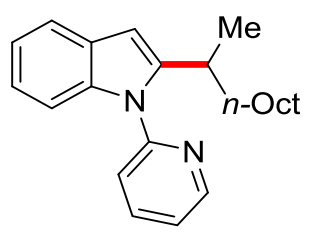

2-(Decan-2-yl)-1-(pyridin-2-yl)-1H-indole (145ad): The general procedure $\mathbf{E}$ was followed using 1(pyridin-2-yl)-1H-indole (105a) $(97.1 \mathrm{mg}, 0.50 \mathrm{mmol})$ and dec-1-ene (143d) $(210 \mathrm{mg}, 1.50 \mathrm{mmol})$. 
Isolation by column chromatography ( $n$-hexane/EtOAc: $25 / 1)$ yielded $145 a d(118 \mathrm{mg}, 71 \%, 94: 6)$ as a colorless oil.

${ }^{1}$ H-NMR $\left(400 \mathrm{MHz}, \mathrm{CDCl}_{3}\right): \delta=8.68(\mathrm{ddd}, J=5.0,2.1,0.9 \mathrm{~Hz}, 1 \mathrm{H}), 7.88(\mathrm{ddd}, J=8.0,7.5,2.1 \mathrm{~Hz}$, $1 \mathrm{H}), 7.65-7.58(\mathrm{~m}, 1 \mathrm{H}), 7.43(\mathrm{ddd}, J=8.0,1.0,0.9 \mathrm{~Hz}, 1 \mathrm{H}), 7.35-7.26(\mathrm{~m}, 2 \mathrm{H}), 7.19-7.11(\mathrm{~m}, 2 \mathrm{H})$, $6.50(\mathrm{dd}, J=0.8,0.8 \mathrm{~Hz}, 1 \mathrm{H}), 3.23(\mathrm{tq}, J=6.8,6.8 \mathrm{~Hz}, 0.94 \mathrm{H}, \mathrm{M}), 2.86$ (t, $J=7.7 \mathrm{~Hz}, 0.12 \mathrm{H}, \mathrm{AM})$, $1.72-1.62(\mathrm{~m}, 1 \mathrm{H}), 1.50-1.40(\mathrm{~m}, 1 \mathrm{H}), 1.34-1.11(\mathrm{~m}, 15 \mathrm{H}), 0.91(\mathrm{t}, J=7.1 \mathrm{~Hz}, 3 \mathrm{H})$.

${ }^{13}$ C-NMR $\left(100 \mathrm{MHz}, \mathrm{CDCl}_{3}\right): \delta=151.7\left(\mathrm{C}_{\mathrm{q}}\right), 149.6(\mathrm{CH}), 147.4\left(\mathrm{C}_{\mathrm{q}}\right), 138.2(\mathrm{CH}), 137.4\left(\mathrm{C}_{\mathrm{q}}\right), 128.6$ $\left(\mathrm{C}_{\mathrm{q}}\right), 122.1(\mathrm{CH}), 121.4(\mathrm{CH}), 121.0(\mathrm{CH}), 120.4(\mathrm{CH}), 119.7(\mathrm{CH}), 109.7(\mathrm{CH}), 99.7(\mathrm{CH}), 37.1$ $\left(\mathrm{CH}_{2}\right), 31.8\left(\mathrm{CH}_{2}\right), 30.7(\mathrm{CH}), 29.5\left(\mathrm{CH}_{2}\right), 29.4\left(\mathrm{CH}_{2}\right), 29.3\left(\mathrm{CH}_{2}\right), 27.1\left(\mathrm{CH}_{2}\right), 22.7\left(\mathrm{CH}_{2}\right), 20.6$ $\left(\mathrm{CH}_{3}\right), 14.1\left(\mathrm{CH}_{3}\right)$.

IR (ATR): 2923, 2852, 1585, 1468, 1435, 780, $735 \mathrm{~cm}^{-1}$.

MS (EI) $m / z$ (relative intensity): 334 ([M] $\left.]^{+}, 45\right), 249$ (30), 235 (100), 221 (45).

HR-MS (EI): $m / z$ calcd. for $\left[\mathrm{C}_{23} \mathrm{H}_{30} \mathrm{~N}_{2}\right]^{+}[\mathrm{M}]^{+} 334.2409$, found 334.2407 .<smiles>CCCCC(C)c1cc2ccccc2n1-c1ccccn1</smiles>

2-(Hexan-2-yl)-1-(pyridin-2-yl)-1H-indole (145ae): The general procedure $\mathbf{E}$ was followed using 1(pyridin-2-yl)-1H-indole (105a) $(97.1 \mathrm{mg}, 0.50 \mathrm{mmol})$ and hex-1-ene (143e) (126 mg, $1.50 \mathrm{mmol})$. Isolation by column chromatography ( $n$-hexane/EtOAc: $25 / 1)$ yielded 145ae $(94.6 \mathrm{mg}, 68 \%, 96: 4)$ as a colorless oil.

${ }^{1}$ H-NMR $\left(400 \mathrm{MHz}, \mathrm{CDCl}_{3}\right): \delta=8.67(\mathrm{ddd}, J=5.0,2.1,0.9 \mathrm{~Hz}, 1 \mathrm{H}), 7.86(\mathrm{ddd}, J=8.0,7.5,2.1 \mathrm{~Hz}$, $1 \mathrm{H}), 7.62-7.56(\mathrm{~m}, 1 \mathrm{H}), 7.42(\mathrm{ddd}, J=8.0,1.0,0.9 \mathrm{~Hz}, 1 \mathrm{H}), 7.36-7.27(\mathrm{~m}, 2 \mathrm{H}), 7.16-7.10(\mathrm{~m}, 2 \mathrm{H})$, $6.46(\mathrm{dd}, J=0.8,0.8 \mathrm{~Hz}, 1 \mathrm{H}), 3.17(\mathrm{tq}, J=7.6,7.6 \mathrm{~Hz}, 0.96 \mathrm{H}, \mathrm{M}), 2.82(\mathrm{t}, J=7.7 \mathrm{~Hz}, 0.08 \mathrm{H}, \mathrm{AM})$, $1.67-1.56(\mathrm{~m}, 1 \mathrm{H}), 1.45-1.35(\mathrm{~m}, 1 \mathrm{H}), 1.24-1.07(\mathrm{~m}, 7 \mathrm{H}), 0.77(\mathrm{t}, J=7.1 \mathrm{~Hz}, 3 \mathrm{H})$.

${ }^{13}$ C-NMR $\left(100 \mathrm{MHz}, \mathrm{CDCl}_{3}\right): \delta=151.7\left(\mathrm{C}_{\mathrm{q}}\right), 149.7(\mathrm{CH}), 147.5\left(\mathrm{C}_{\mathrm{q}}\right), 138.2(\mathrm{CH}), 137.3\left(\mathrm{C}_{\mathrm{q}}\right), 128.6$ $\left(\mathrm{C}_{\mathrm{q}}\right), 122.2(\mathrm{CH}), 121.7(\mathrm{CH}), 121.4(\mathrm{CH}), 120.4(\mathrm{CH}), 119.9(\mathrm{CH}), 110.0(\mathrm{CH}), 99.8(\mathrm{CH}), 36.7$ $\left(\mathrm{CH}_{2}\right), 30.7(\mathrm{CH}), 29.3\left(\mathrm{CH}_{2}\right), 22.6\left(\mathrm{CH}_{2}\right), 20.5\left(\mathrm{CH}_{3}\right), 13.9\left(\mathrm{CH}_{3}\right)$.

IR (ATR): 2928, 2857, 1584, 1469, 1436, 781, 747, $736 \mathrm{~cm}^{-1}$.

MS (EI) $m / z$ (relative intensity): 278 ([M] $\left.]^{+}, 40\right), 221$ (80), 207 (100).

HR-MS (EI): $m / z$ calcd. for $\left[\mathrm{C}_{19} \mathrm{H}_{22} \mathrm{~N}_{2}\right]^{+}[\mathrm{M}]^{+} 278.1783$, found 278.1781 .<smiles>CC(=O)OCCC([14CH3])c1cc2ccccc2n1-c1ccccn1</smiles> 
5-[1-(Pyridin-2-yl)-1H-indol-2-yl]hexyl acetate (145af): The general procedure $\mathbf{E}$ was followed using 1-(pyridin-2-yl)- $1 H$-indole (105a) $(97.1 \mathrm{mg}, 0.50 \mathrm{mmol}$ ) and hex-5-en-1-yl acetate (143f) (213 mg, $1.50 \mathrm{mmol}$ ). Isolation by column chromatography ( $n$-hexane/EtOAc: 10/1) yielded 145af (124 mg, 73\%, 99:1) as a yellowish oil.

${ }^{1}$ H-NMR $\left(300 \mathrm{MHz}, \mathrm{CDCl}_{3}\right.$ ): $\delta=8.68$ (ddd, $J=4.9,2.1,0.9 \mathrm{~Hz}, 1 \mathrm{H}$ ), 7.87 (ddd, $J=8.0,7.6,2.1 \mathrm{~Hz}$, $1 \mathrm{H}), 7.60-7.54(\mathrm{~m}, 1 \mathrm{H}), 7.41(\mathrm{ddd}, J=8.0,1.0,0.9 \mathrm{~Hz}, 1 \mathrm{H}), 7.33(\mathrm{ddd}, J=7.5,4.9,1.0 \mathrm{~Hz}, 1 \mathrm{H})$, 7.25-7.20 (m, 1H), 7.12-7.05 (m, 2H), $6.45(\mathrm{dd}, J=0.8,0.8 \mathrm{~Hz}, 1 \mathrm{H}), 3.94(\mathrm{t}, J=6.7 \mathrm{~Hz}, 2 \mathrm{H}), 3.25-$ $3.13(\mathrm{~m}, 0.99 \mathrm{H}, \mathrm{M}), 2.84$ (t, $J=7.4 \mathrm{~Hz}, 0.02 \mathrm{H}, \mathrm{AM}), 1.99$ (s, 3H), 1.69-1.59 (m, 1H), 1.51-1.39 (m, $3 \mathrm{H}), 1.32-1.21(\mathrm{~m}, 5 \mathrm{H})$.

${ }^{13}$ C-NMR (100 MHz, $\left.\mathrm{CDCl}_{3}\right): \delta=171.1\left(\mathrm{C}_{\mathrm{q}}\right), 151.6\left(\mathrm{C}_{\mathrm{q}}\right), 149.7(\mathrm{CH}), 146.9\left(\mathrm{C}_{\mathrm{q}}\right), 140.3(\mathrm{CH}), 138.3$ $(\mathrm{CH}), 137.3\left(\mathrm{C}_{\mathrm{q}}\right), 128.5\left(\mathrm{C}_{\mathrm{q}}\right), 122.2(\mathrm{CH}), 121.5(\mathrm{CH}), 120.5(\mathrm{CH}), 119.9(\mathrm{CH}), 109.9(\mathrm{CH}), 99.9$ $(\mathrm{CH}), 64.3\left(\mathrm{CH}_{2}\right), 36.5\left(\mathrm{CH}_{2}\right), 30.7(\mathrm{CH}), 28.5\left(\mathrm{CH}_{2}\right), 23.4\left(\mathrm{CH}_{2}\right), 21.0\left(\mathrm{CH}_{3}\right), 20.6\left(\mathrm{CH}_{3}\right)$.

IR (ATR): 2933, 1736, 1585, 1470, 1437, 1242, 782, $748 \mathrm{~cm}^{-1}$.

MS (EI) $\mathrm{m} / \mathrm{z}$ (relative intensity): 336 ([M] $\left.]^{+}, 30\right), 221$ (60), 207 (55), 135 (100).

HR-MS (EI): $\mathrm{m} / z$ calcd. for $\left[\mathrm{C}_{21} \mathrm{H}_{24} \mathrm{~N}_{2} \mathrm{O}_{2}\right]^{+}[\mathrm{M}]^{+} 336.1838$, found 336.1829 .<smiles>CC(O)c1cc2ccccc2n1-c1ccccn1</smiles>

9-[1-(Pyridin-2-yl)-1H-indol-2-yl]decan-1-ol (145ag): The general procedure $\mathbf{E}$ was followed using 1-(pyridin-2-yl)-1H-indole (105a) $(97.1 \mathrm{mg}, \quad 0.50 \mathrm{mmol})$ and dec-9-en-1-ol (143g) (234 mg, $1.50 \mathrm{mmol}$ ). Isolation by column chromatography ( $n$-hexane/EtOAc: $5 / 1)$ yielded 145ag (109 mg, $62 \%, 92: 8)$ as a yellowish oil.

${ }^{1} \mathbf{H}-\mathbf{N M R}\left(400 \mathrm{MHz}, \mathrm{CDCl}_{3}\right): \delta=8.65(\mathrm{ddd}, J=4.9,2.1,0.9 \mathrm{~Hz}, 1 \mathrm{H}), 7.88(\mathrm{ddd}, J=8.0,7.5,2.1 \mathrm{~Hz}$, $1 \mathrm{H}), 7.60-7.54(\mathrm{~m}, 1 \mathrm{H}), 7.40(\mathrm{ddd}, J=8.0,1.0,0.9 \mathrm{~Hz}, 1 \mathrm{H}), 7.34-7.30(\mathrm{ddd}, J=7.5,4.9,1.0 \mathrm{~Hz}, 1 \mathrm{H})$, 7.25-7.21 (m, 1H), 7.13-7.06 (m, 2H), $6.44(\mathrm{dd}, J=0.8,0.8 \mathrm{~Hz}, 1 \mathrm{H}), 3.55(\mathrm{t}, J=6.7 \mathrm{~Hz}, 2 \mathrm{H}), 3.16$ (tq, $J=6.9,6.9 \mathrm{~Hz}, 0.92 \mathrm{H}, \mathrm{M}), 2.82(\mathrm{t}, J=7.6 \mathrm{~Hz}, 0.16 \mathrm{H}, \mathrm{AM}), 1.70(\mathrm{bs}, 1 \mathrm{H}), 1.64-1.36(\mathrm{~m}, 4 \mathrm{H})$, $1.31-1.01(\mathrm{~m}, 13 \mathrm{H})$.

${ }^{13}$ C-NMR $\left(100 \mathrm{MHz}, \mathrm{CDCl}_{3}\right): \delta=151.6\left(\mathrm{C}_{\mathrm{q}}\right), 149.6(\mathrm{CH}), 147.4\left(\mathrm{C}_{\mathrm{q}}\right), 138.1(\mathrm{CH}), 137.3\left(\mathrm{C}_{\mathrm{q}}\right), 128.5$ $\left(\mathrm{C}_{\mathrm{q}}\right), 122.2(\mathrm{CH}), 121.8(\mathrm{CH}), 121.4(\mathrm{CH}), 120.4(\mathrm{CH}), 119.9(\mathrm{CH}), 109.9(\mathrm{CH}), 99.8(\mathrm{CH}), 62.9$ $\left(\mathrm{CH}_{2}\right), 38.6\left(\mathrm{CH}_{2}\right), 37.0\left(\mathrm{CH}_{2}\right), 36.4\left(\mathrm{CH}_{2}\right), 32.7\left(\mathrm{CH}_{2}\right), 30.7(\mathrm{CH}), 29.4\left(\mathrm{CH}_{2}\right), 27.0\left(\mathrm{CH}_{2}\right), 25.6$ $\left(\mathrm{CH}_{2}\right), 20.5\left(\mathrm{CH}_{3}\right)$.

IR (ATR): 3374, 2924, 2852, 1699, 1586, 1468, 1436, 780, $736 \mathrm{~cm}^{-1}$.

MS (EI) $\mathrm{m} / \mathrm{z}$ (relative intensity): 350 ([M] $\left.{ }^{+}, 15\right), 264$ (25), 252 (100), 206 (30). 
HR-MS (EI): $m / z$ calcd. for $\left[\mathrm{C}_{23} \mathrm{H}_{30} \mathrm{~N}_{2} \mathrm{O}\right]^{+}[\mathrm{M}]^{+} 350.2358$, found 350.2351 .

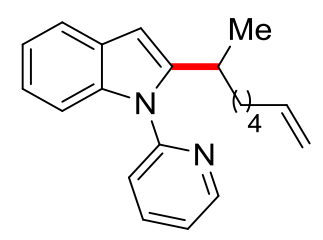

2-(Oct-7-en-2-yl)-1-(pyridin-2-yl)-1H-indole (145ah): The general procedure $\mathbf{E}$ was followed using 1-(pyridin-2-yl)-1H-indole (105a) $(97.1 \mathrm{mg}, 0.50 \mathrm{mmol})$ and octa-1,7-diene (143h) (165 mg, $1.50 \mathrm{mmol}$ ). Isolation by column chromatography ( $n$-hexane/EtOAc: 40/1) yielded 145ah (119 mg, $78 \%, 94: 6)$ as a colorless oil.

${ }^{1}$ H-NMR $\left(300 \mathrm{MHz}, \mathrm{CDCl}_{3}\right): \delta=8.69(\mathrm{ddd}, J=4.9,2.0,0.9 \mathrm{~Hz}, 1 \mathrm{H}), 7.88(\mathrm{ddd}, J=7.8,7.6,2.0 \mathrm{~Hz}$, 1H), 7.64-7.58 (m, 1H), 7.43 (ddd, $J=7.9,0.9,0.9 \mathrm{~Hz}, 1 \mathrm{H}), 7.33$ (ddd, $J=7.2,4.9,0.9 \mathrm{~Hz}, 1 \mathrm{H}$ ), 7.29-7.24 (m, 1H), 7.18-7.10 (m, 2H), 6.49 (dd, $J=0.8,0.8 \mathrm{~Hz}, 1 \mathrm{H}), 5.76$ (ddt, $J=17.2,10.4$, $6.8 \mathrm{~Hz}, 1 \mathrm{H}), 5.08-4.84(\mathrm{~m}, 2 \mathrm{H}), 3.22(\mathrm{tq}, J=6.9,6.9 \mathrm{~Hz}, 0.94 \mathrm{H}, \mathrm{M}), 2.86(\mathrm{t}, J=7.6 \mathrm{~Hz}, 0.12 \mathrm{H}, \mathrm{AM})$, 2.08-1.92 (m, 3H), 1.81-1.54 (m, 2H), 1.53-1.41 (m, 1H), 1.32-1.21 (m, 5H).

${ }^{13}$ C-NMR $\left(125 \mathrm{MHz}, \mathrm{CDCl}_{3}\right): \delta=151.6\left(\mathrm{C}_{\mathrm{q}}\right), 149.6(\mathrm{CH}), 147.3\left(\mathrm{C}_{\mathrm{q}}\right), 138.9(\mathrm{CH}), 138.2(\mathrm{CH}), 137.3$ $\left(\mathrm{C}_{\mathrm{q}}\right), 128.5\left(\mathrm{C}_{\mathrm{q}}\right), 122.1(\mathrm{CH}), 121.7(\mathrm{CH}), 121.4(\mathrm{CH}), 120.4(\mathrm{CH}), 119.9(\mathrm{CH}), 114.1\left(\mathrm{CH}_{2}\right), 109.9$ $(\mathrm{CH}), 102.0(\mathrm{CH}, \mathrm{AM}), 99.8(\mathrm{CH}, \mathrm{M}), 38.6\left(\mathrm{CH}_{2}, \mathrm{AM}\right), 36.8\left(\mathrm{CH}_{2}, \mathrm{M}\right), 36.4\left(\mathrm{CH}_{2}, \mathrm{AM}\right), 33.5\left(\mathrm{CH}_{2}\right.$, M), $30.6(\mathrm{CH}), 28.7\left(\mathrm{CH}_{2}\right), 27.7\left(\mathrm{CH}_{2}, \mathrm{AM}\right), 26.5\left(\mathrm{CH}_{2}\right), 20.5\left(\mathrm{CH}_{3}\right)$.

IR (ATR): 2926, 2854, 1584, 1469, 1455, 1435, 780, $734 \mathrm{~cm}^{-1}$.

MS (EI) $\mathrm{m} / z$ (relative intensity): 304 ([M] $\left.]^{+}, 20\right), 263$ (15), 221 (100), 207 (100).

HR-MS (EI): $m / z$ calcd. for $\left[\mathrm{C}_{21} \mathrm{H}_{24} \mathrm{~N}_{2}\right]^{+}[\mathrm{M}]^{+}$304.1939, found 304.1938 .

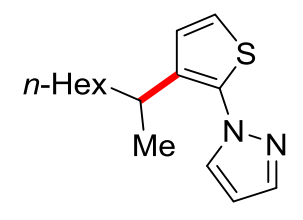

1-[3-(Octan-2-yl)thiophen-2-yl]-1H-pyrazole (148): A modified general procedure $\mathbf{E}$ was followed using 1-(thiophen-2-yl)- $1 H$-pyrazole (131) $(75.0 \mathrm{mg}, 0.50 \mathrm{mmol})$ and oct-1-ene (143a) $(168 \mathrm{mg}$, $1.50 \mathrm{mmol})$ at $80{ }^{\circ} \mathrm{C}$. Isolation by column chromatography ( $n$-hexane/EtOAc: $\left.15 / 1\right)$ yielded 148 (109 $\mathrm{mg}, 83 \%, 79: 21)$ as a yellowish oil.

${ }^{1}$ H-NMR $\left(300 \mathrm{MHz}, \mathrm{CDCl}_{3}\right): \delta=7.68(\mathrm{dd}, J=1.9,0.6 \mathrm{~Hz}, 1 \mathrm{H}), 7.59(\mathrm{dd}, J=2.4,0.6 \mathrm{~Hz}, 0.21 \mathrm{H}$, AM), $7.57(\mathrm{dd}, J=2.4,0.6 \mathrm{~Hz}, 0.79 \mathrm{H}, \mathrm{M}), 7.13(\mathrm{dd}, J=5.7,0.5 \mathrm{~Hz}, 0.79 \mathrm{H}, \mathrm{M}), 7.09$ (dd, $J=5.7$, $0.5 \mathrm{~Hz}, 0.21 \mathrm{H}, \mathrm{AM}), 6.89-6.83(\mathrm{~m}, 1 \mathrm{H}), 6.41-6.38(\mathrm{~m}, 1 \mathrm{H}), 2.73$ (tq, $J=7.1,7.1 \mathrm{~Hz}, 0.79 \mathrm{H}, \mathrm{M}), 2.51$ $(\mathrm{t}, J=7.6 \mathrm{~Hz}, 0.42 \mathrm{H}, \mathrm{AM}), 1.52-1.41(\mathrm{~m}, 2 \mathrm{H}), 1.22-1.13(\mathrm{~m}, 11 \mathrm{H}), 0.83(\mathrm{t}, J=6.9 \mathrm{~Hz}, 3 \mathrm{H})$. 
${ }^{13}$ C-NMR (125 MHz, CDCl $)$ : $\delta=142.1\left(\mathrm{C}_{\mathrm{q}}\right), 140.9(\mathrm{CH}), 135.9\left(\mathrm{C}_{\mathrm{q}}, \mathrm{AM}\right), 135.4\left(\mathrm{C}_{\mathrm{q}}, \mathrm{M}\right), 132.0(\mathrm{CH}$, M), 131.5 (CH, AM), 127.5 (CH, AM), 124.8 (CH, M), 122.5 (CH, M), 121.7 (CH, AM), 106.7 (CH, AM), $106.6(\mathrm{CH}, \mathrm{M}), 37.7\left(\mathrm{CH}_{2}\right), 32.1(\mathrm{CH}), 31.9\left(\mathrm{CH}_{2}, \mathrm{AM}\right), 31.7\left(\mathrm{CH}_{2}, \mathrm{M}\right), 30.2\left(\mathrm{CH}_{2}, \mathrm{AM}\right), 29.3$ $\left(\mathrm{CH}_{2}, \mathrm{AM}\right), 29.3\left(\mathrm{CH}_{2}, \mathrm{AM}\right), 29.2\left(\mathrm{CH}_{2}, \mathrm{M}\right), 27.6\left(\mathrm{CH}_{2}\right), 22.7\left(\mathrm{CH}_{2}\right), 21.8\left(\mathrm{CH}_{3}\right), 14.1\left(\mathrm{CH}_{3}, \mathrm{AM}\right)$, $14.1\left(\mathrm{CH}_{3}, \mathrm{M}\right)$.

IR (ATR): 2957, 2924, 1455, 1389, 919, $746 \mathrm{~cm}^{-1}$.

MS (EI) $m / z$ (relative intensity): 262 ([M] $\left.{ }^{+}, 40\right), 191$ (40), 177 (100), 163 (90), 144 (60).

HR-MS (EI): $m / z$ calcd. for $\left[\mathrm{C}_{15} \mathrm{H}_{22} \mathrm{~N}_{2} \mathrm{~S}\right]^{+}[\mathrm{M}]^{+} 262.1504$, found 262.1515 .

\subsubsection{Removal of the Directing Group}
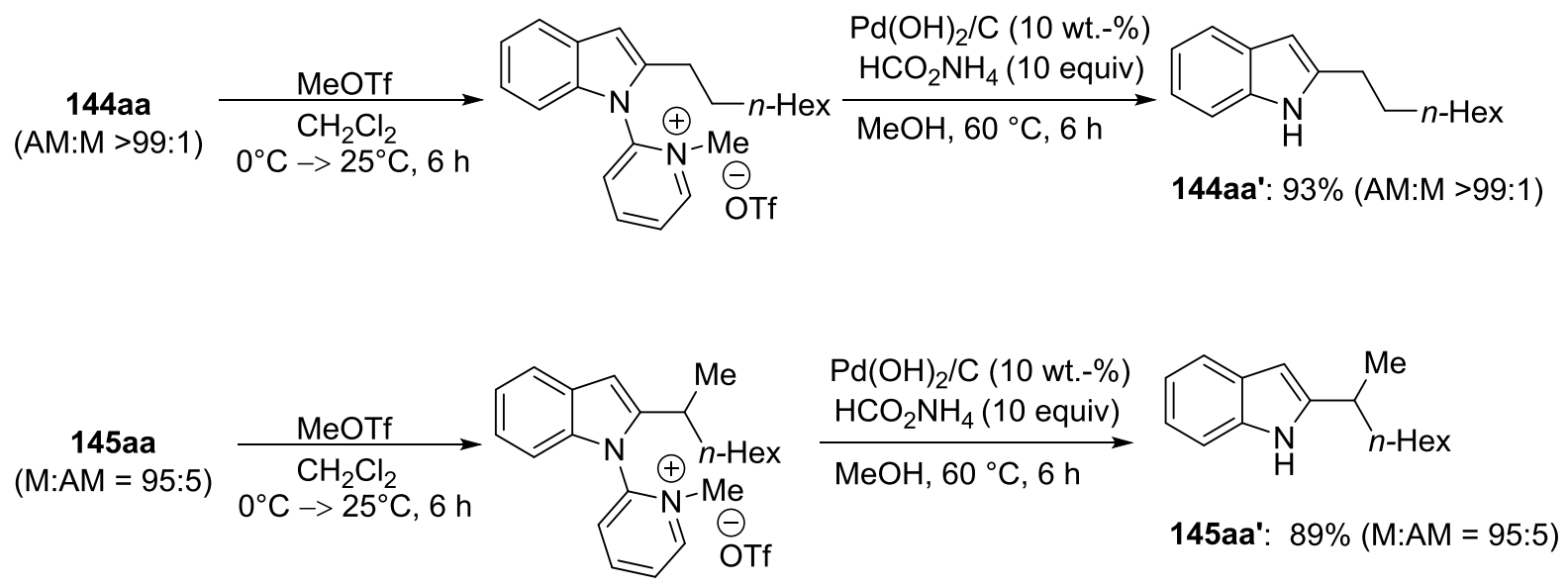

To a solution of 144aa (153 mg, $0.50 \mathrm{mmol}, 1.00$ equiv) or 145aa (153 mg, $0.50 \mathrm{mmol}, 1.00$ equiv) in $\mathrm{CH}_{2} \mathrm{Cl}_{2}(1.0 \mathrm{~mL})$ was added MeOTf $\left(90.3 \mathrm{mg}, 60.0 \mu \mathrm{L}, 0.55 \mathrm{mmol}, 1.10\right.$ equiv) dropwise at $0{ }^{\circ} \mathrm{C}$. After $30 \mathrm{~min}$, the mixture was allowed to warm up to $25^{\circ} \mathrm{C}$ and stirred for $6 \mathrm{~h}$. After removal of the solvent in vacuo, $\mathrm{Pd}(\mathrm{OH})_{2} / \mathrm{C}(19.3 \mathrm{mg}, 10 \mathrm{wt} .-\%)$ and ammonium formate (315 mg, $5.00 \mathrm{mmol}, 10.0$ equiv) were added. The mixture was diluted with $\mathrm{MeOH}(2.0 \mathrm{~mL}, 0.25 \mathrm{M})$ and stirred at $60{ }^{\circ} \mathrm{C}$ for $6 \mathrm{~h}$. After addition of EtOAc $(10 \mathrm{~mL})$ at ambient temperature, the mixture was filtered through a short pad of celite and the solvents were removed in vacuo. The crude mixture was purified by flash column chromatography on silica gel ( $n$-hexane/EtOAc: 8/1) to yield 144aa' (106 mg, 93\%) or 145aa' (102 $\mathrm{mg}, 89 \%, 95: 5)$ as pale yellow solids, respectively. 
<smiles>O=COCc1cc2ccccc2[nH]1</smiles>

\section{2-n-Octyl-1H-indole (144aa'):}

M.p.: $58{ }^{\circ} \mathrm{C}$

${ }^{1}$ H-NMR (300 MHz, $\left.\mathrm{CDCl}_{3}\right): \delta=7.69(\mathrm{bs}, 1 \mathrm{H}), 7.64-7.54(\mathrm{~m}, 1 \mathrm{H}), 7.31-7.26(\mathrm{~m}, 1 \mathrm{H}), 7.23-7.14(\mathrm{~m}$, $2 \mathrm{H}), 6.31(\mathrm{dt}, J=0.8,0.8 \mathrm{~Hz}, 1 \mathrm{H}), 2.74(\mathrm{tt}, J=7.5,7.5 \mathrm{~Hz}, 2 \mathrm{H}), 1.75(\mathrm{tt}, J=7.5,7.5 \mathrm{~Hz}, 2 \mathrm{H}), 1.48-$ $1.34(\mathrm{~m}, 10 \mathrm{H}), 1.00(\mathrm{t}, J=7.0 \mathrm{~Hz}, 3 \mathrm{H})$.

${ }^{13}$ C-NMR $\left(125 \mathrm{MHz}, \mathrm{CDCl}_{3}\right): \delta=139.9\left(\mathrm{C}_{\mathrm{q}}\right), 135.7\left(\mathrm{C}_{\mathrm{q}}\right), 128.7\left(\mathrm{C}_{\mathrm{q}}\right), 120.7(\mathrm{CH}), 119.6(\mathrm{CH}), 119.4$ $(\mathrm{CH}), 110.2(\mathrm{CH}), 99.3(\mathrm{CH}), 31.9\left(\mathrm{CH}_{2}\right), 29.5\left(\mathrm{CH}_{2}\right), 29.4\left(\mathrm{CH}_{2}\right), 29.3\left(\mathrm{CH}_{2}\right), 29.2\left(\mathrm{CH}_{2}\right), 28.2\left(\mathrm{CH}_{2}\right)$, $22.7\left(\mathrm{CH}_{2}\right), 14.2\left(\mathrm{CH}_{3}\right)$.

IR (ATR): 3407, 2925, 2854, 1458, 1288, 779, $748 \mathrm{~cm}^{-1}$.

MS (EI) $\mathrm{m} / z$ (relative intensity): 229 ([M] $\left.{ }^{+}, 30\right), 144$ (30), 130 (100).

HR-MS (EI): $m / z$ calcd. for $\left[\mathrm{C}_{16} \mathrm{H}_{23} \mathrm{~N}\right]^{+}[\mathrm{M}]^{+} 229.1830$, found 229.1829 .<smiles>CCOCCOC(C)c1cc2ccccc2[nH]1</smiles>

\section{2-(Octan-2-yl)-1H-indole (145aa'):}

M.p.: $60{ }^{\circ} \mathrm{C}$

${ }^{1}$ H-NMR $\left(300 \mathrm{MHz}, \mathrm{CDCl}_{3}\right): \delta=7.85(\mathrm{bs}, 1 \mathrm{H}), 7.55-7.49(\mathrm{~m}, 1 \mathrm{H}), 7.32-7.26(\mathrm{~m}, 1 \mathrm{H}), 7.17-7.01(\mathrm{~m}$, $2 \mathrm{H}), 6.23(\mathrm{dd}, J=0.8,0.8 \mathrm{~Hz}, 1 \mathrm{H}), 2.88(\mathrm{tq}, J=7.0,7.0 \mathrm{~Hz}, 0.95 \mathrm{H}), 2.74(\mathrm{t}, J=7.6 \mathrm{~Hz}, 0.05 \mathrm{H}), 1.75-$ $1.57(\mathrm{~m}, 2 \mathrm{H}), 1.37-1.24(\mathrm{~m}, 11 \mathrm{H}), 0.86(\mathrm{t}, J=7.0 \mathrm{~Hz}, 3 \mathrm{H})$.

${ }^{13}$ C-NMR $\left(125 \mathrm{MHz}, \mathrm{CDCl}_{3}\right): \delta=144.9\left(\mathrm{C}_{\mathrm{q}}\right), 135.5\left(\mathrm{C}_{\mathrm{q}}\right), 128.6\left(\mathrm{C}_{\mathrm{q}}\right), 120.8(\mathrm{CH}), 119.8(\mathrm{CH}), 119.5$ $(\mathrm{CH}), 110.2(\mathrm{CH}), 98.2(\mathrm{CH}), 37.3\left(\mathrm{CH}_{2}\right), 33.4(\mathrm{CH}), 31.8\left(\mathrm{CH}_{2}\right), 29.4\left(\mathrm{CH}_{2}\right), 27.4\left(\mathrm{CH}_{2}\right), 22.7\left(\mathrm{CH}_{2}\right)$, $20.7\left(\mathrm{CH}_{3}\right), 14.2\left(\mathrm{CH}_{3}\right)$.

IR (ATR): $3406,2926,2855,1458,781,749 \mathrm{~cm}^{-1}$.

MS (EI) $m / z$ (relative intensity): 229 ([M] $\left.]^{+}, 40\right), 158$ (20), 144 (100), 130 (45).

HR-MS (EI): $m / z$ calcd. for $\left[\mathrm{C}_{16} \mathrm{H}_{23} \mathrm{~N}\right]^{+}[\mathrm{M}]^{+} 229.1830$, found 229.1824 . 


\subsubsection{Mechanistic Studies}

\subsubsection{Kinetic Isotope Effect}

\subsection{KIE for the linear-selective reaction}

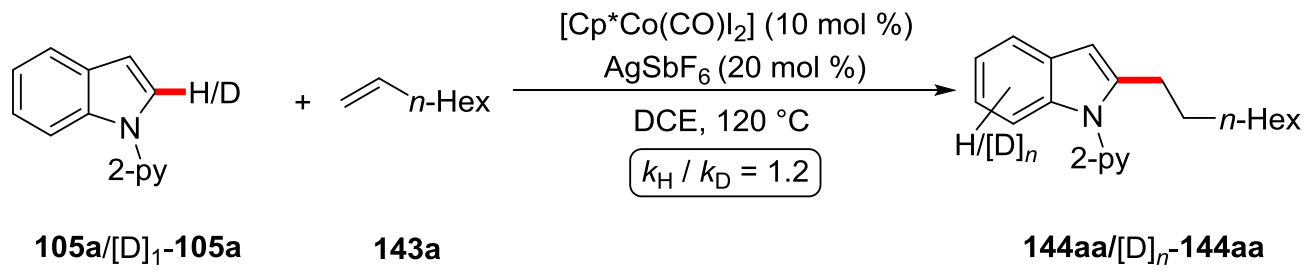

Two parallel reactions of $\mathbf{1 0 5 a}$ or $[\mathrm{D}]_{1}-\mathbf{1 0 5 a}$ with $143 \mathbf{a}$ were performed respectively to determine the KIE value by comparison of the initial reaction rates by GC-analysis with $n$-dodecane as the internal standard. A suspension of 105a (97.1 mg, $0.50 \mathrm{mmol}, 1.00$ equiv) or [D $]_{1}-\mathbf{1 0 5 a}(97.6 \mathrm{mg}, 0.50 \mathrm{mmol}$, 1.00 equiv), 143a (168 mg, $235 \mu \mathrm{L}, 1.50 \mathrm{mmol}, 3.00$ equiv), [Cp*Co(CO) $\left.\mathrm{I}_{2}\right](23.8 \mathrm{mg}, 10.0 \mathrm{~mol} \%)$ and $\mathrm{AgSbF}_{6}(34.4 \mathrm{mg}, 20.0 \mathrm{~mol} \%)$ in $\mathrm{DCE}(2.00 \mathrm{~mL})$ was stirred at $120{ }^{\circ} \mathrm{C}$. Aliquots $(25 \mu \mathrm{L})$ were periodically removed to provide the following conversions as determined by GC-analysis:

Table 32. KIE for the linear-selective reaction.

\begin{tabular}{c|ccccc}
$t / \min$ & 10 & 20 & 30 & 40 & 50 \\
\hline $\mathbf{1 4 4 a a} / \%$ & 2.5 & 5.8 & 11.6 & 18.8 & 25.2 \\
{$[\mathrm{D}]_{n}-\mathbf{1 4 4 a a} / \%$} & 1.0 & 5.4 & 8.7 & 16.3 & 21.0
\end{tabular}

\subsection{KIE for the branched-selective reaction}

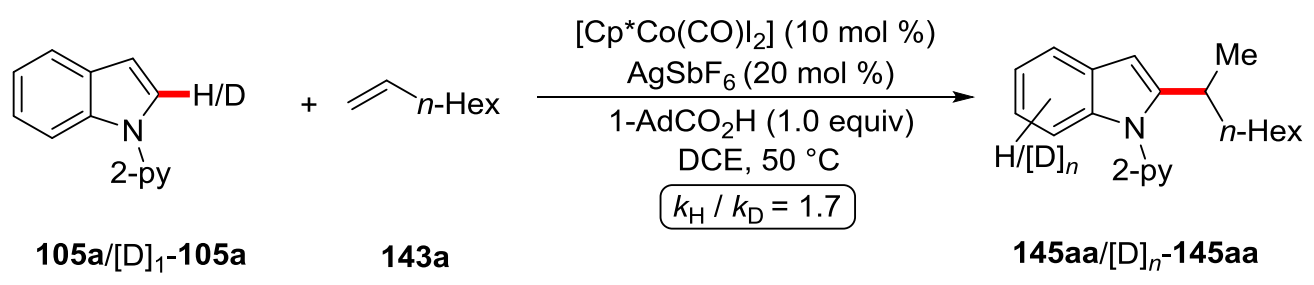

Two parallel reactions of $\mathbf{1 0 5 a}$ or $[D]_{1}-\mathbf{1 0 5 a}$ with $\mathbf{1 4 3 a}$ were performed respectively to determine the KIE value by comparison of the initial reaction rates by GC-analysis with $n$-dodecane as the internal standard. A suspension of 105a (97.1 mg, $0.50 \mathrm{mmol}, 1.00$ equiv) or [D] $]_{1} \mathbf{- 1 0 5 a}(97.6 \mathrm{mg}, 0.50 \mathrm{mmol}$, 1.00 equiv), 143a (168 mg, $235 \mu \mathrm{L}, 1.50 \mathrm{mmol}, 3.00$ equiv), [Cp*Co(CO) $\left.\mathrm{I}_{2}\right](23.8 \mathrm{mg}, 10 \mathrm{~mol} \%)$, $\mathrm{AgSbF}_{6}(34.4 \mathrm{mg}, 20 \mathrm{~mol} \%)$ and $1-\mathrm{AdCO}_{2} \mathrm{H}(90.1 \mathrm{mg}, 0.50 \mathrm{mmol}, 1.00$ equiv) in DCE $(2.00 \mathrm{~mL})$ 
was stirred at $50{ }^{\circ} \mathrm{C}$. Aliquots $(25 \mu \mathrm{L})$ were periodically removed to provide the following conversions as determined by GC-analysis:

Table 33. KIE for the branched-selective reaction.

\begin{tabular}{c|ccccc}
$t / \min$ & 30 & 40 & 50 & 60 & 90 \\
\hline 145aa / \% & 1.64 & 2.37 & 3.06 & 3.44 & 5.92 \\
{$[\mathrm{D}]_{n}-145 \mathbf{a a} / \%$} & 0.76 & 1.36 & 1.67 & 1.98 & 3.38
\end{tabular}

\subsubsection{H/D-Exchange Experiments}

\subsection{H/D-exchange for the linear-selective reaction}

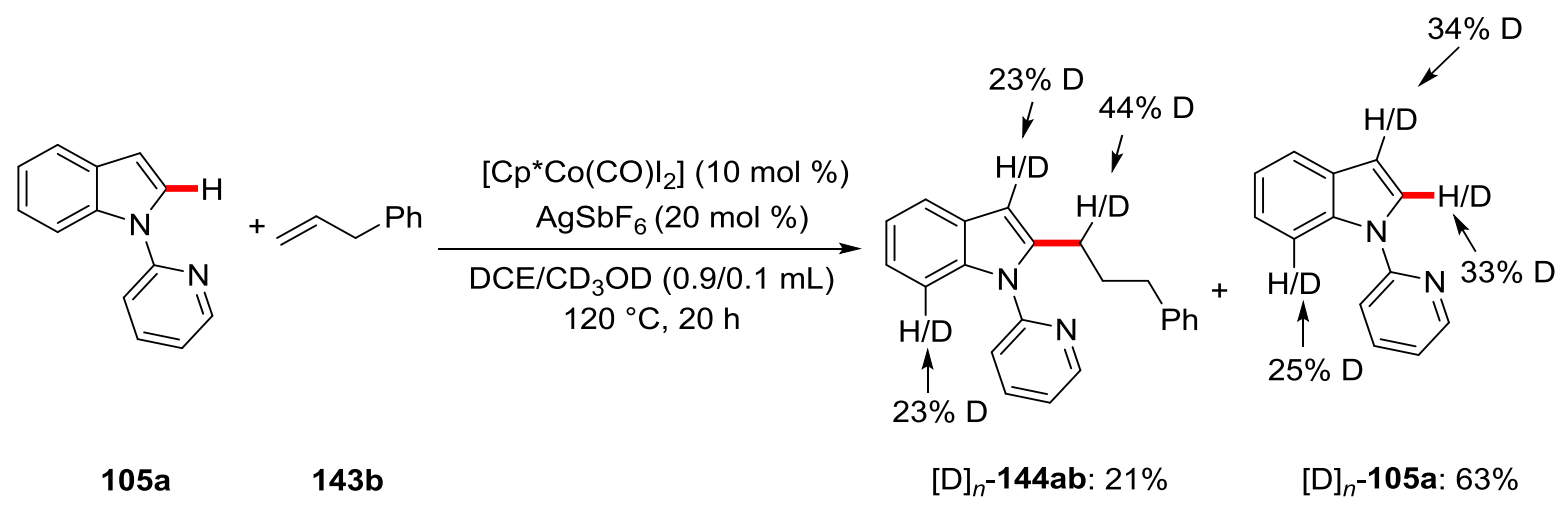

A suspension of 105a (146 mg, $0.75 \mathrm{mmol}, 1.50$ equiv), allylbenzene (143b) $(59.1 \mathrm{mg}, 0.50 \mathrm{mmol}$, 1.00 equiv), $\left[\mathrm{Cp} * \mathrm{Co}(\mathrm{CO}) \mathrm{I}_{2}\right](23.8 \mathrm{mg}, 10.0 \mathrm{~mol} \%)$ and $\mathrm{AgSbF}_{6}(34.4 \mathrm{mg}, 20.0 \mathrm{~mol} \%)$ in $\mathrm{DCE}$ $(0.9 \mathrm{~mL})$ and $\mathrm{CD}_{3} \mathrm{OD}(0.1 \mathrm{~mL})$ was stirred at $120{ }^{\circ} \mathrm{C}$ for $20 \mathrm{~h}$. After removal of the solvents, the crude mixture was purified by column chromatography on silica gel ( $n$-hexane/EtOAc: $20: 1)$ to yield $[\mathrm{D}]_{n^{-}}$ 144ab (32.8 $\mathrm{mg}, 21 \%)$ and $[\mathrm{D}]_{n}-\mathbf{1 0 5 a}(92.0 \mathrm{mg}, 63 \%$ reis.) as yellow oils. 

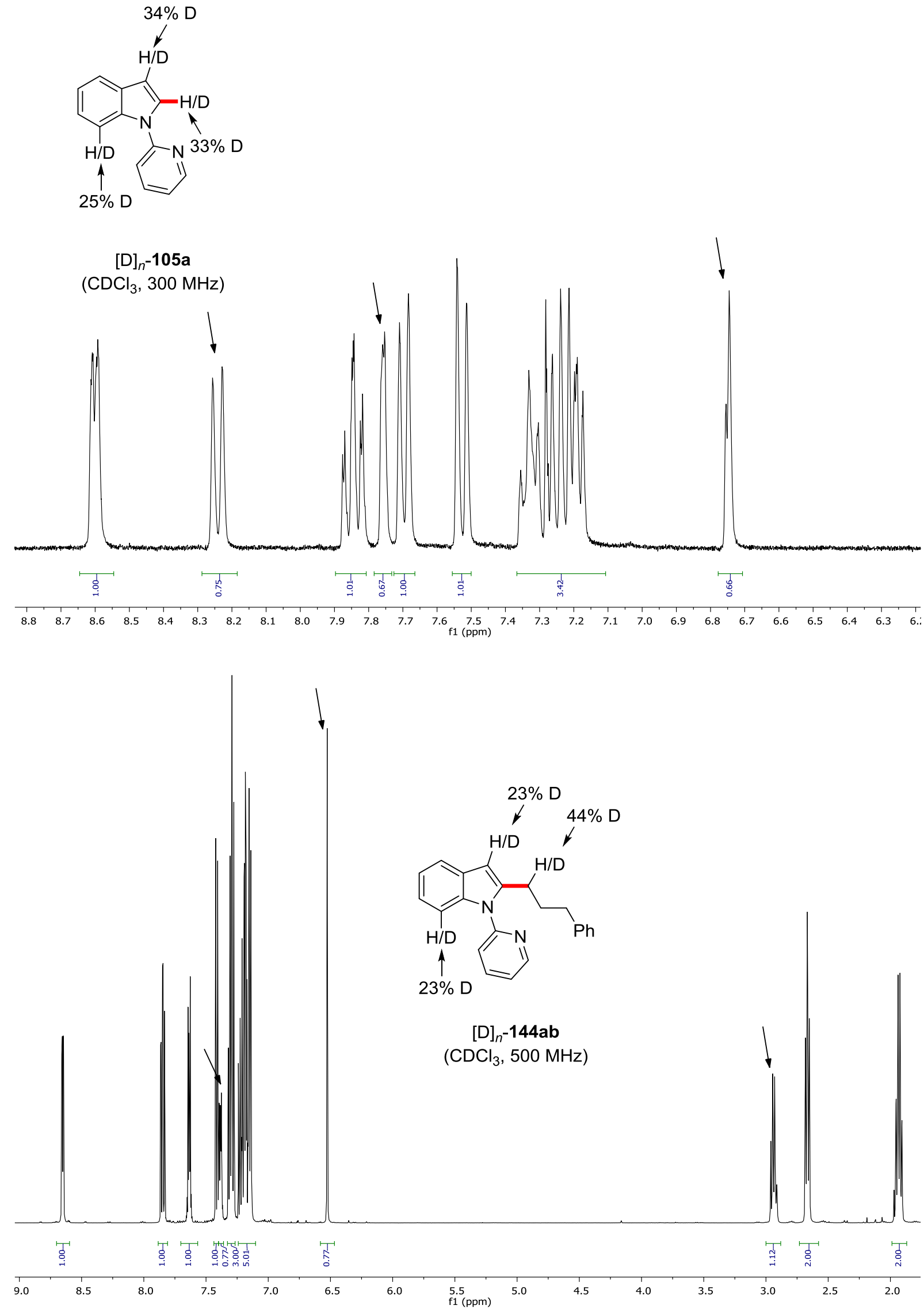


\subsection{H/D-exchange for the branched-selective reaction}

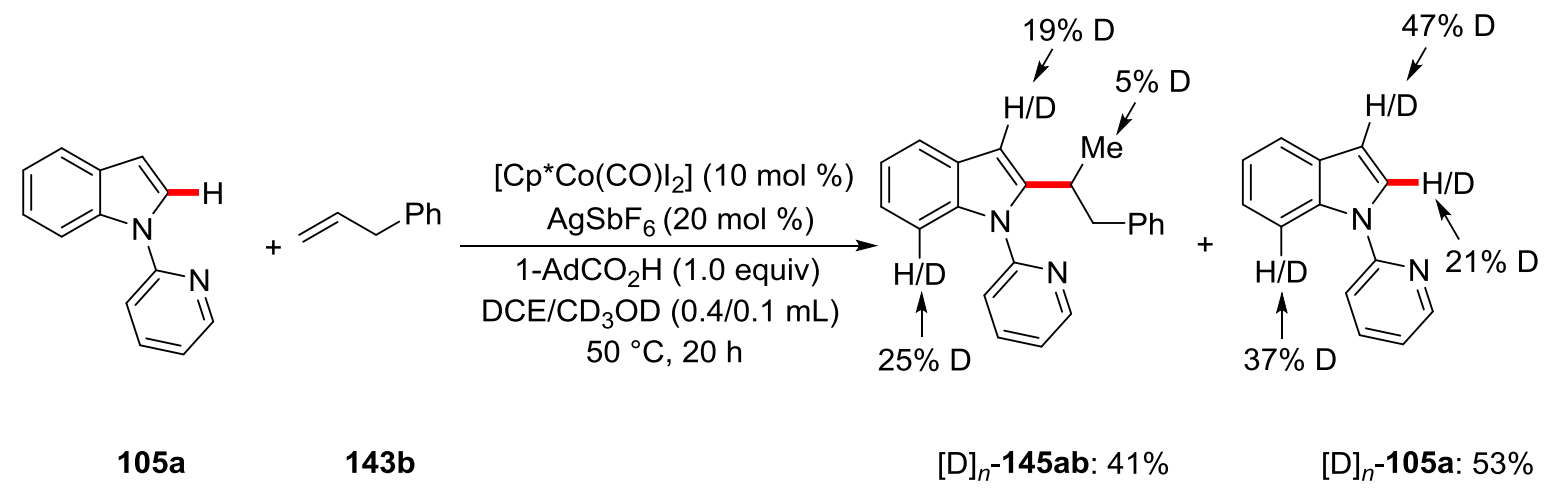

A suspension of 105a (146 mg, $0.75 \mathrm{mmol}, 1.50$ equiv), allylbenzene (143b) (59.1 mg, $0.50 \mathrm{mmol}$, 1.00 equiv), [Cp* $\left.\mathrm{Co}(\mathrm{CO}) \mathrm{I}_{2}\right](23.8 \mathrm{mg}, 10.0 \mathrm{~mol} \%), \mathrm{AgSbF}_{6}(34.4 \mathrm{mg}, 20.0 \mathrm{~mol} \%)$ and $1-\mathrm{AdCO}_{2} \mathrm{H}$ (90.1 mg, $0.50 \mathrm{mmol}, 1.00$ equiv) in DCE $(0.4 \mathrm{~mL})$ and $\mathrm{CD}_{3} \mathrm{OD}(0.1 \mathrm{~mL})$ was stirred at $50{ }^{\circ} \mathrm{C}$ for $20 \mathrm{~h}$. After removal of the solvents, the crude mixture was purified by column chromatography on silica gel ( $n$-hexane/EtOAc: 20:1) to yield $[\mathrm{D}]_{n}$-145ab $(64.0 \mathrm{mg}, 41 \%)$ and $[\mathrm{D}]_{n}-\mathbf{1 0 5 a}(77.1 \mathrm{mg}, 53 \%$ reis.) as yellow oils.
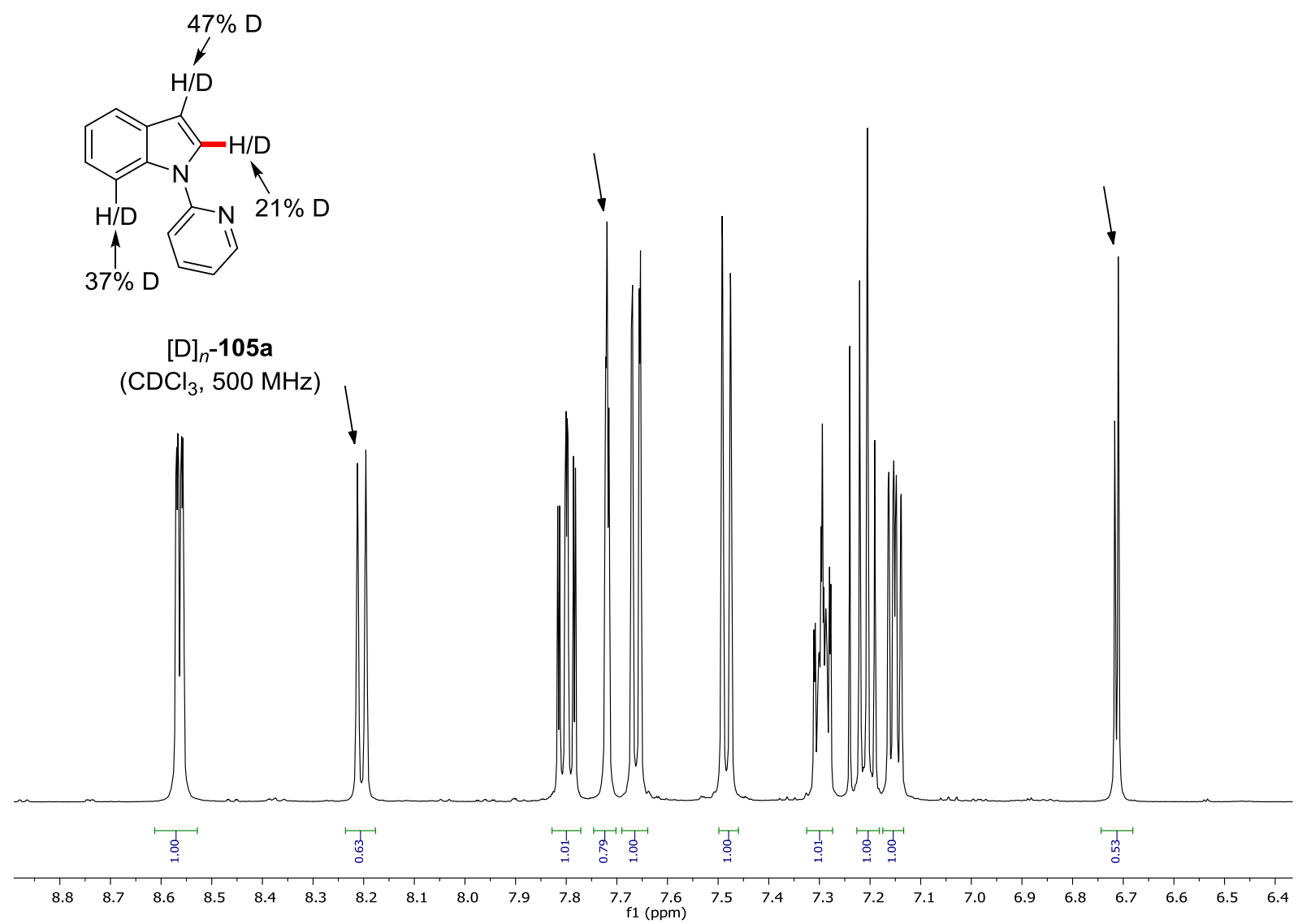


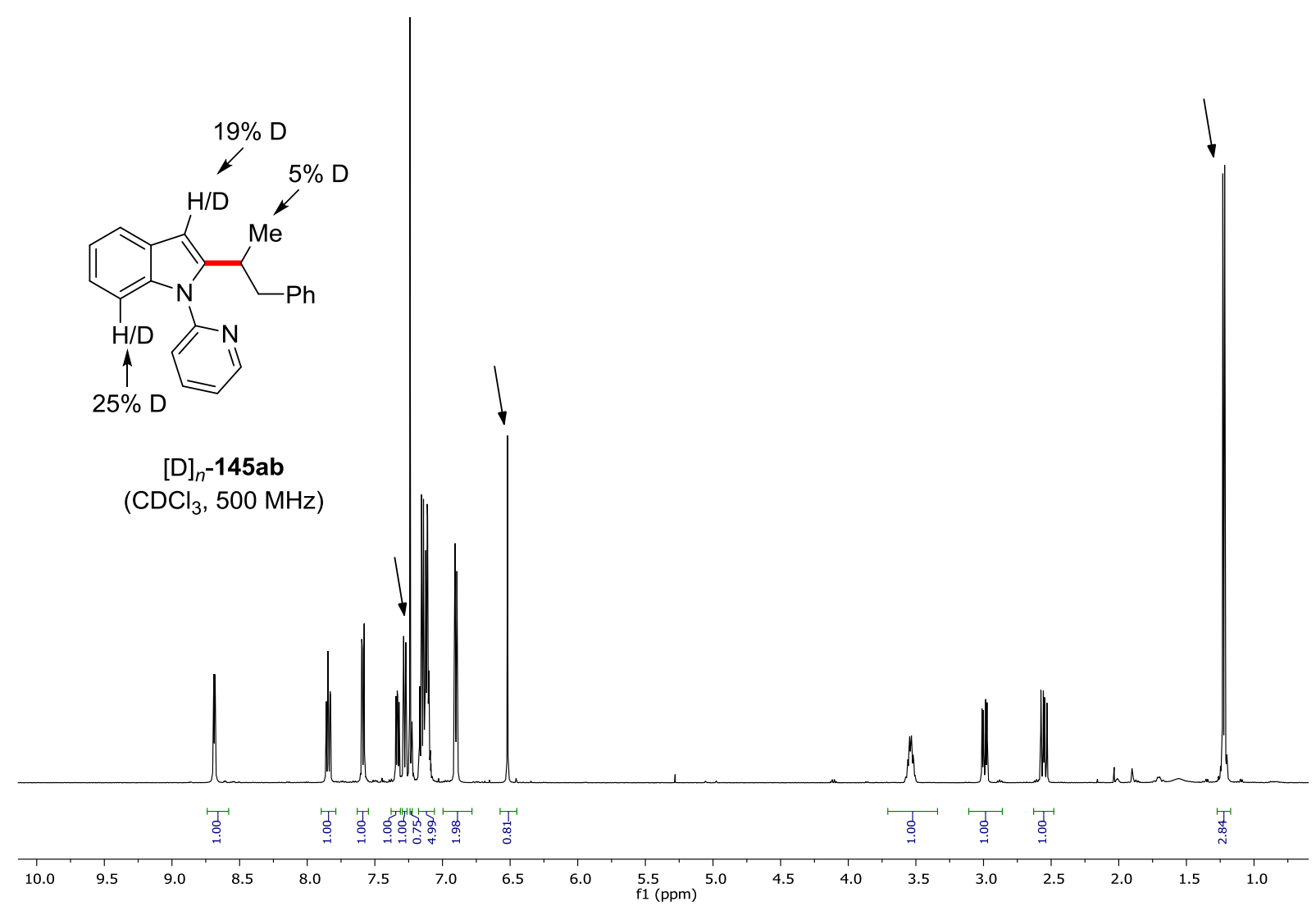

\subsection{H/D-exchange with alkene under the linear-selective regime}
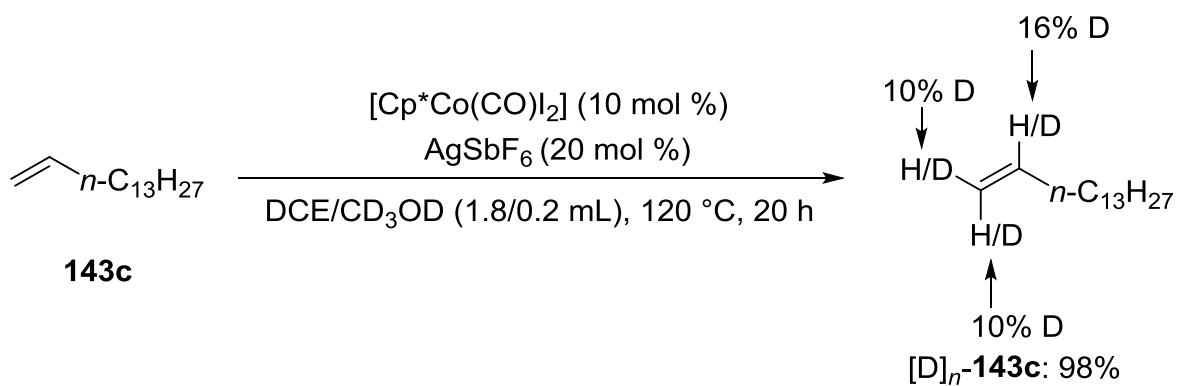

A suspension of 1-pentadecene (143c) $\left(105 \mathrm{mg}, 135 \mu \mathrm{L}, 1.00 \mathrm{mmol}, 1.00\right.$ equiv), [Cp*Co(CO) $\left.\mathrm{I}_{2}\right]$ $(23.8 \mathrm{mg}, 10.0 \mathrm{~mol} \%)$ and $\mathrm{AgSbF}_{6}(34.4 \mathrm{mg}, 20.0 \mathrm{~mol} \%)$ in DCE $(1.8 \mathrm{~mL})$ and $\mathrm{CD}_{3} \mathrm{OD}(0.2 \mathrm{~mL})$ was stirred at $120^{\circ} \mathrm{C}$ for $20 \mathrm{~h}$. After removal of the solvents, the crude mixture was purified by column chromatography on silica gel ( $n$-hexane/EtOAc: $50: 1)$ to yield $[\mathrm{D}]_{n}$-143c $(103 \mathrm{mg}, 98 \%$ reis.) as a colorless oil. 


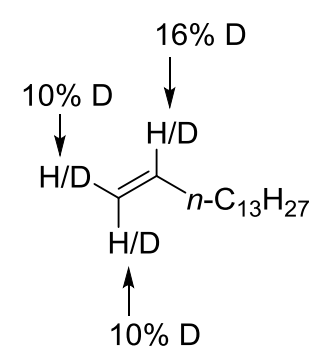

$[\mathrm{D}]_{n}-143 \mathrm{c}$

$\left(\mathrm{CDCl}_{3}, 300 \mathrm{MHz}\right)$

\subsection{H/D-exchange with alkene under the branched-selective regime}
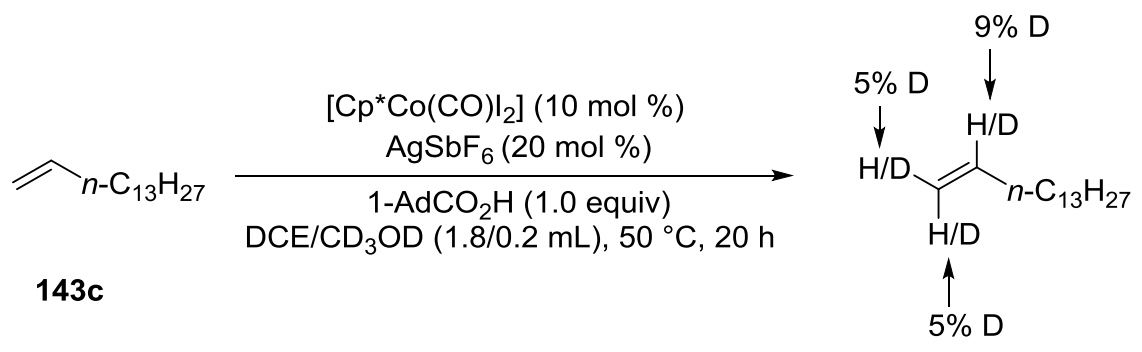

A suspension of 1-pentadecene (143c) $\left(105 \mathrm{mg}, 135 \mu \mathrm{L}, 1.00 \mathrm{mmol}, 1.00\right.$ equiv), [Cp*Co(CO) $\left.\mathrm{I}_{2}\right]$ $(23.8 \mathrm{mg}, 10.0 \mathrm{~mol} \%), \mathrm{AgSbF}_{6}(34.4 \mathrm{mg}, 20.0 \mathrm{~mol} \%)$ and $1-\mathrm{AdCO}_{2} \mathrm{H}(90.1 \mathrm{mg}, 0.50 \mathrm{mmol}$, 1.00 equiv) in $\mathrm{DCE}(1.8 \mathrm{~mL})$ and $\mathrm{CD}_{3} \mathrm{OD}(0.2 \mathrm{~mL})$ was stirred at $50{ }^{\circ} \mathrm{C}$ for $20 \mathrm{~h}$. After removal of the solvents, the crude mixture was purified by column chromatography on silica gel ( $n$-hexane/EtOAc: $50: 1)$ to yield $[\mathrm{D}]_{n}-\mathbf{1 4 3 c}(98.7 \mathrm{mg}, 94 \%$ reisolated) as a colorless oil. 

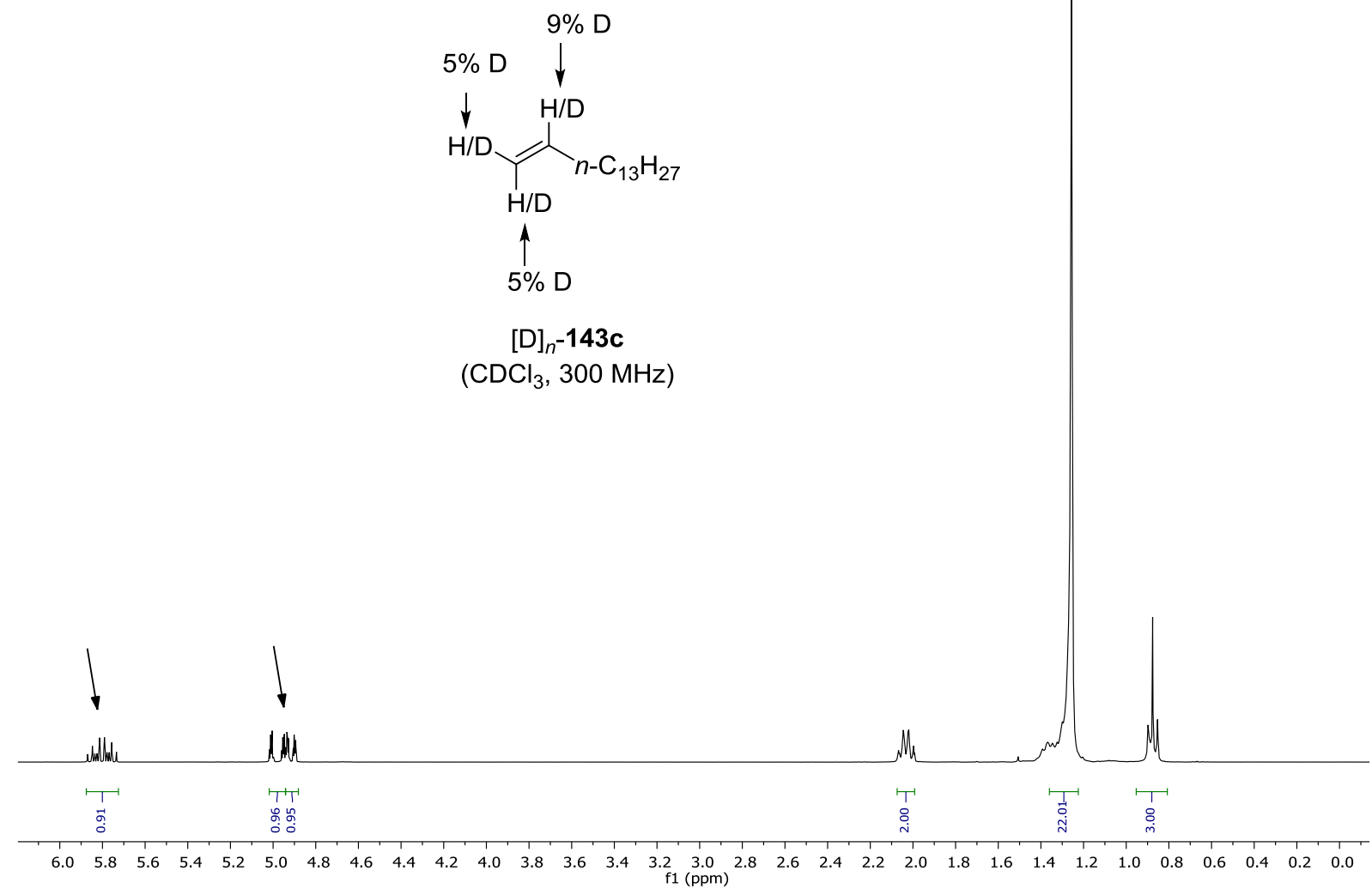

\subsection{H/D-exchange with $[D]_{1}-105 a$}

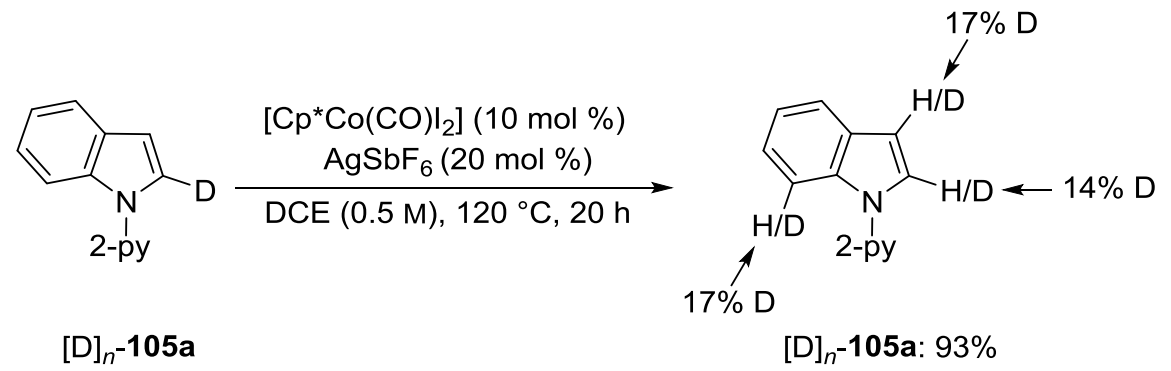

A suspension of $[\mathrm{D}]_{1}-\mathbf{1 0 5 a}$ (97.6 mg, $0.50 \mathrm{mmol}, 1.00$ equiv), [Cp*Co(CO) $\left.\mathrm{I}_{2}\right](23.8 \mathrm{mg}, 10.0 \mathrm{~mol} \%)$, $\mathrm{AgSbF}_{6}(34.4 \mathrm{mg}, 20.0 \mathrm{~mol} \%)$ in DCE $(1.0 \mathrm{~mL})$ was stirred at $120{ }^{\circ} \mathrm{C}$ for $20 \mathrm{~h}$. After removal of the solvents, the crude mixture was purified by column chromatography on silica gel ( $n$-hexane/EtOAc: 20:1) to yield $[\mathrm{D}]_{n}-\mathbf{1 0 5 a}(90.2 \mathrm{mg}, 93 \%$ reisolated) as a colorless oil. 


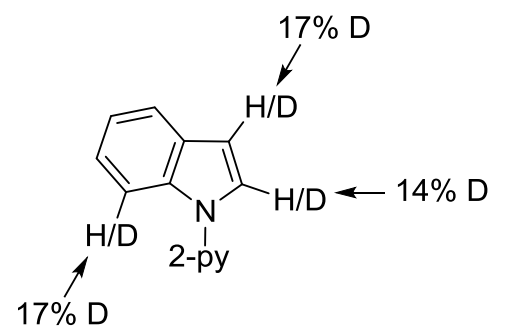

[D] $]^{-105 a}$

$\left(\mathrm{CDCl}_{3}, 500 \mathrm{MHz}\right)$

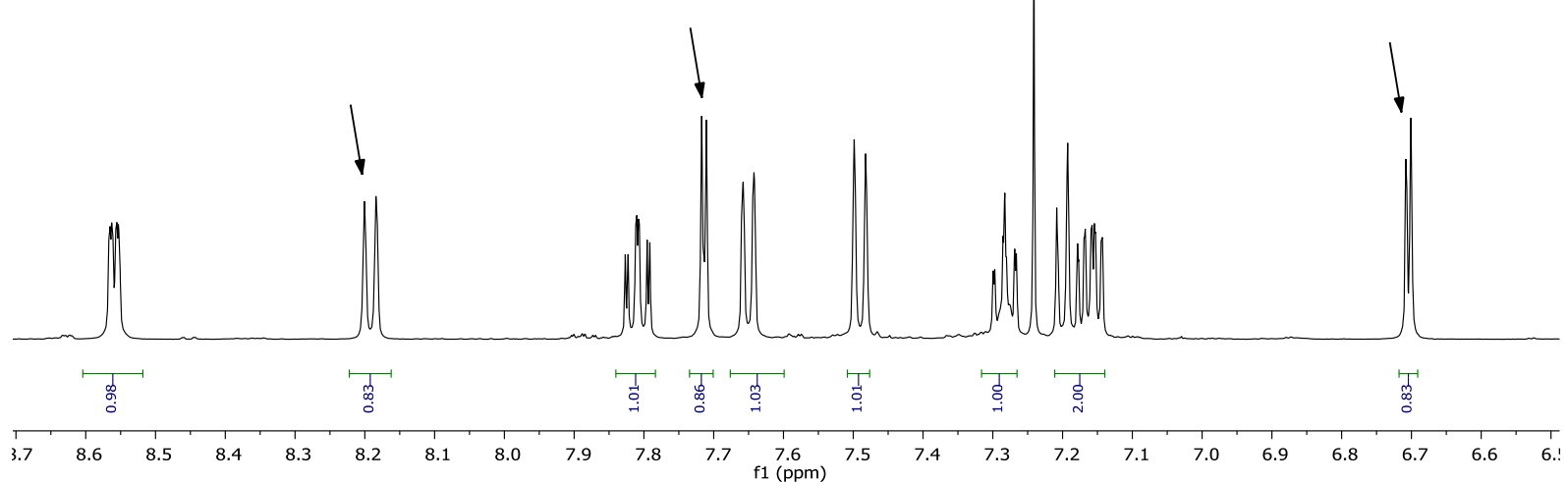

\subsection{H/D-exchange with $[D]_{1}-105 a$ under the reaction conditions}

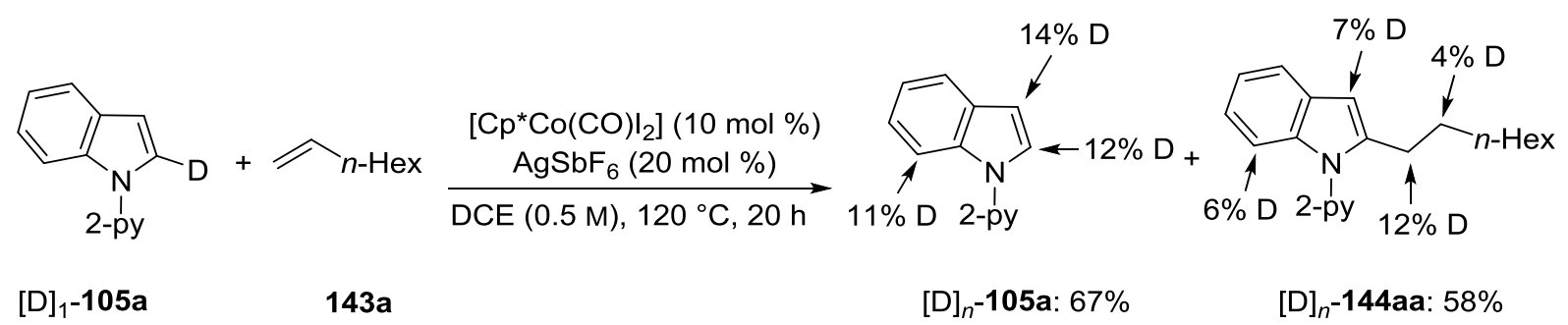

As suspension of $[\mathrm{D}]_{1}-\mathbf{1 0 5 a}$ (146 mg, $0.75 \mathrm{mmol}, 1.50$ equiv), 143a (56.0 mg, $0.50 \mathrm{mmol}, 1.00$ equiv), $\left[\mathrm{Cp} * \mathrm{Co}(\mathrm{CO}) \mathrm{I}_{2}\right](23.8 \mathrm{mg}, 10.0 \mathrm{~mol} \%)$ and $\mathrm{AgSbF}_{6}(34.4 \mathrm{mg}, 20.0 \mathrm{~mol} \%)$ in DCE $(1.0 \mathrm{~mL})$ was stirred at $120{ }^{\circ} \mathrm{C}$ for $20 \mathrm{~h}$. After removal of the solvents, the crude mixture was purified by column chromatography on silica gel ( $n$-hexane/EtOAc: $20: 1)$ to yield $[\mathrm{D}]_{n}{ }^{-144 a a}(88.7 \mathrm{mg}, 58 \%)$ and $[\mathrm{D}]_{n^{-}}$ 105a (97.8 mg, 67\% reisolated) as yellow oils. 


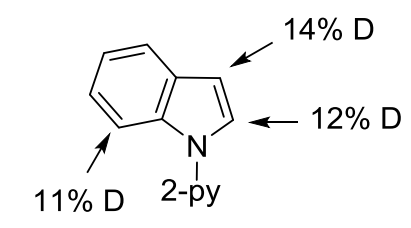

$[\mathrm{D}]_{n}-105 \mathbf{a}$

$\left(\mathrm{CDCl}_{3}, 400 \mathrm{MHz}\right)$
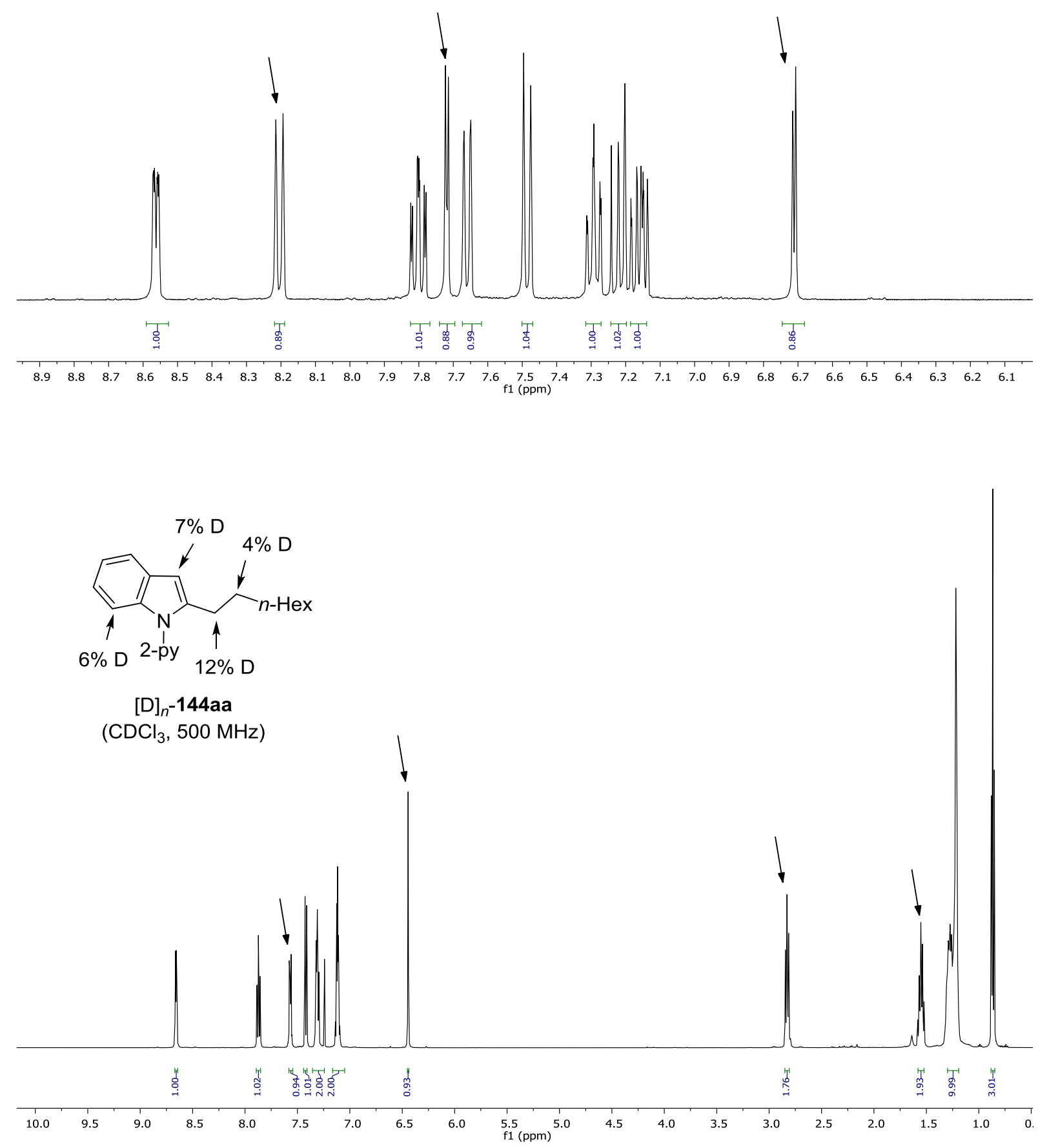


\subsection{H/D-exchange with linear product 144ab}

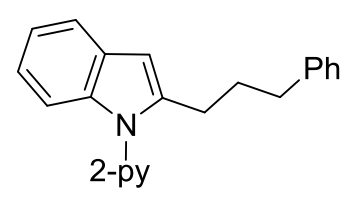

$144 a b$

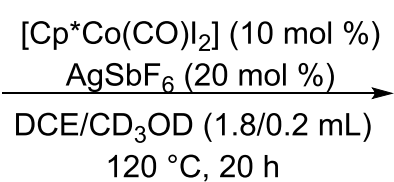

$120^{\circ} \mathrm{C}, 20 \mathrm{~h}$

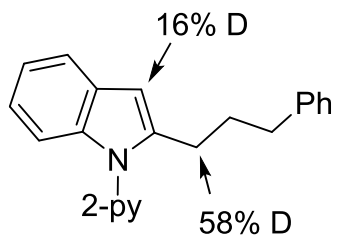

$[D]_{n}-144 a b: 69 \%$

A suspension of 144ab (156 mg, $0.50 \mathrm{mmol}, 1.00$ equiv), [Cp*Co(CO) $\left.\mathrm{I}_{2}\right](23.8 \mathrm{mg}, 10.0 \mathrm{~mol} \%)$, $\mathrm{AgSbF}_{6}(34.4 \mathrm{mg}, 20.0 \mathrm{~mol} \%)$ in DCE $(1.8 \mathrm{~mL})$ and $\mathrm{CD}_{3} \mathrm{OD}(0.2 \mathrm{~mL})$ was stirred at $120{ }^{\circ} \mathrm{C}$ for $20 \mathrm{~h}$. After removal of the solvents, the crude mixture was purified by column chromatography on silica gel ( $n$-hexane/EtOAc: $20: 1)$ to yield [D $]_{n}$-144ab (108 mg, 69\% reisolated) as a colorless oil.

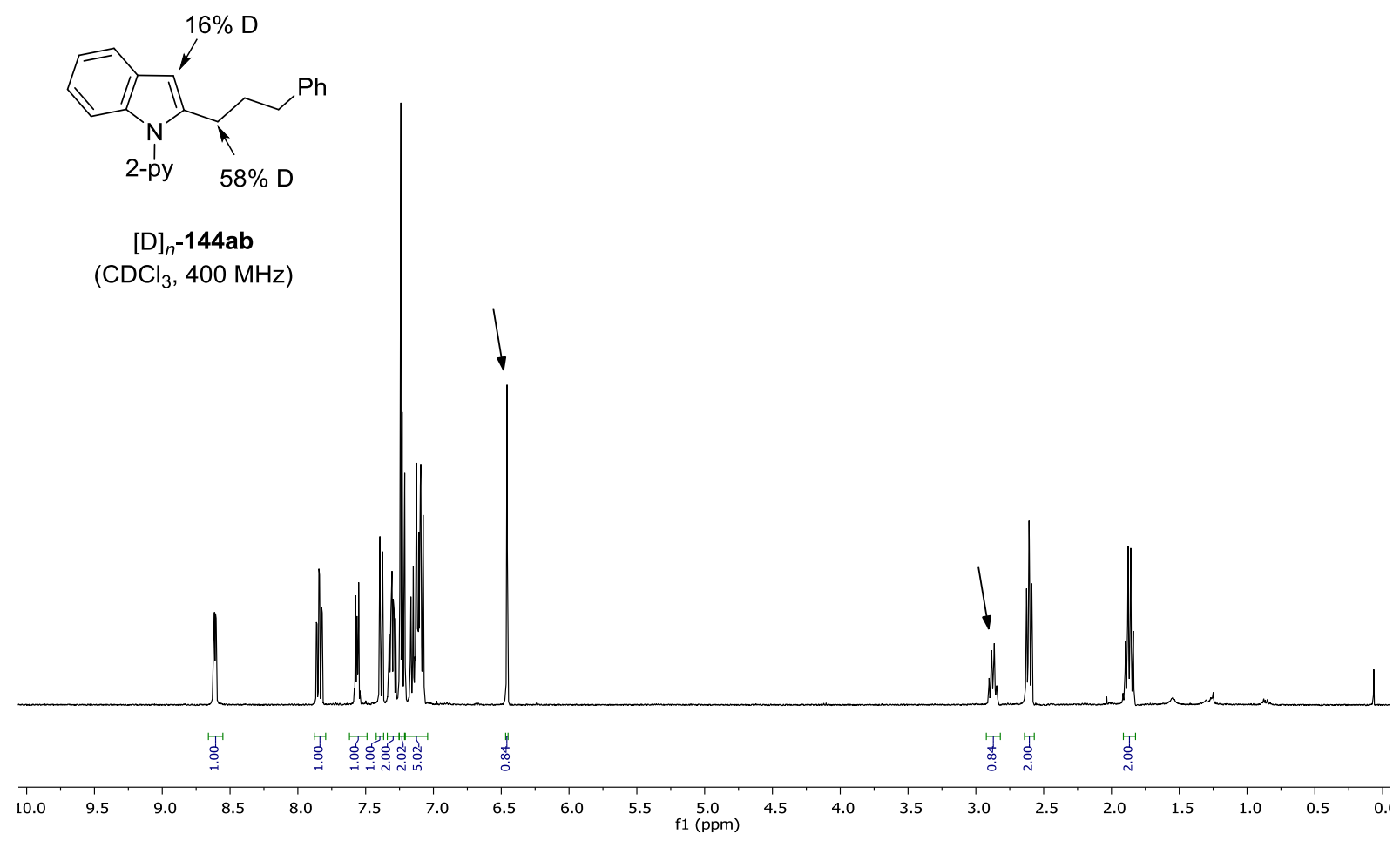




\subsection{H/D-exchange with branched product 145ab}

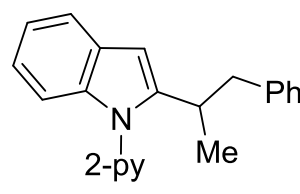

$145 \mathrm{ab}$

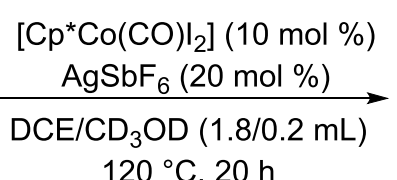

$120^{\circ} \mathrm{C}, 20 \mathrm{~h}$

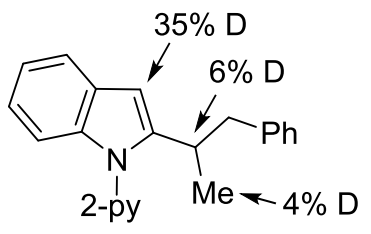

$[\mathrm{D}]_{n}-145 \mathrm{ab}: 76 \%$

A suspension of 145ab (156 mg, $0.50 \mathrm{mmol}, 1.00$ equiv), [Cp*Co(CO) $\left.\mathrm{I}_{2}\right](23.8 \mathrm{mg}, 10.0 \mathrm{~mol} \%)$, $\mathrm{AgSbF}_{6}(34.4 \mathrm{mg}, 20.0 \mathrm{~mol} \%)$ in DCE $(1.8 \mathrm{~mL})$ and $\mathrm{CD}_{3} \mathrm{OD}(0.2 \mathrm{~mL})$ was stirred at $120{ }^{\circ} \mathrm{C}$ for $20 \mathrm{~h}$. After removal of the solvents, the crude mixture was purified by column chromatography on silica gel ( $n$-hexane/EtOAc: $20: 1)$ to yield $[\mathrm{D}]_{n}-\mathbf{1 4 5 a b}(119 \mathrm{mg}, 76 \%$ reisolated) as a colorless oil.
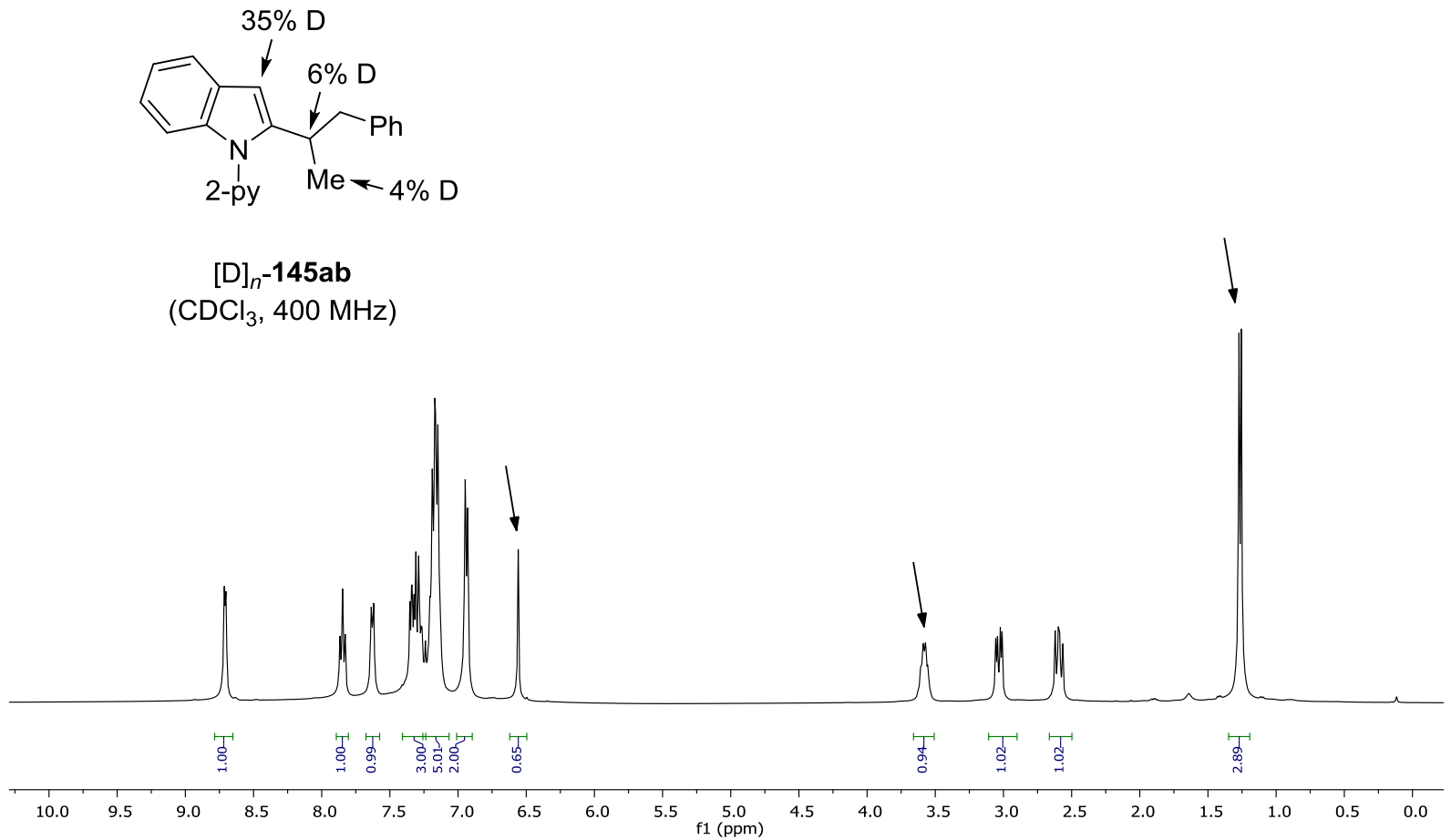


\subsubsection{Intermolecular Competition Experiment}
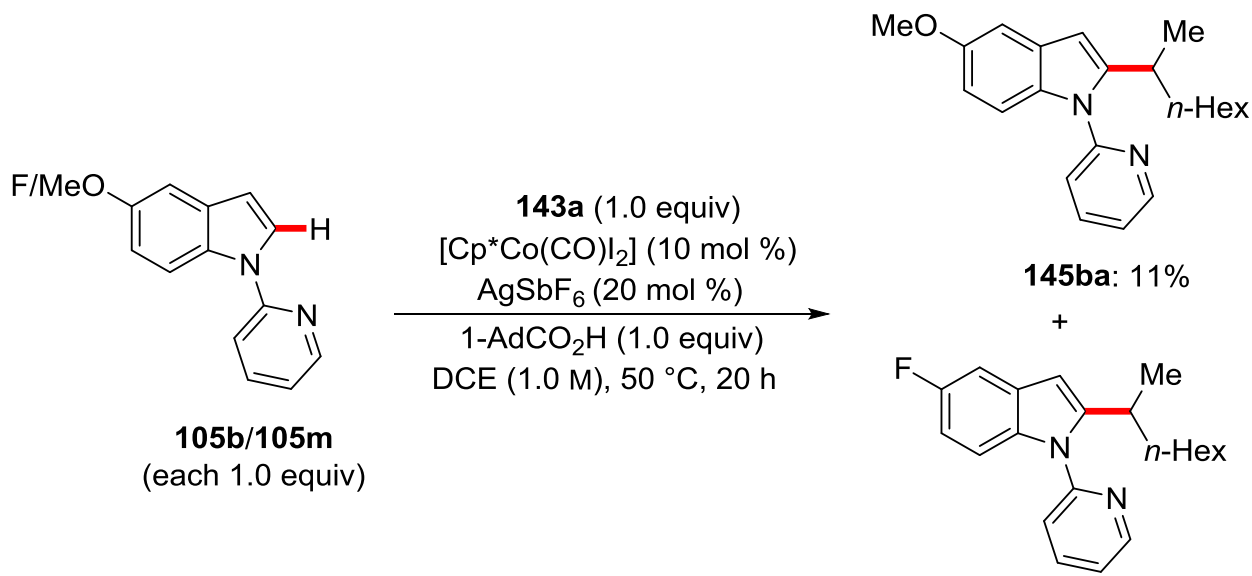

145ma: $7 \%$

A suspension of 5-methoxy-1-(pyridin-2-yl)- $1 H$-indole (105b) (112 mg, $0.50 \mathrm{mmol}, 1.00$ equiv), 5fluoro-1-(pyridin-2-yl)-1H-indole (105m) (106 mg, $0.50 \mathrm{mmol}, 1.00$ equiv), $n$-octene (143a) $(66.1 \mathrm{mg}$, $0.50 \mathrm{mmol}, 1.00$ equiv), [Cp*Co(CO) $\left.\mathrm{I}_{2}\right](23.8 \mathrm{mg}, 10.0 \mathrm{~mol} \%), \mathrm{AgSbF}_{6}(34.4 \mathrm{mg}, 20.0 \mathrm{~mol} \%)$ and 1$\mathrm{AdCO}_{2} \mathrm{H}\left(90.1 \mathrm{mg}, 0.50 \mathrm{mmol}, 1.00\right.$ equiv) in DCE $(0.50 \mathrm{~mL}, 1.00 \mathrm{M})$ was stirred at $50{ }^{\circ} \mathrm{C}$ for $20 \mathrm{~h}$. At ambient temperature, the solvent was removed in vacuo and the remaining residue was purified by column chromatography ( $n$-hexane/EtOAc: $30 / 1)$ to afford the products $145 \mathrm{ba}(18.5 \mathrm{mg}, 11 \%)$ and 145ma (11.3 mg, 7\%).

\subsubsection{Attempted Isomerization Experiments}

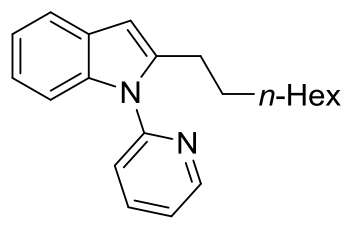

144aa: $A M: M>99: 1$

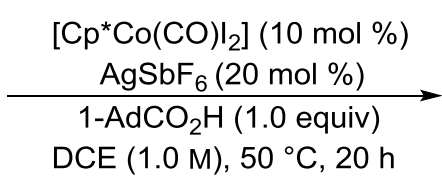

DCE (1.0 M), $50{ }^{\circ} \mathrm{C}, 20 \mathrm{~h}$

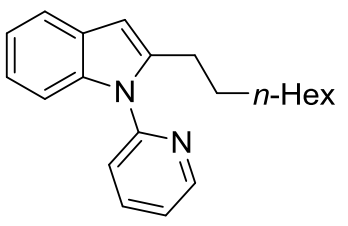

144aa: $95 \%, A M: M>99: 1$

A suspension of 144aa (153 mg, $0.50 \mathrm{mmol}, 1.00$ equiv, AM:M > 99:1), [Cp*Co(CO) $\left.\mathrm{I}_{2}\right](23.8 \mathrm{mg}$, $10.0 \mathrm{~mol} \%), \mathrm{AgSbF}_{6}(34.4 \mathrm{mg}, 20.0 \mathrm{~mol} \%)$ and $1-\mathrm{AdCO}_{2} \mathrm{H}(90.1 \mathrm{mg}, 0.50 \mathrm{mmol}, 1.00$ equiv) in DCE $(0.5 \mathrm{~mL}, 1.0 \mathrm{M})$ was stirred at $50{ }^{\circ} \mathrm{C}$ for $20 \mathrm{~h}$. At ambient temperature, the solvent was removed and the crude mixture was purified by flash column chromatography on silica gel ( $n$-hexane/EtOAc: 10/1) to yield 144aa (145 mg, 95\%, AM:M > 99:1) as a yellow oil. 
<smiles>CC(O)c1cc2ccccc2n1-c1ccccn1</smiles>

145aa: $A M: M=5: 95$

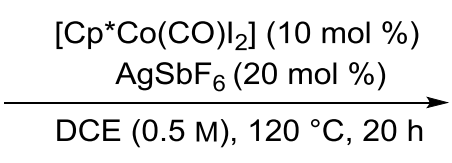

145aa: $94 \%, A M: M=5: 95$

A suspension of $\mathbf{1 4 5 a a}(153 \mathrm{mg}, 0.50 \mathrm{mmol}, 1.00$ equiv, $\mathrm{AM}: \mathrm{M}=5: 95),\left[\mathrm{Cp} * \mathrm{Co}(\mathrm{CO}) \mathrm{I}_{2}\right](23.8 \mathrm{mg}$, $10.0 \mathrm{~mol} \%)$ and $\mathrm{AgSbF}_{6}(34.4 \mathrm{mg}, 20.0 \mathrm{~mol} \%)$ in DCE $(1.0 \mathrm{~mL}, 0.5 \mathrm{M})$ was stirred at $120{ }^{\circ} \mathrm{C}$ for $20 \mathrm{~h}$. At ambient temperature, the solvent was removed and the crude mixture was purified by flash column chromatography on silica gel ( $n$-hexane/EtOAc: 10/1), yielding 145aa (144 mg, 94\%, $\mathrm{AM}: \mathrm{M}=5: 95)$ as a yellow oil.

\subsubsection{Kinetic Studies for the anti-Markovnikov-selective reaction}

\subsection{Determination of the reaction order with respect to the concentration of indole $105 \mathrm{a}$}

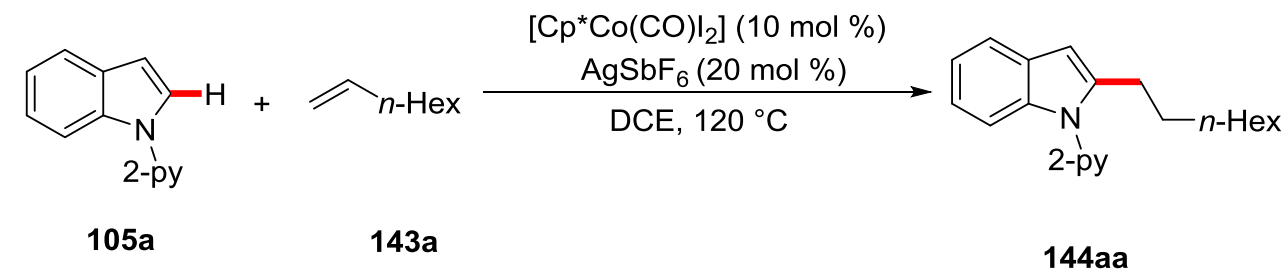

The reaction order was examined using the initial rate method. A suspension of 105a $(0.250,0.400$, $0.500,0.600$ and $0.750 \mathrm{mmol}), \mathbf{1 4 3 a}(168 \mathrm{mg}, 1.50 \mathrm{mmol}),\left[\mathrm{Cp}^{*} \mathrm{Co}(\mathrm{CO}) \mathrm{I}_{2}\right](23.8 \mathrm{mg}, 50.0 \mu \mathrm{mol})$ and $\operatorname{AgSbF}_{6}(34.4 \mathrm{mg}, 100 \mu \mathrm{mol})$ was stirred at $120^{\circ} \mathrm{C}$ in DCE $(2.0 \mathrm{~mL})$. Aliquots up to ca. $10-20 \%$ conversion $(25 \mu \mathrm{L} ; 5,10,15,20,25 \mathrm{~min})$ were periodically removed by a syringe and directly analyzed by GC using $n$-dodecane $(30 \mu \mathrm{L})$ as the internal standard.

Table 34. Reaction order in [105a] for the linear-selective reaction.

\begin{tabular}{|c|c|c|c|c|}
\hline Entry & $c / \mathbf{m o l ~ L}^{-1}$ & $k / \mathrm{mol} \mathrm{L}^{-1} \mathrm{~s}^{-1}$ & $\log \left(c / \mathrm{mol} \mathrm{L}^{-1}\right)$ & $\log \left(k / \mathrm{mol} \mathrm{L}^{-1} \mathrm{~s}^{-1}\right)$ \\
\hline 1 & 0.125 & $7.224 \mathrm{E}-6$ & -0.9031 & -5.141 \\
\hline 2 & 0.200 & $1.154 \mathrm{E}-5$ & -0.6990 & -4.938 \\
\hline 3 & 0.250 & $1.851 \mathrm{E}-5$ & -0.6021 & -4.733 \\
\hline 4 & 0.300 & $2.200 \mathrm{E}-5$ & -0.5229 & -4.658 \\
\hline 5 & 0.375 & $2.643 \mathrm{E}-5$ & -0.4260 & -4.578 \\
\hline
\end{tabular}




\subsection{Determination of the reaction order with respect to the concentration of 1-octene} (143a)

The reaction order was examined using the initial rate method. A suspension of $\mathbf{1 0 5 a}(97.1 \mathrm{mg}$, $0.50 \mathrm{mmol}), 143 \mathrm{a}(0.25,0.38,0.50,0.75,1.00,1.50,2.00 \mathrm{mmol}),\left[\mathrm{Cp}{ }^{*} \mathrm{Co}(\mathrm{CO}) \mathrm{I}_{2}\right](23.8 \mathrm{mg}, 50.0 \mu \mathrm{mol})$ and $\mathrm{AgSbF}_{6}(34.4 \mathrm{mg}, 100 \mu \mathrm{mol})$ was stirred at $120^{\circ} \mathrm{C}$ in DCE $(2.0 \mathrm{~mL})$. Aliquots up to ca. $10-20 \%$ conversion $(25 \mu \mathrm{L} ; 5,10,15,20,25 \mathrm{~min})$ were periodically removed by a syringe and directly analyzed by $\mathrm{GC}$ using $n$-dodecane $(30 \mu \mathrm{L})$ as the internal standard.

Table 35. Reaction order in [143a] for the linear-selective reaction.

\begin{tabular}{ccccc}
\hline Entry & $\boldsymbol{c} / \mathbf{~ m o l ~ L ~ L ~}^{-\mathbf{1}}$ & $\boldsymbol{k} / \mathbf{~ m o l ~ L}^{-\mathbf{1}} \mathbf{s}^{-\mathbf{1}}$ & $\log \left(\boldsymbol{c} / \mathbf{~ m o l ~ L}^{-\mathbf{1}}\right)$ & $\log \left(\boldsymbol{k} / \mathbf{~ m o l ~ L}^{-\mathbf{1}} \mathbf{s}^{-\mathbf{1}}\right)$ \\
\hline 1 & 0.125 & $1.107 \mathrm{E}-5$ & -0.9031 & -4.956 \\
2 & 0.188 & $1.206 \mathrm{E}-5$ & -0.7270 & -4.919 \\
3 & 0.250 & $1.414 \mathrm{E}-5$ & -0.6021 & -4.850 \\
4 & 0.375 & $1.491 \mathrm{E}-5$ & -0.4260 & -4.826 \\
5 & 0.500 & $1.721 \mathrm{E}-5$ & -0.3010 & -4.764 \\
6 & 0.750 & $1.849 \mathrm{E}-5$ & -0.1249 & -4.733 \\
7 & 1.000 & $2.056 \mathrm{E}-5$ & 0 & -4.687 \\
\hline
\end{tabular}

\subsection{Determination of the reaction order with respect to the concentration of $\left[\mathrm{Cp} * \mathrm{Co}(\mathrm{CO}) \mathrm{I}_{2}\right]$}

The reaction order was examined using the initial rate method. A suspension of $\mathbf{1 0 5 a}(97.1 \mathrm{mg}$, $0.50 \mathrm{mmol}), 143 \mathrm{a}(112 \mathrm{mg}, 1.50 \mathrm{mmol}),\left[\mathrm{Cp}^{*} \mathrm{Co}(\mathrm{CO}) \mathrm{I}_{2}\right](3.50,5.00,7.50,10.0,11.5$ and $12.5 \mathrm{~mol} \%)$, $\mathrm{AgSbF}_{6}(34.4 \mathrm{mg}, 20 \mathrm{~mol} \%)$ was stirred at $120{ }^{\circ} \mathrm{C}$ in DCE $(2.0 \mathrm{~mL})$. Aliquots up to ca. $10-20 \%$ conversion $(25 \mu \mathrm{L} ; 5,10,15,20,25 \mathrm{~min})$ were periodically removed by a syringe and directly analyzed by $\mathrm{GC}$ using $n$-dodecane $(30 \mu \mathrm{L})$ as internal standard.

Table 36. Reaction order in $\left[\mathrm{Cp} * \mathrm{Co}(\mathrm{CO}) \mathrm{I}_{2}\right]$ for the linear-selective reaction.

\begin{tabular}{ccccc}
\hline Entry & $\boldsymbol{n} / \mathbf{m o l} \%$ & $\boldsymbol{k} / \mathbf{~ m o l ~ L}^{-1} \mathbf{s}^{-1}$ & $\log (\boldsymbol{n} / \mathbf{m o l} \%)$ & $\log \left(\boldsymbol{k} / \mathbf{~ m o l ~ L}^{-1} \mathbf{s}^{-1}\right)$ \\
\hline 1 & 3.50 & $6.250 \mathrm{E}-6$ & 0.544 & -5.204 \\
2 & 5.00 & $1.100 \mathrm{E}-5$ & 0.699 & -5.018 \\
3 & 7.50 & $1.338 \mathrm{E}-5$ & 0.875 & -4.873 \\
4 & 10.0 & $1.849 \mathrm{E}-5$ & 1.000 & -4.733 \\
5 & 11.5 & $2.451 \mathrm{E}-5$ & 1.061 & -4.678
\end{tabular}




\begin{tabular}{lllll}
6 & 12.5 & $3.055 \mathrm{E}-5$ & 1.097 & -4.580 \\
\hline
\end{tabular}

\subsubsection{Kinetic Studies for the Markovnikov-selective reaction}

\subsection{Determination of the reaction order with respect to the concentration of indole $105 \mathrm{a}$}

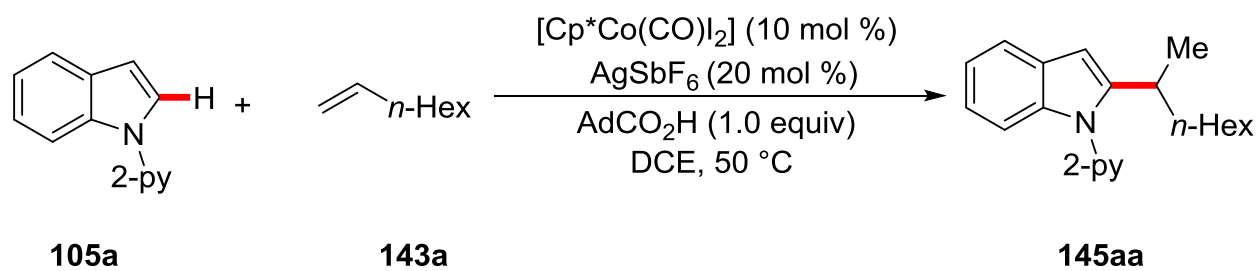

The reaction order was examined using initial rate method. A suspension of 105a $(0.150,0.250,0.400$, 0.500 and $0.750 \mathrm{mmol}), \mathbf{1 4 3 a}(168 \mathrm{mg}, 1.50 \mathrm{mmol}),\left[\mathrm{Cp}^{*} \mathrm{Co}(\mathrm{CO}) \mathrm{I}_{2}\right](23.8 \mathrm{mg}, 50.0 \mu \mathrm{mol}), \mathrm{AgSbF}_{6}$ $(34.4 \mathrm{mg}, 100 \mu \mathrm{mol})$ and $1-\mathrm{AdCO}_{2} \mathrm{H}(90.1 \mathrm{mg}, 0.50 \mathrm{mmol})$ was stirred at $50{ }^{\circ} \mathrm{C}$ in DCE $(2.0 \mathrm{~mL})$. Aliquots up to ca. $10 \%$ conversion $(25 \mu \mathrm{L} ; 15,30,40,50,60 \mathrm{~min})$ were periodically removed by a syringe and directly analyzed by GC using $n$-dodecane ( $30 \mu \mathrm{L})$ as the internal standard.

Table 37. Reaction order in [105a] for the branched-selective reaction.

\begin{tabular}{ccccc}
\hline Entry & $\boldsymbol{c} / \mathbf{~ m o l ~ L ~}^{-\mathbf{1}}$ & $\boldsymbol{k} / \mathbf{~ m o l ~ L}^{-\mathbf{1}} \mathbf{s}^{-\mathbf{1}}$ & $\boldsymbol{l o g}\left(\boldsymbol{c} / \mathbf{~ m o l ~ L}^{-\mathbf{1}}\right)$ & $\log \left(\boldsymbol{k} / \mathbf{~ m o l ~ L}^{-\mathbf{1}} \mathbf{s}^{-\mathbf{1}}\right)$ \\
\hline 1 & 0.075 & $4.332 \mathrm{E}-6$ & -1.125 & -5.338 \\
2 & 0.125 & $4.797 \mathrm{E}-6$ & -0.903 & -5.319 \\
3 & 0.200 & $4.288 \mathrm{E}-6$ & -0.699 & -5.368 \\
4 & 0.250 & $5.473 \mathrm{E}-6$ & -0.602 & -5.262 \\
5 & 0.375 & $4.587 \mathrm{E}-6$ & -0.426 & -5.363 \\
\hline
\end{tabular}

\subsection{Determination of the reaction order with respect to the concentration of 1-octene} (143a)

The reaction order was examined using the initial rate method. A suspension of $\mathbf{1 0 5 a}(97.1 \mathrm{mg}$, $0.50 \mathrm{mmol}), 143 \mathrm{a}(0.50,0.75,1.00,1.25$ and $1.50 \mathrm{mmol}),\left[\mathrm{Cp}^{*} \mathrm{Co}(\mathrm{CO}) \mathrm{I}_{2}\right](23.8 \mathrm{mg}, 50.0 \mu \mathrm{mol})$, $\mathrm{AgSbF}_{6}(34.4 \mathrm{mg}, 100 \mu \mathrm{mol})$ and $1-\mathrm{AdCO}_{2} \mathrm{H}(90.1 \mathrm{mg}, 0.50 \mathrm{mmol})$ was stirred at $50{ }^{\circ} \mathrm{C}$ in DCE $(2.0 \mathrm{~mL})$. Aliquots up to ca. $10 \%$ conversion $(25 \mu \mathrm{L} ; 15,30,40,50,60 \mathrm{~min})$ were periodically removed by a syringe and directly analyzed by $\mathrm{GC}$ using $n$-dodecane $(30 \mu \mathrm{L})$ as the internal standard. 
Table 38. Reaction order in [143a] for the branched-selective reaction.

\begin{tabular}{ccccc}
\hline Entry & $\boldsymbol{n} / \mathbf{~ m o l ~ \%}$ & $\boldsymbol{k} / \mathbf{~ m o l ~ L}^{-1} \mathbf{s}^{-1}$ & $\log (\boldsymbol{n} / \mathbf{~ m o l ~ \%})$ & $\log \left(\boldsymbol{k} / \mathbf{~ m o l ~ L}^{-1} \mathbf{s}^{-\mathbf{1}}\right)$ \\
\hline 1 & 0.250 & $1.547 \mathrm{E}-6$ & -0.6021 & -5.810 \\
2 & 0.375 & $2.261 \mathrm{E}-6$ & -0.4260 & -5.646 \\
3 & 0.500 & $2.834 \mathrm{E}-6$ & -0.3010 & -5.547 \\
4 & 0.625 & $3.689 \mathrm{E}-6$ & -0.2041 & -5.433 \\
5 & 0.750 & $5.473 \mathrm{E}-6$ & -0.1249 & -5.262 \\
\hline
\end{tabular}

\subsection{Determination of the reaction order with respect to the concentration of $\left[\mathrm{Cp}^{*} \mathrm{Co}(\mathrm{CO}) \mathbf{I}_{2}\right]$}

The reaction order was examined using the initial rate method. A suspension of 105a $(97.1 \mathrm{mg}$, $0.50 \mathrm{mmol}), 143 \mathrm{a}(112 \mathrm{mg}, 1.50 \mathrm{mmol}),\left[\mathrm{Cp}^{*} \mathrm{Co}(\mathrm{CO}) \mathrm{I}_{2}\right](2.50,3.50,5.00,7.50,10.0 \mathrm{~mol} \%), \mathrm{AgSbF}_{6}$ $(34.4 \mathrm{mg}, 100 \mu \mathrm{mol})$ and $1-\mathrm{AdCO}_{2} \mathrm{H}(90.1 \mathrm{mg}, 0.50 \mathrm{mmol})$ was stirred at $50{ }^{\circ} \mathrm{C}$ in DCE $(2.0 \mathrm{~mL})$. Aliquots up to ca. $10 \%$ conversion $(25 \mu \mathrm{L} ; 15,30,40,50,60 \mathrm{~min})$ were periodically removed by a syringe and directly analyzed by GC using $n$-dodecane $(30 \mu \mathrm{L})$ as the internal standard.

Table 39. Reaction order in $\left[\mathrm{Cp} * \mathrm{Co}(\mathrm{CO}) \mathrm{I}_{2}\right]$ for the branched-selective reaction.

\begin{tabular}{ccccc}
\hline Entry & $\boldsymbol{n} / \mathbf{~ m o l ~ \%}$ & $\boldsymbol{k} / \mathbf{~ m o l ~ L}^{-\mathbf{1}} \mathbf{s}^{-\mathbf{1}}$ & $\log (\boldsymbol{n} / \mathbf{m o l ~ \%})$ & $\log \left(\boldsymbol{k} / \mathbf{~ m o l ~ L}^{-\mathbf{1}} \mathbf{s}^{-\mathbf{1}}\right)$ \\
\hline 1 & 2.50 & $1.375 \mathrm{E}-6$ & 0.3979 & -5.862 \\
2 & 3.50 & $2.082 \mathrm{E}-6$ & 0.5441 & -5.681 \\
3 & 5.00 & $2.980 \mathrm{E}-6$ & 0.6989 & -5.526 \\
4 & 7.50 & $4.725 \mathrm{E}-6$ & 0.8751 & -5.326 \\
5 & 10.0 & $5.504 \mathrm{E}-6$ & 1.0000 & -5.260
\end{tabular}

\subsubsection{Arrhenius Plot Analysis}

\subsection{Arrhenius plot of anti-Markovnikov-selective reaction}

A suspension of 105a ( $97.1 \mathrm{mg}, 0.50 \mathrm{mmol}, 1.00$ equiv), 143a (112 mg, $1.50 \mathrm{mmol}, 3.00$ equiv), [Cp $\left.{ }^{*} \mathrm{Co}(\mathrm{CO}) \mathrm{I}_{2}\right](23.8 \mathrm{mg}, 10.0 \mathrm{~mol} \%)$ and $\mathrm{AgSbF}_{6}(34.4 \mathrm{mg}, 20 \mathrm{~mol} \%)$ was stirred at different temperatures $\left(105,110,115,120,125\right.$ and $\left.130{ }^{\circ} \mathrm{C}\right)$ in DCE $(2.0 \mathrm{~mL})$. Aliquots up to ca. $10-25 \%$ conversion $\left(25 \mu \mathrm{L} ; 15,20,25,30,35 \mathrm{~min}\right.$ for $115-120{ }^{\circ} \mathrm{C} ; 20,25,30,35,40 \min$ for $105{ }^{\circ} \mathrm{C}$ and $110{ }^{\circ} \mathrm{C}$ ) were periodically removed by a syringe and directly analyzed by GC using $n$-dodecane $(30 \mu \mathrm{L})$ as internal standard. 
Table 40. Arrhenius plot analysis for the linear-selective reaction.

\begin{tabular}{|c|c|c|c|c|}
\hline Entry & $\Theta /{ }^{\circ} \mathbf{C}$ & $k / \mathrm{mol} \mathrm{L}^{-1} \mathrm{~s}^{-1}$ & $1 / T\left(K^{-1}\right)$ & $\ln \left(k / \mathrm{mol} \mathrm{L}^{-1} \mathrm{~s}^{-1}\right)$ \\
\hline 1 & 105 & $6.573 \mathrm{E}-6$ & 0.002644 & -11.93 \\
\hline 2 & 110 & $9.438 \mathrm{E}-6$ & 0.002610 & -11.57 \\
\hline 3 & 115 & $1.642 \mathrm{E}-5$ & 0.002576 & -11.02 \\
\hline 4 & 120 & $1.849 \mathrm{E}-5$ & 0.002544 & -10.90 \\
\hline 5 & 125 & $2.618 \mathrm{E}-5$ & 0.002512 & -10.55 \\
\hline 6 & 130 & $4.398 \mathrm{E}-5$ & 0.002480 & -10.03 \\
\hline
\end{tabular}

\subsection{Arrhenius plot of Markovnikov-selective reaction}

A suspension of 105a (97.1 mg, $0.50 \mathrm{mmol}, 1.00$ equiv), 143a (112 mg, $1.50 \mathrm{mmol}, 3.00$ equiv), [Cp $\left.{ }^{*} \mathrm{Co}(\mathrm{CO}) \mathrm{I}_{2}\right](23.8 \mathrm{mg}, 10.0 \mathrm{~mol} \%), \mathrm{AgSbF}_{6}(34.4 \mathrm{mg}, 20 \mathrm{~mol} \%)$ and $1-\mathrm{AdCO}_{2} \mathrm{H}(90.1 \mathrm{mg}$, $0.50 \mathrm{mmol})$ was stirred at different temperatures $\left(40,45,50,55\right.$ and $\left.60{ }^{\circ} \mathrm{C}\right)$ in DCE $(2.0 \mathrm{~mL})$. Aliquots up to ca. $10 \%$ conversion $(25 \mu \mathrm{L} ; 15,30,40,50,60 \mathrm{~min})$ were periodically removed by a syringe and directly analyzed by GC using $n$-dodecane $(30 \mu \mathrm{L})$ as the internal standard.

Table 41. Arrhenius plot analysis for the branched-selective reaction.

\begin{tabular}{ccccc}
\hline Entry & $\boldsymbol{\Theta} /{ }^{\circ} \mathbf{C}$ & $\boldsymbol{k} / \mathbf{~ m o l ~ L}^{-\mathbf{1}} \mathbf{s}^{-\mathbf{1}}$ & $\mathbf{1} \boldsymbol{T}\left(\mathbf{K}^{-\mathbf{1}}\right)$ & $\ln \left(\boldsymbol{k} / \mathbf{~ m o l ~ L}^{-\mathbf{1}} \mathbf{s}^{-\mathbf{1}}\right)$ \\
\hline 1 & 40 & $2.078 \mathrm{E}-6$ & 0.003193 & -13.08 \\
2 & 45 & $3.156 \mathrm{E}-6$ & 0.003143 & -12.67 \\
3 & 50 & $5.473 \mathrm{E}-6$ & 0.003095 & -12.12 \\
4 & 55 & $5.953 \mathrm{E}-6$ & 0.003047 & -12.03 \\
5 & 60 & $8.525 \mathrm{E}-6$ & 0.003002 & -11.67 \\
\hline
\end{tabular}




\subsection{Cobalt(III)-Catalyzed C-H/C-F Functionalization}

\subsubsection{Characterization Data}

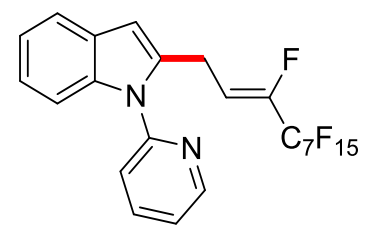

(Z)-2-(1H,1H,2H-Perfluorodec-2-en-1-yl)-1-(pyridin-2-yl)-1H-indole $\quad$ (158aa): $\quad$ The general procedure $\mathbf{F}$ was followed using 1-(pyridin-2-yl)-1H-indole (105a) $(97.1 \mathrm{mg}, 0.50 \mathrm{mmol}$ ) and $1 H, 1 H, 2 H$-perfluorodec-1-ene (127a) $(268 \mathrm{mg}, 0.60 \mathrm{mmol})$. Isolation by column chromatography ( $n$ hexane/EtOAc: 20/1) yielded 158aa (301 $\mathrm{mg}, 97 \%, Z / E=86: 14)$ as a yellow oil.

${ }^{1}$ H-NMR $\left(300 \mathrm{MHz}, \mathrm{CDCl}_{3}\right): \delta=8.64(\mathrm{ddd}, J=4.9,1.9,0.9 \mathrm{~Hz}, 1 \mathrm{H}), 7.88(\mathrm{ddd}, J=7.9,7.6,1.9 \mathrm{~Hz}$, $1 \mathrm{H}), 7.61$ (ddd, $J=7.6,4.9,1.0 \mathrm{~Hz}, 1 \mathrm{H}), 7.41$ (ddd, $J=7.9,1.0,0.9 \mathrm{~Hz}, 1 \mathrm{H}), 7.41-7.35$ (m, 1H), 7.31 (ddd, $J=7.3,4.9,0.9 \mathrm{~Hz}, 1 \mathrm{H}), 7.25-7.12(\mathrm{~m}, 2 \mathrm{H}), 6.50(\mathrm{~d}, J=0.9 \mathrm{~Hz}, 1 \mathrm{H}), 6.16(\mathrm{dt}, J=22.3,8.1 \mathrm{~Hz}$, $0.14 \mathrm{H}, E), 5.82(\mathrm{dt}, J=33.0,7.5 \mathrm{~Hz}, 0.86 \mathrm{H}, Z), 3.90$ (ddt, $J=7.5,2.4,1.8 \mathrm{~Hz}, 1.72 \mathrm{H}, Z$ ), 3.84 (ddt, $J$ $=8.1,2.5,1.8 \mathrm{~Hz}, 0.28 \mathrm{H}, E)$.

${ }^{13}$ C-NMR $\left(125 \mathrm{MHz}, \mathrm{CDCl}_{3}\right): \delta=150.8\left(\mathrm{C}_{\mathrm{q}}\right), 149.6(\mathrm{CH}), 146.1\left(\mathrm{dt},{ }^{1} J_{\mathrm{C}-\mathrm{F}}=261 \mathrm{~Hz},{ }^{2} J_{\mathrm{C}-\mathrm{F}}=29.1 \mathrm{~Hz}\right.$, $\left.\mathrm{C}_{\mathrm{q}}\right), 138.3(\mathrm{CH}), 137.1\left(\mathrm{C}_{\mathrm{q}}\right), 136.1\left(\mathrm{C}_{\mathrm{q}}\right), 128.3\left(\mathrm{C}_{\mathrm{q}}\right), 122.4(\mathrm{CH}), 122.1(\mathrm{CH}), 121.0(\mathrm{CH}), 120.5(\mathrm{CH})$, $120.0(\mathrm{CH}), 117.0\left(\mathrm{dt},{ }^{1} J_{\mathrm{C}-\mathrm{F}}=288 \mathrm{~Hz},{ }^{2} J_{\mathrm{C}-\mathrm{F}}=31.3 \mathrm{~Hz}, \mathrm{C}_{\mathrm{q}}\right), 113.3\left(\mathrm{dt},{ }^{2} J_{\mathrm{C}-\mathrm{F}}=8.1 \mathrm{~Hz},{ }^{3} J_{\mathrm{C}-\mathrm{F}}=4.2 \mathrm{~Hz}\right.$, $\mathrm{CH}), 112.4\left(\mathrm{~m}, \mathrm{C}_{\mathrm{q}}\right), 110.9\left(\mathrm{~m}, \mathrm{C}_{\mathrm{q}}\right), 110.7\left(\mathrm{~m}, \mathrm{C}_{\mathrm{q}}\right), 110.5\left(\mathrm{~m}, \mathrm{C}_{\mathrm{q}}\right), 108.6\left(\mathrm{~m}, \mathrm{C}_{\mathrm{q}}\right), 108.3\left(\mathrm{~m}, \mathrm{C}_{\mathrm{q}}\right), 110.1$ $(\mathrm{CH}), 106.7(\mathrm{CH}), 22.7\left(\mathrm{~d},{ }^{3} J_{\mathrm{C}-\mathrm{F}}=4.3 \mathrm{~Hz}, \mathrm{CH}_{2}\right)$.

${ }^{19}$ F-NMR $\left(282 \mathrm{MHz}, \mathrm{CDCl}_{3}\right): \delta=-80.9(\mathrm{~m}),-115.0(\mathrm{~m}, E),-117.5(\mathrm{~m}, Z),-122.0(\mathrm{~m}),-122.1(\mathrm{~m})$, $-122.8(\mathrm{~m}),-123.0(\mathrm{~m}),-126.3(\mathrm{~m}),-130.8(\mathrm{~m})$.

IR (ATR): 3061, 1589, 1472, 1455, 1439, 1197, 1107, $736 \mathrm{~cm}^{-1}$.

MS (ESI) $m / z$ (relative intensity): $643\left([\mathrm{M}+\mathrm{Na}]^{+}, 20\right), 621\left([\mathrm{M}+\mathrm{H}]^{+}, 100\right)$.

HR-MS (ESI): $\mathrm{m} / z$ calcd. for $\left[\mathrm{C}_{23} \mathrm{H}_{13} \mathrm{~F}_{16} \mathrm{~N}_{2}\right]^{+}[\mathrm{M}+\mathrm{H}]^{+} 621.0818$, found 621.0809 .<smiles>FC(=CCc1cc2ccccc2n1-c1ccccn1)C(F)(F)C(F)(F)C(F)(F)F</smiles>

Z)-2-(1H,1H,2H-Perfluorohex-2-en-1-yl)-1-(pyridin-2-yl)-1H-indole $\quad$ (158ab): $\quad$ The general procedure $\mathbf{F}$ was followed using 1-(pyridin-2-yl)-1H-indole (105a) $(97.1 \mathrm{mg}, 0.50 \mathrm{mmol}$ ) and $1 H, 1 H, 2 H$-perfluorohex-1-ene (127b) $(148 \mathrm{mg}, 0.60 \mathrm{mmol})$. Isolation by column chromatography ( $n$ hexane/EtOAc: 20/1) yielded 158ab (197 $\mathrm{mg}, 94 \%, Z / E=86: 14)$ as a yellow oil. 
${ }^{1} \mathbf{H}-\mathbf{N M R}\left(300 \mathrm{MHz}, \mathrm{CDCl}_{3}\right): \delta=8.64(\mathrm{ddd}, J=4.9,1.9,0.9 \mathrm{~Hz}, 1 \mathrm{H}), 7.88(\mathrm{ddd}, J=7.9,7.6,1.9 \mathrm{~Hz}$, 1H), 7.61 (ddd, $J=7.6,4.9,1.0 \mathrm{~Hz}, 1 \mathrm{H}), 7.45$ (ddd, $J=7.9,1.0,0.9 \mathrm{~Hz}, 1 \mathrm{H}), 7.43-7.35(\mathrm{~m}, 1 \mathrm{H}), 7.31$ (ddd, $J=7.1,4.7,0.9 \mathrm{~Hz}, 1 \mathrm{H}), 7.25-7.14(\mathrm{~m}, 2 \mathrm{H}), 6.50(\mathrm{~d}, J=0.9 \mathrm{~Hz}, 1 \mathrm{H}), 6.16(\mathrm{dt}, J=22.0,7.9 \mathrm{~Hz}$, $0.14 \mathrm{H}, E), 5.82(\mathrm{dt}, J=33.0,7.4 \mathrm{~Hz}, 0.86 \mathrm{H}, Z), 3.90(\mathrm{ddt}, J=7.4,2.4,1.8 \mathrm{~Hz}, 1.72 \mathrm{H}, Z), 3.84$ (ddt, $J$ $=7.9,2.5,1.8 \mathrm{~Hz}, 0.28 \mathrm{H}, E)$.

${ }^{13}$ C-NMR $\left(125 \mathrm{MHz}, \mathrm{CDCl}_{3}\right): \delta=150.8\left(\mathrm{C}_{\mathrm{q}}\right), 149.6(\mathrm{CH}), 145.8\left(\mathrm{dt},{ }^{1} J_{\mathrm{C}-\mathrm{F}}=261 \mathrm{~Hz},{ }^{2} J_{\mathrm{C}-\mathrm{F}}=29.1 \mathrm{~Hz}\right.$, $\left.\mathrm{C}_{\mathrm{q}}\right), 138.3(\mathrm{CH}), 137.0\left(\mathrm{C}_{\mathrm{q}}\right), 136.0\left(\mathrm{C}_{\mathrm{q}}\right), 128.2\left(\mathrm{C}_{\mathrm{q}}\right), 122.4(\mathrm{CH}), 122.1(\mathrm{CH}), 121.0(\mathrm{CH}), 120.5(\mathrm{CH})$, $120.3(\mathrm{CH}), 117.5\left(\mathrm{dt},{ }^{1} J_{\mathrm{C}-\mathrm{F}}=288 \mathrm{~Hz},{ }^{2} J_{\mathrm{C}-\mathrm{F}}=31.3 \mathrm{~Hz}, \mathrm{C}_{\mathrm{q}}\right), 113.3\left(\mathrm{td},{ }^{2} J_{\mathrm{C}-\mathrm{F}}=8.1 \mathrm{~Hz},{ }^{3} J_{\mathrm{C}-\mathrm{F}}=4.2 \mathrm{~Hz}\right.$, $\mathrm{CH}), 110.1(\mathrm{CH}), 109.9\left(\mathrm{~m}, \mathrm{C}_{\mathrm{q}}\right), 108.3\left(\mathrm{~m}, \mathrm{C}_{\mathrm{q}}\right), 103.6(\mathrm{CH}), 22.7\left(\mathrm{~d},{ }^{3} J_{\mathrm{C}-\mathrm{F}}=4.3 \mathrm{~Hz}, \mathrm{CH}_{2}\right)$.

${ }^{19}$ F-NMR $\left(282 \mathrm{MHz}, \mathrm{CDCl}_{3}\right): \delta=-80.8(\mathrm{~m}),-115.9(\mathrm{~m}, E),-118.4(\mathrm{~m}, Z),-127.3(\mathrm{~m}, Z),-127.9(\mathrm{~m}$, E), $-131.0(\mathrm{~m})$.

IR (ATR): 3059, 1587, 1119, 782, $737 \mathrm{~cm}^{-1}$.

MS (ESI) $m / z$ (relative intensity): $421\left([\mathrm{M}+\mathrm{H}]^{+}, 100\right), 197$ (20).

HR-MS (ESI): $m / z$ calcd. for $\left[\mathrm{C}_{19} \mathrm{H}_{13} \mathrm{~F}_{8} \mathrm{~N}_{2}\right]^{+}[\mathrm{M}+\mathrm{H}]^{+} 421.0946$, found 421.0949 .

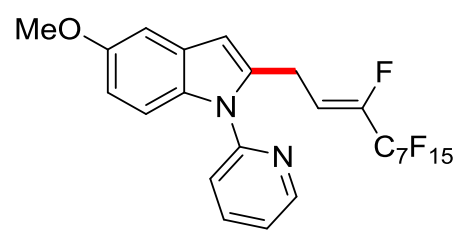

(Z)-2-(1H,1H,2H-Perfluorodec-2-en-1-yl)-5-methoxy-1-(pyridin-2-yl)-1H-indole $\quad(158 \mathrm{ba}): \quad$ The general procedure $\mathbf{F}$ was followed using 5-methoxy-1-(pyridin-2-yl)-1 $H$-indole (105b) (112 mg, $0.50 \mathrm{mmol})$ and $1 \mathrm{H}, 1 \mathrm{H}, 2 \mathrm{H}$-perfluorodec-1-ene (127a) $(268 \mathrm{mg}, 0.60 \mathrm{mmol})$. Isolation by column chromatography ( $n$-hexane/EtOAc: 20/1) yielded 158ba (293 mg, 90\%, Z/E = 86:14) as a colorless solid.

M.p.: $98{ }^{\circ} \mathrm{C}$

${ }^{1} \mathbf{H}-\mathbf{N M R}\left(500 \mathrm{MHz}, \mathrm{CDCl}_{3}\right): \delta=8.61(\mathrm{ddd}, J=4.9,1.9,0.9 \mathrm{~Hz}, 1 \mathrm{H}), 7.87(\mathrm{ddd}, J=7.9,7.6,1.9 \mathrm{~Hz}$, $1 \mathrm{H}), 7.41(\mathrm{ddd}, J=7.9,1.0,0.9 \mathrm{~Hz}, 1 \mathrm{H}), 7.31-7.23(\mathrm{~m}, 2 \mathrm{H}), 7.05(\mathrm{~d}, J=2.5 \mathrm{~Hz}, 1 \mathrm{H}), 6.81(\mathrm{dd}, J=$ 9.0, $2.5 \mathrm{~Hz}, 1 \mathrm{H}), 6.41(\mathrm{~d}, J=0.9 \mathrm{~Hz}, 1 \mathrm{H}), 6.13(\mathrm{dt}, J=22.5,8.0 \mathrm{~Hz}, 0.14 \mathrm{H}, E), 5.76(\mathrm{dt}, J=33.0$, $7.5 \mathrm{~Hz}, 0.86 \mathrm{H}, Z$ ), 3.90 (ddt, $J=7.5,2.4,1.8 \mathrm{~Hz}, 1.72 \mathrm{H}, Z$ ), 3.84 (ddt, $J=8.0,2.5,1.8 \mathrm{~Hz}, 0.28 \mathrm{H}, E$ ), $3.84(\mathrm{~s}, 3 \mathrm{H})$.

${ }^{13}$ C-NMR $\left(125 \mathrm{MHz}, \mathrm{CDCl}_{3}\right): \delta=154.9\left(\mathrm{C}_{\mathrm{q}}\right), 150.9\left(\mathrm{C}_{\mathrm{q}}\right), 149.6(\mathrm{CH}), 145.8\left(\mathrm{dt},{ }^{1} J_{\mathrm{C}-\mathrm{F}}=261 \mathrm{~Hz},{ }^{2} J_{\mathrm{C}-\mathrm{F}}\right.$ $\left.=29.1 \mathrm{~Hz}, \mathrm{C}_{\mathrm{q}}\right), 138.1(\mathrm{CH}), 136.5\left(\mathrm{C}_{\mathrm{q}}\right), 132.1\left(\mathrm{C}_{\mathrm{q}}\right), 128.8\left(\mathrm{C}_{\mathrm{q}}\right), 121.9(\mathrm{CH}), 120.1(\mathrm{CH}), 116.8(\mathrm{dt}$, $\left.{ }^{1} J_{\mathrm{C}-\mathrm{F}}=287 \mathrm{~Hz},{ }^{2} J_{\mathrm{C}-\mathrm{F}}=31.0 \mathrm{~Hz}, \mathrm{C}_{\mathrm{q}}\right), 113.3\left(\mathrm{dt},{ }^{2} J_{\mathrm{C}-\mathrm{F}}=8.4 \mathrm{~Hz},{ }^{3} J_{\mathrm{C}-\mathrm{F}}=4.5 \mathrm{~Hz}, \mathrm{CH}\right), 112.5\left(\mathrm{~m}, \mathrm{C}_{\mathrm{q}}\right)$, $112.1(\mathrm{CH}), 111.0(\mathrm{CH}), 110.5\left(\mathrm{~m}, \mathrm{C}_{\mathrm{q}}\right), 110.2\left(\mathrm{~m}, \mathrm{C}_{\mathrm{q}}\right), 110.0\left(\mathrm{~m}, \mathrm{C}_{\mathrm{q}}\right), 108.6\left(\mathrm{~m}, \mathrm{C}_{\mathrm{q}}\right), 108.2\left(\mathrm{~m}, \mathrm{C}_{\mathrm{q}}\right)$, $103.5(\mathrm{CH}), 102.3(\mathrm{CH}), 55.8\left(\mathrm{CH}_{3}\right), 22.8\left(\mathrm{~d},{ }^{3} J_{\mathrm{C}-\mathrm{F}}=4.3 \mathrm{~Hz}, \mathrm{CH}_{2}\right)$. 
${ }^{19}$ F-NMR $\left(376 \mathrm{MHz}, \mathrm{CDCl}_{3}\right): \delta=-80.9(\mathrm{~m}),-117.5(\mathrm{~m}),-122.0(\mathrm{~m}),-122.8(\mathrm{~m}),-122.9(\mathrm{~m}),-123.4$ (m), $-126.2(\mathrm{~m}),-130.9(\mathrm{~m})$.

IR (ATR): 1474, 1450, 1438, 1237, 1201, 1146, 907, 729, $649 \mathrm{~cm}^{-1}$.

MS (ESI) $m / z$ (relative intensity): $673\left([\mathrm{M}+\mathrm{Na}]^{+}, 20\right), 651\left([\mathrm{M}+\mathrm{H}]^{+}, 100\right), 381(15)$.

HR-MS (ESI): $m / z$ calcd. for $\left[\mathrm{C}_{24} \mathrm{H}_{15} \mathrm{~F}_{16} \mathrm{~N}_{2} \mathrm{O}\right]^{+}[\mathrm{M}+\mathrm{H}]^{+}$651.0923, found 651.0917.

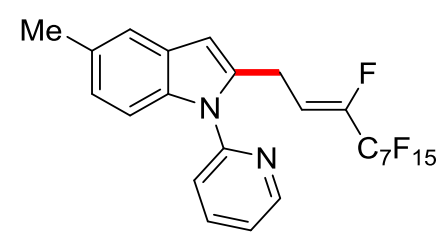

(Z)-2-(1H,1H,2H-Perfluorodec-2-en-1-yl)-5-methyl-1-(pyridin-2-yl)-1H-indole $\quad$ (158fa): $\quad$ The general procedure $\mathbf{F}$ was followed using 5-methyl-1-(pyridin-2-yl)-1H-indole (105f) (104 mg, $0.50 \mathrm{mmol})$ and $1 \mathrm{H}, 1 \mathrm{H}, 2 \mathrm{H}$-perfluorodec-1-ene (127a) $(268 \mathrm{mg}, 0.60 \mathrm{mmol})$. Isolation by column chromatography ( $n$-hexane/EtOAc: 20/1) yielded 158fa (244 mg, 77\%, Z/E= 86:14) as a colorless oil. ${ }^{1}$ H-NMR $\left(400 \mathrm{MHz}, \mathrm{CDCl}_{3}\right): \delta=8.62(\mathrm{ddd}, J=4.9,1.9,0.9 \mathrm{~Hz}, 1 \mathrm{H}), 7.84(\mathrm{ddd}, J=8.0,7.6,1.9 \mathrm{~Hz}$, $1 \mathrm{H}), 7.46(\mathrm{ddd}, J=8.0,1.0,0.9 \mathrm{~Hz}, 1 \mathrm{H}), 7.38(\mathrm{dd}, J=1.7,0.9 \mathrm{~Hz}, 1 \mathrm{H}), 7.31-7.29$ (m, 2H), 7.00 (dd, $J$ $=8.4,1.7 \mathrm{~Hz}, 1 \mathrm{H}), 6.41(\mathrm{~d}, J=0.9 \mathrm{~Hz}, 1 \mathrm{H}), 6.15(\mathrm{dt}, J=22.4,8.0 \mathrm{~Hz}, 0.14 \mathrm{H}, E), 5.80(\mathrm{dt}, J=32.8$, $7.6 \mathrm{~Hz}, 0.86 \mathrm{H}, Z$ ), 3.89 (ddt, $J=7.6,2.5,1.7 \mathrm{~Hz}, 1.72 \mathrm{H}, Z$ ), 3.83 (ddt, $J=8.0,2.4,1.7 \mathrm{~Hz}, 0.28 \mathrm{H}, E$ ), $2.45(\mathrm{~s}, 3 \mathrm{H})$.

${ }^{13}$ C-NMR $\left(100 \mathrm{MHz}, \mathrm{CDCl}_{3}\right): \delta=151.0\left(\mathrm{C}_{\mathrm{q}}\right), 149.6(\mathrm{CH}), 146.1\left(\mathrm{dt},{ }^{1} J_{\mathrm{C}-\mathrm{F}}=261 \mathrm{~Hz},{ }^{2} J_{\mathrm{C}-\mathrm{F}}=28.3 \mathrm{~Hz}\right.$, $\left.\mathrm{C}_{\mathrm{q}}\right), 138.3(\mathrm{CH}), 136.2\left(\mathrm{C}_{\mathrm{q}}\right), 135.5\left(\mathrm{C}_{\mathrm{q}}\right), 130.4\left(\mathrm{C}_{\mathrm{q}}\right), 128.6\left(\mathrm{C}_{\mathrm{q}}\right), 123.9(\mathrm{CH}), 121.9(\mathrm{CH}), 120.3(\mathrm{CH})$, $120.2(\mathrm{CH}), 117.4\left(\mathrm{dt},{ }^{1} J_{\mathrm{C}-\mathrm{F}}=289 \mathrm{~Hz},{ }^{2} J_{\mathrm{C}-\mathrm{F}}=33.9 \mathrm{~Hz}, \mathrm{C}_{\mathrm{q}}\right), 113.4\left(\mathrm{dt},{ }^{2} J_{\mathrm{C}-\mathrm{F}}=8.1 \mathrm{~Hz},{ }^{3} J_{\mathrm{C}-\mathrm{F}}=4.2 \mathrm{~Hz}\right.$, $\mathrm{CH}), 112.9\left(\mathrm{~m}, \mathrm{C}_{\mathrm{q}}\right), 110.9\left(\mathrm{~m}, \mathrm{C}_{\mathrm{q}}\right), 110.6\left(\mathrm{~m}, \mathrm{C}_{\mathrm{q}}\right), 110.2\left(\mathrm{~m}, \mathrm{C}_{\mathrm{q}}\right), 109.8(\mathrm{CH}), 108.3\left(\mathrm{~m}, \mathrm{C}_{\mathrm{q}}\right), 107.8(\mathrm{~m}$, $\left.\mathrm{C}_{\mathrm{q}}\right), 103.4(\mathrm{CH}), 22.8\left(\mathrm{~d},{ }^{3} J_{\mathrm{C}-\mathrm{F}}=4.2 \mathrm{~Hz}, \mathrm{CH}_{2}\right), 21.3\left(\mathrm{CH}_{3}\right)$.

${ }^{19}$ F-NMR $\left(282 \mathrm{MHz}, \mathrm{CDCl}_{3}\right): \delta=-80.8(\mathrm{~m}),-114.9(\mathrm{~m}, E),-117.5(\mathrm{~m}, Z),-122.0(\mathrm{~m}),-122.1(\mathrm{~m})$, $-122.8(\mathrm{~m}),-122.9(\mathrm{~m}),-126.2(\mathrm{~m}),-131.1(\mathrm{~m})$.

IR (ATR): 2921, 1599, 1473, 1439, 1237, 1201, 1146, 908, $731 \mathrm{~cm}^{-1}$.

MS (ESI) $m / z$ (relative intensity): $635\left([\mathrm{M}+\mathrm{H}]^{+}, 100\right)$.

HR-MS (ESI): $m / z$ calcd. for $\left[\mathrm{C}_{24} \mathrm{H}_{15} \mathrm{~F}_{16} \mathrm{~N}_{2}\right]^{+}[\mathrm{M}+\mathrm{H}]^{+}$635.0974, found 635.0984. 


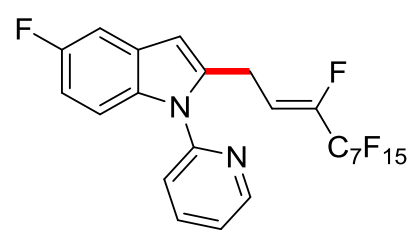

(Z)-2-(1H,1H,2H-Perfluorodec-2-en-1-yl)-5-fluoro-1-(pyridin-2-yl)-1H-indole $\quad$ (158ma): $\quad$ The general procedure $\mathbf{F}$ was followed using 5-fluoro-1-(pyridin-2-yl)-1 $H$-indole (105m) (106 mg, $0.50 \mathrm{mmol})$ and $1 H, 1 H, 2 H$-perfluorodec-1-ene (127a) $(268 \mathrm{mg}, 0.60 \mathrm{mmol})$. Isolation by column chromatography ( $n$-hexane/EtOAc: $20 / 1)$ yielded $\mathbf{1 5 8 m a}(271 \mathrm{mg}, 85 \%, Z / E=86: 14)$ as a yellow oil.

${ }^{1}$ H-NMR $\left(400 \mathrm{MHz}, \mathrm{CDCl}_{3}\right): \delta=8.62(\mathrm{ddd}, J=4.9,1.9,0.9 \mathrm{~Hz}, 1 \mathrm{H}), 7.84(\mathrm{ddd}, J=8.0,7.5,1.9 \mathrm{~Hz}$, $1 \mathrm{H}), 7.43(\mathrm{ddd}, J=8.0,1.0,0.9 \mathrm{~Hz}, 1 \mathrm{H}), 7.33(\mathrm{ddd}, J=7.5,1.9,1.0 \mathrm{~Hz}, 1 \mathrm{H}), 7.29$ (dd, $J=9.1,4.3 \mathrm{~Hz}$, 1H), 7.26 (dd, $J=9.3,2.5 \mathrm{~Hz}, 1 \mathrm{H}), 6.89$ (dt, $J=9.1,2.5 \mathrm{~Hz}, 1 \mathrm{H}), 6.43$ (d, $J=0.9 \mathrm{~Hz}, 1 \mathrm{H}), 6.10$ (dt, $J=22.4,8.2 \mathrm{~Hz}, 0.14 \mathrm{H}, E), 5.77(\mathrm{dt}, J=32.5,7.0 \mathrm{~Hz}, 0.86 \mathrm{H}, Z$ ), 3.79 (ddt, $J=7.0,2.4,1.5 \mathrm{~Hz}$, $1.72 \mathrm{H}, Z), 3.85$ (ddt, $J=8.2,2.5,1.7 \mathrm{~Hz}, 0.28 \mathrm{H}, E)$.

${ }^{13}$ C-NMR $\left(125 \mathrm{MHz}, \mathrm{CDCl}_{3}\right): \delta=158.5\left(\mathrm{~d},{ }^{1} J_{\mathrm{C}-\mathrm{F}}=236 \mathrm{~Hz}, \mathrm{C}_{\mathrm{q}}\right), 150.7\left(\mathrm{C}_{\mathrm{q}}\right), 149.8(\mathrm{CH}), 146.2(\mathrm{dt}$, $\left.{ }^{1} J_{\mathrm{C}-\mathrm{F}}=261 \mathrm{~Hz},{ }^{2} J_{\mathrm{C}-\mathrm{F}}=29.0 \mathrm{~Hz}, \mathrm{C}_{\mathrm{q}}\right), 138.6(\mathrm{CH}), 137.7\left(\mathrm{C}_{\mathrm{q}}\right), 133.7\left(\mathrm{C}_{\mathrm{q}}\right), 128.8\left(\mathrm{~d},{ }^{3} J_{\mathrm{C}-\mathrm{F}}=10.3 \mathrm{~Hz}, \mathrm{C}_{\mathrm{q}}\right)$, $122.4(\mathrm{CH}), 120.4(\mathrm{CH}), 117.2\left(\mathrm{dt},{ }^{1} J_{\mathrm{C}-\mathrm{F}}=285 \mathrm{~Hz},{ }^{2} J_{\mathrm{C}-\mathrm{F}}=33.3 \mathrm{~Hz}, \mathrm{C}_{\mathrm{q}}\right), 113.7\left(\mathrm{~m}, \mathrm{C}_{\mathrm{q}}\right), 113.0\left(\mathrm{dt},{ }^{2} J_{\mathrm{C}-\mathrm{F}}\right.$ $\left.=8.5 \mathrm{~Hz},{ }^{3} J_{\mathrm{C}-\mathrm{F}}=4.4 \mathrm{~Hz}, \mathrm{CH}\right), 112.4\left(\mathrm{~m}, \mathrm{C}_{\mathrm{q}}\right), 110.9\left(\mathrm{~d},{ }^{2} J_{\mathrm{C}-\mathrm{F}}=9.5 \mathrm{~Hz}, \mathrm{CH}\right), 110.5\left(\mathrm{~d},{ }^{2} J_{\mathrm{C}-\mathrm{F}}=26.0 \mathrm{~Hz}\right.$, $\mathrm{CH}), 110.4\left(\mathrm{~m}, \mathrm{C}_{\mathrm{q}}\right), 110.0\left(\mathrm{~m}, \mathrm{C}_{\mathrm{q}}\right), 108.6\left(\mathrm{~m}, \mathrm{C}_{\mathrm{q}}\right), 108.2\left(\mathrm{~m}, \mathrm{C}_{\mathrm{q}}\right), 105.4\left(\mathrm{~d},{ }^{2} J_{\mathrm{C}-\mathrm{F}}=23.7 \mathrm{~Hz}, \mathrm{CH}\right), 103.5$ $\left(\mathrm{d},{ }^{3} J_{\mathrm{C}-\mathrm{F}}=4.3 \mathrm{~Hz}, \mathrm{CH}\right), 22.7\left(\mathrm{~d},{ }^{3} J_{\mathrm{C}-\mathrm{F}}=4.3 \mathrm{~Hz}, \mathrm{CH}_{2}\right)$.

${ }^{19}$ F-NMR $\left(470 \mathrm{MHz}, \mathrm{CDCl}_{3}\right): \delta=-81.0(\mathrm{~m}),-117.6(\mathrm{~m}),-122.1(\mathrm{~m}),-122.9(\mathrm{~m}),-123.0(\mathrm{~m})-123.4$ (m), $-123.5(\mathrm{~m}),-126.3(\mathrm{~m}),-130.5(\mathrm{~m})$.

IR (ATR): 1473, 1450, 1439, 1235, 1143, 776, $663 \mathrm{~cm}^{-1}$.

MS (ESI) $m / z$ (relative intensity): $661\left([\mathrm{M}+\mathrm{Na}]^{+}, 15\right), 639\left([\mathrm{M}+\mathrm{H}]^{+}, 100\right)$.

HR-MS (ESI): $m / z$ calcd. for $\left[\mathrm{C}_{23} \mathrm{H}_{12} \mathrm{~F}_{17} \mathrm{~N}_{2}\right]^{+}[\mathrm{M}+\mathrm{H}]^{+}$639.0724, found 639.0731.

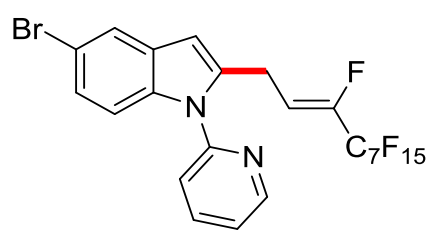

(Z)-2-(1H,1H,2H-Perfluorodec-2-en-1-yl)-5-bromo-1-(pyridin-2-yl)-1H-indole $\quad$ (158ca): The general procedure $\mathbf{F}$ was followed using 5-bromo-1-(pyridin-2-yl)-1H-indole (105c) (136 mg, $0.50 \mathrm{mmol})$ and $1 \mathrm{H}, 1 \mathrm{H}, 2 \mathrm{H}$-perfluorodec-1-ene (127a) $(268 \mathrm{mg}, 0.60 \mathrm{mmol})$. Isolation by column chromatography ( $n$-hexane/EtOAc: $10 / 1)$ yielded 158ca (324 mg, 93\%, Z/E =84:16) as an off-white solid.

M.p.: $59^{\circ} \mathrm{C}$ 
${ }^{1}$ H-NMR $\left(400 \mathrm{MHz}, \mathrm{CDCl}_{3}\right): \delta=8.63(\mathrm{ddd}, J=4.9,1.9,0.8 \mathrm{~Hz}, 1 \mathrm{H}), 7.90(\mathrm{ddd}, J=7.9,7.6,1.9 \mathrm{~Hz}$, $1 \mathrm{H}), 7.70(\mathrm{dd}, J=1.4,0.4 \mathrm{~Hz}, 1 \mathrm{H}), 7.41$ (ddd, $J=7.9,1.0,0.8 \mathrm{~Hz}, 1 \mathrm{H}), 7.34(\mathrm{ddd}, J=7.6,4.9,1.0 \mathrm{~Hz}$, $1 \mathrm{H}), 7.25-7.15(\mathrm{~m}, 2 \mathrm{H}), 6.41(\mathrm{~d}, J=0.9 \mathrm{~Hz}, 1 \mathrm{H}), 6.11(\mathrm{dt}, J=22.5,8.3 \mathrm{~Hz}, 0.16 \mathrm{H}, E), 5.75(\mathrm{dt}, J=$ $32.7,7.3 \mathrm{~Hz}, 0.84 \mathrm{H}, Z$ ), 3.85 (ddt, $J=7.3,2.4,1.8 \mathrm{~Hz}, 1.68 \mathrm{H}, Z$ ), 3.79 (ddt, $J=8.3,2.5,1.8 \mathrm{~Hz}$, $0.32 \mathrm{H}, E)$.

${ }^{13}$ C-NMR $\left(125 \mathrm{MHz}, \mathrm{CDCl}_{3}\right): \delta=150.1\left(\mathrm{C}_{\mathrm{q}}\right), 149.9(\mathrm{CH}), 146.1\left(\mathrm{dt},{ }^{1} J_{\mathrm{C}-\mathrm{F}}=261 \mathrm{~Hz},{ }^{2} J_{\mathrm{C}-\mathrm{F}}=29.5 \mathrm{~Hz}\right.$, $\left.\mathrm{C}_{\mathrm{q}}\right), 138.6(\mathrm{CH}), 137.4\left(\mathrm{C}_{\mathrm{q}}\right), 135.8\left(\mathrm{C}_{\mathrm{q}}\right), 129.9\left(\mathrm{C}_{\mathrm{q}}\right), 125.1(\mathrm{CH}), 122.6(\mathrm{CH}), 122.0(\mathrm{CH}), 120.5(\mathrm{CH})$, $117.2\left(\mathrm{dt},{ }^{1} J_{\mathrm{C}-\mathrm{F}}=288 \mathrm{~Hz},{ }^{2} J_{\mathrm{C}-\mathrm{F}}=31.3 \mathrm{~Hz}, \mathrm{C}_{\mathrm{q}}\right), 114.2\left(\mathrm{C}_{\mathrm{q}}\right), 112.9\left(\mathrm{dt},{ }^{2} J_{\mathrm{C}-\mathrm{F}}=8.1 \mathrm{~Hz},{ }^{3} J_{\mathrm{C}-\mathrm{F}}=4.3 \mathrm{~Hz}\right.$, $\mathrm{CH}), 112.3\left(\mathrm{~m}, \mathrm{C}_{\mathrm{q}}\right), 111.6(\mathrm{CH}), 110.6\left(\mathrm{~m}, \mathrm{C}_{\mathrm{q}}\right), 110.4\left(\mathrm{~m}, \mathrm{C}_{\mathrm{q}}\right), 110.1\left(\mathrm{~m}, \mathrm{C}_{\mathrm{q}}\right), 108.5\left(\mathrm{~m}, \mathrm{C}_{\mathrm{q}}\right), 108.3(\mathrm{~m}$, $\left.\mathrm{C}_{\mathrm{q}}\right), 102.9(\mathrm{CH}), 22.6\left(\mathrm{~d},{ }^{3} J_{\mathrm{C}-\mathrm{F}}=4.3 \mathrm{~Hz}, \mathrm{CH}_{2}\right)$.

${ }^{19}$ F-NMR $\left(376 \mathrm{MHz}, \mathrm{CDCl}_{3}\right): \delta=-80.9(\mathrm{~m}),-114.9(\mathrm{~m}, E),-117.5(\mathrm{~m}, Z),-122.0(\mathrm{~m}),-122.0(\mathrm{~m})$, $-122.7(\mathrm{~m}),-122.9(\mathrm{~m}),-126.2(\mathrm{~m}),-130.4(\mathrm{~m})$.

IR (ATR): 1588, 1472, 1443, 1236, 1200, 1146, 907, $731 \mathrm{~cm}^{-1}$.

MS (ESI) $\mathrm{m} / z$ (relative intensity): $699\left(\left[\mathrm{M}\left({ }^{79} \mathrm{Br}\right)+\mathrm{H}\right]^{+}, 100\right), 275(80)$.

HR-MS (ESI): $m / z$ calcd. for $\left[\mathrm{C}_{23} \mathrm{H}_{12}{ }^{79} \mathrm{BrF}_{16} \mathrm{~N}_{2}\right]^{+}[\mathrm{M}+\mathrm{H}]^{+}$698.9923, found 698.9922 .

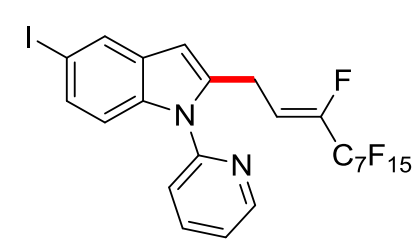

(Z)-2-(1H,1H,2H-Perfluorodec-2-en-1-yl)-5-iodo-1-(pyridin-2-yl)-1H-indole (158da): The general procedure $\mathbf{F}$ was followed using 5-iodo-1-(pyridin-2-yl)-1 $H$-indole (105d) (160 $\mathrm{mg}, 0.50 \mathrm{mmol}$ ) and $1 H, 1 H, 2 H$-perfluorodec-1-ene (127a) $(268 \mathrm{mg}, 0.60 \mathrm{mmol})$. Isolation by column chromatography ( $n$ hexane/EtOAc: $10 / 1)$ yielded 158da (346 $\mathrm{mg}, 93 \%, Z / E=82: 18)$ as a yellow oil.

${ }^{1}$ H-NMR $\left(300 \mathrm{MHz}, \mathrm{CDCl}_{3}\right): \delta=8.62(\mathrm{ddd}, J=4.9,1.9,0.9 \mathrm{~Hz}, 1 \mathrm{H}), 7.98-7.82(\mathrm{~m}, 2 \mathrm{H}), 7.45-7.38$ (m, 2H), $7.33(\mathrm{ddd}, J=7.5,4.9,0.9 \mathrm{~Hz}, 1 \mathrm{H}), 7.10(\mathrm{dd}, J=8.7,0.6 \mathrm{~Hz}, 1 \mathrm{H}), 6.40(\mathrm{~d}, J=0.9 \mathrm{~Hz}$, $0.18 \mathrm{H}, E), 6.39(\mathrm{~d}, J=0.9 \mathrm{~Hz}, 0.82 \mathrm{H}, Z), 6.09(\mathrm{dt}, J=22.5,8.0 \mathrm{~Hz}, 0.18 \mathrm{H}, E), 5.76(\mathrm{dt}, J=33.0$, $7.5 \mathrm{~Hz}, 0.82 \mathrm{H}, Z$ ), 3.90 (ddt, $J=7.5,2.4,1.8 \mathrm{~Hz}, 1.63 \mathrm{H}, Z$ ), 3.84 (ddt, $J=8.0,2.5,1.8 \mathrm{~Hz}, 0.37 \mathrm{H}, E$ ).

${ }^{13}$ C-NMR $\left(125 \mathrm{MHz}, \mathrm{CDCl}_{3}\right): \delta=150.3\left(\mathrm{C}_{\mathrm{q}}\right), 149.8(\mathrm{CH}), 146.1\left(\mathrm{dt},{ }^{1} J_{\mathrm{C}-\mathrm{F}}=261 \mathrm{~Hz},{ }^{2} J_{\mathrm{C}-\mathrm{F}}=29.1 \mathrm{~Hz}\right.$, $\left.\mathrm{C}_{\mathrm{q}}\right), 138.5(\mathrm{CH}), 137.0\left(\mathrm{C}_{\mathrm{q}}\right), 136.3(\mathrm{CH}), 130.6\left(\mathrm{C}_{\mathrm{q}}\right), 130.4\left(\mathrm{C}_{\mathrm{q}}\right), 129.8(\mathrm{CH}), 122.5(\mathrm{CH}), 117.2(\mathrm{dt}$, $\left.{ }^{1} J_{\mathrm{C}-\mathrm{F}}=288 \mathrm{~Hz},{ }^{2} J_{\mathrm{C}-\mathrm{F}}=31.3 \mathrm{~Hz}, \mathrm{C}_{\mathrm{q}}\right), 112.1(\mathrm{CH}), 112.9\left(\mathrm{dt},{ }^{2} J_{\mathrm{C}-\mathrm{F}}=8.1 \mathrm{~Hz},{ }^{3} J_{\mathrm{C}-\mathrm{F}}=4.2 \mathrm{~Hz}, \mathrm{CH}\right), 112.4$ $\left(\mathrm{m}, \mathrm{C}_{\mathrm{q}}\right), 112.1(\mathrm{CH}), 110.7\left(\mathrm{~m}, \mathrm{C}_{\mathrm{q}}\right), 110.3\left(\mathrm{~m}, \mathrm{C}_{\mathrm{q}}\right), 110.2\left(\mathrm{~m}, \mathrm{C}_{\mathrm{q}}\right), 108.5\left(\mathrm{~m}, \mathrm{C}_{\mathrm{q}}\right), 108.2\left(\mathrm{~m}, \mathrm{C}_{\mathrm{q}}\right), 102.7$ $(\mathrm{CH}), 84.5\left(\mathrm{C}_{\mathrm{q}}\right), 22.6\left(\mathrm{~d},{ }^{3} J_{\mathrm{C}-\mathrm{F}}=4.3 \mathrm{~Hz}, \mathrm{CH}_{2}\right)$.

${ }^{19}$ F-NMR $\left(282 \mathrm{MHz}, \mathrm{CDCl}_{3}\right): \delta=-80.9(\mathrm{~m}),-117.6(\mathrm{~m}),-122.1(\mathrm{~m}),-121.9(\mathrm{~m}),-122.9(\mathrm{~m}),-123.5$ (m), $-126.2(\mathrm{~m}),-130.4(\mathrm{~m})$.

IR (ATR): 3044, 1587, 1472, 1443, 1240, 1209, 1150, 904, $725 \mathrm{~cm}^{-1}$. 
MS (ESI) $m / z$ (relative intensity): $769\left([\mathrm{M}+\mathrm{Na}]^{+}, 20\right), 747\left([\mathrm{M}+\mathrm{H}]^{+}, 100\right)$.

HR-MS (ESI): $m / z$ calcd. for $\left[\mathrm{C}_{23} \mathrm{H}_{12} \mathrm{~F}_{16} \mathrm{IN}_{2}\right]^{+}[\mathrm{M}+\mathrm{H}]^{+} 746.9784$, found 746.9786 .

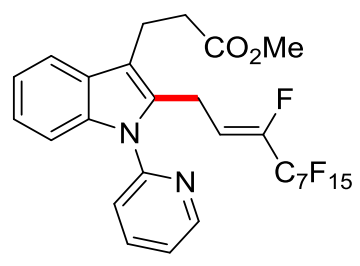

(Z)-Methyl-2-[(1H,1H,2H-Perfluorodec-2-en-1-yl)-1-(pyridin-2-yl)-1H-indole-3-yl]propanoate

(158pa): A modified general procedure $\mathbf{F}$ was followed using methyl 2-(1-(pyridin-2-yl)-1 $H$-indol-3yl)propanoate (105p) (140 mg, $0.50 \mathrm{mmol})$ and $1 H, 1 H, 2 H$-perfluoro-1-decene (127a) (268 mg, 0.60 mmol) at $70{ }^{\circ} \mathrm{C}$. Isolation by column chromatography $(n$-hexane/EtOAc $=10: 1)$ yielded 158pa (237 $\mathrm{mg}, 67 \%, Z / E=80: 20)$ as a white solid.

M.p.: $70{ }^{\circ} \mathrm{C}$.

${ }^{1} \mathbf{H}-\mathbf{N M R}\left(400 \mathrm{MHz}, \mathrm{CDCl}_{3}\right): \delta=8.60(\mathrm{ddd}, J=4.9,1.9,0.8 \mathrm{~Hz}, 1 \mathrm{H}), 7.87(\mathrm{ddd}, J=8.1,7.5,1.9 \mathrm{~Hz}$, 1H), 7.60-7.55 (m, 1H), 7.44 (ddd, $J=8.1,1.0,0.8 \mathrm{~Hz}, 1 \mathrm{H}), 7.34-7.27(\mathrm{~m}, 2 \mathrm{H}), 7.20-7.12(\mathrm{~m}, 2 \mathrm{H})$, $5.89(\mathrm{dt}, J=23.2,8.3 \mathrm{~Hz}, 0.20 \mathrm{H}, E), 5.61(\mathrm{dt}, J=33.3,7.0 \mathrm{~Hz}, 0.80 \mathrm{H}, Z), 3.94-3.90(\mathrm{~m}, 2 \mathrm{H}), 3.65$ (s, $3 \mathrm{H}), 3.12(\mathrm{t}, J=7.8 \mathrm{~Hz}, 2 \mathrm{H}), 2.69(\mathrm{t}, J=7.8 \mathrm{~Hz}, 2 \mathrm{H})$.

${ }^{13}$ C-NMR $\left(100 \mathrm{MHz}, \mathrm{CDCl}_{3}\right): \delta=173.4\left(\mathrm{C}_{\mathrm{q}}\right), 150.9\left(\mathrm{C}_{\mathrm{q}}\right), 149.7(\mathrm{CH}), 144.7\left(\mathrm{dt},{ }^{1} J_{\mathrm{C}-\mathrm{F}}=260 \mathrm{~Hz},{ }^{2} J_{\mathrm{C}-\mathrm{F}}\right.$ $\left.=29.1 \mathrm{~Hz}, \mathrm{C}_{\mathrm{q}}\right), 138.5(\mathrm{CH}), 136.6\left(\mathrm{C}_{\mathrm{q}}\right), 132.2\left(\mathrm{~d},{ }^{4} J_{\mathrm{C}-\mathrm{F}}=2.0 \mathrm{~Hz}, \mathrm{C}_{\mathrm{q}}\right), 127.9\left(\mathrm{C}_{\mathrm{q}}\right), 122.8(\mathrm{CH}), 122.1$ $(\mathrm{CH}), 120.8(\mathrm{CH}), 120.7(\mathrm{CH}), 118.6(\mathrm{CH}), 116.9\left(\mathrm{dt},{ }^{1} J_{\mathrm{C}-\mathrm{F}}=282 \mathrm{~Hz},{ }^{2} J_{\mathrm{C}-\mathrm{F}}=33.0 \mathrm{~Hz}, \mathrm{C}_{\mathrm{q}}\right), 114.4\left(\mathrm{C}_{\mathrm{q}}\right)$, $114.0\left(\mathrm{dt},{ }^{2} J_{\mathrm{C}-\mathrm{F}}=8.1 \mathrm{~Hz},{ }^{3} J_{\mathrm{C}-\mathrm{F}}=4.3 \mathrm{~Hz}, \mathrm{CH}\right), 113.2\left(\mathrm{~m}, \mathrm{C}_{\mathrm{q}}\right), 110.8\left(\mathrm{~m}, \mathrm{C}_{\mathrm{q}}\right), 110.7\left(\mathrm{~m}, \mathrm{C}_{\mathrm{q}}\right), 110.5(\mathrm{~m}$, $\left.\mathrm{C}_{\mathrm{q}}\right), 110.1(\mathrm{CH}), 108.1\left(\mathrm{C}_{\mathrm{q}}\right), 107.4\left(\mathrm{~m}, \mathrm{C}_{\mathrm{q}}\right), 51.6\left(\mathrm{CH}_{3}\right), 34.7\left(\mathrm{CH}_{2}\right), 20.2\left(\mathrm{~d},{ }^{3} J_{\mathrm{C}-\mathrm{F}}=4.2 \mathrm{~Hz}, \mathrm{CH}_{2}\right), 19.6$ $\left(\mathrm{CH}_{2}\right)$.

${ }^{19}$ F-NMR (376 MHz, $\left.\mathrm{CDCl}_{3}\right): \delta=-80.8(\mathrm{~m}),-115.4(\mathrm{~m}, E),-117.3(\mathrm{~m}, Z),-122.0(\mathrm{~m}),-122.1(\mathrm{~m})$, $-122.7(\mathrm{~m}),-122.9(\mathrm{~m}),-126.2(\mathrm{~m}),-131.1(\mathrm{~m})$.

IR (ATR): 2955, 1737, 1586, 1472, 1439, 1200, 1144, $732 \mathrm{~cm}^{-1}$.

MS (ESI) $m / z$ (relative intensity): $707\left([\mathrm{M}+\mathrm{H}]^{+}, 100\right), 687$ (10).

HR-MS (ESI): $m / z$ calcd. for $\left[\mathrm{C}_{27} \mathrm{H}_{19} \mathrm{~F}_{16} \mathrm{~N}_{2} \mathrm{O}_{2}\right]^{+}[\mathrm{M}+\mathrm{H}]^{+}$707.1186, found 707.1188.

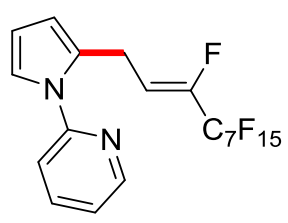

(Z)-2-[2-(1H,1H,2H-Perfluorodec-2-en-1-yl)-1H-pyrrol-1-yl]pyridine (160): The general procedure G was followed using 2-(1H-pyrrol-1-yl)pyridine (146) $(72.1 \mathrm{mg}, 0.50 \mathrm{mmol})$ and $1 H, 1 H, 2 H$ - 
perfluorodec-1-ene (127a) $(268 \mathrm{mg}, 0.60 \mathrm{mmol})$. Isolation by column chromatography ( $n$ hexane/EtOAc: $10 / 1)$ yielded $160(174 \mathrm{mg}, 61 \%, Z / E=84: 16)$ and 160' $(40 \mathrm{mg}, 8 \%, Z / E=84: 16)$ as yellow oils.

${ }^{1} \mathbf{H}-\mathrm{NMR}\left(400 \mathrm{MHz}, \mathrm{CDCl}_{3}\right): \delta=8.47$ (ddd, $\left.J=4.9,1.9,0.9 \mathrm{~Hz}, 1 \mathrm{H}\right), 7.77(\mathrm{ddd}, J=8.2,7.5,1.9 \mathrm{~Hz}$, $1 \mathrm{H}), 7.28(\mathrm{ddd}, J=8.2,1.0,0.9 \mathrm{~Hz}, 1 \mathrm{H}), 7.18(\mathrm{ddd}, J=7.5,4.9,1.0 \mathrm{~Hz}, 1 \mathrm{H}), 7.01(\mathrm{dd}, J=3.1,1.8 \mathrm{~Hz}$, $1 \mathrm{H}), 6.24(\mathrm{dd}, J=3.1,3.0 \mathrm{~Hz}, 1 \mathrm{H}), 6.16(\mathrm{dt}, J=22.7,8.2 \mathrm{~Hz}, 0.16 \mathrm{H}, E), 6.12-6.09(\mathrm{~m}, 1 \mathrm{H}) 5.81(\mathrm{dt}, J$ $=33.4,7.5 \mathrm{~Hz}, 0.84 \mathrm{H}, Z), 3.88(\mathrm{ddt}, J=7.5,3.9,2.0 \mathrm{~Hz}, 1.68 \mathrm{H}, Z), 3.83(\mathrm{ddt}, J=7.5,4.0,2.0 \mathrm{~Hz}$, $0.32 \mathrm{H}, E)$.

${ }^{13} \mathrm{C}-\mathrm{NMR}\left(100 \mathrm{MHz}, \mathrm{CDCl}_{3}\right): \delta=152.5\left(\mathrm{C}_{\mathrm{q}}\right), 148.6(\mathrm{CH}), 145.4\left(\mathrm{dt},{ }^{1} J_{\mathrm{C}-\mathrm{F}}=260 \mathrm{~Hz},{ }^{2} J_{\mathrm{C}-\mathrm{F}}=30.1 \mathrm{~Hz}\right.$, $\left.\mathrm{C}_{\mathrm{q}}\right), 138.5(\mathrm{CH}), 129.1\left(\mathrm{C}_{\mathrm{q}}\right), 121.2(\mathrm{CH}), 121.0(\mathrm{CH}), 116.9\left(\mathrm{dt},{ }^{1} J_{\mathrm{C}-\mathrm{F}}=285 \mathrm{~Hz},{ }^{2} J_{\mathrm{C}-\mathrm{F}}=33.3 \mathrm{~Hz}, \mathrm{C}_{\mathrm{q}}\right)$, $116.6(\mathrm{CH}), 114.6\left(\mathrm{dt},{ }^{2} J_{\mathrm{C}-\mathrm{F}}=8.3 \mathrm{~Hz},{ }^{3} J_{\mathrm{C}-\mathrm{F}}=4.3 \mathrm{~Hz}, \mathrm{CH}\right), 112.2\left(\mathrm{~m}, \mathrm{C}_{\mathrm{q}}\right), 111.3\left(\mathrm{~m}, \mathrm{C}_{\mathrm{q}}\right), 111.2\left(\mathrm{~m}, \mathrm{C}_{\mathrm{q}}\right)$, $110.9\left(\mathrm{~m}, \mathrm{C}_{\mathrm{q}}\right), 110.7(\mathrm{CH}), 109.7(\mathrm{CH}), 108.6\left(\mathrm{~m}, \mathrm{C}_{\mathrm{q}}\right), 107.8\left(\mathrm{C}_{\mathrm{q}}\right), 22.9\left(\mathrm{~d},{ }^{3} J_{\mathrm{C}-\mathrm{F}}=4.2 \mathrm{~Hz}, \mathrm{CH}_{2}\right)$.

${ }^{19} \mathbf{F}-\mathbf{N M R}\left(376 \mathrm{MHz}, \mathrm{CDCl}_{3}\right): \delta=-80.8(\mathrm{~m}),-117.5(\mathrm{~m}),-122.0(\mathrm{~m}),-122.8(\mathrm{~m}),-123.0(\mathrm{~m}),-123.5$ (m), $-126.2(\mathrm{~m}),-132.3(\mathrm{~m})$.

IR (ATR): 1523, 1504, 1325, 1182, 992, $754 \mathrm{~cm}^{-1}$.

MS (ESI) $m / z$ (relative intensity): $593\left([\mathrm{M}+\mathrm{Na}]^{+}, 20\right), 571\left([\mathrm{M}+\mathrm{H}]^{+}, 100\right)$.

HR-MS (ESI): $m / z$ calcd. for $\left[\mathrm{C}_{19} \mathrm{H}_{11} \mathrm{~F}_{16} \mathrm{~N}_{2}\right]^{+}[\mathrm{M}+\mathrm{H}]^{+}$571.0661, found 571.0668.<smiles>FC(=CCc1ccc(CC=C(F)C(F)(F)C(F)(F)C(F)(F)C(F)(F)C(F)(F)F)n1-c1ccccn1)C(F)(F)F</smiles>

\section{(Z)-2-[2,5-Di-(1H,1H,2H-Perfluorodec-2-en-1-yl)-1H-pyrrol-1-yl]pyridine (160'):}

${ }^{1} \mathbf{H}-\mathbf{N M R}\left(400 \mathrm{MHz}, \mathrm{CDCl}_{3}\right): \delta=8.59(\mathrm{ddd}, J=4.9,1.9,0.9 \mathrm{~Hz}, 1 \mathrm{H}), 7.84(\mathrm{ddd}, J=8.0,7.8,1.9 \mathrm{~Hz}$, $1 \mathrm{H}), 7.34$ (ddd, $J=7.8,4.9,1.2 \mathrm{~Hz}, 1 \mathrm{H}), 7.20$ (ddd, $J=8.0,1.2,0.9 \mathrm{~Hz}, 1 \mathrm{H}), 6.00$ (s, 2H), 5.91 (dt, $J$ $=22.5,8.3 \mathrm{~Hz}, 0.32 \mathrm{H}, E), 5.61(\mathrm{dt}, J=33.0,7.6 \mathrm{~Hz}, 1.68 \mathrm{H}, Z), 3.47(\mathrm{ddt}, J=7.6,2.4,1.8 \mathrm{~Hz}, 3.36 \mathrm{H}$, $Z$ ), 3.40 (ddt, $J=8.3,2.5,1.8 \mathrm{~Hz}, 0.64 \mathrm{H}, E$ ).

${ }^{13} \mathrm{C}-\mathrm{NMR}\left(100 \mathrm{MHz}, \mathrm{CDCl}_{3}\right): \delta=150.7\left(\mathrm{C}_{\mathrm{q}}\right), 149.8(\mathrm{CH}), 145.7\left(\mathrm{dt},{ }^{1} J_{\mathrm{C}-\mathrm{F}}=259 \mathrm{~Hz},{ }^{2} J_{\mathrm{C}-\mathrm{F}}=30.1 \mathrm{~Hz}\right.$, $\left.\mathrm{C}_{\mathrm{q}}\right), 138.4(\mathrm{CH}), 129.1\left(\mathrm{C}_{\mathrm{q}}\right), 123.1(\mathrm{CH}), 121.9(\mathrm{CH}), 117.4\left(\mathrm{dt},{ }^{1} J_{\mathrm{C}-\mathrm{F}}=288 \mathrm{~Hz},{ }^{2} J_{\mathrm{C}-\mathrm{F}}=33.3 \mathrm{~Hz}, \mathrm{C}_{\mathrm{q}}\right)$, $112.6\left(\mathrm{dt},{ }^{2} J_{\mathrm{C}-\mathrm{F}}=8.3 \mathrm{~Hz},{ }^{3} J_{\mathrm{C}-\mathrm{F}}=4.3 \mathrm{~Hz}, \mathrm{CH}\right), 111.2\left(\mathrm{~m}, \mathrm{C}_{\mathrm{q}}\right), 110.8\left(\mathrm{~m}, \mathrm{C}_{\mathrm{q}}\right), 110.2\left(\mathrm{~m}, \mathrm{C}_{\mathrm{q}}\right), 109.7(\mathrm{~m}$, $\left.\mathrm{C}_{\mathrm{q}}\right), 108.8\left(\mathrm{~m}, \mathrm{C}_{\mathrm{q}}\right), 108.2\left(\mathrm{~m}, \mathrm{C}_{\mathrm{q}}\right), 107.8(\mathrm{CH}), 21.3\left(\mathrm{~d},{ }^{3} \mathrm{~J}_{\mathrm{C}-\mathrm{F}}=4.1 \mathrm{~Hz}, \mathrm{CH}_{2}\right)$.

${ }^{19} \mathbf{F}-\mathbf{N M R}\left(376 \mathrm{MHz}, \mathrm{CDCl}_{3}\right): \delta=-81.2(\mathrm{~m}),-117.7(\mathrm{~m}),-121.9(\mathrm{~m}),-122.2(\mathrm{~m}),-123.1(\mathrm{~m}),-123.1$ (m), $-126.4(\mathrm{~m}),-131.8(\mathrm{~m})$.

IR (ATR): 2960, 2924, 1473, 1235, 1143, 1047, 735, $559 \mathrm{~cm}^{-1}$.

MS (ESI) $\mathrm{m} / z$ (relative intensity): $997\left([\mathrm{M}+\mathrm{H}]^{+}, 100\right), 573$ (20), 545 (15).

HR-MS (ESI): $m / z$ calcd. for $\left[\mathrm{C}_{29} \mathrm{H}_{13} \mathrm{~F}_{32} \mathrm{~N}_{2}\right]^{+}[\mathrm{M}+\mathrm{H}]^{+} 997.0562$, found: 997.0553 . 
<smiles>CC(C)(C)c1ccc(-n2cccn2)c(C/C=C(\F)C(F)(F)F)c1</smiles>

(Z)-1-[4-(tert-Butyl)-2-(1H,1H,2H-perfluorodec-2-en-1-yl)phenyl]-1H-pyrazole $\quad(161 c): \quad$ A modified general procedure $\mathbf{F}$ was followed using 1-[4-(tert-butyl)phenyl]-1H-pyrazole (18c) (100 mg, $0.50 \mathrm{mmol}), 1 H, 1 H, 2 H$-perfluorodec-1-ene (127a) $(268 \mathrm{mg}, 0.60 \mathrm{mmol})$, [Cp*Co(CO) $\left.\mathrm{I}_{2}\right]$ (23.7 $\mathrm{mg}, 10 \mathrm{~mol} \%$ ) and $\mathrm{AgSbF}_{6}\left(34.4 \mathrm{mg}, 20 \mathrm{~mol} \%\right.$ ) at $70{ }^{\circ} \mathrm{C}$. Isolation by column chromatography ( $n$-hexane/EtOAc: 20/1) yielded 161c $(248 \mathrm{mg}, 79 \%, Z / E=87: 13)$ as a yellow oil.

${ }^{1}$ H-NMR $\left(300 \mathrm{MHz}, \mathrm{CDCl}_{3}\right): \delta=7.70(\mathrm{dd}, J=2.2,1.7 \mathrm{~Hz}, 1 \mathrm{H}), 7.56(\mathrm{~d}, J=4.0,2.4 \mathrm{~Hz}, 1 \mathrm{H}), 7.36$ (dd, $J=8.2,2.2 \mathrm{~Hz}, 1 \mathrm{H}), 7.30(\mathrm{dd}, J=2.4,2.1 \mathrm{~Hz}, 1 \mathrm{H}), 7.23(\mathrm{dd}, J=8.2,1.7 \mathrm{~Hz}, 1 \mathrm{H}), 6.42$ (dd, $J=4.0,2.1 \mathrm{~Hz}, 1 \mathrm{H}), 5.90(\mathrm{dt}, J=21.8,7.9 \mathrm{~Hz}, 0.13 \mathrm{H}, E), 5.67(\mathrm{dt}, J=33.3,7.6 \mathrm{~Hz}, 0.87 \mathrm{H}, Z), 3.79$ (ddt, $J=7.6,4.1,2.5 \mathrm{~Hz}, 2 \mathrm{H}), 1.33$ (s, 9H).

${ }^{13}$ C-NMR $\left(100 \mathrm{MHz}, \mathrm{CDCl}_{3}\right): \delta=152.2\left(\mathrm{C}_{\mathrm{q}}\right), 145.7\left(\mathrm{dt},{ }^{1} J_{\mathrm{C}-\mathrm{F}}=259 \mathrm{~Hz},{ }^{2} J_{\mathrm{C}-\mathrm{F}}=29.1 \mathrm{~Hz}, \mathrm{C}_{\mathrm{q}}\right), 140.5$ $(\mathrm{CH}), 137.1\left(\mathrm{C}_{\mathrm{q}}\right), 132.9\left(\mathrm{C}_{\mathrm{q}}\right), 130.3(\mathrm{CH}), 127.3(\mathrm{CH}), 125.8(\mathrm{CH}), 124.7(\mathrm{CH}), 117.0(\mathrm{dt}$,

$\left.{ }^{1} J_{\mathrm{C}-\mathrm{F}}=289 \mathrm{~Hz},{ }^{2} J_{\mathrm{C}-\mathrm{F}}=31.3 \mathrm{~Hz}, \mathrm{C}_{\mathrm{q}}\right), 114.7\left(\mathrm{dt},{ }^{2} J_{\mathrm{C}-\mathrm{F}}=8.9 \mathrm{~Hz},{ }^{3} J_{\mathrm{C}-\mathrm{F}}=3.6 \mathrm{~Hz}, \mathrm{CH}\right), 113.0\left(\mathrm{~m}, \mathrm{C}_{\mathrm{q}}\right)$, $112.0\left(\mathrm{~m}, \mathrm{C}_{\mathrm{q}}\right), 110.6\left(\mathrm{~m}, \mathrm{C}_{\mathrm{q}}\right), 110.0\left(\mathrm{~m}, \mathrm{C}_{\mathrm{q}}\right), 108.6\left(\mathrm{~m}, \mathrm{C}_{\mathrm{q}}\right), 108.1\left(\mathrm{~m}, \mathrm{C}_{\mathrm{q}}\right), 106.5(\mathrm{CH}), 34.7(\mathrm{CH}), 31.2$ $\left(\mathrm{CH}_{3}\right), 26.3\left(\mathrm{~d},{ }^{3} J_{\mathrm{C}-\mathrm{F}}=3.7 \mathrm{~Hz}, \mathrm{CH}_{2}\right)$.

${ }^{19}$ F-NMR (282 MHz, $\left.\mathrm{CDCl}_{3}\right): \delta=-80.9(\mathrm{~m}),-114.5(\mathrm{~m}, E),-117.5(\mathrm{~m}),-122.1(\mathrm{~m}),-122.1(\mathrm{~m})$, -122.9 (m, Z), -123.1 (m, E), -123.5 (m), -126.2 (m), -132.3 (m).

IR (ATR): 2968, 1522, 1238, 1201, 1146, 909, $732 \mathrm{~cm}^{-1}$.

MS (EI) $\mathrm{m} / z$ (relative intensity): 626 ([M] $\left.{ }^{+}, 100\right), 611$ (20), 598 (10).

HR-MS (ESI): $m / z$ calcd. for $\left[\mathrm{C}_{23} \mathrm{H}_{19} \mathrm{~F}_{16} \mathrm{~N}_{2}\right]^{+}[\mathrm{M}+\mathrm{H}]^{+} 627.1287$, found 627.1289 .<smiles>COc1ccc(-n2cccn2)c(C/C=C(\F)C(F)(F)F)c1</smiles>

(Z)-1-[4-(Methoxy)-2-(1H,1H,2H-perfluorodec-2-en-1-yl)phenyl]-1H-pyrazole (161d): A modified general procedure $\mathbf{F}$ was followed using 1-[4-(methoxy)phenyl]-1 $H$-pyrazole (18d) (100 mg, $0.50 \mathrm{mmol}), 1 \mathrm{H}, 1 \mathrm{H}, 2 \mathrm{H}$-perfluorodec-1-ene (127a) $(268 \mathrm{mg}, 0.60 \mathrm{mmol}),\left[\mathrm{Cp} * \mathrm{Co}(\mathrm{CO}) \mathrm{I}_{2}\right](23.7 \mathrm{mg}$, $10 \mathrm{~mol} \%)$ and $\mathrm{AgSbF}_{6}(34.4 \mathrm{mg}, 20 \mathrm{~mol} \%)$ at $70{ }^{\circ} \mathrm{C}$. Isolation by column chromatography ( $n$ hexane/EtOAc: 10/1) yielded 161d (159 mg, 53\%, Z/E = 82:18) as a colorless oil.

${ }^{1} \mathbf{H}-\mathbf{N M R}\left(400 \mathrm{MHz}, \mathrm{CDCl}_{3}\right): \delta=7.69(\mathrm{dd}, J=1.9,0.7 \mathrm{~Hz}, 1 \mathrm{H}), 7.59(\mathrm{~d}, J=2.3,0.7 \mathrm{~Hz}, 1 \mathrm{H}), 7.24-$ $7.21(\mathrm{~m}, 1 \mathrm{H}), 6.84(\mathrm{dd}, J=8.6,2.9 \mathrm{~Hz}, 1 \mathrm{H}), 6.79(\mathrm{~d}, J=2.9,1 \mathrm{H}), 6.41(\mathrm{dd}, J=2.3,1.9 \mathrm{~Hz}, 1 \mathrm{H}), 5.88$ 
$(\mathrm{dt}, J=21.8,7.9 \mathrm{~Hz}, 0.18 \mathrm{H}, E), 5.62(\mathrm{dt}, J=33.3,7.6 \mathrm{~Hz}, 0.82 \mathrm{H}, Z), 3.82(\mathrm{~s}, 3 \mathrm{H}), 3.45(\mathrm{ddt}, J=7.6$, $4.1,2.5 \mathrm{~Hz}, 2 \mathrm{H})$.

${ }^{13} \mathrm{C}-\mathrm{NMR}\left(100 \mathrm{MHz}, \mathrm{CDCl}_{3}\right): \delta=160.0\left(\mathrm{C}_{\mathrm{q}}\right), 146.0\left(\mathrm{dt},{ }^{1} J_{\mathrm{C}-\mathrm{F}}=259 \mathrm{~Hz},{ }^{2} J_{\mathrm{C}-\mathrm{F}}=29.1 \mathrm{~Hz}, \mathrm{C}_{\mathrm{q}}\right), 140.6$ $(\mathrm{CH}), 135.3\left(\mathrm{C}_{\mathrm{q}}\right), 132.9\left(\mathrm{C}_{\mathrm{q}}\right), 130.7(\mathrm{CH}), 127.8(\mathrm{CH}), 117.4\left(\mathrm{dt},{ }^{1} J_{\mathrm{C}-\mathrm{F}}=289 \mathrm{~Hz},{ }^{2} J_{\mathrm{C}-\mathrm{F}}=31.3 \mathrm{~Hz}, \mathrm{C}_{\mathrm{q}}\right)$, $115.4(\mathrm{CH}), 114.2\left(\mathrm{dt},{ }^{2} J_{\mathrm{C}-\mathrm{F}}=8.9 \mathrm{~Hz},{ }^{3} J_{\mathrm{C}-\mathrm{F}}=3.6 \mathrm{~Hz}, \mathrm{CH}\right), 113.5\left(\mathrm{~m}, \mathrm{C}_{\mathrm{q}}\right), 112.6(\mathrm{CH}), 110.9\left(\mathrm{~m}, \mathrm{C}_{\mathrm{q}}\right)$, $110.8\left(\mathrm{~m}, \mathrm{C}_{\mathrm{q}}\right), 110.6\left(\mathrm{~m}, \mathrm{C}_{\mathrm{q}}\right), 108.6\left(\mathrm{~m}, \mathrm{C}_{\mathrm{q}}\right), 107.7\left(\mathrm{~m}, \mathrm{C}_{\mathrm{q}}\right), 106.5(\mathrm{CH}), 55.5\left(\mathrm{CH}_{3}\right), 26.0(\mathrm{~d}$, $\left.{ }^{3} J_{\mathrm{C}-\mathrm{F}}=3.7 \mathrm{~Hz}, \mathrm{CH}_{2}\right)$.

${ }^{19}$ F-NMR (376 MHz, $\left.\mathrm{CDCl}_{3}\right): \delta=-80.8(\mathrm{~m}),-114.5(\mathrm{~m}, E),-117.4(\mathrm{~m}),-122.0(\mathrm{~m}),-122.0(\mathrm{~m})$, $-122.7(\mathrm{~m}),-122.9(\mathrm{~m}),-126.2(\mathrm{~m}),-131.5(\mathrm{~m})$.

IR (ATR): 2963, 1521, 1235, 1199, 1144, 1044, $735 \mathrm{~cm}^{-1}$.

MS (ESI) $m / z$ (relative intensity): $623\left([\mathrm{M}+\mathrm{Na}]^{+}, 10\right), 601\left([\mathrm{M}+\mathrm{H}]^{+}, 100\right)$.

HR-MS (ESI): $m / z$ calcd. for $\left[\mathrm{C}_{20} \mathrm{H}_{13} \mathrm{~F}_{16} \mathrm{~N}_{2} \mathrm{O}\right]^{+}[\mathrm{M}+\mathrm{H}]^{+}$601.0767, found 601.0768.

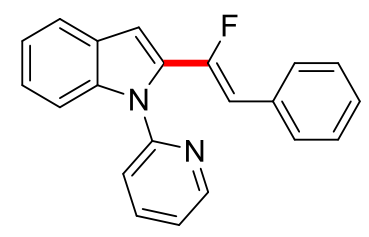

(Z)-2-(1-Fluoro-2-phenylvinyl)-1-(pyridin-2-yl)-1H-indole (159aa): The general procedure $\mathbf{G}$ was followed using 1-(pyridin-2-yl)- $1 H$-indole (105a) $(97.1 \mathrm{mg}, 0.50 \mathrm{mmol}$ ) and 1-(2,2-difluorovinyl)benzene (128a) (105 mg, $0.75 \mathrm{mmol}$ ). Isolation by column chromatography ( $n$-hexane/EtOAc: 10/1) yielded 159aa $(152 \mathrm{mg}, 97 \%, Z / E=99: 1)$ as a colorless solid.

M.p.: $68^{\circ} \mathrm{C}$.

${ }^{1}$ H-NMR $\left(300 \mathrm{MHz}, \mathrm{CDCl}_{3}\right): \delta=8.70(\mathrm{ddd}, J=4.9,2.0,0.9 \mathrm{~Hz}, 1 \mathrm{H}), 7.86(\mathrm{ddd}, J=8.1,7.5,2.0 \mathrm{~Hz}$, $1 \mathrm{H}), 7.72(\mathrm{ddd}, J=8.1,1.0,0.9 \mathrm{~Hz}, 1 \mathrm{H}), 7.56-7.52(\mathrm{~m}, 4 \mathrm{H}), 7.35-7.16(\mathrm{~m}, 6 \mathrm{H}), 7.03(\mathrm{~d}, J=0.9 \mathrm{~Hz}$, $1 \mathrm{H}), 6.48(\mathrm{~d}, J=18.9 \mathrm{~Hz}, 0.03 \mathrm{H}, E), 6.04(\mathrm{~d}, J=38.0 \mathrm{~Hz}, 0.97 \mathrm{H}, Z)$.

${ }^{13} \mathrm{C}-\mathrm{NMR}\left(125 \mathrm{MHz}, \mathrm{CDCl}_{3}\right): \delta=151.8\left(\mathrm{C}_{\mathrm{q}}\right), 151.1\left(\mathrm{~d},{ }^{1} J_{\mathrm{C}-\mathrm{F}}=257 \mathrm{~Hz}, \mathrm{C}_{\mathrm{q}}\right), 149.3(\mathrm{CH}), 138.4\left(\mathrm{C}_{\mathrm{q}}\right)$, $138.2(\mathrm{CH}), 133.3\left(\mathrm{~d},{ }^{4} J_{\mathrm{C}-\mathrm{F}}=3.8 \mathrm{~Hz}, \mathrm{C}_{\mathrm{q}}\right), 132.3\left(\mathrm{~d},{ }^{2} J_{\mathrm{C}-\mathrm{F}}=30.1 \mathrm{~Hz}, \mathrm{C}_{\mathrm{q}}\right), 128.9\left(\mathrm{~d},{ }^{4} J_{\mathrm{C}-\mathrm{F}}=7.8 \mathrm{~Hz}, \mathrm{CH}\right)$, $128.6(\mathrm{CH}), 127.7\left(\mathrm{C}_{\mathrm{q}}\right), 127.6(\mathrm{CH}), 124.4(\mathrm{CH}), 122.4(\mathrm{CH}), 121.7(\mathrm{CH}), 121.3(\mathrm{CH}), 120.7(\mathrm{CH})$, $111.4(\mathrm{CH}), 110.1\left(\mathrm{~d},{ }^{2} J_{\mathrm{C}-\mathrm{F}}=9.4 \mathrm{~Hz}, \mathrm{CH}\right), 107.6\left(\mathrm{~d},{ }^{3} J_{\mathrm{C}-\mathrm{F}}=4.5 \mathrm{~Hz}, \mathrm{CH}\right)$.

${ }^{19}$ F-NMR $\left(376 \mathrm{MHz}, \mathrm{CDCl}_{3}\right): \delta=-91.9(\mathrm{~d}, J=18.9 \mathrm{~Hz}),-105.9(\mathrm{~d}, J=38.0 \mathrm{~Hz})$.

IR (ATR): 3054, 3024, 1586, 1467, 1436, 1382, 1349, 1147, 783, $739 \mathrm{~cm}^{-1}$.

MS (ESI) $m / z$ (relative intensity): $337\left([\mathrm{M}+\mathrm{Na}]^{+}, 100\right), 315\left([\mathrm{M}+\mathrm{H}]^{+}, 20\right)$.

HR-MS (ESI): $m / z$ calcd. for $\left[\mathrm{C}_{21} \mathrm{H}_{16} \mathrm{FN}_{2}\right]^{+}[\mathrm{M}+\mathrm{H}]^{+} 315.1292$, found 315.1300 . 


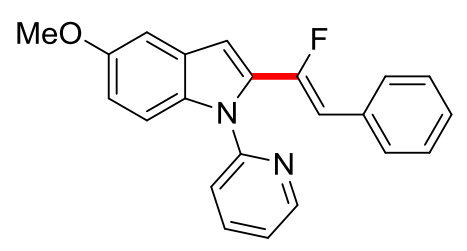

(Z)-2-(1-Fluoro-2-phenylvinyl)-5-methoxy-1-(pyridin-2-yl)-1H-indole $\quad$ (159ba): $\quad$ The general procedure $\mathbf{G}$ was followed using 5-methoxy-1-(pyridin-2-yl)-1 $H$-indole (105b) (112 mg, $0.50 \mathrm{mmol}$ ) and 1-(2,2-difluorovinyl)-benzene (128a) (105 $\mathrm{mg}, 0.75 \mathrm{mmol})$. Isolation by column chromatography ( $n$-hexane/EtOAc: 10/1) yielded 159ba (150 mg, 87\%, Z/E > 99:1) as a colorless oil.

${ }^{1} \mathbf{H}-\mathbf{N M R}\left(300 \mathrm{MHz}, \mathrm{CDCl}_{3}\right): \delta=8.64(\mathrm{ddd}, J=4.9,2.0,0.8 \mathrm{~Hz}, 1 \mathrm{H}), 7.82(\mathrm{ddd}, J=8.0,7.6,2.0 \mathrm{~Hz}$, $1 \mathrm{H}), 7.51-7.46(\mathrm{~m}, 3 \mathrm{H}), 7.58(\mathrm{ddd}, J=8.0,1.0,0.9 \mathrm{~Hz}, 1 \mathrm{H}), 7.35-7.27(\mathrm{~m}, 3 \mathrm{H}), 7.26-7.19(\mathrm{~m}, 1 \mathrm{H})$, $7.09(\mathrm{~d}, J=2.5 \mathrm{~Hz}, 1 \mathrm{H}), 6.94(\mathrm{~d}, J=0.9 \mathrm{~Hz}, 1 \mathrm{H}), 6.92(\mathrm{dd}, J=9.0,2.5 \mathrm{~Hz}, 1 \mathrm{H}), 6.02(\mathrm{~d}, J=37.9 \mathrm{~Hz}$, $1 \mathrm{H}, \mathrm{Z}), 3.86(\mathrm{~s}, 3 \mathrm{H})$.

${ }^{13}$ C-NMR $\left(125 \mathrm{MHz}, \mathrm{CDCl}_{3}\right): \delta=155.2\left(\mathrm{C}_{\mathrm{q}}\right), 151.6\left(\mathrm{C}_{\mathrm{q}}\right), 150.8\left(\mathrm{~d},{ }^{1} J_{\mathrm{C}-\mathrm{F}}=258 \mathrm{~Hz}, \mathrm{C}_{\mathrm{q}}\right), 149.2(\mathrm{CH})$, $138.2(\mathrm{CH}), 133.7\left(\mathrm{C}_{\mathrm{q}}\right), 133.2\left(\mathrm{~d},{ }^{4} J_{\mathrm{C}-\mathrm{F}}=3.9 \mathrm{~Hz}, \mathrm{C}_{\mathrm{q}}\right), 132.3\left(\mathrm{~d},{ }^{2} J_{\mathrm{C}-\mathrm{F}}=30.1 \mathrm{~Hz}, \mathrm{C}_{\mathrm{q}}\right), 128.7\left(\mathrm{~d},{ }^{4} J_{\mathrm{C}-\mathrm{F}}=\right.$ $7.6 \mathrm{~Hz}, \mathrm{CH}), 128.4(\mathrm{CH}), 128.2\left(\mathrm{C}_{\mathrm{q}}\right), 127.4(\mathrm{CH}), 122.0(\mathrm{CH}), 120.3(\mathrm{CH}), 114.5(\mathrm{CH}), 112.2(\mathrm{CH})$, $109.8\left(\mathrm{~d},{ }^{2} J_{\mathrm{C}-\mathrm{F}}=9.7 \mathrm{~Hz}, \mathrm{CH}\right), 107.2\left(\mathrm{~d},{ }^{3} J_{\mathrm{C}-\mathrm{F}}=3.9 \mathrm{~Hz}, \mathrm{CH}\right), 102.3(\mathrm{CH}), 55.7\left(\mathrm{CH}_{3}\right)$.

${ }^{19}$ F-NMR $\left(282 \mathrm{MHz}, \mathrm{CDCl}_{3}\right): \delta=-106.0(\mathrm{~d}, J=37.9 \mathrm{~Hz})$.

IR (ATR): 3056, 2949, 1586, 1467, 1435, 1216, 1201, 1031, $839 \mathrm{~cm}^{-1}$.

MS (ESI) $m / z$ (relative intensity): $345\left([\mathrm{M}+\mathrm{H}]^{+}, 100\right), 325$ (20).

HR-MS (ESI): $m / z$ calcd. for $\left[\mathrm{C}_{22} \mathrm{H}_{18} \mathrm{FN}_{2} \mathrm{O}\right]^{+}[\mathrm{M}+\mathrm{H}]^{+} 345.1398$, found 345.1405 .

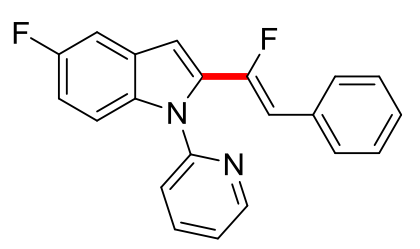

(Z)-2-(1-Fluoro-2-phenylvinyl)-5-fluoro-1-(pyridin-2-yl)-1H-indole $\quad$ (159ma): $\quad$ The general procedure $\mathbf{G}$ was followed using 5-fluoro-1-(pyridin-2-yl)-1H-indole (105m) (106 mg, $0.50 \mathrm{mmol})$ and 1-(2,2-difluorovinyl)-benzene (128a) $(105 \mathrm{mg}, 0.75 \mathrm{mmol})$. Isolation by column chromatography ( $n$-hexane/EtOAc: 10/1) yielded 159ma (153 mg, 92\%, Z/E > 99:1) as a colorless oil.

${ }^{1} \mathbf{H}-\mathbf{N M R}\left(300 \mathrm{MHz}, \mathrm{CDCl}_{3}\right): \delta=8.66(\mathrm{ddd}, J=4.9,2.0,0.8 \mathrm{~Hz}, 1 \mathrm{H}), 7.85(\mathrm{ddd}, J=8.0,7.6,2.0 \mathrm{~Hz}$, $1 \mathrm{H}), 7.52-7.45(\mathrm{~m}, 3 \mathrm{H}), 7.41(\mathrm{ddd}, J=8.0,1.0,0.8 \mathrm{~Hz}, 1 \mathrm{H}), 7.36-7.30(\mathrm{~m}, 3 \mathrm{H}), 7.29-7.21(\mathrm{~m}, 2 \mathrm{H})$, $7.01(\mathrm{ddd}, J=9.0,2.5,0.7 \mathrm{~Hz}, 1 \mathrm{H}), 6.96(\mathrm{dd}, J=0.9,0.9 \mathrm{~Hz}, 1 \mathrm{H}), 6.04(\mathrm{~d}, J=37.8 \mathrm{~Hz}, 1 \mathrm{H}, Z)$.

${ }^{13} \mathbf{C}-\mathbf{N M R}\left(125 \mathrm{MHz}, \mathrm{CDCl}_{3}\right): \delta=158.6\left(\mathrm{~d},{ }^{1} J_{\mathrm{C}-\mathrm{F}}=238 \mathrm{~Hz}, \mathrm{C}_{\mathrm{q}}\right), 151.4\left(\mathrm{C}_{\mathrm{q}}\right), 150.4\left(\mathrm{~d},{ }^{1} J_{\mathrm{C}-\mathrm{F}}=256 \mathrm{~Hz}\right.$, $\left.\mathrm{C}_{\mathrm{q}}\right), 149.3(\mathrm{CH}), 138.4(\mathrm{CH}), 135.0\left(\mathrm{C}_{\mathrm{q}}\right), 133.5\left(\mathrm{~d},{ }^{2} J_{\mathrm{C}-\mathrm{F}}=30.3 \mathrm{~Hz}, \mathrm{C}_{\mathrm{q}}\right), 133.0\left(\mathrm{~d},{ }^{4} J_{\mathrm{C}-\mathrm{F}}=4.0 \mathrm{~Hz}, \mathrm{C}_{\mathrm{q}}\right)$, $128.8\left(\mathrm{~d},{ }^{4} J_{\mathrm{C}-\mathrm{F}}=8.2 \mathrm{~Hz}, \mathrm{CH}\right), 128.5(\mathrm{CH}), 128.1\left(\mathrm{~d},{ }^{3} J_{\mathrm{C}-\mathrm{F}}=10.2 \mathrm{~Hz}, \mathrm{C}_{\mathrm{q}}\right), 127.6(\mathrm{CH}), 122.4(\mathrm{CH})$, 
$120.4(\mathrm{CH}), 112.6\left(\mathrm{~d},{ }^{2} J_{\mathrm{C}-\mathrm{F}}=26.0 \mathrm{~Hz}, \mathrm{CH}\right), 112.2\left(\mathrm{~d},{ }^{3} J_{\mathrm{C}-\mathrm{F}}=9.9 \mathrm{~Hz}, \mathrm{CH}\right), 110.4\left(\mathrm{~d},{ }^{2} J_{\mathrm{C}-\mathrm{F}}=8.8 \mathrm{~Hz}\right.$, $\mathrm{CH}), 107.0\left(\mathrm{~d},{ }^{3} J_{\mathrm{C}-\mathrm{F}}=4.6 \mathrm{~Hz}, \mathrm{CH}\right), 105.9\left(\mathrm{~d},{ }^{2} J_{\mathrm{C}-\mathrm{F}}=23.9 \mathrm{~Hz}, \mathrm{CH}\right)$.

${ }^{19}$ F-NMR $\left(282 \mathrm{MHz}, \mathrm{CDCl}_{3}\right): \delta=-106.4(\mathrm{~d}, J=37.8 \mathrm{~Hz}),-122.2(\mathrm{~m})$.

IR (ATR): 3057, 1585, 1465, 1436, 1382, 1185, 782, 744, $662 \mathrm{~cm}^{-1}$.

MS (EI) $m / z$ (relative intensity): 332 ([M] $\left.]^{+}, 100\right), 311(80), 255$ (35).

HR-MS (EI): $m / z$ calcd. for $\left[\mathrm{C}_{21} \mathrm{H}_{14} \mathrm{~F}_{2} \mathrm{~N}_{2}\right]^{+}[\mathrm{M}]^{+} 332.1125$, found 332.1125 .

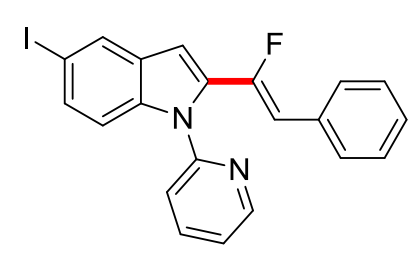

(Z)-2-(1-Fluoro-2-phenylvinyl)-5-iodo-1-(pyridin-2-yl)-1H-indole (159da): A modified procedure $\mathbf{G}$ was followed using 5-iodo-1-(pyridin-2-yl)- $1 H$-indole (105d) $(160 \mathrm{mg}, 0.50 \mathrm{mmol})$ and 1-(2,2difluorovinyl)-benzene (128a) $(105 \mathrm{mg}, 0.75 \mathrm{mmol})$ at $50{ }^{\circ} \mathrm{C}$. Isolation by column chromatography $(n$ hexane/EtOAc: $10 / 1)$ yielded 159da (183 $\mathrm{mg}, 83 \%, Z / E=98: 2)$ as a yellow oil.

${ }^{1}$ H-NMR $\left(300 \mathrm{MHz}, \mathrm{CDCl}_{3}\right.$ ): $\delta=8.64$ (ddd, $\left.J=4.9,2.0,0.8 \mathrm{~Hz}, 1 \mathrm{H}\right), 7.98(\mathrm{dd}, J=1.7,0.5 \mathrm{~Hz}, 1 \mathrm{H})$, 7.84 (ddd, $J=8.0,7.6,2.0 \mathrm{~Hz}, 1 \mathrm{H}), 7.50$ (ddd, $J=4.9,1.0,0.8 \mathrm{~Hz}, 1 \mathrm{H}), 7.47-7.40$ (m, 2H), 7.38 (ddd, $J=8.0,1.7,0.8 \mathrm{~Hz}, 1 \mathrm{H}), 7.36-7.30(\mathrm{~m}, 3 \mathrm{H}), 7.30-7.21(\mathrm{~m}, 2 \mathrm{H}), 6.90(\mathrm{~d}, J=0.9 \mathrm{~Hz}, 1 \mathrm{H}), 6.47(\mathrm{~d}, J=$ $18.7 \mathrm{~Hz}, 0.02 \mathrm{H}, E), 6.03(\mathrm{~d}, J=37.9 \mathrm{~Hz}, 0.98 \mathrm{H}, Z)$.

${ }^{13}$ C-NMR (125 MHz, $\left.\mathrm{CDCl}_{3}\right): \delta=151.1\left(\mathrm{C}_{\mathrm{q}}\right), 150.1\left(\mathrm{~d},{ }^{1} J_{\mathrm{C}-\mathrm{F}}=258 \mathrm{~Hz}, \mathrm{C}_{\mathrm{q}}\right), 149.4(\mathrm{CH}), 138.4(\mathrm{CH})$, $137.6\left(\mathrm{C}_{\mathrm{q}}\right), 132.9\left(\mathrm{~d},{ }^{4} J_{\mathrm{C}-\mathrm{F}}=4.6 \mathrm{~Hz}, \mathrm{C}_{\mathrm{q}}\right), 132.8\left(\mathrm{~d},{ }^{2} J_{\mathrm{C}-\mathrm{F}}=30.3 \mathrm{~Hz}, \mathrm{C}_{\mathrm{q}}\right), 132.4(\mathrm{CH}), 130.0\left(\mathrm{C}_{\mathrm{q}}\right), 129.8$ $(\mathrm{CH}), 128.8\left(\mathrm{~d},{ }^{4} J_{\mathrm{C}-\mathrm{F}}=7.7 \mathrm{~Hz}, \mathrm{CH}\right), 128.4(\mathrm{CH}), 127.6(\mathrm{CH}), 122.5(\mathrm{CH}), 120.4(\mathrm{CH}), 113.2(\mathrm{CH})$, $110.6\left(\mathrm{~d},{ }^{2} J_{\mathrm{C}-\mathrm{F}}=8.8 \mathrm{~Hz}, \mathrm{CH}\right), 106.1\left(\mathrm{~d},{ }^{3} J_{\mathrm{C}-\mathrm{F}}=4.3 \mathrm{~Hz}, \mathrm{CH}\right), 84.4\left(\mathrm{C}_{\mathrm{q}}\right)$.

${ }^{19}$ F-NMR $\left(282 \mathrm{MHz}, \mathrm{CDCl}_{3}\right): \delta=-93.1(\mathrm{~d}, J=18.7 \mathrm{~Hz}, E),-106.7(\mathrm{~d}, J=37.9 \mathrm{~Hz}, Z)$.

IR (ATR): 3056, 1587, 1470, 1469, 1379, 1239, 1207, 732, $693 \mathrm{~cm}^{-1}$.

MS (ESI) $m / z$ (relative intensity): $441\left([\mathrm{M}+\mathrm{H}]^{+}, 100\right), 436(15)$.

HR-MS (ESI): $m / z$ calcd. for $\left[\mathrm{C}_{21} \mathrm{H}_{15} \mathrm{FN}_{2} \mathrm{I}\right]^{+}[\mathrm{M}+\mathrm{H}]^{+} 441.0258$, found 441.0251 .

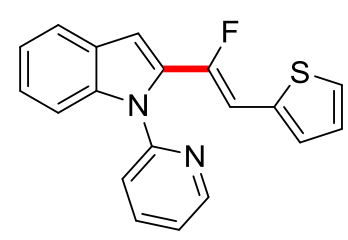

(Z)-2-[1-Fluoro-2-(thiophen-2-yl)vinyl]-1-(pyridin-2-yl)-1H-indole (159ab): The general procedure G was followed using 1-(pyridin-2-yl)-1H-indole (105a) $(97.1 \mathrm{mg}, 0.50 \mathrm{mmol})$ and 2-(2,2- 
difluorovinyl)thiophene (128b) (110 $\mathrm{mg}, 0.75 \mathrm{mmol})$. Isolation by column chromatography ( $n$ hexane/EtOAc: 10/1) yielded 159ab $(154 \mathrm{mg}, 96 \%, Z / E=96: 4)$ as a yellow oil.

${ }^{1}$ H-NMR $\left(300 \mathrm{MHz}, \mathrm{CDCl}_{3}\right): \delta=8.64$ (ddd, $\left.J=4.9,2.0,0.8 \mathrm{~Hz}, 1 \mathrm{H}\right), 7.84(\mathrm{ddd}, J=8.0,7.6,2.0 \mathrm{~Hz}$, $1 \mathrm{H}), 7.67$ (ddd, $J=7.7,1.2,0.9 \mathrm{~Hz}, 1 \mathrm{H}), 7.56-7.51(\mathrm{~m}, 1 \mathrm{H}), 7.42$ (ddd, $J=8.0,1.0,0.8 \mathrm{~Hz}, 1 \mathrm{H}$ ), $7.37-7.35(\mathrm{~m}, 1 \mathrm{H}), 7.32(\mathrm{ddd}, J=7.6,4.9,1.0 \mathrm{~Hz}, 1 \mathrm{H}), 7.29-7.23(\mathrm{~m}, 3 \mathrm{H}), 7.20$ (dd, $J=7.1,1.2 \mathrm{~Hz}$, $1 \mathrm{H}), 6.99(\mathrm{~d}, J=0.9 \mathrm{~Hz}, 1 \mathrm{H}), 6.51(\mathrm{~d}, J=17.9 \mathrm{~Hz}, 0.04 \mathrm{H}, E), 6.11(\mathrm{~d}, J=37.7 \mathrm{~Hz}, 0.96 \mathrm{H}, Z)$.

${ }^{13}$ C-NMR $\left(75 \mathrm{MHz}, \mathrm{CDCl}_{3}\right): \delta=151.8\left(\mathrm{C}_{\mathrm{q}}\right), 150.2\left(\mathrm{~d},{ }^{1} J_{\mathrm{C}-\mathrm{F}}=260 \mathrm{~Hz}, \mathrm{C}_{\mathrm{q}}\right), 149.4(\mathrm{CH}), 138.6\left(\mathrm{C}_{\mathrm{q}}\right)$, $138.3(\mathrm{CH}), 133.9\left(\mathrm{~d},{ }^{4} J_{\mathrm{C}-\mathrm{F}}=3.3 \mathrm{~Hz}, \mathrm{C}_{\mathrm{q}}\right), 131.8\left(\mathrm{~d},{ }^{2} J_{\mathrm{C}-\mathrm{F}}=29.7 \mathrm{~Hz}, \mathrm{C}_{\mathrm{q}}\right), 128.2\left(\mathrm{~d},{ }^{4} J_{\mathrm{C}-\mathrm{F}}=5.8 \mathrm{~Hz}, \mathrm{CH}\right)$, $127.8\left(\mathrm{C}_{\mathrm{q}}\right), 125.3(\mathrm{CH}), 124.1(\mathrm{CH}), 124.0(\mathrm{CH}), 122.3(\mathrm{CH}), 121.6(\mathrm{CH}), 121.2(\mathrm{CH}), 120.6(\mathrm{CH})$, $111.2(\mathrm{CH}), 107.1\left(\mathrm{~d},{ }^{3} J_{\mathrm{C}-\mathrm{F}}=4.4 \mathrm{~Hz}, \mathrm{CH}\right), 104.7\left(\mathrm{~d},{ }^{2} J_{\mathrm{C}-\mathrm{F}}=13.5 \mathrm{~Hz}, \mathrm{CH}\right)$.

${ }^{19}$ F-NMR $\left(282 \mathrm{MHz}, \mathrm{CDCl}_{3}\right): \delta=-93.5(\mathrm{~d}, J=17.9 \mathrm{~Hz}, E),-106.0(\mathrm{~d}, J=37.7 \mathrm{~Hz}, Z)$.

IR (ATR): 3053, 1585, 1467, 1435, 1347, 1294, 1072, 773, $728 \mathrm{~cm}^{-1}$.

MS (EI) $m / z$ (relative intensity): 320 ([M] $\left.]^{+}, 100\right), 299$ (90), 241 (15).

HR-MS (EI): $m / z$ calcd. for $\left[\mathrm{C}_{19} \mathrm{H}_{13} \mathrm{FN}_{2} \mathrm{~S}\right]^{+}[\mathrm{M}]^{+} 320.0783$, found 320.0788 .

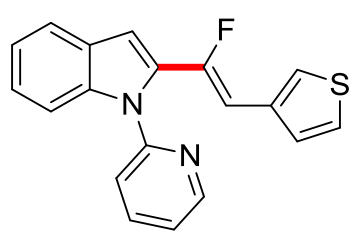

(Z)-2-[1-Fluoro-2-(thiophen-3-yl)vinyl]-1-(pyridin-2-yl)-1H-indole (159ac): The general procedure G was followed using 1-(pyridin-2-yl)-1H-indole (105a) (97.1 mg, $0.50 \mathrm{mmol}$ ) and 3-(2,2difluorovinyl)thiophene $(\mathbf{1 2 8 c})(110 \mathrm{mg}, 0.75 \mathrm{mmol})$. Isolation by column chromatography ( $n$ hexane/EtOAc: $10 / 1)$ yielded 159ac $(159 \mathrm{mg}, 99 \%, Z / E=90: 10)$ as a yellow oil.

${ }^{1} \mathbf{H}-\mathbf{N M R}\left(400 \mathrm{MHz}, \mathrm{CDCl}_{3}\right): \delta=8.64(\mathrm{ddd}, J=4.9,2.0,0.8 \mathrm{~Hz}, 1 \mathrm{H}), 7.87(\mathrm{ddd}, J=8.0,7.6,2.0 \mathrm{~Hz}$, $1 \mathrm{H}), 7.65(\mathrm{ddd}, J=7.9,1.3,0.9 \mathrm{~Hz}, 1 \mathrm{H}), 7.56-7.54(\mathrm{~m}, 1 \mathrm{H}), 7.41(\mathrm{ddd}, J=8.0,1.0,0.8 \mathrm{~Hz}, 1 \mathrm{H}), 7.34$ (ddd, $J=7.6,4.9,1.0 \mathrm{~Hz}, 1 \mathrm{H}), 7.27(\mathrm{dd}, J=5.1,1.0 \mathrm{~Hz}, 1 \mathrm{H}), 7.22-7.16(\mathrm{~m}, 2 \mathrm{H}), 7.03(\mathrm{dd}, J=3.6$, $1.0 \mathrm{~Hz}, 1 \mathrm{H}), 6.99(\mathrm{~d}, J=0.9 \mathrm{~Hz}, 1 \mathrm{H}), 6.98(\mathrm{dd}, J=5.1,3.6 \mathrm{~Hz}, 1 \mathrm{H}), 6.67(\mathrm{~d}, J=17.9 \mathrm{~Hz}, 0.10 \mathrm{H}, E)$, $6.27(\mathrm{~d}, J=37.7 \mathrm{~Hz}, 0.90 \mathrm{H}, Z)$.

${ }^{13}$ C-NMR $\left(75 \mathrm{MHz}, \mathrm{CDCl}_{3}\right): \delta=151.6\left(\mathrm{C}_{\mathrm{q}}\right), 149.4\left(\mathrm{~d},{ }^{1} J_{\mathrm{C}-\mathrm{F}}=260 \mathrm{~Hz}, \mathrm{C}_{\mathrm{q}}\right), 148.4(\mathrm{CH}), 138.5\left(\mathrm{C}_{\mathrm{q}}\right)$, $138.3(\mathrm{CH}), 135.5\left(\mathrm{~d},{ }^{4} J_{\mathrm{C}-\mathrm{F}}=3.3 \mathrm{~Hz}, \mathrm{C}_{\mathrm{q}}\right), 131.4\left(\mathrm{~d},{ }^{2} J_{\mathrm{C}-\mathrm{F}}=29.7 \mathrm{~Hz}, \mathrm{C}_{\mathrm{q}}\right), 127.8\left(\mathrm{C}_{\mathrm{q}}\right), 127.3(\mathrm{CH}), 126.9$ $(\mathrm{CH}), 126.3\left(\mathrm{~d},{ }^{4} J_{\mathrm{C}-\mathrm{F}}=9.5 \mathrm{~Hz}, \mathrm{CH}\right), 124.3(\mathrm{CH}), 122.5(\mathrm{CH}), 121.6(\mathrm{CH}), 121.2(\mathrm{CH}), 120.6(\mathrm{CH})$, $111.2(\mathrm{CH}), 107.3\left(\mathrm{~d},{ }^{3} J_{\mathrm{C}-\mathrm{F}}=4.4 \mathrm{~Hz}, \mathrm{CH}\right), 104.2\left(\mathrm{~d},{ }^{2} J_{\mathrm{C}-\mathrm{F}}=13.5 \mathrm{~Hz}, \mathrm{CH}\right)$.

${ }^{19}$ F-NMR $\left(376 \mathrm{MHz}, \mathrm{CDCl}_{3}\right): \delta=-92.1(\mathrm{~d}, J=17.9 \mathrm{~Hz}, E),-104.6(\mathrm{~d}, J=37.7 \mathrm{~Hz}, Z)$.

IR (ATR): 3058, 1586, 1469, 1450, 1437, 1382, 1214, $780 \mathrm{~cm}^{-1}$.

MS (EI) $\mathrm{m} / \mathrm{z}$ (relative intensity): $320\left([\mathrm{M}]^{+}, 80\right), 299$ (100).

HR-MS (EI): $\mathrm{m} / z$ calcd. for $\left[\mathrm{C}_{19} \mathrm{H}_{13} \mathrm{FN}_{2} \mathrm{~S}\right]^{+}[\mathrm{M}]^{+} 320.0783$, found 320.0775 . 


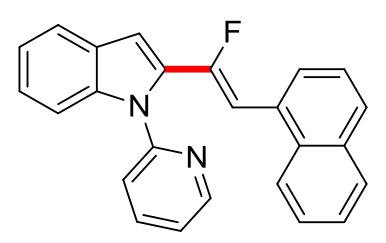

(Z)-2-[1-Fluoro-2-(naphthalen-1-yl)vinyl]-1-(pyridin-2-yl)-1H-indole (159ad): A modified general procedure $\mathbf{G}$ was followed using 1-(pyridin-2-yl)-1H-indole (105a) $(97.1 \mathrm{mg}, 0.50 \mathrm{mmol})$ and 1-(2,2difluorovinyl)naphthalene (128d) $(143 \mathrm{mg}, 0.75 \mathrm{mmol})$ at $70{ }^{\circ} \mathrm{C}$. Isolation by column chromatography ( $n$-hexane/EtOAc: $30 / 1 \rightarrow 20 / 1)$ yielded 159ad $(93.1 \mathrm{mg}, 51 \%, Z / E=92: 8)$ as a white solid.

M.p. $=75^{\circ} \mathrm{C}$.

${ }^{1}$ H-NMR (400 MHz, $\left.\mathrm{CDCl}_{3}\right): \delta=8.72(\mathrm{ddd}, J=4.9,1.9,0.8 \mathrm{~Hz}, 1 \mathrm{H}), 7.90(\mathrm{ddd}, J=8.1,7.6,1.9 \mathrm{~Hz}$, 1H), 7.86-7.77 (m, 4H), $7.72(\mathrm{~d}, J=7.4 \mathrm{~Hz}, 1 \mathrm{H}), 7.54(\mathrm{ddd}, J=8.1,1.0,0.8 \mathrm{~Hz}, 1 \mathrm{H}), 7.51-7.44(\mathrm{~m}$, 4H), 7.34 (ddd, $J=7.6,4.9,1.3 \mathrm{~Hz}, 1 \mathrm{H}$ ), 7.29 (ddd, $J=7.2,7.1,1.3 \mathrm{~Hz}, 1 \mathrm{H}$ ), 7.26-7.24 (m, 1H), 7.15 (d, $J=0.9 \mathrm{~Hz}, 1 \mathrm{H}), 6.92$ (d, $J=17.9 \mathrm{~Hz}, 0.08 \mathrm{H}, E), 6.64$ (d, $J=36.0 \mathrm{~Hz}, 0.92 \mathrm{H}, Z$ ).

${ }^{13} \mathrm{C}-\mathrm{NMR}\left(125 \mathrm{MHz}, \mathrm{CDCl}_{3}\right): \delta=151.9\left(\mathrm{C}_{\mathrm{q}}\right), 151.6\left(\mathrm{~d},{ }^{1} J_{\mathrm{C}-\mathrm{F}}=255 \mathrm{~Hz}, \mathrm{C}_{\mathrm{q}}\right), 149.7(\mathrm{CH}), 138.9\left(\mathrm{C}_{\mathrm{q}}\right)$, $138.6(\mathrm{CH}), 133.7\left(\mathrm{C}_{\mathrm{q}}\right), 132.3\left(\mathrm{~d},{ }^{2} J_{\mathrm{C}-\mathrm{F}}=32.3 \mathrm{~Hz}, \mathrm{C}_{\mathrm{q}}\right), 131.3\left(\mathrm{C}_{\mathrm{q}}\right), 129.2\left(\mathrm{~d},{ }^{4} J_{\mathrm{C}-\mathrm{F}}=3.3 \mathrm{~Hz}, \mathrm{C}_{\mathrm{q}}\right), 128.7$ $\left(\mathrm{d},{ }^{4} J_{\mathrm{C}-\mathrm{F}}=8.2 \mathrm{~Hz}, \mathrm{CH}\right), 128.1(\mathrm{CH}), 127.8\left(\mathrm{C}_{\mathrm{q}}\right), 127.4(\mathrm{CH}), 127.3(\mathrm{CH}), 126.1(\mathrm{CH}), 125.7(\mathrm{CH})$, $125.6(\mathrm{CH}), 124.4(\mathrm{CH}), 123.8(\mathrm{CH}), 121.7(\mathrm{CH}), 121.4(\mathrm{CH}), 121.1(\mathrm{CH}), 111.3(\mathrm{CH}), 107.6\left(\mathrm{~d},{ }^{3} J_{\mathrm{C}-\mathrm{F}}\right.$ $=4.2 \mathrm{~Hz}, \mathrm{CH}), 106.3\left(\mathrm{~d},{ }^{2} J_{\mathrm{C}-\mathrm{F}}=10.5 \mathrm{~Hz}, \mathrm{CH}\right)$.

${ }^{19} \mathbf{F}-\mathbf{N M R}\left(282 \mathrm{MHz}, \mathrm{CDCl}_{3}\right): \delta=-94.2(\mathrm{~d}, J=17.9 \mathrm{~Hz}, E),-107.3(\mathrm{~d}, J=36.0 \mathrm{~Hz}, Z)$.

IR (ATR): 3056, 1586, 1467, 1437, 1213, 1143, 908, $732 \mathrm{~cm}^{-1}$.

MS (EI) $m / z$ (relative intensity): 364 ([M] $\left.]^{+}, 80\right), 343$ (100), 266 (20).

HR-MS (EI): $m / z$ calcd. for $\left[\mathrm{C}_{25} \mathrm{H}_{17} \mathrm{FN}_{2}\right]^{+}[\mathrm{M}]^{+} 364.1376$, found 364.1378 .

\subsubsection{Mechanistic Studies for the Allylative Cobalt(III)-Catalyzed C-H/C-F Activation}

\subsubsection{H/D-Exchange Experiments}

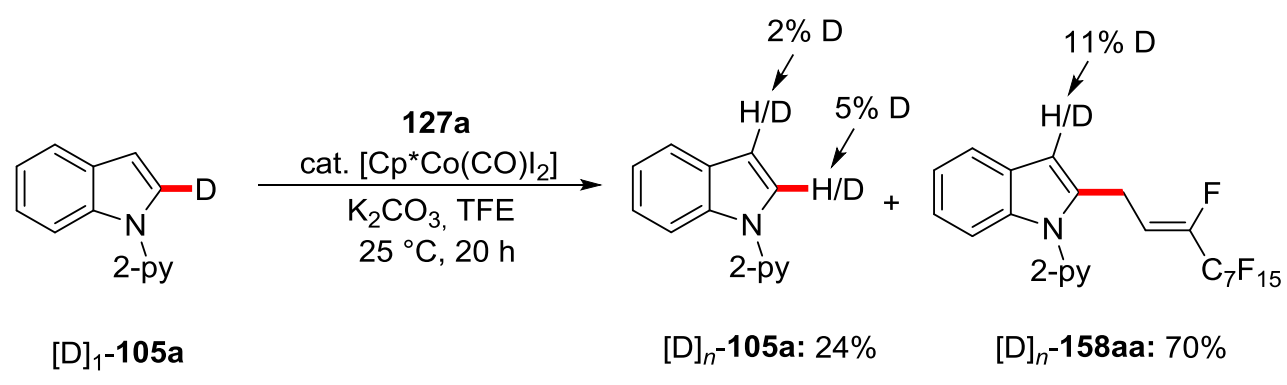

A suspension of $[D]_{1}-\mathbf{1 0 5} \mathbf{a}(117 \mathrm{mg}, 0.60 \mathrm{mmol}, 1.20$ equiv), 127a (223 mg, $0.50 \mathrm{mmol}, 1.00$ equiv), $\left[\mathrm{Cp} * \mathrm{Co}(\mathrm{CO}) \mathrm{I}_{2}\right](6.0 \mathrm{mg}, 2.5 \mathrm{~mol} \%)$ and $\mathrm{K}_{2} \mathrm{CO}_{3}(69.0 \mathrm{mg}, 0.50 \mathrm{mmol}, 1.00$ equiv) in TFE $(0.5 \mathrm{~mL})$ was stirred at $25^{\circ} \mathrm{C}$ for $20 \mathrm{~h}$. After removal of the solvents, the crude mixture was purified by column 
chromatography on silica gel ( $n$-hexane/EtOAc: $30: 1 \rightarrow 20: 1)$ to yield $[\mathrm{D}]_{n}-\mathbf{1 5 8 a a}(214 \mathrm{mg}, 70 \%)$ and $[\mathrm{D}]_{n}$-105a (28.1 mg, 24\% reisolated) as yellow oils.

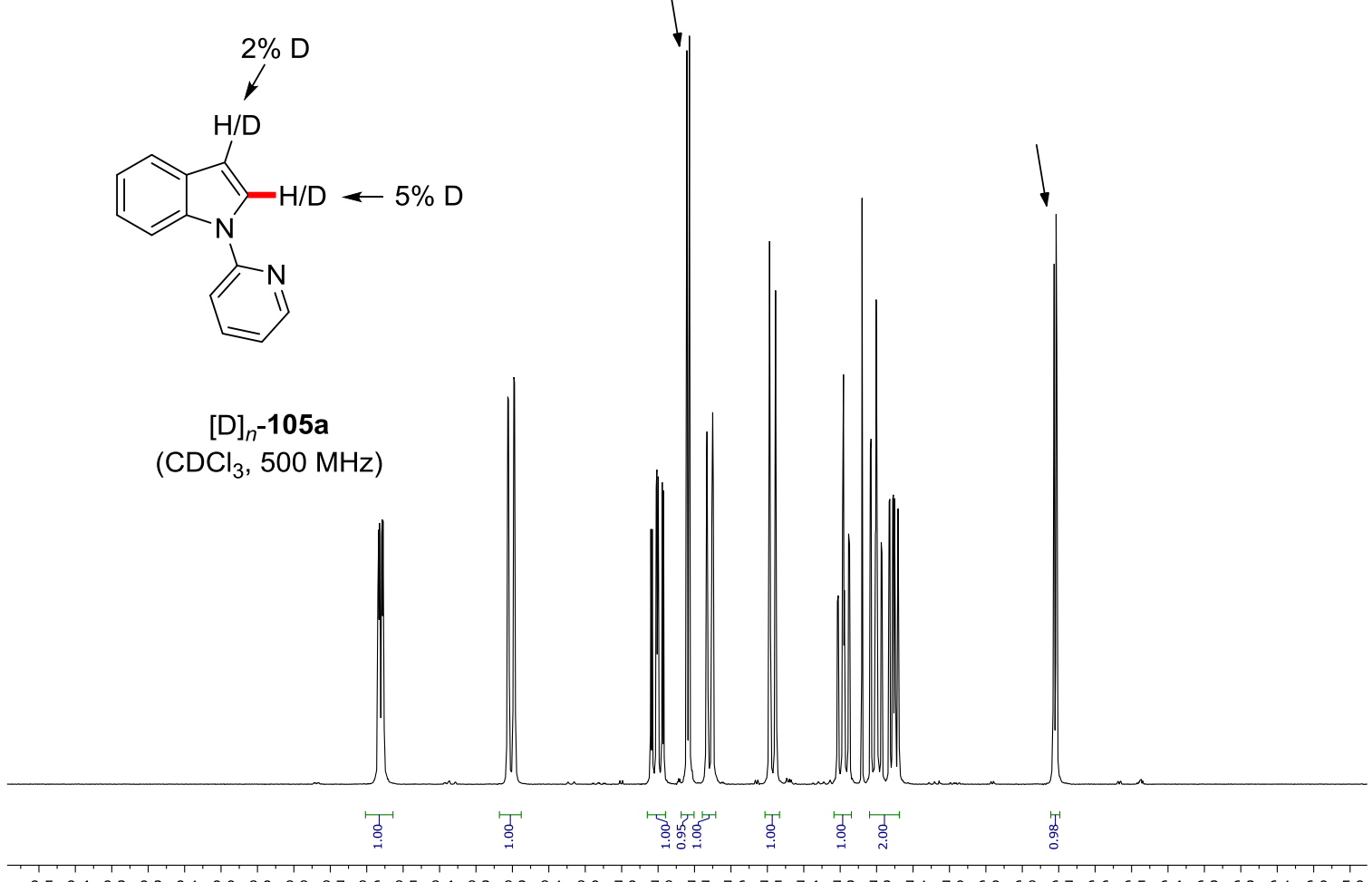

$\begin{array}{llllllllllllllllllllllllllllllllllllllllllllllllll}9.5 & 9.4 & 9.3 & 9.2 & 9.1 & 9.0 & 8.9 & 8.8 & 8.7 & 8.6 & 8.5 & 8.4 & 8.3 & 8.2 & 8.1 & 8.0 & 7.9 & 7.8 & 7.7 & 7.6 & 7.5 & 7.4 & 7.3 & 7.2 & 7.1 & 7.0 & 6.9 & 6.8 & 6.7 & 6.6 & 6.5 & 6.4 & 6.3 & 6.2 & 6.1 & 6.0 & 5.9\end{array}$

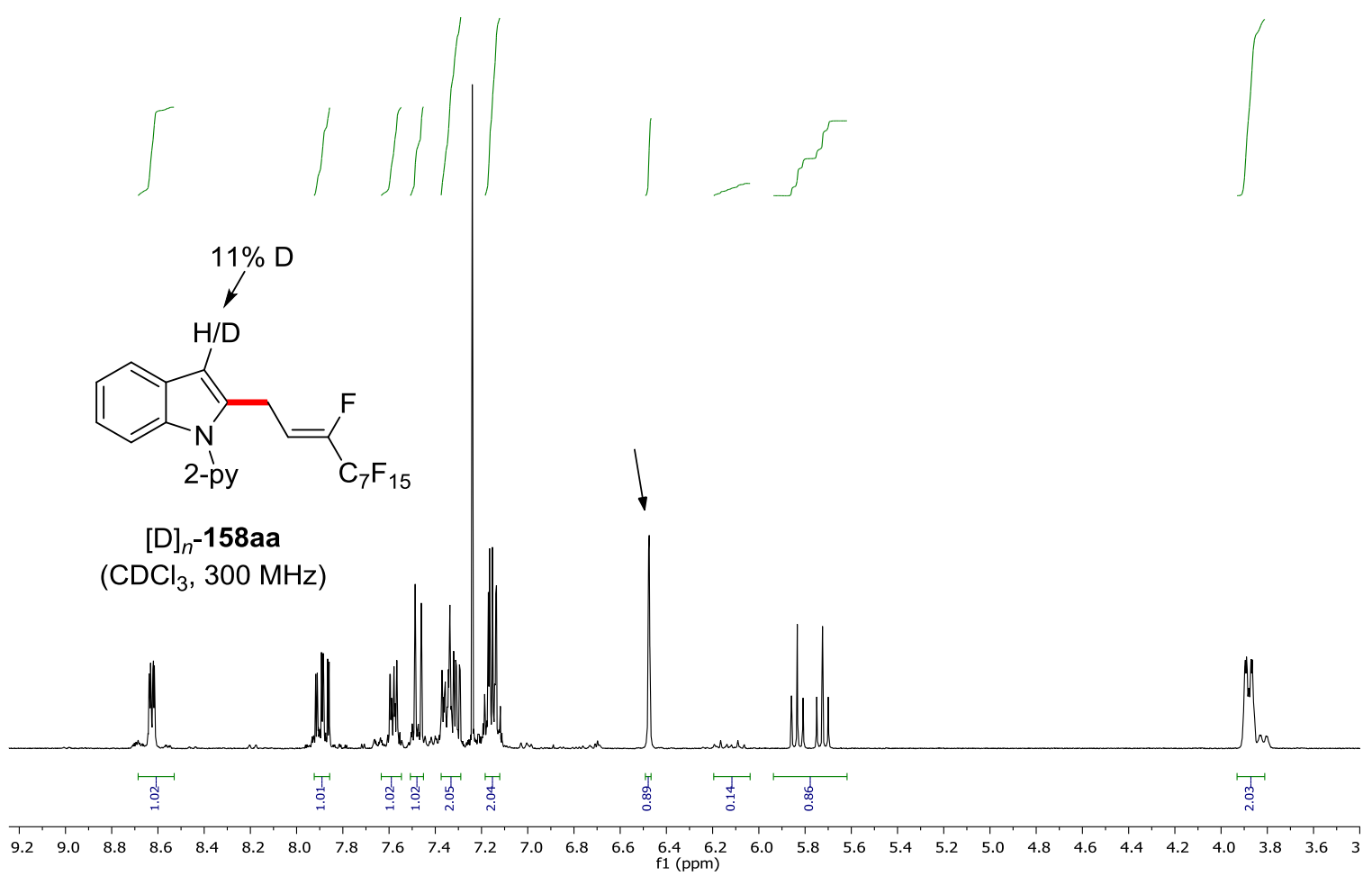




\subsubsection{KIE Studies}

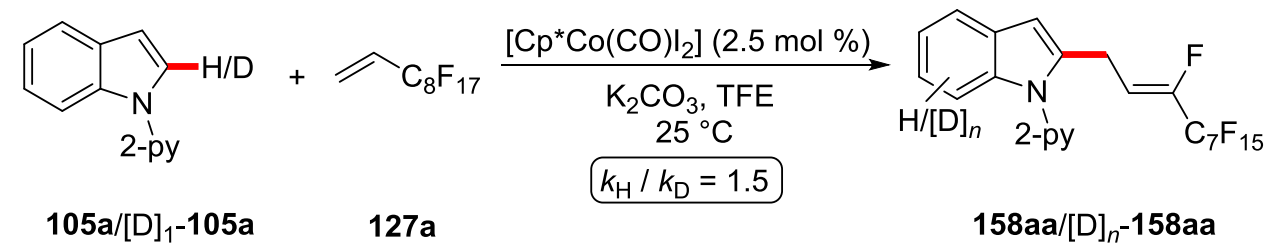

Two parallel reactions of $\mathbf{1 0 5} \mathbf{a}$ and $[D]_{1}-105 \mathbf{a}$ with $\mathbf{1 2 7} \mathbf{a}$ were performed respectively to determine the KIE value by comparison of the initial reaction rates. A suspension of $\mathbf{1 0 5 a}(97.1 \mathrm{mg}, 0.50 \mathrm{mmol}$, 1.00 equiv) or $[\mathrm{D}]_{1}-\mathbf{1 0 5 a}$ ( $97.5 \mathrm{mg}, 0.50 \mathrm{mmol}, 1.00$ equiv), 127a (268 mg, $0.75 \mathrm{mmol}, 1.50$ equiv), $\left[\mathrm{Cp} * \mathrm{Co}(\mathrm{CO}) \mathrm{I}_{2}\right](6.00 \mathrm{mg}, 2.5 \mathrm{~mol} \%), \mathrm{K}_{2} \mathrm{CO}_{3}(69.1 \mathrm{mg}, 1.00$ equiv) and $n$-dodecane $(30 \mu \mathrm{L})$ as the internal standard in TFE $(2.00 \mathrm{~mL})$ was stirred at $25^{\circ} \mathrm{C}$. Aliquots $(25 \mu \mathrm{L})$ were periodically removed to provide the following conversions as determined by GC-analysis:

Table 42. Conversion-time table for determination of the KIE.

\begin{tabular}{c|cccccc}
$t / \min$ & 10 & 20 & 30 & 40 & 50 & 60 \\
\hline $\mathbf{1 5 8 a a} / \%$ & 4.8 & 5.7 & 8.7 & 11.8 & 13.9 & 16.8 \\
{$[\mathrm{D}]_{n}-\mathbf{1 5 8 a a} / \%$} & 3.5 & 5.9 & 6.9 & 9.0 & 10.3 & 12.1
\end{tabular}

\subsubsection{Intermolecular Competition Experiment}

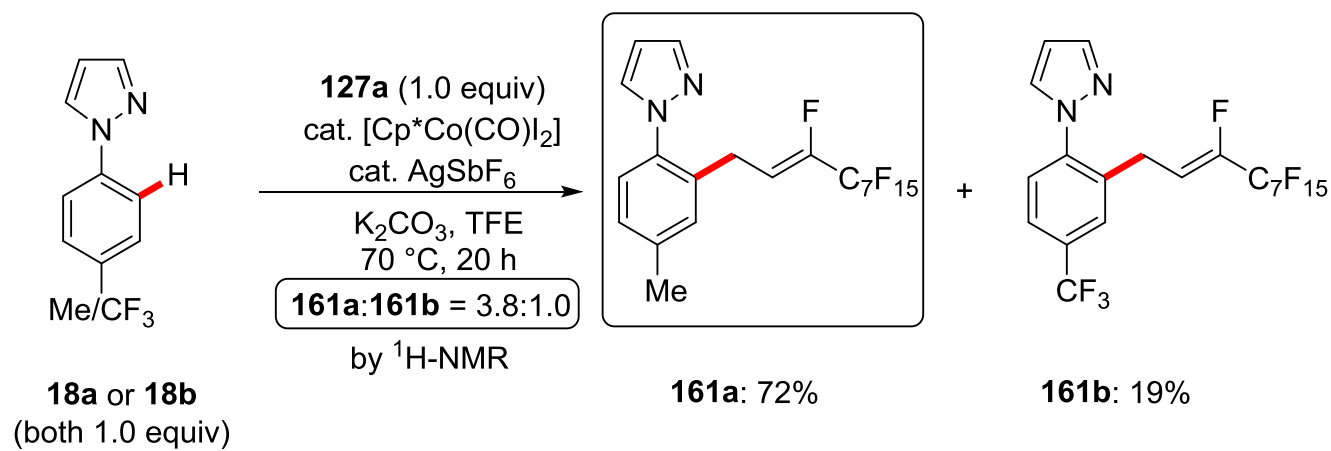

A suspension of 1 -( $p$-tolyl)-1H-pyrazole $\quad(\mathbf{1 8 a}) \quad(79.0 \mathrm{mg}, \quad 0.50 \mathrm{mmol}, \quad 1.00$ equiv), 1 -(4(trifluoromethyl)phenyl)-1 $H$-pyrazole (18b) (106 mg, $0.50 \mathrm{mmol}, 1.00$ equiv), 127a (223 mg, $0.50 \mathrm{mmol}, 1.00$ equiv), [Cp* $\left.\mathrm{Co}(\mathrm{CO}) \mathrm{I}_{2}\right](23.8 \mathrm{mg}, 10.0 \mathrm{~mol} \%), \mathrm{AgSbF}_{6}(34.0 \mathrm{mg}, 20 \mathrm{~mol} \%)$ and $\mathrm{K}_{2} \mathrm{CO}_{3}\left(69.0 \mathrm{mg}, 0.50 \mathrm{mmol}, 1.00\right.$ equiv) in TFE $(0.5 \mathrm{~mL})$ was stirred at $70{ }^{\circ} \mathrm{C}$ for $20 \mathrm{~h}$. After 
filtration over Celite and removal of the solvents, the crude mixture was analyzed by means of ${ }^{1} \mathrm{H}-$ NMR spectroscopy with 1,3,5-trimethoxybenzene $(16.8 \mathrm{mg}, 0.10 \mathrm{mmol})$ as the internal standard to furnish the following conversions: 161a: $72 \%(Z / E=87: 13)$ and 161b: $19 \%(Z / E=86: 14)$. 


\subsection{Manganese(I)-Catalyzed Allylative C-H/C-F Functionalization}

\subsubsection{Characterization Data}<smiles>CC(=O)c1ccccc1C/C=C(\F)C(F)F</smiles>

(Z)-1-[2-(1H,1H,2H-Perfluorodec-2-en-1-yl)phenyl]ethan-1-one (166a): The general procedure $\mathbf{H}$ was followed using $N$-(4-methoxyphenyl)-1-phenylethan-1-imine (6a) (113 mg, 0.50 mmol) and $1 \mathrm{H}, 1 \mathrm{H}, 2 \mathrm{H}$-perfluorodec-1-ene (127a) $(669 \mathrm{mg}, 1.50 \mathrm{mmol})$. Isolation by column chromatography ( $n$ hexane/EtOAc: $25 / 1)$ yielded 166a (197 $\mathrm{mg}, 72 \%, Z / E=97: 3)$ as a yellow oil.

${ }^{1}$ H-NMR $\left(400 \mathrm{MHz}, \mathrm{CDCl}_{3}\right.$ ): $\delta=7.77(\mathrm{dd}, J=7.4,1.4 \mathrm{~Hz}, 1 \mathrm{H}), 7.44(\mathrm{ddd}, J=7.6,7.4,1.4 \mathrm{~Hz}, 1 \mathrm{H})$, $7.34(\mathrm{ddd}, J=7.6,7.5,1.4 \mathrm{~Hz}, 1 \mathrm{H}), 7.27(\mathrm{dd}, J=7.5,1.4 \mathrm{~Hz}, 1 \mathrm{H}), 6.14(\mathrm{dt}, J=23.5,9.5 \mathrm{~Hz}, 0.03 \mathrm{H}$, $E), 5.91(\mathrm{dt}, J=33.5,8.0 \mathrm{~Hz}, 0.97 \mathrm{H}), 3.81(\mathrm{ddt}, J=8.0,4.1,2.1 \mathrm{~Hz}, 2 \mathrm{H}), 2.59(\mathrm{~s}, 3 \mathrm{H})$.

${ }^{13} \mathrm{C}-\mathrm{NMR}\left(100 \mathrm{MHz}, \mathrm{CDCl}_{3}\right): \delta=201.0\left(\mathrm{C}_{\mathrm{q}}\right), 145.9\left(\mathrm{dt},{ }^{1} J_{\mathrm{C}-\mathrm{F}}=257 \mathrm{~Hz},{ }^{2} J_{\mathrm{C}-\mathrm{F}}=29.1 \mathrm{~Hz}, \mathrm{C}_{\mathrm{q}}\right), 137.9$ $\left(\mathrm{C}_{\mathrm{q}}\right), 136.8\left(\mathrm{C}_{\mathrm{q}}\right), 132.4(\mathrm{CH}), 131.5(\mathrm{CH}), 130.1(\mathrm{CH}), 127.1(\mathrm{CH}), 117.4\left(\mathrm{dt},{ }^{1} J_{\mathrm{C}-\mathrm{F}}=288 \mathrm{~Hz},{ }^{2} J_{\mathrm{C}-\mathrm{F}}=\right.$ $\left.34.0 \mathrm{~Hz}, \mathrm{C}_{\mathrm{q}}\right), 115.3\left(\mathrm{dt},{ }^{2} J_{\mathrm{C}-\mathrm{F}}=7.8 \mathrm{~Hz},{ }^{3} J_{\mathrm{C}-\mathrm{F}}=4.1 \mathrm{~Hz}, \mathrm{CH}\right), 113.5\left(\mathrm{~m}, \mathrm{C}_{\mathrm{q}}\right), 113.1\left(\mathrm{~m}, \mathrm{C}_{\mathrm{q}}\right), 111.3(\mathrm{~m}$, $\left.\mathrm{C}_{\mathrm{q}}\right), 110.9\left(\mathrm{~m}, \mathrm{C}_{\mathrm{q}}\right), 110.2\left(\mathrm{~m}, \mathrm{C}_{\mathrm{q}}\right), 108.3\left(\mathrm{~m}, \mathrm{C}_{\mathrm{q}}\right), 29.1\left(\mathrm{CH}_{3}\right), 28.7\left(\mathrm{~d},{ }^{3} J_{\mathrm{C}-\mathrm{F}}=3.5 \mathrm{~Hz}, \mathrm{CH}_{2}\right)$.

${ }^{19} \mathbf{F}-\mathbf{N M R}\left(376 \mathrm{MHz}, \mathrm{CDCl}_{3}\right): \delta=-81.0(\mathrm{~m}),-117.5(\mathrm{~m}),-122.1(\mathrm{~m}),-122.1(\mathrm{~m}),-122.9(\mathrm{~m}),-123.1$ (m), $-126.3(\mathrm{~m}),-132.5(\mathrm{~m})$.

IR (ATR): 1686, 1358, 1236, 1199, 1144, 1106, 759, $732 \mathrm{~cm}^{-1}$.

MS (ESI) $m / z$ (relative intensity): $569\left([\mathrm{M}+\mathrm{Na}]^{+}, 100\right), 547\left([\mathrm{M}+\mathrm{H}]^{+}, 45\right), 373(15)$.

HR-MS (ESI): $\mathrm{m} / z$ calcd. for $\left[\mathrm{C}_{18} \mathrm{H}_{11} \mathrm{~F}_{16} \mathrm{O}\right]^{+}[\mathrm{M}+\mathrm{H}]^{+} 547.0549$, found 547.0543.<smiles>CCC(=O)c1ccccc1C/C=C(\F)C(F)F</smiles>

(Z)-1-[2-(1H,1H,2H-Perfluorodec-2-en-1-yl)phenyl]propan-1-one (166b): The general procedure $\mathbf{H}$ was followed using $N$-(4-methoxyphenyl)-1-phenylpropan-1-imine (6b) (120 mg, $0.50 \mathrm{mmol})$ and $1 H, 1 H, 2 H$-perfluorodec-1-ene (127a) $(669 \mathrm{mg}, 1.50 \mathrm{mmol})$. Isolation by column chromatography ( $n$ hexane/EtOAc: $30 / 1)$ yielded $\mathbf{1 6 6 b}(181 \mathrm{mg}, 65 \%, Z / E=97: 3)$ as a yellow oil.

${ }^{1}$ H-NMR $\left(400 \mathrm{MHz}, \mathrm{CDCl}_{3}\right): \delta=7.71(\mathrm{dd}, J=8.0,1.4 \mathrm{~Hz}, 1 \mathrm{H}), 7.43(\mathrm{ddd}, J=7.7,7.5,1.4 \mathrm{~Hz}, 1 \mathrm{H})$, $7.33(\mathrm{ddd}, J=8.0,7.5,1.3 \mathrm{~Hz}, 1 \mathrm{H}), 7.27(\mathrm{dd}, J=7.7,1.3 \mathrm{~Hz}, 1 \mathrm{H}) 6.13(\mathrm{dt}, J=22.3,8.8 \mathrm{~Hz}, 0.03 \mathrm{H}$, $E), 5.92(\mathrm{dt}, J=33.8,7.7 \mathrm{~Hz}, 0.97 \mathrm{H}, Z), 3.76(\mathrm{ddt}, J=7.7,4.1,2.1 \mathrm{~Hz}, 2 \mathrm{H}), 2.94(\mathrm{q}, J=7.3 \mathrm{~Hz}, 2 \mathrm{H})$, $1.18(\mathrm{t}, J=7.3 \mathrm{~Hz}, 3 \mathrm{H})$. 
${ }^{13}$ C-NMR $\left(100 \mathrm{MHz}, \mathrm{CDCl}_{3}\right): \delta=204.2\left(\mathrm{C}_{\mathrm{q}}\right), 145.2\left(\mathrm{dt},{ }^{1} J_{\mathrm{C}-\mathrm{F}}=259 \mathrm{~Hz},{ }^{2} J_{\mathrm{C}-\mathrm{F}}=28.0 \mathrm{~Hz}, \mathrm{C}_{\mathrm{q}}\right), 137.6$ $\left(\mathrm{C}_{\mathrm{q}}\right), 137.1\left(\mathrm{C}_{\mathrm{q}}\right), 131.9(\mathrm{CH}), 131.4(\mathrm{CH}), 129.1(\mathrm{CH}), 127.0(\mathrm{CH}), 117.4\left(\mathrm{dt},{ }^{1} J_{\mathrm{C}-\mathrm{F}}=288 \mathrm{~Hz},{ }^{2} J_{\mathrm{C}-\mathrm{F}}=\right.$ $\left.34.9 \mathrm{~Hz}, \mathrm{C}_{\mathrm{q}}\right), 115.5\left(\mathrm{dt},{ }^{2} J_{\mathrm{C}-\mathrm{F}}=8.9 \mathrm{~Hz},{ }^{3} J_{\mathrm{C}-\mathrm{F}}=3.6 \mathrm{~Hz}, \mathrm{CH}\right), 113.2\left(\mathrm{~m}, \mathrm{C}_{\mathrm{q}}\right), 110.9\left(\mathrm{~m}, \mathrm{C}_{\mathrm{q}}\right), 110.7(\mathrm{~m}$, $\left.\mathrm{C}_{\mathrm{q}}\right), 110.2\left(\mathrm{~m}, \mathrm{C}_{\mathrm{q}}\right), 108.5\left(\mathrm{~m}, \mathrm{C}_{\mathrm{q}}\right), 107.5\left(\mathrm{~m}, \mathrm{C}_{\mathrm{q}}\right), 34.3\left(\mathrm{CH}_{2}\right), 28.6\left(\mathrm{~d},{ }^{3} J_{\mathrm{C}-\mathrm{F}}=3.6 \mathrm{~Hz}, \mathrm{CH}_{2}\right), 8.20\left(\mathrm{CH}_{3}\right)$. ${ }^{19}$ F-NMR $\left(376 \mathrm{MHz}, \mathrm{CDCl}_{3}\right): \delta=-80.9(\mathrm{~m}),-117.5(\mathrm{~m}),-122.0(\mathrm{~m}),-122.1(\mathrm{~m}),-122.8(\mathrm{~m}),-123.0$ (m), $-126.2(\mathrm{~m}),-132.5(\mathrm{~m})$.

IR (ATR): 1687, 1238, 1203, 1148, 907, $729 \mathrm{~cm}^{-1}$.

MS (ESI) $m / z$ (relative intensity): $583\left([\mathrm{M}+\mathrm{Na}]^{+}, 100\right), 561\left([\mathrm{M}+\mathrm{H}]^{+}, 60\right), 381(15)$.

HR-MS (ESI): $m / z$ calcd. for $\left[\mathrm{C}_{19} \mathrm{H}_{13} \mathrm{~F}_{16} \mathrm{O}\right]^{+}[\mathrm{M}+\mathrm{H}]^{+}$561.0705, found 561.0707.<smiles>COc1ccc(C(=O)[O-])c(C/C=C(\F)C(F)(F)F)c1</smiles>

(Z)-1-[4-Methoxy-2-(1H,1H,2H-perfluorodec-2-en-1-yl)phenyl]ethan-1-one (166c): The general procedure $\mathbf{H}$ was followed using $N$-bis(4-methoxyphenyl)ethan-1-imine (6c) (128 mg, $0.50 \mathrm{mmol}$ ) and $1 H, 1 H, 2 H$-perfluorodec-1-ene (127a) (669 mg, $1.50 \mathrm{mmol}$ ). Isolation by column chromatography ( $n$ hexane/EtOAc: $30 / 1)$ yielded 166c $(181 \mathrm{mg}, 63 \%, Z / E=97: 3)$ as a yellow oil.

${ }^{1} \mathbf{H}-\mathbf{N M R}\left(500 \mathrm{MHz}, \mathrm{CDCl}_{3}\right): \delta=7.81(\mathrm{~d}, J=8.8 \mathrm{~Hz}, 1 \mathrm{H}), 7.82(\mathrm{dd}, J=8.8,2.6 \mathrm{~Hz}, 1 \mathrm{H}), 7.75(\mathrm{~d}, J=$ $2.6 \mathrm{~Hz}, 1 \mathrm{H}), 6.15(\mathrm{dt}, J=23.8,8.6 \mathrm{~Hz}, 0.03 \mathrm{H}, E), 5.92(\mathrm{dt}, J=33.9,7.7 \mathrm{~Hz}, 0.97 \mathrm{H}, Z$ ), 3.86 (ddt, $J=$ 7.7, 4.1, $1.7 \mathrm{~Hz}, 2 \mathrm{H}), 3.84(\mathrm{~s}, 3 \mathrm{H}), 2.55(\mathrm{~s}, 3 \mathrm{H})$.

${ }^{13}$ C-NMR $\left(125 \mathrm{MHz}, \mathrm{CDCl}_{3}\right): \delta=198.9\left(\mathrm{C}_{\mathrm{q}}\right), 162.4\left(\mathrm{C}_{\mathrm{q}}\right), 145.9\left(\mathrm{dt},{ }^{1} J_{\mathrm{C}-\mathrm{F}}=258 \mathrm{~Hz},{ }^{2} J_{\mathrm{C}-\mathrm{F}}=29.0 \mathrm{~Hz}\right.$, $\left.\mathrm{C}_{\mathrm{q}}\right), 141.1\left(\mathrm{C}_{\mathrm{q}}\right), 133.1(\mathrm{CH}), 128.8\left(\mathrm{C}_{\mathrm{q}}\right), 116.9(\mathrm{CH}), 116.5\left(\mathrm{dt},{ }^{1} J_{\mathrm{C}-\mathrm{F}}=287 \mathrm{~Hz},{ }^{2} J_{\mathrm{C}-\mathrm{F}}=31.3 \mathrm{~Hz}, \mathrm{C}_{\mathrm{q}}\right)$, $115.1\left(\mathrm{dt},{ }^{2} J_{\mathrm{C}-\mathrm{F}}=8.9 \mathrm{~Hz},{ }^{3} J_{\mathrm{C}-\mathrm{F}}=3.6 \mathrm{~Hz}, \mathrm{CH}\right), 112.8\left(\mathrm{~m}, \mathrm{C}_{\mathrm{q}}\right), 111.5(\mathrm{CH}), 110.5\left(\mathrm{~m}, \mathrm{C}_{\mathrm{q}}\right), 110.3\left(\mathrm{~m}, \mathrm{C}_{\mathrm{q}}\right)$, $110.0\left(\mathrm{~m}, \mathrm{C}_{\mathrm{q}}\right), 108.2\left(\mathrm{~m}, \mathrm{C}_{\mathrm{q}}\right), 107.9\left(\mathrm{~m}, \mathrm{C}_{\mathrm{q}}\right), 55.2\left(\mathrm{CH}_{3}\right), 29.2\left(\mathrm{~d},{ }^{3} J_{\mathrm{C}-\mathrm{F}}=3.7 \mathrm{~Hz}, \mathrm{CH}_{2}\right), 28.7\left(\mathrm{CH}_{3}\right)$.

${ }^{19}$ F-NMR $\left(470 \mathrm{MHz}, \mathrm{CDCl}_{3}\right): \delta=-81.0(\mathrm{~m}),-117.5(\mathrm{~m}),-122.1(\mathrm{~m}),-122.2(\mathrm{~m}),-122.9(\mathrm{~m}),-123.1$ (m), $-126.3(\mathrm{~m}),-132.6(\mathrm{~m})$.

IR (ATR): 1676, 1604, 1585, 1235, 1199, 1144, 1106, 733, $706 \mathrm{~cm}^{-1}$.

MS (ESI) $m / z$ (relative intensity): $599\left([\mathrm{M}+\mathrm{Na}]^{+}, 90\right), 577\left([\mathrm{M}+\mathrm{H}]^{+}, 100\right)$.

HR-MS (ESI): $m / z$ calcd. for $\left[\mathrm{C}_{19} \mathrm{H}_{13} \mathrm{~F}_{16} \mathrm{O}_{2}\right]^{+}[\mathrm{M}+\mathrm{H}]^{+} 577.0655$, found 577.0661 . 
<smiles>CC(=O)c1ccc(C(F)(F)F)cc1C/C=C(\F)C(F)(F)F</smiles>

(Z)-1-[4-Trifluoromethyl-2-(1H,1H,2H-perfluorodec-2-en-1-yl)phenyl]ethan-1-one (166d): The general procedure $\mathbf{H}$ was followed using 1-(4-trifluoromethylphenyl)- $N$-(4-methoxyphenyl)ethan-1imine (6d) (147 mg, $0.50 \mathrm{mmol})$ and $1 H, 1 H, 2 H$-perfluorodec-1-ene (127a) $(669 \mathrm{mg}, 1.50 \mathrm{mmol})$. Isolation by column chromatography ( $n$-hexane/EtOAc: $30 / 1)$ yielded 166d $(157 \mathrm{mg}, 51 \%, \mathrm{Z} / \mathrm{E}=$ 97:3) as a yellow oil.

${ }^{1}$ H-NMR $\left(400 \mathrm{MHz}, \mathrm{CDCl}_{3}\right): \delta=7.83(\mathrm{~d}, J=8.0 \mathrm{~Hz}, 1 \mathrm{H}), 7.61(\mathrm{dd}, J=8.0,1.4 \mathrm{~Hz}, 1 \mathrm{H}), 7.51(\mathrm{~d}, J=$ $1.4 \mathrm{~Hz}, 1 \mathrm{H}), 6.10(\mathrm{dt}, J=22.6,8.5 \mathrm{~Hz}, 0.03 \mathrm{H}, E), 5.87(\mathrm{dt}, J=33.4,7.8 \mathrm{~Hz}, 0.97 \mathrm{H}, Z), 3.83$ (ddt, $J=$ 7.8, 4.0, 2.0 Hz, 2H), 2.62 (s, 3H).

${ }^{13} \mathrm{C}-\mathrm{NMR}\left(100 \mathrm{MHz}, \mathrm{CDCl}_{3}\right): \delta=200.3\left(\mathrm{C}_{\mathrm{q}}\right), 146.5\left(\mathrm{dt},{ }^{1} J_{\mathrm{C}-\mathrm{F}}=261 \mathrm{~Hz},{ }^{2} J_{\mathrm{C}-\mathrm{F}}=29.5 \mathrm{~Hz}, \mathrm{C}_{\mathrm{q}}\right), 139.9$ $\left(\mathrm{C}_{\mathrm{q}}\right), 138.5\left(\mathrm{C}_{\mathrm{q}}\right), 133.7\left(\mathrm{q},{ }^{2} J_{\mathrm{C}-\mathrm{F}}=33.0 \mathrm{~Hz}, \mathrm{C}_{\mathrm{q}}\right), 130.0(\mathrm{CH}), 128.0\left(\mathrm{q},{ }^{3} J_{\mathrm{C}-\mathrm{F}}=3.8 \mathrm{~Hz}, \mathrm{CH}\right), 124.1(\mathrm{q}$, $\left.{ }^{3} J_{\mathrm{C}-\mathrm{F}}=3.7 \mathrm{~Hz}, \mathrm{CH}\right), 123.4\left(\mathrm{~d},{ }^{1} J_{\mathrm{C}-\mathrm{F}}=273 \mathrm{~Hz}, \mathrm{C}_{\mathrm{q}}\right), 117.0\left(\mathrm{dt},{ }^{1} J_{\mathrm{C}-\mathrm{F}}=286 \mathrm{~Hz},{ }^{2} J_{\mathrm{C}-\mathrm{F}}=22.3 \mathrm{~Hz}, \mathrm{C}_{\mathrm{q}}\right)$, $114.2\left(\mathrm{dt},{ }^{2} J_{\mathrm{C}-\mathrm{F}}=7.8 \mathrm{~Hz},{ }^{3} J_{\mathrm{C}-\mathrm{F}}=3.7 \mathrm{~Hz}, \mathrm{CH}\right), 113.2\left(\mathrm{~m}, \mathrm{C}_{\mathrm{q}}\right), 111.1\left(\mathrm{~m}, \mathrm{C}_{\mathrm{q}}\right), 110.7\left(\mathrm{~m}, \mathrm{C}_{\mathrm{q}}\right), 110.2(\mathrm{~m}$, $\left.\mathrm{C}_{\mathrm{q}}\right), 108.6\left(\mathrm{~m}, \mathrm{C}_{\mathrm{q}}\right), 107.7\left(\mathrm{~m}, \mathrm{C}_{\mathrm{q}}\right), 29.3\left(\mathrm{CH}_{3}\right), 28.3\left(\mathrm{~d},{ }^{3} J_{\mathrm{C}-\mathrm{F}}=3.7 \mathrm{~Hz}, \mathrm{CH}_{2}\right)$.

${ }^{19}$ F-NMR $\left(376 \mathrm{MHz}, \mathrm{CDCl}_{3}\right): \delta=-63.5(\mathrm{~m}),-80.9(\mathrm{~m}),-117.6(\mathrm{~m}),-122.1(\mathrm{~m}),-122.1(\mathrm{~m}),-122.8$ (m), $-123.1(\mathrm{~m}),-126.3(\mathrm{~m}),-130.6(\mathrm{~m})$.

IR (ATR): 1695, 1334, 1238, 1201, 1172, 1130, 1093, 833, $720 \mathrm{~cm}^{-1}$.

MS (EI) $m / z$ (relative intensity): $614\left([\mathrm{M}]^{+}, 100\right), 599$ (85), 579 (20).

HR-MS (ESI): $m / z$ calcd. for $\left[\mathrm{C}_{19} \mathrm{H}_{9} \mathrm{~F}_{19} \mathrm{O}+\mathrm{Na}\right]^{+} 637.0242$, found 637.0222 .<smiles>CC(=O)c1ccc(C#N)cc1C/C=C(\F)C(F)F</smiles>

(Z)-4-Acetyl-3-(1H,1H,2H-perfluorodec-2-en-1-yl)benzonitrile (166e): The general procedure $\mathbf{H}$ was followed using 4-\{1-[(4-methoxyphenyl)imino]ethyl $\}$ benzonitrile $(\mathbf{6 e})(125 \mathrm{mg}, 0.50 \mathrm{mmol})$ and $1 H, 1 H, 2 H$-perfluorodec-1-ene (127a) $(669 \mathrm{mg}, 1.50 \mathrm{mmol})$. Isolation by column chromatography ( $n$ hexane/EtOAc: 20/1) yielded 166e (134 $\mathrm{mg}, 47 \%, Z / E=97: 3)$ as a yellow oil.

${ }^{1}$ H-NMR $\left(400 \mathrm{MHz}, \mathrm{CDCl}_{3}\right): \delta=7.81(\mathrm{~d}, J=7.7 \mathrm{~Hz}, 1 \mathrm{H}), 7.66(\mathrm{dd}, J=7.7,1.9 \mathrm{~Hz}, 1 \mathrm{H}), 7.56(\mathrm{~d}, J=$ $1.9 \mathrm{~Hz}, 1 \mathrm{H}), 6.07(\mathrm{dt}, J=23.8,8.6 \mathrm{~Hz}, 0.03 \mathrm{H}, E), 5.86(\mathrm{dt}, J=33.0,7.7 \mathrm{~Hz}, 0.97 \mathrm{H}, Z), 3.79$ (ddt, $J=$ 7.7, 4.2, $2.1 \mathrm{~Hz}, 2 \mathrm{H}), 2.61$ (s, 3H). 
${ }^{13}$ C-NMR $\left(100 \mathrm{MHz}, \mathrm{CDCl}_{3}\right): \delta=199.9\left(\mathrm{C}_{\mathrm{q}}\right), 146.6\left(\mathrm{dt},{ }^{1} J_{\mathrm{C}-\mathrm{F}}=261 \mathrm{~Hz},{ }^{2} J_{\mathrm{C}-\mathrm{F}}=28.7 \mathrm{~Hz}, \mathrm{C}_{\mathrm{q}}\right), 140.5$ $\left(\mathrm{C}_{\mathrm{q}}\right), 138.8\left(\mathrm{C}_{\mathrm{q}}\right), 134.7(\mathrm{CH}), 130.9(\mathrm{CH}), 130.0(\mathrm{CH}), 117.4\left(\mathrm{C}_{\mathrm{q}}\right), 116.8\left(\mathrm{dt},{ }^{1} J_{\mathrm{C}-\mathrm{F}}=289 \mathrm{~Hz},{ }^{2} J_{\mathrm{C}-\mathrm{F}}=\right.$ $\left.33.8 \mathrm{~Hz}, \mathrm{C}_{\mathrm{q}}\right), 115.8\left(\mathrm{dt},{ }^{2} J_{\mathrm{C}-\mathrm{F}}=8.9 \mathrm{~Hz},{ }^{3} J_{\mathrm{C}-\mathrm{F}}=3.6 \mathrm{~Hz}, \mathrm{CH}\right), 113.9\left(\mathrm{~m}, \mathrm{C}_{\mathrm{q}}\right), 113.0\left(\mathrm{~m}, \mathrm{C}_{\mathrm{q}}\right), 111.2(\mathrm{~m}$, $\left.\mathrm{C}_{\mathrm{q}}\right), 110.7\left(\mathrm{~m}, \mathrm{C}_{\mathrm{q}}\right), 110.3\left(\mathrm{~m}, \mathrm{C}_{\mathrm{q}}\right), 108.3\left(\mathrm{~m}, \mathrm{C}_{\mathrm{q}}\right), 107.6\left(\mathrm{~m}, \mathrm{C}_{\mathrm{q}}\right), 29.4\left(\mathrm{CH}_{3}\right), 28.2\left(\mathrm{~d},{ }^{3} \mathrm{~J}_{\mathrm{C}-\mathrm{F}}=3.7 \mathrm{~Hz}\right.$, $\mathrm{CH}_{2}$ ).

${ }^{19}$ F-NMR $\left(376 \mathrm{MHz}, \mathrm{CDCl}_{3}\right): \delta=-80.8(\mathrm{~m}),-117.5(\mathrm{~m}),-122.0(\mathrm{~m}),-122.0(\mathrm{~m}),-122.8(\mathrm{~m}),-122.9$ (m), $-126.1(\mathrm{~m}),-130.0(\mathrm{~m})$.

IR (ATR): 3051, 2920, 1585, 1467, 1435, 1380, $1348 \mathrm{~cm}^{-1}$.

MS (ESI) $m / z$ (relative intensity): $594\left([\mathrm{M}+\mathrm{Na}]^{+}, 20\right), 572\left([\mathrm{M}+\mathrm{H}]^{+}, 100\right)$.

HR-MS (ESI): $m / z$ calcd. for $\left[\mathrm{C}_{19} \mathrm{H}_{10} \mathrm{~F}_{16} \mathrm{NO}\right]^{+}[\mathrm{M}+\mathrm{H}]^{+} 572.0501$, found 572.0498.<smiles>CC(=O)c1ccc(F)cc1C/C=C(\F)C(F)F</smiles>

(Z)-1-[4-Fluoro-2-(1H,1H,2H-perfluorodec-2-en-1-yl)phenyl]ethan-1-one (166f): The general procedure $\mathbf{H}$ was followed using 1-(4-fluorophenyl)- $N$-(4-methoxyphenyl)ethan-1-imine (6f) (122 mg, $0.50 \mathrm{mmol})$ and $1 \mathrm{H}, 1 \mathrm{H}, 2 \mathrm{H}$-perfluorodec-1-ene (127a) $(669 \mathrm{mg}, 1.50 \mathrm{mmol})$. Isolation by column chromatography ( $n$-hexane/EtOAc: 30/1) yielded 166f (195 mg, 69\%, Z/E = 97:3) as a colorless oil.

${ }^{1} \mathbf{H}-\mathbf{N M R}\left(500 \mathrm{MHz}, \mathrm{CDCl}_{3}\right): \delta=7.82(\mathrm{dd}, J=8.4,5.7 \mathrm{~Hz}, 1 \mathrm{H}), 7.02(\mathrm{ddd}, J=10.1,8.4,2.4 \mathrm{~Hz}, 1 \mathrm{H})$, $6.96(\mathrm{dd}, J=9.4,2.4 \mathrm{~Hz}, 1 \mathrm{H}), 6.11(\mathrm{dt}, J=23.5,8.5 \mathrm{~Hz}, 0.03 \mathrm{H}, E), 5.89(\mathrm{dt}, J=33.6,7.6 \mathrm{~Hz}, 0.97 \mathrm{H}$, Z), 3.83 (ddt, $J=7.6,4.1,1.7 \mathrm{~Hz}, 2 \mathrm{H}), 2.58$ (s, 3H).

${ }^{13} \mathrm{C}-\mathrm{NMR}\left(125 \mathrm{MHz}, \mathrm{CDCl}_{3}\right): \delta=200.3\left(\mathrm{C}_{\mathrm{q}}\right), 161.6\left(\mathrm{~d},{ }^{1} J_{\mathrm{C}-\mathrm{F}}=254 \mathrm{~Hz}, \mathrm{C}_{\mathrm{q}}\right), 145.7\left(\mathrm{dt},{ }^{1} J_{\mathrm{C}-\mathrm{F}}=260 \mathrm{~Hz}\right.$, $\left.{ }^{2} J_{\mathrm{C}-\mathrm{F}}=29.5 \mathrm{~Hz}, \mathrm{C}_{\mathrm{q}}\right), 139.3\left(\mathrm{~d},{ }^{3} J_{\mathrm{C}-\mathrm{F}}=3.2 \mathrm{~Hz}, \mathrm{C}_{\mathrm{q}}\right), 128.3\left(\mathrm{~d},{ }^{3} J_{\mathrm{C}-\mathrm{F}}=8.6 \mathrm{~Hz}, \mathrm{CH}\right), 125.2\left(\mathrm{~d},{ }^{4} J_{\mathrm{C}-\mathrm{F}}=\right.$ $3.8 \mathrm{~Hz}, \mathrm{CH}), 124.6\left(\mathrm{~d},{ }^{2} J_{\mathrm{C}-\mathrm{F}}=16.8 \mathrm{~Hz}, \mathrm{CH}\right), 118.9\left(\mathrm{~d},{ }^{2} J_{\mathrm{C}-\mathrm{F}}=23.7 \mathrm{~Hz}, \mathrm{CH}\right), 116.8\left(\mathrm{dt},{ }^{1} J_{\mathrm{C}-\mathrm{F}}=287 \mathrm{~Hz}\right.$, $\left.{ }^{2} J_{\mathrm{C}-\mathrm{F}}=32.8 \mathrm{~Hz}, \mathrm{C}_{\mathrm{q}}\right), 113.9\left(\mathrm{dt},{ }^{2} J_{\mathrm{C}-\mathrm{F}}=8.9 \mathrm{~Hz},{ }^{3} J_{\mathrm{C}-\mathrm{F}}=3.6 \mathrm{~Hz}, \mathrm{CH}\right), 113.2\left(\mathrm{~m}, \mathrm{C}_{\mathrm{q}}\right), 111.3\left(\mathrm{~m}, \mathrm{C}_{\mathrm{q}}\right), 110.8$ $\left(\mathrm{m}, \mathrm{C}_{\mathrm{q}}\right), 110.2\left(\mathrm{~m}, \mathrm{C}_{\mathrm{q}}\right), 108.3\left(\mathrm{~m}, \mathrm{C}_{\mathrm{q}}\right), 107.5\left(\mathrm{~m}, \mathrm{C}_{\mathrm{q}}\right), 29.4\left(\mathrm{CH}_{3}\right), 20.3\left(\mathrm{dd},{ }^{3} J_{\mathrm{C}-\mathrm{F}}=5.8,4.4 \mathrm{~Hz}, \mathrm{CH}_{2}\right)$.

${ }^{19}$ F-NMR $\left(470 \mathrm{MHz}, \mathrm{CDCl}_{3}\right): \delta=-80.8(\mathrm{~m}),-105.8(\mathrm{~m}),-117.4(\mathrm{~m}),-122.0(\mathrm{~m}),-122.0(\mathrm{~m}),-122.8$ (m), $-123.0(\mathrm{~m}),-126.2(\mathrm{~m}),-131.4(\mathrm{~m})$.

IR (ATR): 1692, 1456, 1237, 1199, 1144, 791, $730 \mathrm{~cm}^{-1}$.

MS (ESI) $m / z$ (relative intensity): $587\left([\mathrm{M}+\mathrm{Na}]^{+}, 100\right), 565\left([\mathrm{M}+\mathrm{H}]^{+}, 15\right)$.

HR-MS (ESI): $m / z$ calcd. for $\left[\mathrm{C}_{18} \mathrm{H}_{10} \mathrm{~F}_{17} \mathrm{O}\right]^{+}[\mathrm{M}+\mathrm{H}]^{+} 565.0455$, found 565.0457 . 
<smiles>CC(=O)c1ccc(Br)cc1CC=C(F)C(F)(F)F</smiles>

(Z)-1-[4-Bromo-2-(1H,1H,2H-perfluorodec-2-en-1-yl)phenyl]ethan-1-one $\quad(166 \mathrm{~g}): \quad$ The general procedure $\mathbf{H}$ was followed using 1-(4-bromophenyl)- $N$-(4-methoxyphenyl)ethan-1-imine (6g) (152 mg, $0.50 \mathrm{mmol})$ and $1 H, 1 H, 2 H$-perfluorodec-1-ene (127a) $(669 \mathrm{mg}, 1.50 \mathrm{mmol})$. Isolation by column chromatography ( $n$-hexane/EtOAc: 30/1) yielded $\mathbf{1 6 6 g}(218 \mathrm{mg}, 71 \%, Z / E=97: 3)$ as a yellow oil.

${ }^{1}$ H-NMR $\left(300 \mathrm{MHz}, \mathrm{CDCl}_{3}\right): \delta=7.64(\mathrm{~d}, J=8.5 \mathrm{~Hz}, 1 \mathrm{H}), 7.49(\mathrm{dd}, J=8.5,2.0 \mathrm{~Hz}, 1 \mathrm{H}), 7.42(\mathrm{~d}, J=$ $2.0 \mathrm{~Hz}, 1 \mathrm{H}), 6.11(\mathrm{dt}, J=22.3,8.8 \mathrm{~Hz}, 0.03 \mathrm{H}, E), 5.86(\mathrm{dt}, J=33.3,7.8 \mathrm{~Hz}, 0.97 \mathrm{H}, Z), 3.79$ (ddt, $J=$ 7.8, 4.1, 2.0 Hz, 2H), 2.57 (s, 3H).

${ }^{13} \mathrm{C}-\mathrm{NMR}\left(125 \mathrm{MHz}, \mathrm{CDCl}_{3}\right): \delta=199.7\left(\mathrm{C}_{\mathrm{q}}\right), 145.8\left(\mathrm{dt},{ }^{1} J_{\mathrm{C}-\mathrm{F}}=259 \mathrm{~Hz},{ }^{2} J_{\mathrm{C}-\mathrm{F}}=28.4 \mathrm{~Hz}, \mathrm{C}_{\mathrm{q}}\right), 140.0$ $\left(\mathrm{C}_{\mathrm{q}}\right), 135.3\left(\mathrm{C}_{\mathrm{q}}\right), 134.4(\mathrm{CH}), 131.4(\mathrm{CH}), 130.2(\mathrm{CH}), 126.9\left(\mathrm{C}_{\mathrm{q}}\right), 117.0\left(\mathrm{dt},{ }^{1} J_{\mathrm{C}-\mathrm{F}}=288 \mathrm{~Hz},{ }^{2} J_{\mathrm{C}-\mathrm{F}}=\right.$ $\left.32.3 \mathrm{~Hz}, \mathrm{C}_{\mathrm{q}}\right), 114.4\left(\mathrm{dt},{ }^{2} J_{\mathrm{C}-\mathrm{F}}=8.9 \mathrm{~Hz},{ }^{3} J_{\mathrm{C}-\mathrm{F}}=3.6 \mathrm{~Hz}, \mathrm{CH}\right), 112.5\left(\mathrm{~m}, \mathrm{C}_{\mathrm{q}}\right), 110.8\left(\mathrm{~m}, \mathrm{C}_{\mathrm{q}}\right), 110.7(\mathrm{~m}$, $\left.\mathrm{C}_{\mathrm{q}}\right), 110.3\left(\mathrm{~m}, \mathrm{C}_{\mathrm{q}}\right), 108.7\left(\mathrm{~m}, \mathrm{C}_{\mathrm{q}}\right), 107.4\left(\mathrm{~m}, \mathrm{C}_{\mathrm{q}}\right), 29.1\left(\mathrm{CH}_{3}\right), 28.6\left(\mathrm{~d},{ }^{3} J_{\mathrm{C}-\mathrm{F}}=3.5 \mathrm{~Hz}, \mathrm{CH}_{2}\right)$.

${ }^{19}$ F-NMR $\left(282 \mathrm{MHz}, \mathrm{CDCl}_{3}\right): \delta=-80.9(\mathrm{~m}),-117.5(\mathrm{~m}),-122.0(\mathrm{~m}),-122.1(\mathrm{~m}),-122.8(\mathrm{~m}),-123.1$ (m), $-126.3(\mathrm{~m}),-131.3(\mathrm{~m})$.

IR (ATR): 1686, 1238, 1203, 1148, 906, $729 \mathrm{~cm}^{-1}$.

MS (ESI) $m / z$ (relative intensity): $647\left(\left[\mathrm{M}\left({ }^{79} \mathrm{Br}\right)+\mathrm{Na}\right]^{+}, 20\right), 625\left(\left[\mathrm{M}\left({ }^{79} \mathrm{Br}\right)+\mathrm{H}\right]^{+}, 100\right), 450(50)$.

HR-MS (ESI): $m / z$ calcd. for $\left[\mathrm{C}_{18} \mathrm{H}_{10}{ }^{79} \mathrm{BrF}_{16} \mathrm{O}\right]^{+}[\mathrm{M}+\mathrm{H}]^{+}$624.9654, found 624.9643 .<smiles>CC(=O)c1ccc(I)cc1CC=C(F)C(F)(F)F</smiles>

(Z)-1-[4-Iodo-2-(1H,1H,2H-perfluorodec-2-en-1-yl)phenyl]ethan-1-one $\quad(166 h): \quad$ The general procedure $\mathbf{H}$ was followed using 1-(4-iodophenyl)- $N$-(4-methoxyphenyl)ethan-1-imine (6h) (176 mg, $0.50 \mathrm{mmol})$ and $1 \mathrm{H}, 1 \mathrm{H}, 2 \mathrm{H}$-perfluorodec-1-ene (127a) $(669 \mathrm{mg}, 1.50 \mathrm{mmol})$. Isolation by column chromatography ( $n$-hexane/EtOAc: 30/1) yielded 166h (248 mg, 74\%, Z/E = 97:3) as a yellow oil.

${ }^{1}$ H-NMR $\left(300 \mathrm{MHz}, \mathrm{CDCl}_{3}\right): \delta=7.74(\mathrm{dd}, J=8.2,1.7 \mathrm{~Hz}, 1 \mathrm{H}), 7.66(\mathrm{~d}, J=1.7 \mathrm{~Hz}, 1 \mathrm{H}), 7.50(\mathrm{~d}, J=$ $8.2 \mathrm{~Hz}, 1 \mathrm{H}), 6.10(\mathrm{dt}, J=22.3,8.8 \mathrm{~Hz}, 0.03 \mathrm{H}, E), 5.88(\mathrm{dt}, J=33.3,7.7 \mathrm{~Hz}, 0.97 \mathrm{H}, Z), 3.78(\mathrm{ddt}, J=$ 7.7, 4.1, 2.1 Hz, 2H), $2.62(\mathrm{~s}, 0.09 \mathrm{H}, E), 2.58(\mathrm{~s}, 2.91 \mathrm{H}, \mathrm{Z})$.

${ }^{13}$ C-NMR $\left(75 \mathrm{MHz}, \mathrm{CDCl}_{3}\right): \delta=199.9\left(\mathrm{C}_{\mathrm{q}}\right), 145.9\left(\mathrm{dt},{ }^{1} J_{\mathrm{C}-\mathrm{F}}=260 \mathrm{~Hz},{ }^{2} J_{\mathrm{C}-\mathrm{F}}=28.9 \mathrm{~Hz}, \mathrm{C}_{\mathrm{q}}\right), 140.4$ $(\mathrm{CH}), 139.6\left(\mathrm{C}_{\mathrm{q}}\right), 136.3(\mathrm{CH}), 135.9\left(\mathrm{C}_{\mathrm{q}}\right), 131.2(\mathrm{CH}), 117.0\left(\mathrm{dt},{ }^{1} J_{\mathrm{C}-\mathrm{F}}=288 \mathrm{~Hz},{ }^{2} J_{\mathrm{C}-\mathrm{F}}=32.3 \mathrm{~Hz}, \mathrm{C}_{\mathrm{q}}\right)$, 
$114.5\left(\mathrm{dt},{ }^{2} J_{\mathrm{C}-\mathrm{F}}=8.9 \mathrm{~Hz},{ }^{3} J_{\mathrm{C}-\mathrm{F}}=3.6 \mathrm{~Hz}, \mathrm{CH}\right), 113.0\left(\mathrm{~m}, \mathrm{C}_{\mathrm{q}}\right), 111.1\left(\mathrm{~m}, \mathrm{C}_{\mathrm{q}}\right), 110.6\left(\mathrm{~m}, \mathrm{C}_{\mathrm{q}}\right), 110.3(\mathrm{~m}$, $\left.\mathrm{C}_{\mathrm{q}}\right), 108.7\left(\mathrm{~m}, \mathrm{C}_{\mathrm{q}}\right), 107.4\left(\mathrm{~m}, \mathrm{C}_{\mathrm{q}}\right), 99.6\left(\mathrm{C}_{\mathrm{q}}\right), 29.1\left(\mathrm{CH}_{3}\right), 28.4\left(\mathrm{~d},{ }^{3} J_{\mathrm{C}-\mathrm{F}}=3.5 \mathrm{~Hz}, \mathrm{CH}_{2}\right)$.

${ }^{19}$ F-NMR $\left(376 \mathrm{MHz}, \mathrm{CDCl}_{3}\right): \delta=-80.7(\mathrm{~m}),-117.4(\mathrm{~m}),-122.0(\mathrm{~m}),-122.0(\mathrm{~m}),-122.9(\mathrm{~m}),-123.0$ (m), $-126.1(\mathrm{~m}),-131.0(\mathrm{~m})$.

IR (ATR): 1687, 1581, 1551, 1235, 1198, 1143, 720, $707 \mathrm{~cm}^{-1}$.

MS (ESI) $m / z$ (relative intensity): $695\left([\mathrm{M}+\mathrm{Na}]^{+}, 100\right), 673\left([\mathrm{M}+\mathrm{H}]^{+}, 20\right)$.

HR-MS (ESI): $m / z$ calcd. for $\left[\mathrm{C}_{18} \mathrm{H}_{10} \mathrm{~F}_{16} \mathrm{IO}\right]^{+}[\mathrm{M}+\mathrm{H}]^{+} 672.9515$, found 672.9506 .<smiles>CC(=O)c1cc2ccccc2cc1CC=C(F)C(F)(F)F</smiles>

(Z)-1-[3-(1H,1H,2H-Perfluorodec-2-en-1-yl)naphthalen-2-yl]ethan-1-one $\quad(166 i): \quad$ The general procedure $\mathbf{H}$ was followed using $N$-(4-methoxyphenyl)-1-(naphthalen-2-yl)ethan-1-imine (6i) $(138 \mathrm{mg}, 0.50 \mathrm{mmol})$ and $1 H, 1 H, 2 H$-perfluorodec-1-ene (127a) $(669 \mathrm{mg}, 1.50 \mathrm{mmol})$. Isolation by column chromatography ( $n$-hexane/EtOAc: $30 / 1)$ yielded $\mathbf{1 6 6 i}(179 \mathrm{mg}, 60 \%, Z / E=98: 2)$ as a red oil. ${ }^{1}$ H-NMR $\left(300 \mathrm{MHz}, \mathrm{CDCl}_{3}\right): \delta=8.32(\mathrm{~s}, 1 \mathrm{H}), 7.89(\mathrm{dd}, J=8.0,1.3 \mathrm{~Hz}, 1 \mathrm{H}), 7.79$ (dd, $J=7.9$, $1.4 \mathrm{~Hz}, 1 \mathrm{H}), 7.67(\mathrm{~s}, 1 \mathrm{H}), 7.59(\mathrm{ddd}, J=8.0,6.9,1.4 \mathrm{~Hz}, 1 \mathrm{H}), 7.52(\mathrm{ddd}, J=7.9,6.9,1.3 \mathrm{~Hz}, 1 \mathrm{H})$, $6.24(\mathrm{dt}, J=33.2,8.2 \mathrm{~Hz}, 0.02 \mathrm{H}, E), 5.99$ (dt, $J=33.5,7.6 \mathrm{~Hz}, 0.98 \mathrm{H}, Z$ ), 3.99 (ddt, $J=7.6,4.1$, $2.1 \mathrm{~Hz}, 2 \mathrm{H}), 2.73(\mathrm{~s}, 3 \mathrm{H})$.

${ }^{13}$ C-NMR $\left(125 \mathrm{MHz}, \mathrm{CDCl}_{3}\right): \delta=200.5\left(\mathrm{C}_{\mathrm{q}}\right), 145.5\left(\mathrm{dt},{ }^{1} J_{\mathrm{C}-\mathrm{F}}=258 \mathrm{~Hz},{ }^{2} J_{\mathrm{C}-\mathrm{F}}=28.3 \mathrm{~Hz}, \mathrm{C}_{\mathrm{q}}\right), 134.8$ $\left(\mathrm{C}_{\mathrm{q}}\right), 134.7\left(\mathrm{C}_{\mathrm{q}}\right), 134.0\left(\mathrm{C}_{\mathrm{q}}\right), 131.8(\mathrm{CH}), 131.4\left(\mathrm{C}_{\mathrm{q}}\right), 130.1(\mathrm{CH}), 128.7(\mathrm{CH}), 128.6(\mathrm{CH}), 127.2(\mathrm{CH})$, $126.7(\mathrm{CH}), 117.1\left(\mathrm{dt},{ }^{1} J_{\mathrm{C}-\mathrm{F}}=287 \mathrm{~Hz},{ }^{2} J_{\mathrm{C}-\mathrm{F}}=32.8 \mathrm{~Hz}, \mathrm{C}_{\mathrm{q}}\right), 115.5\left(\mathrm{dt},{ }^{2} J_{\mathrm{C}-\mathrm{F}}=8.9 \mathrm{~Hz},{ }^{3} J_{\mathrm{C}-\mathrm{F}}=3.6 \mathrm{~Hz}\right.$, $\mathrm{CH}), 113.2\left(\mathrm{~m}, \mathrm{C}_{\mathrm{q}}\right), 110.9\left(\mathrm{~m}, \mathrm{C}_{\mathrm{q}}\right), 110.8\left(\mathrm{~m}, \mathrm{C}_{\mathrm{q}}\right), 109.9\left(\mathrm{~m}, \mathrm{C}_{\mathrm{q}}\right), 108.7\left(\mathrm{~m}, \mathrm{C}_{\mathrm{q}}\right), 108.2\left(\mathrm{~m}, \mathrm{C}_{\mathrm{q}}\right), 29.0$ $\left(\mathrm{CH}_{3}\right), 28.9\left(\mathrm{~d},{ }^{3} J_{\mathrm{C}-\mathrm{F}}=3.1 \mathrm{~Hz}, \mathrm{CH}_{2}\right)$.

${ }^{19}$ F-NMR $\left(282 \mathrm{MHz}, \mathrm{CDCl}_{3}\right): \delta=-80.9(\mathrm{~m}),-117.4(\mathrm{~m}),-122.0(\mathrm{~m}),-122.1(\mathrm{~m}),-122.8(\mathrm{~m}),-123.0$ (m), $-126.2(\mathrm{~m}),-132.3(\mathrm{~m})$.

IR (ATR): 1681, 1238, 1201, 1149, 906, $728 \mathrm{~cm}^{-1}$.

MS (ESI) $m / z$ (relative intensity): $619\left([\mathrm{M}+\mathrm{Na}]^{+}, 100\right), 597\left([\mathrm{M}+\mathrm{H}]^{+}, 40\right)$.

HR-MS (ESI): $m / z$ calcd. for $\left[\mathrm{C}_{22} \mathrm{H}_{13} \mathrm{~F}_{16} \mathrm{O}\right]^{+}[\mathrm{M}+\mathrm{H}]^{+}$597.0705, found 597.0704. 
<smiles>CC(=O)c1cccc(F)c1CC=C(F)C(F)F</smiles>

(Z)-1-[3-Fluoro-2-(1H,1H,2H-perfluorodec-2-en-1-yl)phenyl]ethan-1-one $\quad(\mathbf{1 6 6 j}): \quad$ The general procedure $\mathbf{H}$ was followed using 1-(3-fluorophenyl)- $N$-(4-methoxyphenyl)ethan-1-imine (6j) (122 mg, $0.50 \mathrm{mmol})$ and $1 \mathrm{H}, 1 \mathrm{H}, 2 \mathrm{H}$-perfluorodec-1-ene (127a) $(669 \mathrm{mg}, 1.50 \mathrm{mmol})$. Isolation by column chromatography ( $n$-hexane/EtOAc: 30/1) yielded 166j (149 mg, 53\%, Z/E=97:3) as a yellow oil.

${ }^{1}$ H-NMR $\left(400 \mathrm{MHz}, \mathrm{CDCl}_{3}\right): \delta=7.53(\mathrm{dd}, J=7.8,0.9 \mathrm{~Hz}, 1 \mathrm{H}), 7.33(\mathrm{ddd}, J=7.8,7.8,5.3 \mathrm{~Hz}, 1 \mathrm{H})$, $7.21(\mathrm{ddd}, J=8.4,7.8,0.9 \mathrm{~Hz}, 1 \mathrm{H}), 5.98(\mathrm{dt}, J=23.5,8.1 \mathrm{~Hz}, 0.03 \mathrm{H}, E), 5.79(\mathrm{dt}, J=33.0,7.3 \mathrm{~Hz}$, $0.97 \mathrm{H}, Z), 3.81$ (ddt, $J=7.3,4.1,2.0 \mathrm{~Hz}, 2 \mathrm{H}), 2.59$ (s, $3 \mathrm{H})$.

${ }^{13}$ C-NMR $\left(100 \mathrm{MHz}, \mathrm{CDCl}_{3}\right): \delta=200.3\left(\mathrm{C}_{\mathrm{q}}\right), 161.6\left(\mathrm{~d},{ }^{1} J_{\mathrm{C}-\mathrm{F}}=254 \mathrm{~Hz}, \mathrm{C}_{\mathrm{q}}\right), 145.7\left(\mathrm{dt},{ }^{1} J_{\mathrm{C}-\mathrm{F}}=260 \mathrm{~Hz}\right.$, $\left.{ }^{2} J_{\mathrm{C}-\mathrm{F}}=29.5 \mathrm{~Hz}, \mathrm{C}_{\mathrm{q}}\right), 139.3\left(\mathrm{~d},{ }^{3} J_{\mathrm{C}-\mathrm{F}}=3.2 \mathrm{~Hz}, \mathrm{C}_{\mathrm{q}}\right), 128.3\left(\mathrm{~d},{ }^{3} J_{\mathrm{C}-\mathrm{F}}=8.6 \mathrm{~Hz}, \mathrm{CH}\right), 125.2\left(\mathrm{~d},{ }^{4} J_{\mathrm{C}-\mathrm{F}}=\right.$ $3.8 \mathrm{~Hz}, \mathrm{CH}), 124.6\left(\mathrm{~d},{ }^{2} J_{\mathrm{C}-\mathrm{F}}=16.8 \mathrm{~Hz}, \mathrm{C}_{\mathrm{q}}\right), 118.9\left(\mathrm{~d},{ }^{2} J_{\mathrm{C}-\mathrm{F}}=23.7 \mathrm{~Hz}, \mathrm{CH}\right), 116.8\left(\mathrm{dt},{ }^{1} J_{\mathrm{C}-\mathrm{F}}=287 \mathrm{~Hz}\right.$, $\left.{ }^{2} J_{\mathrm{C}-\mathrm{F}}=32.8 \mathrm{~Hz}, \mathrm{C}_{\mathrm{q}}\right), 113.9\left(\mathrm{dt},{ }^{2} J_{\mathrm{C}-\mathrm{F}}=8.9 \mathrm{~Hz},{ }^{4} J_{\mathrm{C}-\mathrm{F}}=3.6 \mathrm{~Hz}, \mathrm{CH}\right), 113.2\left(\mathrm{~m}, \mathrm{C}_{\mathrm{q}}\right), 111.3\left(\mathrm{~m}, \mathrm{C}_{\mathrm{q}}\right), 110.8$ $\left(\mathrm{m}, \mathrm{C}_{\mathrm{q}}\right), 110.2\left(\mathrm{~m}, \mathrm{C}_{\mathrm{q}}\right), 108.3\left(\mathrm{~m}, \mathrm{C}_{\mathrm{q}}\right), 107.5\left(\mathrm{~m}, \mathrm{C}_{\mathrm{q}}\right), 29.4\left(\mathrm{CH}_{3}\right), 20.3\left(\mathrm{dd},{ }^{3} J_{\mathrm{C}-\mathrm{F}}=5.8,4.4 \mathrm{~Hz}, \mathrm{CH}_{2}\right)$.

${ }^{19} \mathbf{F}-\mathbf{N M R}\left(376 \mathrm{MHz}, \mathrm{CDCl}_{3}\right): \delta=-80.8(\mathrm{~m}),-115.1(\mathrm{~m}),-117.5(\mathrm{~m}),-122.0(\mathrm{~m}),-122.1(\mathrm{~m}),-122.8$ (m), $-123.0(\mathrm{~m}),-126.2(\mathrm{~m}),-131.0(\mathrm{~m})$.

IR (ATR): 1692, 1456, 1237, 1199, 1144, 791, $730 \mathrm{~cm}^{-1}$.

MS (ESI) $m / z$ (relative intensity): $587\left([\mathrm{M}+\mathrm{Na}]^{+}, 40\right), 441$ (100), 419 (30).

HR-MS (ESI): $m / z$ calcd. for $\left[\mathrm{C}_{18} \mathrm{H}_{10} \mathrm{~F}_{17} \mathrm{O}\right]^{+}[\mathrm{M}+\mathrm{H}]^{+} 565.0455$, found 565.0452 .<smiles>COC(=O)c1ccc2c(c1CC=C(F)C(F)(F)F)OCO2</smiles>

(Z)-1-[4-(1H,1H,2H-Perfluorodec-2-en-1-yl)benzo[d][1,3]dioxol-5-yl]ethan-1-one $\quad(166 \mathrm{k})$ : The general procedure $\mathbf{H}$ was followed using 1-(benzo[ $d][1,3]$ dioxol-5-yl)- $N$-(4-methoxyphenyl)ethan-1imine (6k) (135 mg, $0.50 \mathrm{mmol})$ and $1 \mathrm{H}, 1 \mathrm{H}, 2 \mathrm{H}$-perfluorodec-1-ene (127a) (669 mg, $1.50 \mathrm{mmol})$. Isolation by column chromatography ( $n$-hexane/EtOAc: 20/1) yielded 166k $(193 \mathrm{mg}, 65 \%, \mathrm{Z} / \mathrm{E}=$ 98:2) as a yellow oil.

${ }^{1}$ H-NMR $\left(300 \mathrm{MHz}, \mathrm{CDCl}_{3}\right): \delta=7.43(\mathrm{~d}, J=8.2 \mathrm{~Hz}, 1 \mathrm{H}), 6.75(\mathrm{~d}, J=8.2 \mathrm{~Hz}, 1 \mathrm{H}), 6.02(\mathrm{~s}, 2 \mathrm{H}), 5.85$ (dt, $J=33.5,7.4 \mathrm{~Hz}, 1 \mathrm{H}), 3.85$ (ddt, $J=7.4,4.1,2.1 \mathrm{~Hz}, 2 \mathrm{H}), 2.53$ (s, 3H).

${ }^{13} \mathrm{C}-\mathrm{NMR}\left(125 \mathrm{MHz}, \mathrm{CDCl}_{3}\right): \delta=198.6\left(\mathrm{C}_{\mathrm{q}}\right), 150.2\left(\mathrm{C}_{\mathrm{q}}\right), 147.8\left(\mathrm{C}_{\mathrm{q}}\right), 145.3\left(\mathrm{dt},{ }^{1} J_{\mathrm{C}-\mathrm{F}}=260 \mathrm{~Hz},{ }^{2} J_{\mathrm{C}-\mathrm{F}}=\right.$ $\left.29.5 \mathrm{~Hz}, \mathrm{C}_{\mathrm{q}}\right), 130.8\left(\mathrm{C}_{\mathrm{q}}\right), 126.5(\mathrm{CH}), 119.6\left(\mathrm{C}_{\mathrm{q}}\right), 116.8\left(\mathrm{dt},{ }^{1} J_{\mathrm{C}-\mathrm{F}}=287 \mathrm{~Hz},{ }^{2} J_{\mathrm{C}-\mathrm{F}}=32.8 \mathrm{~Hz}, \mathrm{C}_{\mathrm{q}}\right), 114.1$ 
$\left(\mathrm{dt},{ }^{2} J_{\mathrm{C}-\mathrm{F}}=8.9 \mathrm{~Hz},{ }^{3} J_{\mathrm{C}-\mathrm{F}}=3.6 \mathrm{~Hz}, \mathrm{CH}\right), 113.2\left(\mathrm{~m}, \mathrm{C}_{\mathrm{q}}\right), 110.9\left(\mathrm{~m}, \mathrm{C}_{\mathrm{q}}\right), 110.8\left(\mathrm{~m}, \mathrm{C}_{\mathrm{q}}\right), 109.9\left(\mathrm{~m}, \mathrm{C}_{\mathrm{q}}\right)$, $108.7\left(\mathrm{~m}, \mathrm{C}_{\mathrm{q}}\right), 108.2\left(\mathrm{~m}, \mathrm{C}_{\mathrm{q}}\right), 106.2(\mathrm{CH}), 101.8\left(\mathrm{CH}_{2}\right), 28.8\left(\mathrm{CH}_{3}\right), 21.8\left(\mathrm{~d},{ }^{3} J_{\mathrm{C}-\mathrm{F}}=4.0 \mathrm{~Hz}, \mathrm{CH}_{2}\right)$.

${ }^{19}$ F-NMR $\left(282 \mathrm{MHz}, \mathrm{CDCl}_{3}\right): \delta=-80.9(\mathrm{~m}),-117.5(\mathrm{~m}),-122.0(\mathrm{~m}),-122.1(\mathrm{~m}),-122.8(\mathrm{~m}),-123.1$ (m), $-126.2(\mathrm{~m}),-131.7(\mathrm{~m})$.

IR (ATR): 1675, 1453, 1237, 1200, 1146, 1058, 939, $731 \mathrm{~cm}^{-1}$.

MS (ESI) $m / z$ (relative intensity): $613\left([\mathrm{M}+\mathrm{Na}]^{+}, 80\right), 591\left([\mathrm{M}+\mathrm{H}]^{+}, 100\right)$.

HR-MS (ESI): $m / z$ calcd. for $\left[\mathrm{C}_{19} \mathrm{H}_{11} \mathrm{~F}_{16} \mathrm{O}_{3}\right]^{+}[\mathrm{M}+\mathrm{H}]^{+} 591.0447$, found 591.0451 .<smiles>CC(C)(C)c1ccc(-n2cccn2)c(C/C=C(\F)C(F)(F)F)c1</smiles>

(Z)-1-[4-(tert-Butyl)-2-(1H,1H,2H-perfluorodec-2-en-1-yl)phenyl]-1H-pyrazole $\quad(161 c): \quad$ The general procedure $\mathbf{H}$ was followed using 1-[4-(tert-butyl)phenyl]-1H-pyrazole (18c) (100 mg, $0.50 \mathrm{mmol}$ ) and $1 \mathrm{H}, 1 \mathrm{H}, 2 \mathrm{H}$-perfluorodec-1-ene (127a) $(315 \mathrm{mg}, 0.75 \mathrm{mmol})$. Isolation by column chromatography ( $n$-hexane/EtOAc: $20 / 1)$ yielded 161c $(241 \mathrm{mg}, 77 \%, Z / E=87: 13)$ as a colorless solid.

M.p.: $80{ }^{\circ} \mathrm{C}$.

${ }^{1} \mathbf{H}-\mathbf{N M R}\left(300 \mathrm{MHz}, \mathrm{CDCl}_{3}\right): \delta=7.70(\mathrm{dd}, J=2.2,1.7 \mathrm{~Hz}, 1 \mathrm{H}), 7.56(\mathrm{dd}, J=4.0,2.4 \mathrm{~Hz}, 1 \mathrm{H}), 7.36$ $(\mathrm{dd}, J=8.2,2.2 \mathrm{~Hz}, 1 \mathrm{H}), 7.30(\mathrm{dd}, J=2.4,2.1 \mathrm{~Hz}, 1 \mathrm{H}), 7.23(\mathrm{dd}, J=8.2,1.7 \mathrm{~Hz}, 1 \mathrm{H}), 6.42(\mathrm{dd}, J=$ 4.0, $2.1 \mathrm{~Hz}, 1 \mathrm{H}), 5.90$ (dt, $J=21.8,7.9 \mathrm{~Hz}, 0.13 \mathrm{H}, E), 5.67$ (dd, $J=33.3,7.6 \mathrm{~Hz}, 0.87 \mathrm{H}, Z$ ), 3.79 (ddt, $J=7.6,4.1,2.5 \mathrm{~Hz}, 2 \mathrm{H}), 1.33$ (s, 9H).

${ }^{13}$ C-NMR $\left(125 \mathrm{MHz}, \mathrm{CDCl}_{3}\right): \delta=152.2\left(\mathrm{C}_{\mathrm{q}}\right), 145.7\left(\mathrm{dt},{ }^{1} J_{\mathrm{C}-\mathrm{F}}=259 \mathrm{~Hz},{ }^{2} J_{\mathrm{C}-\mathrm{F}}=29.1 \mathrm{~Hz}, \mathrm{C}_{\mathrm{q}}\right), 140.5$ $(\mathrm{CH}), 137.1\left(\mathrm{C}_{\mathrm{q}}\right), 132.9\left(\mathrm{C}_{\mathrm{q}}\right), 130.3(\mathrm{CH}), 127.3(\mathrm{CH}), 125.8(\mathrm{CH}), 124.7(\mathrm{CH}), 117.0\left(\mathrm{dt},{ }^{1} J_{\mathrm{C}-\mathrm{F}}=\right.$ $\left.289 \mathrm{~Hz},{ }^{2} J_{\mathrm{C}-\mathrm{F}}=31.3 \mathrm{~Hz}, \mathrm{C}_{\mathrm{q}}\right), 114.7\left(\mathrm{dt},{ }^{2} J_{\mathrm{C}-\mathrm{F}}=8.9 \mathrm{~Hz},{ }^{3} J_{\mathrm{C}-\mathrm{F}}=3.6 \mathrm{~Hz}, \mathrm{CH}\right), 113.0\left(\mathrm{~m}, \mathrm{C}_{\mathrm{q}}\right), 112.0(\mathrm{~m}$, $\left.\mathrm{C}_{\mathrm{q}}\right), 110.6\left(\mathrm{~m}, \mathrm{C}_{\mathrm{q}}\right), 110.0\left(\mathrm{~m}, \mathrm{C}_{\mathrm{q}}\right), 108.6\left(\mathrm{~m}, \mathrm{C}_{\mathrm{q}}\right), 108.1\left(\mathrm{~m}, \mathrm{C}_{\mathrm{q}}\right), 106.5(\mathrm{CH}), 34.7(\mathrm{CH}), 31.2\left(\mathrm{CH}_{3}\right)$, $26.3\left(\mathrm{~d},{ }^{3} J_{\mathrm{C}-\mathrm{F}}=3.7 \mathrm{~Hz}, \mathrm{CH}_{2}\right)$.

${ }^{19}$ F-NMR $\left(282 \mathrm{MHz}, \mathrm{CDCl}_{3}\right): \delta=-80.9(\mathrm{~m}),-114.5(\mathrm{~m}, E),-117.5(\mathrm{~m}, Z),-122.1(\mathrm{~m}),-122.1(\mathrm{~m}),-$ $122.9(\mathrm{~m}, Z),-123.1(\mathrm{~m}, E),-123.5(\mathrm{~m}),-126.2(\mathrm{~m}),-132.3(\mathrm{~m})$.

IR (ATR): 2968, 1522, 1238, 1201, 1146, 909, $732 \mathrm{~cm}^{-1}$.

MS (EI) $m / z$ (relative intensity): 626 ([M] $\left.]^{+}, 100\right), 611$ (20), 598 (10).

HR-MS (ESI): $m / z$ calcd. for $\left[\mathrm{C}_{23} \mathrm{H}_{19} \mathrm{~F}_{16} \mathrm{~N}_{2}\right]^{+}[\mathrm{M}+\mathrm{H}]^{+}$627.1287, found 627.1289. 


\subsubsection{Mechanistic Studies}

\subsubsection{H/D-Exchange Experiments}

\subsection{H/D-Exchange Experiment with $\mathrm{CD}_{3} \mathrm{OD}$}

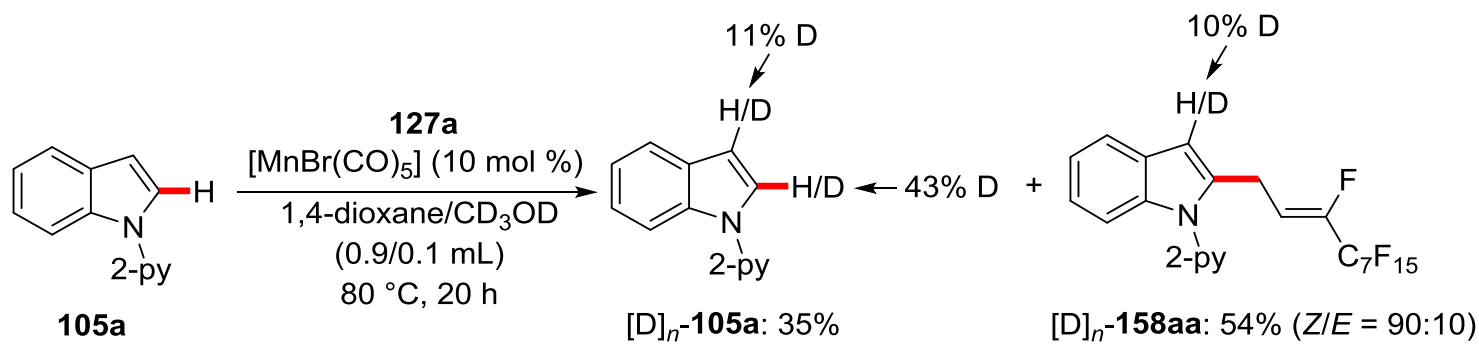

A suspension of $105 \mathrm{a}$ (116 mg, $0.60 \mathrm{mmol}, 1.20$ equiv), 127a (224 mg, $0.50 \mathrm{mmol}, 1.00$ equiv), $\left[\mathrm{MnBr}(\mathrm{CO})_{5}\right](13.7 \mathrm{mg}, 10.0 \mathrm{~mol} \%)$ and $\mathrm{K}_{2} \mathrm{CO}_{3}(69.0 \mathrm{mg}, 0.50 \mathrm{mmol}, 1.00$ equiv) in 1,4-dioxane $(0.9 \mathrm{~mL})$ and $\mathrm{CD}_{3} \mathrm{OD}(0.1 \mathrm{~mL})$ was stirred at $80^{\circ} \mathrm{C}$ for $20 \mathrm{~h}$. After removal of the solvents, the crude mixture was purified by column chromatography on silica gel ( $n$-hexane/EtOAc: 30:1 $\rightarrow$ 20:1) to yield $[\mathrm{D}]_{n}$-158aa (167 mg, 54\%, Z/E $\left.=90: 10\right)$ and $[\mathrm{D}]_{n}-\mathbf{1 0 5 a}(40.7 \mathrm{mg}, 35 \%$ reisolated) as yellow oils.

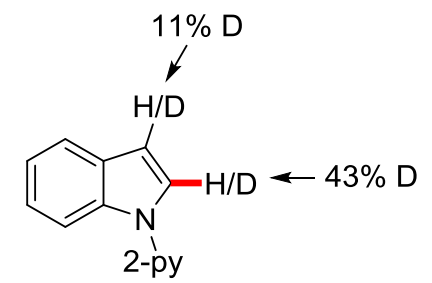

$[\mathrm{D}]_{n}-105 \mathbf{a}$ $\left(\mathrm{CDCl}_{3}, 300 \mathrm{MHz}\right)$

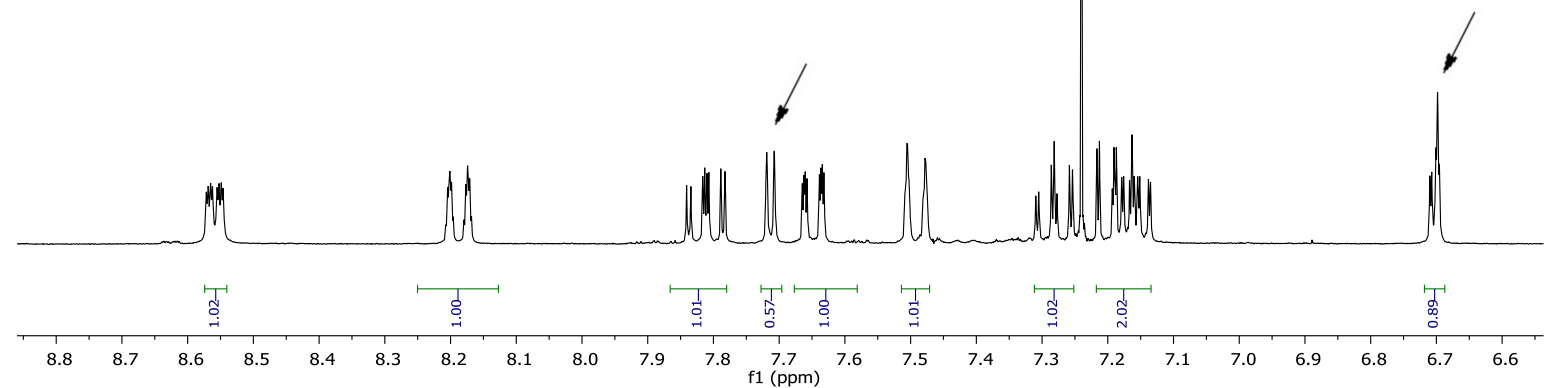




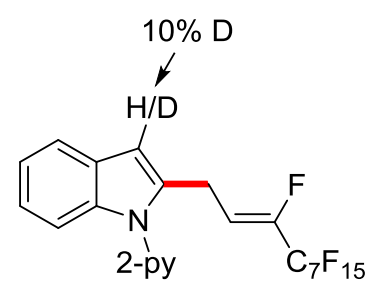

$[\mathrm{D}]_{n}-158 \mathrm{aa}$

$\left(\mathrm{CDCl}_{3}, 500 \mathrm{MHz}\right)$

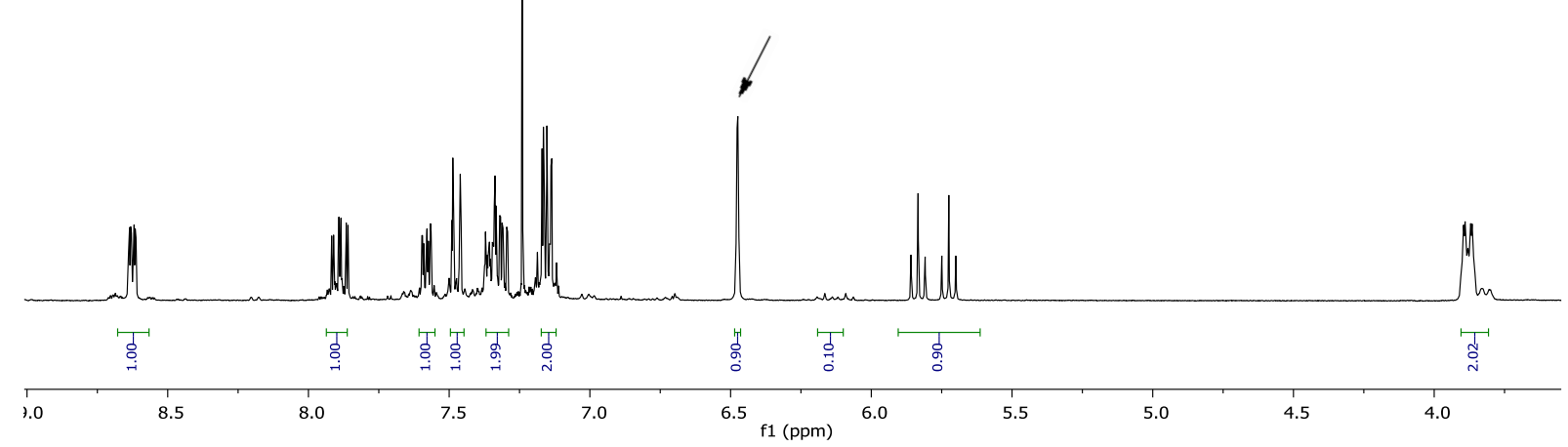

\subsection{H/D-Exchange Experiment with $\mathrm{CD}_{3} \mathrm{OH}$}<smiles>[Pb]n1ccc2ccccc21</smiles>

$105 a$

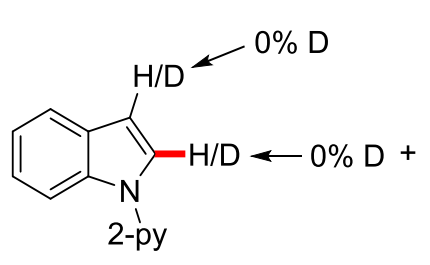

105a: $37 \%$

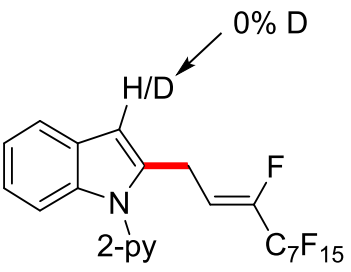

158aa: $56 \%(Z / E=90: 10)$

A suspension of 105a ( $97.0 \mathrm{mg}, 0.50 \mathrm{mmol}, 1.00$ equiv), 127a (268 mg, $0.60 \mathrm{mmol}, 1.20$ equiv), $\left[\mathrm{MnBr}(\mathrm{CO})_{5}\right](13.7 \mathrm{mg}, 10.0 \mathrm{~mol} \%)$ and $\mathrm{K}_{2} \mathrm{CO}_{3}(69.0 \mathrm{mg}, 0.50 \mathrm{mmol}, 1.00$ equiv) in 1,4-dioxane $(0.9 \mathrm{~mL})$ and $\mathrm{CD}_{3} \mathrm{OH}(0.1 \mathrm{~mL})$ was stirred at $80^{\circ} \mathrm{C}$ for $20 \mathrm{~h}$. After removal of the solvents, the crude mixture was purified by column chromatography on silica gel ( $n$-hexane/EtOAc: $30: 1 \rightarrow 20: 1)$ to yield 158aa (171 mg, 56\%, Z/E = 90:10) and 105a (43.0 mg, 37\% reisolated) as yellow oils. 


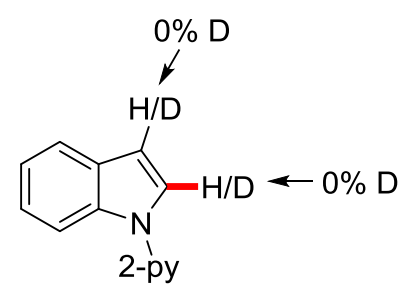

105a

$\left(\mathrm{CDCl}_{3}, 300 \mathrm{MHz}\right)$
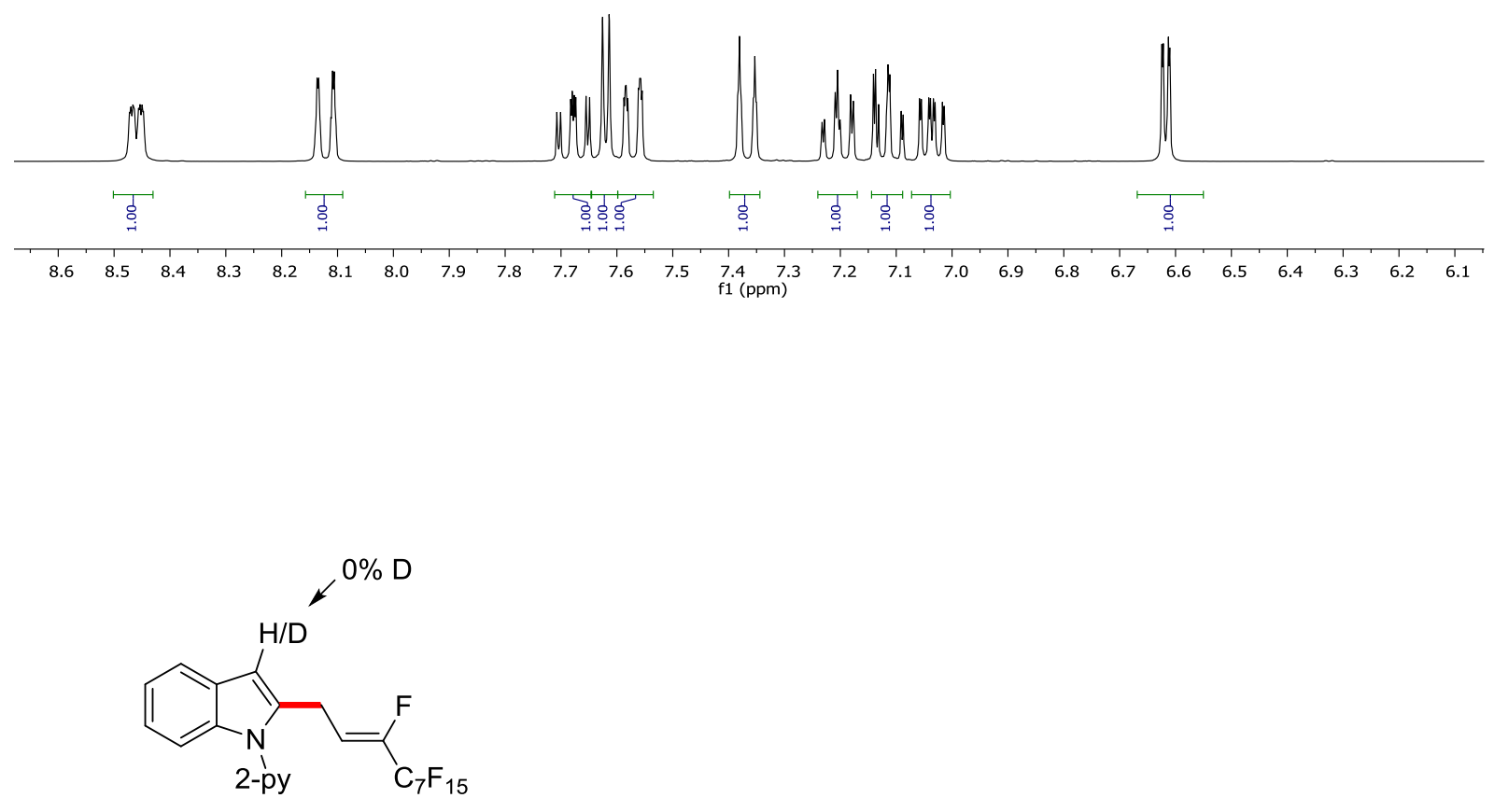

158aa

$\left(\mathrm{CDCl}_{3}, 300 \mathrm{MHz}\right)$

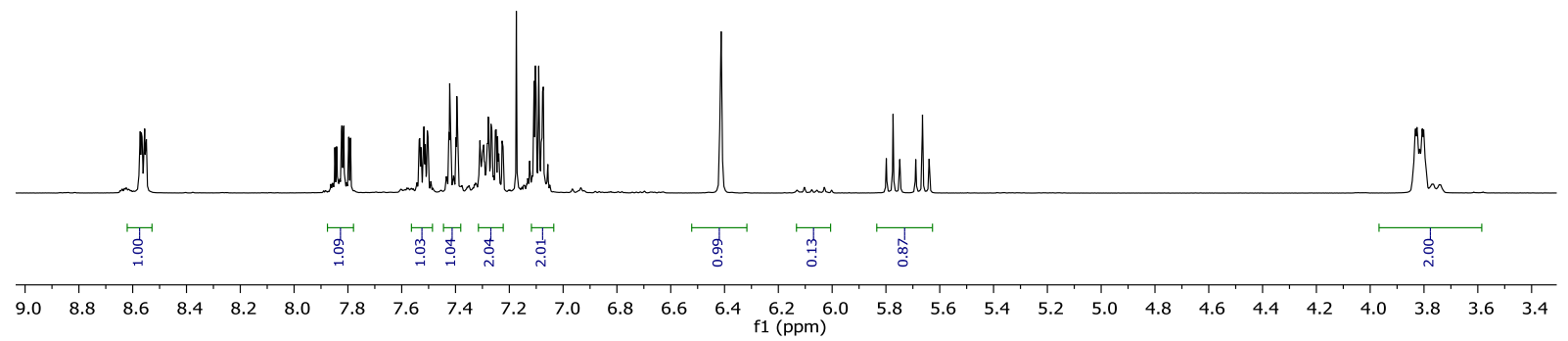




\subsubsection{KIE Studies}

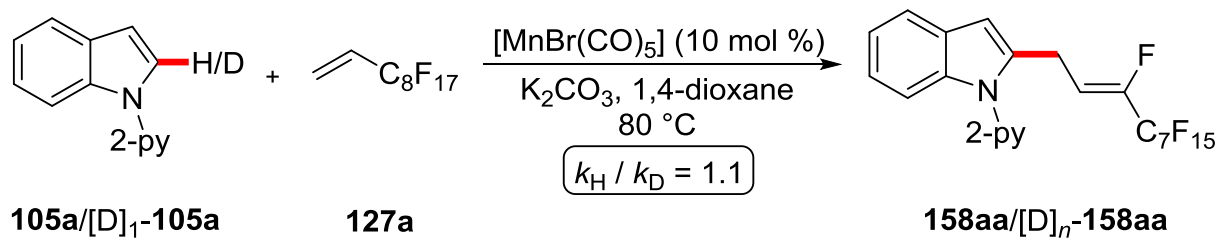

Two parallel reactions of $\mathbf{1 0 5 a}$ or $[D]_{1}-\mathbf{1 0 5 a}$ with $127 \mathbf{a}$ were performed respectively to determine the KIE value by comparison of the initial reaction rates by GC-analysis with $n$-dodecane as the internal standard. A suspension of 105a (97.1 mg, $0.50 \mathrm{mmol}, 1.00$ equiv) or [D $]_{1}-\mathbf{1 0 5 a}(97.6 \mathrm{mg}, 0.50 \mathrm{mmol}$, 1.00 equiv), 127a (268 mg, $0.60 \mathrm{mmol}, 1.20$ equiv), $\left[\mathrm{MnBr}(\mathrm{CO})_{5}\right](13.7 \mathrm{mg}, 10 \mathrm{~mol} \%), \mathrm{K}_{2} \mathrm{CO}_{3}$ (69.1 mg, 1.00 equiv) and $n$-dodecane $(30 \mu \mathrm{L})$ in 1,4-dioxane $(2.00 \mathrm{~mL})$ was stirred at $80{ }^{\circ} \mathrm{C}$. Aliquots $(30 \mu \mathrm{L})$ were periodically removed to provide the following conversions as determined by GCanalysis:

Table 43. Conversion-time table for determination of the KIE.

\begin{tabular}{c|ccccc}
$t / \min$ & 40 & 60 & 80 & 100 & 120 \\
\hline 158aa / \% & 5.19 & 8.00 & 13.2 & 17.9 & 20.9 \\
{$[\mathrm{D}]_{n}-\mathbf{1 5 8 a a} / \%$} & 3.25 & 5.69 & 9.98 & 14.4 & 17.7
\end{tabular}

\subsubsection{Experiments with Cyclometalated Complex 167}

\subsection{Complex 167 as Catalyst}

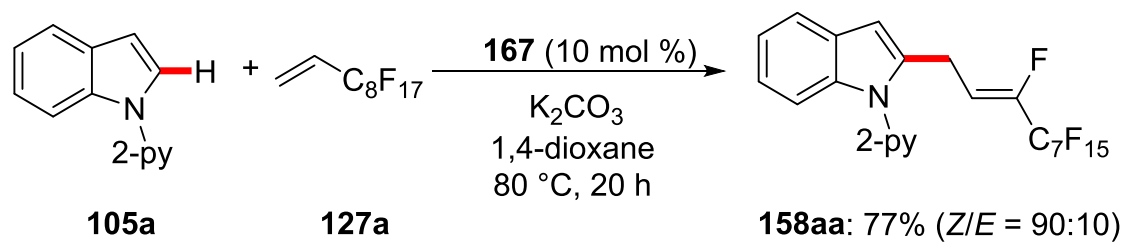

A suspension of indole $\mathbf{1 0 5 a}(97.1 \mathrm{mg}, 0.50 \mathrm{mmol}, 1.00$ equiv), $1 \mathrm{H}, 1 \mathrm{H}, 2 \mathrm{H}$-perfluorodecene (127a) (268 mg, $0.60 \mathrm{mmol}, 1.20$ equiv), 167 (18.0 mg, $10.0 \mathrm{~mol} \%$ ) and $\mathrm{K}_{2} \mathrm{CO}_{3}(69.1 \mathrm{mg}, 0.50 \mathrm{mmol}$, 1.00 equiv) in 1,4-dioxane $(0.50 \mathrm{~mL}, 1.00 \mathrm{M})$ was stirred at $80^{\circ} \mathrm{C}$ for $20 \mathrm{~h}$. At ambient temperature, the solvent was removed in vacuo and the remaining residue was purified by column chromatography on silica gel to afford the desired product 158aa (240 mg, 77\%, $Z / E=90: 10)$. 


\subsection{Stoichiometric Reaction with Complex 167}

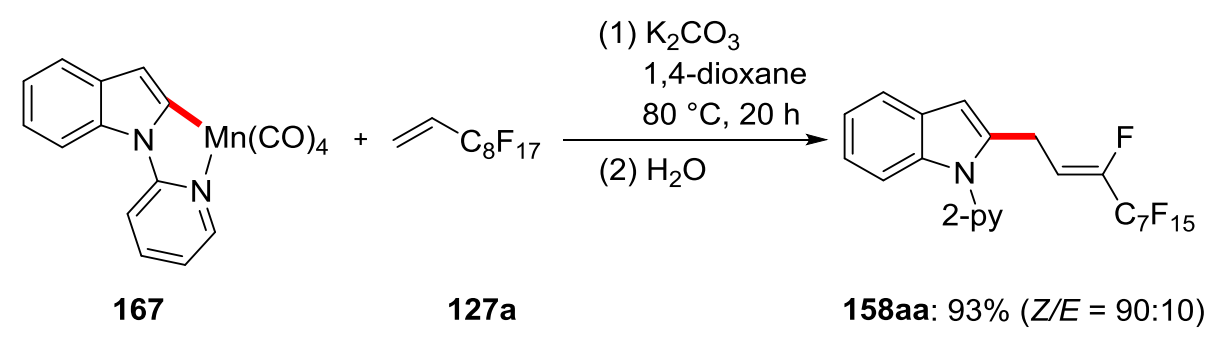

A suspension of complex 167 (72.0 mg, $0.20 \mathrm{mmol}, 1.00$ equiv), $1 \mathrm{H}, 1 \mathrm{H}, 2 \mathrm{H}$-perfluorodecene (127a) (107 mg, $0.24 \mathrm{mmol}, 1.20$ equiv), $\mathrm{K}_{2} \mathrm{CO}_{3}(27.6 \mathrm{mg}, 0.20 \mathrm{mmol}, 1.00$ equiv) in 1,4-dioxane $(0.20 \mathrm{~mL}$, $1.00 \mathrm{M}$ ) was stirred at $80{ }^{\circ} \mathrm{C}$ for $20 \mathrm{~h}$. At ambient temperature, the reaction mixture was diluted with water $(5.0 \mathrm{~mL})$ and extracted with EtOAc $(3 \times 10 \mathrm{~mL})$. After removal of the solvents in vacuo, the remaining residue was purified by column chromatography on silica gel to afford the desired product 158aa $(115 \mathrm{mg}, 93 \%, Z / E=90: 10)$.

\subsection{Stoichiometric Reaction with Complex 167 in the absence of $\mathrm{K}_{2} \mathrm{CO}_{3}$}

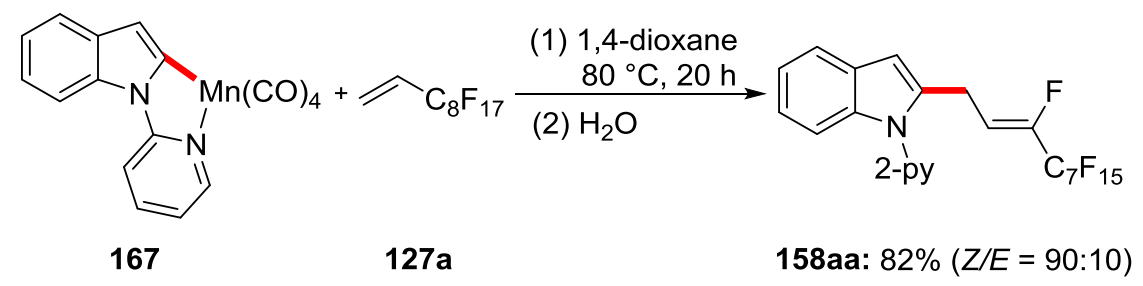

A suspension of complex 167 (72.0 mg, $0.20 \mathrm{mmol}, 1.00$ equiv), $1 \mathrm{H}, 1 \mathrm{H}, 2 \mathrm{H}$-perfluorodecene (127a) (107 mg, $0.24 \mathrm{mmol}, 1.20$ equiv) in 1,4-dioxane $(0.20 \mathrm{~mL}, 1.00 \mathrm{M})$ was stirred at $80{ }^{\circ} \mathrm{C}$ for $20 \mathrm{~h}$. At ambient temperature, the reaction mixture was diluted with water $(5.0 \mathrm{~mL})$ and extracted with EtOAc $(3 \times 10 \mathrm{~mL})$. The solvents were removed in vacuo and the remaining residue was purified by column chromatography on silica gel to afford the desired product 158aa (102 mg, 82\%, Z/E = 90:10) 


\section{References}

[1] a) M. Fujita, T. Hiyama, J. Org. Chem. 1988, 53, 5415-5421; b) T. Hiyama, M. Obayashi, I. Mori, H. Nozaki, J. Org. Chem. 1983, 48, 912-914.

[2] a) R. J. P. Corriu, J. P. Masse, J. Chem. Soc., Chem. Commun. 1972, 144; b) K. Tamao, K. Sumitani, M. Kumada, J. Am. Chem. Soc. 1972, 94, 4374-4376.

[3] R. F. Heck, J. P. Nolley, J. Org. Chem. 1972, 37, 2320-2322.

[4] S. Baba, E. Negishi, J. Am. Chem. Soc. 1976, 98, 6729-6731.

[5] K. Sonogashira, J. Organomet. Chem. 2002, 653, 46-49.

[6] a) D. Milstein, J. K. Stille, J. Am. Chem. Soc. 1978, 100, 3636-3638; b) M. Kosugi, Y. Shimizu, T. Migita, Chem. Lett. 1977, 6, 1423-1424; c) D. Azarian, S. S. Dua, C. Eaborn, D. R. M. Walton, J. Organomet. Chem. 1976, 117, C55-C57.

[7] a) N. Miyaura, K. Yamada, A. Suzuki, Tetrahedron Lett. 1979, 20, 3437-3440; b) N. Miyaura, A. Suzuki, J. Chem. Soc., Chem. Commun. 1979, 866-867.

[8] a) C. A. Busacca, D. R. Fandrick, J. J. Song, C. H. Senanayake, Adv. Synth. Catal. 2011, 353, 1825-1864; b) C. Torborg, M. Beller, Adv. Synth. Catal. 2009, 351, 3027-3043; c) K. Nicolaou, P. G. Bulger, D. Sarlah, Angew. Chem. Int. Ed. 2005, 44, 4442-4489.

[9] a) L. Ackermann, Modern Arylation Methods, Wiley-VCH, Weinheim, 2009; b) A. de Meijere, F. Diederich, Metal-Catalyzed Cross-Coupling Reactions, Wiley-VCH, Weinheim, 2004.

[10] a) B. M. Trost, Angew. Chem. Int. Ed. 1995, 34, 259-281; b) B. M. Trost, Science 1991, 254, 1471-1477.

[11] a) P. A. Wender, B. L. Miller, Nature 2009, 460, 197-201; b) P. A. Wender, M. P. Croatt, B. Witulski, Tetrahedron 2006, 62, 7505-7511.

[12] a) L. Ackermann, A. R. Kapdi, H. K. Potukuchi, S. I. Kozhushkov, in Handbook of Green Chemistry, Ed.: P. T. Anastas, Wiley-VCH, Weinheim 2012, pp. 259-305; b) P. Anastas, J. Warner, Green Chemistry: Theory and Practice, Oxford University Press, New York, 1998.

[13] For reviews on $\mathrm{C}-\mathrm{H}$ functionalization: a) L. Vaccaro, S. Santoro, L. Ackermann, S. Kozhushkov, Green Chem. 2016, 18, 5025-5030; b) C. Borie, L. Ackermann, M. Nechab, Chem. Soc. Rev. 2016, 45, 1368-1386; c) B. Ye, N. Cramer, Acc. Chem. Res. 2015, 48, 13081318; d) K. Shin, H. Kim, S. Chang, Acc. Chem. Res. 2015, 48, 1040-1052; e) Y. Segawa, T. Maekawa, K. Itami, Angew. Chem. Int. Ed. 2015, 54, 66-81; f) B. Liu, F. Hu, B.-F. Shi, ACS Catal. 2015, 5, 1863-1881; g) O. Daugulis, J. Roane, L. D. Tran, Acc. Chem. Res. 2015, 48, 1053-1064; h) V. S. Thirunavukkarasu, S. I. Kozhushkov, L. Ackermann, Chem. Commun. 2014, 50, 29-39; i) S. A. Girard, T. Knauber, C.-J. Li, Angew. Chem. Int. Ed. 2014, 53, 74100; j) S. De Sarkar, W. Liu, S. I. Kozhushkov, L. Ackermann, Adv. Synth. Catal. 2014, 356, 1461-1479; k) J. Wencel-Delord, F. Glorius, Nat. Chem. 2013, 5, 369-375; 1) G. Rouquet, N. Chatani, Angew. Chem. Int. Ed. 2013, 52, 11726-11743; m) J. Yamaguchi, A. D. Yamaguchi, K. Itami, Angew. Chem. Int. Ed. 2012, 51, 8960-9009; n) C. S. Yeung, V. M. Dong, Chem. Rev. 2011, 111, 1215-1292; o) T. Satoh, M. Miura, Chem. Eur. J. 2010, 16, 11212-11222; p) R. Giri, B.-F. Shi, K. M. Engle, N. Maugel, J.-Q. Yu, Chem. Soc. Rev. 2009, 38, 3242-3272; q) R. G. Bergman, Nature 2007, 446, 391-393; r) D. Alberico, M. E. Scott, M. Lautens, Chem. Rev. 2007, 107, 174-238; s) L. Ackermann, Synlett 2007, 507-526; t) L. Ackermann, in Chelation-Assisted Arylation via $C-H$ Bond Cleavage, Ed.: N. Chatani, Springer Berlin, Heidelberg, 2007, pp. 35-60.

[14] a) W. Liu, L. Ackermann, ACS Catal. 2016, 6, 3743-3752; b) C. Wang, Synlett 2013, 24, $1606-1613$.

[15] a) M. Moselage, J. Li, L. Ackermann, ACS Catal. 2015, 6, 498-525; b) L. Ackermann, J. Org. Chem. 2014, 79, 8948-8954; c) K. Gao, N. Yoshikai, Acc. Chem. Res. 2014, 47, 1208-1219.

[16] a) D. Balcells, E. Clot, O. Eisenstein, Chem. Rev. 2010, 110, 749-823; b) Y. Boutadla, D. L. Davies, S. A. Macgregor, A. I. Poblador-Bahamonde, Dalton Trans. 2009, 5820-5831; c) Z. Lin, Coord. Chem. Rev. 2007, 251, 2280-2291. 
[17] a) T. R. Cundari, T. R. Klinckman, P. T. Wolczanski, J. Am. Chem. Soc. 2002, 124, 14811487; b) J. L. Bennett, P. T. Wolczanski, J. Am. Chem. Soc. 1997, 119, 10696-10719.

[18] J. Kua, X. Xu, R. A. Periana, W. A. Goddard, Organometallics 2002, 21, 511-525.

[19] J. Oxgaard, W. J. Tenn, R. J. Nielsen, R. A. Periana, W. A. Goddard, Organometallics 2007, $26,1565-1567$.

[20] a) L. Ackermann, Chem. Rev. 2011, 111, 1315-1345; b) D. Lapointe, K. Fagnou, Chem. Lett. 2010, 39, 1118-1126; c) S. I. Gorelsky, D. Lapointe, K. Fagnou, J. Am. Chem. Soc. 2008, 130, 10848-10849.

[21] a) Y. Boutadla, D. L. Davies, S. A. Macgregor, A. I. Poblador-Bahamonde, Dalton Trans. 2009, 5887-5893; b) D. L. Davies, S. M. Donald, S. A. Macgregor, J. Am. Chem. Soc. 2005, 127, 13754-13755.

[22] a) D. García-Cuadrado, A. A. Braga, F. Maseras, A. M. Echavarren, J. Am. Chem. Soc. 2006, 128, 1066-1067; b) L.-C. Campeau, M. Parisien, A. Jean, K. Fagnou, J. Am. Chem. Soc. 2006, 128, 581-590; c) L.-C. Campeau, M. Parisien, M. Leblanc, K. Fagnou, J. Am. Chem. Soc. 2004, 126, 9186-9187.

[23] a) K. Raghuvanshi, D. Zell, L. Ackermann, Org. Lett. 2017, 19, 1278-1281; b) K. Raghuvanshi, D. Zell, K. Rauch, L. Ackermann, ACS Catal. 2016, 6, 3172-3175; c) H. Wang, M. Moselage, M. J. González, L. Ackermann, ACS Catal. 2016, 6, 2705-2709; d) R. Mei, J. Loup, L. Ackermann, ACS Catal. 2016, 6, 793-797; e) W. Ma, R. Mei, G. Tenti, L. Ackermann, Chem. Eur. J. 2014, 20, 15248-15251.

[24] S. De Sarkar, W. Liu, S. I. Kozhushkov, L. Ackermann, Adv. Synth. Catal. 2014, 356, 14611479 .

[25] a) W. Ma, P. Gandeepan, J. Li, L. Ackermann, Org. Chem. Front. 2017, DOI: 10.1039/C1037QO00134G; b) F. Zhang, D. R. Spring, Chem. Soc. Rev. 2014, 43, 6906-6919.

[26] a) M. Seki, Org. Process Res. Dev. 2016, 20, 867-877; b) L. Ackermann, Org. Process Res. Dev. 2015, 19, 260-269; c) J. Yamaguchi, A. D. Yamaguchi, K. Itami, Angew. Chem. Int. Ed. 2012, 51, 8960-9009; d) J.-Q. Yu, Z. Shi, C-H Activation, Springer Berlin, Heidelberg, 2010.

[27] a) L. Ackermann, Acc. Chem. Res. 2014, 47, 281-295; b) P. B. Arockiam, C. Bruneau, P. H. Dixneuf, Chem. Rev. 2012, 112, 5879-5918.

[28] S. Oi, S. Fukita, N. Hirata, N. Watanuki, S. Miyano, Y. Inoue, Org. Lett. 2001, 3, 2579-2581.

[29] S. Oi, R. Funayama, T. Hattori, Y. Inoue, Tetrahedron 2008, 64, 6051-6059.

[30] S. Oi, Y. Ogino, S. Fukita, Y. Inoue, Org. Lett. 2002, 4, 1783-1785.

[31] S. Oi, E. Aizawa, Y. Ogino, Y. Inoue, J. Org. Chem. 2005, 70, 3113-3119.

[32] S. Oi, K. Sakai, Y. Inoue, Org. Lett. 2005, 7, 4009-4011.

[33] S. G. Ouellet, A. Roy, C. Molinaro, R. Angelaud, J.-F. Marcoux, P. D. O'Shea, I. W. Davies, J. Org. Chem. 2011, 76, 1436-1439.

[34] F. Kakiuchi, S. Kan, K. Igi, N. Chatani, S. Murai, J. Am. Chem. Soc. 2003, 125, 1698-1699.

[35] F. Kakiuchi, Y. Matsuura, S. Kan, N. Chatani, J. Am. Chem. Soc. 2005, 127, 5936-5945.

[36] a) L. Ackermann, R. Vicente, A. Althammer, Org. Lett. 2008, 10, 2299-2302; b) L. Ackermann, A. Althammer, R. Born, Angew. Chem. Int. Ed. 2006, 45, 2619-2622; c) L. Ackermann, Org. Lett. 2005, 7, 3123-3125.

[37] a) S. I. Kozhushkov, H. K. Potukuchi, L. Ackermann, Catal. Sci. Technol. 2013, 3, 562-571; b) J. Kerr, Chem. Rev. 1966, 66, 465-500.

[38] V. Sokolov, L. L. Troitskaya, O. A. Reutov, J. Organomet. Chem. 1979, 182, 537-546.

[39] a) M. Lafrance, S. I. Gorelsky, K. Fagnou, J. Am. Chem. Soc. 2007, 129, 14570-14571; b) M. Lafrance, K. Fagnou, J. Am. Chem. Soc. 2006, 128, 16496-16497.

[40] a) H. Nandivada, X. Jiang, J. Lahann, Adv. Mater. 2007, 19, 2197-2208; b) Y. L. Angell, K. Burgess, Chem. Soc. Rev. 2007, 36, 1674-1689.

[41] F. Požgan, P. H. Dixneuf, Adv. Synth. Catal. 2009, 351, 1737-1743.

[42] L. Ackermann, R. Vicente, H. K. Potukuchi, V. Pirovano, Org. Lett. 2010, 12, 5032-5035.

[43] L. Ackermann, A. V. Lygin, Org. Lett. 2011, 13, 3332-3335.

[44] a) P. B. Arockiam, C. Fischmeister, C. Bruneau, P. H. Dixneuf, Angew. Chem. Int. Ed. 2010, 122, 6629-6632; b) L. Ackermann, J. Pospech, H. K. Potukuchi, Org. Lett. 2012, 14, 21462149. 
[45] a) M. Seki, Synthesis 2012, 44, 3231-3237; b) M. Seki, M. Nagahama, J. Org. Chem. 2011, 76, 10198-10206.

[46] M. K. Lakshman, A. C. Deb, R. R. Chamala, P. Pradhan, R. Pratap, Angew. Chem. Int. Ed. 2011, 50, 11400-11404.

[47] a) L. Ackermann, A. Althammer, R. Born, Tetrahedron 2008, 64, 6115-6124; b) L. Ackermann, A. Althammer, R. Born, Synlett 2007, 2833-2836.

[48] N. Luo, Z. Yu, Chem. Eur. J. 2010, 16, 787-791.

[49] O. Roelen, Chemische Verwertungsgesellschaft Oberhausen mbH, DE 849548, 1938/1952.

[50] F. Hebrard, P. Kalck, Chem. Rev. 2009, 109, 4272-4282.

[51] I. Khand, G. Knox, P. Pauson, W. Watts, J. Chem. Soc. D, Chem. Commun. 1971, 36.

[52] A. Y. Khodakov, W. Chu, P. Fongarland, Chem. Rev. 2007, 107, 1692-1744.

[53] M. Hawkins, Appl. Earth Sci. 2001, 110, 66-70.

[54] D. S. Avila, R. L. Puntel, M. Aschner, in Interrelations between Essential Metal Ions and Human Diseases, Vol. 13, Eds.: A. Sigel, H. Sigel, K. O. R. Sigel, Springer Netherlands, Dordrecht, 2013, pp. 199-227.

[55] a) S. Murahashi, S. Horiie, J. Am. Chem. Soc. 1956, 78, 4816-4817; b) S. Murahashi, J. Am. Chem. Soc. 1955, 77, 6403-6404.

[56] H. F. Klein, S. Schneider, M. He, U. Floerke, H. J. Haupt, Eur. J. Inorg. Chem. 2000, 22952301.

[57] a) R. Beck, H. Sun, X. Li, S. Camadanli, H. F. Klein, Eur. J. Inorg. Chem. 2008, 3253-3257;

b) S. Camadanli, R. Beck, U. Flörke, H.-F. Klein, Dalton Trans. 2008, 5701-5704; c) H. F. Klein, S. Camadanli, R. Beck, D. Leukel, U. Flörke, Angew. Chem. Int. Ed. 2005, 44, 975977; d) H. F. Klein, R. Beck, U. Flörke, H. J. Haupt, Eur. J. Inorg. Chem. 2003, 1380-1387.

[58] U. Koelle, B. Fuss, M. Rajasekharan, B. Ramakrishna, J. Ammeter, M. Böhm, J. Am. Chem. Soc. 1984, 106, 4152-4160.

[59] a) G. Song, F. Wang, X. Li, Chem. Soc. Rev. 2012, 41, 3651-3678; b) F. W. Patureau, J. Wencel-Delord, F. Glorius, Aldrichim. Acta 2012, 45, 31-41.

[60] T. Yoshino, H. Ikemoto, S. Matsunaga, M. Kanai, Angew. Chem. Int. Ed. 2013, 52, $2207-$ 2211.

[61] a) M. E. Tauchert, C. D. Incarvito, A. L. Rheingold, R. G. Bergman, J. A. Ellman, J. Am. Chem. Soc. 2012, 134, 1482; b) Y. Li, X.-S. Zhang, H. Li, W.-H. Wang, K. Chen, B.-J. Li, Z.J. Shi, Chem. Sci. 2012, 3, 1634-1639.

[62] A. Allred, J. Inorg. Nucl. Chem. 1961, 17, 215-221.

[63] T. Yoshino, H. Ikemoto, S. Matsunaga, M. Kanai, Chem. Eur. J. 2013, 19, 9142-9146.

[64] J. R. Hummel, J. A. Ellman, J. Am. Chem. Soc. 2015, 137, 490-498.

[65] H. Wang, J. Koeller, W. Liu, L. Ackermann, Chem. Eur. J. 2015, 21, 15525-15528.

[66] J. Park, S. Chang, Angew. Chem. Int. Ed. 2015, 54, 14103-14107.

[67] Y. Liang, Y. F. Liang, C. Tang, Y. Yuan, N. Jiao, Chem. Eur. J. 2015, 21, 16395-16399.

[68] B. Sun, T. Yoshino, M. Kanai, S. Matsunaga, Angew. Chem. Int. Ed. 2015, 54, 12968-12972.

[69] M. Sen, D. Kalsi, B. Sundararaju, Chem. Eur. J. 2015, 21, 15529-15533.

[70] N. R. Candeias, L. C. Branco, P. M. P. Gois, C. A. Afonso, A. F. Trindade, Chem. Rev. 2009, 109, 2703-2802.

[71] B. Sun, T. Yoshino, S. Matsunaga, M. Kanai, Adv. Synth. Catal. 2014, 356, 1491-1495.

[72] B. Sun, T. Yoshino, S. Matsunaga, M. Kanai, Chem. Commun. 2015, 51, 4659-4661.

[73] P. Anbarasan, T. Schareina, M. Beller, Chem. Soc. Rev. 2011, 40, 5049-5067.

[74] J. Li, L. Ackermann, Angew. Chem. Int. Ed. 2015, 54, 3635-3638.

[75] D.-G. Yu, T. Gensch, F. de Azambuja, S. Vásquez-Céspedes, F. Glorius, J. Am. Chem. Soc. 2014, 136, 17722-17725.

[76] U. Kazmaier, Transition Metal Catalyzed Enantioselective Allylic Substitution in Organic Synthesis, Vol. 38, Springer Berlin, Heidelberg, 2011.

[77] M. Moselage, N. Sauermann, J. Koeller, W. Liu, D. Gelman, L. Ackermann, Synlett 2015, 26, $1596-1600$.

[78] Y. Suzuki, B. Sun, K. Sakata, T. Yoshino, S. Matsunaga, M. Kanai, Angew. Chem. Int. Ed. 2015, 54, 9944-9947.

[79] T. Gensch, S. Vásquez-Céspedes, D.-G. Yu, F. Glorius, Org. Lett. 2015, 17, 3714-3717. 
[80] G. Halbritter, F. Knoch, A. Wolski, H. Kisch, Angew. Chem. Int. Ed. 1994, 33, 1603-1605.

[81] K. Gao, P.-S. Lee, T. Fujita, N. Yoshikai, J. Am. Chem. Soc. 2010, 132, 12249-12251.

[82] P.-S. Lee, T. Fujita, N. Yoshikai, J. Am. Chem. Soc. 2011, 133, 17283-17295.

[83] Z. Ding, N. Yoshikai, Angew. Chem. Int. Ed. 2012, 51, 4698-4701.

[84] K. Gao, N. Yoshikai, J. Am. Chem. Soc. 2011, 133, 400-402.

[85] L. Ilies, Q. Chen, X. Zeng, E. Nakamura, J. Am. Chem. Soc. 2011, 133, 5221-5223.

[86] V. Galamb, G. Palyi, F. Ungvary, L. Marko, R. Boese, G. Schmid, J. Am. Chem. Soc. 1986, $108,3344-3351$.

[87] Q. Chen, L. Ilies, E. Nakamura, J. Am. Chem. Soc. 2011, 133, 428-429.

[88] P.-S. Lee, N. Yoshikai, Org. Lett. 2015, 17, 22-25.

[89] J. F. Teichert, B. L. Feringa, Angew. Chem. Int. Ed. 2010, 49, 2486-2528.

[90] D. J. Schipper, M. Hutchinson, K. Fagnou, J. Am. Chem. Soc. 2010, 132, 6910-6911.

[91] H. Ikemoto, T. Yoshino, K. Sakata, S. Matsunaga, M. Kanai, J. Am. Chem. Soc. 2014, 136, $5424-5431$.

[92] S. B. Goldhaber, Regul. Toxicol. Pharm. 2003, 38, 232-242.

[93] J. Emsley, Oxygen. Nature's Building Blocks: An A-Z Guide to the Elements, Oxford University Press, Oxford, 2001.

[94] M. Bruce, M. Iqbal, F. Stone, J. Chem. Soc. A: Inorg. Phys. Theor. 1970, 3204-3209.

[95] L. S. Liebeskind, J. R. Gasdaska, J. S. McCallum, S. J. Tremont, J. Org. Chem. 1989, 54, 669-677.

[96] a) G. J. Depree, L. Main, B. K. Nicholson, J. Organomet. Chem. 1998, 551, 281-291; b) W. Tully, L. Main, B. K. Nicholson, J. Organomet. Chem. 1995, 503, 75-92.

[97] R. C. Cambie, M. R. Metzler, P. S. Rutledge, P. D. Woodgate, J. Organomet. Chem. 1992, $429,41-57$.

[98] Y. Kuninobu, Y. Nishina, T. Takeuchi, K. Takai, Angew. Chem. Int. Ed. 2007, 46, 6518-6520.

[99] B. Zhou, Y. Hu, C. Wang, Angew. Chem. Int. Ed. 2015, 54, 13659-13663.

[100] W. Liu, J. Bang, Y. Zhang, L. Ackermann, Angew. Chem. Int. Ed. 2015, 54, 14137-14140.

[101] W. Liu, S. C. Richter, R. Mei, M. Feldt, L. Ackermann, Chem. Eur. J. 2016, 22, 1795817961.

[102] P. Anbarasan, H. Neumann, M. Beller, Angew. Chem. Int. Ed. 2011, 50, 519-522.

[103] B. Zhou, H. Chen, C. Wang, J. Am. Chem. Soc. 2013, 135, 1264-1267.

[104] B. Zhou, P. Ma, H. Chen, C. Wang, Chem. Commun. 2014, 50, 14558-14561.

[105] R. He, Z. T. Huang, Q. Y. Zheng, C. Wang, Angew. Chem. Int. Ed. 2014, 53, 4950-4953.

[106] W. Liu, D. Zell, M. John, L. Ackermann, Angew. Chem. Int. Ed. 2015, 54, 4092-4096.

[107] a) L. Kiss, F. Fülöp, Chem. Rev. 2013, 114, 1116-1169; b) D. Seebach, J. Gardiner, Acc. Chem. Res. 2008, 41, 1366-1375.

[108] W. Liu, S. C. Richter, Y. Zhang, L. Ackermann, Angew. Chem. Int. Ed. 2016, 55, 7747-7750.

[109] a) L. Ackermann, Org. Process Res. Dev. 2015, 19, 260-269; b) L. Ackermann, Acc. Chem. Res. 2013, 47, 281-295.

[110] a) L. Souillart, N. Cramer, Chem. Rev. 2015, 115, 9410-9464; b) M. Murakami, T. Matsuda, Chem. Commun. 2011, 47, 1100-1105; c) V. Ritleng, C. Sirlin, M. Pfeffer, Chem. Rev. 2002, 102, 1731-1770.

[111] a) T. F. Schneider, J. Kaschel, D. B. Werz, Angew. Chem. Int. Ed. 2014, 53, 5504-5523; b) M. Rubin, M. Rubina, V. Gevorgyan, Chem. Rev. 2007, 107, 3117-3179.

[112] N. A. Foley, J. P. Lee, Z. Ke, T. B. Gunnoe, T. R. Cundari, Acc. Chem. Res. 2009, 42, 585597.

[113] a) G. M. Crisenza, O. O. Sokolova, J. F. Bower, Angew. Chem. Int. Ed. 2015, 54, 1486614870; b) G. M. Crisenza, N. G. McCreanor, J. F. Bower, J. Am. Chem. Soc. 2014, 136, $10258-10261$.

[114] K. Gao, N. Yoshikai, J. Am. Chem. Soc. 2010, 133, 400-402.

[115] H. Amii, K. Uneyama, Chem. Rev. 2009, 109, 2119-2183.

[116] a) W. K. Hagmann, J. Med. Chem. 2008, 51, 4359-4369; b) K. Müller, C. Faeh, F. Diederich, Science 2007, 317, 1881-1886; c) P. Kirsch, Modern Fluoroorganic Chemistry: Synthesis, Reactivity, Applications, Wiley-VCH, Weinheim, 2004. 
[117] a) Q. Lu, F. J. Klauck, F. Glorius, Chem. Sci. 2017, 8, 3379-3383; b) D. Zell, Q. Bu, M. Feldt, L. Ackermann, Angew. Chem. Int. Ed. 2016, 55, 7408-7412; c) J.-Q. Wu, Z.-P. Qiu, S.-S. Zhang, J.-G. Liu, Y.-X. Lao, L.-Q. Gu, Z.-S. Huang, J. Li, H. Wang, Chem. Commun. 2015, 51, 77-80; d) T. H. Meyer, W. Liu, M. Feldt, A. Wuttke, R. A. Mata, L. Ackermann, Chem. Eur. J. 2017, 23, 5443-5447.

[118] Selected examples: a) G. Yin, X. Mu, G. Liu, Acc. Chem. Res. 2016, 49, 2413-2423; b) T. Ahrens, J. Kohlmann, M. Ahrens, T. Braun, Chem. Rev. 2015, 115, 931-972; c) H. Egami, M. Sodeoka, Angew. Chem. Int. Ed. 2014, 53, 8294-8308; d) T. Besset, T. Poisson, X. Pannecoucke, Chem. Eur. J. 2014, 20, 16830-16845; e) T. Liang, C. N. Neumann, T. Ritter, Angew. Chem. Int. Ed. 2013, 52, 8214-8264; f) J. Wang, M. Sánchez-Roselló, J. L. Aceña, C. del Pozo, A. E. Sorochinsky, S. Fustero, V. A. Soloshonok, H. Liu, Chem. Rev. 2013, 114, 2432; g) T. Stahl, H. F. T. Klare, M. Oestreich, ACS Catal. 2013, 3, 1578-1587; h) T. Furuya, A. S. Kamlet, T. Ritter, Nature 2011, 473, 470-477; i) T. Braun, F. Wehmeier, Eur. J. Inorg. Chem. 2011, 2011, 613-625; j) H. Amii, K. Uneyama, Chem. Rev. 2009, 109, 2119-2183; k) K. Müller, C. Faeh, F. Diederich, Science 2007, 317, 1881-1886; 1) T. Braun, R. N. Perutz, Chem. Commun. 2002, 2749-2757.

[119] a) D. J. Harrison, G. M. Lee, M. C. Leclerc, I. Korobkov, R. T. Baker, J. Am. Chem. Soc. 2013, 135, 18296-18299; b) M. F. Kühnel, D. Lentz, Angew. Chem. Int. Ed. 2010, 49, $2933-$ 2936; c) A. A. Peterson, K. McNeill, Organometallics 2006, 25, 4938-4940; d) K. Sakoda, J. Mihara, J. Ichikawa, Chem. Commun. 2005, 4684-4686; e) L. A. Gharat, A. R. Martin, Heterocycles 1996, 1, 185-189; f) W. Heitz, A. Knebelkamp, Macromol. Rapid Commun. 1991, 12, 69-75.

[120] Y. F. Liang, L. Massignan, W. Liu, L. Ackermann, Chem. Eur. J. 2016, 22, 14856-14859.

[121] a) J.-R. Pouliot, F. Grenier, J. T. Blaskovits, S. Beaupré, M. Leclerc, Chem. Rev. 2016, 116, 14225-14274; b) Y. Segawa, T. Maekawa, K. Itami, Angew. Chem. Int. Ed. 2015, 54, 66-81; c) L. G. Mercier, M. Leclerc, Acc. Chem. Res. 2013, 46, 1597-1605; d) W. Lu, J. Kuwabara, T. Kanbara, Macromol. Rapid Commun. 2013, 34, 1151-1156.

[122] a) E. Tomás-Mendivil, V. Cadierno, M. I. Menéndez, R. López, Chem. Eur. J. 2015, 21, 16874-16886; b) S. M. M. Knapp, T. J. Sherbow, R. B. Yelle, J. J. Juliette, D. R. Tyler, Organometallics 2013, 32, 3744-3752; c) E. Y. Chan, Q.-F. Zhang, Y.-K. Sau, S. M. Lo, H. H. Sung, I. D. Williams, R. K. Haynes, W.-H. Leung, Inorg. Chem. 2004, 43, 4921-4926.

[123] D. Zell, S. Warratz, D. Gelman, S. J. Garden, L. Ackermann, Chem. Eur. J. 2016, 22, 1248 1252.

[124] J. P. Wagner, P. R. Schreiner, Angew. Chem. Int. Ed. 2015, 54, 12274-12296, and references cited therein.

[125] L. Ackermann, P. Novák, R. Vicente, V. Pirovano, H. K. Potukuchi, Synthesis 2010, 2010, $2245-2253$.

[126] A. B. Flynn, W. W. Ogilvie, Chem. Rev. 2007, 107, 4698-4745, and references cited therein.

[127] S. Warratz, PhD thesis, Georg-August-Universität Göttingen 2016.

[128] T. Tsou, J. Kochi, J. Am. Chem. Soc. 1979, 101, 6319-6332.

[129] G. Manolikakes, P. Knochel, Angew. Chem. Int. Ed. 2009, 48, 205-209.

[130] T. L. Hall, M. F. Lappert, P. W. Lednor, J. Chem. Soc., Dalton Trans. 1980, 1448-1456.

[131] J. Hubrich, L. Ackermann, Eur. J. Org. Chem. 2016, 3700-3704.

[132] P. L. Anelli, F. Montanari, S. Quici, Org. Synth. 1990, 49, 212.

[133] L. P. Hammett, J. Am. Chem. Soc. 1937, 640, 96-103.

[134] C. Hansch, A. Leo, Substituent Constants for Correlation Analysis in Chemistry and Biology, John Wiley \& Sons Inc., Hoboken, New Jersey, 1979.

[135] a) Z. Ruan, S. Lackner, L. Ackermann, Angew. Chem. Int. Ed. 2016, 55, 3153-3157; b) Y. Aihara, N. Chatani, Chem. Sci. 2013, 4, 664-670.

[136] E. M. Simmons, J. F. Hartwig, Angew. Chem. Int. Ed. 2012, 51, 3066-3072.

[137] a) M. Brasse, J. Cámpora, J. A. Ellman, R. G. Bergman, J. Am. Chem. Soc. 2013, 135, 6427; b) Y. Tan, F. Barrios-Landeros, J. F. Hartwig, J. Am. Chem. Soc. 2012, 134, 3683; c) E. V. Anslyn, D. A. Dougherty, Modern Physical Organic Chemistry, University Science Books, Sausalito, 2006. 
[138] M. Simonetti, G. Perry, X. C. Cambeiro, F. Juliá-Hernández, J. N. Arokianathar, I. Larrosa, J. Am. Chem. Soc. 2016, 138, 3596-3606.

[139] C. Wang, Synlett 2013, 24, 1606-1613.

[140] a) X. Wang, A. Lerchen, F. Glorius, Org. Lett. 2016, 18, 2090-2093; b) D. Zhao, J. H. Kim, L. Stegemann, C. A. Strassert, F. Glorius, Angew. Chem. Int. Ed. 2015, 54, 4508-4511.

[141] X.-G. Liu, S.-S. Zhang, J.-Q. Wu, Q. Li, H. Wang, Tetrahedron Lett. 2015, 56, 4093-4095.

[142] S. Grimme, A. Hansen, J. G. Brandenburg, C. Bannwarth, Chem. Rev. 2016, 116, 5105-5154.

[143] M. Feldt, L. Ackermann, unpublished results.

[144] H. McNab, Chem. Soc. Rev. 1978, 7, 345-358, and references cited therein.

[145] P. Gandeepan, J. Koeller, L. Ackermann, ACS Catal. 2017, 7, 1030-1034.

[146] a) T. Zhou, Y. Wang, B. Li, B. Wang, Org. Lett. 2016, 18, 5066-5069; b) L. Xu, C. Zhang, Y. He, L. Tan, D. Ma, Angew. Chem. Int. Ed. 2016, 55, 321-325.

[147] L. Xu, L. Tan, D. Ma, J. Org. Chem. 2016, 81, 10476-10483.

[148] I. Krossing, I. Raabe, Angew. Chem. Int. Ed. 2004, 43, 2066-2090.

[149] S. Kozuch, S. Shaik, Acc. Chem. Res. 2010, 44, 101-110.

[150] a) G. M. Crisenza, J. F. Bower, Chem. Lett. 2016, 45, 2-9; b) F. Kakiuchi, S. Murai, Acc. Chem. Res. 2002, 35, 826-834.

[151] a) Y. Ebe, T. Nishimura, J. Am. Chem. Soc. 2015, 137, 5899-5902; b) B. Ye, P. A. Donets, N. Cramer, Angew. Chem. Int. Ed. 2014, 53, 507-511; c) P. S. Lee, N. Yoshikai, Angew. Chem. Int. Ed. 2013, 52, 1240-1244; d) S. Pan, N. Ryu, T. Shibata, J. Am. Chem. Soc. 2012, 134, 17474-17477; e) Y. Nakao, N. Kashihara, K. S. Kanyiva, T. Hiyama, Angew. Chem. Int. Ed. 2010, 49, 4451-4454; f) T. Mukai, K. Hirano, T. Satoh, M. Miura, J. Org. Chem. 2009, 74, 6410-6413; g) Y. Uchimaru, Chem. Commun. 1999, 1133-1134.

[152] V. Müller, L. Ackermann, unpublished results.

[153] E. V. Dehmlow, M. Lissel, Synthesis 1979, 5, 372-374.

[154] A. Isidro Llobet, M. Álvarez Domingo, F. Albericio Palomera, Chem. Rev. 2009, 109 24552504.

[155] J. Li, S. Warratz, D. Zell, S. De Sarkar, E. E. Ishikawa, L. Ackermann, J. Am. Chem. Soc. 2015, 137, 13894-13901.

[156] a) G.-J. Cheng, Y.-F. Yang, P. Liu, P. Chen, T.-Y. Sun, G. Li, X. Zhang, K. Houk, J.-Q. Yu, Y.-D. Wu, J. Am. Chem. Soc. 2014, 136, 894-897; b) G. Li, D. Leow, L. Wan, J. Q. Yu, Angew. Chem. Int. Ed. 2013, 52, 1245-1247.

[157] B. F. Shi, N. Maugel, Y. H. Zhang, J. Q. Yu, Angew. Chem. Int. Ed. 2008, 47, 4882-4886.

[158] B. Ye, N. Cramer, Angew. Chem. Int. Ed. 2014, 53, 7896-7899.

[159] J. Zheng, S.-B. Wang, C. Zheng, S.-L. You, J. Am. Chem. Soc. 2015, 137, 4880-4883.

[160] S. Reddy Chidipudi, D. J. Burns, I. Khan, H. W. Lam, Angew. Chem. Int. Ed. 2015, 54, 13975-13979.

[161] D. Zell, M. Bursch, V. Müller, S. Grimme, L. Ackermann, Angew. Chem. Int. Ed. 2017, 56, under revision.

[162] a) D. Zell, F. Pescialioli, V. Müller, L. Ackermann, unpublished results; b) V. Müller, MasterThesis, Georg-August-Universität Göttingen, 2016.

[163] a) M. Sen, B. Emayavaramban, N. Barsu, J. R. Premkumar, B. Sundararaju, ACS Catal. 2016, 6, 2792-2796; b) J. Zhang, H. Chen, C. Lin, Z. Liu, C. Wang, Y. Zhang, J. Am. Chem. Soc. 2015, 137, 12990-12996.

[164] a) G. 1. Erdogan, D. B. Grotjahn, J. Am. Chem. Soc. 2009, 131, 10354-10355; b) J. Zhou, J. F. Hartwig, Angew. Chem. Int. Ed. 2008, 47, 5783-5787; c) C. M. Yung, M. B. Skaddan, R. G. Bergman, J. Am. Chem. Soc. 2004, 126, 13033-13043.

[165] K. J. Laidler, in Chemical Kinetics, McGraw-Hill, New York, 1977, pp. 310-311.

[166] S. Arrhenius, Z. Phys. Chem. 1889, 4, 226-248.

[167] J. Wang, M. Sánchez-Roselló, J. L. Aceña, C. del Pozo, A. E. Sorochinsky, S. Fustero, V. A. Soloshonok, H. Liu, Chem. Rev. 2014, 114, 2432-2506.

[168] a) C.-Q. Wang, L. Ye, C. Feng, T.-P. Loh, J. Am. Chem. Soc. 2017, 139, 1762-1765; b) J.-Q. Wu, S.-S. Zhang, H. Gao, Z. Qi, C.-J. Zhou, W.-W. Ji, Y. Liu, Y. Chen, Q. Li, X. Li, J. Am. Chem. Soc. 2017, 139, 3537-3545; c) L. Kong, X. Zhou, X. Li, Org. Lett. 2016; d) P. Tian, C. Feng, T.-P. Loh, Nat. Commun. 2015, 6, 7472. 
[169] J. A. Gladysz, D. P. Curran, I. T. Horváth, Handbook of Fluorous Chemistry, John Wiley \& Sons Inc., Hoboken, New Jersey, 2006.

[170] A. Berkessel, J. A. Adrio, D. Hüttenhain, J. M. Neudörfl, J. Am. Chem. Soc. 2006, 128, 84218426.

[171] D. A. Wynn, M. M. Roth, B. D. Pollard, Talanta 1984, 31, 1036-1040.

[172] a) T. K. Hyster, D. M. Dalton, T. Rovis, Chem. Sci. 2015, 6, 254-258; b) T. Piou, T. Rovis, J. Am. Chem. Soc. 2014, 136, 11292.

[173] a) G. Landelle, M. Bergeron, M.-O. Turcotte-Savard, J.-F. Paquin, Chem. Soc. Rev. 2011, 40, 2867-2908; b) C. E. Jakobsche, G. Peris, S. J. Miller, Angew. Chem. Int. Ed. 2008, 47, 67076711.

[174] D. Zell, V. Müller, U. Dhawa, M. Bursch, R. R. Presa, S. Grimme, L. Ackermann, Chem. Eur. J. 2017, 23, submitted.

[175] L. Ackermann, N. Hofmann, R. Vicente, Org. Lett. 2011, 13, 1875-1877.

[176] D. Zell, U. Dhawa, V. Müller, M. Bursch, S. Grimme, L. Ackermann, ACS Catal. 2017, 7, 4209-4213.

[177] a) M. Moselage, J. Li, F. Kramm, L. Ackermann, Angew. Chem. Int. Ed. 2017, 56, 53415344; b) C. Kashima, S. Hibi, T. Maruyama, K. Harada, Y. Omote, J. Heterocycl. Chem. 1987, 24, 637-639.

[178] D. García-Cuadrado, P. de Mendoza, A. A. Braga, F. Maseras, A. M. Echavarren, J. Am. Chem. Soc. 2007, 129, 6880-6886.

[179] a) H. Clavier, S. P. Nolan, Chem. Commun. 2010, 46, 841-861; b) A. C. Hillier, W. J. Sommer, B. S. Yong, J. L. Petersen, L. Cavallo, S. P. Nolan, Organometallics 2003, 22, 4322-4326.

[180] J. L. R. Williams, R. E. Adel, J. M. Carlson, G. A. Reynolds, D. G. Borden, J. A. Ford Jr., J. Org. Chem. 1963, 28, 387-390.

[181] G. Ghattas, D. Chen, F. Pan, J. Klankermayer, Dalton Trans. 2012, 41, 9026-9028.

[182] a) V. K. Tiwari, N. Kamal, M. Kapur, Org. Lett. 2015, 17, 1766-1769; b) S. Xu, X. Huang, X. Hong, B. Xu, Org. Lett. 2012, 14, 4614-4617.

[183] a) J. Y. Kim, S. H. Park, J. Ryu, S. H. Cho, S. H. Kim, S. Chang, J. Am. Chem. Soc. 2012, 134, 9110-9113; b) V. P. Böhm, T. Weskamp, C. W. Gstöttmayr, W. A. Herrmann, Angew. Chem. Int. Ed. 2000, 39, 1602-1604.

[184] L. Ackermann, R. Born, P. Álvarez-Bercedo, Angew. Chem. Int. Ed. 2007, 46, 6364-6367.

[185] B. Punji, W. Song, G. A. Shevchenko, L. Ackermann, Chem. Eur. J. 2013, 19, 10605-10610.

[186] V. Soni, R. A. Jagtap, R. G. Gonnade, B. Punji, ACS Catal. 2016, 6, 5666-5672. 



\section{Danksagung}

Mein ganz herzlicher Dank gilt Herrn Prof. Dr. Lutz Ackermann dafür, dass er mir die Möglichkeit gegeben hat, meine Doktorarbeit in einem exzellenten Umfeld durchzuführen. Dabei sind vor allem seine höchst interessante Aufgabenstellung sowie die stets sehr freundliche, motivierende und fachlich hervorragende Betreuung meiner Arbeit hervorzuheben.

Bei Herrn PD Dr. Alexander Breder möchte ich mich für die Übernahme des Korreferats bedanken und die hilfreichen Anregungen während der Arbeitskreisseminare. Des Weiteren danke ich Prof. Dr. Konrad Koszinowksi, Prof. Dr. D. Stalke, Dr. Shoubik Das und Dr. Franziska Thomas für bereitwillige Teilnahme an der Prüfungskomission.

Schließlich möchte ich mich bei Tjark Meyer, Valentin Müller, Dr. Gandeepan Parthasarathy, Torben Rogge, Nicolas Sauermann, Dr. Svenja Warratz und Dr. Santhi Vardhana Yetra für das akribische Korrekturlesen der Arbeit bedanken.

Für alle administrativen und organisatorischen Fragen möchte ich Frau Gabriele Keil-Knepel danken. Schließlich gilt mein Dank auch Karsten Rauch und Stefan Beußhausen für ihre mühevollen und wichtigen Beiträge, die Laborarbeit deutlich effizienter zu gestalten.

An dieser Stelle möchte ich mich ausdrücklich und ganz herzlich bei Dr. Jonathan Hubrich, Dr. Weiping Liu, Valentin Müller sowie allen ehemaligen Mitgliedern des Labors 309 für die freundschaftliche Arbeitsatmosphäre und die interessanten Gespräche während der regelmäßigen ,coffee break“ bedanken.

Insbesondere aber möchte ich meinen Eltern und Großeltern ganz herzlich für die ständige Unterstützung jeder Art in den letzten Jahren danken. Ganz besonders danke ich meiner Freundin Doreen, die mir durch ihre wundervolle Art Kraft und Motivation für diese Arbeit gegeben hat. 



\section{Curriculum Vitae}

\section{Personal Information}

Name:

Date of Birth:

Place of Birth:

Nationality:
Daniel Zell

19.11.1988

Bad Karlshafen

German

\section{Academic Education}

$11 / 2013-06 / 2017$

10/2011-09/2013

10/2008-07/2011
Ph.D. thesis (Mentor: Prof. Dr. L. Ackermann) at the Georg-August-University Göttingen

Title: C-H Activation by Ruthenium(II), Cobalt(III) and Manganese(I) Catalysis

Master of Science in chemistry at the Georg-AugustUniversity Göttingen

Master thesis (Mentor: Prof. Dr. L. Ackermann) at the Georg-August-University Göttingen

Title: Mechanistic Studies of Ruthenium-Catalyzed C-H Functionalizations grade: 1.2 (awarded with distinction)

Bachelor of Science in chemistry at the Georg-August University Göttingen

grade: 1.7

\section{School Education}

09/2005-07/2008

09/1999-06/2005

08/1994-07/1999
Friedrichsgymnasium Kassel

Abitur grade: 1.2

High School in Beverungen

Primary School in Trendelburg 


\begin{tabular}{|c|c|}
\hline $11 / 2013-03 / 2014$ & Lecture assistant of the course "Heterocyclic Chemistry" \\
\hline 04/2014-09/2014 & Lecture assistant of the course "Reaction Mechanisms" \\
\hline $10 / 2014-03 / 2015$ & $\begin{array}{l}\text { Lecture assistant of the course "Synthetic Methods of } \\
\text { Organic Chemistry" }\end{array}$ \\
\hline 04/2015-09/2015 & Lecture assistant of the course "Reaction Mechanisms" \\
\hline $10 / 2015-03 / 2016$ & $\begin{array}{l}\text { Lecture assistant of the course "Synthetic Methods of } \\
\text { Organic Chemistry" }\end{array}$ \\
\hline 04/2016-09/2016 & Lecture assistant of the course "Organic Chemistry I" \\
\hline $10 / 2016-03 / 2017$ & Lecture assistant of the course "Heterocyclic Chemistry" \\
\hline 04/2017-present & Lecture assistant of the course "Organic Chemistry I" \\
\hline
\end{tabular}

\section{Publications}

11. D. Zell, V. Müller, U. Dhawa, M. Bursch, R. R. Presa, S. Grimme, L. Ackermann, "Mild Cobalt(III)-Catalyzed Allylative $\mathrm{C}-\mathrm{F} / \mathrm{C}-\mathrm{H}$ Functionalizations at Room Temperature" Chem. Eur. J. 2017, 23, submitted.

10. D. Zell, M. Bursch, V. Müller, S. Grimme, L. Ackermann, "Switch of C-H Activation Mechanism for Full Selectivity Control in Cobalt(III)-Catalyzed C-H Alkylations" Angew. Chem. Int. Ed. 2017, 56, under revision.

9. D. Zell,' U. Dhawa,' V. Müller, M. Bursch, S. Grimme, L. Ackermann, "C-F/C-H Functionalization by Manganese(I) Catalysis: Expedient (Per)Fluoro-Allylations and Alkenylations" ACS Catal. 2017, 7, 4209-4213.

8. K. Raghuvanshi, D. Zell, L. Ackermann, "Ruthenium(II)-Catalyzed $C-H$ Oxygenations of Reusable Sulfoximine Benzamides" Org. Lett. 2016, 18, 1278-1281.

7. D. Tonin,' D. Zell,' V. Müller, L. Ackermann, "Ruthenium(II)-Catalyzed C-H Methylation with Trifluoroborates" Synthesis 2017, 49, 127-134.

6. D. Zell,' Q. Bu,' M. Feldt, L. Ackermann, "Mild C-H/C-C Activation by (Z)-Selective Cobalt-Catalysis" Angew. Chem. Int. Ed. 2016, 55, 7408-7412.

5. K. Raghuvanshi, D. Zell, K. Rauch, L. Ackermann, "Ketone-Assisted Ruthenium(II)Catalyzed $C-H$ Imidation: Access to Primary Aminoketones by Weak Coordination" ACS Catal. 2016, 6, 3172-3175.

4. D. Zell,' S. Warratz,' D. Gelman, S. J. Garden, L. Ackermann, "Single-Component Phosphinous Acid Ruthenium(II) Catalysts for Versatile C-H Activations by MetalLigand Cooperation" Chem. Eur. J. 2016, 22, 1248-1252. 
3. J. Li, S. Warratz, D. Zell, S. De Sarkar, E. E. Ishikawa, L. Ackermann, "N-Acyl Amino Acid Ligands for Ruthenium(II)-catalyzed meta-C-H tert-Alkylation with Removable Auxiliaries" J. Am. Chem. Soc. 2015, 137, 13894-13901.

2. T. Niklas, C. Steinmetzger, W. Liu, D. Zell, D. Stalke, L. Ackermann, M. John, "Determination of the Relative Configuration of $\beta$-Amino Acid Esters Based on Residual Dipolar Couplings" Eur. J. Org. Chem. 2015, 6801-6805.

1. W. Liu, D. Zell, M. John, L. Ackermann, "Manganese-Catalyzed Synthesis of cis- $\beta$ Amino Acid Esters through Organometallic $C-H$ Activation of Ketimines" Angew. Chem. Int. Ed. 2015, 54, 4092-4096.

' both authors contributed equally.

\section{Conferences}

$07 / 2014$

$10 / 2014$

$09 / 2015$

$10 / 2015$

$05 / 2016$

$07 / 2016$

$10 / 2016$

$02 / 2017$
Second Symposium on C-H Activation, Rennes, France

(Poster Presentation), Green Chemistry Poster Prize

Lower Saxony Catalysis Symposium, Göttingen

(Poster Presentation)

GDCh-Wissenschaftsforum, Dresden

(Poster Presentation)

Workshop DFG SPP 1807, Göttingen

(Poster Presentation)

Summerschool DFG SPP 1807, Bremen

(Oral Presentation)

XXVII European Colloquium on Heterocyclic

Chemistry, Amsterdam (Poster Presentation)

Workshop DFG SPP 1807, Köln (Poster Presentation)

CaRLa Winterschool, Heidelberg

(Oral Presentation) 